\title{
Two-in-one Pincer Type Ligands and Their Metal Complexes for Catalysis
}

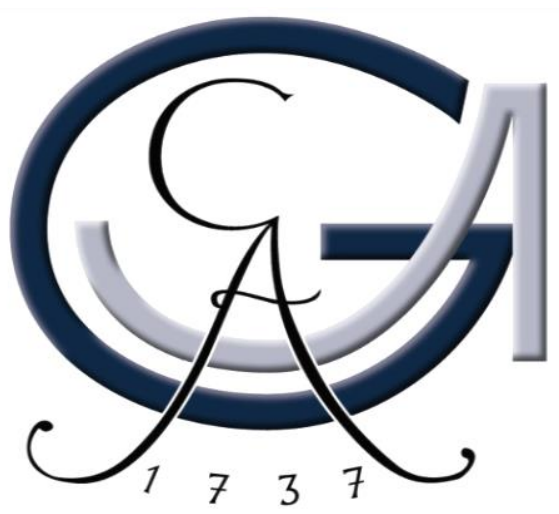

Dissertation

zur Erlangung des mathematisch-naturwissenschaftlichen Doktorgrades

"Doctor rerum naturalium"

der Georg-August-Universität Göttingen

im Promotionsprogramm der Georg-August University School of Science (GAUSS)

vorgelegt von

Alexander Gers-Barlag

aus Soest

Göttingen 2016 



\section{Betreuungsausschuss}

Prof. Dr. F. Meyer

Institut für Anorganische Chemie, Georg-August-Universität Göttingen

Prof. Dr. S. Schneider

Institut für Anorganische Chemie, Georg-August-Universität Göttingen

\section{Mitglieder der Prüfungskommission}

Referent:

Prof. Dr. F. Meyer

Institut für Anorganische Chemie, Georg-August-Universität Göttingen

Korreferent:

Prof. Dr. S. Schneider

Institut für Anorganische Chemie, Georg-August-Universität Göttingen

Weitere Mitglieder der Prüfungskommission:

Prof. Dr. M. Alcarazo

Institut für Organische und Biomolekulare Chemie, Georg-August-Universität Göttingen Prof. Dr. K. Koszinowski

Institut für Organische und Biomolekulare Chemie, Georg-August-Universität Göttingen Jun.-Prof. Dr. S. Demir

Institut für Anorganische Chemie, Georg-August-Universität Göttingen

Dr. A. Breder

Institut für Organische und Biomolekulare Chemie, Georg-August-Universität Göttingen

Tag der mündlichen Prüfung: 24.11.2016 



\section{Affidavit}

Hereby, I declare that the present dissertation 'Two-in-one Pincer Type Ligands and Their Metal Complexes for Catalysis' has been written independently and with no other sources and aids than quoted.

(Alexander Gers-Barlag)

This dissertation has been completed between November 2012 and November 2016 at the Institute of Inorganic Chemistry at the Georg-August University Göttingen under the supervision of Prof. Dr. Franc Meyer. 

for Janina, Felix, Paul and Frida 



\section{Foreword}

This thesis covers the work done on three different topics. At first, the synthesis of a new pyrazole bridged tetraamine ligand and its high valent molybdenum complexes was targeted. The second topic regarded the isolation and characterization of high valent nickel and copper (oxo) compounds supported by a macrocyclic tetracarbene ligand which was known to allow the synthesis of a terminal iron(IV)oxo species. The last part of this thesis deals with dinuclear Two-in-one pincer ligands and its metal complexes. The synthesis of different metal complexes and their possible application for catalysis were investigated.

Although not related on a first glance, the projects may be put in the wide background of the activation of small molecules and its relevance in catalysis and the understanding of enzymatic efficiency. Mononuclear molybdenum complexes exhibit a remarkable dinitrogen activation chemistry and Sснвоск has shown that the homogeneous catalytic fixation of dinitrogen at ambient conditions is possible. The use of dinuclear complexes exhibiting similar coordination geometries around the two metal ions might lead to beneficial synergisms towards the activation of the inert nitrogen triple bond. A very similar approach is applied in the pincer complex catalysis topic. Mononuclear pincer complexes are widely used in catalysis for the transformation of various substrates under mild conditions. 'Doubling' these systems might enable metal metal cooperativity which could be interesting for the activation of rather difficult substrates. In this work dihydrogen, as a small molecule, was activated by a dinuclear rhodium(I) complex to hydrogenate alkynes under mild conditions. The study of high valent intermediates which occur during the activation of another small molecule, namely dioxygen, should give new insights in enzymatic mechanisms and a further understanding of the underlying chemistry. 



\section{Table of Contents}

1 A Pyrazole-Based Tetraamine Ligand and Its Metal Complexes ....................... 1

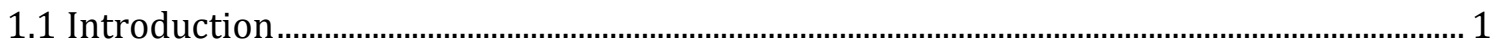

1.2 Objective

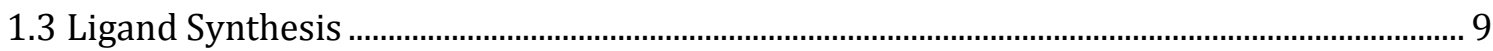

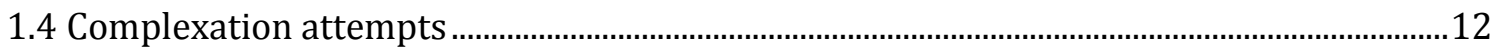

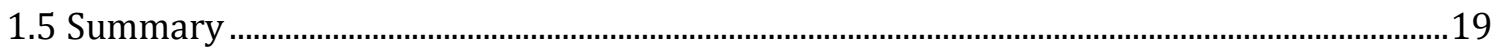

2 Macrocyclic Tetracarbene Ligands for High Valent Metal Complexes ............. 21

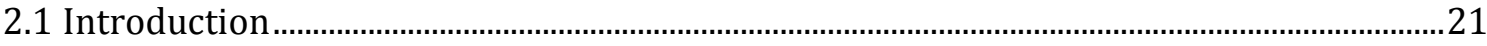

2.2 Objective

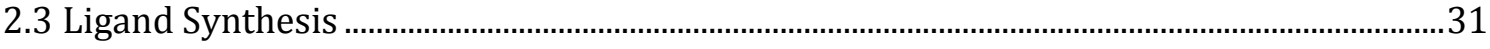

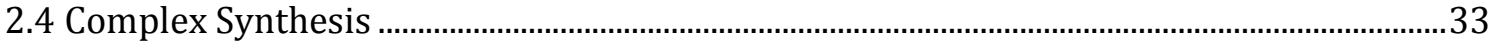

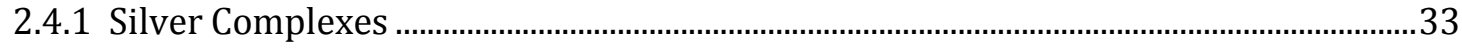

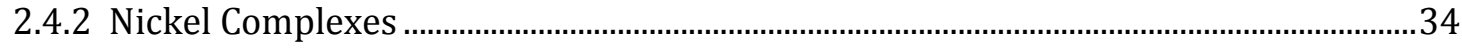

2.4.3 Complexation Attempts with Copper and Manganese......................................................42

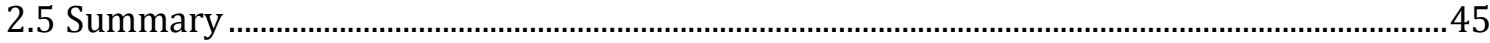

3 Two-in-one Pincer Ligands and Their Metal Complexes for Catalysis ............. 47

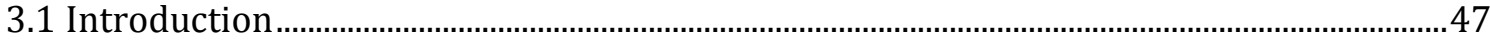

3.1.1 Metal-Ligand and Metal-Metal Cooperativity .....................................................................48

3.1.2 Activation of Small Molecules .............................................................................................51

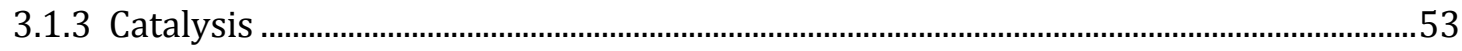

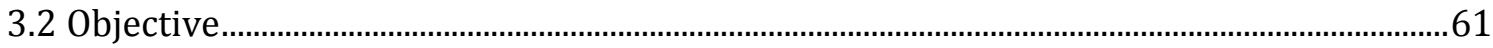

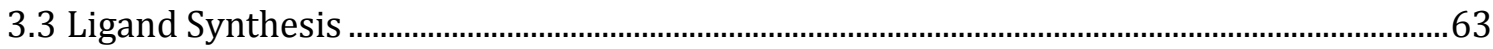

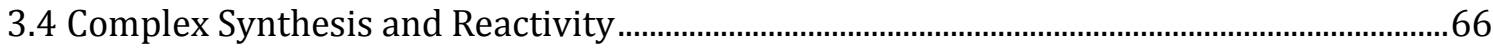

3.4.1 Zinc Complexes .....................................................................................................................66

3.4.2 Cobalt Complexes ................................................................................................................

3.4.3 Ruthenium and Molybdenum Complexes ...............................................................................

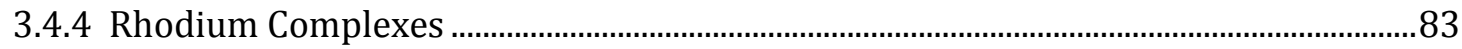

3.5 Dearomatization/Aromatization in a Rhodium Carbonyl Complex ................................... 109

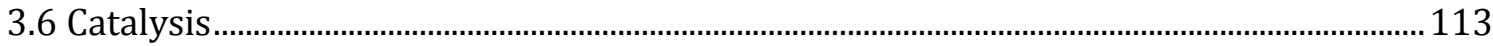

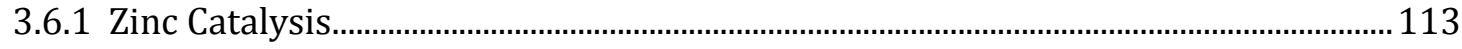

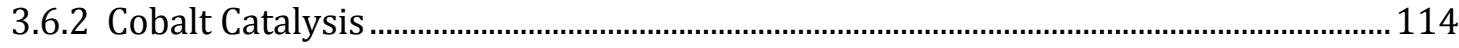


4 Conclusion

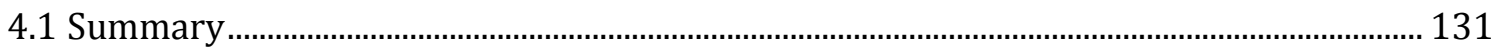

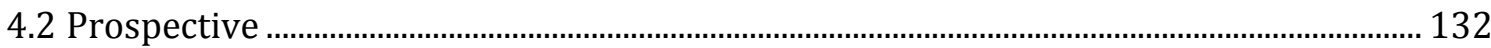

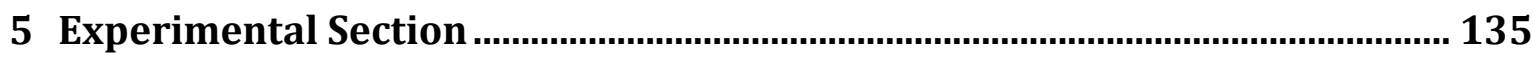

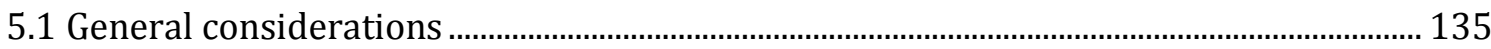

5.2 Methods

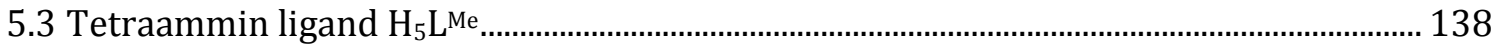

5.3.1 Ligand Synthesis................................................................................................................. 138

5.4 Macrocylic Tetracarbene Ligands and Complexes ................................................................... 142

5.4.1 Ligand Synthesis.......................................................................................................... 142

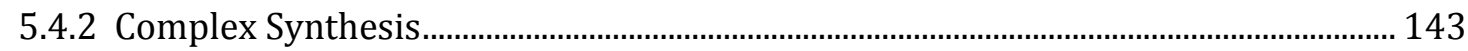

5.5 Two-in-one Pincer Ligands and Complexes ............................................................................ 148

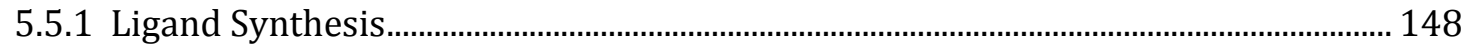

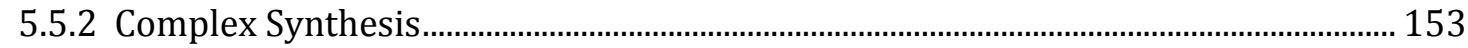

5.5.3 Ligand Flexibility and Exchange Studies ........................................................................ 163

5.5.4 Catalysis .............................................................................................................................. 164

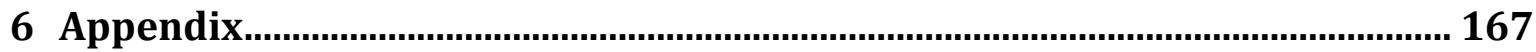

6.1 Two-in-one Pincer Ligands and their Metal Complexes ....................................................... 167

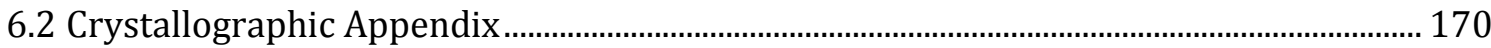

Biblography ................................................................................................................. 177

Structures of Ligand precursors, Ligands and Complexes................................... 191

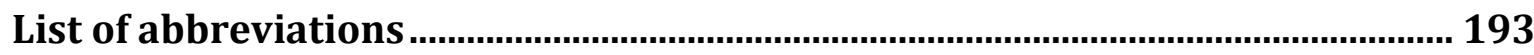

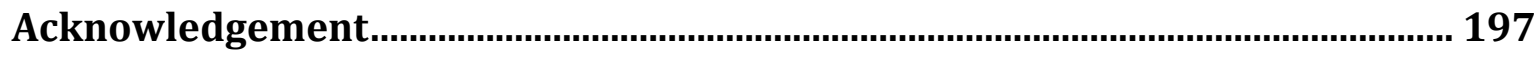

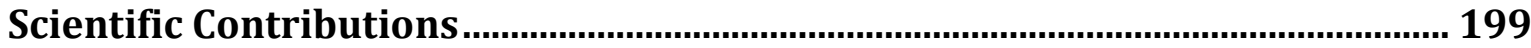

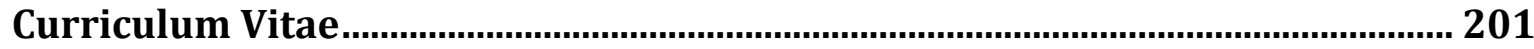




\section{A Pyrazole-Based Tetraamine Ligand and Its Metal Complexes}

\subsection{Introduction}

In the context of a worldwide expanding population, the optimization of the food production is already a great topic of recent studies. Due to regional, meteorological circumstances and political as well as environmental restraints it is not possible to enlarge the limited cultivation area. One aspect of hight interest is the utilization of fertilizers since the availability of essential nutrients is a limiting factor in plant cultivation. Thereby, nitrogen based, artificial fertilizers are important, since biologically available nitrogen sources usually contain ammonium salts. For most living species the usage of dinitrogen itself is not possible, because of the high thermodynamic stability of the nitrogen-nitrogen triple bond. In nature, some bacteria and archaea are able to convert dinitrogen into ammonia by using the nitrogenase enzyme complexes. Industrial nitrogen fixation is realized on an annual multi-hundred million tons' scale during the Haber-Bosch process where dinitrogen is reduced to ammonia over a heterogeneous iron catalyst. The fixation of nitrogen is defined as the reduction of dinitrogen to ammonia (Scheme 1.1).

$$
\mathrm{N}_{2}+6 \mathrm{H}^{+}+6 \mathrm{e}^{-} \longrightarrow 2 \mathrm{NH}_{3}
$$

Scheme 1.1: General equation of nitrogen fixation.

\section{Natural nitrogen fixation}

In nature, only a few species, e.g. the bacterium azotobacter vinelandii, are able to fix dinitrogen by using nitrogenase enzyme complexes. The need of eight electrons during the catalytic cycle sets the nitrogenase apart from essentially all other enzymatic catalyzed redox processes. The enzyme contains two metalloproteins as subunits (iron protein and molybdenum iron protein). The active site contains an iron molybdenum cofactor (FeMo cofactor) and an iron sulfur cluster (P cluster).[1] It was found that the molybdenum iron cofactor consists of an unsual [ $\left.\mathrm{Fe}_{7} \mathrm{MoS}_{9} \mathrm{X}\right]$ cluster with an interstitial light atom in the center of the iron sulfur cluster. ${ }^{[2-4]}$ With increasing resolution of the crystallographic analyses and the use of enhanced spectroscopic methods the nature of the interstitial light atom could be resolved to be a carbon atom..$^{[5-7]}$ The actual mechanism of the enzymatic reduction of dinitrogen is not fully understood, ${ }^{[8]}$ especially the role of the molybdenum atom is uncertain since also vanadium iron and only iron forms of the nitrogenase are known. ${ }^{[9,10]}$ Under physiological conditions, the reduction of dinitrogen to ammonium is thermodynamically favored by having a $\Delta G$ value of $-63.6 \mathrm{~kJ} / \mathrm{mol}$ when ferredoxin is used as the reductant.[11] Although the free energy is largely negative, the 
activation barrier is high and additional energy is needed for this reaction. For that reason, the nitrogenase uses at least eight instead of the theoretically six electrons needed for the reduction of one molecule of dinitrogen. The high number of electrons, which have to be accumulated for one turnover, and the requirement of exact timing of the electron transfer make this enzyme unique in nature. Aside from dinitrogen, many other substrates like carbon dioxide (to $\mathrm{CO}$ ) are known to be reduced by nitrogenase.[12]

\section{Artificial nitrogen fixation}

Artificial nitrogen fixation plays an important role in both academic and industrial research in order to cover the great demand of ammonia in industry and agriculture. For more than 100 years, ammonia is produced in the heterogeneous iron catalyzed Haber-Bosch process, which is by far the largest industrial process in the world.[13] In 1910 MITTASCH already found by screening of more than 3000 metal oxides the most applicable catalyst $\left(\mathrm{Fe}_{3} \mathrm{O}_{4}, \mathrm{~K}_{2} \mathrm{O}, \mathrm{CaO}, \mathrm{Al}_{2} \mathrm{O}_{3}\right.$ and $\mathrm{SiO}_{2}$ ), which is still in use. ${ }^{[14,15]}$ Due to economic reasons, mixed iron oxide is used, although osmium oxide results to be more active. The active species is formed by reduction of $\mathrm{Fe}_{3} \mathrm{O}_{4}$ with dihydrogen to ferrite. The detailed mechanism of this heterogeneous reaction was intensively studied by ERTL. For the determination of the molecular steps at the $\alpha$-iron surface ERTL was awarded the Nobel Prize in 2007.[16]

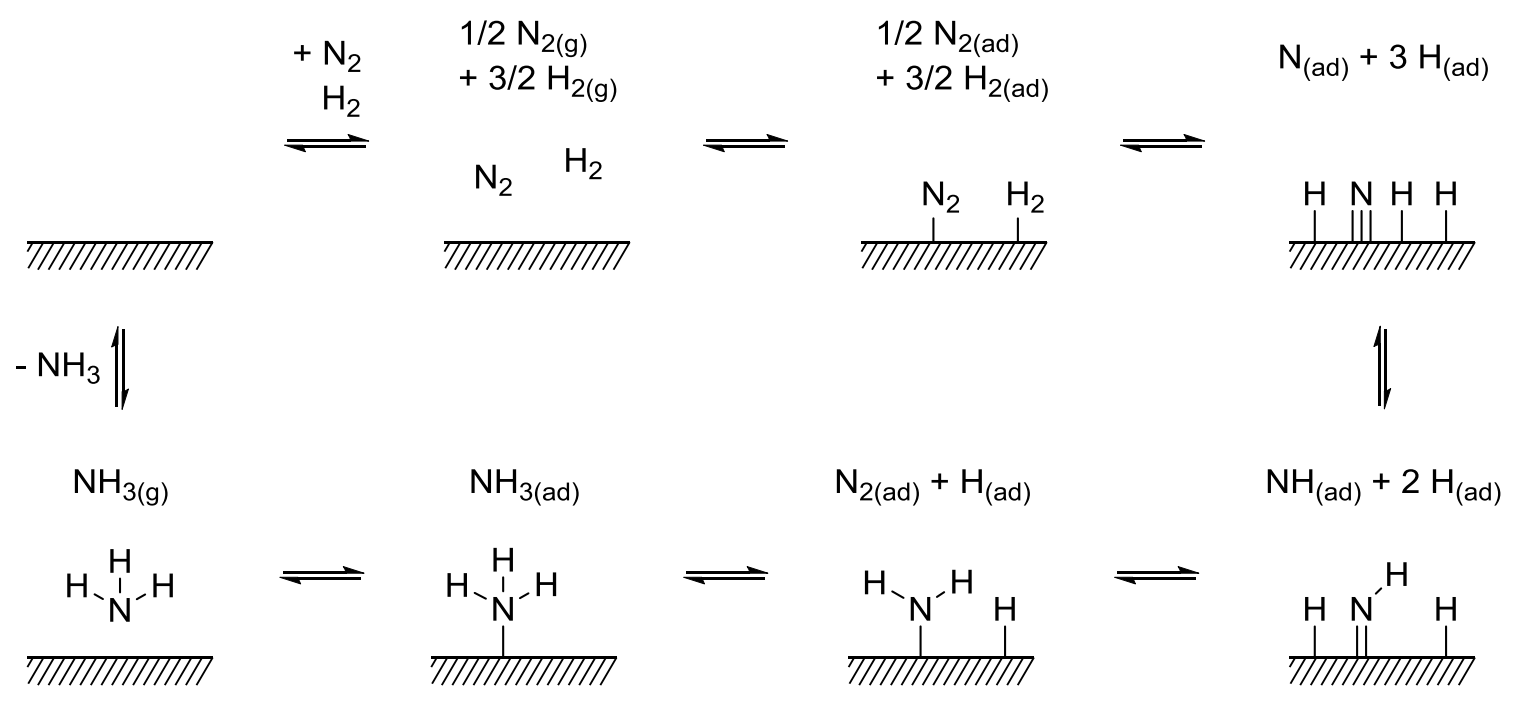

Figure 1.1: Proposed mechanism of the $\mathrm{NH}_{3}$ formation on an iron surface in the Haber-Bosch process.[16,17]

The crucial step of the mechanism was found to be the dissociative chemisorption of dinitrogen.[16] The sticking coefficient (probability of chemisorption) of dinitrogen is the rate determining step and is in the order of $10^{-6}$ and therefore relatively low.[18] The proposed mechanism is depicted in Figure 1.1. The first step is the physisorption of $\mathrm{N}_{2}$ on the active catalyst surface. The intermediate $\mathrm{N}_{2, \text { ad }}$ is stabilized by $\mathrm{K}_{2} \mathrm{O}$ to enhance the dissociation 
probability. The triple bond is broken and chemisorbed nitrides $\left(\mathrm{N}_{\mathrm{ad}}\right)$ on the surface are obtained. Dihydrogen is also chemisorbed and a stepwise reaction of $\mathrm{H}_{\mathrm{ad}}$ atoms with the nitrides leads to physisorbed ammonia, which then has to be desorbed. All reactions are equilibrium reactions, which can be influenced by external parameters such as temperature or pressure. Removing ammonia from the reaction mixture drives the reaction to the product site and enables a continuous reaction. The main issue of this process is the production and purification of dihydrogen. To date the dihydrogen for the Haber-Bosch process is produced by steam reforming of methane. However, the removal of remaining methane, carbon monoxide and other catalyst poisons such as sulfur compounds is expensive and energy consuming.

Due to these drawbacks in combination with the harsh reactions conditions of the Haber-Bosch process, the development of new catalytic systems is highly desirable. For several decades, chemists are searching for new approaches for the reduction of dinitrogen at ambient conditions.

Since the first dinitrogen complex was obtained by ALLEN and SENOFF[19] in 1965 many $\mathrm{N}_{2}$ complexes with different degrees of activation of the triple bond were synthesized.[20-24] However, only few examples in which dinitrogen was reduced in a catalytic way were reported during the early years. ${ }^{[25,26]}$ First catalytic systems were presented by SHILOV and co-workers in the 1970's and 1980's, using different $d^{2}$ and $d^{3}$ metal ions, such as Mo ${ }^{\text {III }}$ or VII.[27-29] The reactions were conducted in alkaline solutions to avoid proton reduction to dihydrogen. Under these conditions mainly hydrazine was produced. It was assumed that the catalytically active species is a cluster compounds, but it could not be identified conclusively. Later, SHILOV et al. further introducted a vanadium(II) system reducing dinitrogen to ammonia. ${ }^{[30]}$ However, the reaction was non-catalytic.

The work of Cummins on three coordinated molybdenum amido complexes Mo(NRAr) 3 attracted a lot of attention, because it was the first reported example of the splitting of a dinitrogen molecule by a well-defined metal complex (Scheme 1.2).[31,32] At low temperature the molybdenum(III) species readily reacts with dinitrogen to form a linear $\mathrm{N}_{2}$-bridged dinuclear complex 3. Upon warming, the N-N bond is cleaved via a 'zigzag' intermediate $\mathbf{4}$ and two molecules of a terminal nitrido molybdenum (VI) complex $\mathbf{5}$ are formed. ${ }^{[33]}$ So far complex 5 proved to be relatively stable and the nitride ligand could not be protonated to form ammonia. Nevertheless, Cummins showed that molybdenum(III) is able to supply three electrons for the reduction of dinitrogen. 


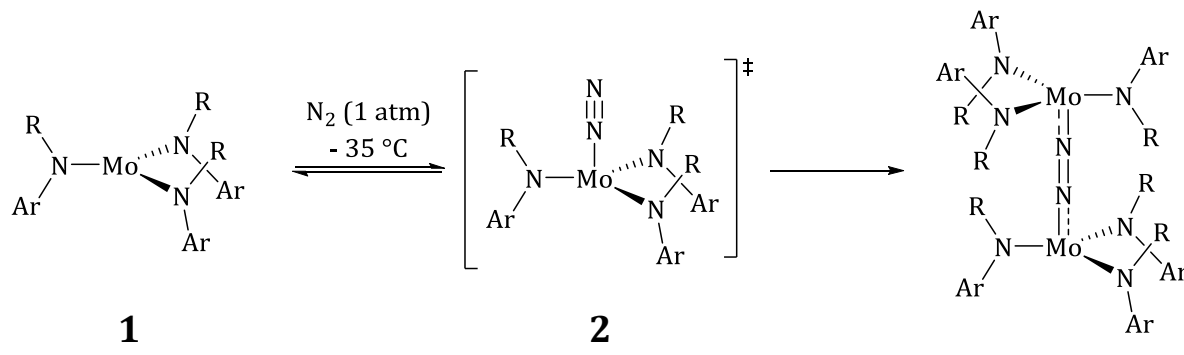

1

2

3
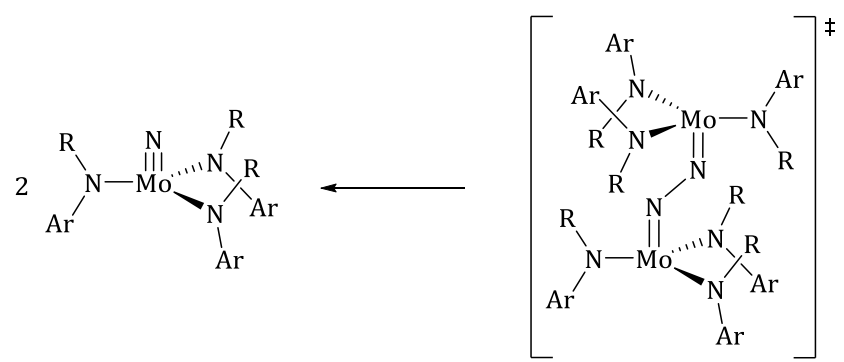

4

5

Scheme 1.2: Proposed mechanism of reductive dinitrogen splitting by a Mo(NRAr) 1 complex.[17,33]

A milestone in the artificial nitrogen fixation at ambient conditions was reached in 2003, when ScHRоск published the first well defined, triamidoamine based catalyst which can fixate dinitrogen homogeneously.[34] Similar to Cummins they used a molybdenum(III) nitrogen complex 6 with sterically demanding hexaisopropylterphenyl groups (HIPT) to ensure a protected cavity above the metal center (Scheme 1.3, left).[34] Catalytic formation of ammonia from dinitrogen was achieved by the use of $\left[\mathrm{CrCp}_{2}^{*}\right]$ as an electron source and [2,6-lutidinium $] \mathrm{BAr}_{4}$ as a proton source (Scheme 1.2, right). The reductant was slowly added over hours to a suspension of acid and $\mathbf{6}$ in heptane to decrease the amount of complex degradation. Under these conditions an averaged TON of 7.9 (of maximum 12 equivalents $\mathrm{NH}_{3}$ ) was obtained. The formed ammonia was detected by the indophenol method used for the detection of small amounts of $\mathrm{NH}_{3}{ }^{[35]}$

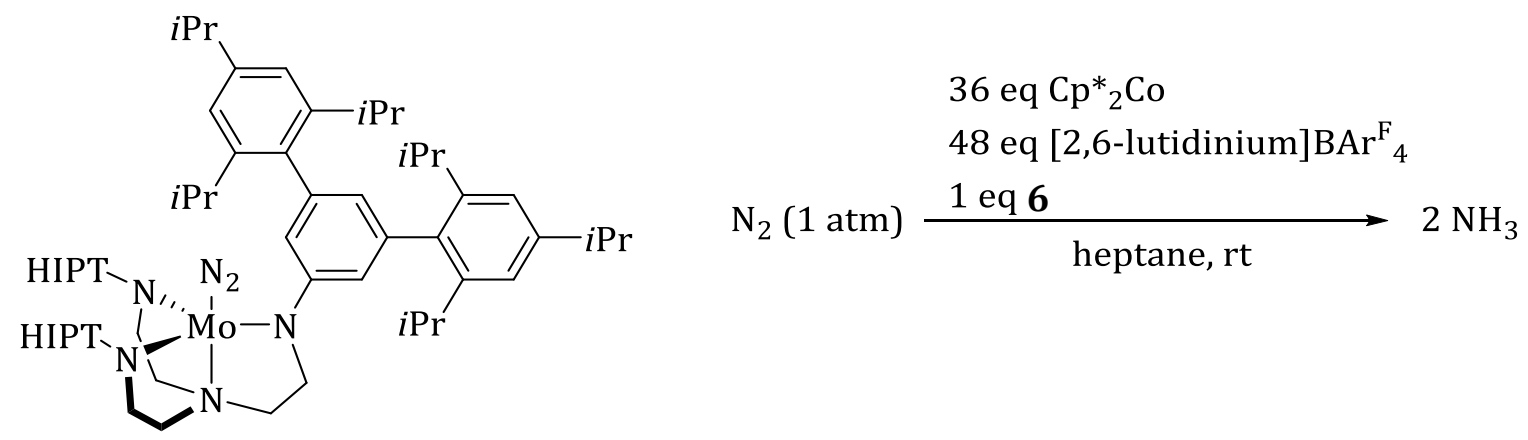

6

Scheme 1.3: Schematic structure of ScHRock's highly shielded triamido molybdenum(III) complex 6 (left) and catalytic reduction of dinitrogen with 6.[36]

Detailed studies were undertaken to evaluate the mechanism of the reaction and several possible intermediates could be isolated and characterized. In addition, with theoretical 
calculations it was shown that the mechanism of the reductions is very similar to the one CHATT proposed already in 1978.[37-40] However, catalyst 6 was found to rapidly decompose due to ligand protonation and consequently SснRоск et al. changed the side arms to three pyrrole moieties.[41] But changing of the electronical and sterical environment of the molybdenum center with the introduction of three new donor sites did not lead to a catalytically active compound. In 2010 the group of Sснгоск presented an intermediate ligand where only one of the amido side arms was replaced by a pyrrolyl side arm (Figure 1.2).[42]
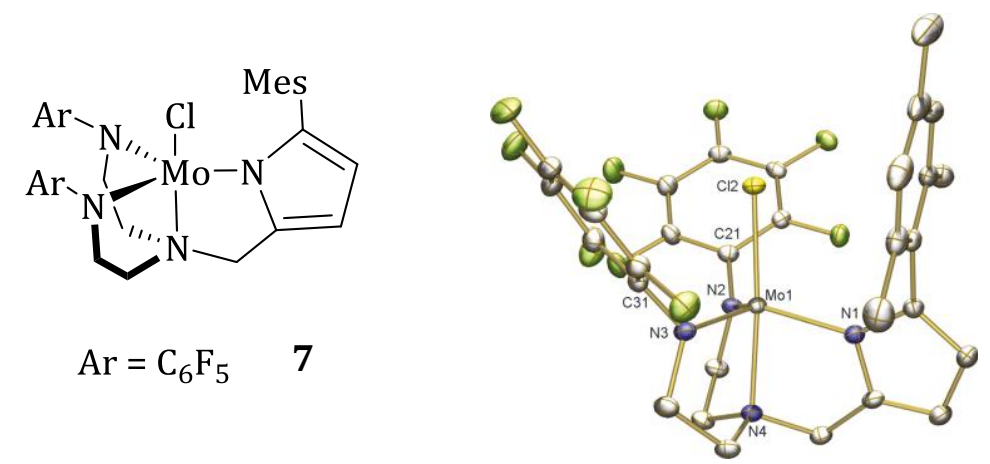

Figure 1.2: Schematic, general structure of diamidopyrrolyl $\mathrm{MoCl}$ complexes (left, for $\mathrm{Ar}=\mathrm{C}_{6} \mathrm{~F}_{5}$ complex 7) and thermal displacement ellipsoids (shown at $50 \%$ probability) of the molecular structure of $\left[\left(\mathrm{C}_{6} \mathrm{~F}_{5}\right)_{2} \mathrm{Pyr}\right] \mathrm{MoCl} 7$ (right). Hydrogen atoms were omitted for clarity.[17,42]

Although being structurally very similar to the initial trisamido system, the bisamidopyrrolyl molybdenum complexes showed not the desired enhanced activity. When the nitrido complex (obtained by the reaction of $\mathbf{7}$ with $\mathrm{NaN}_{3}$ ) is reacted under the same conditions as complex 6 $1.02 \pm 0.12$ eq. of ammonia were formed. So in the range of the experimental error this system may produce ammonia, but did not turn over. The formation of ammonia was achieved, but it could not be released from the catalyst.[42]

In recent years many new and interesting systems for the activation and reduction of dinitrogen were published. The focus was not only set on the direct reduction of dinitrogen to ammonia but also other strategies were applied such as nitrogen carbon bond formation to incorporate the nitrogen into organic scaffolds. To name just a few, the groups of NiSHIBAYASHI[43-48], PETERS[49-53], Holland[54-56], SCHNEIDER[57-60] and TuCZEK[61-64] made a lot of effort and provided great input and inspiration in these fields. 



\subsection{Objective}

Inspired by the work of SCHROCK on the bisamidopyrrolyl ligand system for mononuclear molybdenum complexes, ${ }^{[42]}$ and based on previous work in the group of MEYER,[65] the aim of this work is the synthesis of new dinuclear transition metal complexes, especially with molybdenum, as possible candidates for the activation of small molecules, in particular dinitrogen. Dinuclear complexes with two metal centers in close proximity are known to show metal metal cooperativity.[66] The use of 3,5-substituted pyrazoles as bridging unit with suitable metal metal distances are established.[67] Therefore, the synthesis of a combination of the pyrazole bridging unit often used in the group of MEYER and the bisamido side arm of the SCHROCK system is targeted (Figure 1.3).<smiles>Cc1cc(C)cc(NCCN(CCNc2cc(C)cc(C)c2)Cc2cc(CN(CCNc3cc(C)cc(C)c3)CCNc3cc(C)cc(C)c3)[nH]n2)c1</smiles>

Figure 1.3: Targeted pyrazole based tetraamine ligand $\mathbf{H}_{5} \mathbf{L}^{\text {Me. }}$.

To ensure a shielded binding pocket for potential substrates, a large residue for the amido donor site is chosen. In a next step, the synthesis of molybdenum or other metal complexes is planned. If possible, reduction of the molybdenum(IV) complex in the presence of dinitrogen might lead to a dinitrogen complex (Figure 1.4). The binding properties as well as the electronic structure of such a complex would be highly interesting.
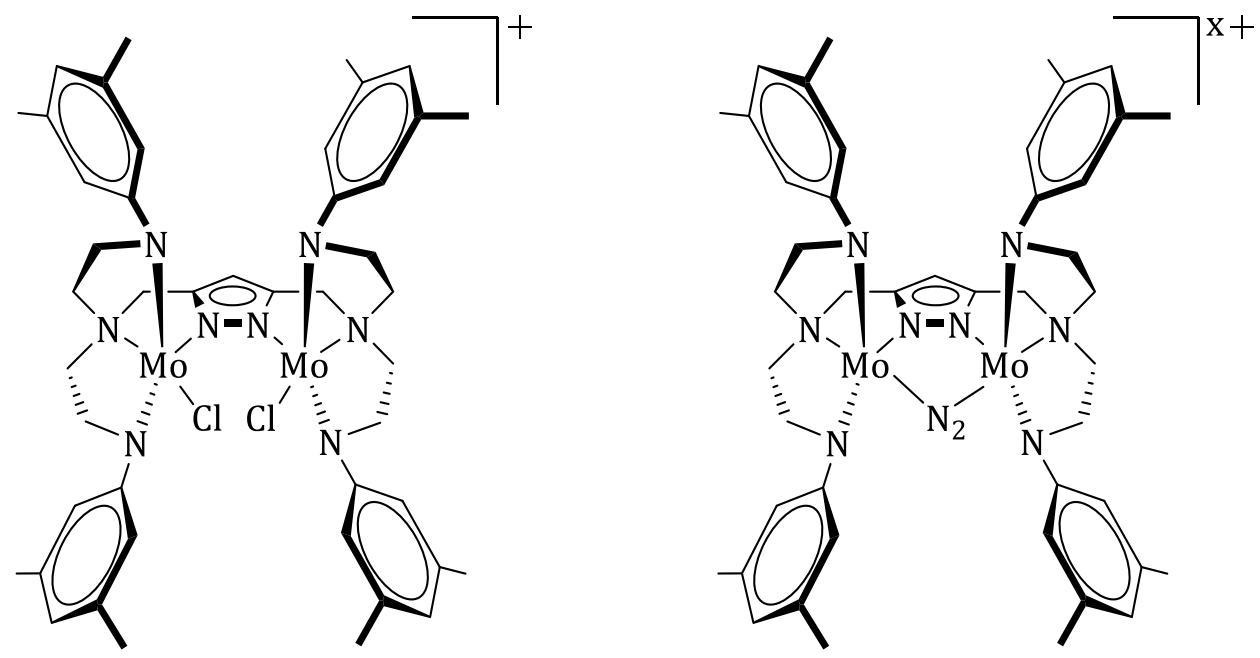

Figure 1.4: Targeted pyrazole bridged dinuclear molybdenum complexes: dichloro $\mathrm{Mo}^{\mathrm{IV}}$ complex (left) and Mo dinitrogen complex (right). 



\subsection{Ligand Synthesis}

Previos work ${ }^{[17]}$ showed, that the well-established standard procedure for the synthesis of pyrazole bridged ligand systems was not successful for side arm II (Scheme 1.4).[68,69] It could be shown, that the central amine moiety in II exhibited a similar reactivity in the coupling reaction as the aniline moieties giving rise to a mixture of different oligomers observed by ESI mass spectrometry. Due to the large amount of amine functions in these molecules, it was impossible to separate the desired proligand $\mathbf{H}_{5} \mathbf{L}^{\text {Me }}$ from the side products.

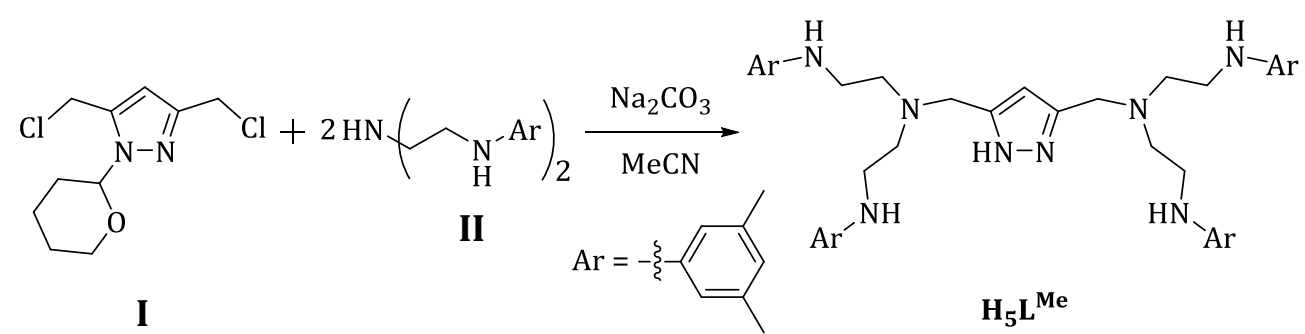

Scheme 1.4: Attempted synthesis of $\mathbf{H}_{5} \mathbf{L}^{\text {Me }}$ which proved to be not selective due to different amine moieties in side arm II yielding various unseparable side products.

To increase the differences in activity a new synthetic strategy was applied. If bisamide side arm VI (Scheme 1.5) was used for the coupling reaction followed by subsequent reduction of the formed amide proligand VIII, the desired product $\mathbf{H}_{5} \mathbf{L}^{\text {Me }}$ was obtained (Scheme 1.7).<smiles>Cc1cc(C)cc(N)c1</smiles><smiles>Cc1cc(C)cc(NC(=O)CNCC(=O)Nc2cc(C)cc(C)c2)c1</smiles>

Scheme 1.5: Three step synthesis of bisamide side arm VI from iminodiacetic acid III.

In a first step the amine of the iminodiacetic acid III was protected with a tert-butyloxy carbonyl group (Boc) under basic conditions in aqueous 1,4-dioxane. $N, N^{\prime}$-Dicyclohexylcarbodiimid (DCC) was used to enhance the coupling of the carboxylate groups of the protected iminodiacetic acid IV with 3,5-dimethylaniline to yield the protected side arm $\mathbf{V}$ in moderate yields of approximately $50 \%$. Concentrated hydrochloric acid was used to deprotect the central amine function. The deprotected bisamide amine side arm VI was purified by recrystallization from ethanol to yield $53 \%$ as fine, colorless needles. 


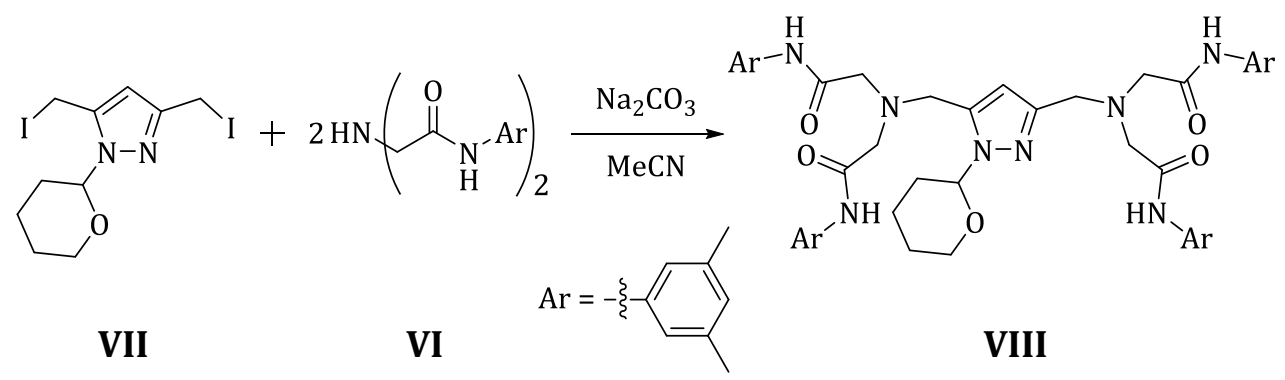

Scheme 1.6: Coupling reaction of the pyrazole building block VII and the bisamide side arm VI to yield VIII.

Due to a low conversion rate when using the standard 3,5-bis(chloromethyl)-1tetrahydropyran-2-yl-pyrazole building block ${ }^{[0,71]} \mathbf{I}$ in the coupling reaction with the side arm, the iodo analogue VII was used instead. It was synthesized in a Finkelstein reaction by suspending the chloro compound I with an excess of potassium iodide in acetone. The use of the THP (tetrahydropyranyl) protected building block was needed to avoid undesired side reactions of the pyrazole NH function. The coupling reaction was carried out according to the standard conditions developted in the MEYER group earlier.[72] At ambient temperature the reaction was slow, therefore the reaction mixture was heated to reflux for two days to accelerate the coupling. To obtain the product VIII in pure form column chromatography was tested, but resulted in low yields. It was then found that the product could be isolated in good purity by extracting the crude product with chloroform. The insoluble salt was separated by centrifugation. The product contained still traces of sodium iodide which could not be separated without a dramatic decrease of the yield. Therefore, the mixture was used for the reduction without further purification. Figure 1.5 shows the ${ }^{1} \mathrm{H}$ NMR spectrum of VIII displaying an asymmetric species in solution due to the THP protecting group.

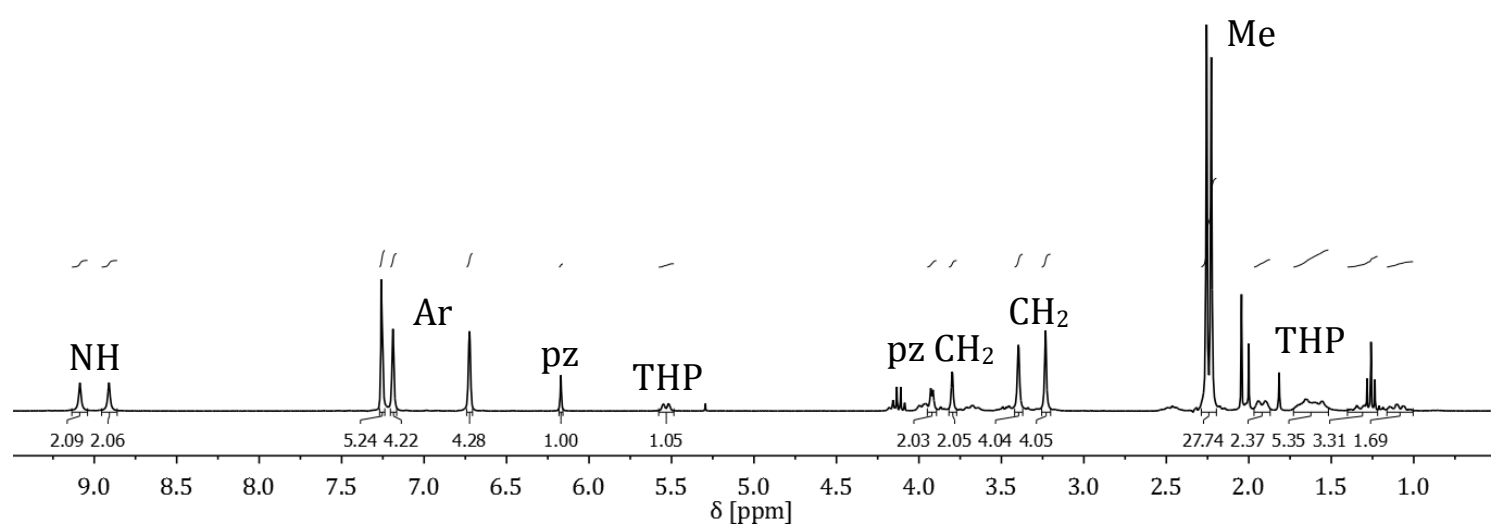

Figure 1.5: ${ }^{1} \mathrm{H}$ NMR spectrum of compound $\mathrm{H}_{4} \mathrm{LC}$ CMe-THP VIII $\left(\mathrm{CDCl}_{3}, 298 \mathrm{~K}\right)$.

The reduction of the four amides as well as the purification of the final proligand $\mathbf{H}_{5} \mathbf{L}^{\text {Me }}$ proofed to be challenging. The reduction could be finally achieved with $\mathrm{BH}_{3} \cdot \mathrm{THF}$, while other reducing agents were too weak (DIBAL or K-selectride) or led to decomposition of the compound $\left(\mathrm{LiAlH}_{4}\right)$. 
<smiles>O=C(CN(CC(=O)N[Al])Cc1cc(CN(CC(=O)N[Al])CC(=O)N[Al])n(C2CCCCO2)n1)N[Al]</smiles>

VIII

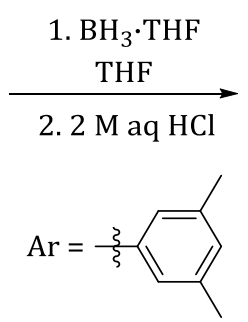

\section{$\mathbf{H}_{5} \mathbf{L}^{\text {Me }}$}

Scheme 1.7: Synthesis of the ligand $\mathbf{H}_{5} \mathbf{L}^{\text {Me }}$ by reduction of compound VIII.

In addition, the amount of borane had to be sufficiently high to ensure a complete reduction of all four amide moieties. If less than 10 equivalents of the borane were used, a mixture of different partially reduced species was observed in the corresponding ESI mass spectra. The reaction could be monitored by ${ }^{1} \mathrm{H}$ NMR spectroscopy, where the amide signals around $9 \mathrm{ppm}$ vanished and a second $\mathrm{CH}_{2}$ peak for the side arm arose at $2.8 \mathrm{ppm}$ (Figure 1.7). The target product IX was identified via ESI mass spectrometry by the presence of the main peak at $\mathrm{m} / \mathrm{z}=715.3$ corresponding to the protonated ligand $\left[\mathrm{H}_{5} \mathrm{~L}^{\mathrm{Me}}+\mathrm{H}\right]^{+}$(Figure 1.6). The acidic workup had the beneficial effect that the THP protection group is cleaved during workup. However, the product could not be further purified after the reaction due to the five $\mathrm{NH}$ functions, which made column chromatography impossible. For this reason, the workup conditions were optimized to yield $\mathbf{H}_{5} \mathbf{L}^{\mathbf{M e}}$ in a reasonable purity after extraction (Figure 1.7). The ligand was obtained in an overall yield of $40 \%$ for all six steps. Crystalline material of $\mathbf{H}_{5} \mathbf{L}^{\mathbf{M}}$ could not be isolated.

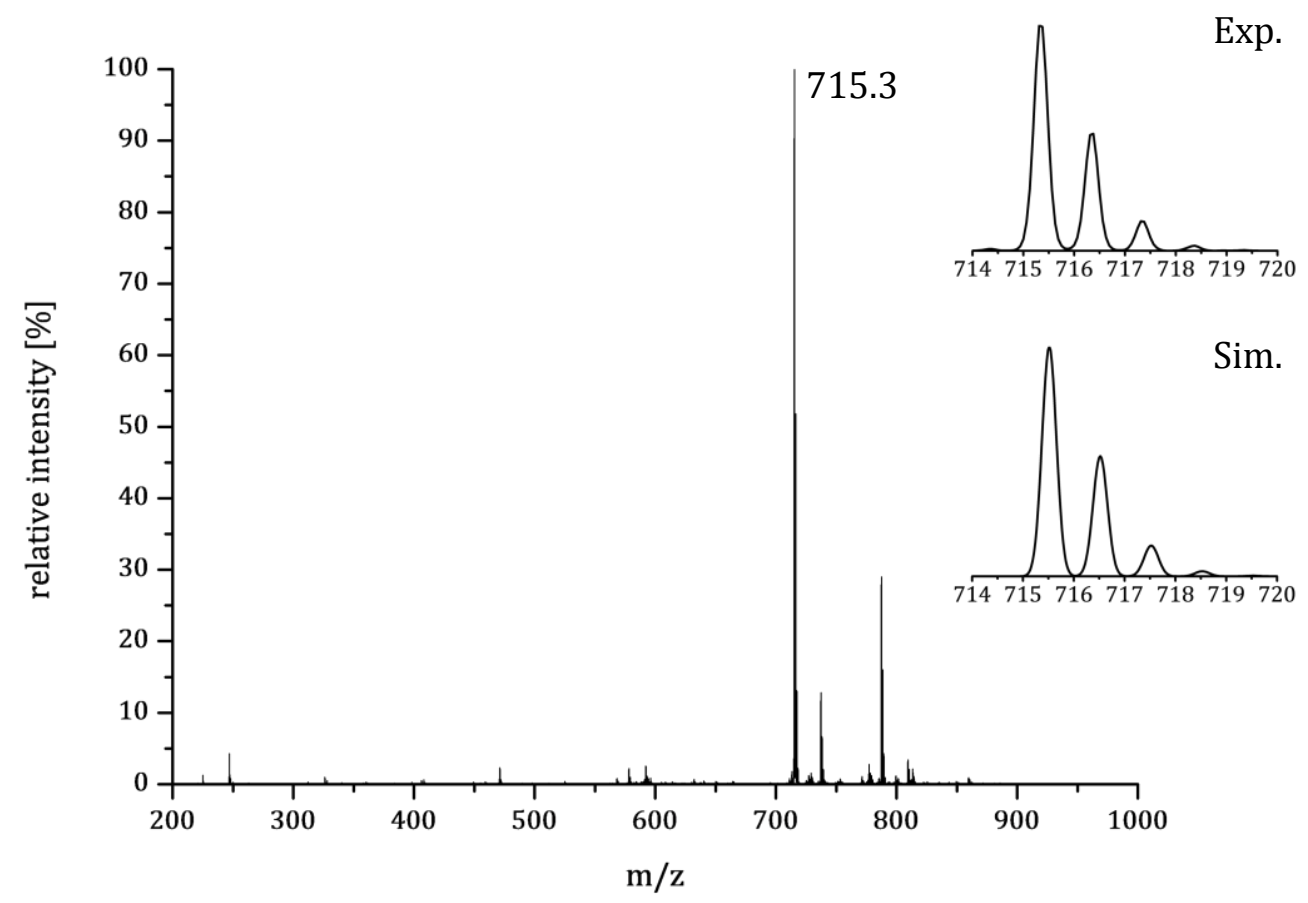

Figure 1.6: $\mathrm{m} / \mathrm{z}$ 200-1000 range of the ESI(+)-mass spectrum of $\mathbf{H}_{5} \mathbf{L}^{\mathrm{Me}}$. The insets show the experimental and simulated isotopic distribution patterns for $\left[\mathrm{H}_{5} \mathrm{~L}^{\mathrm{Me}}+\mathrm{H}\right]^{+}$. 


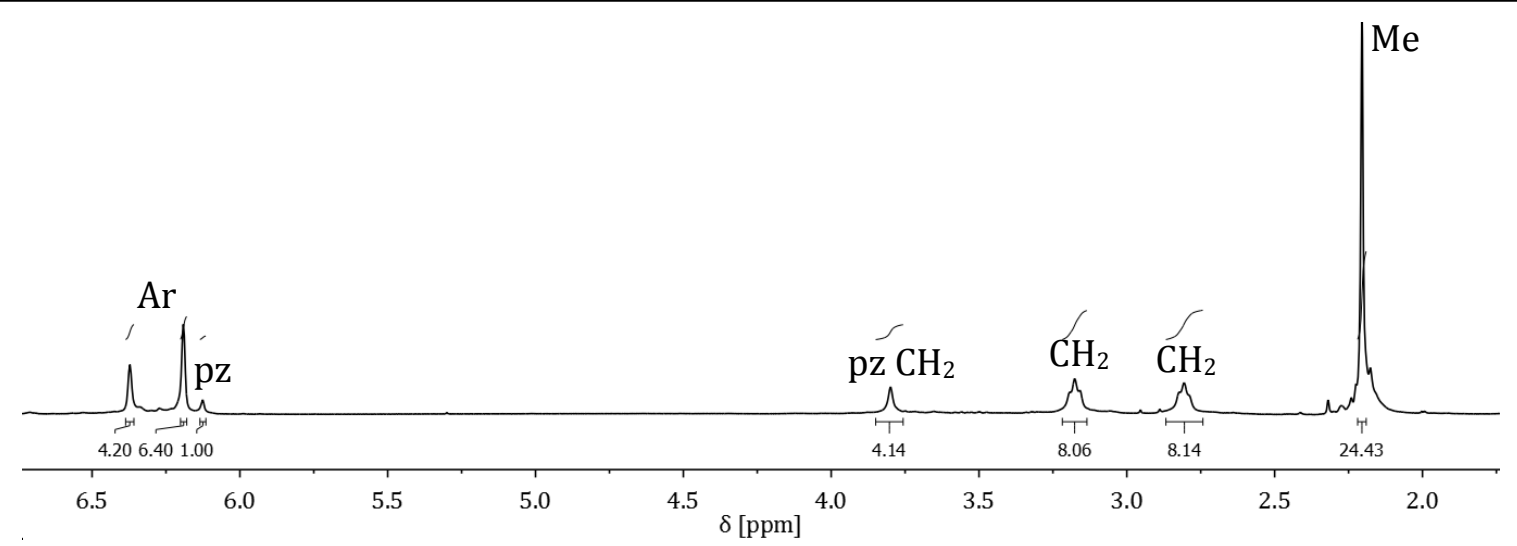

Figure 1.7: ${ }^{1} \mathrm{H}$ NMR spectrum of ligand $\mathrm{H}_{5} \mathrm{~L}^{\mathrm{Me}} \mathbf{I X}\left(\mathrm{CDCl}_{3}, 298 \mathrm{~K}\right)$.

\subsection{Complexation Attempts}

Although it was not possible to purify the proligand $\mathbf{H}_{5} \mathbf{L}^{\text {Me }}$ completely, the synthesis of high valent molybdenum complexes was investigated. The assumed general coordination behavior for the fully deprotonated ligand $\mathbf{H}_{5} \mathbf{L}^{\text {Me }}$ is depicted in Scheme 1.8. From earlier studies on similar but less bulky tetraamine ligands it was known that the state of deprotonation is crucial on the formation of one single product. [65,69] In addition, the coordination of all four side arms to the two metal centers proved to be difficult. To ensure a complete deprotonation of all five $\mathrm{NH}$ moieties (the pyrazole one and the four on the side arms) strong bases such as potassium hexamethyldisilazide (KHMDS) and potassium tert-butoxide $\left(\mathrm{KO}^{t} \mathrm{Bu}\right)$ were applied although structurally similar triamidoamine complexes of early transition metals can by synthesized with the use of the weaker base triethylamine.[73,74] These complexes are known to be very sensitive towards fast decomposition, therefore strictly anaerobic conditions are mandatory.

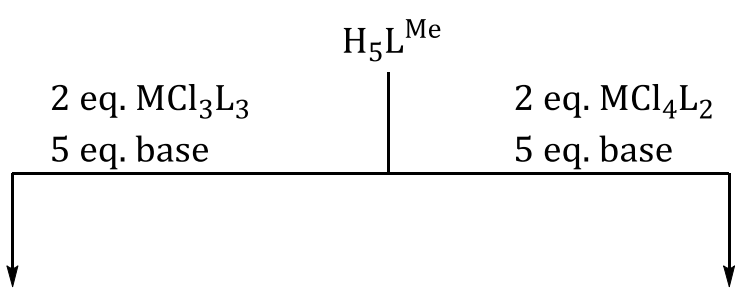

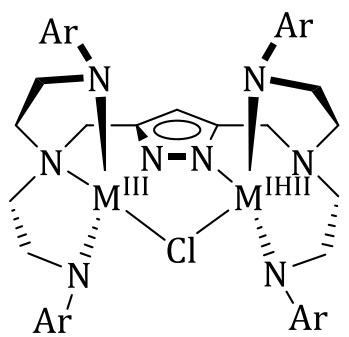

A

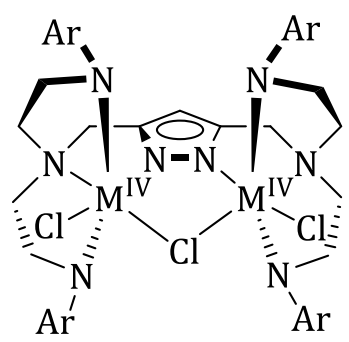

B

Scheme 1.8: Assumed coordination behavior of $\mathbf{H}_{5} \mathbf{L}^{\mathrm{Me}}$ towards high valent early transition metals with different oxidations states: Type A for $\mathrm{M}^{\mathrm{III}}$ and type B for $\mathrm{M}^{\mathrm{IV}}$ metal centers. 
Metal chloride precursors were chosen, because it was shown that in related tripodal amido complexes an axial chloride could be substituted by more reactive alkyl, alkynyl or hydride ligands. ${ }^{[75-78]}$ Two possible coordination motives are shown in Scheme 1.8. Type A represents a neutral complex for metals in the oxidation state of +III with a trigonal bipyramidal coordination environment, which is often found for triamidoamine complexes. ${ }^{[79-81]}$ The distance between the two metals should be short enough to enable a bridging chloride ligand in the binding pocket. For metals in the oxidation state +IV type B displays a potential coordination geometry with two additional chlorides filling the octahedral coordination sphere of the metal ions.

$\mathbf{H}_{5} \mathbf{L}^{\text {Me }}$ was added to a suspension of $\mathrm{MoCl}_{4}(\text { thf })_{2}$ in THF followed by the addition of either triethylamine or KHMDS. In both cases dark brown, almost black solutions were obtained which were analyzed by inert ESI mass spectrometry. Depending on the conditions of the reaction (reaction time, solvent, concentration, added counterions) in most samples only peaks related to the free ligand were found $\left(\mathrm{m} / \mathrm{z}=715.3\left[\mathrm{H}_{5} \mathrm{~L}^{\mathrm{Me}}+\mathrm{H}\right]^{+}\right.$or its adducts with $\mathrm{Li}^{+}, \mathrm{Na}^{+}$or $\mathrm{THF}+\mathrm{H}^{+}$). In a few cases molybdenum species were observed, showing a characteristic isotopic distribution patterns. One of those spectra is depicted in Figure 1.8 and exhibits, aside the ligand peak with the highest intensity among the signals from the sample, at least four peaks with isotopic distribution patterns typical for $\mathrm{Mo}_{2} \mathrm{~L}^{\mathrm{Me}} \mathrm{Cl}_{\mathrm{x}} \mathrm{O}_{\mathrm{y}}$ compounds. Unfortunately, none of these peaks could be assigned to a chemically reasonable species. Crystallization attempts were unsuccessful. Similar results were obtained for complexation reactions with $\mathrm{MoCl}_{3}(\mathrm{thf})_{3}$ as metal precursor.
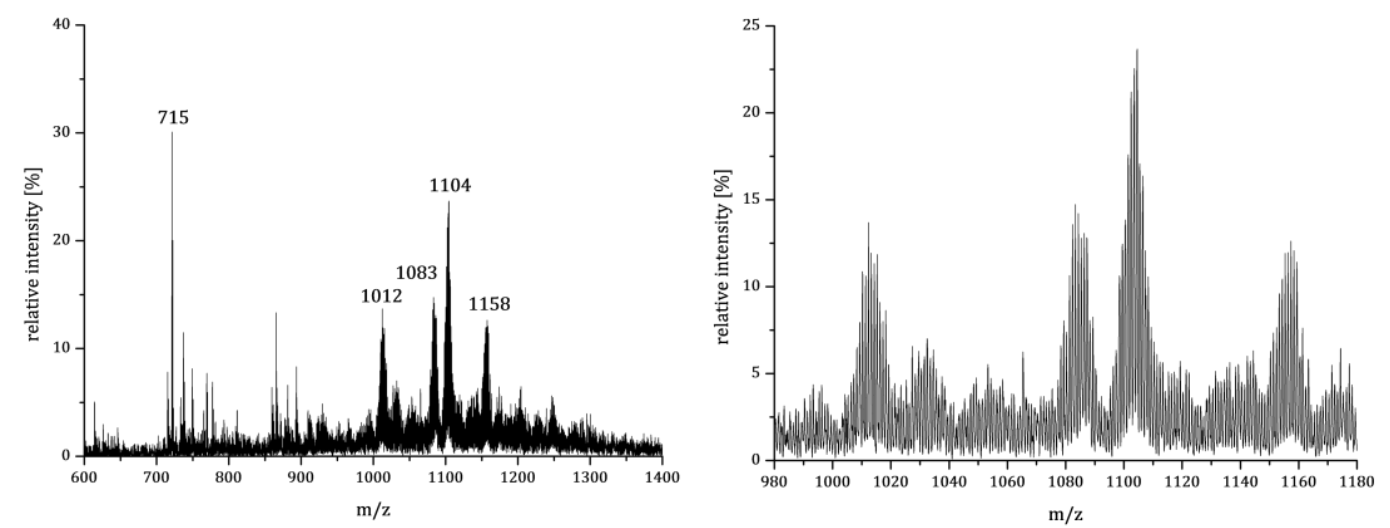

Figure 1.8: ESI $(+)$ mass spectrum of the reaction mixture of a reaction between $\mathbf{H}_{5} \mathbf{L}^{\mathrm{Me}}, \mathrm{MoCl}_{4}(\mathrm{thf})_{2}$, $\mathrm{KHMDS}$ and NaOTf. Left: range of $\mathrm{m} / \mathrm{z} 600-1400$ showing the free ligand $(\mathrm{m} / \mathrm{z}=715)$ as the peak with the highest intensity. Right: range of $\mathrm{m} / \mathrm{z}$ 980-1180 showing the isotopic distribution patterns of the found $\mathrm{Mo}_{2}$ species in detail.

The amount of free ligand in the products of these experiments could not be decreased by using an excess of metal precursor, even if the crude products were washed with toluene to remove unreacted ligand before the measurement. Because of that it was assumed that the free ligand is generated during the measurement of the ESI-MS spectra indicating a rather weak binding 
to the metal centers. It was not possible to obtain reasonably good NMR spectra of those reaction mixtures. The spectra exhibited broad signals and in total less resonances as expected. Nonetheless, diffusion ordered spectroscopy (DOSY) experiments were performed and a mixture of several species with different diffusion coefficients was found (Figure 1.9). The results were ambiguous, since compounds of a certain diffusion coefficient were found to have cross peaks with the aliphatic region of the proton NMR spectrum but none with the aromatic region. Thus, a decomposition of the ligand could not be excluded.

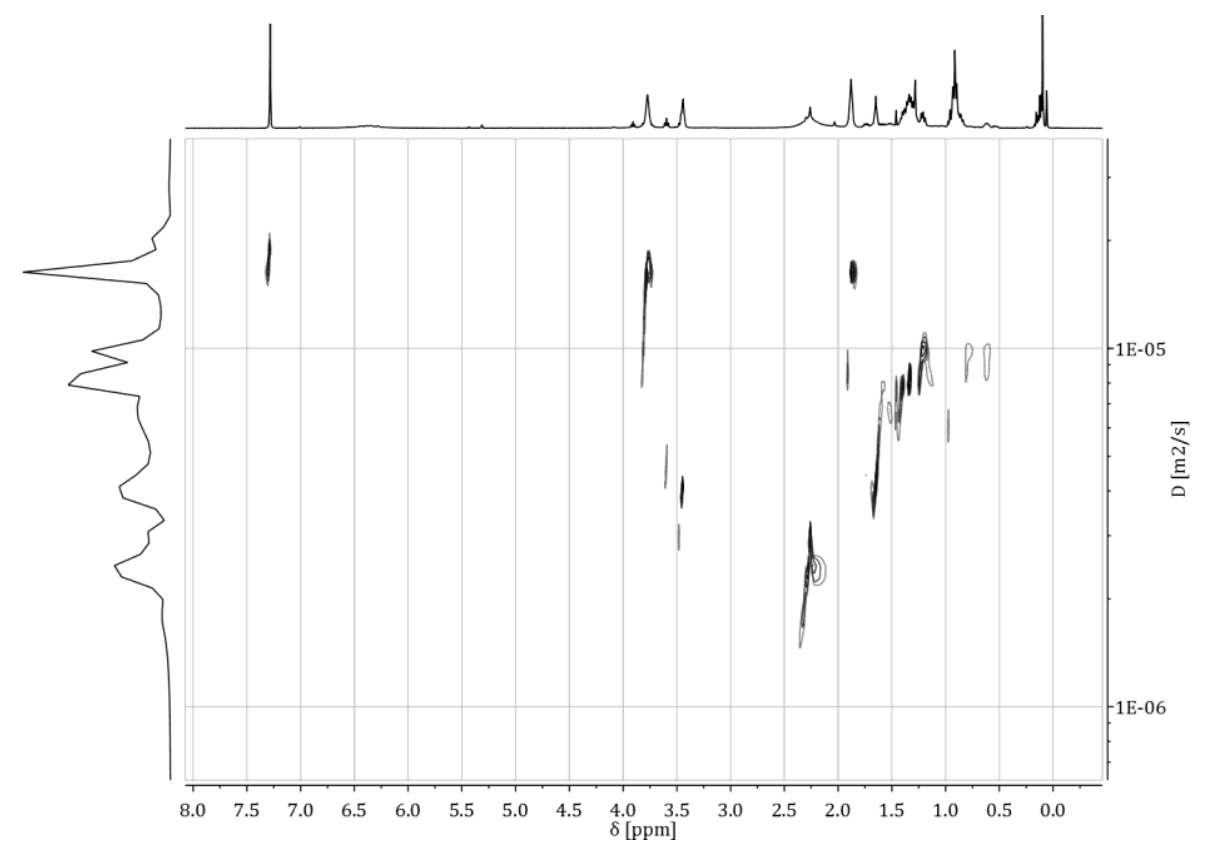

Figure 1.9: DOSY NMR spectrum $\left(\mathrm{CDCl}_{3}, 298 \mathrm{~K}\right)$ of the crude product of a reaction between $\mathbf{H}_{5} \mathbf{L}^{\mathrm{Me}}, \mathrm{MoCl}_{4}(\mathrm{thf})_{2}$ and $n \mathrm{BuLi}$ in THF.

The formation of molybdenum complexes of $\mathrm{H}_{5} \mathrm{~L}^{\text {Me }}$ seemed to be in principle possible, although major drawbacks were faced. For stability reasons the metal precursors were changed to $\operatorname{MoN}\left(\mathrm{O}^{t} \mathrm{Bu}\right)_{3}{ }^{[82]}$ and to $\left[\mathrm{MoN}(\mathrm{Cl})_{3}(\mathrm{MeCN})\right]_{4}{ }^{[83]}$, with molybdenum in the oxidation state $+\mathrm{VI}$. Further, the diamagnetism of these compounds enables the use of NMR spectroscopy as an additional analytical method. $\mathbf{H}_{5} \mathbf{L}^{\mathrm{Me}}$ and $\mathrm{KHMDS}$ were combined with $\left[\mathrm{MoN}(\mathrm{Cl})_{3}(\mathrm{MeCN})\right]_{4}$ in THF, which resulted in a dark brown solution in which free ligand and mainly one species with a molybdenum isotopic distribution pattern were identified by ESI mass spectrometry (Figure 1.10 , left). Although the peak at $\mathrm{m} / \mathrm{z}=1107.5$ could not be assigned in a reasonable fashion, fragmentation of this peak with MS-MS techniques gave rise to one major peak with a mass to charge ratio of 930.4 (Figure 1.10, right). This isotopic distribution pattern might be well simulated with $\left[(\mathrm{MoN})_{2} \mathrm{~L}^{\mathrm{Me}}\right]^{+}$, the desired cation of the dinitrido molybdenum(VI) complex. The mass difference between the fragmentation product and the small peak at $\mathrm{m} / \mathrm{z}=1107.5$ could not be explained. 

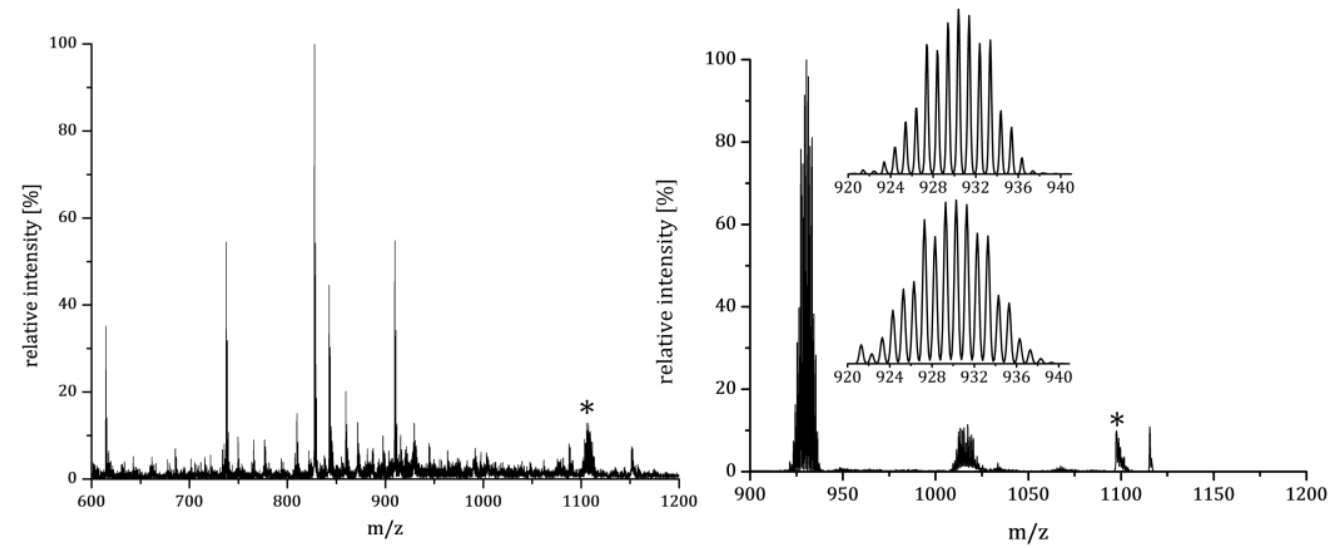

Figure 1.10: $\mathrm{m} / \mathrm{z}$ 600-1200 range of the ESI(+)-MS spectrum of a reaction between $\mathbf{H}_{5} \mathbf{L}^{\mathrm{Me}},\left[\mathrm{MoN}(\mathrm{Cl})_{3}(\mathrm{MeCN})\right]_{4}$, and KHMDS (left) and the spectrum after fragmentation of the peak at $\mathrm{m} / \mathrm{z}=1107.5$ marked with an asterisk (right). The insets show the experimental and simulated isotopic distribution patterns for $\left[(\mathrm{MoN})_{2} \mathrm{~L}^{\mathrm{Me}}\right]^{+}$.

Comparison of the ATR-IR spectra of the crude reaction product with those of the starting materials revealed the disappearance of the acetonitrile bands of the precursor around $2300 \mathrm{~cm}^{-1}$ (Figure 1.11). In the reaction mixture bands of the ligand between $3000 \mathrm{~cm}^{-1}$ and $2800 \mathrm{~cm}^{-1}$ were found. In the precursor the molybdenum-nitrogen virbration was found to appear at $1038 \mathrm{~cm}^{-1}$. Due to the presence of ligand bands in that region it remained uncertain whether a MoN vibration band was present in the crude product. However, it was not possible to isolate the unknown compound for further characterization by NMR spectroscopy.

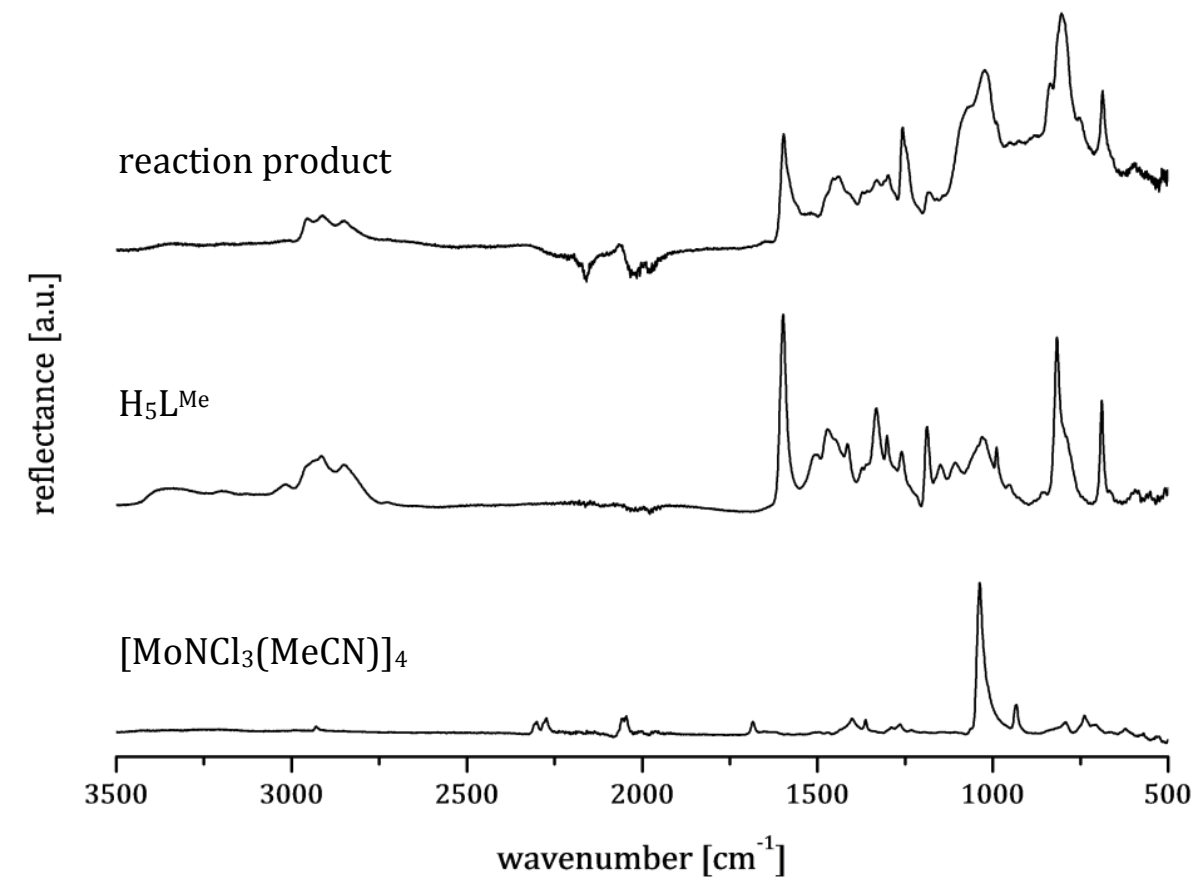

Figure 1.11: ATR-IR spectrum of the reaction product of a reaction between $\left[\mathrm{MoNCl}_{3}(\mathrm{MeCN})\right]_{4}, \mathrm{H}_{5} \mathrm{~L}^{\mathrm{Me}}$ and $\mathrm{KHMDS}$ in THF (top). To compare the spectra of $\mathrm{H}_{5} \mathrm{~L}^{\mathrm{Me}}$ (middle) and the $\left[\mathrm{MoNCl}_{3}(\mathrm{MeCN})\right]_{4}$ (bottom). 
When $\mathrm{MoN}\left(\mathrm{O}^{t} \mathrm{Bu}\right)_{3}$ was used as a metal source, one equivalent of acid had to be added to neutralize the sixth tert-butoxide, which was not needed for the deprotonation of the ligand. Upon addition of the metal salt to a solution of the ligand in THF, a color change from almost colorless to dark blue was observed immediately. Then one equivalent of ammonium chloride was added. An ESI-MS spectrum showed again the free ligand as one of the major species. Thus just one peak with an isotopic distribution pattern, which is typical for molybdenum, was observed at $\mathrm{m} / \mathrm{z}=1040.4$ (Figure 1.12). A simulation of $\left[(\mathrm{MoN})_{2} \mathrm{H}_{4} \mathrm{~L}^{\mathrm{Me}} \mathrm{Cl}_{3}\right]^{+}$fitted well to the measured isotopic distribution pattern. Nevertheless, the ligand would have been only deprotonated once.

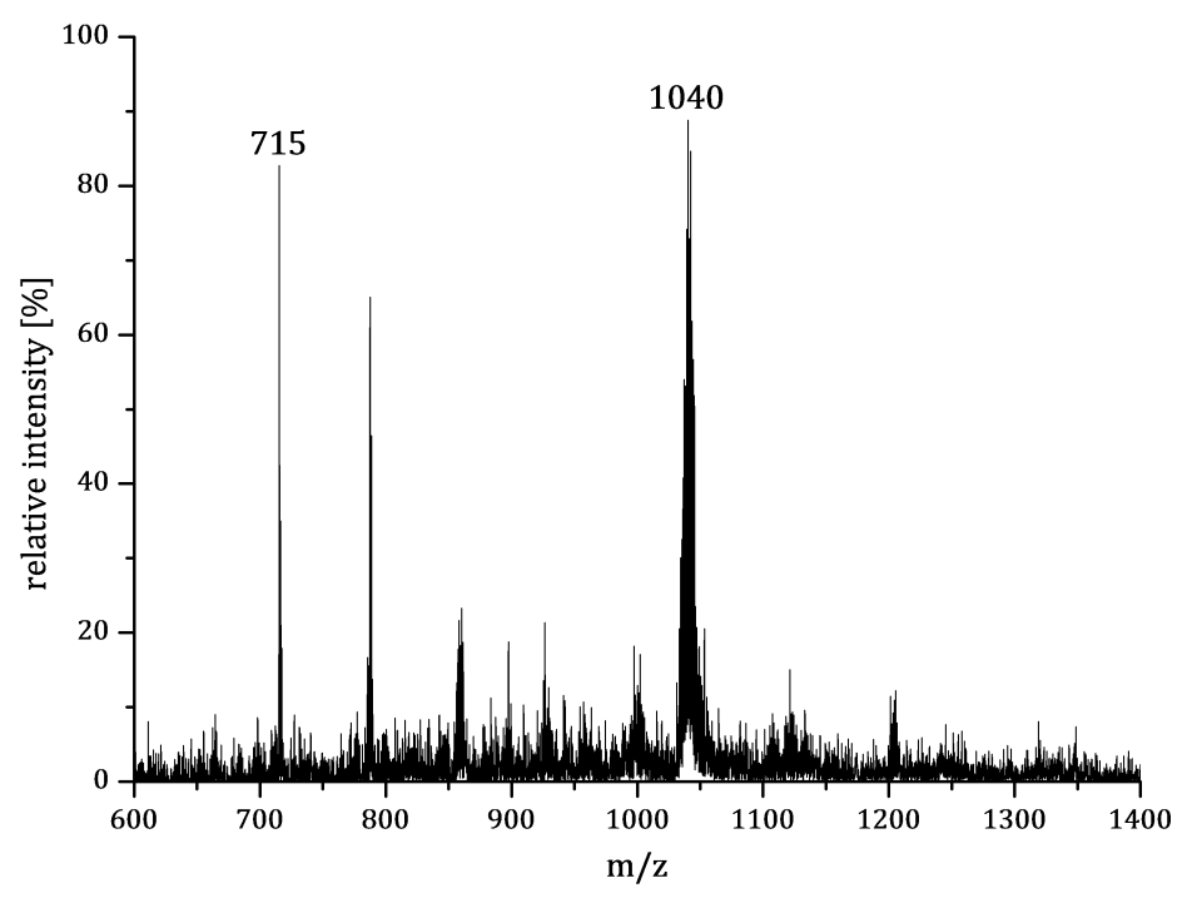

Figure 1.12: $\mathrm{m} / \mathrm{z}$ 600-1400 range of the ESI(+)-MS spectrum of the reaction mixture of a reaction between $\mathbf{H}_{5} \mathbf{L}^{\mathrm{Me}}$, $\mathrm{MoN}\left(\mathrm{O}^{t} \mathrm{Bu}\right)_{3}$ and $\mathrm{NH}_{4} \mathrm{Cl}$.

So either the formed complex was very labile towards protonation and readily picked up four protons during the ionization process, or in the reaction the ligand was not deprotonated completely. Despite numerous attempts it was not possible to obtain any product of these reaction in single crystalline form. Hence, it was not possible to get cleaner material to gain structural insight.

Next the scope of the used metal ions was extended to other high valent transition metals such as tungsten, titanium and zirconium. Chloride salts $\left(\mathrm{WCl}_{4}(\mathrm{dme}), \mathrm{ZrCl}_{4}(\mathrm{thf})_{2}, \mathrm{VCl}_{3}(\mathrm{thf})_{3}\right)$ as well as tetramido precursors $\left(\mathrm{Ti}\left(\mathrm{NMe}_{2}\right)_{4}, \mathrm{Zr}\left(\mathrm{NEt}_{2}\right)_{4}\right)$ were tested. Again the identification and isolation of a single product failed. Peaks of possibly metal containing species could not be assigned in a chemically reasonable sense in recorded ESI-MS spectra. One exception was the reaction of the ligand $\mathbf{H}_{5} \mathbf{L}^{\mathrm{Me}}$ with $\mathrm{Ti}\left(\mathrm{NMe}_{2}\right)_{4}$ in THF: in an ESI-MS spectrum of the dark orange 
reaction mixture at least one titanium containing species was assumed together with two peaks of similar intensity related to $\mathbf{H}_{5} \mathbf{L}^{\mathrm{Me}}(\mathrm{m} / \mathrm{z}=715.6$ and 721.6$)$. The peak at $\mathrm{m} / \mathrm{z}=826.7$ was in good agreement with the simulated isotopic distribution pattern of $\left[\mathrm{Ti}\left(\mathrm{NMe}_{2}\right) \mathrm{H}_{2} \mathrm{LMe}^{\mathrm{Me}} \mathrm{Na}\right]^{+}$ (Figure 1.13). If the assignment was correct it showed that in this compound only one metal was incorporated in the ligand cavity. The pyrazole would be deprotonated while two of the four side arms remain protonated. Since no dinuclear titanium species were assigned, it was tried to react $\mathbf{H}_{5} \mathbf{L}^{\text {Me }}$ with an excess of Ti( $\left.\mathrm{NMe}_{2}\right)_{4}$ leading to an ESI-MS spectrum with even more unidentified peaks. In general, titanium(IV) complexes are known to be reactive compounds, which might degenerate the ligand itself.[84,85]

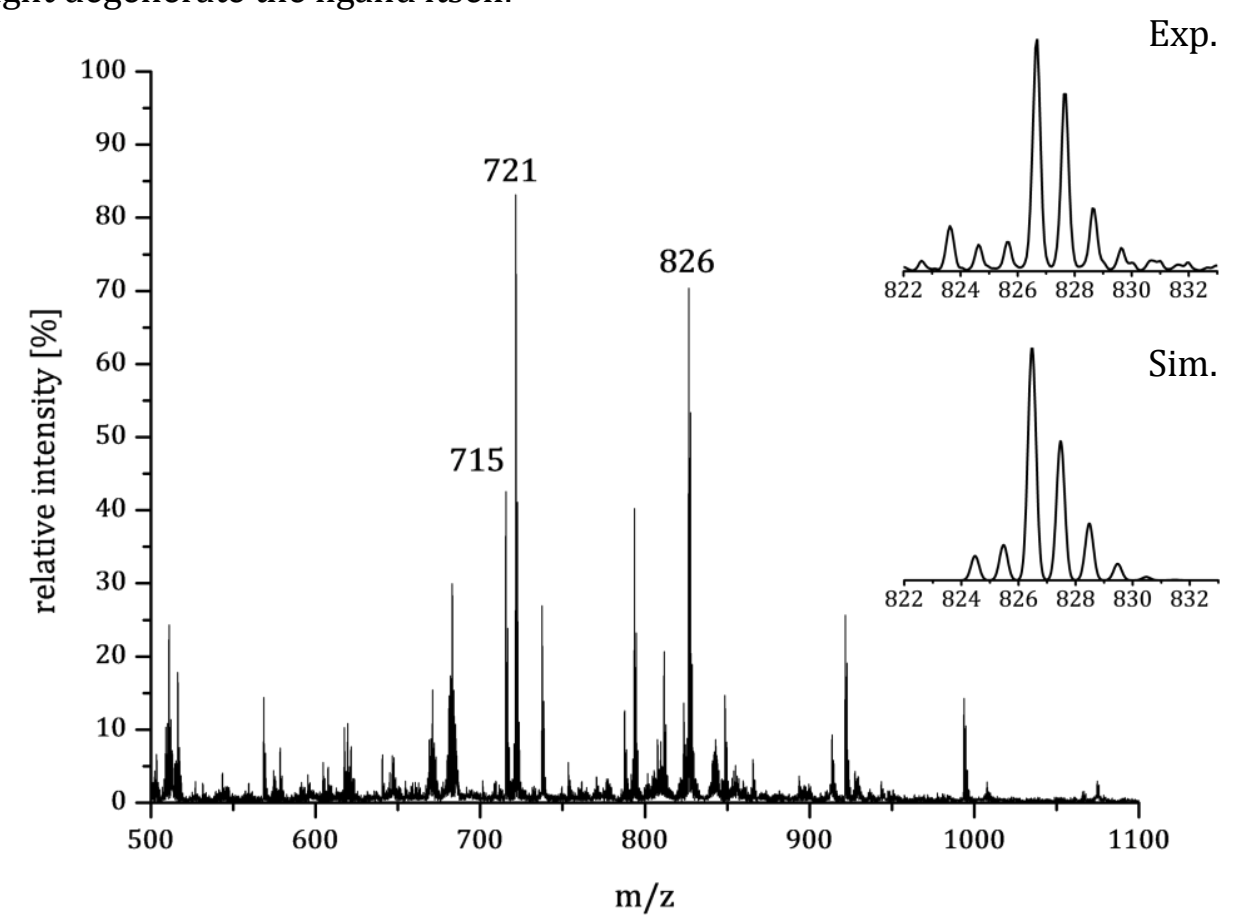

Figure 1.13: $\mathrm{m} / \mathrm{z}$ 500-1100 range of the ESI(+)-MS spectrum of the reaction mixture of a reaction between $\mathbf{H}_{5} \mathbf{L}^{\mathrm{Me}}$, $\mathrm{Ti}\left(\mathrm{NMe}_{2}\right)_{4}$ and $\mathrm{NH}_{4} \mathrm{PF}_{6}$. The insets show the experimental and simulated isotopic distribution patterns for $\left[\mathrm{Ti}\left(\mathrm{NMe}_{2}\right) \mathrm{H}_{2} \mathrm{~L}^{\mathrm{Me}}+\mathrm{Na}\right]^{+}$.

Since the complete deprotonation of all four amine side arms seemed to be challenging although metals ions were present, the synthesis of tetraamine complexes of $\mathbf{H}_{5} \mathbf{L}^{\text {Me }}$ was attempted (Figure 1.14). For this purpose, divalent 3d transitions metals were chosen.

a)

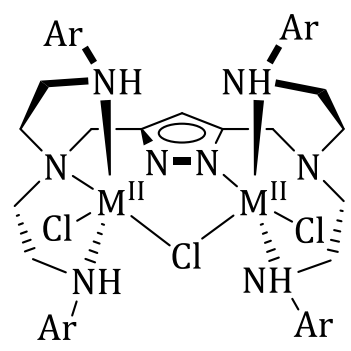

b)

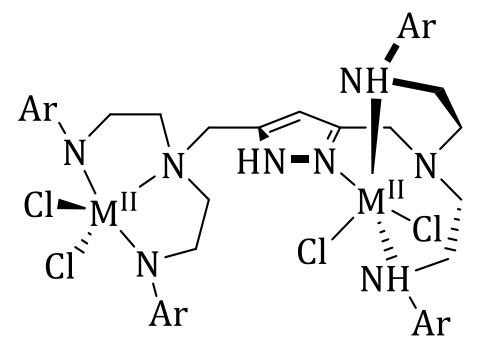

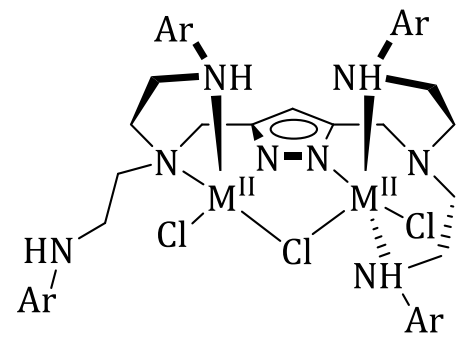

Figure 1.14: a) Targeted tetramine complex with divalent 3d transition metals. b) Possible reaction products: if the ligand is not deprotonated at all (left) and if one side arm is not bound to the metal center (right). 
$\mathrm{NiCl}_{2} \cdot 6 \mathrm{H}_{2} \mathrm{O}, \mathrm{CuCl}_{2} \cdot 2 \mathrm{H}_{2} \mathrm{O}, \mathrm{MnCl}_{2}$ and $\mathrm{Zn}(\mathrm{OAc})_{2} \cdot 2 \mathrm{H}_{2} \mathrm{O}$ were investigated as metal salts using triethylamine as a base. The reaction mixtures were filtered and the crude product was thoroughly washed with ether to remove remaining free ligand. However, in the resulting ESI-MS spectra the ligand $\mathbf{H}_{5} \mathbf{L}^{\text {Me }}$ was found as the dominant species except from the nickel chloride attempt. Here, peaks were found which contained nickel ions due to the isotopic distribution patterns and no ligand related peaks were observed. The ESI-MS spectrum features multiple peaks having the typical $\mathrm{Ni}_{2}$ isotopic distribution pattern. Selected assigned peaks are depicted in Table 1.1. The exact $\mathrm{m} / \mathrm{z}$ ratios are different for the simulated and found isotopic distribution pattern, since it was not an HR-MS measurement and additionally the oxidation states of the nickel atoms remained uncertain, since the total charges of the simulated complexes are not in agreement with two $\mathrm{Ni}^{2+}$ ions. It was shown before that in those systems several different constitutions of metal complexes are possible. ${ }^{[69]}$ And since the base was used in an excess, also different states of deprotonation probably occur resulting in even more possible products making the isolation and crystallization of a single, clean product almost impossible. Indeed, neither crystals nor a cleaner product after precipitation in the crystallization attempt were obtained.

Table 1.1: Comparison of selected peaks of a crude product ESI-MS spectrum after a reaction between $\mathrm{H}_{5} \mathrm{~L}^{\mathrm{Me}}$, $\mathrm{NiCl}_{2} \cdot 6 \mathrm{H}_{2} \mathrm{O}$ and $\mathrm{NEt}_{3}$ in THF with possible simulated species.

\begin{tabular}{ccccc}
\hline entry & $\begin{array}{c}\text { found mass } \\
\mathrm{m} / \mathrm{z}\end{array}$ & $\begin{array}{c}\text { simulated } \\
\text { mass } \mathrm{m} / \mathrm{z}\end{array}$ & simulated formula & potential complex \\
\hline 1 & 977.4 & 977.3 & $\mathrm{C}_{46} \mathrm{H}_{59} \mathrm{~F}_{3} \mathrm{~N}_{8} \mathrm{Ni}_{2} \mathrm{O}_{3} \mathrm{~S}$ & {$\left[\mathrm{Ni}_{2}\left(\mathrm{H}_{2} \mathrm{~L}^{\mathrm{Me}}\right)(\mathrm{OTf})\right]^{+}$} \\
\hline 2 & 1017.5 & 1017.3 & $\mathrm{C}_{48} \mathrm{H}_{61} \mathrm{~F}_{3} \mathrm{~N}_{9} \mathrm{Ni}_{2} \mathrm{O}_{3} \mathrm{~S}$ & {$\left[\mathrm{Ni}_{2}(\mathrm{HLMe})(\mathrm{OTf})(\mathrm{MeCN})\right]^{+}$} \\
\hline 3 & 1031.5 & 1031.3 & $\mathrm{C}_{46} \mathrm{H}_{65} \mathrm{~F}_{3} \mathrm{~N}_{8} \mathrm{Ni}_{2} \mathrm{O}_{6} \mathrm{~S}$ & {$\left[\mathrm{Ni}_{2}\left(\mathrm{H}_{2} \mathrm{~L}^{\mathrm{Me}}\right)(\mathrm{OTf})\left(\mathrm{H}_{2} \mathrm{O}\right)_{3}\right]^{+}$} \\
\hline 4 & 1089.6 & 1089.4 & $\mathrm{C}_{52} \mathrm{H}_{69} \mathrm{~F}_{3} \mathrm{~N}_{9} \mathrm{Ni}_{2} \mathrm{O}_{4} \mathrm{~S}$ & {$\left[\mathrm{Ni}_{2}\left(\mathrm{HL}^{\mathrm{Me}}\right)(\mathrm{OTf})(\mathrm{MeCN})(\mathrm{thf}]^{+}\right.$} \\
\hline 5 & 1103.6 & 1103.4 & $\mathrm{C}_{50} \mathrm{H}_{73} \mathrm{~F}_{3} \mathrm{~N}_{8} \mathrm{Ni}_{2} \mathrm{O}_{7} \mathrm{~S}$ & {$\left[\mathrm{Ni}_{2}\left(\mathrm{H}_{2} \mathrm{~L}^{\mathrm{Me}}\right)(\mathrm{OTf})(\mathrm{thf})\left(\mathrm{H}_{2} \mathrm{O}\right)_{3}\right]^{+}$} \\
\hline
\end{tabular}




\subsection{Summary}

A new synthetic strategy was developed to avoid the formation of oligomers in the synthesis of $\mathbf{H}_{5} \mathbf{L}^{\text {Me }}$. Eventually it was possible to synthesize the desired ligand $\mathbf{H}_{5} \mathbf{L}^{\text {Me }}$ (IX) in acceptable purity by using a bisamide side arm (VI), which leads to a tetraamide ligand precursor. In the last step, this compound VIII was reduced with an excess of borane and deprotected during the acidic work up. The compound could be identified with NMR spectroscopy as well as by ESI mass spectrometry. A solid state structure of $\mathbf{H}_{5} \mathbf{L}^{\text {Me }}$ could not be obtained.

In conclusion, the synthesis of metal complexes of the new ligand $\mathbf{H}_{5} \mathbf{L}^{\text {Me }}$ was not achieved. Different strategies were applied to synthesize metal complexes of this ligand with high valent transition metals but also with divalent late transition metals. However, all attempts to isolate a single species proved to be unsuccessful. Furthermore, in the majority of these reactions it was not clear, if stable complexes were formed during the synthesis. Instead of the expected complex related signals, peaks with high intensity related to the ligand were found in ESI-MS measurements. From the results above two major problems were faced: Firstly, the selective deprotonation of the ligand and secondly the stability of the potentially formed complexes. If the ligand would not be deprotonated completely a variety of different products are concevable. The stability of the formed complexes might be rather low due to steric interactions of the relatively bulky 3,5-dimethylphenyl substituents at the amine/amide donor sites in the side arms. In addition, four aryl groups should be beneficial for crystallization issues, but this seemed to be not true for these systems. ${ }^{[65]}$ 



\section{Macrocyclic Tetracarbene Ligands for High Valent Metal Complexes}

\subsection{Introduction}

High valent metal compounds, especially metal oxo species, are often known as reactive intermediates in enzyme cofactors. The oxygenation of unactivated C-H bonds (by insertion of an oxygen atom) is of high importance in nature. ${ }^{[86,87]} \mathrm{A}$ lot of different classes of enzymes are expected to use high valent metal species for the transformation of a broad substrate scope. To name just a few examples the cytochrome P450 oxygenases (CYPs) ${ }^{[88,89]}$, soluble methane monooxygenase (sMMO) [90,91], particular methane monooxygenase (pMMO) ${ }^{[92]}$ and superoxide dimutases (SODs) ${ }^{[93]}$ are mentioned. The synthesis and characterization of structural and/or functional model complexes for these active centers already provided a lot of insights, which helped to understand the basic chemistry behind these complex systems. But still many mechanisms remain uncertain or even unknown.

Nickel atoms were not found in metalloproteins as often as iron or copper, but some of them show fascinating and rather unusual coordination environments, stabilizing high valent nickel(III) species featuring extraordinary reactivity. For example, the bifunctional enzyme nickel carbon monoxide dehydrogenase/acetyl-cofactor A (CoA) synthase (CODH/ACS) catalyzes in total the acetylation of CoA. ${ }^{[94]}$ Thereby the CODH part mediates the reversible reaction of carbon monoxide and water to carbon dioxide, protons and electrons, which is somehow the biological equivalent to the water gas shift reaction (WGSR).[94] The cytotoxic carbon monoxide from this equilibrium is used in the production of acetyl-CoA. Both enzymes contain a dinuclear nickel species in the active site.[95,96] For the acetyl-CoA synthase two possible mechanisms are discussed: One mechanism contains a $\mathrm{Ni}^{\mathrm{II}} \mathrm{Ni}^{0}$ cycle, whereas the other proceeds via $\mathrm{Ni}{ }^{I I I}$ and $\mathrm{Ni}^{\mathrm{I}}$ intermediates. ${ }^{[96,97]}$

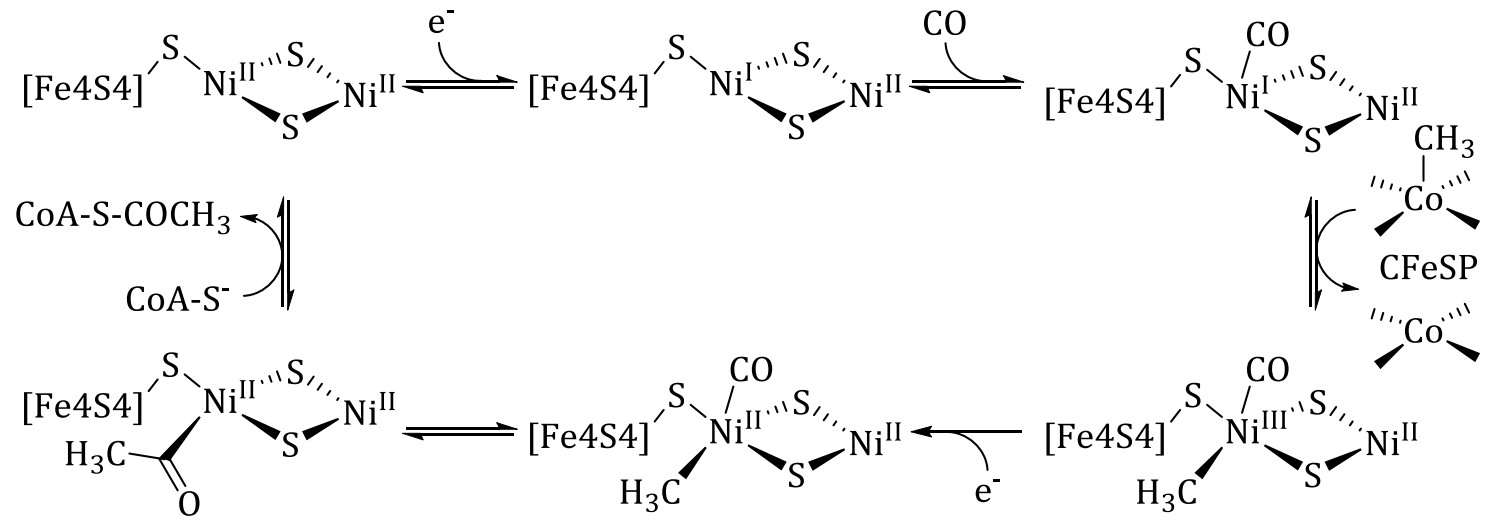

Scheme 2.1: Proposed mechanism for the acetyl-cofactor A (CoA) synthase including high valent nickel(III) intermediate which is generated by nucleophilic attack of a nickel(I) species on a CFeSP.[94] 
In the latter case shown in Scheme 2.1, $\mathrm{CO}$ coordinates to a $\mathrm{Ni}^{\mathrm{I}}$ species, which afterwards performs a nucleophilic attack on a methylated corrinoid iron sulfur protein (CFeSP) to generate a $\mathrm{Ni}^{\mathrm{III}}$ methyl species, which is then rapidly reduced to a $\mathrm{Ni}^{\mathrm{II}}$ methyl compound. Either a methyl migration or a $\mathrm{CO}$ insertion yields a $\mathrm{Ni}^{\mathrm{II}}$ acetyl species, which can be attacked by a deprotonated $\mathrm{CoA}^{-}$molecule. Acetyl-CoA is formed by reductive elimination in order to reform the starting $\mathrm{Ni}^{\mathrm{I}}$ complex. ${ }^{[94]}$

A high valent organometallic nickel species is discussed to take part in the methane formation reaction catalyzed by the $\mathrm{F} 430$ cofactor of the methyl coenzyme $\mathrm{M}$ reductase (the proposed $\mathrm{Ni} \mathrm{i}^{\mathrm{III}}$ methyl porphinoid species is shown in Figure 2.1,8).[98,99] Nickel superoxide dismutase catalyzes the disproportionation of superoxide radicals to hydrogen peroxide and molecular oxygen and thus protects cells from oxidative damage. ${ }^{[93,100]}$ After addition of the substrate, a nickel(III) center is square pyramidally five-coordinated by the $\mathrm{N}_{2} \mathrm{~S}_{2}$ donor set and an axial imidazole of a histidine stabilizes the high oxidation state (Figure 2.1, 9). A third enzyme is the [NiFe] hydrogenase being responsible for the generation of protons from the cleavage of dihydrogen.[101,102] The mechanism proceeds via a heterolytic splitting of dihydrogen producing a nickel(III) species with a bridging hydride (Figure $2.1,10$ ). The cycle is closed with the release of another proton and electron so that in total dihydrogen is split into two protons and two electrons.
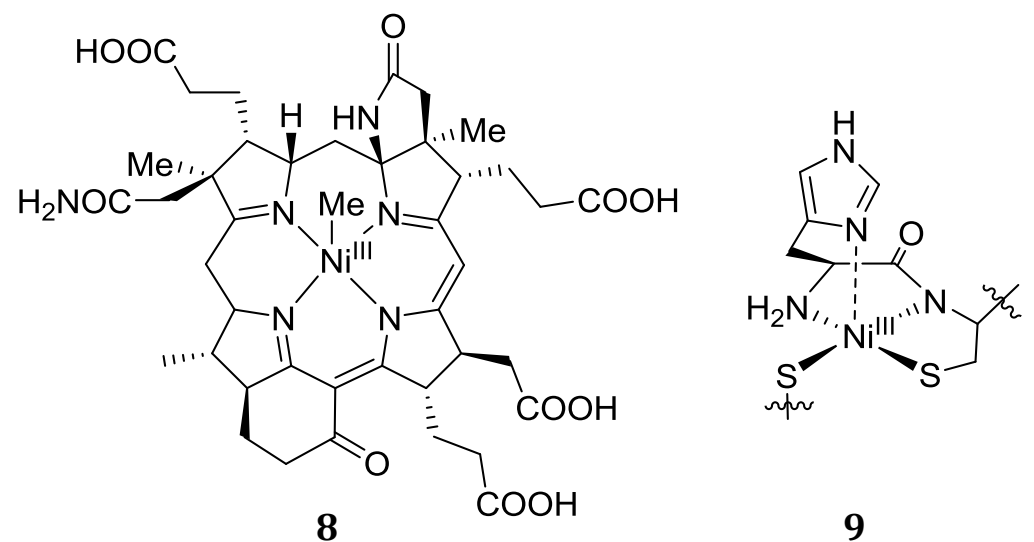

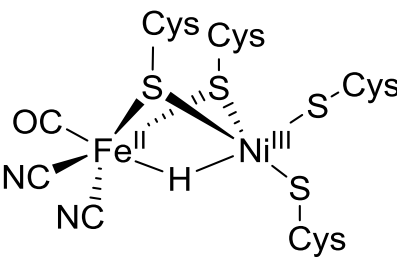

10

Figure 2.1: Proposed high valent nickel cofactors from methyl coenzyme M reductase (F430) 8, nickel superoxide dismutase (NiSOD) 9 and [NiFe] hydrogenase 10.

Nickel complexes are also used for catalysis in which nickel(III) species are discussed as intermediates.[103-105] The mechanistic aspects of these reactions are less well understood compared to the well-established palladium analogues. ${ }^{[106-109]}$ In the case of nickel catalysis not only $\mathrm{M}^{0} \mathrm{M}^{\mathrm{II}}$ oxidative addition/reductive elimination cycles are assumed to take part, but also $\mathrm{Ni}^{\mathrm{I}} \mathrm{Ni}^{\mathrm{III}}$ cycles or radical species may be involved.[110,111] Thus, a general mechanism for nickel catalyzed coupling reactions remains elusive. 
Since the understanding of mechanistic aspects of both nature's enzymatic reactions and organometallic catalysis is of great interest for research, high valent nickel(III) model complexes have been prepared. First examples were already presented back in the 1960s, when e.g. macrocyclic tetraaza (cyclam type) ligands were used.[112] More recently, nickel(III) complexes with a phospasalen ligand or organometallic aryl or alkyl ligands were reported (Figure 2.2, 11).[113-116] The group of HILLHOUSE reported that high valent nickel ions can be coordinated by a carbene ligand (Figure 2.2, 12).[117] This was the first and only example in literature for the stabilization of $\mathrm{Ni}^{\mathrm{III}}$ by a carbene.

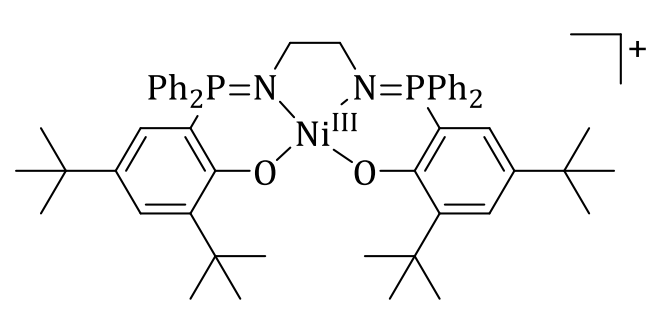

11

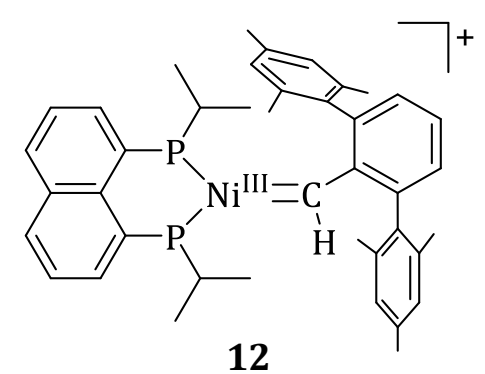

12

Figure 2.2: Selected examples for high valent nickel(III) complexes: phosphasalen $\mathrm{Ni}^{\mathrm{III}}$ complex $\mathbf{1 1}$ and the only example of a carbene supported nickel(III) complex 12.

Although nickel(III) aryl or alkyl species are discussed in catalytic cycles of nickel mediated coupling reactions, the synthesis of compounds with relevant reactivity remained unknown until 2014. MIRICA et al. demonstrated 2014 that the halide aryl nickel(III) complex $\mathbf{1 3}$ can undergo a transmetallation reaction with a Grignard reagent. Subsequent reductive elimination from the nickel(III) alkyl aryl species $\mathbf{1 4}$ yielding the C-C coupled product was observed after warming to ambient temperature (Scheme 2.2).[118] The produced nickel(I) species was not found due to disproportionation into $\mathrm{Ni}^{0}$ and $\mathrm{Ni}^{\mathrm{II}}$.

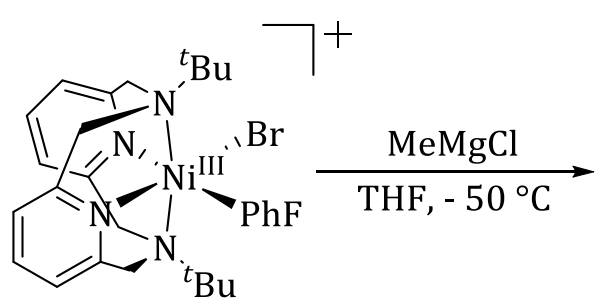

13

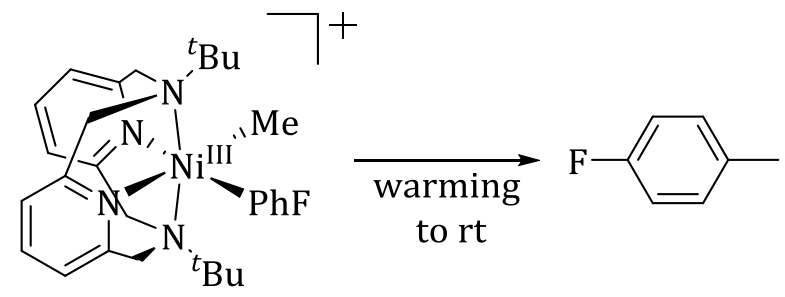

14

Scheme 2.2: Reductive elimination from a nickel(III) aryl alkyl complex 14.[118]

Copper containing cofactors are often found in enzymes relevant for dioxygen transport such as hemocyanine ${ }^{[119,120]}$ or for the activation of substrates in, e.g., different oxidoreductases like tyrosinase or cytochrome-c-oxidase.[121] In general, copper proteins are classified into different types of proteins due to their spectroscopic and structural features,[122] and most often they are using the $\mathrm{Cu}^{\mathrm{I}} / \mathrm{Cu}^{\mathrm{II}}$ redox couple. For example in the case of the activation of dioxygen mononuclear active copper(I) sites donate one electron to $\mathrm{O}_{2}$ which results in an end-on bound superoxo species [123], while dinuclear copper sites can bind dioxygen in a peroxo or bis- $\mu$-oxo fashion ${ }^{[124,125]}$ transferring several electrons to dioxygen. Aside the above mentioned binding 
motives there are other binding modes that have been found in model systems and structurally characterized. ${ }^{[92,126-128]}$ High valent copper species in nature remain uncharacterized to date. Nevertheless, for the particular methane monooxygenase (pMMO) a copper(III) intermediate is supposed to be part of the catalytic cycle.[129,130] This protein is found in methanotrophic bacteria, which uses methane as the only source of carbon and energy. The active center $\mathbf{1 5}$ of this protein consist of two different binding sites for the two copper ions ${ }^{[131]}$ : one featuring two histidine ligands while the second copper ion is bound by a primary amine and one histidine residue (Figure 2.3). As the only known example, the active species of the pMMO is proposed to contain either a dicopper(III) bis( $\mu$-oxido), a dicopper(III) $\mu$-oxido core or a dicopper(II)$\mu$-peroxido species.[125,132] This high valent compound is able to oxidize the very strong $\mathrm{C}-\mathrm{H}$ bond (104 kcal/mol) of methane to methanol.[92] From calculations it was assumed that a $\mathrm{Cu}^{\mathrm{I}} \mathrm{Cu}^{\mathrm{II}}$ species, rather than a $\mathrm{Cu}^{\mathrm{II}} \mathrm{Cu}^{\mathrm{II}}$ species, is oxidized by dioxygen resulting in an active $\mathrm{Cu}^{\mathrm{II}} \mathrm{Cu}^{\mathrm{III}}$ species for oxidation reactions, since the reorganization energy was lower for the mixed valent compound.[133] However recently presented model complexes by STACK rather indicate the potential presence of the dicopper(III) bis( $\mu$-oxido) core in the active site of the pMMO.[134]

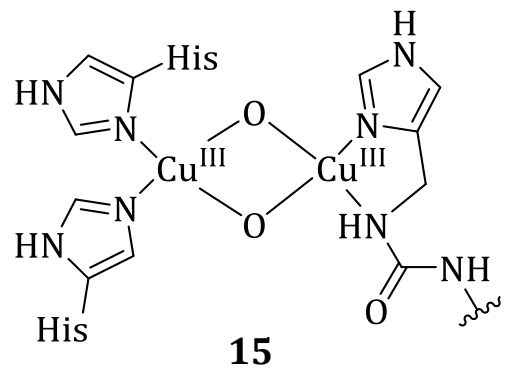

Figure 2.3: Proposed active center of the pMMO featuring a dicopper(III) bis( $\mu$-oxido) core.[131]

Since copper(III) intermediates also of high interest in catalysis,[135] many copper(III) complexes have been reported. These literature known complexes are mostly supported by a variety of classical N, O or S donor ligands varying from amine[136] or amide ${ }^{[137]}$ to salen type ${ }^{[138]}$ or $\mathrm{N}_{2} \mathrm{~S}_{2}{ }^{[139,140]}$ donor sets. All complexes featured a square planar coordination geometry with $\mathrm{Cu}^{\mathrm{III}}$-ions in the low spin ground state. The redox potentials proved to be highly dependent on the supporting ligand and the used solvent.[140,141] In C-H activation and related coupling reactions organometallic copper(III) complexes are proposed as intermediates in the catalytic cycles.[142-146] However, well-defined organometallic $\mathrm{Cu}^{\mathrm{III}}$ complexes as well as reactivity studies on such systems are rarely known. In Figure 2.4 examples of few structurally characterized copper(III) complexes are shown. Complex 16 was one of the first reported organometallic copper compounds. ${ }^{[147]}$ Complex 17 showed an interesting switch between a diamagnetic and a paramagnetic ground state upon addition of a second chloride ion.[148] Macrocyclic ligands such as doubly N-confused porphyrins or triazamacrocyles are represented for example by $\mathbf{1 8}$ and 19, respectively.[132,149,150] 


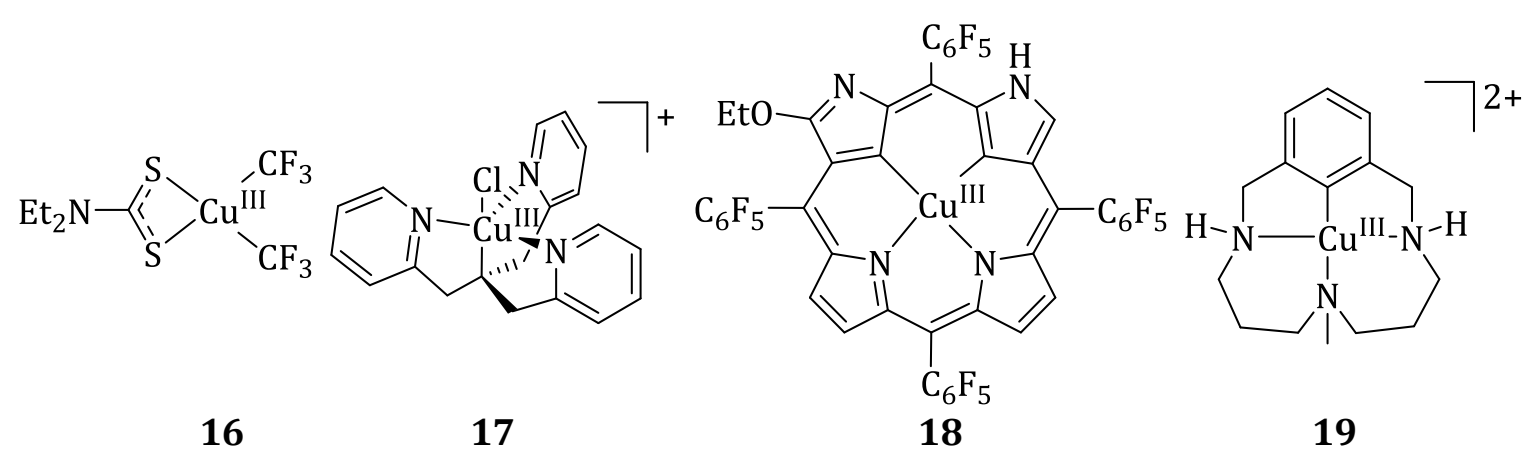

Figure 2.4: Examples of well-defined organometallic copper(III) complexes.[147-150]

In order to use base metals for catalytic transformations, copper is used for coupling reactions and copper(III) species are assumed to be part of catalytic cycles of such reactions. ${ }^{[151,152]}$ The reversible oxidative addition of aryl halides is believed to be the key step in the catalytic cycle of copper mediated coupling reactions. The two electron process features a switch between $\mathrm{Cu}^{\mathrm{I}}$ and $\mathrm{Cu}^{\mathrm{III}}$ species. The direct observation of such a step was reported in 2010 by the group of STAHL[153] based on a macrocyclic ligand system, which was earlier shown to stabilize $\mathrm{Cu}^{\mathrm{III}}$ complexes (Figure 2.4 19).[150] The reaction of the ligand with a $\mathrm{Cu}^{\text {II }}$ salt led to a disproportionation into equimolar amounts of $\mathrm{Cu}^{\mathrm{I}}$ and $\mathrm{Cu}^{\mathrm{III}}$ complexes. It was shown that halide copper(III) complex $\mathbf{2 0}$ can undergo acid triggered reductive elimination yielding the aryl halide $\mathbf{2 1}$ and a copper(I) species.[153] Upon addition of base this reaction is reversible. It was also shown that addition of a nitrogen nucleophile to this $\mathrm{Cu}^{\mathrm{III}}$ complex led to $\mathrm{C}-\mathrm{N}$ bond formation with the release of a $\mathrm{Cu}^{\mathrm{I}}$ complex.[154] In this example the revesible reductive elimination included the ligand of the copper ion revealing the need for further studies on the essential step of copper catalyzed coupling reactions.

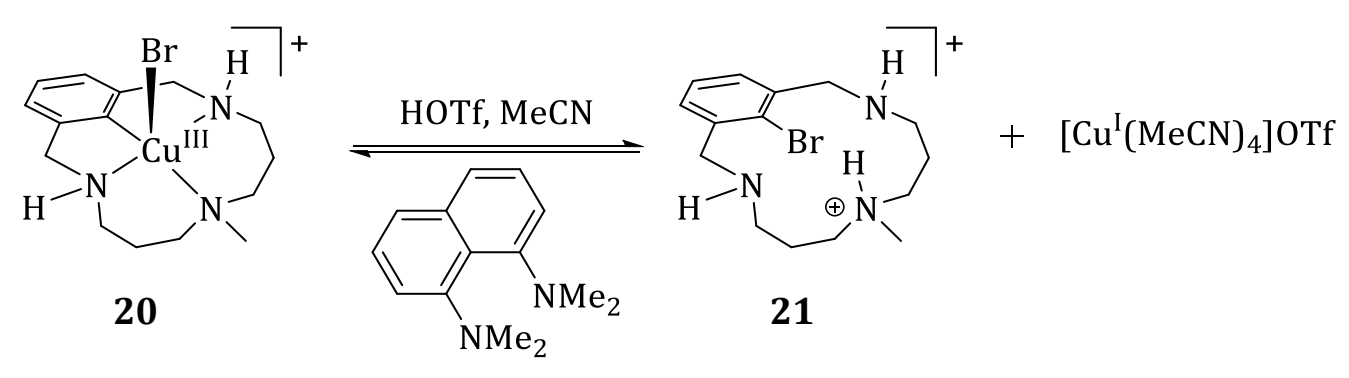

Scheme 2.3: Reversible reductiv elimination of an aryl halide from a copper(III) compound.[153]

Carbenes, in particular N-heterocyclic carbenes (NHCs), are widely used as ligands in catalysis.[155-159] Since the discovery of the first metal carbene complexes by WANZLICK and ÖFELE and the isolation of the first free carbene by ARDUENGO the applications of this class of ligands expanded to almost all aspects of organometallic chemistry and catalysis. ${ }^{[160-162]}$ NHCs offer a high complex stability, a broad range of different functional groups to tune e.g. steric environment around a metal center, and easy synthetic access. Imidazolium, imidazoline or triazole scaffolds are commonly used to synthesize metal carbene compounds. Synthetic 
strategies include deprotonation in presence of a metal ion, the transmetallation of silver or coppers complexes, direct reaction of imidazolium salts with elemental metals or the oxidative addition of imidazolium salts to low valent metal complexes.[163-165]

Carbene ligands are usually regarded as two electron donors exhibiting strong $\sigma$-donor character and rather weak $\pi$-acceptor properties.[159] In singlet carbenes these electrons are located both in the $\sigma$-orbital of the $\mathrm{sp}^{2}$ hybridized carbon while in triplet carbenes they singly occupy the $\sigma$-orbital and the $\pi$-orbital. Due to steric and electronic effects NHCs are only known as singlet carbenes.[165] A metal-carbene bond can be seen as the sum of three interactions, namely a NHC $\rightarrow$ metal $\sigma$ donation, metal $\rightarrow$ NHC $\pi^{*}$ backdonation and NHC $\rightarrow$ metal $\pi$ donation.[166] Similar to phosphines and cyclopentadienyls at a first glance, the electronic structures and steric demands of NHCs are distinct from these of other powerful classes of ligands in organometallic chemistry. Changing substituents of e.g. phosphine ligands not only changes the steric parameters, but also affects the electronic structure of the ligand.[167] An independent tuning of this two factors is hardly possible. In contrast to that, the substituents on the NHC periphery have only minor influence on the electronic structure, hence a fine tuning of the size of an open binding site is possible without changing the electronic properties of the ligand too much. To change the electronic structure in a NHC ligand, the central heterocycle can be modified e.g. by the use of benzimidazole, imidazoline or triazole instead of an imidazole ring. Also the steric influence is different. While the substituents of phosphines are pointing away from the metal center, they are classified mainly by their cone angles (Tolman parameter).[168,169] In NHC complexes the substituents on the heteroatoms are located much closer to the coordination sphere of the metal and have therefore a more crucial influence on the catalytic activity.[159]

Using chelating NHC ligands is one way of increasing the stability of the corresponding metal complexes. Thus many bidentate ${ }^{[164,170-173]}$, tridentate ${ }^{[174-179]}$ and tetradentate ${ }^{[180-185]}$ ligand scaffolds were developed and used for the synthesis of transition metal complexes (selected examples of these ligand scaffolds $\mathbf{X}$ - XIII are depicted in Figure 2.5).

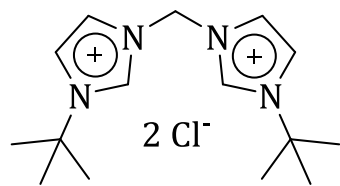

$\mathbf{X}$

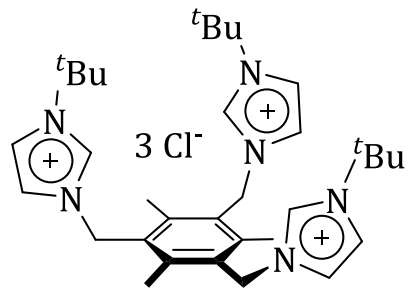

XI

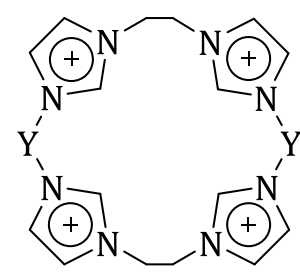

$$
\mathrm{Y}=\mathrm{CH}_{2} \quad \mathrm{XII}(\mathrm{OTf})_{4}
$$$$
\mathrm{BMe}_{2} \mathrm{XIII}(\mathrm{Br})_{2}
$$

Figure 2.5: Selected examples of bidentate (X), tridentate (XI) and macrocyclic tetradentate (XII and XIII) NHC ligands. $[173,175,184,185]$ 
The macrocyclic tetracarbene XII was found to be a suitable ligand for the isolation of an terminal iron(IV) oxo complex 23 (Scheme 2.4).[186] Upon addition of 2-(tert-butylsulfonyl)iodosobenzene XIV (sPhIO) at low temperature to iron(II) complex 22 in MeCN the green iron(IV) oxo species $\mathbf{2 3}$ was formed. At higher temperatures it decomposed to form a dimeric iron(III) $\mu$-oxo compound 24. This was also the product of the reaction of $\mathbf{2 2}$ with dioxygen. $\mathbf{2 3}$ was reformed by addition of sPhIO to the ferric dimer. Complex $\mathbf{2 3}$ proved to be quite stable at low temperatures in contrast to other reported non-heme iron oxo complexes.[187] This was also represented in the low reactivity of $\mathbf{2 3}$. Several substrates were tested, however only the hydrogen abstraction in 1,4-cyclohexadiene and 9,10-dihydroanthracene XV (DHA) was successful.[188] By treating 23 with an excess of sPhIO and DHA approximately six turnovers after $3 \mathrm{~h}$ at $25^{\circ} \mathrm{C}$ were observed. The chemistry of iron nitric oxide, nitrido and disulfido complexes was thoroughly investigated by the group of MEYER.[189-192] The octaphenyl derivative of the iron(II) complex was used for the catalytic aziridation of various alkenes.[193,194]

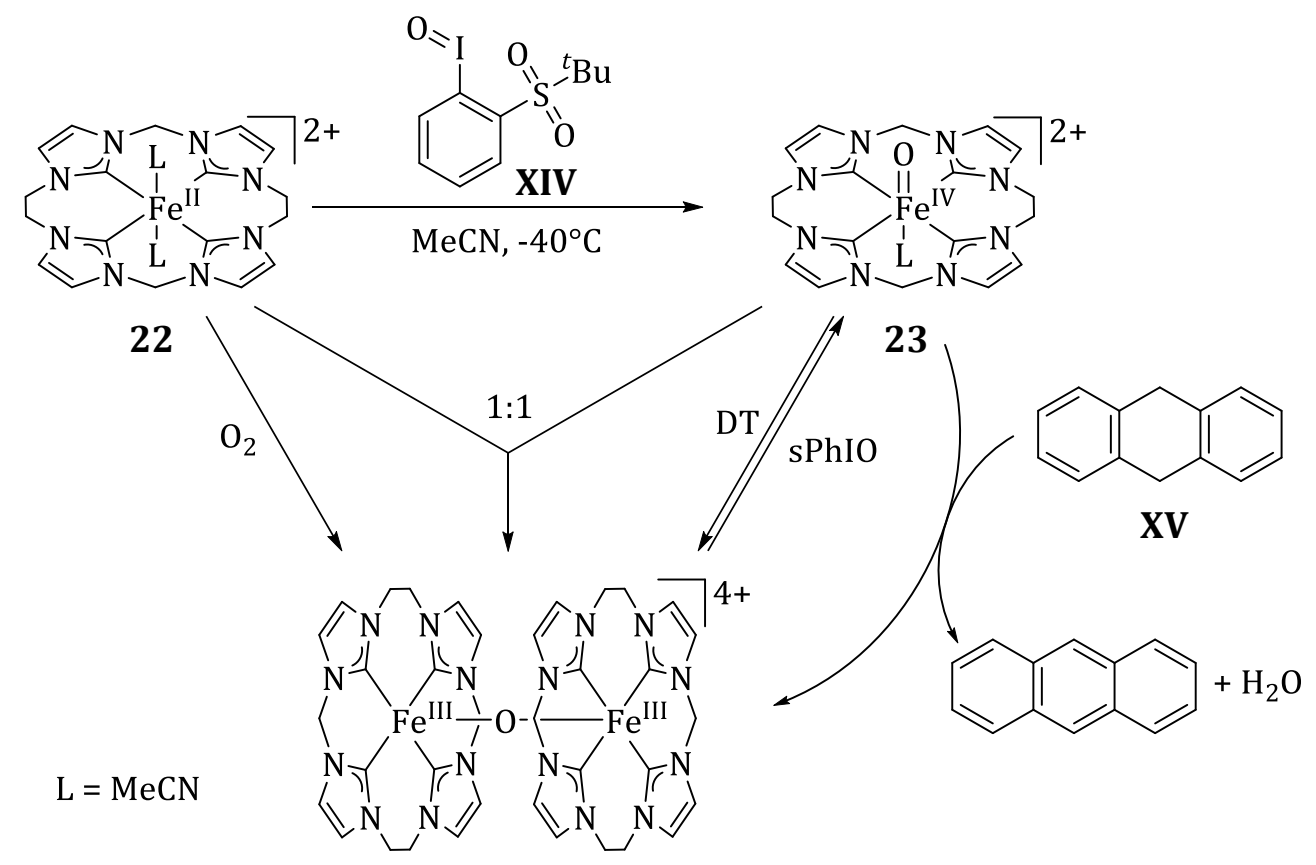

24

Scheme 2.4: Overview of the synthesis and reactivity of tetracarbene supported iron(IV) oxo complex 23.[188] 



\subsection{Objective}

The isolation and characterization of $\mathbf{2 3}$ was the first example of an terminal iron(IV) oxo complex, which was not supported by nitrogen donor ligands but by strong $\sigma$-donor carbene ligands.[186] It showed a remarkable stability for such reactive compounds. Therefore, the tetracarbene ligand system was thought to be suitable for the stabilization of other high valent metal species such as copper(III), nickel(III) and manganese in different oxidation states. In addition, the strong $\pi$-backbonding ability of the tetracarbene ligands might enable a break through of the "oxo-wall" by lowering the substantial destabilization of the degenerated $\mathrm{d}_{\mathrm{xy}}$ and $\mathrm{d}_{\mathrm{yz}}$ orbitals (this destabilization is caused by $\pi$-bonding of the metal and the oxo ligand).[195] Starting from the MII precursors, new complexes of higher oxidation states were planned to be synthesized using different oxidation-, oxygenation- and nitride transfer agents (Figure 2.6). These high valent metal complexes should be investigated by various spectroscopic methods to gain insights into their electronic structures. The comparison of the electronic and structural parameters of these organometallic metal complexes can lead to a deeper understanding of bioinorganic reactions. In addition, reactive intermediates in catalysis can be investigated.

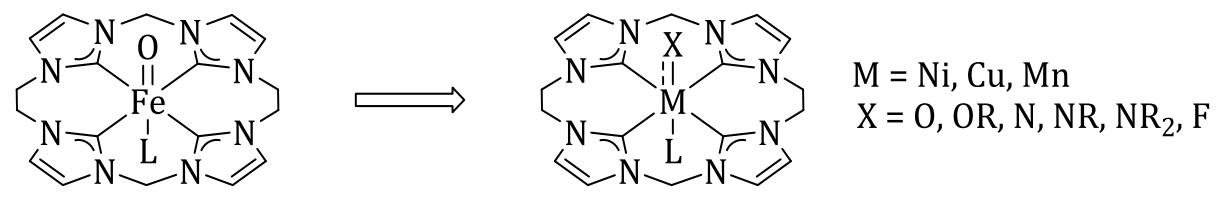

Figure 2.6: Possible structure of the attempted high valent metal complexes of a tetracarbene ligand.

Furthermore, the ligand system should be extended to enhance the solubility of the complexes and to investigate the influences of the backbone substitution on the electronic structure of the metal sites (Figure 2.7). The backbone protons of the imidazolium rings may be replaced by methyl groups. Also the size of the cavity of the macrocycle should be decreased by using shorter linkers between the imidazoles $\left(\mathrm{CH}_{2}\right.$ instead of $\left.\mathrm{CH}_{2} \mathrm{CH}_{2}\right)$.
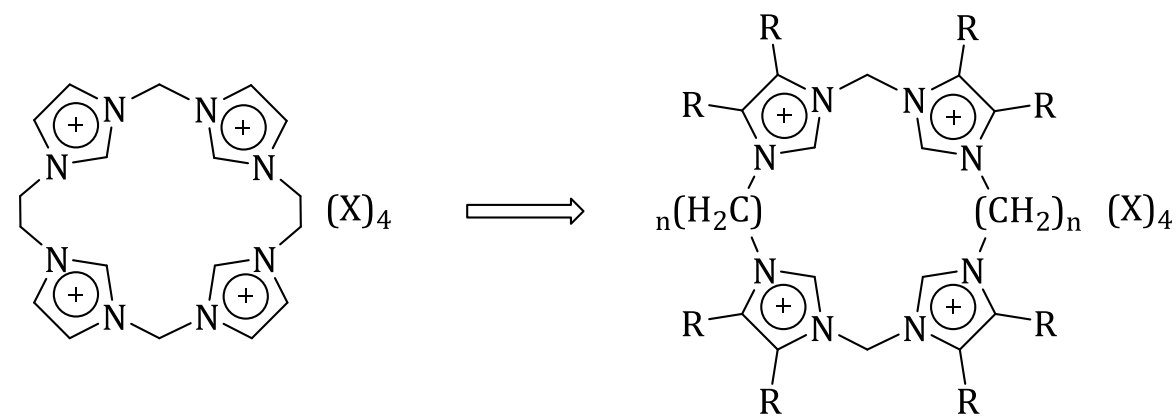

$$
\begin{aligned}
& \mathrm{R}=\mathrm{H}, \mathrm{Me} \\
& \mathrm{n}=1,2
\end{aligned}
$$

Figure 2.7: Modification of the tetracarbene ligand system by changing the size of the macrocycle and the backbone substituents.[182,185,196] 



\subsection{Ligand Synthesis}

The macrocyclic tetracarbene ligand scaffolds $\mathbf{H}_{4} \mathbf{L}^{\mathbf{1}}(\mathrm{OTf})_{4} \mathbf{X I I}$ and $\mathbf{H}_{4} \mathbf{L}^{3}\left(\mathrm{PF}_{6}\right)_{4} \mathbf{X X I I}$ with protons in the imidazole backbone were synthesized according to optimized literature procedures from a bis-imidazole precursor (Scheme 2.5).[182,184] The new octamethyl analogues $\mathbf{H}_{4} \mathbf{L}^{2}(\mathrm{OTf})_{4} \mathbf{X X I}$ and $\mathbf{H}_{4} \mathbf{L}^{4}\left(\mathrm{PF}_{6}\right)_{4} \mathbf{X X I I I}$ were synthesized by using the same conditions. For the 18 membered rings $\left(\mathbf{H}_{4} \mathbf{L}^{\mathbf{1}}(\mathrm{OTf})_{4}\right.$ and $\left.\mathbf{H}_{4} \mathbf{L}^{2}(\mathrm{OTf})_{4}\right)$ bis-imidazoles $\mathbf{X I X}$ or $\mathbf{X X}$ were reacted with ethan-1,2-diylbis-(trifluormethanesulfonate) XVI in diluted MeCN solutions. The isolated yields were found to increase from $15 \%$ to more than $24 \%$, when diluted XVI was added to a more concentrated solution of the bis-imidazole in MeCN (instead of adding pure XVI to a diluted solution of XIX as descripted by JenKINS[184]). For $\mathbf{H}_{4} \mathbf{L}^{2}(\mathrm{OTf})_{4}$ the yield was even higher (33\%).

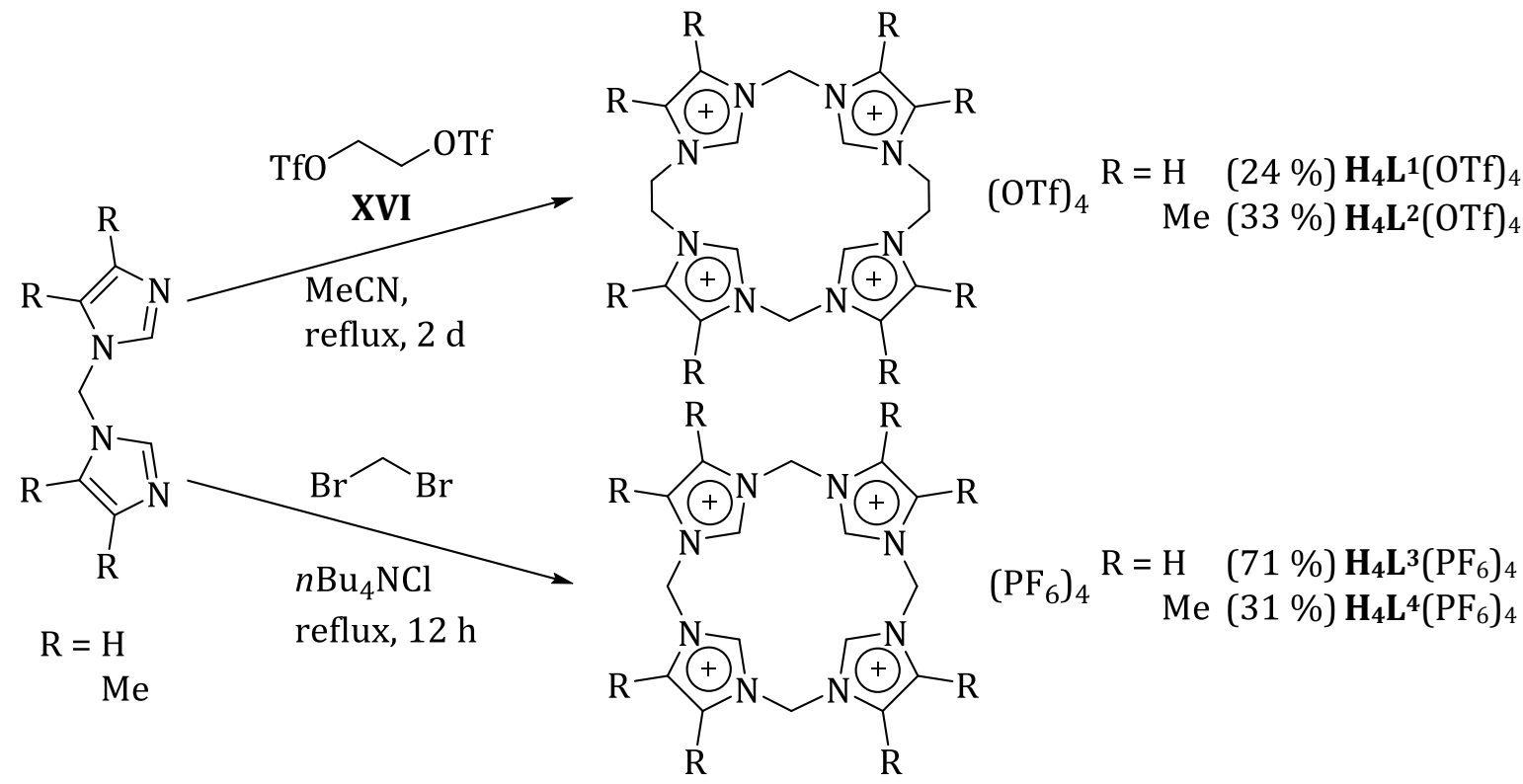

Scheme 2.5: Synthetic routes to macrocyclic tetracarbene ligand precursors XII, XXI, XXII and XXIII.[182,184]

A salt metathesis of the triflate compound XII was performed by adding tetra- $n$ butylammonium chloride to a solution of XII in a mixture of MeCN and DMSO (Scheme 2.6). The resulting white precipitate was filtered off and washed with DCM to remove tetra- $n$ butylammonium triflate. The reaction was quantitative and XXIV was found to be only soluble in water. For the octamethyl derivative XXI analogous salt metatheses did not lead to the desired products since always mixtures of different counterions were found. This might be due to a different solubility of XXI.

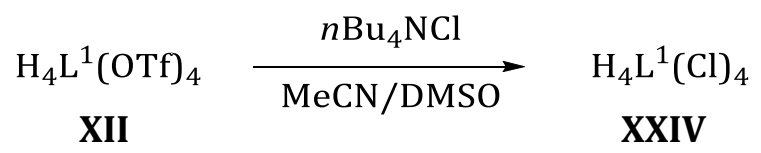

Scheme 2.6: Synthesis of $\mathbf{H}_{4} \mathbf{L}^{1}(\mathrm{Cl})_{4}$ XXIV. 
The smaller, 16 membered ring $\mathbf{H}_{4} \mathbf{L}^{3}\left(\mathrm{PF}_{6}\right)_{4}$ and the new methylated analogue $\mathbf{H}_{4} \mathbf{L}^{4}\left(\mathrm{PF}_{6}\right)_{4}$ were synthesized by a template assisted ring closing reaction in dibromomethane according to a literature-known procedure.[182]

The formation of imidazolium salts was assumed by the appearance of characteristic signals of the $\mathrm{C}-2$ position in the down field region of ${ }^{1} \mathrm{H}$ NMR spectra (Table 2.1). In comparison to $\mathbf{H}_{4} \mathbf{L}^{\mathbf{1}}(\mathrm{OTf})_{4}$ this signal was shifted to higher field in the octamethyl derivative $\mathbf{H}_{4} \mathbf{L}^{2}(\mathrm{OTf})_{4}$ representing a higher electron density in the imidazolium units due to the $+\mathrm{M}$ effect of the methyl groups. This effect was not found for the smaller rings, where the shift of the octamethyl derivative $\mathbf{H}_{4} \mathbf{L}^{4}\left(\mathrm{PF}_{6}\right)_{4}$ is essentially the same as for $\mathbf{H}_{4} \mathbf{L}^{3}\left(\mathrm{PF}_{6}\right)_{4}$. The resonances of the larger rings ( $\mathbf{H}_{4} \mathbf{L}^{1}(\mathrm{OTf})_{4}$ and $\mathbf{H}_{4} \mathbf{L}^{2}(\mathrm{OTf})_{4}$ ) showed a smaller shifts compared to the 16 membered ring analogues $\mathbf{H}_{4} \mathbf{L}^{3}\left(\mathrm{PF}_{6}\right)_{4}$ and $\mathbf{H}_{4} \mathbf{L}^{4}\left(\mathrm{PF}_{6}\right)_{4}$. This trend was also found for the methylene bridges. As expected, no influence of the backbone substitution for the $\mathrm{CH}_{2}$ groups was observed.

Table 2.1: ${ }^{1} \mathrm{H}$ NMR shifts of the imidazolium 2-H and $\mathrm{CH}_{2}$ protons of ligand precursors XII, XXI, XXII and XXIII in DMSO-d6 at $298 \mathrm{~K}$.

\begin{tabular}{ccccc}
\hline ring size & \multicolumn{2}{c}{18 membered } & \multicolumn{2}{c}{16 membered } \\
\hline $\mathrm{R}$ & $\mathrm{H}$ & $\mathrm{Me}$ & $\mathrm{H}$ & $\mathrm{Me}$ \\
\hline ligand & $\mathbf{H}_{4} \mathbf{L}^{\mathbf{1}}(\mathrm{OTf})_{4} \mathbf{X I I}$ & $\mathbf{H}_{4} \mathbf{L}^{2}(\mathrm{OTf})_{4} \mathbf{X X I}$ & $\mathbf{H}_{4} \mathbf{L}^{3}\left(\mathrm{PF}_{6}\right)_{4} \mathbf{X X I I}$ & $\mathbf{H}_{4} \mathbf{L}^{4}\left(\mathrm{PF}_{6}\right)_{4} \mathbf{X X I I I}$ \\
\hline 2-H $[\mathrm{ppm}]$ & 9.00 & 8.77 & 9.80 & 9.85 \\
\hline $\mathrm{CH}_{2}[\mathrm{ppm}]$ & 6.51 & 6.50 & 6.79 & 6.81 \\
\hline
\end{tabular}

Colorless crystals of XXI were obtained by slow evaporation of a MeCN solution. The crystallographic data was not sufficiently good for a complete resolution of the molecular structure (too many unresolved and disordered solvent molecules), but the formation of the macrocyclic ring structure could be proven (Figure 2.8).
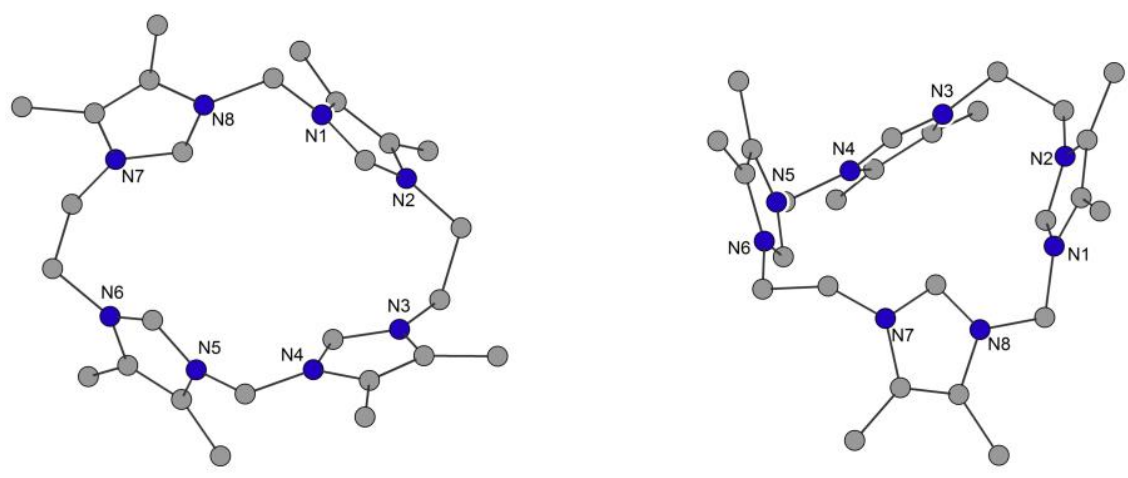

Figure 2.8: Stick-and-ball representation of the molecular structure of XXI in two orientations. Triflate counterions, hydrogen atoms and solvent molecules were omitted for clarity.

In all reactions also the formation of a cyclic bis-imidazolium compound ( $\mathbf{X X V}$ and $\mathbf{X X V I )}$ as side product was observed by ${ }^{1} \mathrm{H}$ NMR spectroscopy, that could not be separated from the 
target molecule. ${ }^{[180,197]}$ This side product did not effect the reaction of different metal ions in complexation attempts with the macrocyclic ligands.

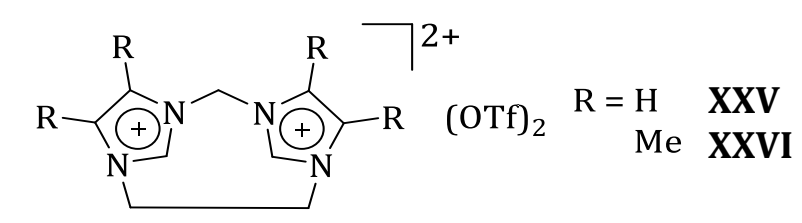

Scheme 2.7: Side products XXV and XXVI which occurred in the ring closing reaction.

\subsection{Complex Synthesis}

To synthesize the desired third row transition metal complexes of the macrocyclic tetracarbene ligand scaffold, mainly two strategies were applied. For the direct route the imidazolium salts were combined with four equivalents of a suitable base and one equivalent of a metal salt. The disadvantage of this reaction pathway is the simultaneous formation of two equivalents of triflate salt, which are well soluble and difficult to separate from the complex. Additionally, the transmetallation route via silver(I) complexes with subsequent transmetallation was applied. Therefore, the ligand precursors were reacted with silver oxide, yielding tetrameric silver carbene complexes, which were then used for transmetallation reactions with the corresponding metal halide salts. A practical issue in these reactions was the complete separation of the formed silver halide as an insoluble salt.

\subsubsection{Silver Complexes}

In order to follow the transmetallation route, ligand precursors $\mathbf{H}_{4} \mathbf{L}^{\mathbf{1}}(\mathrm{OTf})_{4}, \mathbf{H}_{4} \mathbf{L}^{2}(\mathrm{OTf})_{4}$, $\mathbf{H}_{4} \mathbf{L}^{3}\left(\mathrm{PF}_{6}\right)_{4}$ and $\mathbf{H}_{4} \mathbf{L}^{4}\left(\mathrm{PF}_{6}\right)_{4}$ were used to synthesize tetranuclear silver(I) complexes $\mathbf{2 5}$ - $\mathbf{2 8}$ with the general formula $\left[\mathrm{Ag}_{4}\left(\mathrm{Lx}_{2}\right](\mathrm{X})_{4}\right.$ (Scheme 2.8). As already observed by KLAWITTER, the use of silver triflate and triethylamine as reported by JENKINS led not to the formation of the targeted complexes.[163,192] In consequence, silver oxide, was reacted with the tetraimidazolium salts in $\mathrm{MeCN}$ at elevated temperatures. Important to note, it was found that above an oil bath temperature of $70^{\circ} \mathrm{C}$ no silver complexes were observed due to decomposition of the formed products. Remaining $\mathrm{Ag}_{2} \mathrm{O}$ was removed by filtration and the desired products were precipitated with $\mathrm{Et}_{2} \mathrm{O}$. The structure of the tetrameric compounds is assumed to be analogous to the reported silver complex of the octaphenyl ligand.[163] 


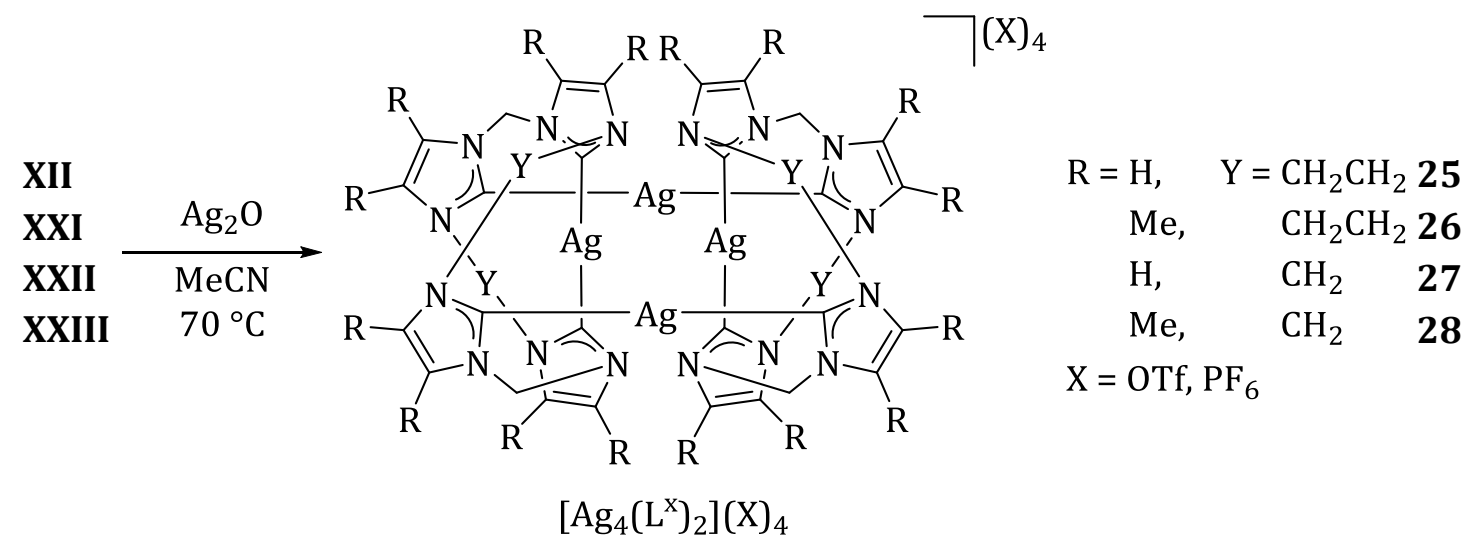

Scheme 2.8: Synthesis of tetranuclear silver carbene complexes 25 - 28.

The formation of silver carbene complexes was proven by ${ }^{1} \mathrm{H}$ NMR spectroscopy, where the signals of the imidazolium 2-H (between 8 - 10 ppm, Table 2.1) vanished. Carbon resonances around $180 \mathrm{ppm}$ were found in ${ }^{13} \mathrm{C}$ NMR spectra indicative for metal carbene complexes.[163] ESI-MS measurements of the tetranuclear complexes showed that the complex cation was not stable under the ESI conditions and only fragments like $\left[\mathrm{Ag}_{2} \mathrm{~L}^{\mathrm{x}}(\mathrm{OTf})\right]^{+}$and $\left[\mathrm{Ag}_{2} \mathrm{~L}^{\mathrm{x}}\right]^{2+}$ were found. The purification proved to be difficult and even visually crystalline material gave no clean NMR spectra. Since the silver complexes could be seen as intermediates during the synthesis of the transition metal complexes, the crude products were used without further purification for the transmetallation reactions.

High valent silver(III) complexes are rarely described in literature mainly with nitrogen based donor sets such as ethylene-bis-(guanide) or macrocyclic porphyrin or corrole derivatives.[198200] They were synthesized by reacting the silver(I) complexes with an excess of a silver(I) salt or strong oxidants like potassium peroxidisulfate. To probe the ability of the tetracarbene ligand system of stabilising high valent silver complexes, silver(I) complex $\mathbf{2 6}$ was reacted with xenon difluoride $\left(\mathrm{XeF}_{2}\right)$ as strong oxidant at low temperature in an UV-vis cuvette. However, no change in the absorption bands could be observed even with an excess of $\mathrm{XeF}_{2}$. A problem might be the required reorganization of the cluster.[163] In $\mathbf{2 6}$ a silver to ligand ratio of 2:1 is assumed, but in an expected silver(III) complex each silver ion should be coordinated by just one ligand.

\subsubsection{Nickel Complexes}

Nickel(II) complexes of the larger macrocyclic tetracarbene ligands XII and XXI were synthesized, characterized and the redox reactivity of the resulting complexes was evaluated. A nickel(II) complex of the octaphenyl derivative of this ligand system was previously reported by the group of JENKINS and synthesized via transmetallation of the corresponding tetranuclear silver complex.[163] 


\subsubsection{Synthesis}

Nickel(II) complex 29 of the unsubstituted tetracarbene ligand XII was synthesized via transmetallation of the corresponding silver complex 25 with $\mathrm{NiBr}_{2}(\mathrm{dme})$ (dme = 1,2-dimethoxyethane) or $\mathrm{NiCl}_{2}$ in $\mathrm{MeCN}$ (Scheme 2.9). The resulting silver salts were removed by filtration and polycrystalline 29 was obtained by layering the yellow filtrate with $\mathrm{Et}_{2} \mathrm{O}$ in yields up to $66 \%$. Complex 30 with chlorides instead of triflates as counterions was synthesized via a direct complexation of $\mathbf{H}_{4} \mathbf{L}^{1} \mathrm{Cl}_{4}$ XXIV with nickel(II) acetate and sodium acetate in refluxing DMSO according to a literature procedure for a similar nickel tetracarbene complex containing a larger ring size.[201]

$$
\left[\mathrm{Ag}_{4}\left(\mathrm{~L}^{1}\right)_{2}\right](\mathrm{OTf})_{4}+\mathrm{NiCl}_{2}
$$

Scheme 2.9: Synthesis of $\mathbf{2 9}$ and $\mathbf{3 0}$ containing the unsubstituted tetracarbene ligand XII via transmetallation of $\left[\mathrm{Ag}_{4}\left(\mathrm{~L}^{1}\right)_{2}\right](\mathrm{OTf})_{4}$ and direct synthesis from $\mathbf{H}_{4} \mathbf{L}^{\mathbf{1}} \mathrm{Cl}_{4}$.

For the synthesis of $\mathbf{3 1}$ both strategies were used (Scheme 2.10): The transmetallation pathway by combining silver complex 26 and $\mathrm{NiBr}_{2}(\mathrm{dme})$ in $\mathrm{MeCN}$ and the direct route by reacting $\mathrm{H}_{4} \mathrm{~L}^{2}(\mathrm{OTf})_{4} \mathrm{XXI}$ with $\mathrm{NiBr}_{2}(\mathrm{dme})$ and cesium carbonate $\left(\mathrm{Cs}_{2} \mathrm{CO}_{3}\right)$ as base. The direct route worked only with $\mathrm{Cs}_{2} \mathrm{CO}_{3}$ as base, neither with stronger bases like sodium hydride or $\mathrm{KO}{ }^{t} \mathrm{Bu}$ nor with other weak bases such as sodium carbonate and triethylamine. Both strategies gave $\mathbf{3 1}$ in similar yields (50 to $60 \%$ ). However, the transmetallation reaction with an easy and fast workup could only be done on a small scale, otherwise the yield decreased dramatically. On the other hand, the direct reaction of the ligand with a nickel salt and base required a more time consuming purification, which was possible with a larger amount of substance.

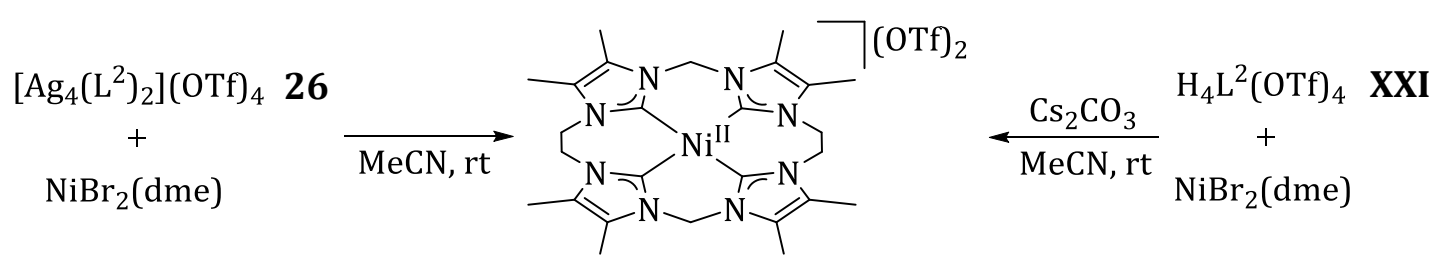

31

Scheme 2.10: Synthesis of 31 via transmetallation (left side) or direct route with addition base (right side).

All three nickel(II) salts were air, water and temperature (tested up to $120^{\circ} \mathrm{C}$ ) stable and they showed different solubility properties. The unsubstituted complexes 29 and $\mathbf{3 0}$ were only soluble in water, $\mathrm{MeOH}$ and $\mathrm{MeCN}$, whereas the methylated complex 31 could be dissolved in 
less polar solvents. The substitution of the protons by methyl groups in the imidazole backbone led to the desired effect: complex $\mathbf{3 1}$ with the methylated ligand was also soluble in acetone, DCM and slightly in THF. This provides practical advantages for further reactions.
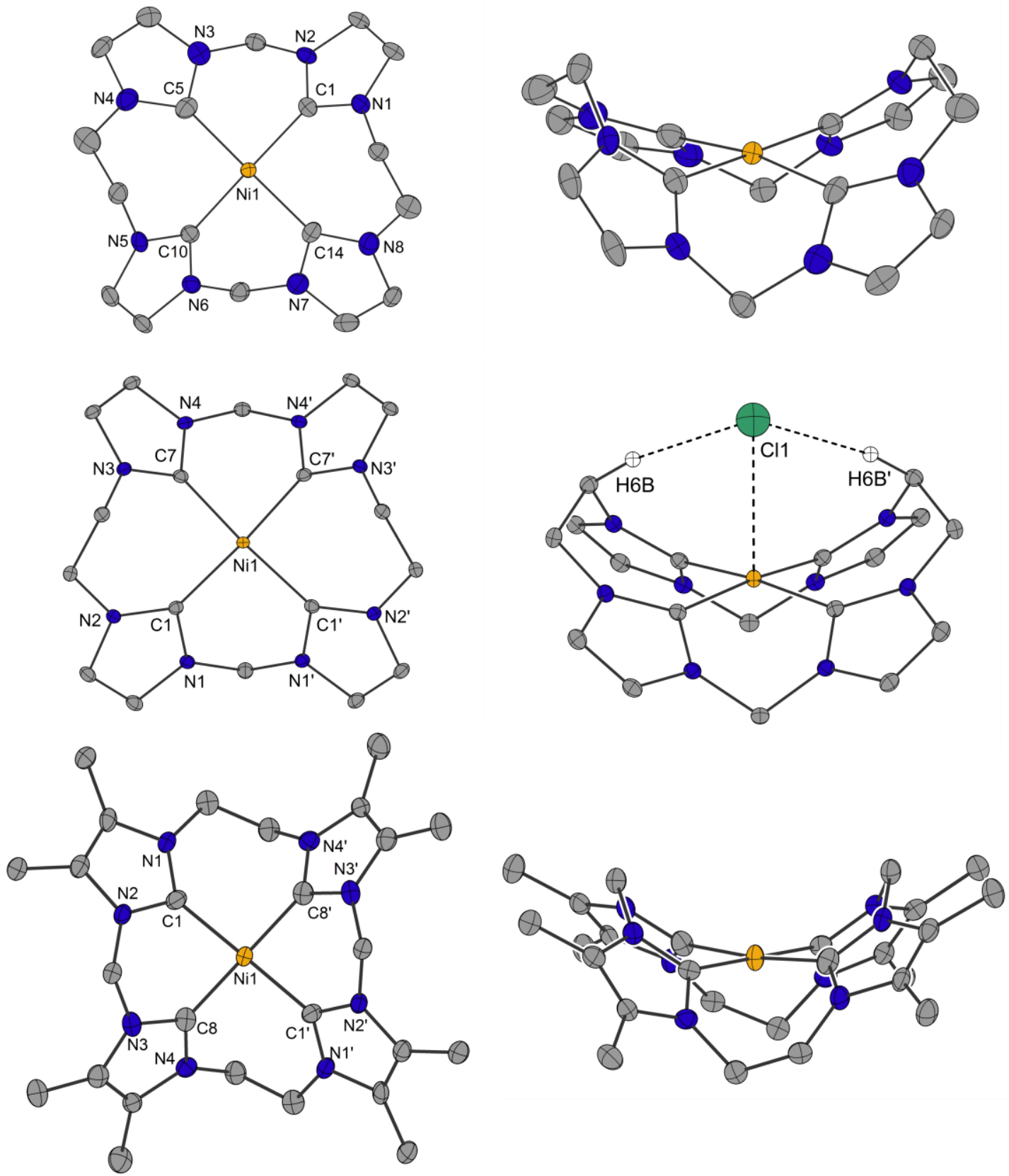

Figure 2.9: Thermal displacement ellipsoids (shown at $30 \%$ probability) of the molecular structures of [NiL $\left.{ }^{1}\right]\left(\mathrm{OTf}_{2}\right.$ 29 (top), [ $\left.\mathrm{NiL}^{1}\right](\mathrm{Cl})_{2} 30$ (middle) and [ $\left.\mathrm{NiL}^{2}\right](\mathrm{OTf})_{2} \mathbf{3 1}$ (bottom) in two orientations (left: top view, right: side view). Counterions, hydrogen atoms and solvent molecules were omitted for clarity.

In the solid state very similar structures for the three complexes were found (Figure 2.9). 29 crystallizes in the triclinic space group $\mathrm{P} \overline{1}, \mathbf{3 0}$ and $\mathbf{3 1}$ in monoclinic space groups $\mathrm{P} 12_{1} / \mathrm{m} 1$ and C12/c1. The nickel ions are coordinated in a distorted square planar fashion by the four carbene moieties of the ligands (Figure 2.9). The macrocyclic ring is bent and the imidazole ring planes are twisted against the central carbon plane. To calculate the distortion from ideal square planar geometry the methods by HOUSER and a modified version of it by KUBIAK were 36 
applied, using equations 1 and 2 with $\alpha$ and $\beta$ being the largest and second largest $\mathrm{C}-\mathrm{Ni}-\mathrm{C}$ angles.[202,203]

$$
\begin{gathered}
\tau_{4}=\frac{360^{\circ}-(\alpha+\beta)}{141^{\circ}} \\
\tau_{\delta}=\frac{360^{\circ}-(\alpha+\beta)}{141^{\circ}} \cdot \frac{\beta}{\alpha}
\end{gathered}
$$

With $\tau_{4}$ and $\tau_{\delta}$ values smaller 0.15 all complexes can be categorized as distorted square planar (Table 2.2). The carbon nickel bond lengths are in the range of $1.881-1.940 \AA$ (29), $1.878-1.940 \AA$ (30) and $1.873-1.914 \AA$ (31). These distances are in good agreement with similar literature known nickel(II) tetracarbene complexes.[163,201] The metal atom positions are perfectly in the plane of the carbon donor atoms in complex 31 and almost perfectly in 29, with a distance of $0.0036 \AA$ to the plane. In 30 this distance to the carbon atom plane is longer $(0.126 \AA)$ due to weak, axial contact to a chloride counterion. The $\mathrm{Ni} \cdots \mathrm{Cl}$ distance is $3.265 \AA$. There are also two weak hydrogen bond interactions between the ethylene linkers each and the chloride ( $2.568 \AA$ A , Figure 2.9 bottom center). Comparable hydrogen bond interactions were also found in the iron(IV) oxo complex 23 with weak contacts between the oxygen atom and protons of the ethylene linkers. ${ }^{[186]}$

Table 2.2: Selected bond lengths, angles and structural parameter $\tau_{4}$ and $\tau_{\delta}$ for the deviation from ideal square planar coordination geometry of complexes 29 - 31.

\begin{tabular}{ccccccc}
\hline & $\mathrm{dNi}-\mathrm{C}[\AA]$ & $\mathrm{dNi}$-plane $[\AA]$ & $\alpha\left[^{\circ}\right]$ & $\beta\left[^{\circ}\right]$ & $\tau_{4}$ & $\tau_{\delta}$ \\
\hline $\mathbf{2 9}$ & $\begin{array}{l}1.881,1.882 \\
1.914,1.940\end{array}$ & 0.126 & 178.8 & 169.5 & 0.083 & 0.079 \\
\hline $\mathbf{3 0}$ & $\begin{array}{l}1.878,1.878 \\
1.940,1.940\end{array}$ & 0.004 & 171.6 & 171.6 & 0.119 & 0.119 \\
\hline $\mathbf{3 1}$ & $\begin{array}{l}1.873,1.873 \\
1.914,1.914\end{array}$ & 0 & 179.7 & 173.6 & 0.048 & 0.046 \\
\hline
\end{tabular}

The bend structure in the solid state was also present in solution. ${ }^{1} \mathrm{H}$ NMR spectra showed the protons of the methylene and ethylene groups to be inequivalent due to slow boat-chair interconversion on the NMR timescale. The methylene protons are split into two doublets while the two signals for the ethylene protons appeared as complicated multiplets (Figure 2.10). This rigidity remained present even at elevated temperature of $80^{\circ} \mathrm{C}$ (Figure 2.11). Almost no change of the spectrum was observed at high temperatures compared to the measurement at ambient conditions. This effect was also observed for other nickel tetracarbene complexes.[184,201,204,205] 


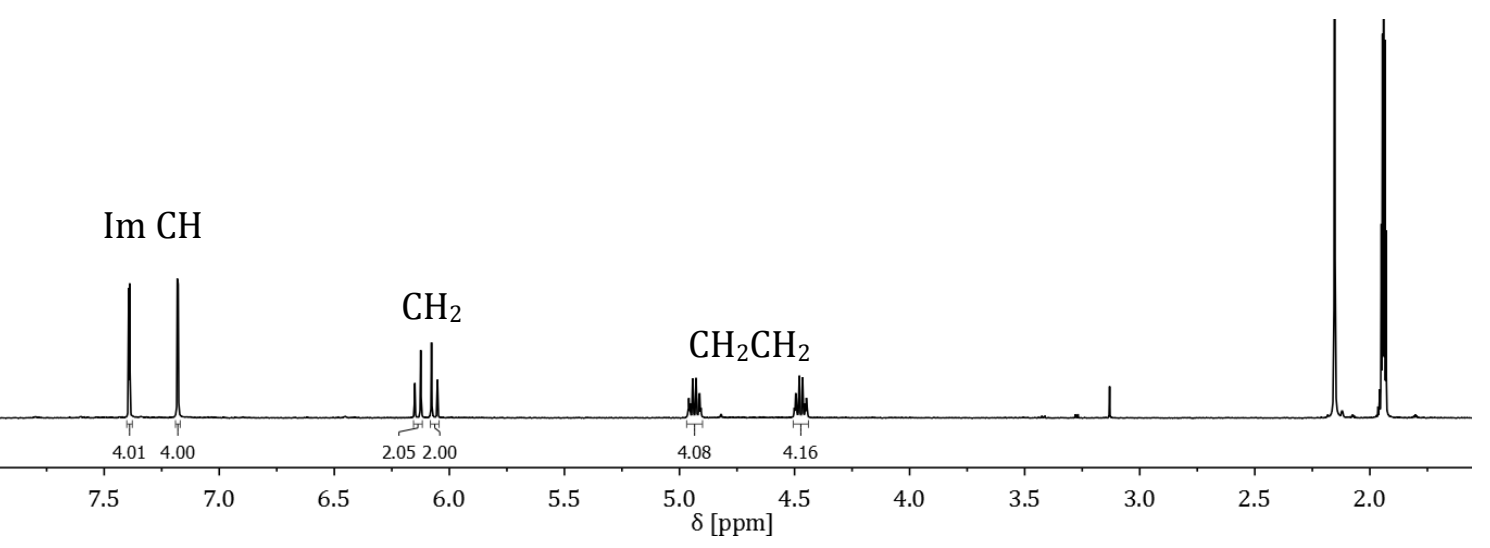

Figure 2.10: ${ }^{1} \mathrm{H}$ NMR spectrum of $\mathbf{3 0}$ showing the rigidity of the methylene and ethylene linkers on the NMR timescale (acetonitrile- $\mathrm{d}_{3}, 298 \mathrm{~K}, 500 \mathrm{MHz}$ ).

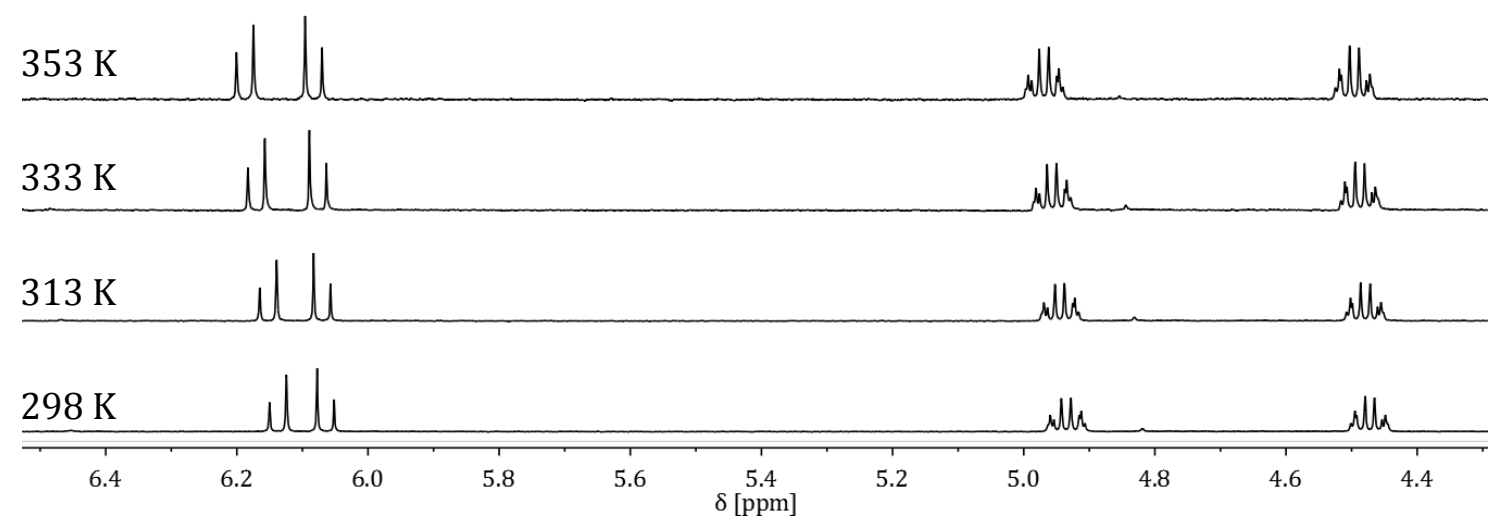

Figure 2.11: Temperature dependent ${ }^{1} \mathrm{H}$ NMR spectra of $\mathbf{3 0}$ (acetonitrile- $\mathrm{d}_{3}$ ) showing the resonances for the methylene (6.1 ppm) and ethylene (4.9 ppm and $4.5 \mathrm{ppm})$ linkers.

For investigation of the redox properties of the complexes, cyclic voltammetry experiments were carried out (Figure 2.12). In general, in all three complexes redox processes at high potentials for both reductions and oxidations were found, reflecting the high stability of these compounds. In addition, only irreversible redox events were observed, which indicates the instability of potential reduction or oxidation products. The values of the redox potentials are listed in Table 2.3.

Table 2.3: Oxidation $\left(\mathbf{E}_{\mathbf{o x}}\right)$ and reduction $\left(\mathbf{E}_{\text {red }}\right)$ peak potentials for complexes $\mathbf{2 9}-\mathbf{3 1}$ measured in MeCN at $100 \mathrm{mV} / \mathrm{s}$ scan rate and referenced versus $\mathrm{Fc} / \mathrm{Fc}^{+}$.

\begin{tabular}{cccccc}
\hline complex & $\mathrm{E}_{\text {ox }}^{1}[\mathrm{~V}]$ & $\mathrm{E}_{\text {ox }}^{2}[\mathrm{~V}]$ & $\mathrm{E}_{\text {red }}^{1}[\mathrm{~V}]$ & $\mathrm{E}_{\text {red }}^{2}[\mathrm{~V}]$ & $\mathrm{E}_{\text {red }}^{3}[\mathrm{~V}]$ \\
\hline $\mathbf{2 9}$ & 1.67 & 1.89 & -1.48 & & \\
\hline $\mathbf{3 0}$ & 0.426 & 0.833 & -1.34 & -1.69 & \\
\hline $\mathbf{3 1}$ & 0.748 & 1.20 & -1.25 & -2.41 & -2.51 \\
\hline
\end{tabular}

For all tree complexes two oxidation processes were found. However, the first one was less prominent, especially for $\mathbf{3 1}$. The highest peak potentials were obtained for $\mathbf{2 9}$ (higher than 
$1.6 \mathrm{~V} v s . \mathrm{Fc} / \mathrm{Fc}^{+}$), wheras in $\mathbf{3 0}$ both oxidation waves were found at peak potentials lower than $1 \mathrm{~V} v s$. Fc/Fc $\mathrm{Fc}^{+}$. For 29 only one reduction event could be observed at $-1.48 \mathrm{~V}$, while two and three events were found for $\mathbf{3 0}$ and 31, respectively. Due to the eight methyl groups of 31, more electron density is located on the metal and thus the reduction of the nickel(II) is more difficult. This is reflected by the low potentials for the second and third reduction in 31, although the first reduction was found at $-1.25 \mathrm{~V}$.
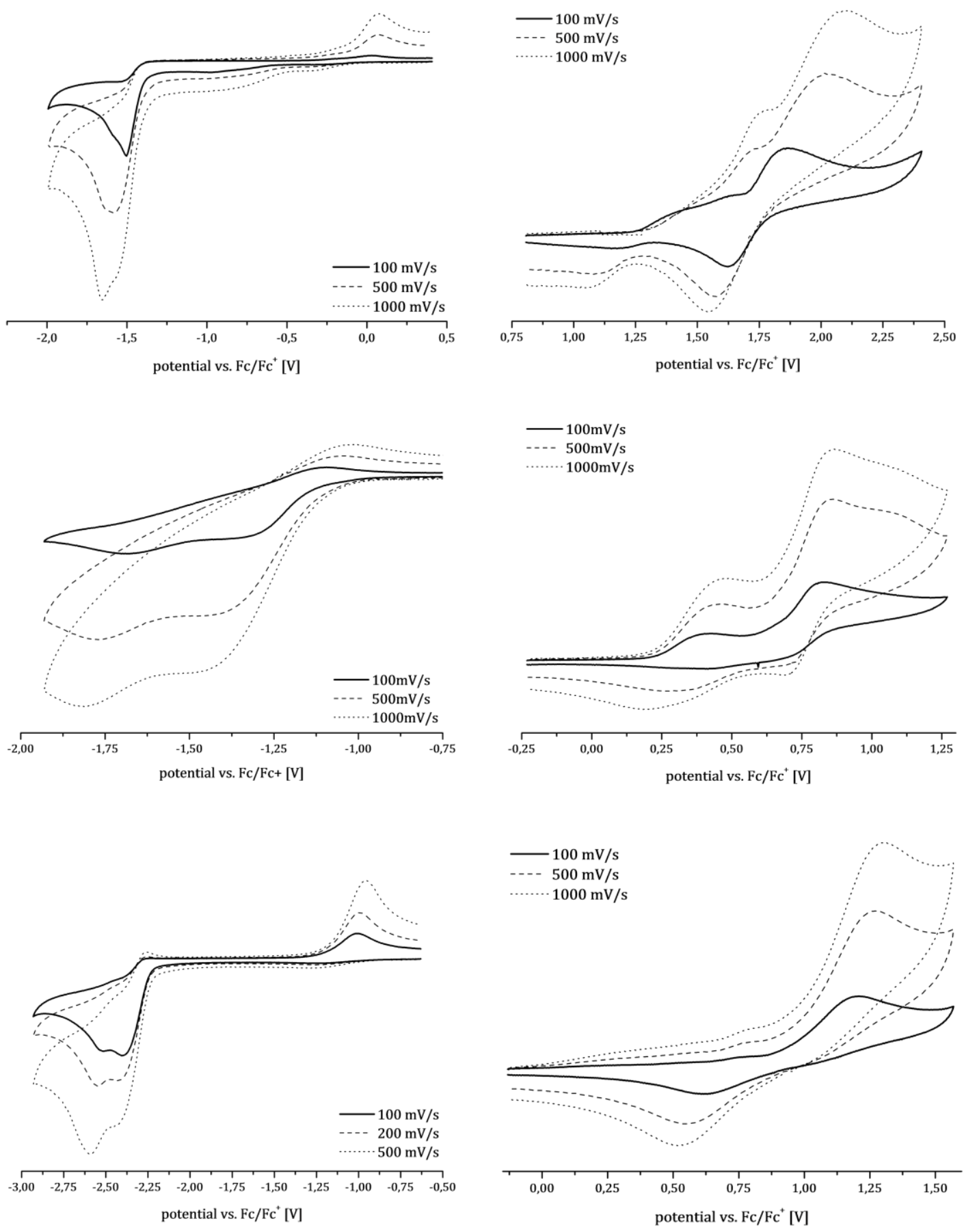

Figure 2.12: Cyclic voltammograms (measured in $0.1 \mathrm{M} n \mathrm{Bu}_{4} \mathrm{NPF}_{6}$ in $\mathrm{MeCN}$ ) of nickel(II) complexes 29 (top), $\mathbf{3 0}$ (middle) and $\mathbf{3 1}$ (bottom). The reduction (left) and oxidation (right) events are shown separately at different scan rates. 
For the smaller ring size ligands XXII and XXIII the same reaction conditions were applied, but different conditions seemed to be required for the synthesis of those complexes since no isolation of nickel(II) complexes was possible. In crude reaction mixtures the formation of $\left[\mathrm{NiL}^{3}\right]^{2+}$ was evidenced by an intense peak in an ESI-MS spectrum at $\mathrm{m} / \mathrm{z}=189.1$ correlating well with a simulated isotopic distribution pattern of $\left[\mathrm{C}_{16} \mathrm{H}_{16} \mathrm{~N}_{8} \mathrm{Ni}\right]^{2+}$. However, the ${ }^{1} \mathrm{H}$ NMR spectrum showed very broad, but not paramagnetically shifted, signals, which did not sharpen upon lowering the temperature. Crystals were not obtained from various attempts, so that the exact structure remained uncertain.

\subsubsection{Reactivity}

In order to archieve high valent nickel complexes, compounds 29 - 31 were treated with different oxidants. The reactions with oxygen atom transfer agents including 2-(tertbutylsulfonyl)-iodosobenzene (sPhIO), metachlorperoxybenzoic acid (mCPBA), Oxone ${ }^{\circledR}$ $\left(\mathrm{KHSO}_{5}\right)$, dimethyloxyrane,[206] ceric ammonium nitrate (CAN) and potassium superoxide $\left(\mathrm{KO}_{2}\right)$ did not yield any oxidation products. The complexes proved to be very stable since no decomposition was observed. Also large excesses of the oxidants led to no oxidation products. Strong LEWIS acids such as scandium(III) are known to stabilized oxo species.[207,208] Therefore, $\mathrm{Sc}(\mathrm{OTf})_{3}$ was added to a suspension of 29 and sPhIO at $-35^{\circ} \mathrm{C}$. No change in the UV-vis absorptions was detected. The only reagent causing a reaction was xenon difluoride $\left(\mathrm{XeF}_{2}\right)$ with a high oxidation potential (no values for MeCN solutions are available, but for aqueous acidic solution the $\mathrm{Xe}^{0} / \mathrm{Xe}^{2+}$ potential was estimated to be higher than $2 \mathrm{~V}$ although no reference was mentioned).[209] When solid $\mathrm{XeF}_{2}$ was added to a precooled solution of 29 at $-35^{\circ} \mathrm{C}$ an instant color change from yellow to orange was observed. The reaction could be followed by UV-vis spectroscopy (Figure 2.13, left).
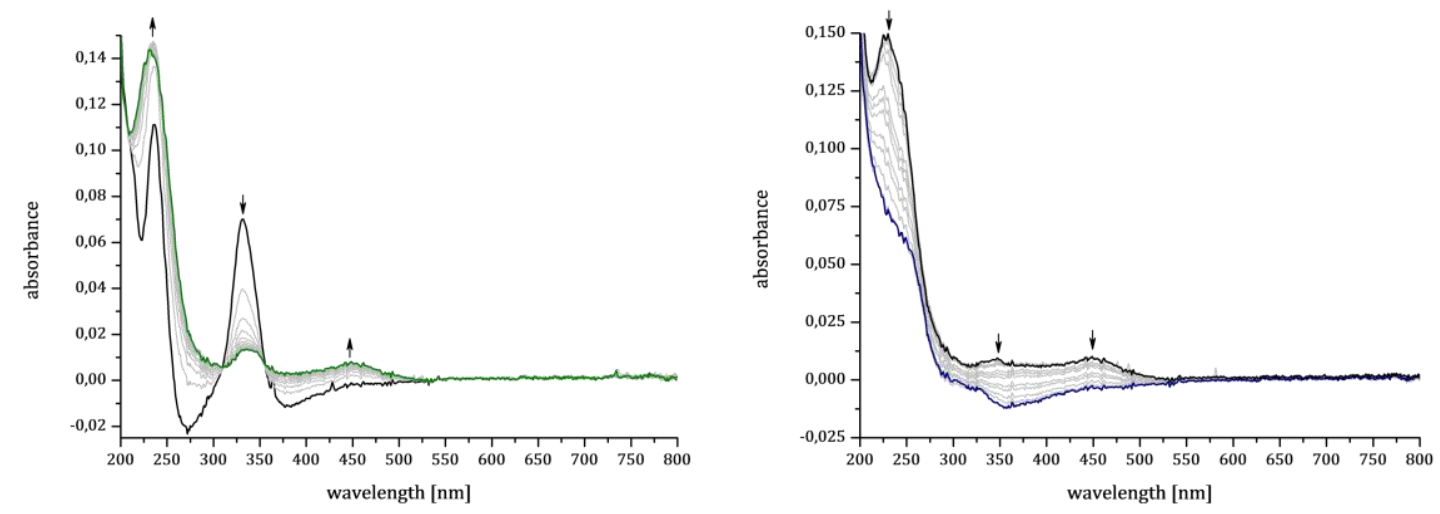

Figure 2.13: Oxidation of 29 with $\mathrm{XeF}_{2}$ at $-35^{\circ} \mathrm{C}$ in $\mathrm{MeCN}$ monitored by UV-vis spectroscopy (left). Decay of the formed $\mathrm{Ni}{ }^{I I I}$ species at $-10{ }^{\circ} \mathrm{C}$ (right).

Upon addition of $\mathrm{XeF}_{2}$ the band at $332 \mathrm{~nm}$ decreased while a new band around $450 \mathrm{~nm}$ arose. Additionally, the UV band at $230 \mathrm{~nm}$ increased in intensity. When the temperature was raised 40 
to $-10^{\circ} \mathrm{C}$ all bands decreased indicating a decomposition of the complex species (Figure 2.13 , right). An EPR spectrum of a frozen complex solution was recorded directly after addition of $\mathrm{XeF}_{2}$ (Figure 2.14). The spectrum measured at $161 \mathrm{~K}$ exhibits broad lines and it was not possible to simulate the splitting pattern in a reasonable way at this temperature.

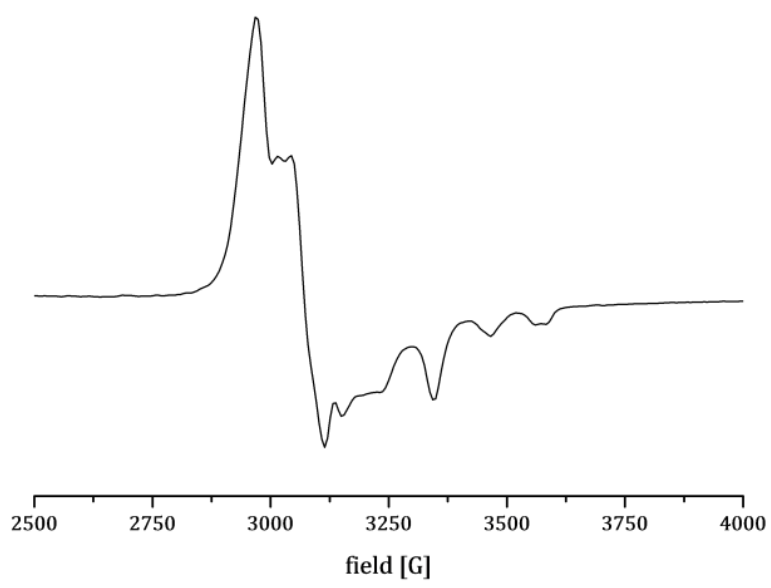

Figure 2.14: EPR spectrum of a frozen solution recorded directly after addition of $\mathrm{XeF}_{2}$ to $\left[\mathrm{NiL}^{1}\right](\mathrm{OTf})_{2} 29$ in MeCN $(161 \mathrm{~K})$.

Despite these promising observations, it was not possible to further characterize or isolate the reaction product due to its low stability. The color of a nickel(III) solution changed back to yellow within two hours at low temperature even in the presence of an excess of $\mathrm{XeF}_{2}$. Upon slow diffusion of $\mathrm{Et}_{2} \mathrm{O}$ into a concentrated reaction mixture at $-80{ }^{\circ} \mathrm{C}$, the yellow starting nickel(II) complex precipitated.

Azides are versatile reagents to produce metal nitrides or related species.[192,210] The reaction of 29 with one equivalent of tetra- $n$-butylammonium azide $\left(n \mathrm{Bu}_{4} \mathrm{~N}-\mathrm{N}_{3}\right)$ led to no change in an ATR-IR spectrum except for the appearance of the free azide band at $1999 \mathrm{~cm}^{-1}$. Thus, a coordination of the azide to the nickel was not indicative. An exchange of one counterion is likely, but this led not to a reaction at the nickel center not even after irradiation of the sample with a UV lamp. 3,5-Dimethylphenyl azide and the nitride transfer agent $N$-tosyl-iminophenyliodinane led also to no desired products.

Since the nickel(II) complexes were hard to oxidize, the opposite reactions were tried.[203] Starting from a nickel(I) species oxidative addition could lead to a nickel(III) species. Hence, 31 was reacted with different amounts of potassium graphite $\left(\mathrm{KC}_{8}\right)$ in THF at low temperatures. ${ }^{[203]}$ A color change of the mixture was not detected, due to the formed black graphite. However, after removing the graphite by filtration a yellow solution was obtained and the nickel(II) complex was found by ESI mass spectrometry. It was assumed, that no stable complex could be formed, since the reduction waves in cyclic voltammograms were completely irreversible (Figure 2.12). 
In summary, a variety of strong oxidants as well as $\mathrm{KC}_{8}$ as reductant were reacted with the synthesized nickel(II) complexes 29 - 31, but only in the case of $\mathrm{XeF}_{2}$ a reaction was observed that could not be quantified. The potential nickel(III) product could be observed by EPR and UV-vis measurements, however it proved to be very instable and decomposed after short periods of time so that an isolation or further characterization was not possible.

\subsubsection{Complexation Attempts with Copper and Manganese}

To synthesize copper complexes of the macrocyclic tetracarbene ligand system, both the direct and the transmetallation route were tried. When using the transmetallation approach, redox activity of silver and copper ions might lead to mixtures of different oxidation states. In fact, in such reactions different color changes were observed, although no copper complexes were identified or isolated. An oxidation of the ligand was observed if the direct complexation reactions were carried out under aerobic conditions, but no copper containing species could be observed in ESI-MS measurements. Generally, carbene complexes are known for their stability against oxidation, but free carbenes are oxidized by dioxygen at the $\mathrm{C}-2$ position forming cyclic urea derivatives, which are not able to coordinate a metal ion.[211] Many different copper salts and bases were tested under various reaction conditions, but in only a very few experiments analytical evidence for copper containing products were found. Routinely ESI-MS spectra of dried crude products were recorded and in the majority of cases no peaks with an isotopic distribution pattern specific for copper were observed. An exception was the reaction of $\mathbf{H}_{4} \mathbf{L}^{2}(\mathrm{OTf})_{4}$ with anhydrous copper bromide in the presence of four equivalents of sodium tert-butanolate as base. After a salt metathesis from bromide/triflate to tetraphenylborate $\left(\mathrm{BPh}_{4}^{-}\right)$the crude mixture contained at least two peaks, which could be assigned to a copper(I) and a copper(III) dibromide species. However, the intensity was really low and these two species may also be formed in the ionization process of the measurement. By taking a closer look at the other peaks of the spectrum, it was noticeable that there were several peaks for singly charged ions with a rather low $\mathrm{m} / \mathrm{z}$ ratio exhibiting an organic isotopic distribution pattern. For example, a peak at $\mathrm{m} / \mathrm{z}=231.1$ was also observed frequently before in other spectra, also in reactions with manganese salts. It was excluded that these peaks came from the background. A reasonable explanation was the decomposition of the macrocyclic ligand to smaller units with two imidazole rings bridged by methylene or ethylene groups. The isotopic distribution pattern of a peak at $\mathrm{m} / \mathrm{z}=231.1$ was well simulated with a formula of $\mathrm{C}_{12} \mathrm{H}_{14} \mathrm{~N}_{4} \mathrm{O}$. Since the methylene groups should be more reactive, a possible decomposition product might be an ethylene bridged bis-imidazole (formula: $\mathrm{C}_{12} \mathrm{H}_{18} \mathrm{~N}_{4}$ ). After all it remained uncertain how the number of hydrogen atoms should have decreased to fit to the found formula $\left(\mathrm{C}_{12} \mathrm{H}_{14} \mathrm{~N}_{4} \mathrm{O}\right)$ although the oxidation of the $\mathrm{C}-2$ carbon is chemical reasonable. 
It was tested whether dioxygen or water might have an influence on the reaction. Therefore, a crude reaction mixture of $\mathbf{H}_{4} \mathbf{L}^{2}(\mathrm{OTf})_{4}, \mathrm{CuCl}_{2}$ and $\mathrm{NaO}{ }^{t} \mathrm{Bu}$ in $\mathrm{MeCN}$ was portioned into two Schlenk tubes. Then degassed water was added to one sample and dioxygen was bubbled through the second sample. In both cases no color change occurred. Crystallization attempts of both samples led to the same small, intense blue cubic crystals. Unexpectedly, the macroscopic geometry was found to represent also the molecular structure. The cubic structure of $\left[\mathrm{Cu}_{4}{ }_{4}\left(\mu_{3}-\mathrm{OH}\right)_{4} \mathrm{~L}_{4}\right](\mathrm{OTf})_{4} 32$ with a $\left[\mathrm{Cu}_{4} \mathrm{O}_{4}\right]$ core is depicted in Figure 2.15. The $\left[\mathrm{Cu}_{4}\left(\mu_{3}-\mathrm{OH}\right)_{4}\right]$ cubane like core is coordinated by four bis-(4,5-dimethylimidazol-1-yl)-ethane XXVII ligands capping a $\mathrm{Cu}_{2} \mathrm{O}_{2}$ face each. The $\mathrm{C}-2$ atoms of the imidazoles were protonated and the coordination took place via the unsubstituted nitrogen atoms of the imidazole rings. The geometric form of the core is a distorted rectangular cuboid, having $\mathrm{Cu}-\mathrm{O}-\mathrm{Cu}$ angles between $77.6^{\circ}$ and $81.5^{\circ}$ and the sum of angles in each plane is almost ideally $360^{\circ}\left(357.8^{\circ}-358.6^{\circ}\right)$. The $\mathrm{Cu}-\mathrm{O}$ distances in the square planes were very similar and in the range of $1.959 \AA$ and $1.981 \AA$, whereas the other Cu-O distances were longer (averaged $2.394 \AA$ ). Cluster 32 showed structurally similarity to nickel and cobalt cubane type complexes with 2-(1-(2-hydroxyethyl)1- $H$-pyrazole-3-yl)-phenols as ligands, which have shown interesting solvomagnetic properties.[212,213] Since only very few clean crystals were obtained, investigations regarding those properties were not possible so far.
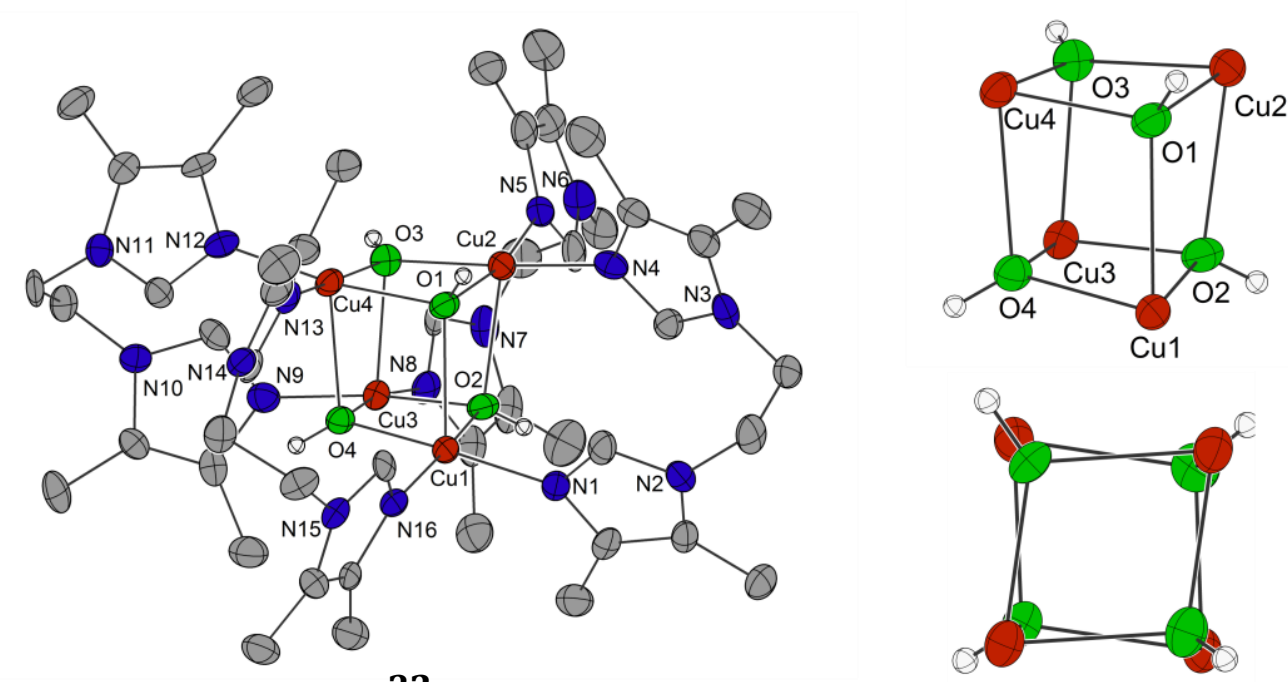

32

Figure 2.15: Thermal displacement ellipsoids (shown at $30 \%$ probability) of the molecular structure of $\left[\mathrm{Cu}_{4}\left(\mu_{3}-\mathrm{OH}\right)_{4} \mathrm{~L}_{4}\right]^{4+}(32$, left, with $\mathrm{L}=\mathbf{X X V I I})$ and the inner $\left[\mathrm{Cu}_{4} \mathrm{O}_{4}\right]$ core without $\mathrm{L}$ in two orientations (right). Triflate counterions, hydrogen atoms and solvent molecules were omitted for clarity.

In conclusion, the isolation of this cubane type complex $\mathbf{3 2}$ with XXVII as ligands showed that the macrocyclic ligand $\mathbf{X X I}$, at least in parts, decomposed in mixtures with copper(II) salts and base. However, is was not possible to work out whether these crystals were just a minor decomposition product due to an elongated time in solution or whether it represented a general chemical problem of the attempted synthesis of tetracarbene copper(II) complexes, 
namely the decomposition of the ligand in the presence of $\mathrm{Cu}^{2+}$. Therefore, Chelex $100^{\circledR}, \mathrm{a}$ styrene-divenylbenzene co-polymer containing iminodiacetic acid groups to bind transition metal ions ${ }^{[214]}$, was added to a crude reaction mixture of a reaction between copper triflate, base and $\mathbf{H}_{4} \mathbf{L}^{2}(\mathrm{OTf})_{4}$ in MeCN. After several hours the color of the polymer changed to blue indicating the uptake of $\mathrm{Cu}^{2+}$ ions. A ${ }^{1} \mathrm{H}$ NMR spectrum of the dried filtrate showed minimal amounts of ligand XXI and various resonances between 1 and 3 ppm characteristic for small organic molecules (Figure 2.16). The decomposition of the ligand occurred also at low temperatures.

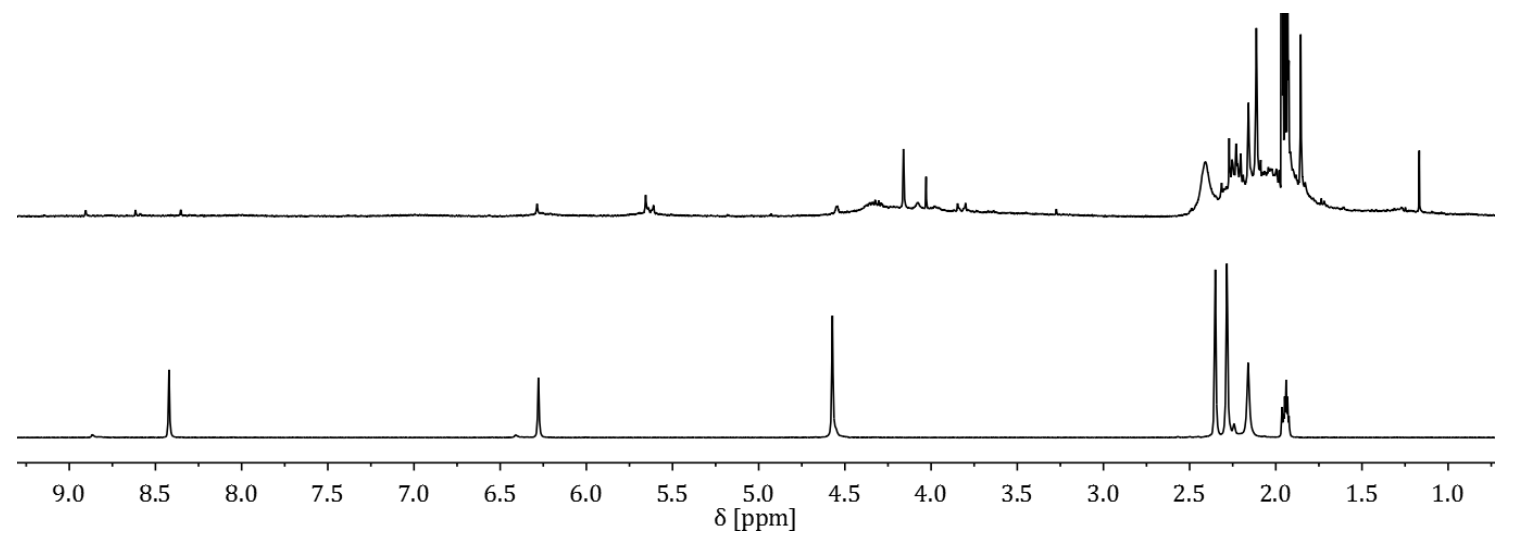

Figure 2.16: ${ }^{1} \mathrm{H}$ NMR spectra of a reaction mixture of copper triflate, base and $\mathrm{H}_{4} \mathrm{~L}^{2}(\mathrm{OTf})_{4}$ in $\mathrm{MeCN}$ after treatment with Chelex 100 (top) and ligand XXI (bottom), both in acetonitrile-d $\mathrm{d}_{3}$ at $298 \mathrm{~K}$.

According to CHEN et al. also the reaction of the ligand precursors with elemental copper powder was tried.[164] The metal powder and the ligand were suspended in MeCN and heated to reflux for several hours. According to ESI mass spectrometry and ${ }^{1} \mathrm{H}$ NMR spectroscopy no formation of any copper complex containing the carbene ligand was observed.

Very similar results as for the copper were also obtained for manganese. The ligand precursors and the silver(I) complexes were reacted with various manganese(II) and manganese(III) salts and manganese powder $\left(\mathrm{MnCl}_{2}, \mathrm{MnCl}_{2} \text { (thf }\right)_{2}, \mathrm{MnI}_{2}, \mathrm{Mn}(\mathrm{OAc})_{2}, \mathrm{MnSO}_{4},\left[\mathrm{Mn}\left(\mathrm{N}\left(\mathrm{SiMe}_{3}\right)_{2}\right)_{2}\right]_{2}$, $\mathrm{MnF}_{3}$ ). Neither the variation of the reaction conditions nor the change of the used base led to the formation of a manganese species, which could have been detected in ESI-MS measurements. In addition, often a decomposition of the ligand was found to occur. 


\subsection{Summary}

Three nickel(II) complexes (29 - 31) of the macrocyclic tetracarbene ligands were synthesized and characterized by different spectroscopic methods. The complexes exhibit a bend ring structure in the solid state, which is preserved in solution. Although different attempts were tried it was not possible to isolate any high valent nickel(III) compound, due to the high stability of the low spin square planar $\mathrm{d}_{8}$ metal starting complexes. The four strong $\sigma$-donor ligands lead to a strong separation of the orbital energy levels and making especially reductions more difficult. But also oxidations were found to be unfavored. Only when $\mathrm{XeF}_{2}$ was used as strong oxidant, a reaction was observed, which could not be quantified and the potentially formed nickel(III) species was not stable enough to be isolated or further characterized and decomposed over a short period of time at low temperatures.

Copper or manganese complexes analogous to the presented new nickel complexes could not be synthesized. It was shown that under several reaction conditions the macrocyclic ligand decomposed into bisimidazole units, which are capable of stabilizing a cubane type cluster structure. Suspiciously, the group of JENKINS reported no copper or manganese complexes among a large variety of different transition metal complexes with macrocyclic tetracarbene ligands, which they were able to synthesize via the transmetallation route.[163] 



\section{Two-in-one Pincer Ligands and Their Metal Complexes for Catalysis}

\subsection{Introduction}

Meriodinally coordinating tridentate 'pincer' ligands and the corresponding metal complexes are well known and widely used in catalysis. ${ }^{[215,216]}$ The complexes have been shown to enable small molecule activation as well as catalytic substrate transformations.[217]

Although known for longer time, systematic development of this class of ligands started in the late 1970s with pioneering work of SHAW, while the name 'pincer' was coined by VAN KOTEN a decade later.[218-220] Since then, the concept of pincer ligands was developed into a concept of multifunctional building blocks, which are used in numerous complexes for a wide range of applications like homogeneous catalysis, activation of small molecules and almost all other areas of inorganic chemistry. ${ }^{[216,221-223]}$ From the general scheme (Figure 3.1, left) of pincer ligands with three donor atoms arranged in a meridional fashion, countless symmetric as well as asymmetric combinations of different donor atoms such as carbon $\left(\mathrm{C}_{\mathrm{NHC}}{ }^{[224,225]}\right.$ and $\left.\mathrm{C}_{\text {aryl }}[223,226]\right)$, nitrogen (pyridine ${ }^{[227,228]}$, amine ${ }^{[229-234]}$ and imine ${ }^{[235-237]}$ ), phosphorus (phosphine ${ }^{[238,239]}$ and phosphinidene ${ }^{[240]}$ ), sulphur[241,242], silicon ${ }^{[243,244]}$ and boron[245,246] were synthesized (selected examples: Figure 3.1, right).
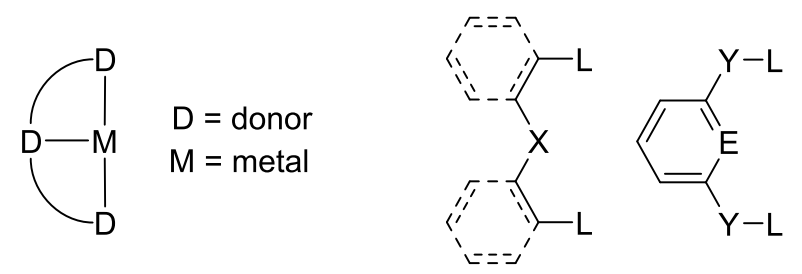

$X=N R, P R, \operatorname{SiR}$

$\mathrm{E}=\mathrm{N}, \mathrm{CH}$

$\mathrm{L}=\mathrm{PR}_{2}, \mathrm{PR}, \mathrm{NR}_{2}, \mathrm{NH}, \mathrm{SR}, \mathrm{C}_{\mathrm{NHC}}, \mathrm{C}_{\text {aryl }}$

$\mathrm{Y}=\mathrm{CH}_{2}, \mathrm{NH}, \mathrm{O}$

Figure 3.1: General scheme of pincer ligands (left) and schematic representation of selected examples of different types of pincer ligands (right) with various donor sites such as carbenes ( $\left.\mathrm{C}_{\mathrm{NHC}}\right)$, aryl carbon (Caryl), nitrogen ( $\mathrm{NR}_{2}$, $\mathrm{NR}$ ), phosphorus (PR2, PR), sulfphur (SR) and silicon (SiR) donor atoms.

The versatility of this ligand scaffold is based on the easy and fast synthetic accessibility, the robustness and in some cases the non-innocent behavior which enables unusual reactivity (see Chapter 3.1.1).[224] Due to this simple tuneability of electronic and steric properties of these ligands, they are used in many catalytic applications, since a control of both activity and selectivity is possible. Pyridine-based pincer ligands are synthesized by lithiation of 2,6lutidine followed by subsequent reaction with a nucleophile such as chlorophosphines or by substitution of 2,6-bis-(chloromethyl)-pyridine with phosphides or phosphines. ${ }^{[247]}$ 


\subsubsection{Metal-Ligand and Metal-Metal Cooperativity}

In metalloenzyme active sites two metal ions are often preorganized in close proximity to each other, which leads to remarkable cooperativity in activating various substrates (e.g. pMMO and [FeNi] hydrogenase, see Chapter 2.1).[248] This metal-metal cooperativity (MMC) was introduced into several pyrazole based dinuclear complexes,[67,249,250] which were applied in the activation of various substrates such as dioxygen [128] or for water oxidation catalysis. ${ }^{[251,252]}$ By introducing redox active metal ions such as iron, copper or cobalt, further reactions, which need more electrons than a single metal alone can supply, are possible. If the electrons are provided by the ligand, metal-ligand cooperativity (MLC) is present and the ligands are denoted as non-innocent ligands. ${ }^{[253,254]}$ This effect was found in nature, where tyrosin or phorphyrin residues might donate electrons during catalytic transformations.[255,256] Redox non-innocent ligands are utilized e.g. in $\mathrm{C}_{\mathrm{sp} 3}-\mathrm{H}$ amination catalysis.[257,258]

\section{Metal-Metal Cooperativity}

For pincer complexes this combination of two metal centers in close proximity was only sparely found and utilized so far. Only a few of such systems are known, which use different bridging units. The dinuclear complexes might be formed by dimerization of mononuclear complexes ${ }^{[259]}$ or by the connection of two pincer subunits by a linker in one molecule. Longer connections such as ferrocene[260], pyridazine [261] or 1,4-phenylene[262] lead to a quite large separation of the metal centers of more than $6 \AA$ which lowers the interaction of the metal centers. To yield closer distances the length of the linker was shortened but still flexibility in the metal-metal distance remained.[263,264]

Another approach to ensure cooperativity is the fusing of two pincer subunits with a bridging unit that coordinates to both metal ions.[265,266] The group of FIEDLER synthesized the redox active pentadentate ligand $\mathbf{H}_{3} \mathbf{L}^{\mathbf{N 3 0 2}}$, which forms upon coordination of several transition metals fused $\{\mathrm{NNO}\}_{2}$ pincer complexes. The two subunits are bridged by a diarylamido unit (Figure 3.2 , left). The separation of the metal centers was short (2.7 - 3.3 $\AA$ ). It was shown, that one of the observed oxidations in electrochemical measurements is ligand based. A cobalt(II) complex (33) of this ligand was found to activate dioxgen by forming a $\mu$-peroxo dicobalt(III) complex (34, Figure 3.2, right).[266] 

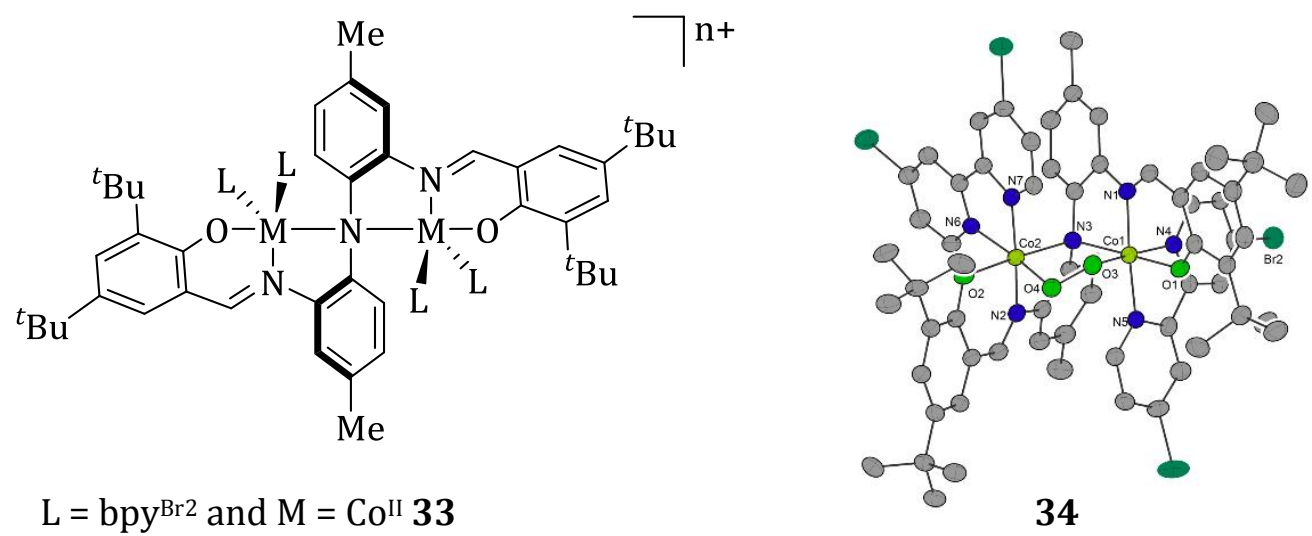

Figure 3.2: Left: Fused dinuclear $\{\mathrm{NNO}\}_{2}$ pincer complex with different chelating auxiliary nitrogen donor ligands $\mathrm{L}$ (e.g. $\mathrm{L}=$ bpy ${ }^{\mathrm{Br} 2}$ and $\mathrm{M}=\mathrm{Co}^{\mathrm{II}}$ 33). Right: Thermal displacement ellipsoids (shown at $30 \%$ probability) of the molecular structure of $\left[\mathrm{Co}_{2}\left(\mathrm{O}_{2}\right) \mathrm{L}^{\mathrm{N} 302}\left(\mathrm{bpy}^{\mathrm{Br} 2}\right)_{2}\right]\left(\mathrm{ClO}_{4}\right)$ 34. Counterions, hydrogen atoms and solvent molecules were omitted for clarity.[266]

A similar methodology was pursued by the MEYER group before, using 3,5-substituted pyrazoles as bridging unit between two metal ions.[128,265,267] For example in water oxidation catalysis an intramolecular interaction of two high valent Ru-O units was found as the crucial step in the O-O bond formation. ${ }^{[268,269]}$ In 2015, the synthesis of a fused $\{\mathrm{PNN}\}_{2}$ ligand $\mathbf{H L}^{t \mathrm{Bu}}$ was presented, which is the first report that denotes these systems as fused ('Two-in-one') pincer ligands.[265] The found separation of the iron centers of $4.195 \AA$ was expected to enable MMC, and indeed the diiron complex 35 exhibited cooperativity by undergoing a reversible and temperature induced spin transition. Upon lowering the temperature, a high-spin/low-spin transition was found, caused by a multistep ligand exchange between $\mathrm{MeCN}$ and triflate ions in solution (Scheme 3.1).

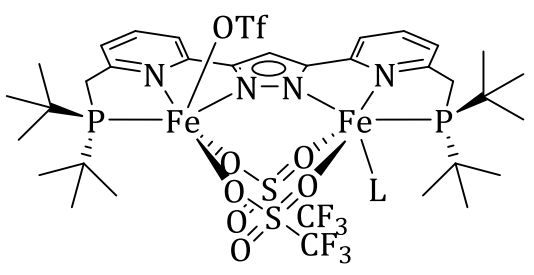

high temp.: hs $\mathrm{Fe}^{\mathrm{II}}$

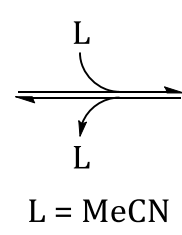

$\mathrm{L}=\mathrm{MeCN}$

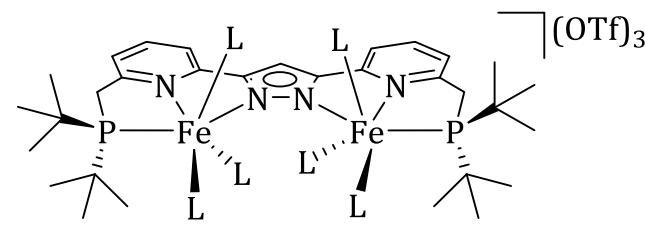

low temp.: ls Fe ${ }^{\mathrm{II}}$

Scheme 3.1: Reversible, temperature induced spin state switching and MeCN/trfilate ligand exchange in 35.[265]

Recently, MMC was also found in exciting new ligand systems developed by MURRAY and LU in which close metal metal distances were found and also bimetallic systems were synthesized.[270-273]

\section{Metal-Ligand Cooperativity}

Aside the above discussed metal-metal cooperativity and the redox innocence of certain ligands, another effect was found for some pyridine-based and aliphatic secondary amine 
ligand systems. MILSTEIN reported in 2005 that upon addition of base to complex 36 the methylene side arm was deprotonated instead of the expected reduction of the ruthenium center. ${ }^{[229]}$ The deprotonation of the side arm led to a dearomatization of the pyridine ring and resulted in a negative charge on the nitrogen atom (Scheme 3.2).

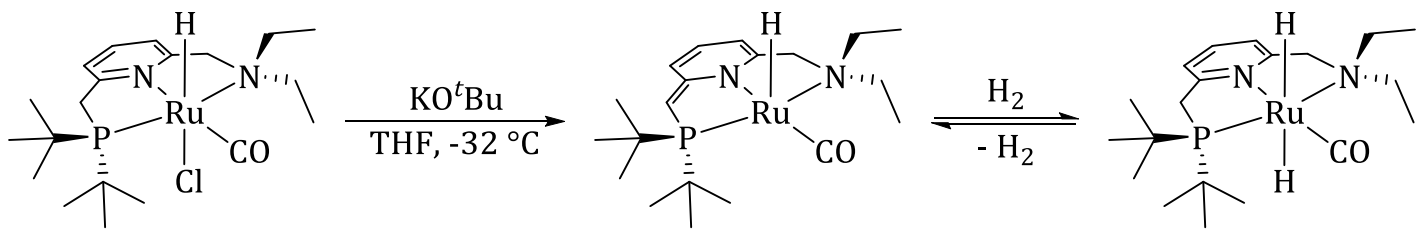

36

37

38

Scheme 3.2: Dearomatization/aromatization pathway for ruthenium complex 36. First deprotonation of the side arm and dearomatization of the pyrindin ring (37) followed by the bifunctional activation of dihydrogen with rearomatization (38).[229]

Dearomatization of the pyridine ring in $\mathbf{3 7}$ was indicated by a drastic shift of the pyridine ${ }^{1} \mathrm{H}$ NMR resonances $1-2 \mathrm{ppm}$ to higher field. Although the electronic structure of the trans standing pyridine to the CO ligand changed, the CO stretching frequency was not affected by the dearomatization. ${ }^{[229]}$ In only very few cases the isolation and structural characterization by means of X-ray diffraction analysis was reported, reflecting somehow the high reactivity of these compounds.[274-276] The dearomatization effect was also found for other pincer ligand systems with $\mathrm{NH}$ linkers between the donor sides[277-279] and for systems having an acridine backbone. For benzene based PCP pincer ligands this effect was not observed due to a higher resonance energy of the ring.[280,281] Complex 39 is able to provide an unusual long range MLC involving an interaction of the distal C9 carbon of the acridine backbone and the metal center (Scheme 3.3). The high flexibility of the ligand enables this interaction as it was suggested by DFT calculations. A decoordination of the acridine nitrogen seemed to be involved in the formation of $\mathbf{4 0}$,in which the central ring of the acridine is dearomatized.[282]

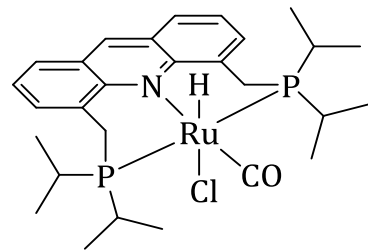

39

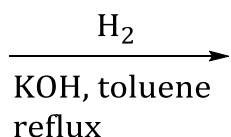

reflux

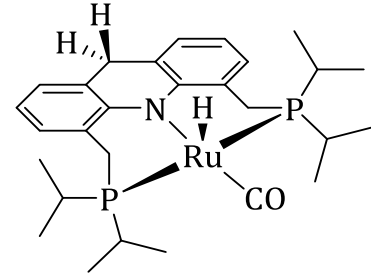

40

Scheme 3.3: Unusual long-range MLC found for acridine base PNP pincer complex $\mathbf{3 9}$ upon deprotonation in the presence of dihydrogen.[280]

The group of SCHNEIDER showed that MLC could also be observed in aliphatic PNP pincer systems.[234,283-285] As an example, the formation of ammonia from hydrogenolysis of a ruthenium nitrido complex is depicted in Scheme 3.4.[57] In contrast to catalytic ammonia formation in other systems (see Chapter 1.1), for the first time dihydrogen directly was used 
for the hydrogenation yielding $\mathrm{NH}_{3}$. The cyclic pathway showed MLC by deprotonation and protonation of the amine donor function to activate dihydrogen.

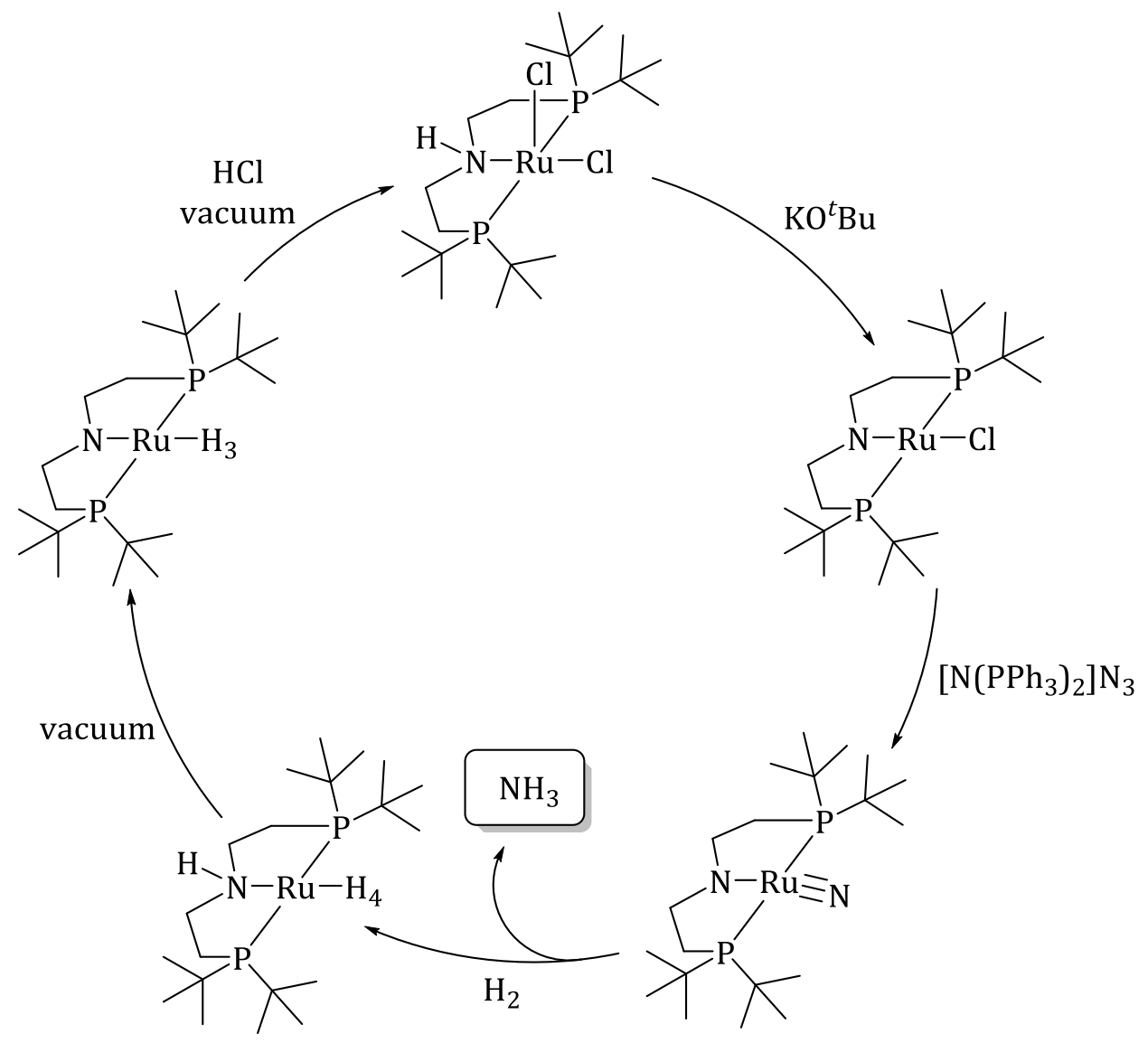

Scheme 3.4: Proposed mechanism of the MLC assisted formation of $\mathrm{NH}_{3}$ from hydrogenolysis of a ruthenium PNP nitrido complex.[57]

\subsubsection{Activation of Small Molecules}

The discovery of metal-ligand cooperativity by a dearomatization/aromatization mechanism in pyridine and acridine PNP systems opened a new field of reactivity towards the activation of different H-X bonds.[286-289] Complex 37 was found to activate various H-X bonds by reprotonation of the side arm and incorporation of the resulting anionic substrate in the coordination sphere of the metal center. ${ }^{[290]}$ As another example, the dearomatized complex $\mathbf{3 7}$ facilitated the heterolytic splitting of dihydrogen to form a dihydride complex $\mathbf{3 8}$ (Scheme 3.2).[229] Further studies revealed that the ligand was involved in this dihydrogen activation, since the trans product was formed exclusively.[291] This was not expected for reactions not involving a metal-ligand cooperativity. Also the activation of N-H bonds was shown for ammine and aniline derivatives.[292,293] An exceptional example for MLC was presented by MILSTEIN in 2009 , showing the splitting of water into dihydrogen and dioxygen by a cascade of thermal $\mathrm{H}_{2}$ and light induced $\mathrm{O}_{2}$ evolution at a ruthenium(II) center (Scheme 3.5).[294] Addition of water to 
37 led to the formation of a hydrido-hydroxo complex $\mathbf{3 7 0 H}$, which reacts with a second molecule of water to yield a cis-dihydroxo complex $37(\mathrm{OH})_{2}$ upon heating with simultaneous release of $\mathrm{H}_{2}$. Under irridiation complex $37(\mathrm{OH})_{2}$ liberated hydrogen peroxide by reductive elimination, which is then catalytically decomposed to water and dioxygen by $\mathbf{3 7}$. The cycle is closed by migration of a proton of the methylene group to the metal center.[294]

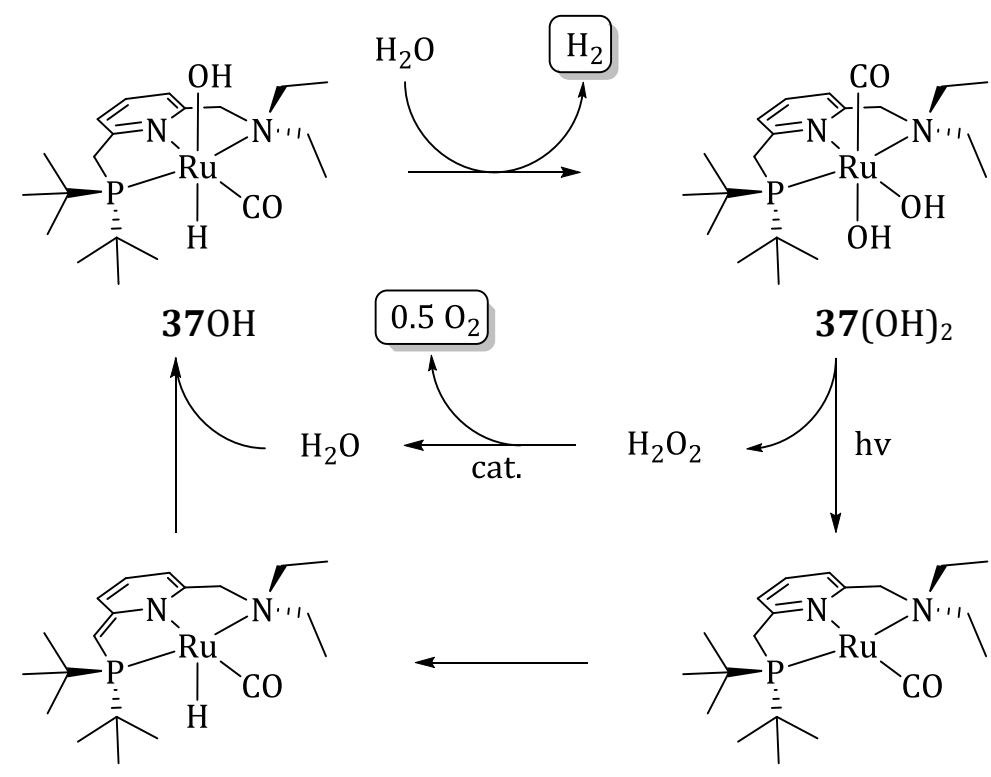

37

Scheme 3.5: Proposed mechanism of the stepwise water splitting promoted by ruthenium PNN complex $\mathbf{3 7}$ including thermal $\mathrm{H}_{2}$ and light-induced $\mathrm{O}_{2}$ evolution. ${ }^{[294]}$

A wide range of substrate activation chemistry was found for rhodium(I) pyridine-based pincer complexes.[295-297] The rhodium(I) complex 41 underwent facile $\mathrm{N}-\mathrm{H}$ activation of aniline to form 42 (Scheme 3.6). Interestingly, upon addition of carbon monoxide, the reverse reaction was observed yielding the starting aniline again and a dearomatized Rh-CO complex 43.[298]

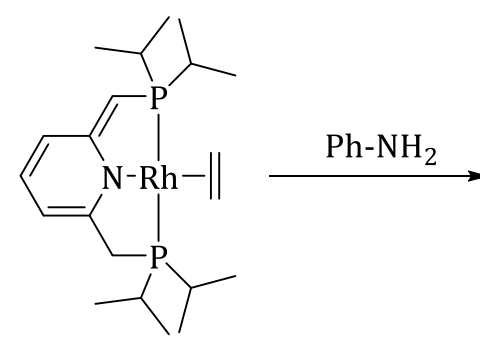

41

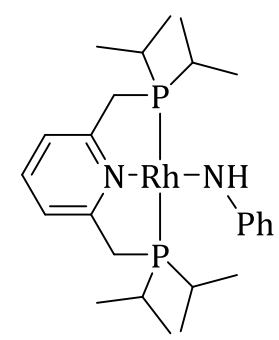

42
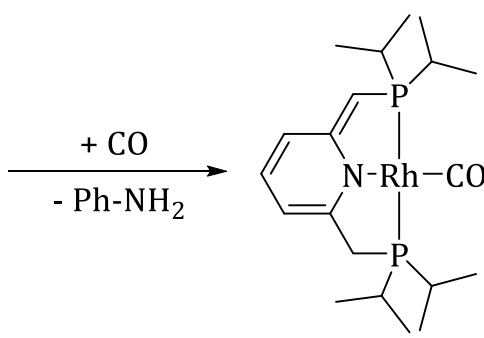

43

Scheme 3.6: N-H bond activation by dearomatized RhIPNP complex 41 and subsequent N-H bond formation upon addition of CO.[298]

Pincer complexes were also applied for the activation of small molecules without using metal ligand cooperativity by using reactive metal hydrido or nitrido species.[59,299-302] 


\subsubsection{Catalysis}

Catalysis in general is a field of chemical research which is in close contact with industrial production of both base and fine chemicals such as fertilizers or drugs. Most large scale reactions are either heterogeneously or homogeneously catalyzed. Catalysts increase the rate of a certain reaction by lowering the activation barrier and sometimes enable reaction pathways, which are not possible without a catalyst.

Against the background of sustainability of catalytic reactions, the atom economical use of compounds and the reduction of waste products is highly desired. In addition, the use of compounds from renewable sources such as biomass converted products would help to decrease the dependence on limited fossil resources. Thus the development of more reactive but also more stable catalysts is a great future challenge. Today pincer ligands, as one class aside NHC and cyclopentadienyl ligands, are widely used in homogeneous catalysis and make a great impact in the conversion of organic substrates to value-added compounds. ${ }^{[303,304]}$ In the last decade chemists started to use MLC for environmentally benign catalytic reactions to discover new synthetic pathways to convert biomass and basic chemicals into high valued products by using earth abundant base metals as catalytically active centers. ${ }^{[305-308]}$ A huge number of catalysts for other conversions were reported in the field of pincer catalysis, so that only a few selected examples will be discussed in the following.

Apart from many mono- and di-phosphines also pincer ligands were applied in carbon-carbon and carbon-heteroatom coupling reactions, in which $\mathrm{d}^{10}$ transition metal ions like palladium often were used (e.g. SUZUKI, KUMADA or HECK reactions).[224,309-315]

\section{Dimerization of Alkynes}

Pincer and other metal complexes were shown to dimerize terminal alkynes to conjugated enynes, which are versatile building blocks for organic synthesis.[316] $\mathrm{One} \mathrm{C}_{\mathrm{sp}}$ - $\mathrm{H}$ bond is formally added across the triple bond of a second alkyne in a perfectly atom economical route. In principle three isomers can be formed during the dimerization of terminal alkynes: $E, Z$ and gem isomers (Scheme 3.7) which are often formed in mixtures of different ratios. Although the control of the selectivity was a problematic issue in some reports,[316,317] there are also catalytic systems that selectively form the $E^{[318,319]}, Z^{[320,321]}$ or gem $^{[322,323]}$ isomer. For this type of reaction usually noble metals like ruthenium and rhodium were applied, but very recently an iron pincer complex was shown to selectively form the $Z$ isomer even at ambient conditions. ${ }^{[324]}$ The cross dimerization of two different alkynes is also possible, but the two alkynes have to have a distinct electronic structure to prefer the cross coupled products.[325,326] 


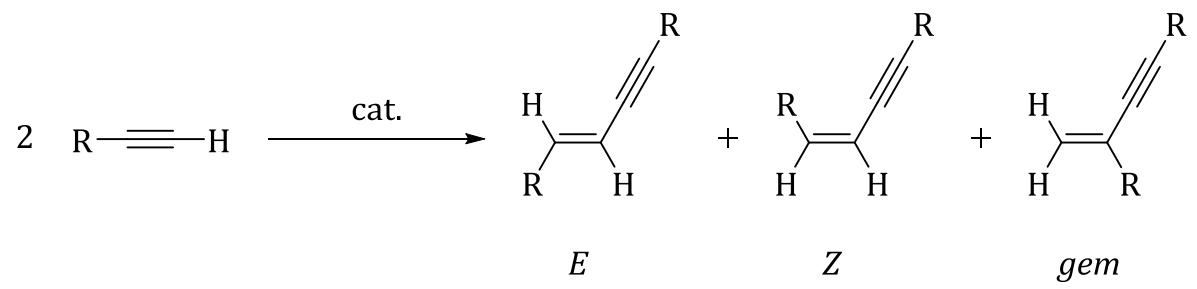

Scheme 3.7: Alkyne dimerization to three possible isomers ( $E, Z$ and $g e m) .[316]$

\section{Hydrofunctionalization of Alkenes and Alkynes}

Another important transformation of unsaturated substrates such as alkenes and alkynes is the hydrofunctionationalization (e.g. hydroamination[327,328], hydroboration[329,330] and hydrosilylation ${ }^{[331,332]}$ ). The use of non-noble metals for such reactions is desirable in the sense of lower costs and sustainability, but iron and nickel complexes often show limited functional group tolerance (especially carbonyl groups proved to be problematic) and low stability. Thus superhydride ${ }^{\circledR}$ was used to generate the active catalyst species from more stable catalyst precursors in several cases. ${ }^{[333-335]} \mathrm{Hu}$ presented a hydrosilylation protocol with non-precious \{NNN\} nickel methoxide complex $\mathbf{4 4}$ without the need for an activating agent, giving a very high reactivity (Scheme 3.8, max. TOF $\approx 83000 \mathrm{~h}^{-1}$ ).[336] In addition, 44 selectively hydrosilylated alkenes in the presence of ketone and formyl groups, although the hydrosilylation of ketones and aldehydes is well known.[332,337]

$$
\mathrm{R} \curvearrowright+\mathrm{Ph}_{2} \mathrm{SiH}_{2} \underset{\mathrm{THF}, \mathrm{rt}, 6 \mathrm{~h}}{\stackrel{1 \mathrm{~mol} \% 44}{\longrightarrow}} \overbrace{\mathrm{SiHPh}}
$$

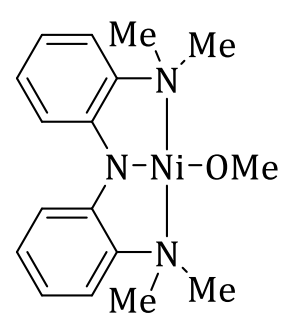

44

Scheme 3.8: Hydrosilylation by \{NNN\} nickel pincer complex 44 to form the anti-Markovnikov product.[336]

\section{Hydrogenation Catalysis}

Hydrogenation of various unsaturated substrates are important synthetic tools in organic synthesis. Catalytic reactions can be divided into two main categories: the direct hydrogenation with dihydrogen gas and the transfer hydrogenation by using a hydrogen donor agent such as iso-propanol or formic acid. The latter systems are practically more easy to handle but produce one equivalent of waste per turnover. The famous asymmetric transfer hydrogenation developed by NOYORI utilizing ruthenium complex 46 was an early example for the versatility of this reaction.[338,339] Two mechanistic pathways can be distinguished: the inner sphere 
mechanism features the coordination and reaction of the substrate at a metal dihydride complex, while the outer sphere mechanism shows MLC activity with a bifunctional activation of the hydrogen donor molecule as it was shown for 46.[238,340] Selected examples for catalysts used for hydrogenation, transfer hydrogenation and dehydrogenation reactions are depicted in Figure 3.3.

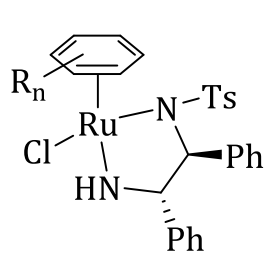

NOYORI 46

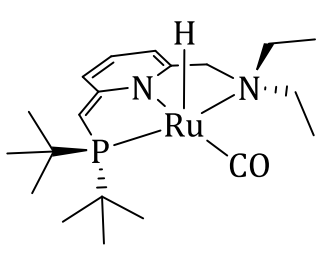

37

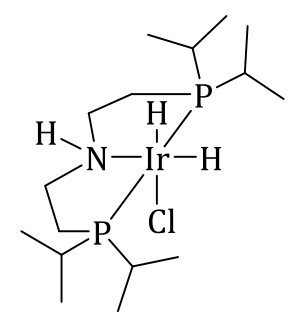

BELLER

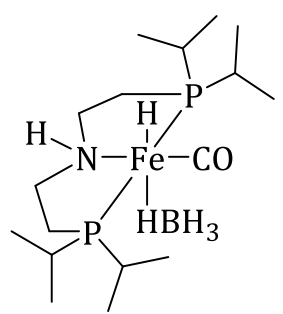

BELLER

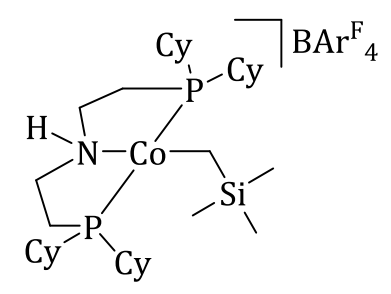

HANSON<smiles>[13CH2][15NH2]</smiles>

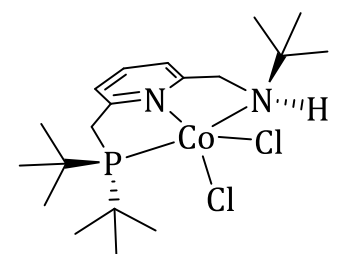

MILSTEIN

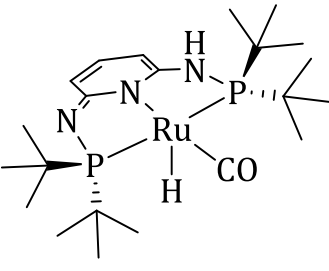

HUANG

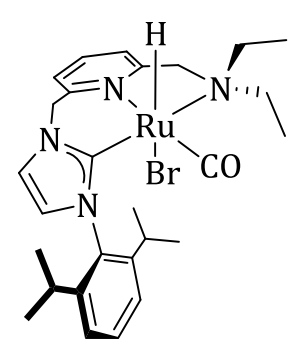

SONG

Figure 3.3: Overview of selected complexes used as catalysts in hydrogenation, transfer hydrogenation and dehydrogenation reactions.[279,306,338,341-345]

The probably most famous hydrogenation catalyst was developed by WILKINSON in the 1960s.[346,347] $\left[\mathrm{RhCl}\left(\mathrm{PPh}_{3}\right)_{3}\right]$ (45) was shown to be outstanding active and versatile in the hydrogenation of numerous substrates. Alkynes are hydrogenated to alkanes because the observed rates for the hydrogenation of alkynes and alkenes are very similar. ${ }^{[348]}$ Mechanistic investigations by NMR spectroscopy and considerations of well-known phosphine dissociation/association reactions lead to the proposal of a two-electron redox cycle (Scheme 3.9).[349-352] Dissociation of one triphenylphosphine ligand from the 16 electron rhodium(I) complex $\mathbf{4 5}$ forms the catalytically active, 14 electron species featuring an open coordination site. This first equilibrium was assumed since reactions with additional ligand equivalents exhibit slower reaction rates. ${ }^{[348]}$ The oxidative addition of dihydrogen to form a rhodium(III) cis-dihydride is reversible as well as the subsequent coordination of the substrate. A 1,2 insertion step forms an alkyl-hydride compound from which the alkane is released by reductive elimination, while the active rhodium(I) species is reformed. By using chiral mono or bisphosphine ligands stereoselective transformations are possible. ${ }^{[38]}$ Recently, REPO reported the addition of a strong base, such as $2{ }^{-}{ }^{t}$ BuTMG (2-tert-butyl-1,1,3,3-tetramethylguanidine, XXVIII) to drastically increase the activity of $\mathbf{4 5}$ by the formation of [RhH( $\left.\left.\mathrm{PPh}_{3}\right)_{3}\right]$. [353] 


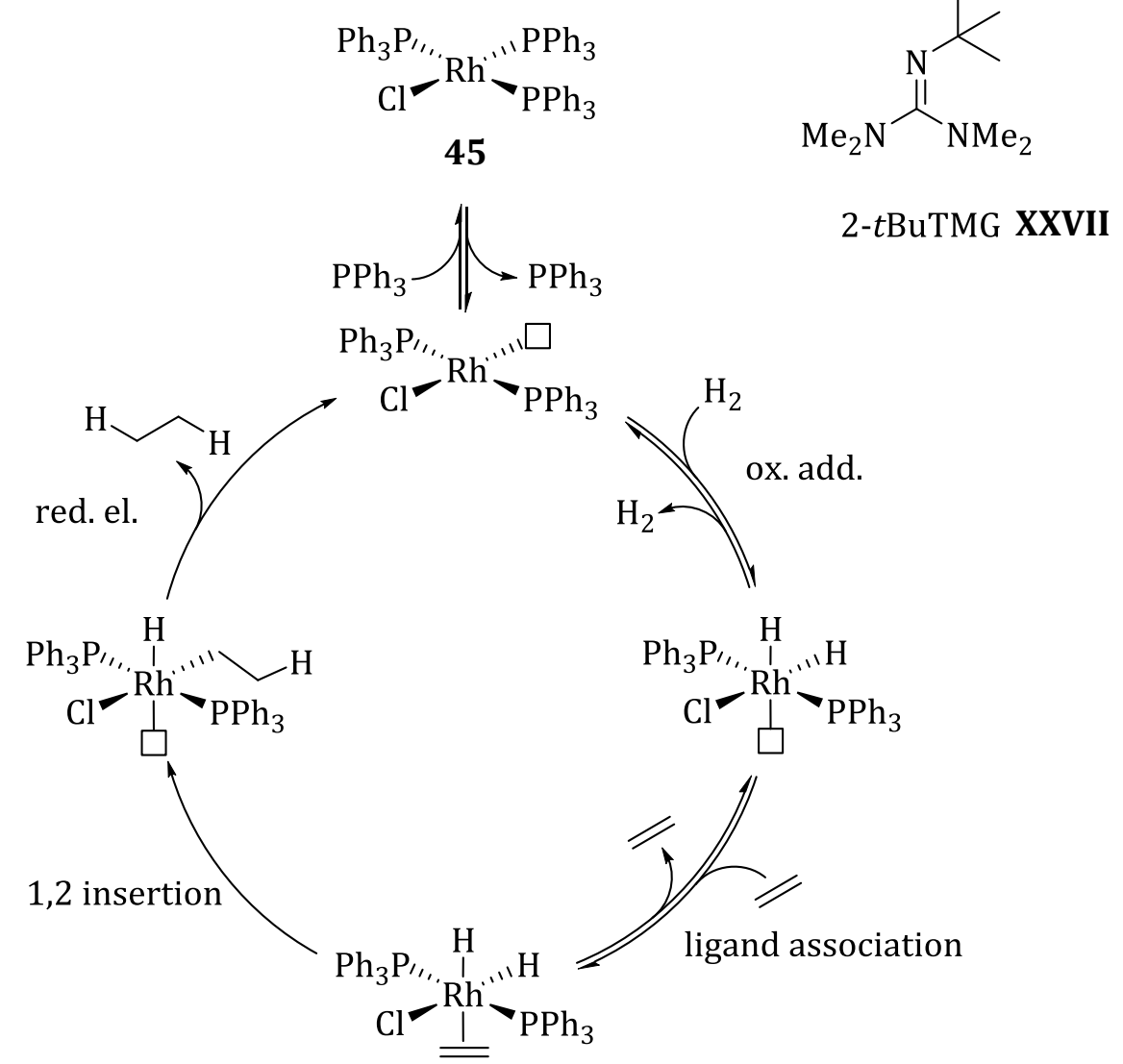

Scheme 3.9: Proposed catalytic cycle of hydrogenation reactions with the WILKINSON's catalyst 45 using the example of ethylene.[349,354]

\section{Hydrogenation of Carbon Dioxide}

A greatly emerging field is the catalytic hydrogenation of carbon dioxide or related compounds. ${ }^{[355-357]}$ The atmospheric amount of carbon dioxide, which is a final product of combustion, drastically increased in the past century due to an unlimited use of fossil fuels, which is belived to cause crucial changes for the global climate.[358-361] The decrease of $\mathrm{CO}_{2}$ emission is one worldwide major issue and without any alternative. Therefore, the capture and storage of carbon dioxide becomes more and more a fundamentally important field of research, not only in chemistry. ${ }^{[362-364]}$ This should happen preferably at its point of generation. The storage and recycling of the greenhouse gas $\mathrm{CO}_{2}$ on a large scale would be beneficial not only for decreasing the amount of atmospheric $\mathrm{CO}_{2}$, but also carbon dioxide is a non-toxic, everywhere abundant and versatile $\mathrm{C}_{1}$ building block for the use as chemical feedstock.[365-368] A possible, carbon dioxide based economy is depicted in Figure 3.4, featuring the capture of $\mathrm{CO}_{2}$ by means of hydrogenation for the utilization as storage of dihydrogen and the use as fuel. The transformations to formic acid (FA) and methanol both contribute to a change in the energy source for future mobility. The reversible hydrogenation and dehydrogenation of $\mathrm{CO}_{2}$ and $\mathrm{FA}$ can be used as energy storage for a hydrogen economy with FA fuel cells for the energy 56 
generation. ${ }^{[369]}$ Although the hydrogen density in FA (4.4 wt \%) is lower than in methanol (12.6 wt \%) the reversible transformations are more developed for FA to date.[355] A main drawback is still the production of dihydrogen from fossil resources. Thus, processes have to be developed in order to use only dihydrogen generated from water and not, as today, steam reformed dihydrogen. The synthesis of basic chemicals from $\mathrm{CO}_{2}$ with different reducing agents and alcohols, amines or epoxides was already shown before. However, this chemistry is underrepresented in literature in relation to its importance for future carbon cycles and processes for generating basic chemicals from renewable resources.[370,371] The reduction of carbon dioxide can also be done by electrocatalysis under an atmosphere of $\mathrm{CO}_{2 \cdot}{ }^{[372-375]}$

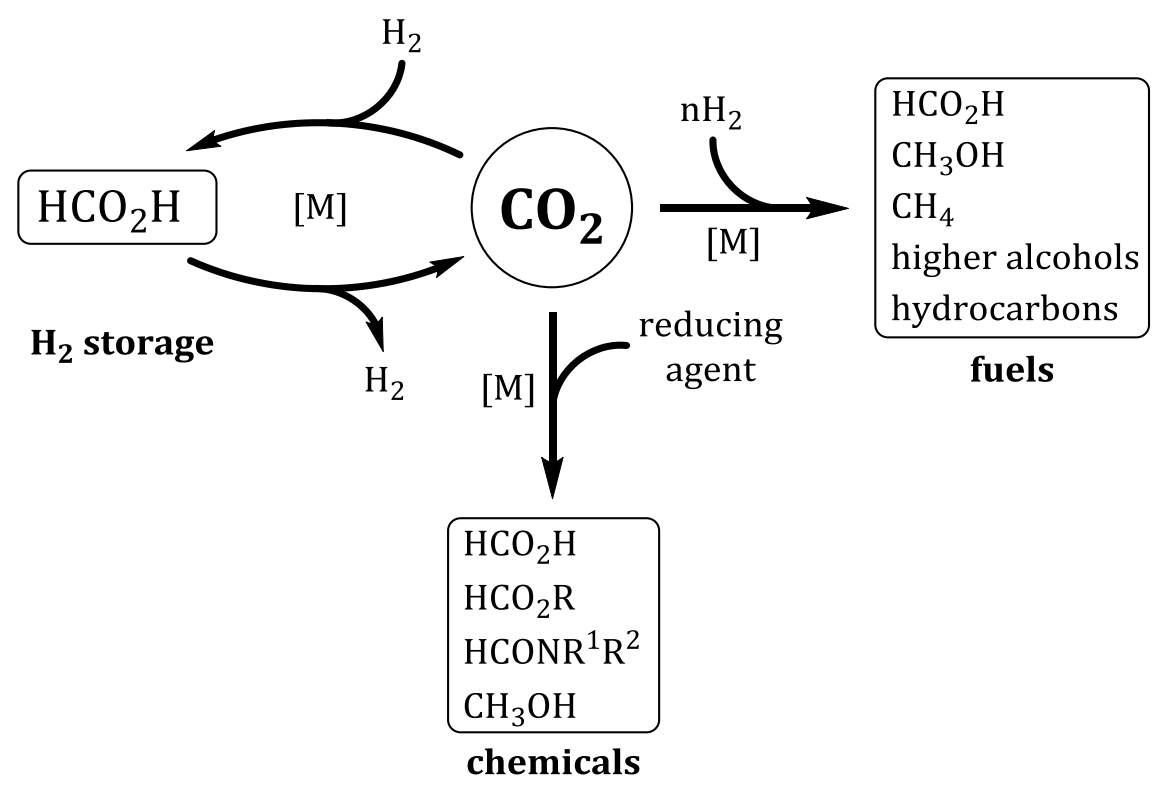

Figure 3.4: Possible utilization of carbon dioxide as chemical feedstock for the generation of fuels and chemical building blocks as well as for the storage of dihydrogen. [355,357]

The activation of $\mathrm{CO}_{2}$ is rather difficult due to its thermodynamic stability and kinetic inertness. ${ }^{[35]}$ The hydrogenation of carbon dioxide to various products (FA, formaldehyde, methanol and methane) is in general entropically disfavoured.[376] To lower this entropic barrier, often the use of additives such as bases was applied, which stabilized the produced formic acid. In organic solvents mostly $\mathrm{NEt}_{3}$ or DBU (1,8-diazabicyclo[5,4,0]undec-7-ene) are used, in aqueous systems hydroxide or carbonate are applied.[355] It was found that the choice of the solvent for the hydrogenation to formic acid is of high importance, because it can stabilize the product in a way that additional base is not necessary (Scheme 3.10).[377,378] A highly active ruthenium pincer complex based catalytic system was developed in the group of LEITNER, which reached TONs of 16000 and TOFs of $1000 \mathrm{~h}^{-1}$ in buffered DMSO solutions.[379] 


$$
\mathrm{H}_{2}+\mathrm{CO}_{2}+\text { base } \rightleftharpoons \mathrm{CO}_{2}+\text { solvent } \rightleftharpoons \text { Hbase }{ }^{+}
$$

Scheme 3.10: Stabilization of formic acid by suitable solvent molecules (bottom) to avoid the use of base (top).[379]

Pioneering work on homogenous hydrogenation of carbon dioxide to formic acid and methanol was done by INOUE with different noble metal phosphine complexes.[380] Later on, the WILKINSON catalyst and water soluble derivatives ${ }^{[381-384]}$ as well as various ruthenium complexes were shown to catalyze this reaction. ${ }^{[385,386]}$ In recent years also the development of catalysts with earth abundant metals such as iron and cobalt was achieved.[387-390] Due to the high solubility of dihydrogen in super critical carbon dioxide $\left(\mathrm{scCO}_{2}\right)$, high catalytic activities were found when the hydrogenation was conducted in $\mathrm{scCO}_{2 .}{ }^{[391,392]}$ Some selected catalysts, which are used in the hydrogenation of carbon dioxide, are presented in Figure 3.5.

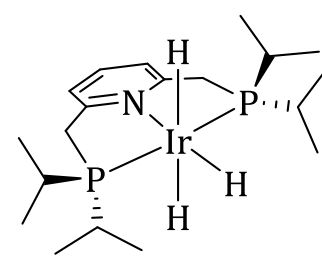

NOZAKI<smiles></smiles>

HIMEDA

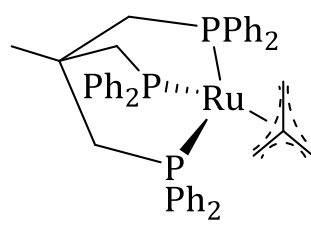

KLANKERMAYER

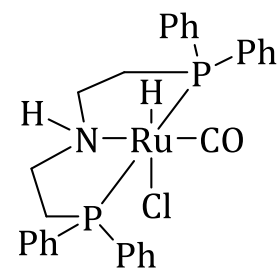

DING

Figure 3.5: Selected catalysts used for the hydrogenation of carbon dioxide to formiate or methanol and the reversed reaction.[393-396]

The iridium PNP trihydride complex of NOZAKI reached an impressive turnover number (TON) of more than 3.5 million and high turnover frequencies (max. TOF $=150000 \mathrm{~h}^{-1}$ ) at high $\mathrm{CO}_{2}$ and $\mathrm{H}_{2}$ pressures. ${ }^{[393]}$ The proposed mechanism involves $\mathrm{CO}_{2}$ insertion into the iridium hydride bond along with a dearomatization/aromatization of the pyridine ring (MLC).[397] Another example in which non-innocent behavior was shown to be important for the catalytic activity was reported by HIMEDA. ${ }^{[394]}$ Based on proton responsive hydroxy bipyridine or bipyrimidine ligands, which can also act as electron donor sites, iridium, rhodium, ruthenium and cobalt metal ions were applied in the reversible storage of dihydrogen in formiate. ${ }^{[398-400]}$ The presence of one or more hydroxy groups not only increased the solubility of the catalysts in water, but also the initial rate was positively affected due to deprotonation of the hydroxyl group. The hydrogenation of carbon dioxide to methanol was possible by using a ruthenium triphos ligand. ${ }^{[395]}$ LEITNER and KLANKERMAYER proposed a ruthenium(II) hydride species as active catalyst. DING et al. indirectly hydrogenated carbon dioxide by using ethylene oxide to synthesize ethylene carbonate, which then can be hydrogenated by a ruthenium pincer complex to form methanol and ethylene glycol..[396] A similar concept was developed by MILSTEIN in utilizing organic carbonates, carbamates and formates as substrates for the 
hydrogenation.[401] These compound can be easily produced from carbon dioxide. Dearomatized PNN pincer complexes of ruthenium efficiently produce methanol and provide a new route for methanol production.

Not only the hydrogenation but also the dehydrogenation of substrates is an important reaction in the context of hydrogen storage and organic synthesis. To use FA as a hydrogen storage material, the efficient dehydrogenation is necessary to release $\mathrm{H}_{2}$ for further use. Often catalysts for the hydrogenation also work for dehydrogenation reactions with similar activities. A highly active iron catalyst was synthesized and tested in the groups of SCHNEIDER and HAZARI (Scheme 3.11).[283] For this system it was found that the addition of LEWIS acids enhanced the activity of $\mathbf{4 7}$ by assisting in the decarboxylation. The affinity for carboxylates of the LEWIS acids are well correlated to the activity of the catalytic system and thus highest for $\mathrm{Li}^{+}$. This effect was also found to take place in the hydrogenation of carbon dioxide.[390]
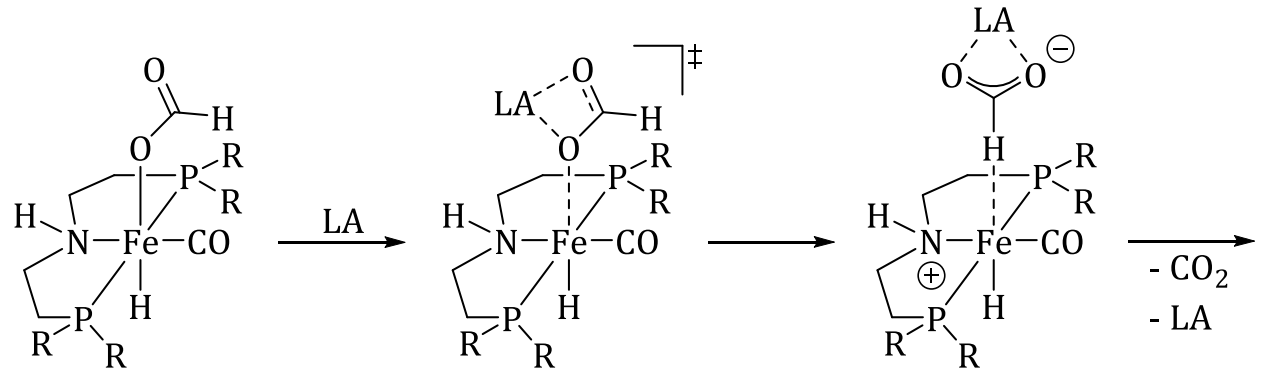

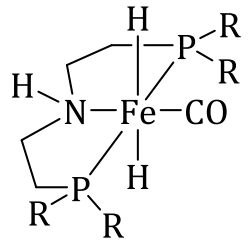

47

Scheme 3.11: Proposed mechanism for the catalytic, LEWIS acid assisted dehydrogenation of formic acid with PNP iron pincer complex 47.[283]

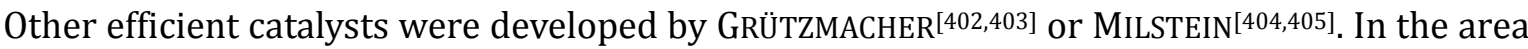
of green chemistry, the group of BELLER used carbohydrates and bioethanol to produce dihydrogen from renewable resources. [306,307,341] 



\subsection{Objective}

In order to thoroughly investigate the properties and the potential applications of the new Two-in-one pincer ligand $\mathbf{H L}^{\mathbf{B} \mathbf{B u}}$, the ligand system will be extended to other phosphine side arms aside the ${ }^{\mathrm{Bu}}$ derivative synthesized by SAMANTA (Figure 3.6).[265] The change of the phosphine substituents is expected to vary the electronic and steric properties of the ligand. Therefore, not only $\mathrm{ClP}^{t} \mathrm{Bu}_{2}$ but also $\mathrm{ClP}^{i} \mathrm{Pr}_{2}, \mathrm{ClPPh}_{2}$ and $\mathrm{ClP}(\mathrm{pyr})_{2}$ (pyr = pyrrolyl) should be used for the phosphorylations to enhance the catalytic activity of the corresponding complexes.

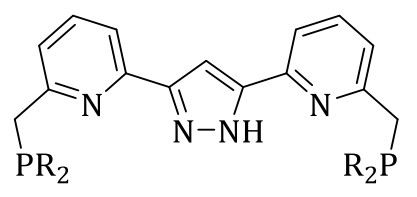

$\mathrm{R}={ }^{t} \mathrm{Bu},{ }^{i} \mathrm{Pr}, \mathrm{Ph}, \mathrm{pyr}$

Figure 3.6: Attempted extension of the Two-in-one pincer ligand system with tert-butyl, iso-propyl, phenyl and pyrroyl residues at the phosphorus side arms.

For mononuclear, pyridine based pincer complexes an unusual metal ligand cooperativity (MLC) was observed by MILSTEIN, namely the deprotonation of the methylene group with concurrent dearomatization of the pyridine ring.[229] This effect was utilized for the activation of various substrates during catalytic conversions. For dinuclear complexes a metal metal cooperativity (MMC) was observed in several complexes, in which metal ions are hosted in close proximity in the ligand cavities, especially for pyrazole-bridged complexes. Combining these two effects, namely MLC and MMC, in one well-defined metal pincer complex offers a great perspective for multifunctional substrate activation in catalysis, while at the same time making use of the beneficial properties of pincer ligands (Figure 3.7).

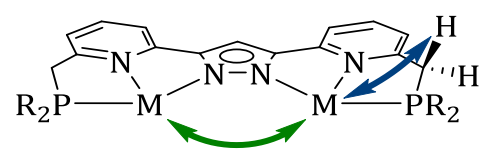

Figure 3.7: Potential MMC (green arrow) and MLC (blue arrow) in Two-in-one pincer complexes.

The aim of this work is to synthesize new transition metal complexes of the Two-in-one pincer ligand system and to investigate the dearomatization of the pyridine rings upon deprotonation followed by subsequent substrate activation. Zinc, cobalt and rhodium are chosen as metals, since zinc(II) as an inert metal ion might provide structural and electrochemical information about the ligand system itself, cobalt is quite versatile in catalysis and rhodium as a noble metal is suitable for NMR spectroscopy $\left(\mathrm{Rh}^{\mathrm{I}}\right.$ and $\left.\mathrm{Rh}^{\mathrm{III}}\right)$ and features a broad range of possible applications in catalysis such as hydrogenations. 



\subsection{Ligand Synthesis}

The ligands were synthesized in a six step synthesis based on literature procedures.[265,406,407] The conditions of single steps were optimized to increase the yield or to simplify the workup procedure. Scheme 3.12 shows an overview of the synthetic pathway to Two-in-one pincer ligands $\mathbf{H L}^{t \mathrm{Bu}}(\mathbf{X X X V I I})$ and $\mathbf{H L}^{\mathbf{P r}}$ (XXXVIII). To obtain the pyridine-pyrazole precursor XXXIII, 2,6-lutidine XXIX was singly oxidized with potassium permanganate in water to obtain 6-methyl-2-picolinic acid XXX in ca. $50 \%$ yield. The carboxylic acid was converted to the corresponding ester (methyl-6-methyl-2-picolinate XXXI) under acidic conditions in methanol. Parts of the ester were transformed into 2-acetyl-6-methylpyridine XXXII by a CLAISEN ester condensation with sodium ethoxide and ethyl acetate in dry toluene with subsequent hydrolysis of the formed $\beta$-keto ester. Another CLAISEN condensation of the ester XXXI and the ketone XXXII produced $\beta$-diketone 1,3-bis-[2-(6-methyl)-pyridyl]-1,3-propanedione, which was used without isolation for a ring closure with hydrazine monohydrate to yield 3,5-bis-2(6-methylpyridyl)-pyrazole XXXIII.

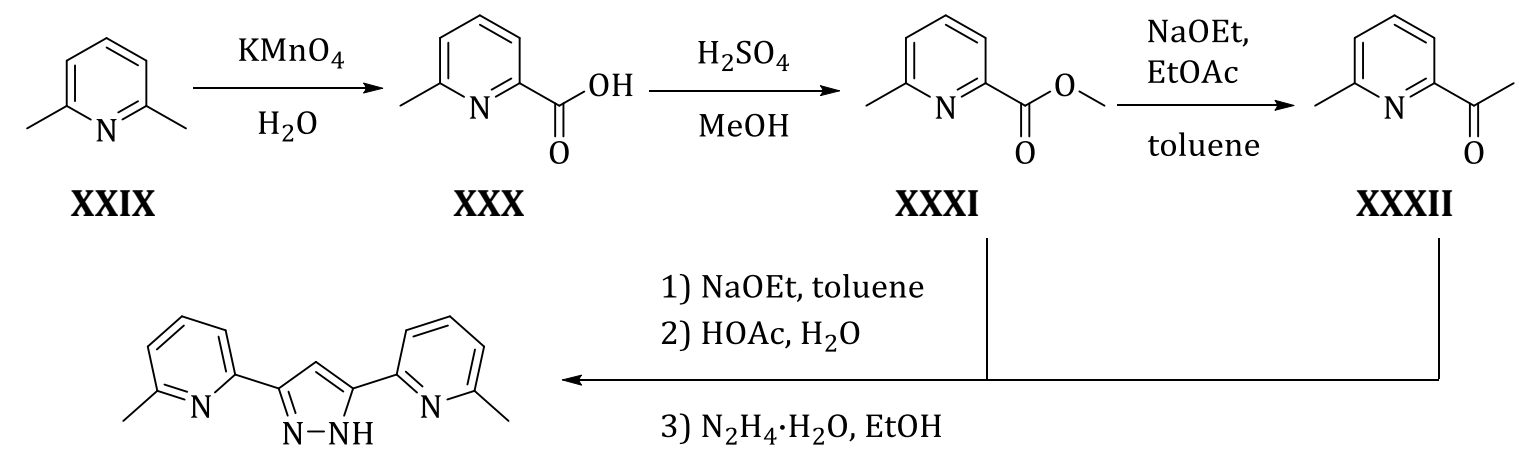

XXXIII

1) 2.5 eq $n B u L i$, THF

2) $\mathrm{Cl}-\mathrm{PR}_{2}$
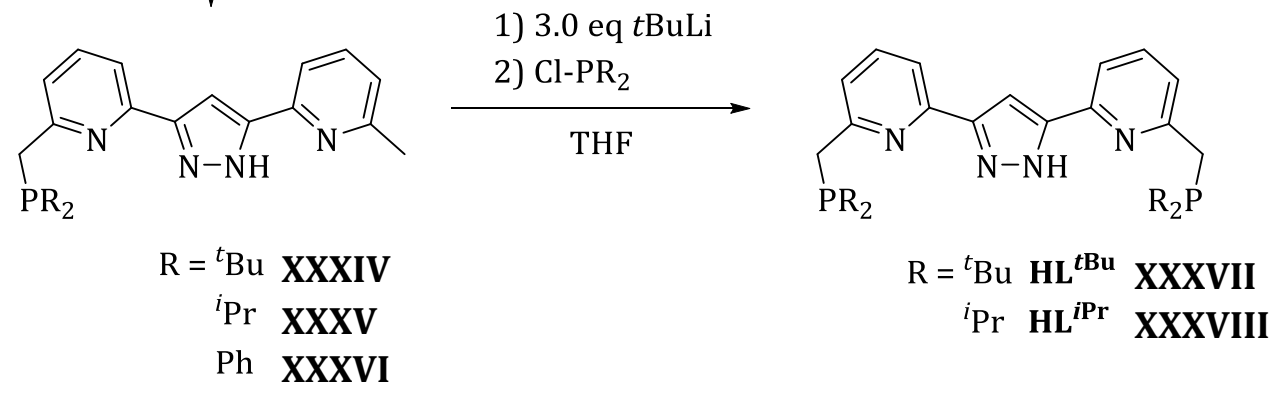

Scheme 3.12: Synthetic pathway of the synthesis the Two-in-one pincer ligand scaffold.

A two-fold phosphorylation in one step was not possible, since P-P bond formation occurred.[265] Hence, a two-step procedure was applied. XXXIII was deprotonated by $n$-butyl lithium (2.5 eq) and one equivalent of chloro-dialkylphosphine was added. After the aqueous 
workup mono phosphorylated compounds XXXIV, XXXV and XXXVI were obtained in high yields of approximately $90 \%$. NMR spectroscopy showed asymmetric species in ${ }^{1} \mathrm{H}$ and single resonances in ${ }^{31} \mathrm{P}$ spectra. The ${ }^{31} \mathrm{P}$ NMR signals were observed at $36.25,12.20$ and $-11.43 \mathrm{ppm}$ for the ${ }^{t} \mathrm{Bu}, i \mathrm{Pr}$ and phenyl derivative, respectively, showing an increased in electron density at the phosphorus atom from tert-butyl to phenyl substituents. For the second deprotonation a stronger base ( $t$-butyl lithium, $3.0 \mathrm{eq}$ ) was used, since it was found that the second deprotonation is much more difficult than the first one. Addition of a second equivalent of chloro-dialkylphosphine in the same way as before yielded crude products $\mathbf{H L}^{\mathbf{t B u}}$ and $\mathbf{H L}^{i \mathbf{P r}}$ which were purified by filtration through silica under anaerobic conditions and subsequent recrystallization from $\mathrm{Et}_{2} \mathrm{O}\left(\mathbf{H L}^{t \mathbf{B u}}\right)$ and pentane $\left(\mathbf{H L}^{i \mathbf{P r}}\right)$. The yield of the second phosphorylation was significantly lower than for the first step and was further decreased by the recrystallization step. In total $\mathbf{H L}^{\mathrm{tBu}}$ was isolated in $34 \%$ yield over the last two steps as offwhite powder. Since $\mathbf{H L}^{i \mathbf{P r}}$ was considerably better soluble in apolar solvents, only $7 \%$ of the ligand precipitated from a concentrated pentane solution after months at low temperature. Additionally, it was found that the monophosphine educt could not be separated completely in each batch.

A broad signal for the $\mathrm{NH}$ proton of the pyrazole is observed around $12.7 \mathrm{ppm}$ and the $\mathrm{CH}_{2}$ signals were found at $3.13 \mathrm{ppm}\left(\mathbf{H L}^{t \mathrm{Bu}}\right)$ and at $3.06 \mathrm{ppm}\left(\mathbf{H L}^{i \mathrm{Pr}}\right)$ in ${ }^{1} \mathrm{H}$ NMR spectra (Figure 3.8). Interestingly, the signals of the phosphorus atoms are not shifted in the mono phosphorylated species compared to the final ligands (Figure 3.9). For $\mathbf{H L}^{t \mathbf{B u}}$ a resonance was observed at $36.3 \mathrm{ppm}$ and for $\mathbf{H L}^{\mathbf{i P r}}$ at $12.1 \mathrm{ppm}$. At low temperatures, two sets of pyridine and methylene resonances were found due to slow $\mathrm{N}_{\mathrm{pz}} \mathrm{H}$ tautomerism.

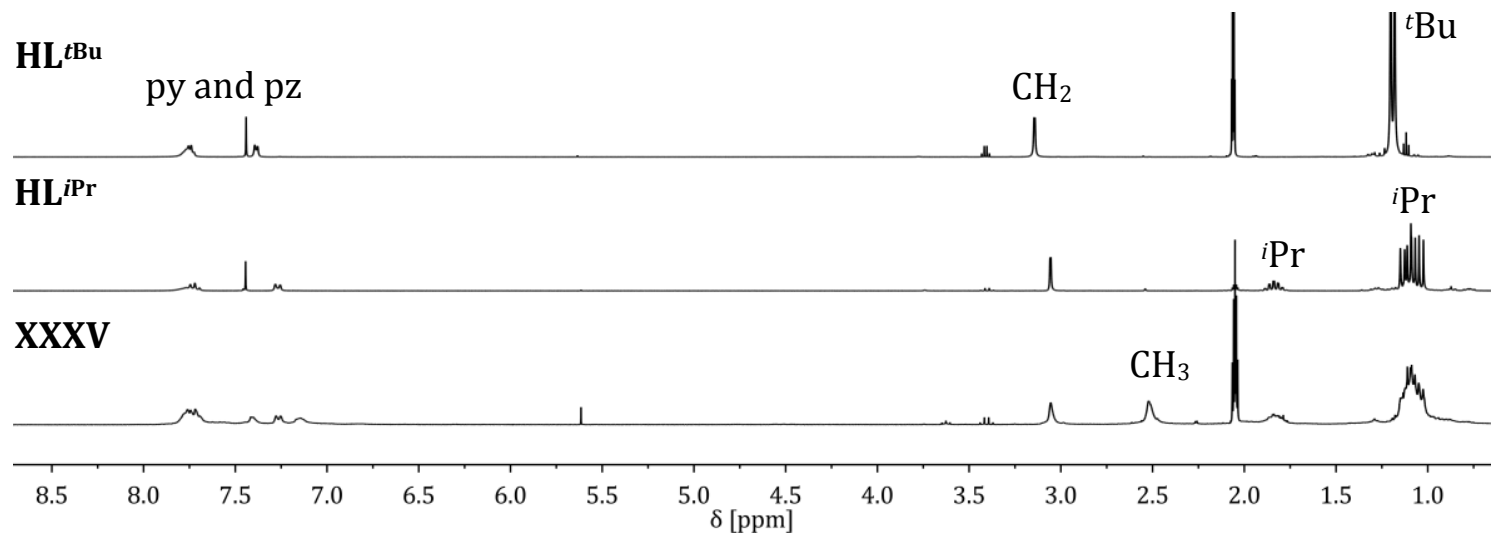

Figure 3.8: ${ }^{1} \mathrm{H}$ NMR spectra of ligands HL $^{t \mathbf{B u}}$ (XXXVII), HL ${ }^{i P r}$ (XXXVIII) and crude ${ }^{i}$ Pr monophosphine XXXV (acetone-d6, $298 \mathrm{~K}$ ). 


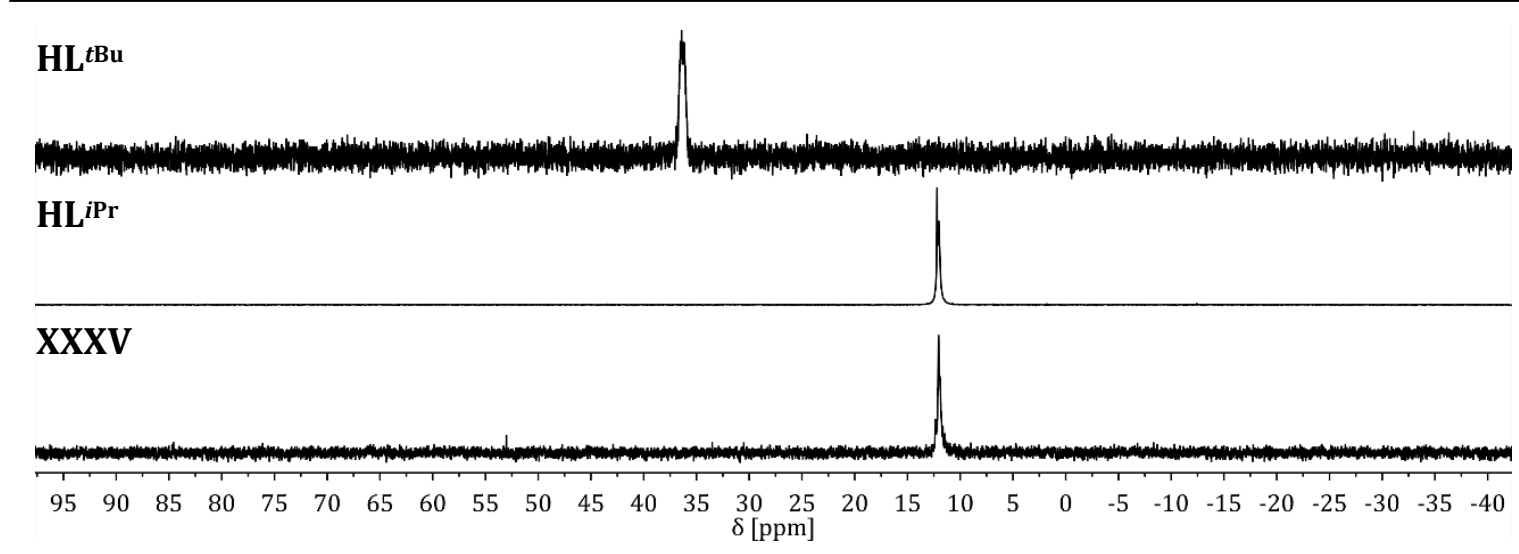

Figure 3.9: ${ }^{31} \mathrm{P}\left\{{ }^{1} \mathrm{H}\right\}$ NMR spectra of ligands $\mathbf{H L}^{t \mathbf{B u}}(\mathbf{X X X V I I}), \mathbf{H L}^{i \mathbf{P r}}(\mathbf{X X X V I I I})$ and crude ${ }^{i} \mathrm{Pr}$ monophosphine $\mathbf{X X X V}$ (acetone-d6, $298 \mathrm{~K}$ ).

Unfortunately, the second phosphorylation was not possible for the diphenyl substituted chlorophosphine. With different amounts of base and in different solvents in all cases the monophosphine compound XXXVI was isolated aside the diphosphane product $\left(\mathrm{Ph}_{2} \mathrm{P}-\mathrm{PPh}_{2}\right)$.. Either the deprotonation of the second methyl group was too slow in comparison to the reductive P-P coupling, or even not possible. Aside the lithiation of 2,6-lutidine and reaction with chlorophosphines, mononuclear PNP pincer ligands could also be prepared by halide substitution with lithium diphenylphosphide.[408] The analoguous reaction was tested by reacting 3,5-bis[6-(bromomethyl)-2-pyridyl]-1H-pyrazole XXXIX, which was used for the synthesis of other pyrazole based ligands before,[267] with in situ prepared $\mathrm{LiPPh}_{2}$ (Scheme 3.13). Again the monophospine was detected as the only compound in the crude reaction mixture.<smiles>BrCc1cccc(-c2cc(-c3cccc(CBr)n3)[nH]n2)n1</smiles><smiles>CC(C)P(C)[PH2+]</smiles><smiles>c1ccc(Cc2cccc(-c3cc(-c4cccc(Cc5ccccc5)n4)[nH]n3)n2)cc1</smiles>

\section{XXXIX}

Scheme 3.13: Alternative synthetic route tested to obtain $\mathbf{H L}^{\mathrm{Ph}}$.

Dipyrrolochlorophosphine $\left(\mathrm{Pyr}_{2} \mathrm{PCl}\right)$ was used as another phosphine to extend the ligand system towards highly electron deficient phosphorus donor side arms. [409] The synthesis from $\mathrm{PCl}_{3}$ and pyrrole in the presence of $\mathrm{NEt}_{3}$ as a base was straight forward and distillation gave pure chlorophosphine.[110] However, the coupling to the pyrazole building block XXXIII under conditions used for the alkyl substituted phosphines gave various unidentified products. Since the development of suitable reaction and workup conditions for this type of ligands is very time consuming, this project was left for future studies due to limited time for this work. 


\subsection{Complex Synthesis and Reactivity}

For the synthesis of dinuclear pyrazolate based complexes of the Two-in-one pincer ligands the deprotonation of the pyrazole is necessary. The use of excess triethylamine was observed to hamper a facile isolation of pure complex since one equivalent of triethylammonium salt was formed in the reaction. Pure compound was only obtained by crystallization of the complexes. To facilitate the purification procedure, one equivalent of a strong base such as KOtBu was used to deprotonate the pyrazole $\mathrm{NH}$ group. The formed potassium salts could be separated more easily due to a lower solubility in apolar solvents. Alternatively, a metal salt with an internal base (metal acetate) was used. The reactivity of the synthesized complexes was tested regarding the ability of deprotonation of the side arm and the formation of reactive species such as hydrides or alkyl compounds, which might activate small molecules.

\subsubsection{Zinc Complexes}

Pincer complexes with zinc are only barely known and a complex with a phosphine side arm is not described so far. ${ }^{[411-413]}$ Hence, zinc complexes were synthesized with both ligands, $\mathbf{H L}^{t \mathbf{B u}}$ and $\mathbf{H L} \mathbf{L P r}^{\mathbf{P r}}$ and with triflate and acetate as bridging units between the two metal centers. Zinc(II) complexes are useful for investigating structural properties due to their air stability, redox inertness and diamagnetism (for NMR spectroscopy). When $\mathbf{H L}^{t \mathbf{B u}}$ was reacted with $\mathrm{Zn}(\mathrm{OAc})_{2} \cdot 2 \mathrm{H}_{2} \mathrm{O}$ in $\mathrm{MeCN}$, no additional base was needed to deprotonate the pyrazole and potassium hexafluorophosphate $\left(\mathrm{KPF}_{6}\right)$ was added to introduce a non-coordinating counterion (Scheme 3.14).

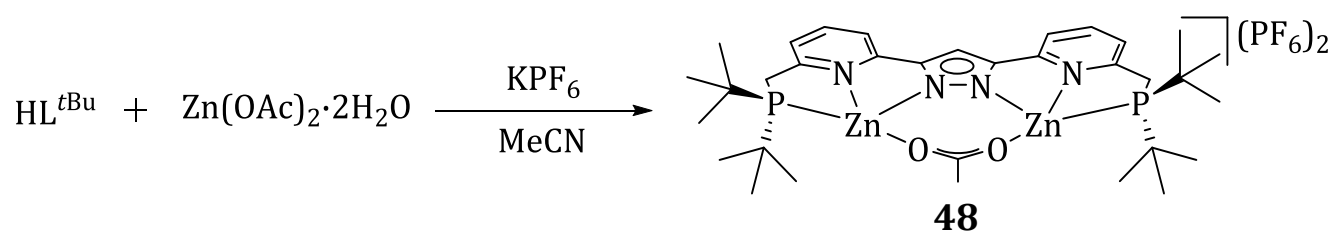

Scheme 3.14: Synthesis of acetate bridged $\left[\mathrm{Zn}_{2}(\mu-\mathrm{OAc}) \mathrm{L}^{t \mathrm{Bu}}\right]\left(\mathrm{PF}_{6}\right) \mathbf{4 8}$ from zinc acetate and $\mathbf{H L}^{t \mathrm{Bu}}$.

The reaction gave the symmetric, acetate bridged complex 48 in high yield. The ${ }^{1} \mathrm{H}$ NMR spectrum is depicted in Figure 3.10 showing the symmetry by having three resonances for the pyridine protons between $7.96 \mathrm{ppm}$ and $7.42 \mathrm{ppm}$, the singulet for the pyrazole $4-\mathrm{H}$ proton at $7.21 \mathrm{ppm}$, one doublet for the methylene group at $3.48 \mathrm{ppm}$ and one doublet for the tert-butyl groups at $1.35 \mathrm{ppm}$ resulting from a coupling to the phosphorus nuclei. The signal of the acetate is superimposed by the MeCN signal. In a ${ }^{31} \mathrm{P}$ NMR spectrum a shift of $19.48 \mathrm{ppm}$ was observed for the phosphorus side arms. Crystals of this compound were not obtained, thus the zinc salt 
was changed to zinc triflate in order to vary the counterion and to change the crystallization properties.

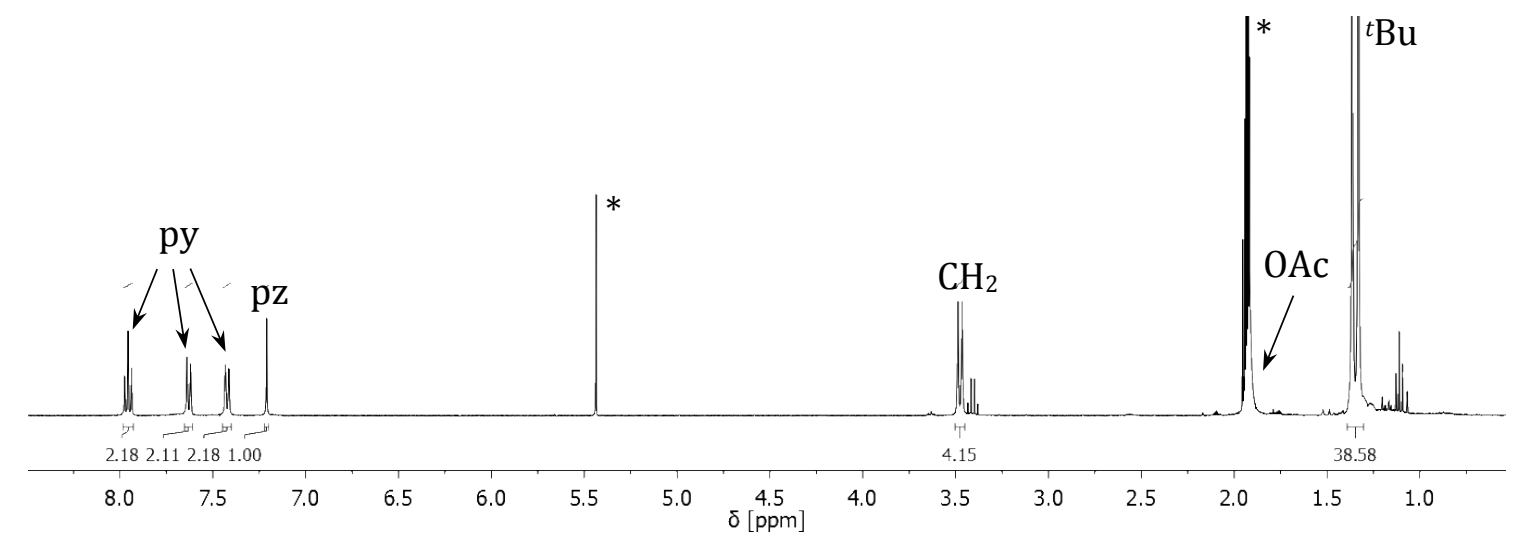

Figure 3.10: ${ }^{1} \mathrm{H}$ NMR spectrum of $\left[\mathrm{Zn}_{2}(\mu-\mathrm{OAc}) \mathrm{L}^{t \mathrm{Bu}}\right]\left(\mathrm{PF}_{6}\right)_{2} 48$ (acetonitrile- $\left.\mathrm{d}_{3}, 298 \mathrm{~K}\right)$. Residual solvent resonances are marked with an asterisk.

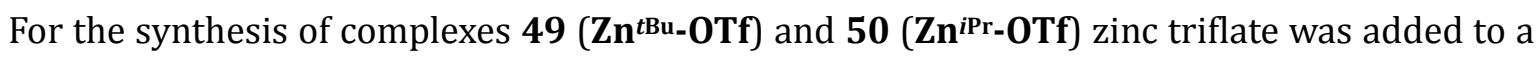
suspension of ligand $\mathbf{H L}^{\mathbf{x}}$ and $\mathrm{KO}{ }^{t} \mathrm{Bu}$ in MeCN. The crude products were extracted with DCM to separate the formed potassium triflate from the complexes.

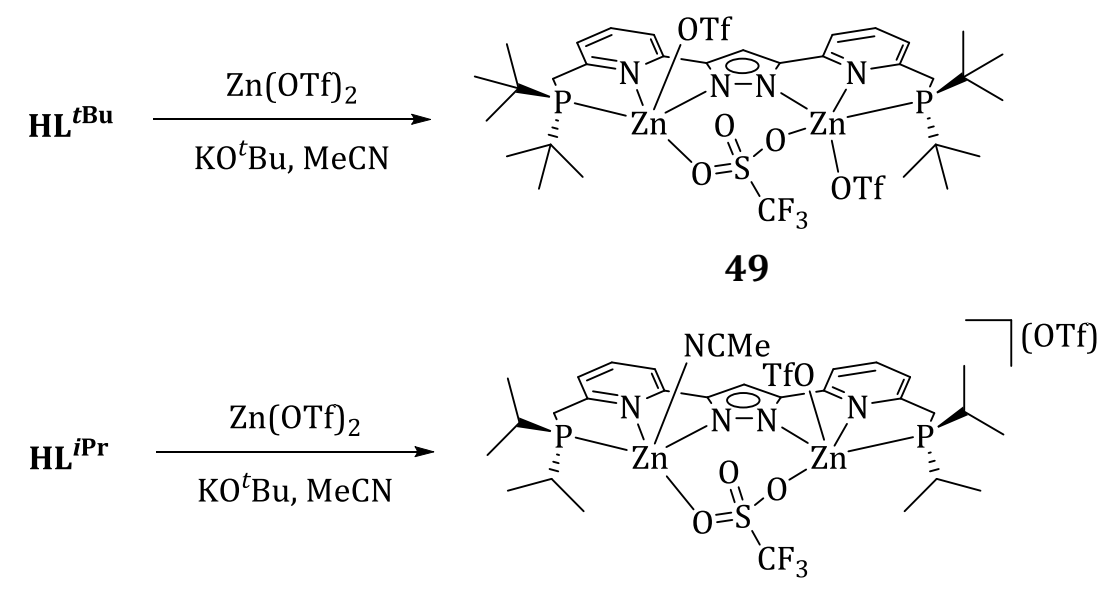

\section{0}

Scheme 3.15: Synthesis of $\left[(\operatorname{Zn}(\mathrm{OTf}))_{2}(\mu-\mathrm{OTf}) \mathrm{L}^{\mathrm{tB} u}\right] \mathbf{4 9}$ and $\left[(\mathrm{Zn}(\mathrm{MeCN}))(\mathrm{Zn}(\mathrm{OTf}))(\mu-\mathrm{OTf}) \mathrm{L}^{\mathrm{iPr}}\right](\mathrm{OTf}) \mathbf{5 0}$ by reacting

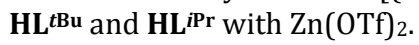

The formation of the desired, air stable complexes was shown by NMR spectroscopy. In ${ }^{31} \mathrm{P}$ spectra only single resonances at $22.55 \mathrm{ppm}$ and $6.04 \mathrm{ppm}$ were observed for the ${ }^{\mathrm{B}} \mathrm{Bu}$ and the ${ }^{i}$ Pr ligand, respectively. Both ${ }^{1} \mathrm{H}$ NMR spectra exhibit one doublet for the $\mathrm{CH}_{2}$ groups evidencing symmetric species in solution (Figure 3.11). These signals of $\mathbf{H L}^{t \mathbf{B u}}$ (3.62 ppm) are slightly more deshielded as in $\mathbf{H L}^{\mathbf{i P r}}$ (3.50 ppm), which was also visible in the ${ }^{13} \mathrm{C}$ NMR resonances (29.03 ppm vs. $26.39 \mathrm{ppm}$ ). ESI-MS spectra showed several species which could be assigned to different oxygenated species. Even under inert conditions, results showing only one species were not detected. It was found that complex $\mathbf{5 0}$ was not stable in solution under aerobic conditions for longer time. In NMR measurements a shift of the ${ }^{31} \mathrm{P}$ NMR resonance from $6.04 \mathrm{ppm}$ to $71.56 \mathrm{ppm}$ for the oxidized species was observed. In addition, the signal for the 
$\mathrm{CH}_{2}$ groups is located at $3.66 \mathrm{ppm}$, a shift of $0.17 \mathrm{ppm}$ to lower field. This instability was not found for the tert-butyl complex, which may indicate that the steric crowd of the substituents on the phosphine play an important role in the corresponding oxygenation reaction.

$49(\mathrm{Bu})$

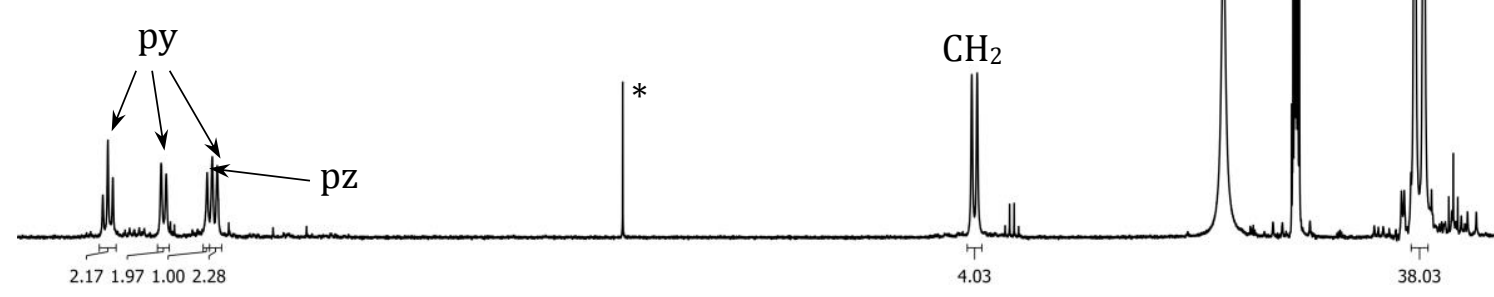

$50\left({ }^{i} \operatorname{Pr}\right)$

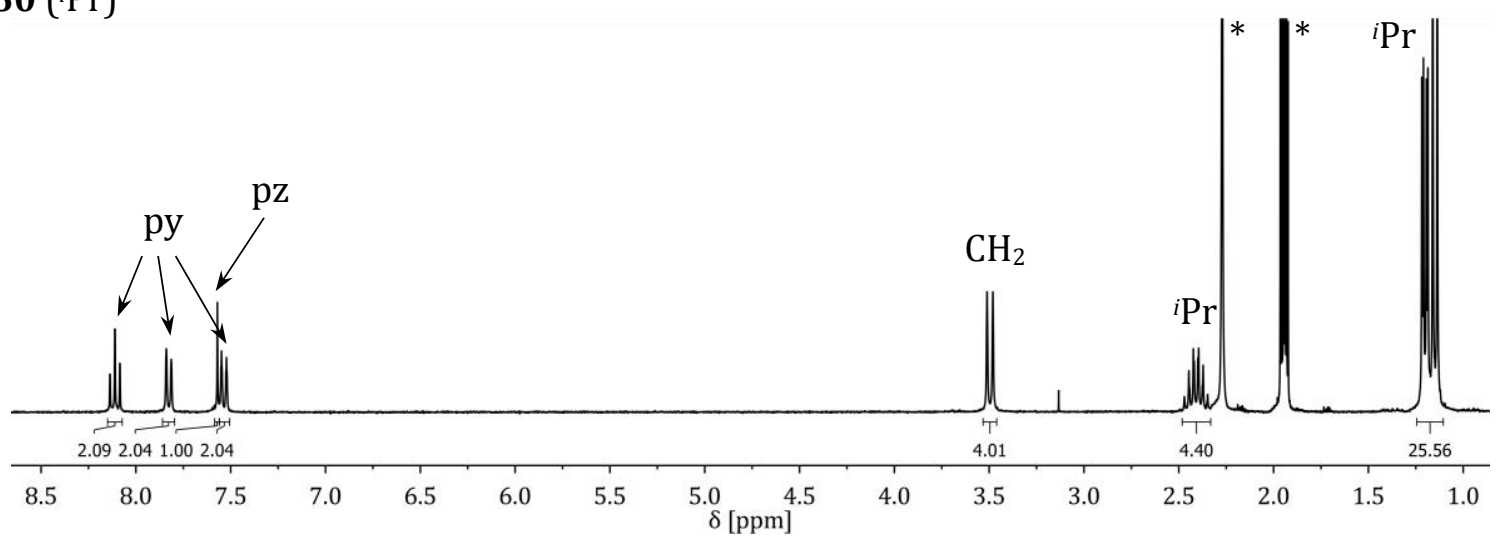

Figure 3.11: ${ }^{1} \mathrm{H}$ NMR spectra of $\mathbf{4 9}$ and $\mathbf{5 0}$ (acetonitrile-d 3 , $298 \mathrm{~K}$ ). Residual solvent resonances are marked with an asterisk.

Crystals for 49 and $\mathbf{5 0}$ were obtained by slow $\mathrm{Et}_{2} \mathrm{O}$ diffusion into $\mathrm{MeCN}$ solutions. Both complexes crystallize in the triclinic space group $\mathrm{P} \overline{1}$ with two molecules in the unit cell.
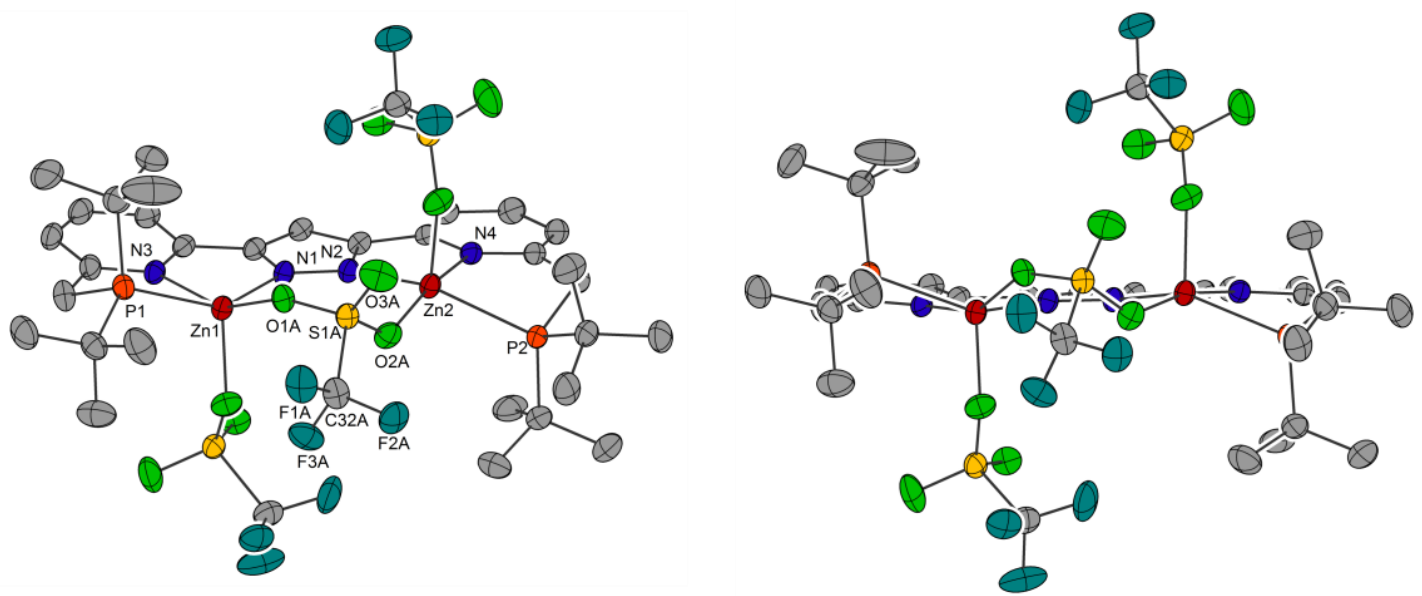

Figure 3.12: Thermal displacement ellipsoids (shown at $30 \%$ probability) of the molecular structure of $\left[(\mathrm{Zn}(\mathrm{OTf}))_{2}(\mu-\mathrm{OTf}) \mathrm{L}^{t \mathrm{Bu}}\right] \mathbf{4 9}$ in two orientations. Hydrogen atoms and solvent molecules were omitted for clarity.

In 49 both zinc atoms are coordinated by the meridional $\{\mathrm{PNN}\}$ ligand pocket and two oxygen atoms of two triflate ions, giving a distorted square pyramidal geometry around the metal 68 
centers with triflate in the axial positions (Figure 3.12). According to the method of ADDISON, the geometric parameter $\tau_{5}$ is 0.14 and 0.38 reflecting the found geometry. ${ }^{[414]}$ The zinc atoms are bridged by one of the triflates and separated by $d_{\mathrm{Zn}-\mathrm{Zn}}=4.326 \AA$. The metal centers are only slightly above and below the pyrazole-pyridine-methylene backbone plane, whereas the phosphorus atoms are located $0.604 \AA$ and $-0.447 \AA$ outside of that plane. The Zn-P bond lengths are $\mathrm{d}_{\mathrm{Zn} 1-\mathrm{P} 1}=2.432 \AA$ and $\mathrm{d}_{\mathrm{Zn} 2-\mathrm{P} 2}=2.462 \AA$, which is slightly longer compared to zinc phosphine complexes reported in literature. ${ }^{[415]}$ Weak $\pi$-stacking interactions can be found between the pyridine $\mathrm{C}-3$ carbon atoms of the ligand backbones. The minimum distances were found to be $\mathrm{d}_{\mathrm{C}-\mathrm{C}}=3.351 \AA$ A (Figure 3.13).
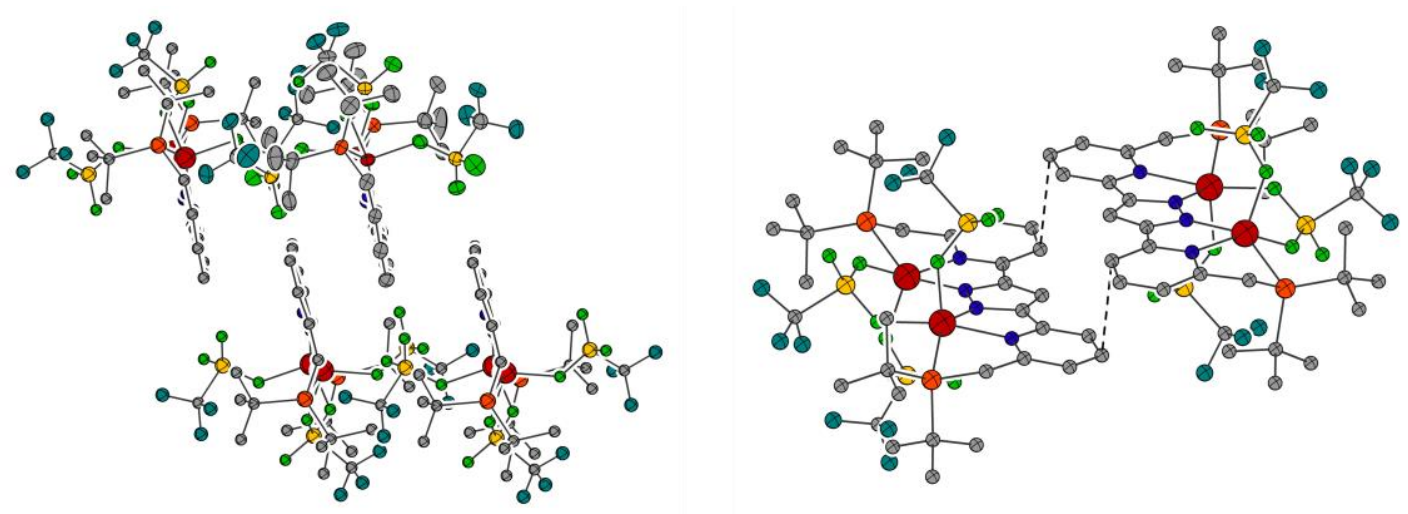

Figure 3.13: Thermal displacement ellipsoids (shown at $30 \%$ probability) of the molecular structure of 49: The packing diagram in the solid state (left) and the two molecules of the unit cell showing weak interactions of the pyrazole-pyridine backbones (right). Hydrogen atoms and solvent molecules were omitted for clarity.

50 was crystallized under inert conditions. The molecular structure is depicted in Figure 3.14, showing a non-symmetric arrangement with one acetonitrile molecule coordinated to one of the zinc atoms. On the same side of the backbone plane, a triflate ion is bound to the second zinc ion. A second triflate ion is bridging the metal centers, while the third is found as counterion hampering a stacking of the molecules as observed in $\mathbf{4 9}$.
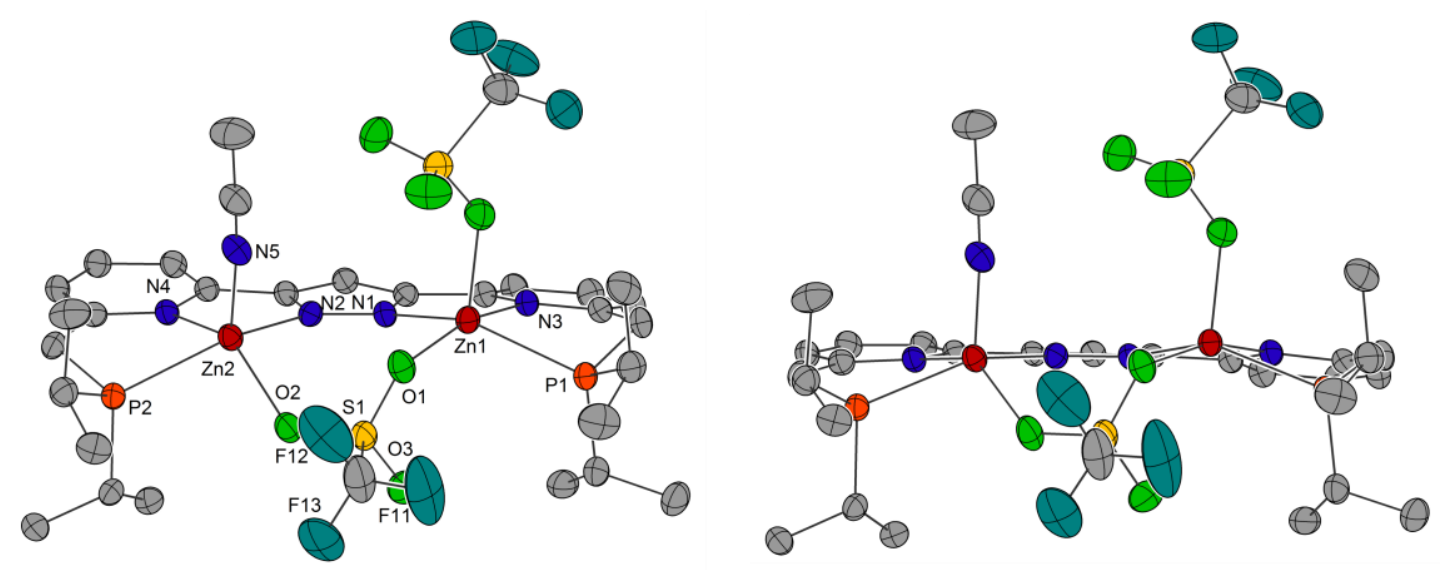

Figure 3.14: Thermal displacement ellipsoids (shown at $30 \%$ probability) of the molecular structure of $\left[(\mathrm{Zn}(\mathrm{MeCN}))(\mathrm{Zn}(\mathrm{OTf}))(\mu-\mathrm{OTf}) \mathrm{L}^{\mathrm{iPr}}\right](\mathrm{OTf}) \mathbf{5 0}$ in two different orientations. Counterions, hydrogen atoms and noncoordinating solvent molecules were omitted for clarity. 
The metal-metal distance is essentially the same $\left(\mathrm{d}_{\mathrm{Zn}-\mathrm{Zn}}=4.342 \AA\right)$ and also the lengths of the Zn-P bonds $\left(\mathrm{d}_{\mathrm{Zn} 1-\mathrm{P} 1}=2.431 \AA\right.$ and $\mathrm{d}_{\mathrm{Zn2}-\mathrm{P} 2}=2.445 \AA$ ) are very similar. The high structurally similarity is also reflected by the $\tau_{5}$ values, which are close to those of the ${ }^{\mathrm{B}} \mathrm{Bu}$ analogue $(0.16$ and 0.30 in 50; 0.14 and 0.38 in $\mathbf{4 9}$ ).

If $\mathbf{5 0}$ was crystallized under aerobic conditions, oxidation of the phosphines was observed. Although the quality of the crystallographic data was not sufficient to completely refine the

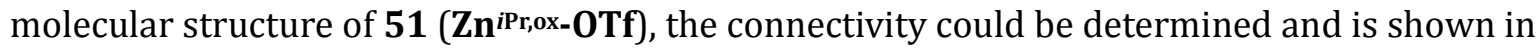
Figure 3.15 .

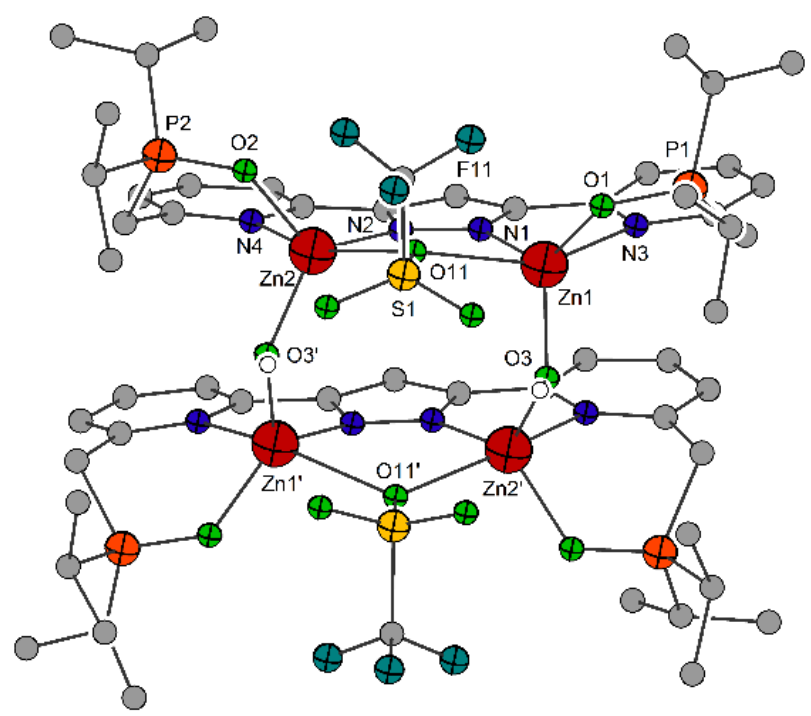

Figure 3.15: Stick-and-ball representation of the molecular structure of $\left[\left(\mathrm{Zn}_{2}(\mu-\mathrm{OTf}) \mathrm{L}^{t \mathrm{Bu}, \mathrm{ox}}\right)_{2}(\mu-\mathrm{OH})_{2}\right](\mathrm{OTf})_{2} \mathbf{5 1}$ Triflate counterions, hydrogen atoms and solvent molecules were omitted for clarity.

The phosphorus atoms were oxidized and the zinc ions are coordinated via the oxygen atoms, which resulted in a more clinched arrangement. This is represented by the shorter metal separation $\left(d_{\mathrm{zn}-\mathrm{Zn}}=3.920 \AA\right)$ and the fact that the triflate ions are bridging via only one oxygen atom. The two subunits of the dimer are connected by hydroxides, producing a short distance between the zinc atoms of the two subunits $\left(\mathrm{d}_{\mathrm{Zn}-\mathrm{Zn}}=3.398 \AA\right.$ ). A summary of selected bond lengths and angles is depicted in Table 3.1. For the non-oxidized complexes $\mathbf{4 9}$ and $\mathbf{5 0}$ similar bond length and angles were found, although the coordination environment of one zinc ion is different in the iso-propyl complex. 
Table 3.1: Selected bond lengths, angles and structural parameter $\tau_{5}$ for the deviation from ideal square pyramidal coordination geometry of complexes 49 - $\mathbf{5 1}$.

\begin{tabular}{|c|c|c|c|c|c|c|c|}
\hline & $\mathrm{d}_{\mathrm{Zn}-\mathrm{Zn}}[\AA]$ & $\mathrm{d}_{\mathrm{Zn}-\mathrm{P}}[\AA]$ & $\left.\mathrm{d}_{\mathrm{Zn-pz} \mathrm{N}}[\AA]\right]$ & $\left.\mathrm{d}_{\mathrm{Zn} \text {-py N }}[\AA]\right]$ & $\alpha\left[^{\circ}\right]$ & $\beta\left[^{\circ}\right]$ & $\tau_{5}$ \\
\hline \multirow{2}{*}{49} & \multirow{2}{*}{4.326} & 2.432 & 2.042 & 2.159 & 154.2 & 145.8 & 0.14 \\
\hline & & 2.462 & 2.050 & 2.151 & 167.4 & 144.9 & 0.38 \\
\hline \multirow{2}{*}{50} & \multirow{2}{*}{4.342} & 2.431 & 2.041 & 2.151 & 148.3 & 138.6 & 0.16 \\
\hline & & 2.445 & 2.058 & 2.161 & 162.3 & 144.4 & 0.30 \\
\hline \multirow{2}{*}{$51^{\mathrm{a}}$} & 3.920 & \multirow[t]{2}{*}{ 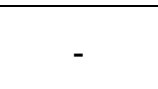 } & 1.966 & 2.218 & 151.7 & 131.7 & 0.33 \\
\hline & $3.398^{\mathrm{b}}$ & & 1.983 & 2.246 & 156.6 & 126.6 & 0.50 \\
\hline
\end{tabular}

a) For the symmetric structure of $\mathbf{5 1}$ values for one subunit are depicted for clarity. Values should be considered with caution because of the low quality of the structure determination. b) Metal-metal distance between zinc ions of the two subunits.

In order to investigate the electronic properties of the Two-in-one ligand system, electrochemical measurements of the two zinc triflate complexes ( $\mathbf{Z n}^{\text {tBu-OTf }}$ and $\mathbf{Z n}^{\text {iPr-OTf}}$ ) were performed. Since zinc(II) is an almost redox inert metal ion, reduction or oxidation events in cyclic voltammograms can be assigned to the ligand itself. Cyclic voltammograms of the reduction (left) and oxidation (right) of $\mathbf{Z n}^{\text {tBu-OTf }}$ in MeCN are depicted in Figure 3.16. Two irreversible reductions were observed at low potentials of approx. $-1.40 \mathrm{~V}$ and $-1.65 \mathrm{~V}$ (peak potentials at $100 \mathrm{mV} / \mathrm{s}$ ). In contrast, no distinct oxidation was found. These findings indicate that the ligand system can be reduced, but not oxidized in the measured potential range. Very similar results were also observed for $\mathbf{Z n}^{\text {iPr-OTf. }}$
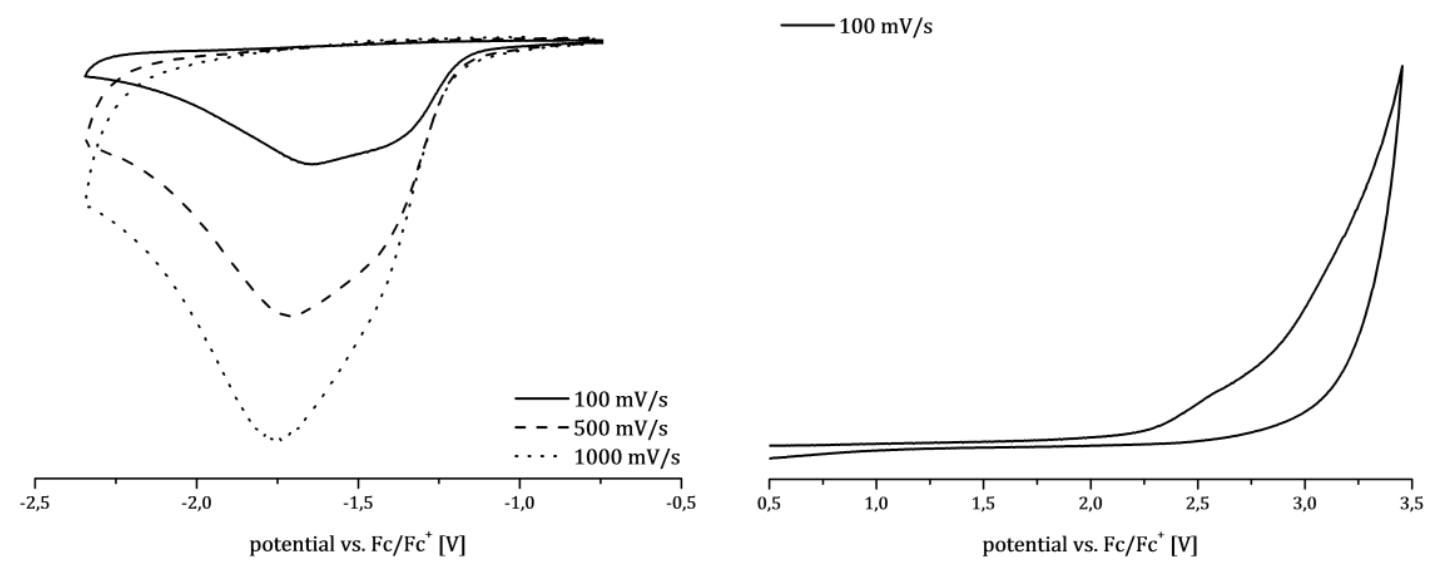

Figure 3.16: Cyclic voltammogram (measured in $0.1 \mathrm{M} n \mathrm{Bu}_{4} \mathrm{NPF}_{6}$ in $\mathrm{MeCN}$ ) of $\mathbf{Z n}^{\text {tBu}}$-OTf at different scan rates for the reduction (left) and at $100 \mathrm{mV} / \mathrm{s}$ for the oxidation (right).

Reactions of the synthesized zinc(II) complexes with different strong bases in order to deprotonate the ligand side arm were not successful (Scheme 3.16). Upon addition of KO ${ }^{t} \mathrm{Bu}$ or $\mathrm{KH}$, the triflate complexes $\mathbf{4 9}$ and $\mathbf{5 0}$ decomposed and no products could be identified by ESI mass spectrometry or NMR spectroscopy. 


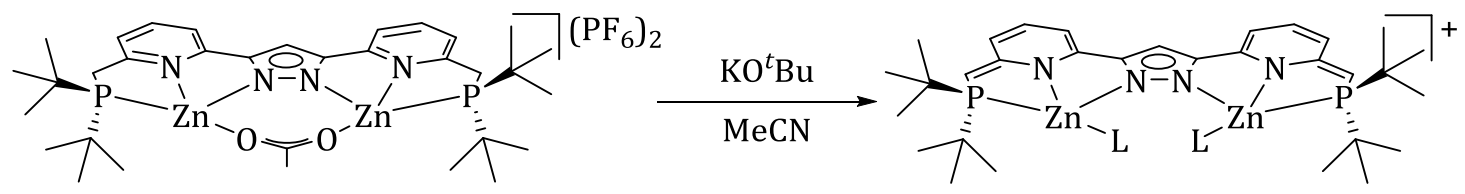

48

Scheme 3.16: Attempted deprotonation of complex 48 with $\mathrm{KO}^{t} \mathrm{Bu}$ with neutral ligands $\mathrm{L}$ in the binding pocket.

A crude ${ }^{1} \mathrm{H}$ NMR spectrum of a reaction of the acetate complex 48 with $\mathrm{KO}{ }^{\mathrm{t}} \mathrm{Bu}$ showed some indications that a deprotonation took place (Figure 3.17), however further investigations were not possible, since the signals of the formed species vanished after one day completely and the resonances of the free ligand appeared. The signal for the bridged acetate disappeared and the integral of the former $\mathrm{CH}_{2}$ group was lower and shifted to higher field which is in agreement with a higher electron density at this position. In contrast, the signals for the pyridine protons were only shifted less than $0.25 \mathrm{ppm}$ to higher field, so that a dearomatization was not very likely. But this dearomatization might be crucial for the stabilization of the deprotonated complex. Unfortunately, a ${ }^{13} \mathrm{C}$ NMR spectrum exhibited too low intensity of the resonances to safely assign them and support the assumption of deprotonation without a dearomatization. The ${ }^{31 P}$ NMR signal shifted from $6.04 \mathrm{ppm}$ to $32.11 \mathrm{ppm}$ upon addition of base.

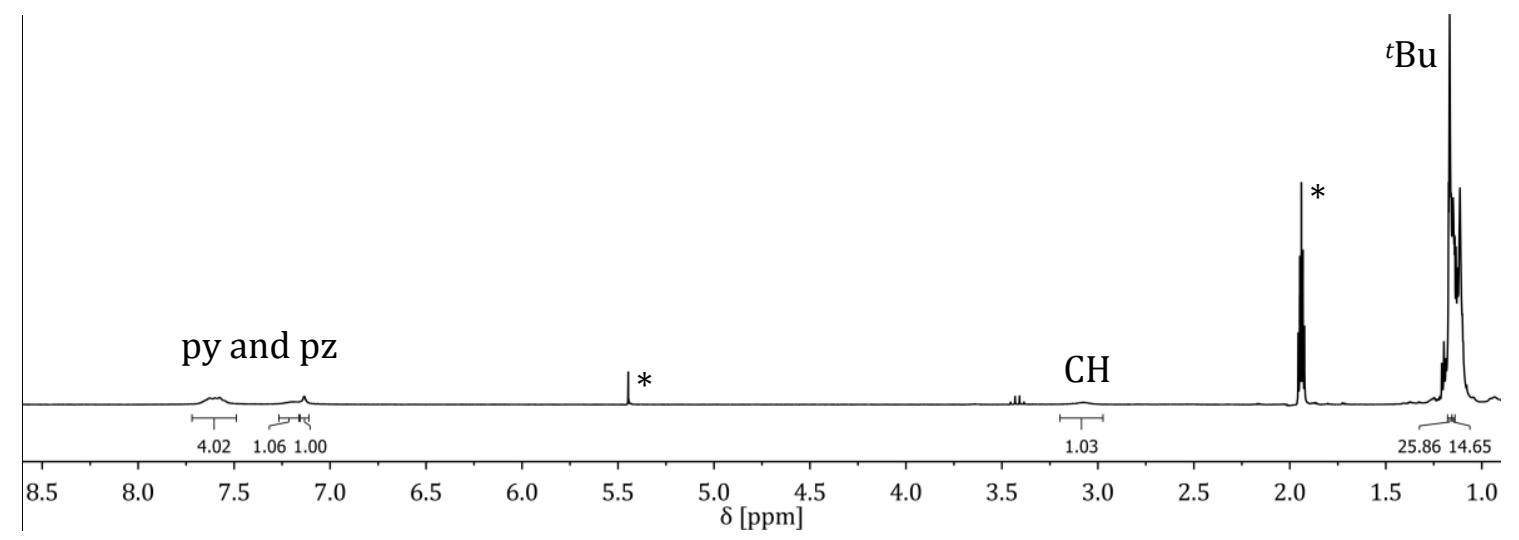

Figure 3.17: ${ }^{1} \mathrm{H}$ NMR spectra of a reaction mixture of zinc acetate complex 48 and $\mathrm{KO}^{t} \mathrm{Bu}$ with prosposed assignment of the resonances (acetonitrile- $\mathrm{d}_{3}, 298 \mathrm{~K}$ ). Residual solvent resonances are marked with an asterisk.

It was tried to follow the deprotonation reaction by UV-vis spectroscopy but the observations were ambiguous. The spectrum of $\mathbf{Z n}^{\mathrm{tBu}} \mathbf{\text { OT }} \mathbf{\text { Tf }}$ in MeCN exhibited two intensive $\pi$ - $\pi^{*}$ absorption bands in the UV region $\left(\lambda_{\max , 1}=259 \mathrm{~nm} \quad\left(\varepsilon_{1}=38800 \mathrm{M}^{-1} \mathrm{~cm}^{-1}\right)\right.$ and $\quad \lambda_{\max , 2}=306 \mathrm{~nm}$ $\left.\left(\varepsilon_{2}=38100 \mathrm{M}^{-1} \mathrm{~cm}^{-1}\right)\right)$. Upon addition of $\mathrm{KO}{ }^{t} \mathrm{Bu}$, a shift of the absorption maxima was observed ( 259 and $306 \mathrm{~nm}$ to 272 and $340 \mathrm{~nm}$ ), indicating a change in the electronic structure of the ligand, since these bands were assigned to ligand related $\pi-\pi^{*}$ transitions (Figure 3.18, left). This effect was expected for a deprotonation and dearomatization of the ligand. Unfortunately, stable products in a reaction on a larger scale could not be isolated, although the formed species in the cuvettes seemed to be quite stable. In methylene chloride as solvent and using $\mathrm{LiO}^{\text {t }} \mathrm{Bu}$ as 
a base, a different behavior was found. Here, the absorption maxima shifted only slightly, which might be a consequence of a ligand exchange with tert-butoxide anions (Figure 3.18, right).
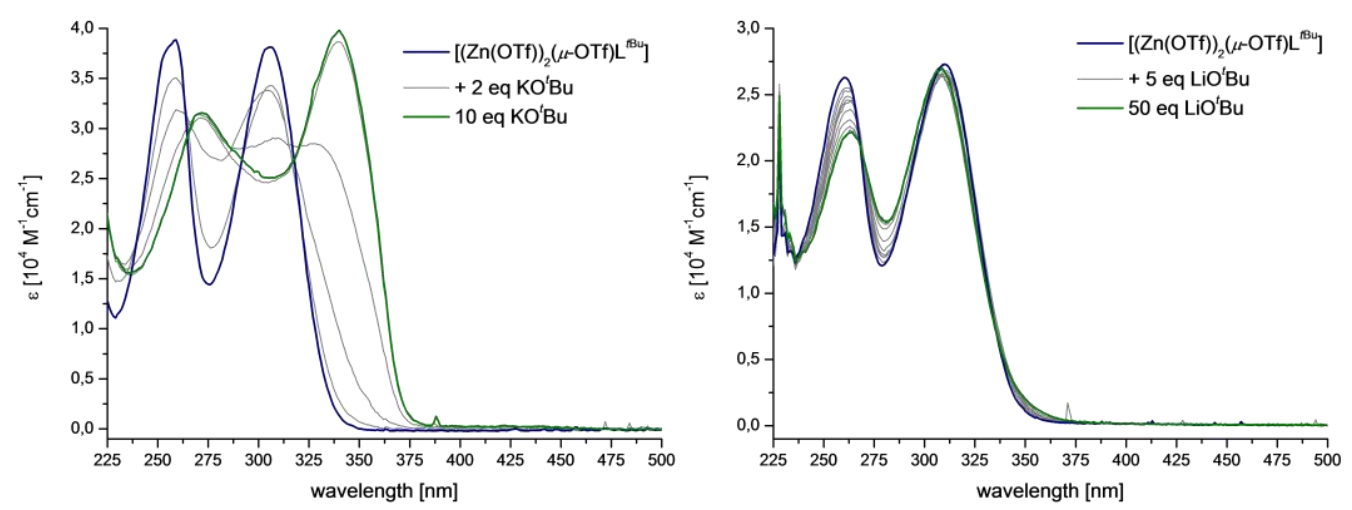

Figure 3.18: UV-vis spectra of reactions of $\left[(\mathrm{Zn}(\mathrm{OTf}))_{2}(\mu\right.$-OTf $\left.) \mathrm{L}^{t \mathrm{Bu}}\right] \mathbf{4 9}$ with $\mathrm{KO}{ }^{\mathrm{t} B u}$ in $\mathrm{MeCN}$ at $0{ }^{\circ} \mathrm{C}$ (left) and with $\mathrm{LiO}^{t} \mathrm{Bu}$ in $\mathrm{CH}_{2} \mathrm{Cl}_{2}$ at ambient temperature (right).

The behavior of $\mathbf{Z n}^{\text {tBu-OTf }}$ upon addition of base was found to be ambiguous and highly dependent on the reaction conditions. In fact, a deprotonation and a concomitant dearomatization could not be proved.

Zinc alkyl or alkoxy complexes are known as active initiators for the polymerization of rac-lactide and other cyclic esters.[416,417] Thus, different ways to synthesize zinc alkyl complexes were tried, but both the use of diethylzinc in combination with ligand $\mathbf{H L}^{\mathrm{tBu}}$ and the reaction of 49 with methyl magnesium bromide did not lead to succesful isolation of the desired zinc alkyl complexes. With the zinc alkyl precursor, a mixture of various species was formed as indicated by ${ }^{31} \mathrm{P}$ NMR spectroscopy. The reaction with a Grignard reagent (Scheme 3.17) seemed to be cleaner, the ${ }^{31} \mathrm{P}$ NMR spectrum of the crude reaction products revealed only one resonance in at 71.01 ppm (49: $22.55 \mathrm{ppm})$.

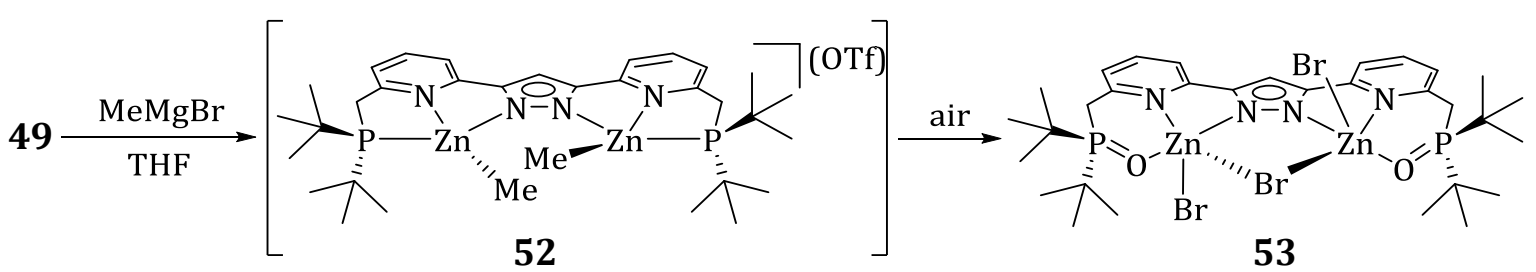

Scheme 3.17: Possible reaction pathway for the formation of ligand oxidized zinc bromide complex $\mathbf{5 3}$ via the alkyl zinc intermediate $\mathbf{5 2}$ upon reaction of $\mathbf{4 9}$ with methyl magnesium bromide.

However, the signals of the methylene groups could not be reliably assigned. In addition the signals of the methyl groups were not found in the expected regions $(0--1 \mathrm{ppm}) .{ }^{[416,418]}$ The spectrum is shown in Figure 3.19, exhibiting three signals for the pyridine protons, revealing a symmetric compound. Further purification steps led to fast decomposition of the product. 


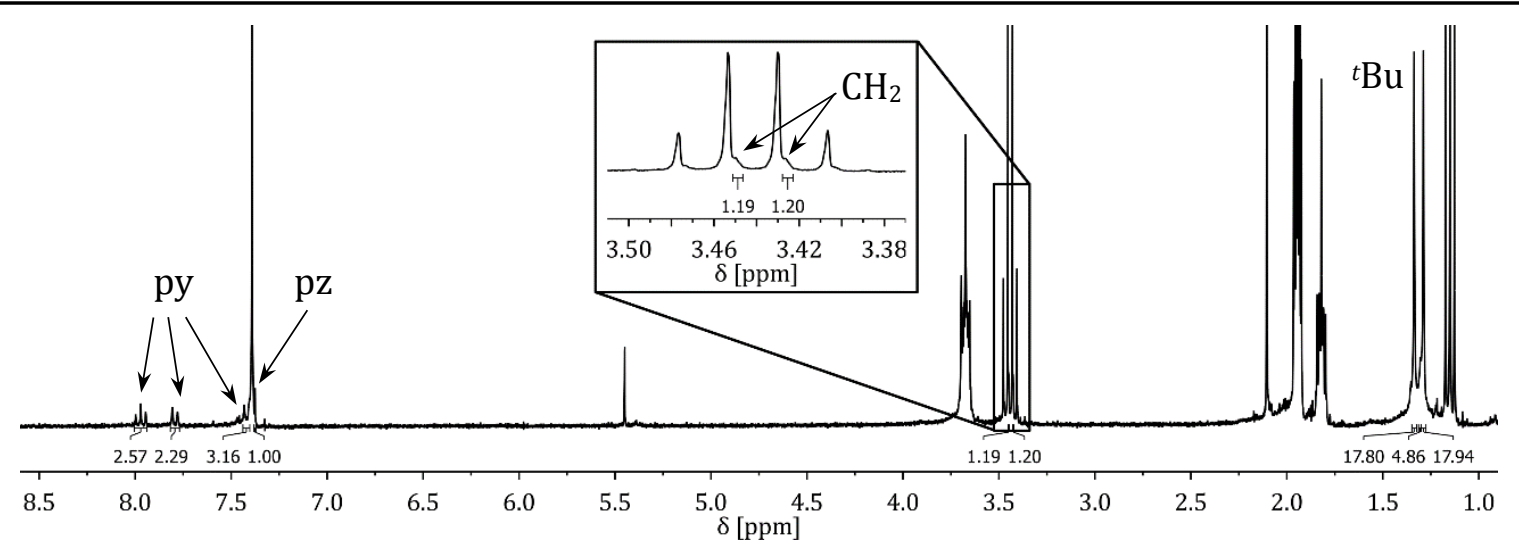

Figure 3.19: ${ }^{1} \mathrm{H}$ NMR spectrum of a crude product of a reaction of 49 and $\mathrm{MeMgBr}$ (acetonitrile- $\mathrm{d}_{3}, 298 \mathrm{~K}$ ). The inset is showing the $\mathrm{CH}_{2}$ resonance, which is superimposed by the $\mathrm{Et}_{2} \mathrm{O}$ signal.

Crystallisization attempts were conducted in the presences of a slight excess of methyl magnesium bromide and colorless crystals of complex $53\left(\mathbf{Z n}^{t \mathbf{B u}, 0 x-B r}\right)$ were obtained. 53 crystallized in the monoclinic space group P12 $1 / \mathrm{c} 1$ with eight molecules in the unit cell. The molecular structure of $\mathbf{5 3}$ featured an oxidized ligand with three bromides as coligands presumably due to air in the crystallization flask (Figure 3.20). The metal metal separation $\left(d_{\mathrm{zn}-\mathrm{Zn}}=3.971 \AA\right.$ A $)$ was found to be shorter than for $\mathbf{4 9}$ due to the smaller brigding unit (bromide $v s$. triflate). The geometry around the zinc ions can be described as an intermediate between square pyramidal and trigonal bipyramidal with $\tau_{5}$ values of $0.53(\mathrm{Zn} 1)$ and $0.48(\mathrm{Zn} 2)$.
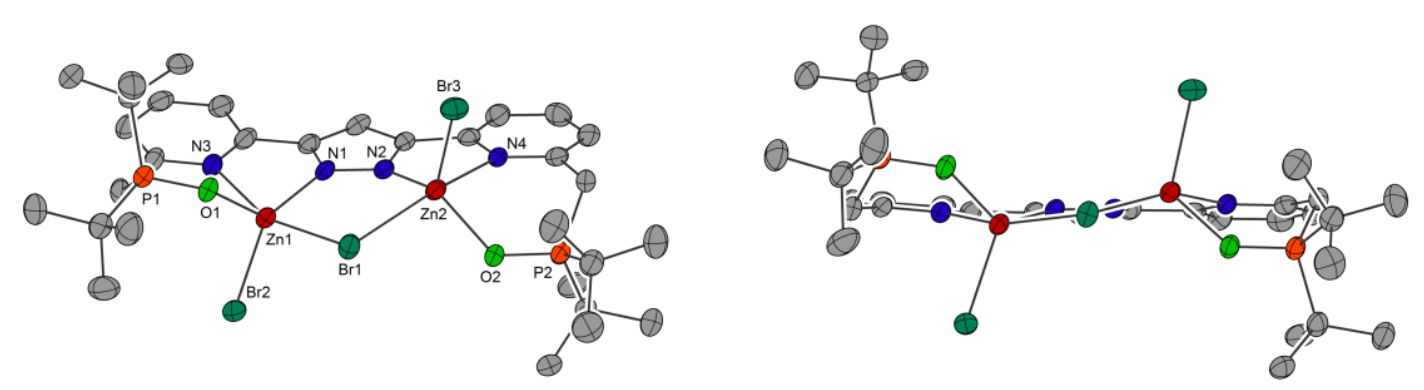

Figure 3.20: Thermal displacement ellipsoids (shown at $30 \%$ probability) of the molecular structure of $\left[(\mathrm{ZnBr})_{2}(\mu-\mathrm{Br}) \mathrm{L}^{t \mathrm{Bu} \text {, ox }}\right] \mathbf{5 3}$ in two different orientations. Hydrogen atoms and solvent molecules were omitted for clarity.

\subsubsection{Cobalt Complexes}

Cobalt(II) complex 54 (Co $\left.{ }^{\text {tBu-}-M e C N}\right)$ was first synthesized and crystallized by SAMANTA. ${ }^{[419]}$ In this work the synthetic and crystallization procedures were optimized and reactivity studies as well as catalysis experiments were conducted. 


\subsubsection{Synthesis}

Co ${ }^{t \mathrm{Bu}}$-MeCN was synthesized according to the reaction depicted in Scheme 3.18. Cobalt(II) tetrafluoroborate was combined with a suspension of $\mathbf{H L}^{t \mathbf{B u}}$ and $\mathrm{KO}^{t} \mathrm{Bu}$ in MeCN. Pure material was obtained by layering a MeCN solutions with $\mathrm{Et}_{2} \mathrm{O}$ as red-brown plates in up to $52 \%$ yield.

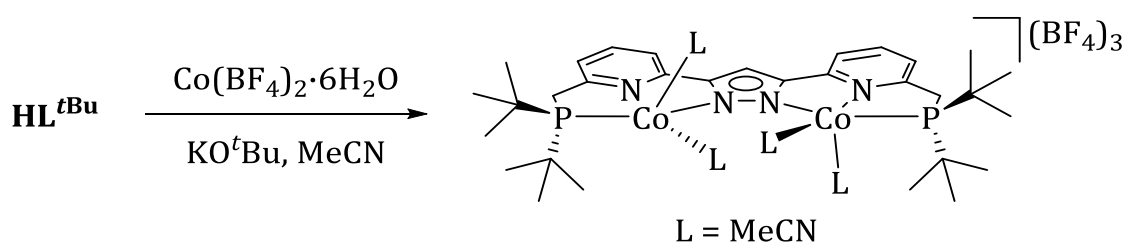

54

Scheme 3.18: Synthesis of $\left[\left(\mathrm{Co}(\mathrm{MeCN})_{2}\right)_{2} \mathrm{~L}^{\mathrm{LBu} u}\left(\mathrm{BF}_{4}\right)_{3} \mathbf{5 4}\right.$ from $\mathbf{H L}^{t} \mathbf{B u}$ and $\mathrm{KO}{ }^{t} \mathrm{Bu}$.

Co $^{t B u}-$ MeCN crystallized in the monoclinic space group P12/n1 with two molecules in the unit cell. Similar to the zinc complexes the cobalt ions are coordinated in a distorted square pyramidal geometry $\left(\tau_{5}=0.10\right)$, having one acetonitrile ligand in the axial and one in the equatorial position of each cobalt ion (Figure 3.21). The bonds to the axial ligands are longer (2.083 $\AA$ vs. $1.893 \AA$ ) and the metal atoms are slightly above the plane of the four donor atoms in the base plane of the pyramidal arrangement $\left(\mathrm{d}_{\text {Co-plane }}=0.242 \AA\right)$. The metal separation in the

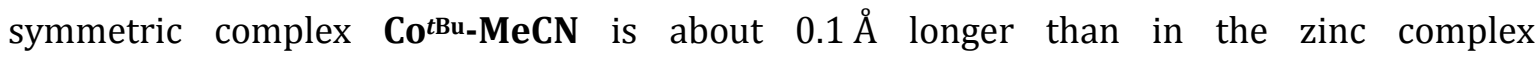
$\left(\mathrm{d}_{\mathrm{Co}-\mathrm{Co}_{0}}=4.437 \AA\right.$ ). The separation of the phosphorus atoms and the backbone plane including the pyrazole, the pyridines and methylene groups is larger than that of the metal ions.
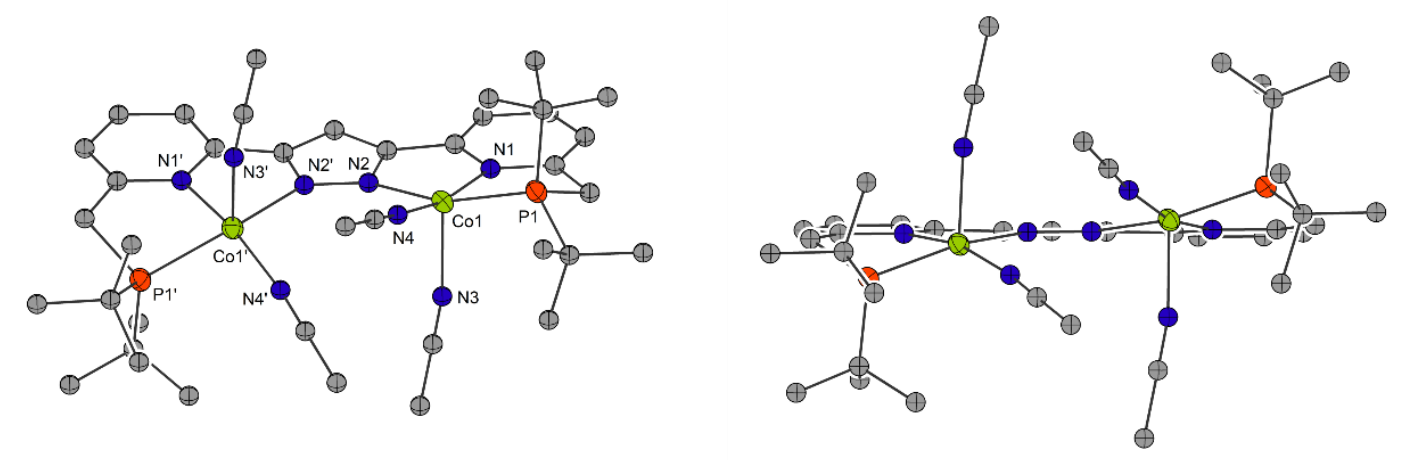

Figure 3.21: Thermal displacement ellipsoids (shown at $30 \%$ probability) of the molecular structure of $\left[\left(\mathrm{Co}(\mathrm{MeCN})_{2}\right)_{2} \mathrm{~L}^{t B u}\right]\left(\mathrm{BF}_{4}\right)_{3} \mathbf{5 4}$ in two different orientations. Counterions, hydrogen atoms and solvent molecules were omitted for clarity.

Further characterization of the cobalt(II) complex proved to be difficult in the way that NMR spectroscopy and mass spectrometry were not suitable methods for this complex. Paramagnetic NMR measurements showed some rather broad lines, but less than expected and not assignable (Figure 3.25). Other methods such as UV-vis or IR spectroscopy provided no structural information. This limitation became especially a problem when the reactivity of this complex was investigated (see chapter 3.4.2.2). To enhance the crystallization properties, the 
large non-coordinating anion tetraphenylborate was introduced, but no crystals of the product of the salt metathesis could be obtained. Also the structural characterization of cobalt complexes with chloride or bromide or with ligand $\mathbf{H L} \mathbf{i P r}^{\mathbf{P r}}$ failed, although many reaction conditions and crystallization attempts were tested.

The magnetic properties of CotBu-MeCN were investigated by EPR spectroscopy (frozen MeCN solution) and by temperature dependent measurements of the magnetic susceptibility with a SQUID magnetometer (solid material, Figure 3.22, left). The magnetic data revealed that the cobalt(II) ions have a low-spin configuration with one unpaired electron each $(S=1 / 2)$. Via superexchange through the pyrazole unit the two spins can interact with each other leading to possible spin states $S_{t}=0$ and $S_{t}=1$. The energetic separation of these two states is represented by the coupling parameter $J$ for the isotropic interactions $\left(E_{S t=1}-E_{S t}=0=-2 J\right)$. The magnetic susceptibility was recorded in the temperature range from $295 \mathrm{~K}$ to $2 \mathrm{~K}$ and the data was fitted using the HeISENBERG-DiRAC-VAN-VLECK Hamiltonian $\widehat{H}=-2 J \hat{S}_{1} \hat{S}_{2}+g \mu_{B}\left(\vec{S}_{1}+\vec{S}_{2}\right) \vec{B}$. At ambient temperature $(295 \mathrm{~K})$ a $X_{M} T$ value of $1.24 \mathrm{~cm}^{3} \mathrm{Kmol}^{-1}$ was observed, which is larger than the calculated spin only value for two uncoupled $S=1 / 2$ spins $\left(0.75 \mathrm{~cm}^{3} \mathrm{Kmol}^{-1}\right)$. This indicated also an impact of the orbital angular momentum and with that a larger LANDÉ factor than two. Upon lowering the temperature, the $\mathrm{X}_{M} T$ value decreased to $0.58 \mathrm{~cm}^{3} \mathrm{Kmol}^{-1}$. The data was best fitted with a small antiferromagnetic coupling constant $J=-1.25 \mathrm{~cm}^{-1}$ and temperature independent paramagnetism (TIP, $453 \cdot 10^{-6} \mathrm{~cm}^{3} \mathrm{~mol}^{-1}$ ).
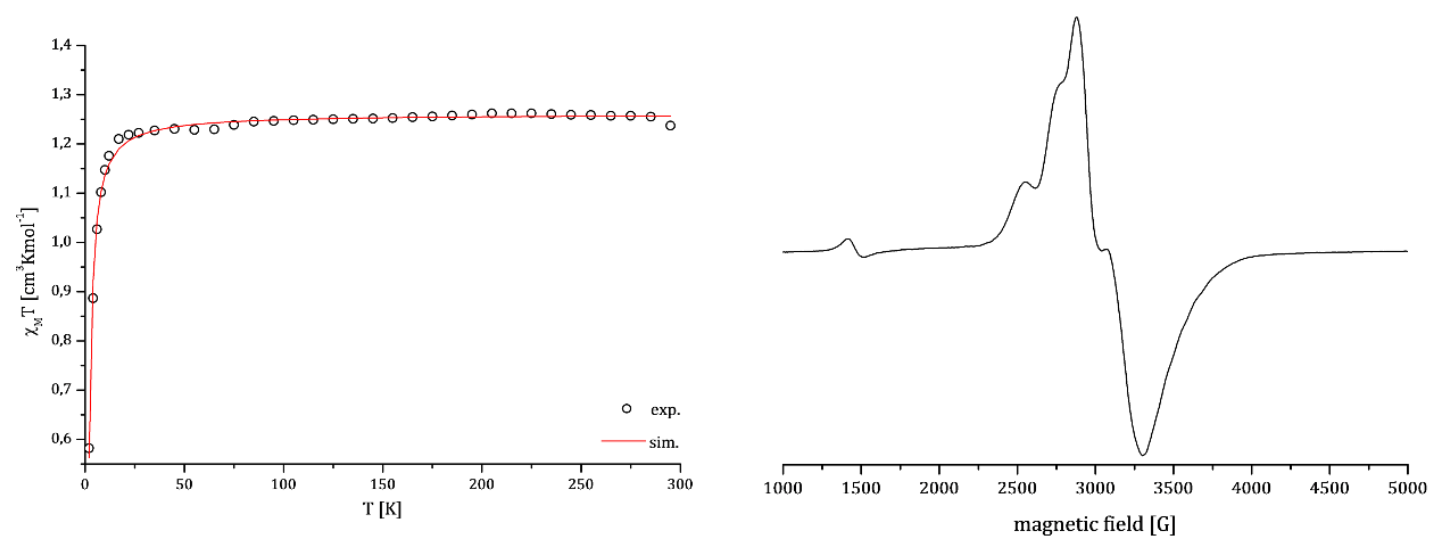

Figure 3.22: Temperature dependent measurement of the magnetic susceptibility (left)[419] and EPR spectrum in $\mathrm{MeCN}$ at $161 \mathrm{~K}$ (right) of $\left[\left(\mathrm{Co}(\mathrm{MeCN})_{2}\right)_{2} \mathrm{~L}^{\mathrm{tBu}}\right]\left(\mathrm{BF}_{4}\right)_{3} \mathbf{5 4}$.

Electron paramagnetic resonance spectroscopy (EPR) showed less than expected and extremely broad signals at $161 \mathrm{~K}$ which could not be fitted in an adequate way (Figure 3.22 , right). The g-values of the rhombic spectrum were estimated to $g_{x}=1.97, g_{y}=2.28$ and $\mathrm{g}_{\mathrm{z}}=2.72$. These values are in sharp contrast to the fused pincer dinuclear cobalt complex of FIEDLER (Figure 3.2), which exhibited diamagnetic behavior.[266] To increase the resolution of the signal the temperature has to be lowered, but this data was not available to date. 
The redox properties of $\mathbf{C o}^{t \mathrm{Bu}}-\mathbf{M e C N}$ were investigated by electrochemistry measurements. The cyclic voltammogram and the square wave voltammogram showed four irreversible reduction events (Figure 3.23, left). The first two are well separated while the third and fourth step are close together with a separation of only $80 \mathrm{mV}$. The first reduction is much easier to access with a potential of almost $1 \mathrm{~V}$ less negative compared to the second reduction. A shoulder of the first reduction wave could not be explained so far. It was assumed that the first two reductions are metal centered producing a dinuclear cobalt(I) compound. The third and fourth event was assigned to be ligand-based, since the zinc complexes exhibited events at similar potentials (Figure 3.16).
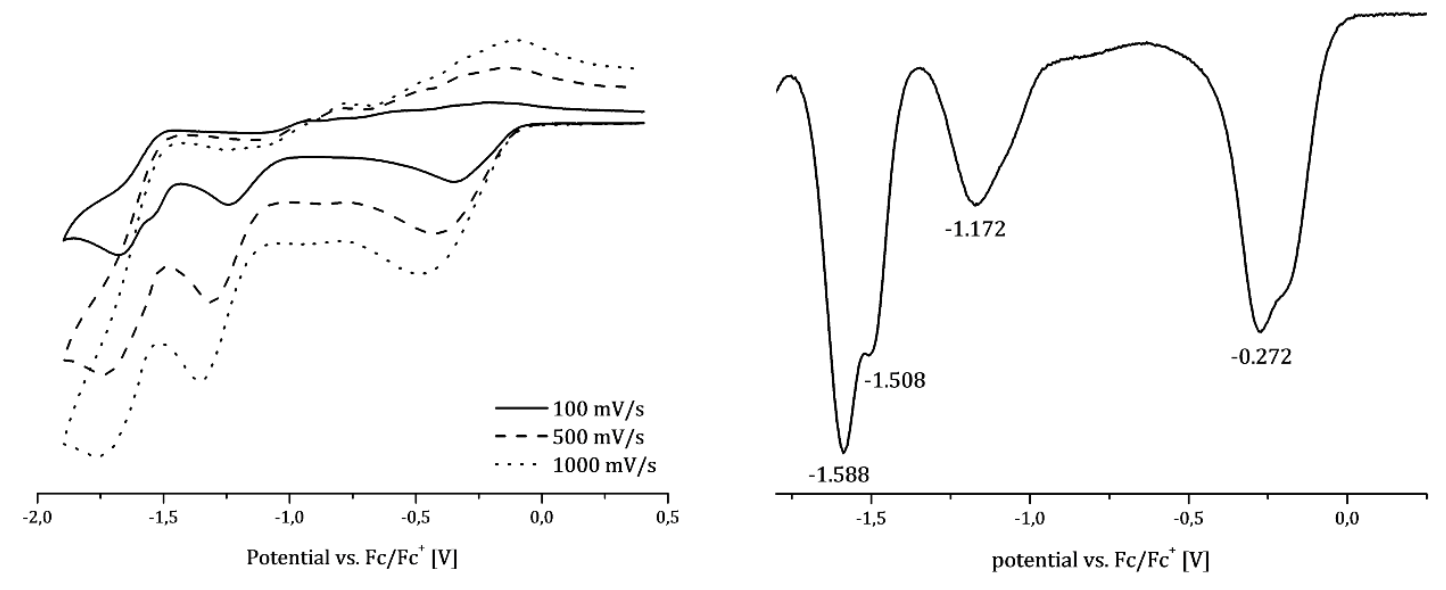

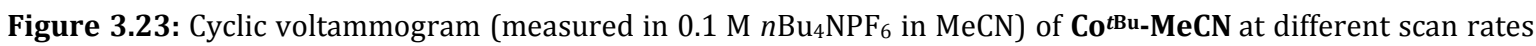
(left) and square wave voltammogram (at $100 \mathrm{mV} / \mathrm{s}$ ) with the assigned reduction potentials (right).

An elemental analyses performed with carefully dried single crystalline material showed that loss of almost one acetonitrile per molecule could not be avoided. However, the best values are in good agreement with the formula $\left[\mathrm{Co}_{2} \mathrm{~L}^{t \mathrm{Bu}}(\mathrm{MeCN})_{3}\right]\left(\mathrm{BF}_{4}\right)_{3}$ (see Chapter 5.5.2.4).

\subsubsection{Reactivity}

To study the reactivity of the cobalt(II) complex $\mathbf{C o}^{t \mathrm{Bu}}$-MeCN, different reagents such as bases, reductants and hydride or alkyl transfer agents were applied. With regards to the concept of the activation of small molecules, especially the reduced cobalt(I) and the deprotonated species (Scheme 3.19, pathways A and B) are of interest. 


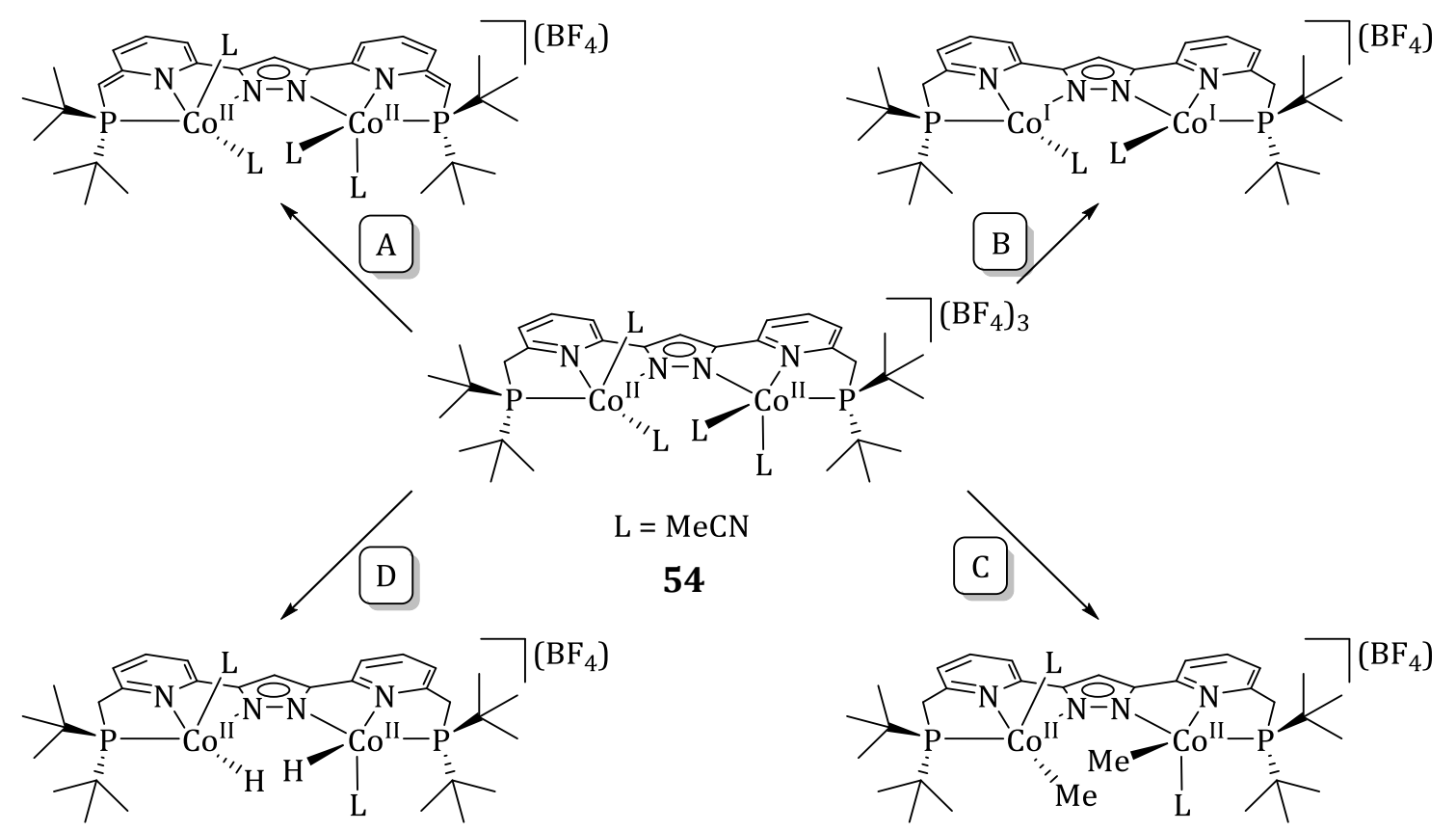

Scheme 3.19: Potential reactivity pathways for $\left[\left(\mathrm{Co}(\mathrm{MeCN})_{2}\right)_{2} \mathrm{~L}^{t \mathrm{Bu}}\right]\left(\mathrm{BF}_{4}\right)_{3}$ 54: deprotonation of the side $\operatorname{arm}(\mathrm{s})(\mathrm{A})$; reduction to cobalt(I) or even further (B); alkylation of the cobalt centers (C) and formation of a cobalt hydride complex for the activation of small molecules (D).

Hence, $\mathrm{Co}^{t \mathrm{Bu}}-\mathrm{MeCN}$ was treated with different strong bases $\left(\mathrm{KO}^{t} \mathrm{Bu}, \mathrm{LiO}^{t} \mathrm{Bu}\right.$ and $\left.\mathrm{KH}\right)$ in $\mathrm{MeCN}$ or $\mathrm{CH}_{2} \mathrm{Cl}_{2}$ and the reaction was followed by UV-vis spectroscopy. When the reaction was conducted in $\mathrm{MeCN}$, a slight color change from a red-brown solution to a rather brown solution was observed. The maximum of the absorption band, however, did not change. Somehow the intensity of the $\pi-\pi^{*}$ ligand band at $258 \mathrm{~nm}$ increased upon each addition of $\mathrm{KO}{ }^{t} \mathrm{Bu}$, without ending in a saturation (Figure 3.24, left). Changing the base to $\mathrm{LiO}{ }^{t} \mathrm{Bu}$, similar absorption changes were observed. The reactions were slower at lower temperature, but showed the same behavior. This indicated that a deprotonation reaction of $\mathbf{C o}^{\mathbf{t B u}}-\mathbf{M e C N}$ did not take place. The reason for that might be that strong bases may deprotonate bound acetonitrile and with that totally different reactions may occur. ${ }^{[420]}$ Unfortunately, the poor solubility of $\mathbf{C o}^{\text {tBu-}} \mathbf{M e C N}$ in other solvents than $\mathrm{MeCN}$ and $\mathrm{CH}_{2} \mathrm{Cl}_{2}$ rendered the use of different solvents impossible. It was thought that the dearomatization as a consequence of the deprotonation would drastically change the electronic structure of the complex and therefore a much distinct absorption maximum was expected. 

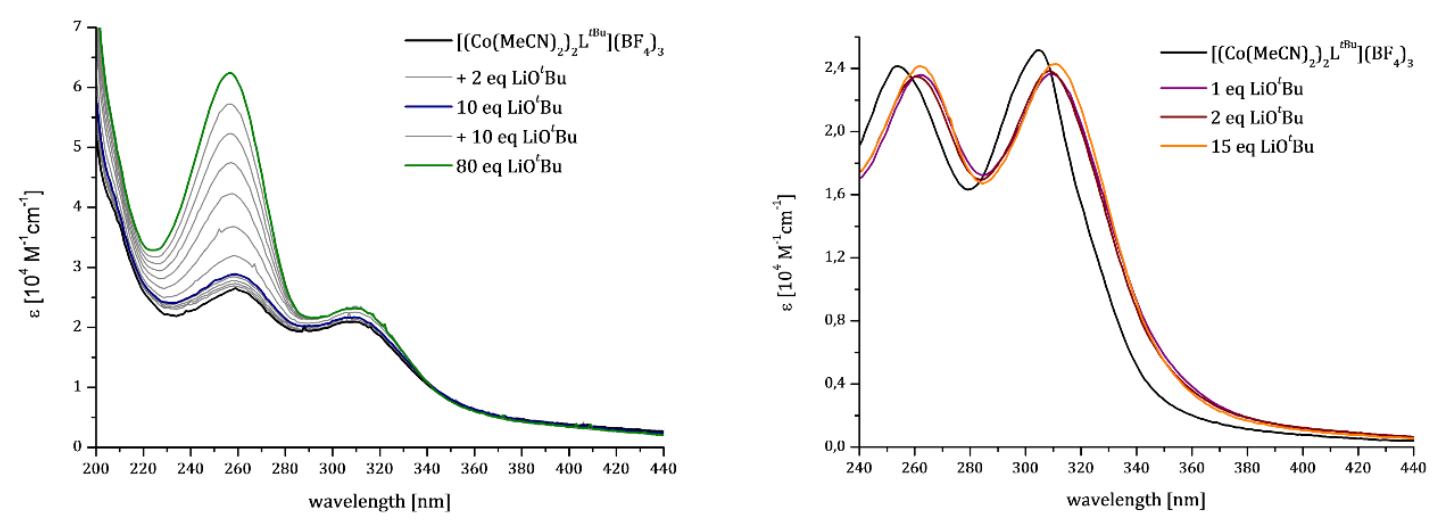

Figure 3.24: UV-vis spectra of reactions of $\left[\left(\mathrm{Co}(\mathrm{MeCN})_{2}\right)_{2} \mathrm{~L}^{\mathrm{LBu}}\right]\left(\mathrm{BF}_{4}\right)_{3} \mathbf{5 4}$ with LiOtBu in MeCN (left) and in DCM (right).

A reaction in dichloromethane (with $\mathrm{LiO}^{t} \mathrm{Bu}$ for solubility reasons) supported this hypothesis, since in contrast to the reaction in $\mathrm{MeCN}$, in $\mathrm{CH}_{2} \mathrm{Cl}_{2}$ no increase of the absorption bands was observed (Figure 3.24, right). In this experiment a slight shift of the absorption maximum by $7 \mathrm{~nm}$ was observed. The reason for that might be a change in the coordination sphere of the cobalt centers. The use of potassium hydride as base yielded intense dark blue reaction mixtures but no products could be identified by different spectroscopic techniques.

The cyclic voltammogram of $\mathbf{C o}^{\text {BBu}}-\mathbf{M e C N}$ (Figure 3.23) exhibited well separated reduction events. The second reduction at a potential of approx. $-1.17 \mathrm{~V}$ versus $\mathrm{Fc} / \mathrm{Fc}^{+}$is perfectly in line with the reduction potential of cobaltocene $\left(-1.33 \mathrm{~V}\right.$ versus $\mathrm{Fc} / \mathrm{Fc}^{+}$in $\left.\mathrm{CH}_{2} \mathrm{Cl}_{2}\right) \cdot{ }^{[421]}$ Assuming the reduction to take place at the cobalt centers, the resulting species should be a dinuclear cobalt(I) $\mathrm{d}^{8}$ species, which is diamagnetic in the low spin case. Reduction of the cobalt(II) complex Co ${ }^{t \mathrm{Bu}}-\mathbf{M e C N}$ with cobaltocene in $\mathrm{MeCN}$ resulted in an instant color change of the solution to intense purple. The reaction products were very sensitive to oxygen and moisture. Upon crystallization yellow needles of the cobaltocenium salt were obtained, but single crystals suitable for X-ray diffraction analysis of a product resulting from Co $^{\text {tBu-MeCN }}$ could not be isolated. ${ }^{1} \mathrm{H}$ NMR spectroscopy revealed the presence of paramagnetic compounds, since strongly shifted resonances were observed (Figure 3.25). This might be explained by the formation of a high spin cobalt(I) complex. Like for $\mathbf{C o}^{t \mathrm{Bu}}$-MeCN the resonances could not be assigned and therefore the exact number as well as the structures of reaction products remained unknown. In ${ }^{31} \mathrm{P}$ NMR spectra neither resonances for the phosphine side arm nor of free ligand were observed. 


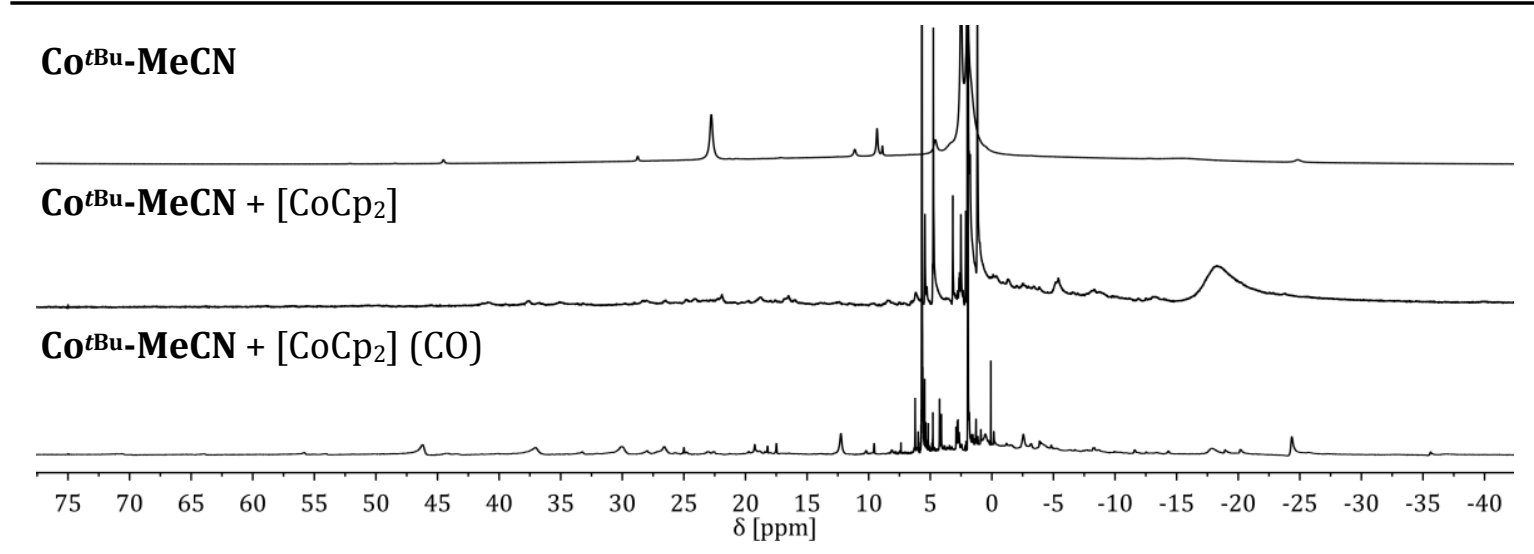

Figure 3.25: ${ }^{1} \mathrm{H}$ NMR spectra of $\mathrm{Co}^{t \mathrm{Bu}}-\mathbf{M e C N}$ (top) and crude products of reactions of $\mathbf{C o}^{t \mathrm{Bu}}-\mathbf{M e C N}$ and [CoCp 2 under Ar (middle) and CO atmospheres (bottom)(acetonitrile-d3, $298 \mathrm{~K}$ ).

To avoid the formation of high spin complexes, the reduction was carried out under an atmosphere of carbon monoxide, which can act as a strong donor ligand supporting low spin configurations. Unfortunately, ${ }^{1} \mathrm{H}$ NMR spectra of crude products still showed paramagnetic character, although a carbonyl vibration at $1893 \mathrm{~cm}^{-1}$ was found in ATR-IR measurements showing the presence of a CO ligand in the reaction product. The presence of paramagnetic impurities was assumed. If the paramagnetically shifted resonances resulted from a formed carbonyl cobalt(I) complex, it has to have a distorted geometry so that the unusual high spin state is favored. No high spin cobalt(I) complexes bearing a CO ligand were found in literature and also only a very few high spin cobalt(I) complexes with other ligands are known. ${ }^{[422,423]}$

It was also tried to reduce complex $\mathbf{C o}^{t \mathrm{Bu}}-\mathbf{M e C N}$ with a stronger reductant, namely potassium graphite $\left(\mathrm{KC}_{8}\right)$. The same purple color of the reaction mixture and similar ${ }^{1} \mathrm{H}$ NMR data were found for the reduction with $\mathrm{KC}_{8}$ in a mixture of cold MeCN and THF. However, the low stability of the product(s) prevented the isolation and identification of the formed species. This fast decomposition (on air within few seconds, under inert conditions at low temperatures within several hours) supports the assumption of the synthesis of a reactive, low valent cobalt species. Reaction of $\mathbf{C o}^{\mathrm{tBu}}-\mathbf{M e C N}$ with a Grignard reagent $(\mathrm{MeMgBr}$ ) or sodium borohydride yielded almost the same purple solutions like the stronger reductants before. Grignard solutions are also used for reduction reactions, therefore the reduction to cobalt(I) might be favored over the transmetallation reaction (Scheme 3.19, pathway C). With hydrazine or azides a rather slight color change of the reaction mixtures was observed indicative for a simple ligand exchange at the cobalt centers, but the products could not be isolated.

The synthesis of cobalt hydride species was attempted (Scheme 3.19 pathway D). The relatively large metal-metal distance should lead to a dihydride compound rather than a bridged monohydride one. Indeed, a color change of the reaction mixture was observed until approx. two equivalents of superhydride ${ }^{\circledR}$ solution $\left(\mathrm{KHBEt}_{3}\right)$ were added. Further equivalents did not 
lead to a further color change. Again the identification of the product failed, since single crystals of the compound could not be obtained and other spectroscopic methods provided no structural information. Nevertheless, the formation of a reactive species upon addition of superhydride ${ }^{\circledR}$ to $\mathbf{C o}^{t \mathrm{Bu}}-\mathbf{M e C N}$ was taken as initiation to investigate of the catalytic activity of this mixture (see Chapter 3.6.2).

\subsubsection{Ruthenium and Molybdenum Complexes}

Since in literature many mononuclear ruthenium and molybdenum pincer complexes are known, which show very interesting reactivity in the activation of small molecules and in catalysis, the synthesis of dinuclear analogues was attempted.[45,300,305,424-427] The synthesis of similar pyrazolate bridged ruthenium(II) complexes and their use for water oxidation catalysis was shown before.[251,252] For ruthenium pincer complexes a wide range of synthetic protocols were developed by using various different ruthenium precursors, and some of them were used in this work for the complexation with the Two-in-one pincer ligand.[215] The reaction of ruthenium precursors and the ligand had to be performed at elevated temperatures due to slow ligand exchange at the ruthenium centers. The combination of metal, ligand, base and in some cases of additional bridging moieties resulted in orange-brown reaction mixtures. In ESI-MS measurements numerous species with isotopic distribution patterns indicative for dinuclear ruthenium species were found and some of them could be assigned as desired products. For example, the reaction of $\mathbf{H L}^{t \mathrm{Bu}}$ with $\mathrm{Ru}(\mathrm{dmso})_{4} \mathrm{Cl}_{2}$ in THF in the presence of triethylamine as base gave among others a peak at $\mathrm{m} / \mathrm{z}=969.2$, which could be simulated with a species containing one ligand, two ruthenium ions, two dimethylsulfoxides and two chlorides (Figure 3.26). The other two dominant species at $\mathrm{m} / \mathrm{z}=817.3$ and $\mathrm{m} / \mathrm{z}=858.3$ could not be assigned in a reasonable way, but they were assigned to contain one ruthenium ion based on the isotopic distribution pattern. The ${ }^{31} \mathrm{P}$ NMR spectrum of this material showed the presence of the free ligand and three new species shifted to lower field compared to $\mathbf{H L}^{\boldsymbol{t B u}}$ (approx. 50 - $80 \mathrm{ppm}$ ). Unfortunately, a purification via column chromatography in using different stationary phases or crystallization was not successful, so that the exact structure of the complex remained elusive. 


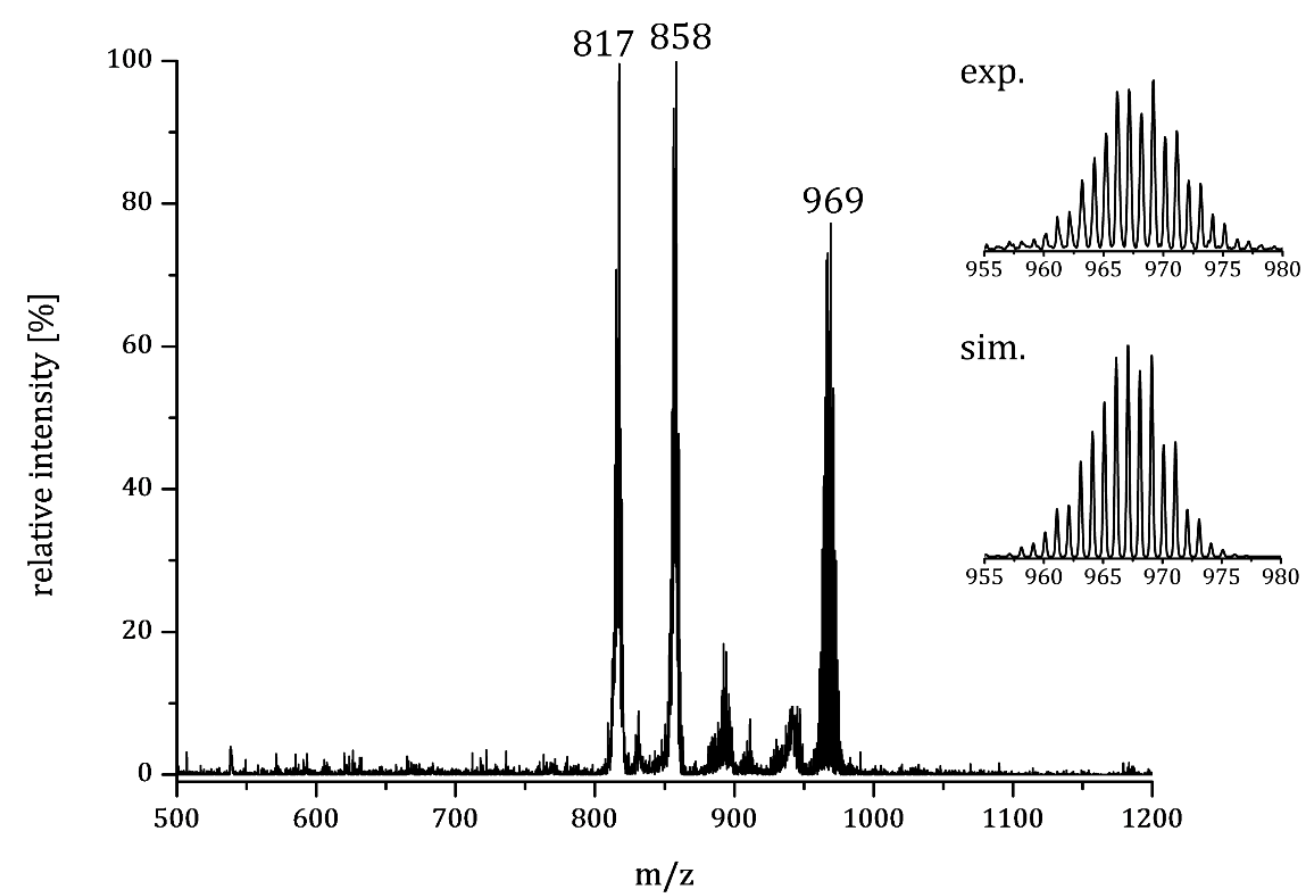

Figure 3.26: m/z 500-1200 range of the ESI(+)-MS spectrum of the crude product of a reaction between $\mathbf{H L}^{t \mathrm{Bu}}$, $\left[\mathrm{Ru}(\mathrm{dmso})_{4} \mathrm{Cl}_{2}\right]$ and $\mathrm{NEt}_{3}$ in THF. The insets show the experimental and simulated isotopic distribution patterns for $\left[\mathrm{Ru}_{2} \mathrm{~L}^{\mathrm{tBu}}(\mathrm{Cl})_{2}(\mathrm{dmso})_{2}\right]^{+}$.

Other ruthenium sources were tried like $\mathrm{RuCl}_{3}$, $\left[\mathrm{Ru}(p \text {-cymene }) \mathrm{Cl}_{2}\right]_{2}, \quad\left[\mathrm{RuCl}_{2}\left(\mathrm{PPh}_{3}\right)_{3}\right]$, $\left[\mathrm{Ru}(\mathrm{H})_{2}(\mathrm{CO})\left(\mathrm{PPh}_{3}\right)_{3}\right], \quad\left[\mathrm{RuCl}(\mathrm{H})(\mathrm{CO})\left(\mathrm{PPh}_{3}\right)_{3}\right]$. The addition of pyridine or acetonitrile as potential ligands did not lead to the formation of a single species. It was tried to saturate the coordination sphere of the ruthenium centers with acetonitrile, so that a symmetric species would be formed. It was known from other projects in the group, that MeCN binds strongly to ruthenium(II). Therefore, acetonitrile was added to a reaction mixture and afterwards a salt metathesis with $\mathrm{NaBPh}_{4}$ was conducted (Scheme 3.20). A single resonance in the ${ }^{31} \mathrm{P}$ NMR spectrum was found at $24.96 \mathrm{ppm}$ and also the ${ }^{1} \mathrm{H}$ NMR spectrum showed just one peak for the ${ }^{t} \mathrm{Bu}$ groups and one for the MeCN ligands, indicating a high symmetry. In contrast, more peaks in the aromatic region as expected were observed and $\mathrm{CH}_{2}$ peaks could not be assigned (Figure 3.27). Furthermore, ESI-MS of the NMR sample solution revealed the presence of several $\mathrm{Ru}_{2}$ species identified by their typical isotopic distribution patterns, but these peaks could not be matched with potential products.

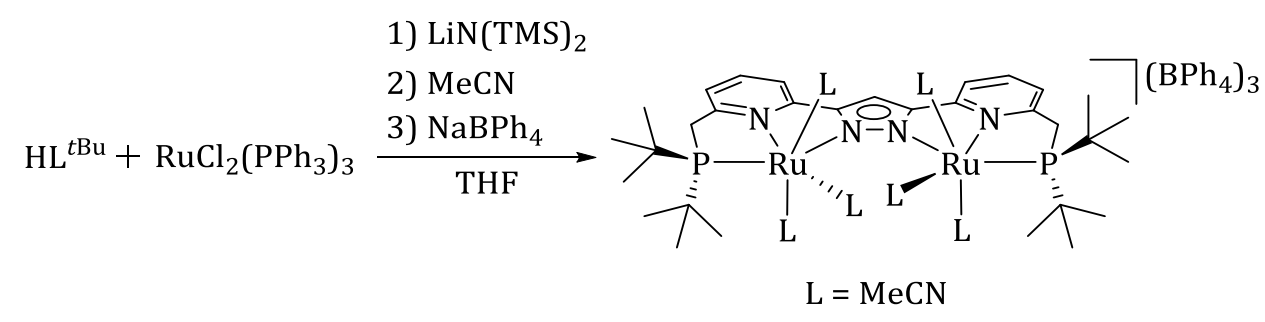

Scheme 3.20: Example for an attempted synthesis of dinuclear ruthenium(II) complexes of $\mathbf{H L}^{t} \mathbf{B u}$. 


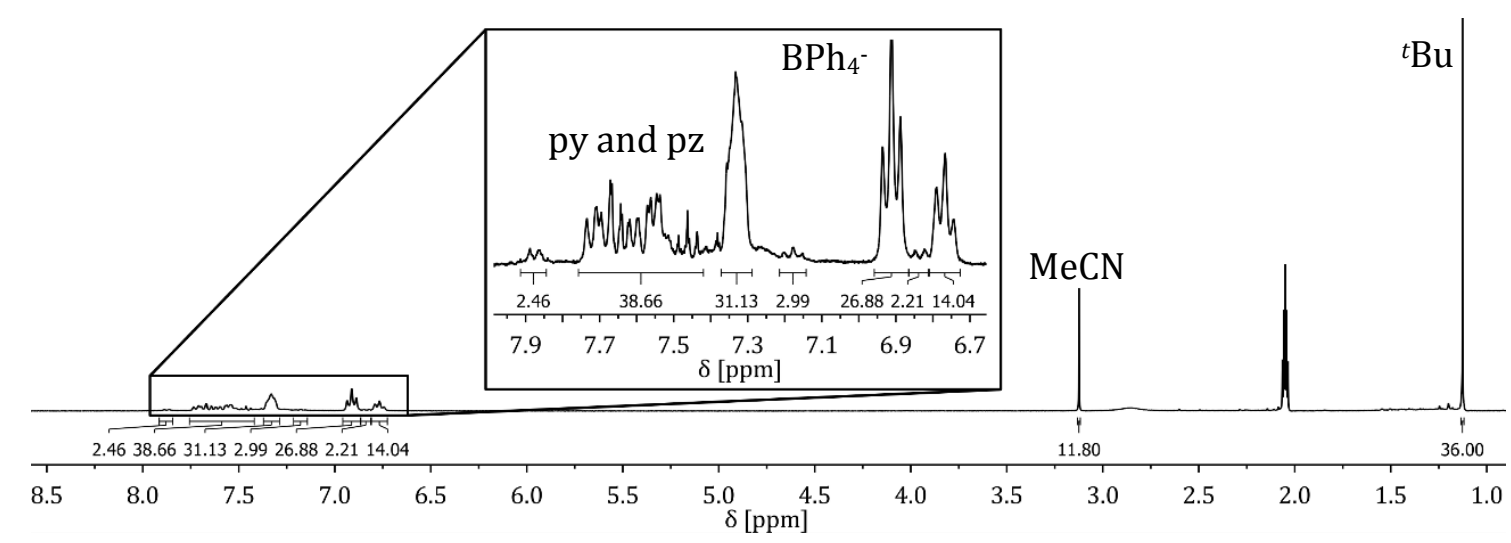

Figure 3.27: ${ }^{1} \mathrm{H}$ NMR spectrum of a crude product of a reaction of $\mathbf{H L}^{t \mathbf{B u}},\left[\mathrm{RuCl}_{2}(\mathrm{PPh})_{3}\right]$ and lithium hexamethyldisilazane (LiN(TMS) 2 ) in THF/MeCN and subsequent salt metathesis with $\mathrm{NaBPh}_{4}$ (acetone-d 6 , $298 \mathrm{~K}$ ). The inset shows the aromatic region of the spectrum in detail.

Although the complexation reactions of ruthenium(II) with $\mathbf{H L}^{t \mathbf{B u}}$ showed promising indications for the formation of the corresponding dinuclear complexes, it was not possible to eventually isolate and identify a reaction product in pure form. Numerous crystallization attempts failed so far and thus the work on ruthenium complexes was not continued.

For synthesizing molybdenum complexes of $\mathbf{H L}^{t \mathbf{B u}}$, the same metal precursor as for the tetramine ligand $\mathbf{H}_{5} \mathbf{L}^{\mathrm{Me}}$ (Chapter 1.4 ) was used $\left(\mathrm{MoCl}_{3}(\mathrm{thf})_{3}\right)$ and additionally molybdenum hexacarbonyl $\mathrm{Mo}(\mathrm{CO})_{6}$ as zero valent metal source. Upon addition of the molybdenum(III) complex to deprotonated ligand $\mathbf{H L}^{t \mathbf{B u}}$, an instant color change to dark brown was observed. However, neither the addition of potential bridging units (hydrazine, sodium acetate and benzoate) or the change of the reaction conditions nor the subsequent reduction with potassium graphite or sodium amalgam resulted in the identification or isolation of welldefined dinuclear molybdenum complexes of the Two-in-one pincer ligand. In ESI-MS spectra of crude reaction mixtures often the ligand was found as most intense peak along with some possible molybdenum species (assigned by the typically broad isotopic distribution patterns for $\mathrm{Mo}_{1}$ or $\mathrm{Mo}_{2}$ compounds). However, none of these peaks could be simulated with ligand containing dinuclear molybdenum species in a chemically reasonable fashion. No reaction could be detected via NMR spectroscopy when $\mathbf{H L}^{t \mathbf{B u}}$ was reacted with $\mathrm{Mo}(\mathrm{CO})_{6}$.

\subsubsection{Rhodium Complexes}

The synthesis of rhodium(I) complexes of the Two-in-one pincer ligand was attempted, since rhodium complexes allow facile analysis due to the low spin character of these $\operatorname{Rh}^{1}\left(\mathrm{~d}^{8}\right)$ complexes. Also products of an oxidative addition are diamagnetic $\left(\mathrm{Rh}^{\mathrm{III}}, \mathrm{d}^{6}\right)$, which is a typical reaction for rhodium(I) complexes. In addition, rhodium(I) complexes were often used for 
numerous catalytic transformations and therefore many examplary reactions are available, which might be tested.

\subsubsection{Synthesis of Rhodium(I) Complexes}

Addition of two equivalents of the rhodium source $\left[\mathrm{Rh}(\mathrm{acac})(\mathrm{CO})_{2}\right]$ to a suspension of ligand $\mathrm{HL}^{t \mathrm{Bu}}, \mathrm{KO}{ }^{t} \mathrm{Bu}$ as base and $\mathrm{KPF}_{6}$ led to the formation of two products, which were identified by NMR spectroscopy and ESI mass spectrometry. The desired dinuclear complex $\mathbf{5 5}$ (Rh $\left.{ }^{t \mathrm{Bu}}-\mathbf{C O}\right)$ and a mononuclear rhodium species 56 were identified. In $\mathbf{5 6}$ one phosphine side arm was abstracted (Scheme 3.21). The use of impure and thus monophosphine XXXIV containing ligand material was excluded by recording a ${ }^{1} \mathrm{H}$ NMR spectrum of the ligand batch prior to use. Variation of the reaction time did not change the observed ratio of the two species of ca. 1:1. The two compounds were separated by crystallization of $\mathbf{R} \mathbf{h}^{t \mathrm{Bu}}-\mathbf{C O}$ as yellow, air stable needles. This purification procedure diminished the yield of the complexation reaction to $28 \%$.

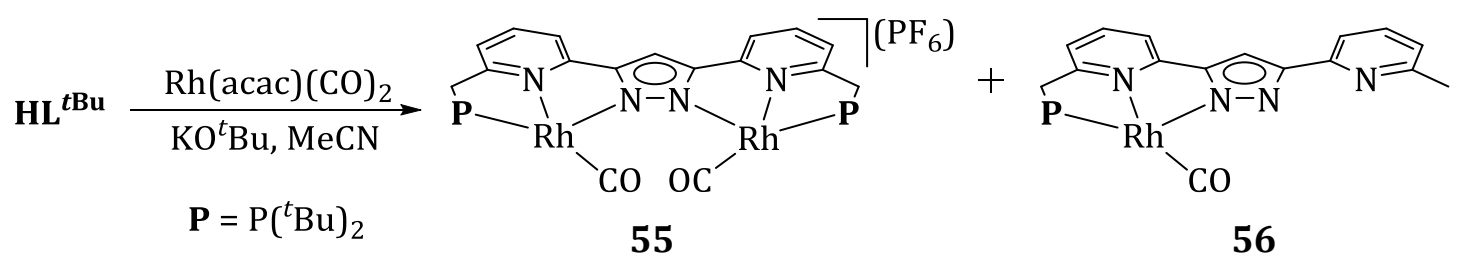

Scheme 3.21: Synthesis of rhodium carbonyl complex 55 with formation of side product 56.

The ${ }^{1} \mathrm{H}$ NMR spectrum of $\mathbf{R h}^{t \mathrm{Bu}}-\mathrm{CO}$ in MeCN- $\mathrm{d}_{3}$ proved the formation of a symmetric species by the existence of only three signals for the pyridine protons as well as one doublet for the methylene spacer at $3.81 \mathrm{ppm}$ (Figure 3.28). The ${ }^{31} \mathrm{P}$ spectrum shows a sharp doublet at $99.13 \mathrm{ppm}\left({ }^{1} J_{\mathrm{RhP}}=148.2 \mathrm{~Hz}\right)$ for the phosphorus atoms coupled to rhodium centers. The carbonyl carbon atoms gave a doublet of doublets at $\delta=194.4 \mathrm{ppm}$ in a ${ }^{13} \mathrm{C}$ NMR spectrum with coupling constants of $J_{R h-C}=73.8 \mathrm{~Hz}, J_{P-C}=17.5 \mathrm{~Hz}$ similar to other reported rhodium(I) pincer complexes.[298,316]

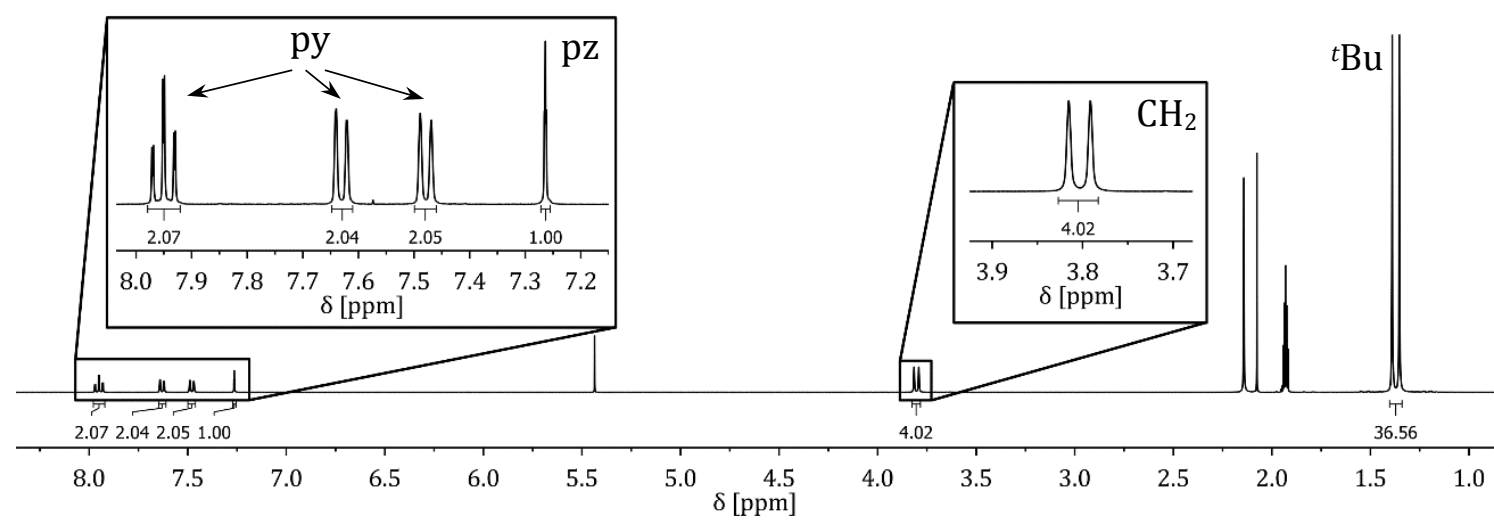

Figure 3.28: ${ }^{1} \mathrm{H}$ NMR spectrum of $\left[(\mathrm{Rh}(\mathrm{CO}))_{2} \mathrm{~L}^{t \mathrm{Bu}}\right]\left(\mathrm{PF}_{6}\right) 55$ (acetonitrile- $\left.\mathrm{d}_{3}, 298 \mathrm{~K}\right)$. The insets show the aromatic region of the spectrum and the $\mathrm{CH}_{2}$ resonance in detail. 
ESI-MS measurements showed the cation $\left[(\mathrm{Rh}(\mathrm{CO}))_{2} \mathrm{~L}^{t \mathrm{Bu}}\right]^{+}$as dominant peak at $\mathrm{m} / \mathrm{z}=799.1$ and the CO stretching frequencies were found at $1964 \mathrm{~cm}^{-1}$ and $1978 \mathrm{~cm}^{-1}$ in an ATR-IR spectrum, slightly lower than reported pyrazole-bridged rhodium (I) carbonyl complexes. ${ }^{[70]}$

Crystals suitable for X-ray diffraction analysis were grown by slow ether diffusion into a MeCN solution of $\mathbf{R h}^{t \mathbf{B u}}$-CO. The complex crystallized in the orthorhombic space group Cmca with eight molecules in the unit cell. The molecular structure is depicted in Figure 3.29 and shows the two rhodium centers in a distorted square planar coordination geometry. Very similar to the zinc and cobalt complexes the ligand backbone is only slightly bend and the metal ions are found above and below this plane $\left(\mathrm{d}_{\mathrm{Rh} \text {-plane }}=0.386 \AA\right.$ ). The carbonyl ligands come in steric clash and thus they are pointing up and down, respectively. In solution the carbonyl ligands are less rigid. Thus, an apparent $\mathrm{C}_{2 \mathrm{~V}}$ symmetry was found on the NMR timescale at ambient temperature with a fast interconversion of the two enantiomeric isomers $(P$ and $M)$. The metal-metal distance was found $0.06 \AA$ longer than in Co ${ }^{t \mathrm{Bu}}$-MeCN $\left(\mathrm{d}_{\mathrm{Rh}-\mathrm{Rh}}=4.494 \AA\right.$ 的. $\mathrm{d}_{\mathrm{Co}-\mathrm{Co}_{0}}=4.437 \AA$ ) and longer compared to the zinc complexes, which have triflate ions as bridging units. The bond length of the donor atoms to the metal centers are in the same range as for the zinc and cobalt complexes and the Rh-CO $\left(\mathrm{d}_{\mathrm{Rh}-\mathrm{CO}}=1.827 \AA\right)$ and C-O $\left(\mathrm{d}_{\mathrm{C}-\mathrm{O}}=1.149 \AA\right)$ bonds are essentially as long as in the mononuclear analogue $[(\mathrm{PNP}) \mathrm{Rh}(\mathrm{CO})]\left(\mathrm{BF}_{4}\right) \cdot{ }^{[428]}$
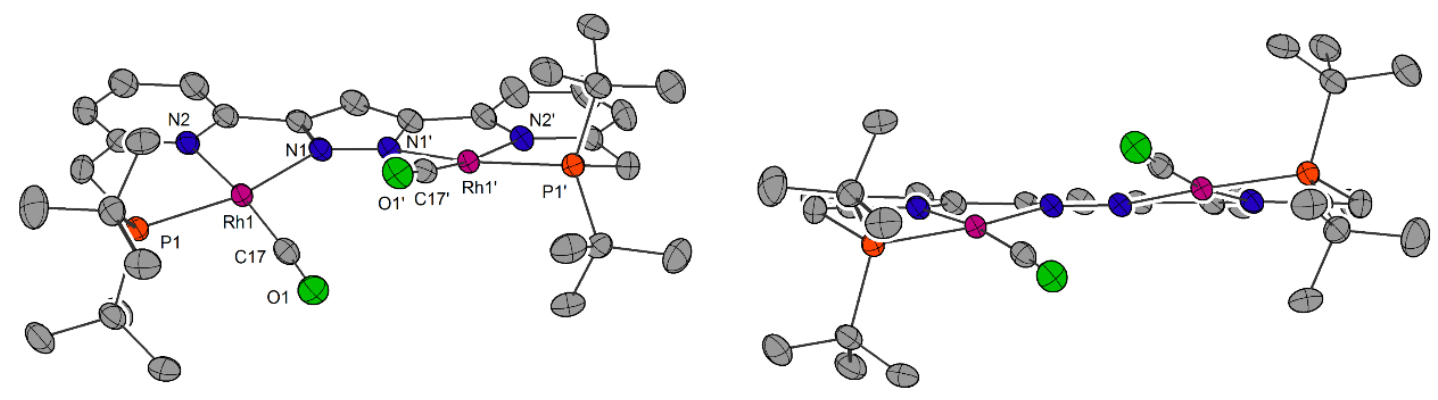

Figure 3.29: Thermal displacement ellipsoids (shown at $30 \%$ probability) of the molecular structure of $\left[(\mathrm{Rh}(\mathrm{CO}))_{2} \mathrm{~L}^{t \mathrm{Bu}}\right]\left(\mathrm{PF}_{6}\right) 55$ in two different orientations. Counterions, hydrogen atoms and solvent molecules were omitted for clarity.

Using the same procedure, the rhodium carbonyl complex $\mathbf{5 7}$ (R/Pr-CO) of ligand $\mathbf{H L}^{\mathbf{i P r}}$ was prepared but here no formation of a second product was observed (Scheme 3.22). The complex could be isolated as yellow solid in up to $62 \%$ yield, but the growing of single crystals, suitable for X-ray diffraction analysis, failed so far. Interestingly, the solubility of $\mathbf{R h}^{\mathbf{i P r}} \mathbf{- C O}$ was limited to $\mathrm{MeCN}$, while $t \mathrm{Bu}$ analogue $\mathbf{5 5}$ was also soluble in acetone and methanol. This is in contrast to the solubility behavior of the ligands themselves. 


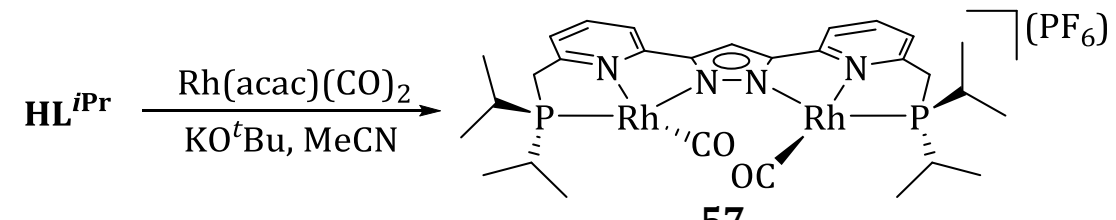

57

Scheme 3.22: Synthesis of complex $\left[(\mathrm{Rh}(\mathrm{CO}))_{2} \mathrm{~L}^{i \mathrm{Pr}}\right]\left(\mathrm{PF}_{6}\right) 57$.

Complex $\mathbf{5 7}$ was characterized by ESI mass spectrometry with a single main peak (m/z = 743.1, $\left.\left[(\mathrm{Rh}(\mathrm{CO}))_{2} \mathrm{~L}^{i \mathrm{Pr}}\right]^{+}\right)$as well as by NMR spectroscopy. The doublet of the phosphorus donors in the 31P NMR spectrum was found at a chemical shift of $84.90 \mathrm{ppm}, \sim 15 \mathrm{ppm}$ high field shifted compared to $\mathbf{R h}^{\text {tBu-CO, }}$, whereas the coupling constant was essentially the same $\left(1 J_{\mathrm{RhP}}=144.5 \mathrm{~Hz}\right)$. The ${ }^{1} \mathrm{H}$ NMR spectrum revealed a symmetric compound in solution and the shift of the methylene resonance in $\mathbf{R h}^{\text {iPr-}} \mathbf{C O}(\delta=3.76 \mathrm{ppm})$ is only slightly different from

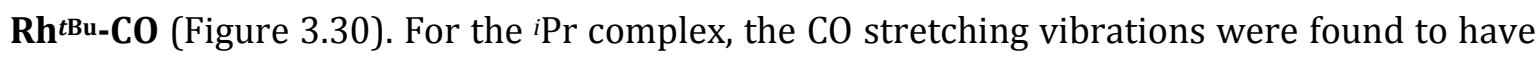
the same energy $\left(1965 \mathrm{~cm}^{-1}\right.$ and $\left.1977 \mathrm{~cm}^{-1}\right)$ as the ${ }^{t} \mathrm{Bu}$ complex showing, that the difference of the two ligands $\mathbf{H L}^{\boldsymbol{t} \mathbf{B u}}$ and $\mathbf{H L}^{i \mathbf{P r}}$ in rhodium(I) carbonyl complexes is only of steric nature.

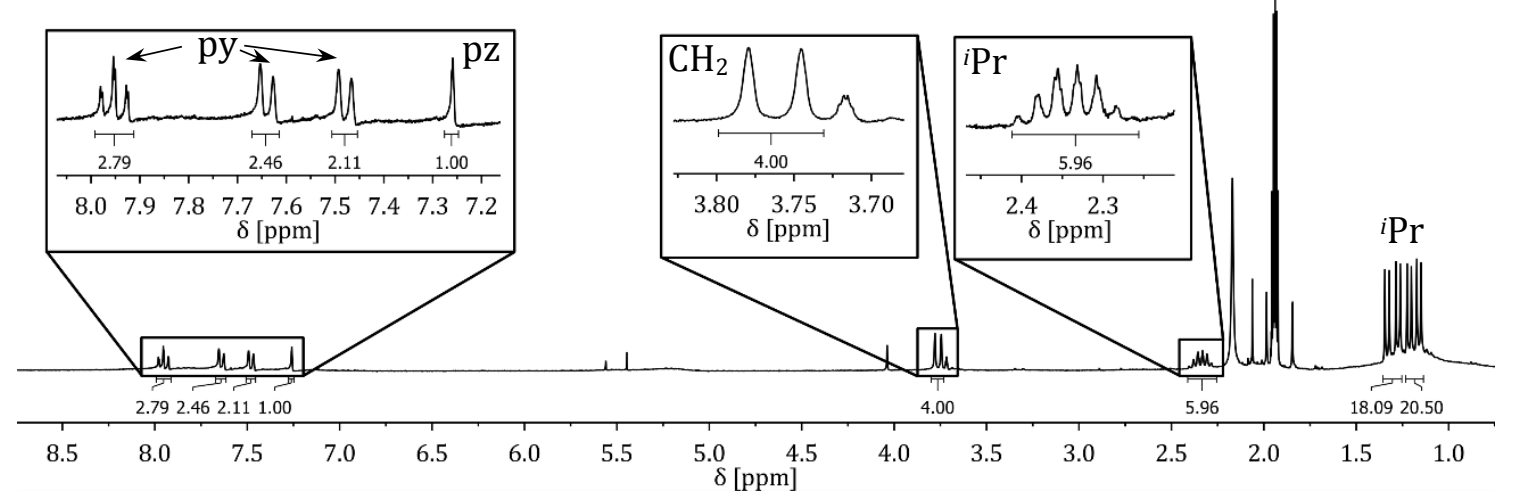

Figure 3.30: ${ }^{1} \mathrm{H}$ NMR spectrum of $\left[(\mathrm{Rh}(\mathrm{CO}))_{2} \mathrm{~L}^{i \mathrm{Pr}}\right]\left(\mathrm{PF}_{6}\right) \mathbf{5 7}$ (acetonitrile- $\left.\mathrm{d}_{3}, 298 \mathrm{~K}\right)$. The insets show the aromatic region of the spectrum, the $\mathrm{CH}_{2}$ and the $\mathrm{CH}$ group of the ${ }^{i}$ Pr residue resonances in detail.

The carbonyl complexes were air and water stable and assumed to exhibit a low reactivity, since the exchange of the CO ligand is not favored. Therefore, another synthetic strategy was applied in order to synthesize complexes with more labile coligands. $\left.{ }^{2298}\right]\left[\mathrm{RhCl}(\mathrm{coe})_{2}\right]_{2}$ (coe = cyclooctene) was chosen as metal precursor, because the alkene ligands are more easily substituted than dienes in ,e.g., $[\mathrm{RhCl}(\operatorname{cod})]_{2}$ ( $\operatorname{cod}=$ cyclooctadiene). A direct use of $\left[\mathrm{RhCl}(\mathrm{coe})_{2}\right]_{2}$ was not successful and produced a mixture of several species as it was observed via ${ }^{31 P}$ NMR spectroscopy. Thus, the precursor was treated with silver hexafluorophosphate $\left(\mathrm{AgPF}_{6}\right)$ to abstract the chloride, forming $\left[\mathrm{Rh}(\mathrm{L})_{2}(\mathrm{coe})_{2}\right]^{+}$in which $\mathrm{L}$ is depending on the solvent used for the reaction. The formed silver chloride was separated and the solution of the rhodium complex was added to a mixture of ligand and base. When the complexation reactions were 
performed in MeCN, one solvent molecule was coordinated to each metal center (Scheme 3.23, $\left.\mathbf{5 8}=\mathbf{R h}^{t \mathrm{Bu}}-\mathrm{MeCN}\right)$.

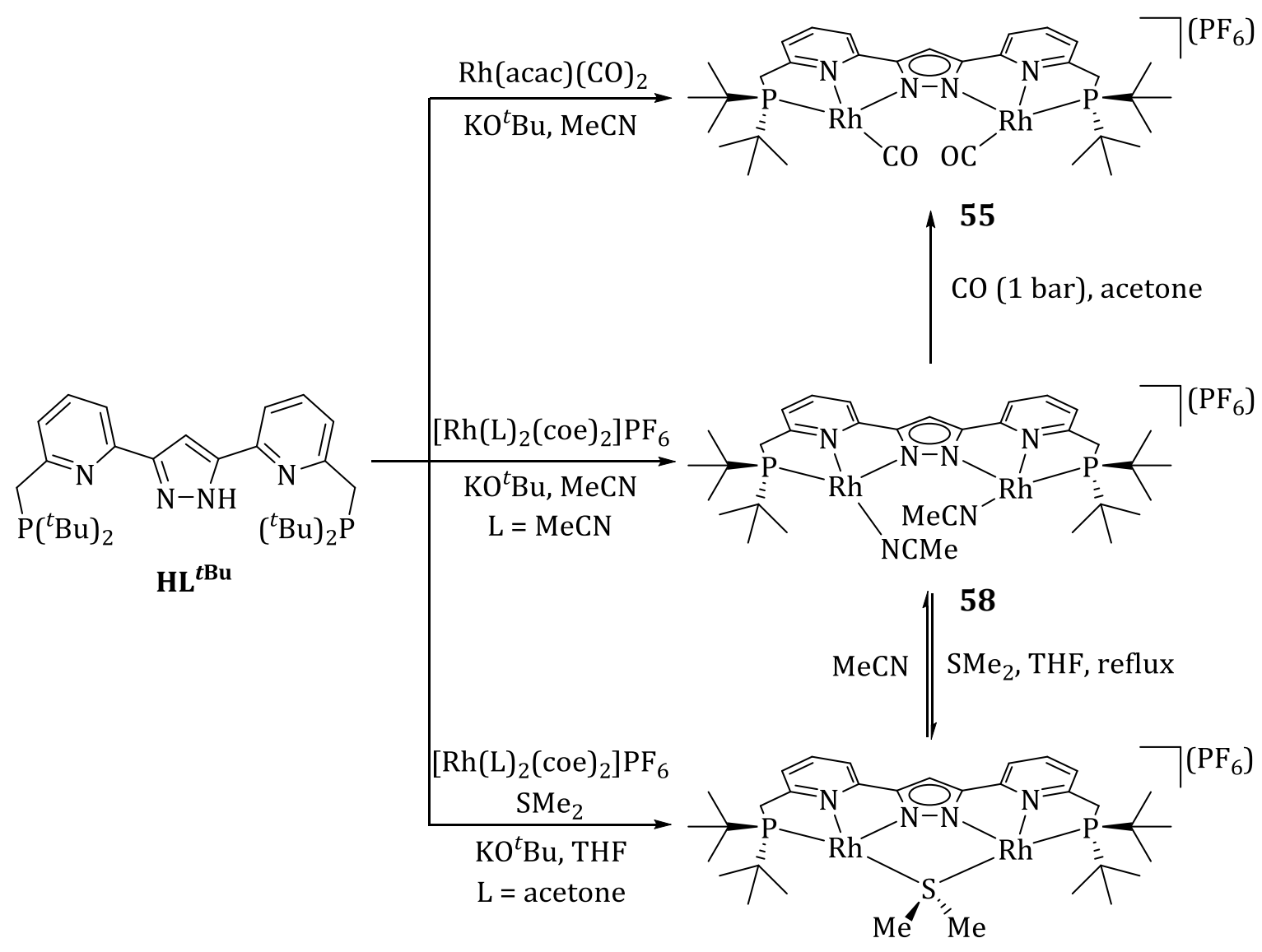

61

Scheme 3.23: Synthesis of complexes $\left[(\mathrm{Rh}(\mathrm{CO}))_{2} \mathrm{~L}^{t \mathrm{Bu} u}\right]\left(\mathrm{PF}_{6}\right) \quad \mathbf{5 5}, \quad\left[(\mathrm{Rh}(\mathrm{MeCN}))_{2} \mathrm{~L}^{t \mathrm{Bu}}\right]\left(\mathrm{PF}_{6}\right) \quad \mathbf{5 8}$ and $\left[\left(\mathrm{Rh}_{2}\left(\mu-\mathrm{SMe}_{2}\right) \mathrm{L}^{\mathrm{tBu}}\right]\left(\mathrm{PF}_{6}\right) \mathbf{6 1}\right.$ and the transformations between them.

Analytically pure substance of $\mathbf{5 8}$ was obtained after extraction with a mixture of benzene and MeCN (8:1), so that the formed $\mathrm{KPF}_{6}$ was separated. The identity of $\mathbf{R h}^{\mathrm{tBu}}-\mathbf{M e C N}$ was proven by ESI-MS showing a single main peak at $\mathrm{m} / \mathrm{z}=825.3$ corresponding to the desired cation $\left[(\mathrm{Rh}(\mathrm{MeCN}))_{2} \mathrm{~L}^{\mathrm{tBu}}\right]_{+}^{+}$(Figure 3.31). 


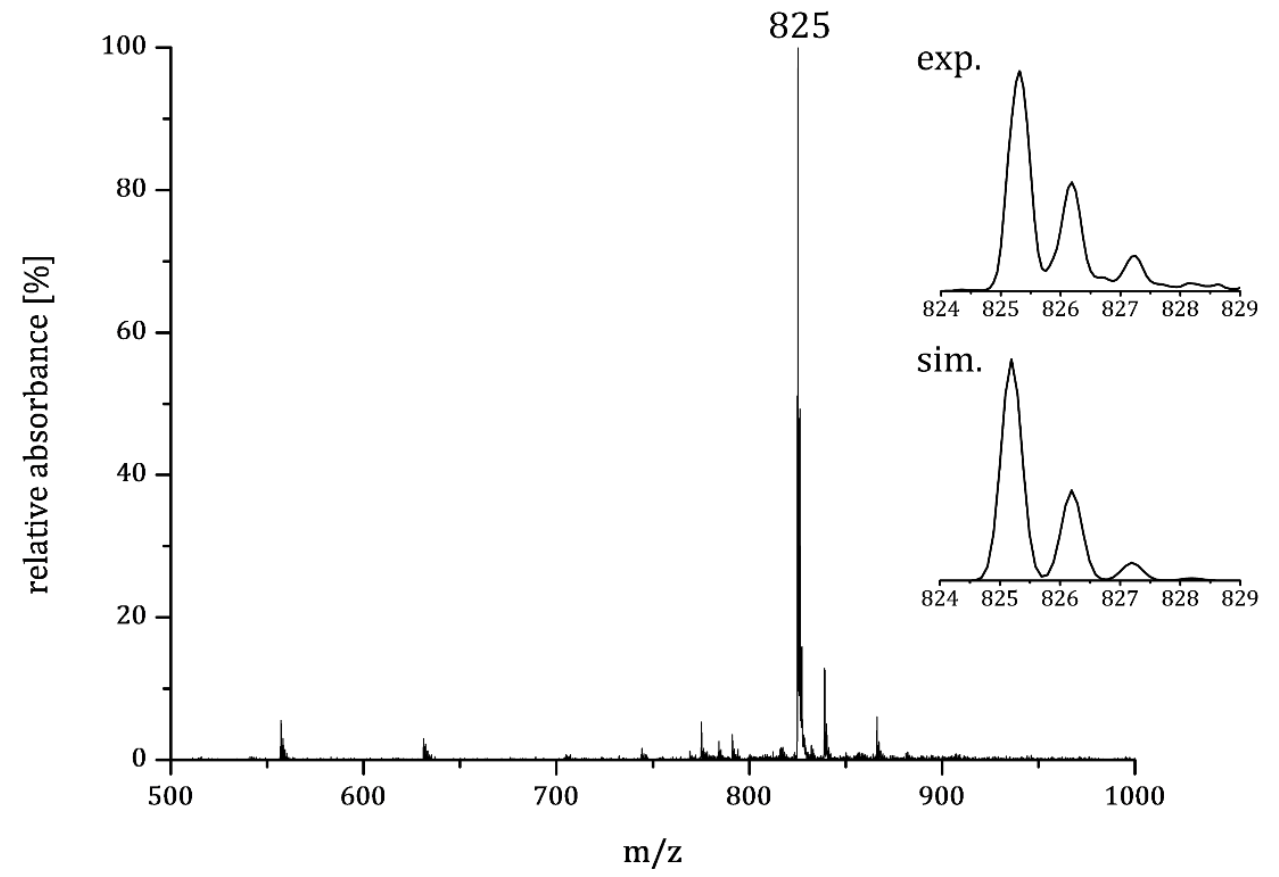

Figure 3.31: m/z 500-1000 range of the ESI(+)-MS spectrum of $\mathbf{R h}^{t \mathbf{B u}-M e C N}$ in MeCN. The insets show the experimental and simulated isotopic distribution patterns for $\left[(\mathrm{Rh}(\mathrm{MeCN}))_{2} \mathrm{~L}^{t \mathrm{Bu}}\right]^{+}$.

The ${ }^{1} \mathrm{H}$ NMR spectrum at ambient temperature in $\mathrm{MeCN}-\mathrm{d}_{3}$ (Figure 3.32) revealed again a species with apparent $\mathrm{C}_{2 \mathrm{~V}}$ symmetry on the NMR timescale with three resonances corresponding to the pyridine protons, one pyrazole proton and one doublet each for the $\mathrm{CH}_{2}$ $(\delta=3.28 \mathrm{ppm})$ and the tert-butyl groups $(\delta=1.38 \mathrm{ppm})$. The signal of the methylene groups in

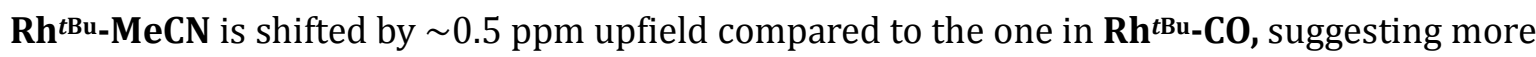
electron density at the ligand. A sharp doublet at $90.13 \mathrm{ppm}$ was observed in the ${ }^{31} \mathrm{P}$ NMR spectrum with a significantly larger coupling constant of $J_{\mathrm{Rh}-\mathrm{P}}=178.2 \mathrm{~Hz}$, which was not observed for the mononuclear PNP rhodium(I) complexes in which the coupling constants of the $\mathrm{CO}$ and MeCN complex were similar $\left(U_{\mathrm{Rh}-\mathrm{P}}=120 \mathrm{~Hz}\right.$ for the $\mathrm{CO}$ complex and $J_{\mathrm{Rh}-\mathrm{P}}=137 \mathrm{~Hz}$ for the MeCN complex).[428,429]

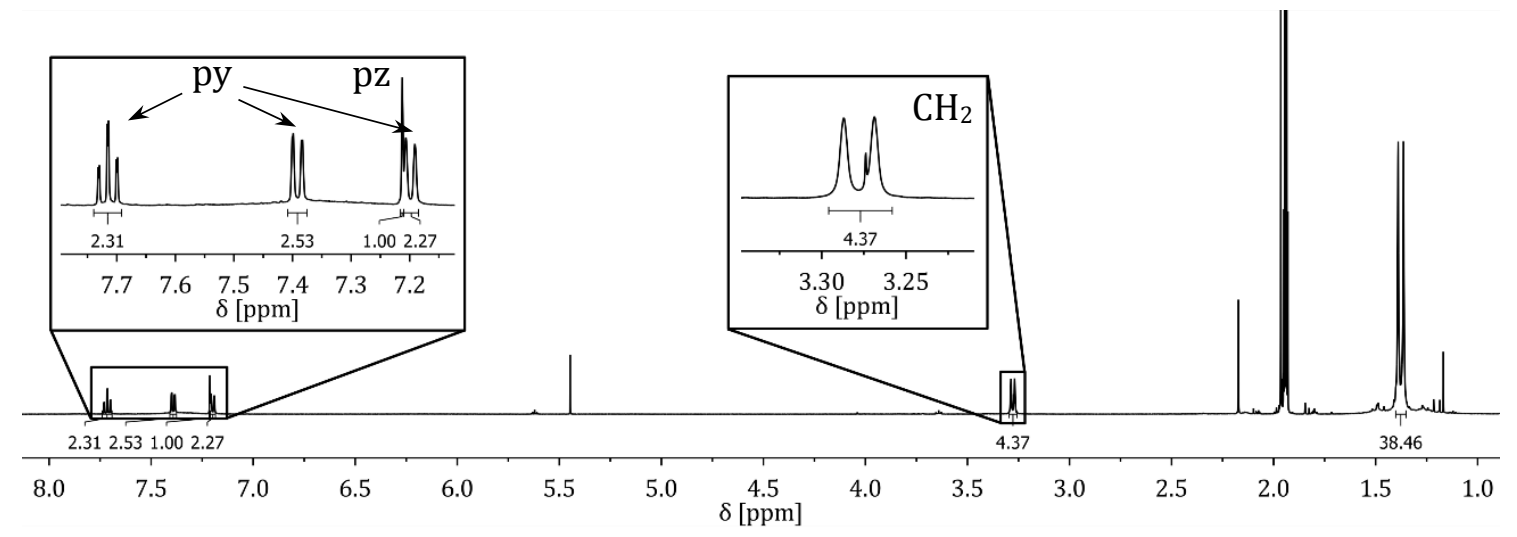

Figure 3.32: ${ }^{1} \mathrm{H}$ NMR spectrum of $\left[(\mathrm{Rh}(\mathrm{MeCN}))_{2} \mathrm{~L}^{t \mathrm{Bu}}\right]\left(\mathrm{PF}_{6}\right) \mathbf{5 8}$ (acetonitrile- $\left.\mathrm{d}_{3}, 298 \mathrm{~K}\right)$. The insets show the aromatic region of the spectrum and the $\mathrm{CH}_{2}$ resonance in detail. 
When performing the NMR experiments in acetone- $\mathrm{d}_{6}$, THF- $\mathrm{d}_{8}, \mathrm{CD}_{2} \mathrm{Cl}_{2}$ or benzene (with one drop of MeCN- $\mathrm{d}_{3}$ for solubility reasons) the resonance for the MeCN ligands was observed at 2.40 ppm, $2.48 \mathrm{ppm}, 2.40 \mathrm{ppm}$ and $0.89 \mathrm{ppm}$, respectively. In MeCN- $\mathrm{d}_{3}$ this resonance was not found due to fast exchange with deuterated MeCN. The chemical shifts of the proton resonances like the methylene groups or the pyrazole H-4 vary in different solvents due to different magnetic susceptibilities of the solvents. However, the ${ }^{31} \mathrm{P}$ NMR shifts and $J_{\mathrm{Rh}-\mathrm{P}}$ coupling constants are almost not affected (Table 3.2). In all solvents a dissociation of the MeCN ligand was not observed, but a slow oxidative addition occured in $\mathrm{CD}_{2} \mathrm{Cl}_{2}$ (see Chapters 3.4.4.2 and 3.4.4.3).

Table 3.2: Selected ${ }^{1} \mathrm{H}$ and ${ }^{31} \mathrm{P}$ chemical shift and $J_{\mathrm{Rh}-\mathrm{P}}$ coupling constants of $\mathbf{R h}^{\mathrm{tBu}}-\mathbf{M e C N}$ in different solvents. The shifts are given in [ppm] and the coupling constants in $[\mathrm{Hz}]$.

\begin{tabular}{ccccccc}
\hline solvent & ${ }^{1} \mathrm{H} \mathrm{CH}_{2}$ & ${ }^{1} \mathrm{H} \mathrm{pz} \mathrm{4-H}$ & ${ }^{1} \mathrm{H}{ }^{t} \mathrm{Bu}$ & ${ }^{1} \mathrm{H} \mathrm{MeCN}$ & ${ }^{31} \mathrm{P}$ & $J_{\text {Rh-P }}$ \\
\hline $\mathrm{MeCN} \mathrm{d}_{3}$ & 3.28 & 7.21 & 1.38 & - & 91.51 & 178.2 \\
\hline $\mathrm{C}_{6} \mathrm{D}_{6}{ }^{\mathrm{a}}$ & 2.74 & 6.80 & 1.26 & 0.89 & 90.67 & 179.8 \\
\hline acetone- $\mathrm{d}_{6}$ & 3.40 & 7.40 & 1.44 & 2.40 & 91.27 & 179.3 \\
\hline $\mathrm{THF} \mathrm{d}_{8}$ & 3.28 & 7.21 & 1.42 & 2.48 & 91.19 & 180.2 \\
\hline $\mathrm{CD}_{2} \mathrm{Cl}_{2}$ & 3.20 & 7.05 & 1.40 & 2.40 & 91.49 & 178.0 \\
\hline
\end{tabular}

a One drop MeCN-d 3 was added for solubility reasons.

Single crystals of $\mathbf{R h}^{\mathrm{tBu}}$-MeCN were obtained by layering a concentrated acetone solution with pentane. The complex crystallized in the monoclinic space group $\mathrm{P} 12_{1} / \mathrm{n} 1$ with four molecules in the unit cell. The molecular structure is shown in Figure 3.33, featuring very similar structural properties of $\mathbf{R h}^{t \mathrm{Bu}}-\mathbf{M e C N}$ and $\mathbf{R h}^{t \mathrm{Bu}}-\mathbf{C O}$.
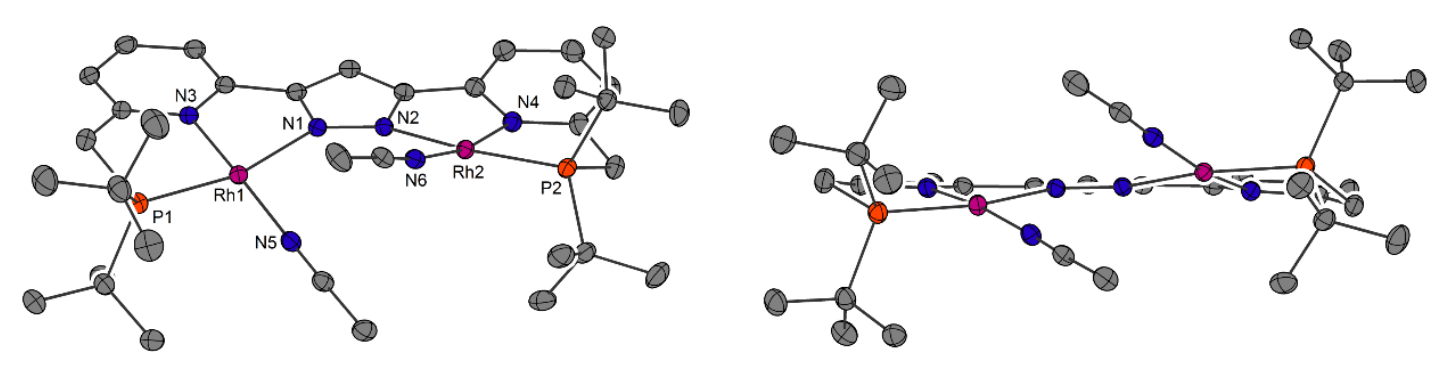

Figure 3.33: Thermal displacement ellipsoids (shown at $30 \%$ probability) of the molecular structure of $\left[(\mathrm{Rh}(\mathrm{MeCN}))_{2} \mathrm{~L}^{t \mathrm{Bu} u}\right]\left(\mathrm{PF}_{6}\right) \mathbf{5 8}$ in two different orientations. Counterions, hydrogen atoms and solvent molecules were omitted for clarity.

Again the coligands in the binding pocket as well as the metal centers and the phosphine donor atoms are laying above and below the ligand backbone plane, respectively. Due to more steric interactions of the MeCN ligands in $\mathbf{R h}^{\mathrm{tBu}}-\mathbf{M e C N}$ compared to CO ligands in $\mathbf{R h}^{\mathrm{tBu}} \mathbf{C} \mathbf{C O}$, the distances between the plane and the rhodium and phosphorus atoms are larger 
$\left(\bar{d}_{\mathrm{Rh}-\text { plane }}=0.530 \AA\right.$ and $\bar{d}_{\mathrm{P} \text {-plane }}=0.838 \AA$ ). This is also reflected in a longer metal-metal separation of $4.612 \AA$. The Rh-N $\mathrm{N}_{\mathrm{MeCN}}$ bond length was found to be $\bar{d}_{\mathrm{Rh}-\mathrm{N}}=1.987 \AA$.

When the chloride abstraction of $\left[\mathrm{RhCl}(\mathrm{coe})_{2}\right]_{2}$ was performed in acetone with subsequent addition of the filtrate to a solution of ligand and base in THF, the formation of the acetone complex 60 was assumed based on ${ }^{1} \mathrm{H}$ NMR spectroscopy (Scheme 3.24). However, isolation as a pure compound failed several times due to extremely fast ligand exchange with stronger binding ligands, which might have been present as impurities in the used solvents.

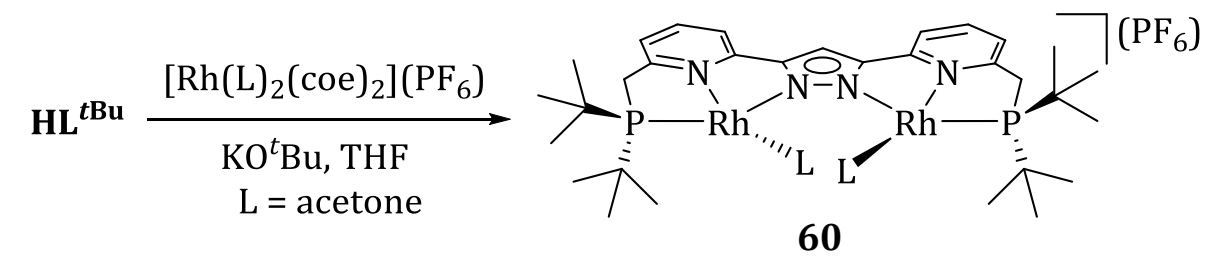

Scheme 3.24: Attempted synthesis of complex $\left[(\mathrm{Rh}(\text { acetone }))_{2} \mathrm{~L}^{t \mathrm{Bu}}\right]\left(\mathrm{PF}_{6}\right) 60$.

To circumvent the observed fast exchange, additional ligands were used, namely dimethyl sulfide $\left(\mathrm{SMe}_{2}\right)$ and ethylene $\left(\mathrm{C}_{2} \mathrm{H}_{4}\right)$. Such neutral ligands were known to potentially dissociate from rhodium(I) centers and to create an open binding site for subsequent catalysis.[316,428] $\mathrm{SMe}_{2}$ was introduced by simply adding an excess to the reaction mixture (Scheme 3.23). Complex 61 ( $\mathbf{R h}^{\text {tBu-SMe}}$ ) exhibited one sharp doublet for the phosphine side arms at $80.77 \mathrm{ppm}$ with $J_{\mathrm{Rh}-\mathrm{P}}=163.4 \mathrm{~Hz}$ in the ${ }^{31} \mathrm{P}$ NMR spectrum. The ${ }^{1} \mathrm{H}$ NMR spectrum revealed a symmetric species with one resonance for the $\mathrm{SMe}_{2}$ unit at $3.26 \mathrm{ppm}$, which is more than one ppm low field shifted compared to the signals of free $\mathrm{SMe}_{2}$ (Figure 3.34). The pyridine resonances are more low field shifted than in $\mathbf{R h}^{\mathrm{tBu}}-\mathbf{M e C N}$. This might be explained by a stronger trans effect of $\mathrm{SMe}_{2}$.

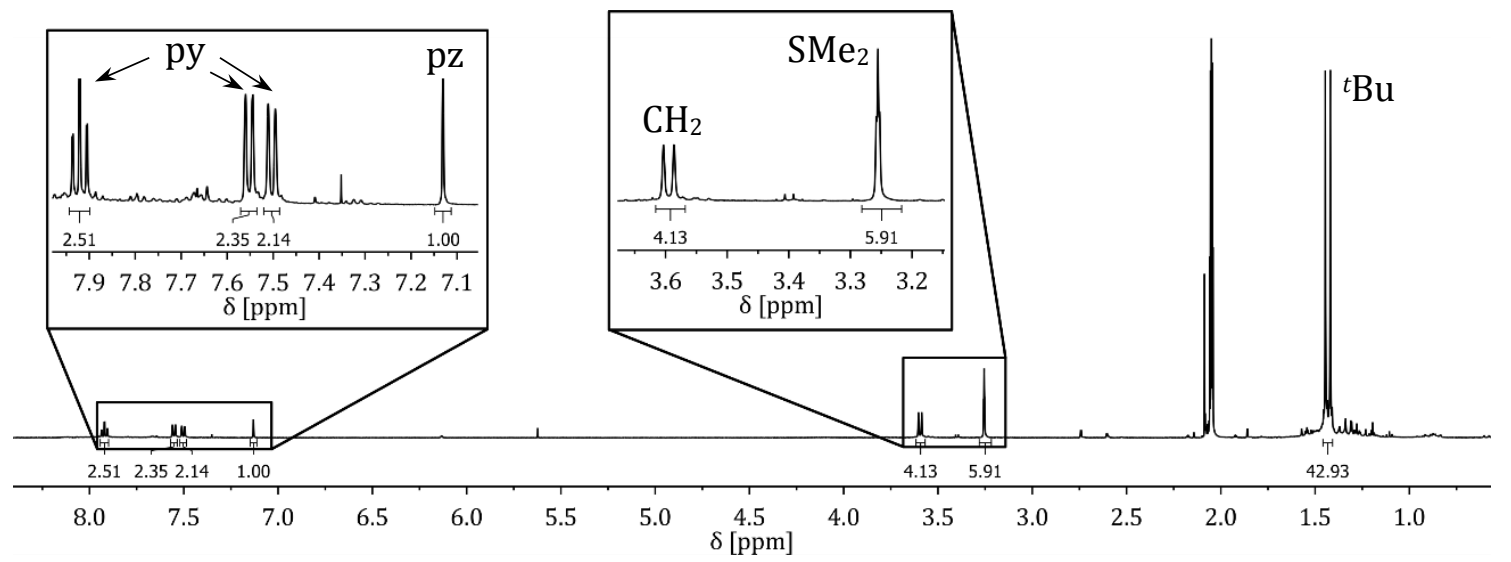

Figure 3.34: ${ }^{1} \mathrm{H}$ NMR spectrum of $\left[\mathrm{Rh}_{2}\left(\mu-\mathrm{SMe}_{2}\right) \mathrm{L}^{t \mathrm{Bu}}\right]\left(\mathrm{PF}_{6}\right) 61$ (acetone- $\left.\mathrm{d}_{6}, 298 \mathrm{~K}\right)$. The insets show the aromatic region of the spectrum and the $\mathrm{CH}_{2}$ and $\mathrm{SMe}_{2}$ resonances in detail.

From the integral of the $\mathrm{SMe}_{2}$ signal which equals six protons, the presence of only a single $\mathrm{SMe}_{2}$ and a bridging binding situation was assumed. Indeed, this situation was found in the solid state by X-ray diffraction analysis. Red single crystals formed upon standing of a concentrated 90 
acetone solution of $\mathbf{R h}^{t \mathrm{Bu}}-\mathbf{S M e}_{2}$ in the course of two days. The molecular structure offered some differences compared to the rhodium complexes with $\mathrm{CO}$ or MeCN ligands (Figure 3.35). The rhodium ions were still coordinated in a square planar fashion, although the parameter for the distortion from ideal square planar geometry $\tau_{\delta}$ is higher as for the other two complexes (Table 3.3). Interestingly, the distance between the two metal centers is remarkably shorter $\left(\mathrm{d}_{\mathrm{Rh}-\mathrm{Rh}}=3.823 \AA\right)$ indicating that the ligand systems is more flexible than expected and even a monoatomic bridging unit is possible.
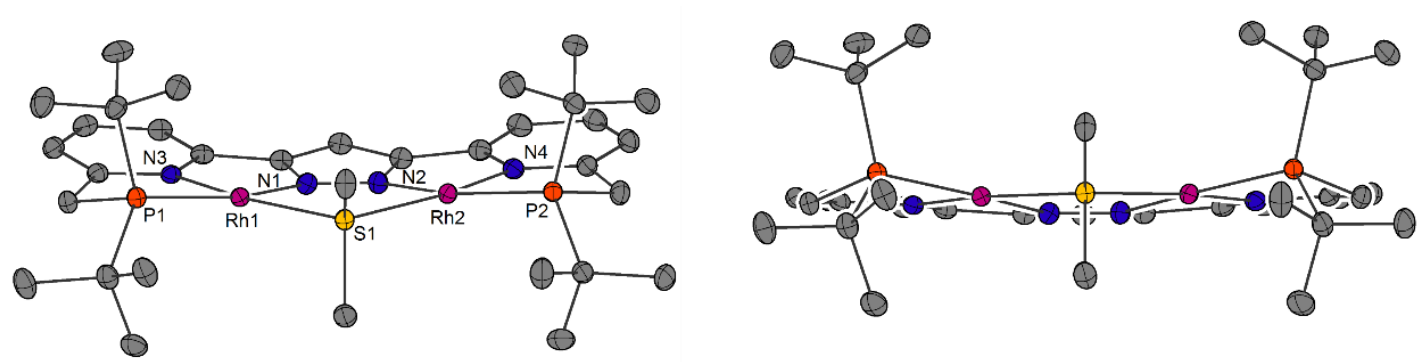

Figure 3.35: Thermal displacement ellipsoids (shown at $30 \%$ probability) of the molecular structure of $\left[\mathrm{Rh}_{2}\left(\mu-\mathrm{SMe}_{2}\right) \mathrm{L}^{t \mathrm{Bu}}\right]\left(\mathrm{PF}_{6}\right) \mathbf{6 1}$ in two different orientations. Counterions, hydrogen atoms and solvent molecules were omitted for clarity.

Both metal centers and phosphine side arms are located on one side of the backbone plane in $\mathbf{R h}^{t \mathrm{Bu}-\mathrm{SMe}_{2}}\left(\bar{d}_{\mathrm{Rh}-\text { plane }}=0.322 \AA\right.$ and $\left.\bar{d}_{\mathrm{P}-\text { plane }}=0.703 \AA\right)$, giving the complex a slightly bowlshape overall structure. The bridging sulfur atom is located almost exactly in the middle between the two rhodium ions. The Rh-P bond lengths are marginally elongated $\left(\bar{d}_{\mathrm{Rh}-\mathrm{P}}=2.272 \AA\right)$, while the Rh-N $\mathrm{N}_{\mathrm{pz}}$ bonds are shorter in $\mathbf{R h}^{t \mathrm{Bu}-S M \mathbf{S}_{2}}\left(\bar{d}_{\mathrm{Rh}-\mathrm{pz} \mathrm{N}}=1.976 \AA\right)$ than in $\mathbf{R h}^{\text {tBu}}-\mathbf{M e C N}$ and $\mathbf{R h}^{\mathrm{tBu}}$-CO (Table 3.3). The distance between the rhodium center and the pyridine nitrogen atom is about $0.03 \AA$ longer in $\mathbf{R} \mathbf{h}^{t \mathrm{Bu}}-\mathbf{S} \mathbf{M} \mathbf{e}_{2}$ than in $\mathbf{R h} \mathbf{h}^{\mathrm{tBu}}-\mathbf{M e C N}$ as a consequence of a stronger of a stronger trans effect of $\mathrm{SMe}_{2}$.

Table 3.3: Selected bond lengths in $[\AA]$, angles in $\left[{ }^{\circ}\right]$ and structural parameter $\tau_{\delta}$ for the deviation from ideal square planar coordination geometry of complexes $\left[(\mathrm{Rh}(\mathrm{CO}))_{2} \mathrm{~L}^{t \mathrm{Bu}}\right](\mathrm{PF} 6) \mathbf{5 5}, \quad\left[(\mathrm{Rh}(\mathrm{MeCN}))_{2} \mathrm{~L}^{t \mathrm{Bu}}\right]\left(\mathrm{PF}_{6}\right) \quad \mathbf{5 8}$ and $\left[\left(\mathrm{Rh}_{2}\left(\mu-\mathrm{SMe}_{2}\right) \mathrm{L}^{t \mathrm{Bu}}\right]\left(\mathrm{PF}_{6}\right) \mathbf{6 1}\right.$.

\begin{tabular}{|c|c|c|c|c|c|c|c|}
\hline & $\mathrm{d}_{\mathrm{Rh}-\mathrm{Rh}}$ & $\mathrm{d}_{\mathrm{Rh}-\mathrm{P}}$ & $d_{\text {Rh-pz N }}$ & $\mathrm{d}_{\text {Rh-py N }}$ & $\alpha$ & $\beta$ & $\tau_{\delta}$ \\
\hline $\mathbf{R h}^{\mathrm{tBu}-\mathrm{CO}}$ & 4.494 & 2.244 & 2.091 & 2.072 & 172.8 & 161.6 & 0.17 \\
\hline $\mathrm{Rh}^{\mathrm{tBu}}-\mathrm{MeCN}$ & 4.612 & $\begin{array}{l}2.218 \\
2.221\end{array}$ & $\begin{array}{l}2.098 \\
2.105\end{array}$ & $\begin{array}{l}2.001 \\
2.003\end{array}$ & $\begin{array}{l}169.5 \\
171.8\end{array}$ & $\begin{array}{l}163.2 \\
163.4\end{array}$ & $\begin{array}{l}0.19 \\
0.17\end{array}$ \\
\hline $\mathrm{Rh}^{\mathrm{tBu}}-\mathrm{SMe}_{2}$ & 3.823 & $\begin{array}{l}2.270 \\
2.274 \\
\end{array}$ & $\begin{array}{l}1.975 \\
1.977 \\
\end{array}$ & $\begin{array}{l}2.035 \\
2.036 \\
\end{array}$ & $\begin{array}{l}162.6 \\
162.9 \\
\end{array}$ & $\begin{array}{l}160.9 \\
160.9 \\
\end{array}$ & $\begin{array}{l}0.26 \\
0.25 \\
\end{array}$ \\
\hline
\end{tabular}

The ethylene complex $\mathbf{6 2}$ was tried to synthesize by using the rhodium precursor $\left[\mathrm{RhCl}\left(\mathrm{C}_{2} \mathrm{H}_{4}\right)_{2}\right]_{2}$ under otherwise equal reaction conditions (Scheme 3.25) but it was not possible to isolate one single product, according to ${ }^{31} \mathrm{P}$ NMR spectroscopy. From a reaction with an impure ligand batch a mononuclear complex 63 of monophosphine ligand XXXIV was 
crystallized (Figure 3.36) showing that the formation of a rhodium(I) ethylene complex is possible. The structural parameters for the single rhodium ion are similar to those of the dinuclear complexes discussed before. The $\mathrm{C}=\mathrm{C}$ bond of the ethylene ligand is slightly elongated as compared with free ethylene. ${ }^{[430]}$ For further studies, the use of gaseous ethylene in combination with $\left[\mathrm{RhCl}(\mathrm{coe})_{2}\right]_{2}$ similar to the synthesis of $\mathbf{R h}^{t \mathbf{B u}}-\mathbf{S M e} \mathbf{e}_{2}$ might result in the isolation of the desired compound $\mathbf{6 2}$.

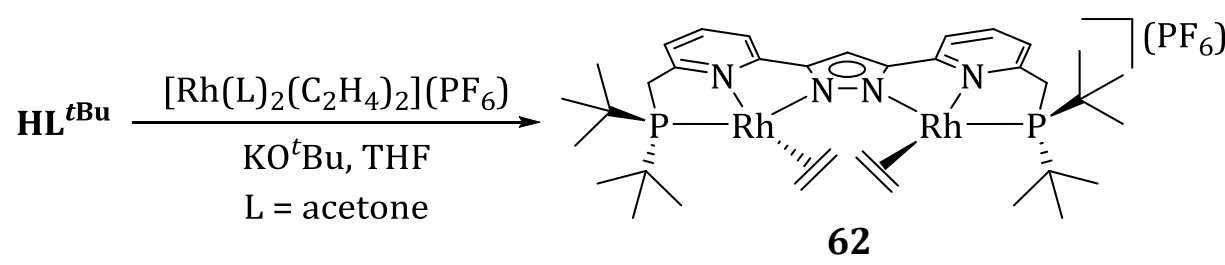

Scheme 3.25: Attempted synthesis of rhodium(I) ethylene complex 62.
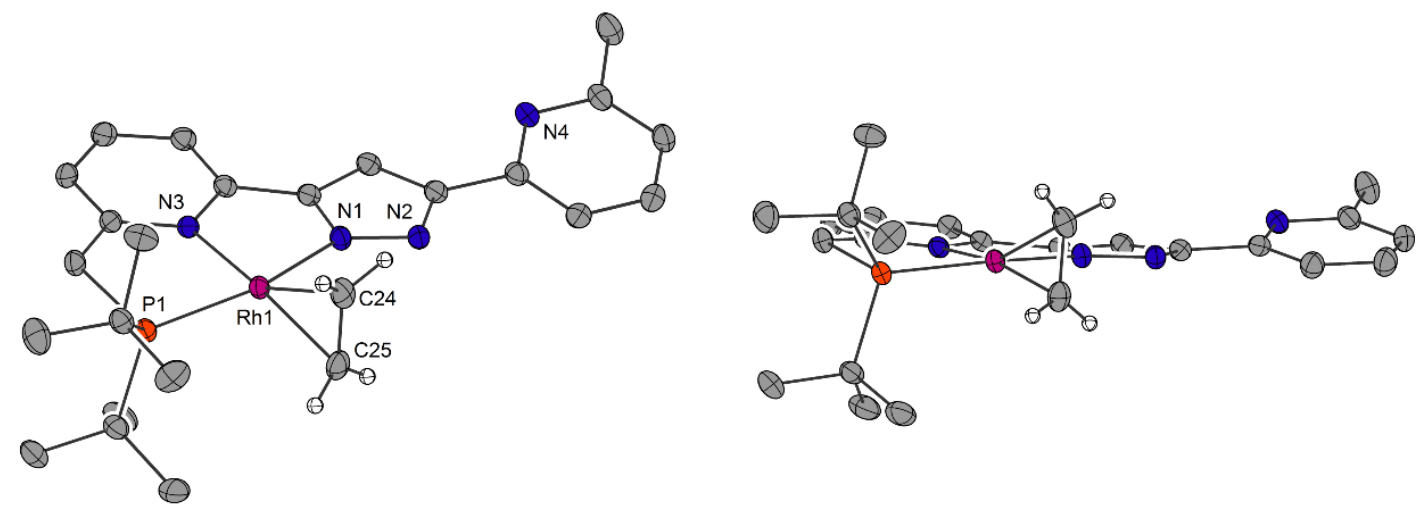

Figure 3.36: Thermal displacement ellipsoids (shown at $30 \%$ probability) of the molecular structure of $\left[\mathrm{Rh}\left(\mathrm{C}_{2} \mathrm{H}_{4}\right)(\mathrm{XXXIV})\right] 63$ in two different orientations. Hydrogen atoms except for the $\mathrm{C}_{2} \mathrm{H}_{4}$ ligand were omitted for clarity.

The molecular structure of $\mathbf{6 3}$ showed the ethylene coligand to occupy significant space of the ligand binding cavity, which might be a reason for the formation of several products in the above discussed reaction of $\mathbf{H L}{ }^{t \mathbf{B u}}$ with $\left[\mathrm{RhCl}\left(\mathrm{C}_{2} \mathrm{H}_{4}\right)_{2}\right]_{2}$. To lower the steric demand of two ethylene units it was considered to combine both binding sites in one ligand. Therefore,

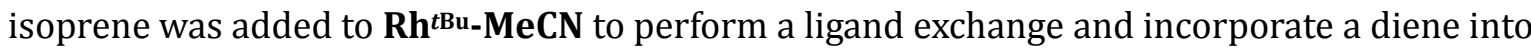
the binding pocket between the two rhodium centers (Scheme 3.26).

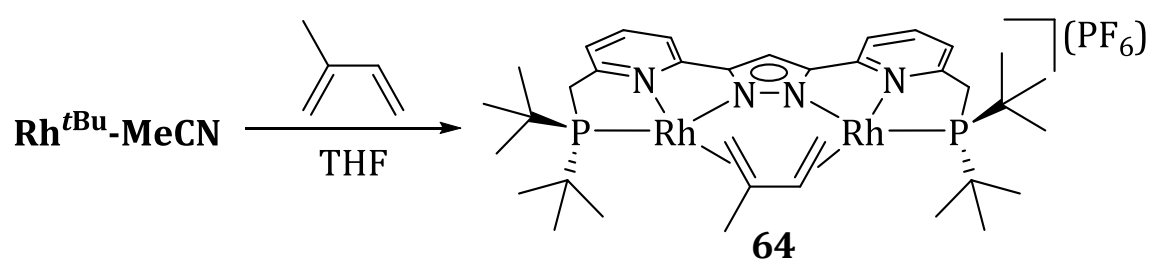

Scheme 3.26: Attempted synthesis of rhodium isoprene complex $\left[\mathrm{Rh}_{2}(\mu\right.$-isoprene $\left.) \mathrm{L}^{t \mathrm{~B} u}\right]\left(\mathrm{PF}_{6}\right) 64$.

The successful synthesis of the isoprene complex $\mathbf{6 4}$ was indicated by NMR spectroscopy, showing only two doublets in a ${ }^{31} \mathrm{P}$ NMR spectrum at $75.90 \mathrm{ppm}$ and $77.13 \mathrm{ppm}$ as expected for 
an unsymmetric complex. The ${ }^{1} \mathrm{H}$ NMR spectrum revealed the presence of some minor impurities (Figure 3.37) but the incorporation of isoprene in the complex was demonstrated by the shift of the corresponding ${ }^{1} \mathrm{H}$ NMR resonances by ca. 1 ppm to higher field as compared to free isoprene. Crystalline material could not be isolated and also crystallization attempts in the presence of an excess of isoprene did not lead to suitable crystals for X-ray diffraction analysis.

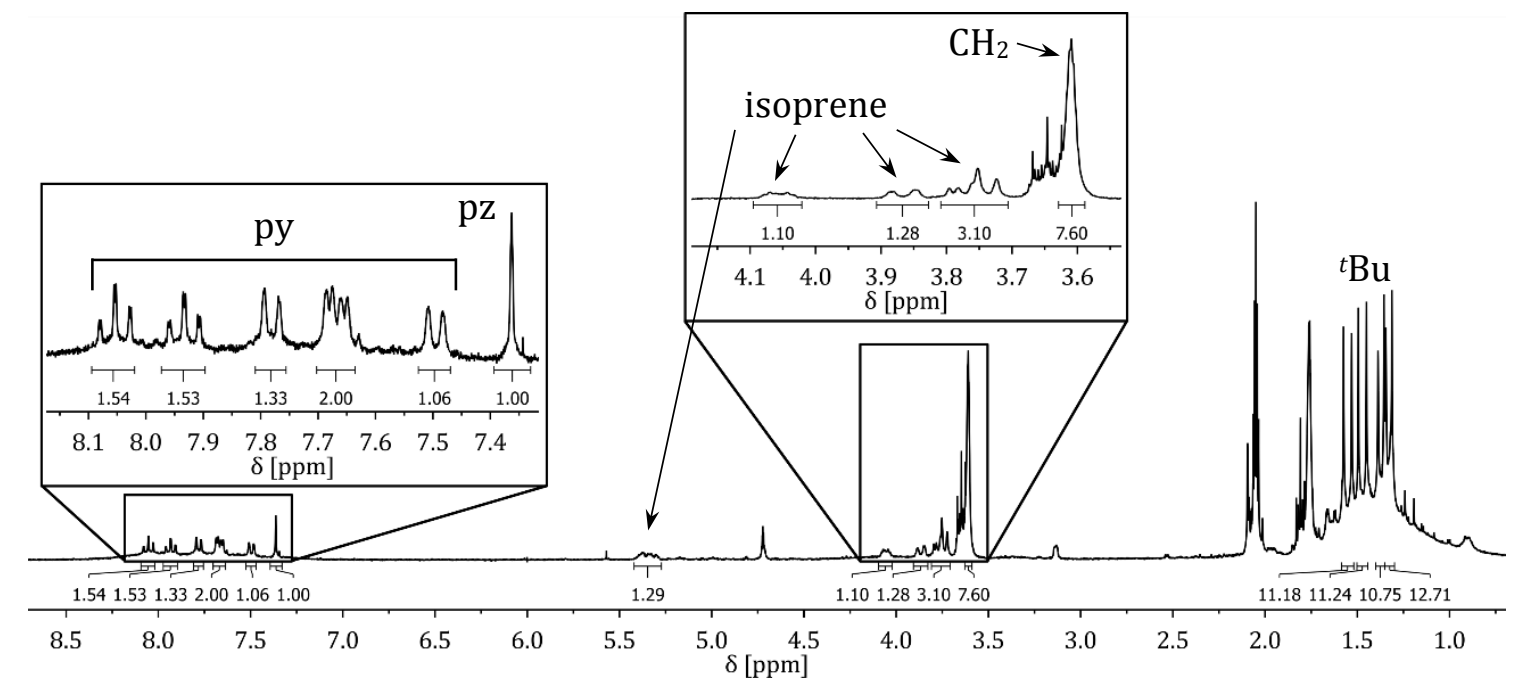

Figure 3.37: ${ }^{1} \mathrm{H}$ NMR spectrum of crude $\left[\mathrm{Rh}_{2}\left(\mu\right.\right.$-isoprene) $\left.\mathrm{L}^{t \mathrm{tBu}}\right]\left(\mathrm{PF}_{6}\right) 64$ (acetone- $\left.\mathrm{d}_{6}, 298 \mathrm{~K}\right)$. The insets show the aromatic region of the spectrum and the $\mathrm{CH}_{2}$ and isoprene resonances in detail.

As for the carbonyl complexes also a ${ }^{i} \operatorname{Pr}$ analogue of $\mathbf{R} \mathbf{h}^{t \mathrm{Bu}}$-MeCN was synthesized (Scheme 3.27). Under identical reaction conditions a mixture of different species was detected by ${ }^{31} \mathrm{P}$ NMR spectroscopy. When the reaction mixture was heated to reflux for several hours, a clean reaction was observed and $\left[(\mathrm{Rh}(\mathrm{MeCN}))_{2} L^{i \mathrm{Pr}}\right]\left(\mathrm{PF}_{6}\right) 65\left(\mathbf{R h}^{i \mathrm{Pr}}-\mathrm{MeCN}\right)$ was isolated as the only product. It was assumed that for the sterically less bulky $\mathbf{H L}^{\mathbf{i P r}}$ the complete dissociation of the cyclooctene ligand of the precursor was not required in contrast to the more crowded $\mathbf{H L}^{t \mathbf{B u}}$. For that reason, the reaction mixture was heated to reflux to fully remove the cyclooctene ligands from the rhodium centers.

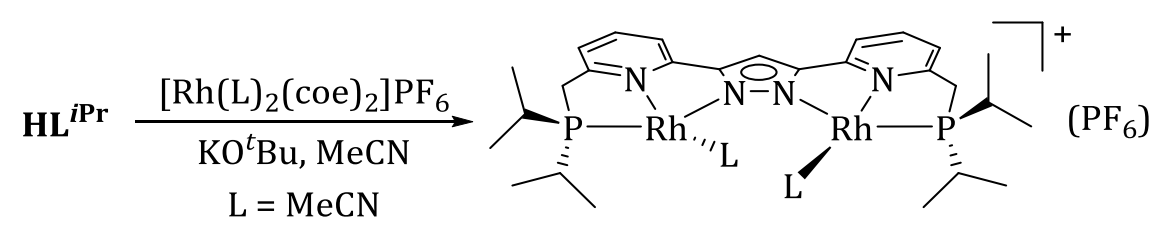

Scheme 3.27: Synthesis of $\left[(\mathrm{Rh}(\mathrm{MeCN}))_{2} \mathrm{~L}^{i \mathrm{Pr}}\right]\left(\mathrm{PF}_{6}\right) 65$.

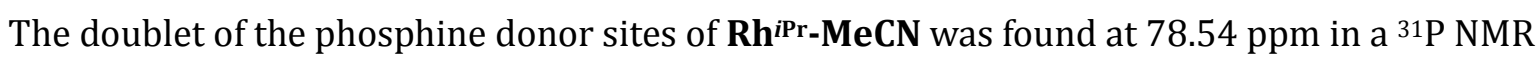
spectrum with a coupling constant of $J_{\mathrm{Rh}-\mathrm{P}}=176.0 \mathrm{~Hz}$, showing the same effect of a high field shift from ${ }^{t} \mathrm{Bu}$ to $i \operatorname{Pr}$ as found already for the carbonyl complexes, while the coupling constants remain similar. The proton resonances of $\mathbf{R h}{ }^{i P r}-\mathbf{M e C N}$ for the ligand backbone and for the $\mathrm{CH}_{2}$ group are essentially the same as for $\mathbf{R h}^{\text {tBu}}$-MeCN, again showing that the difference between 
the two ligands is only of steric nature (Figure 3.38). Unfortunately, the crystallization of compound 65 was not successful.

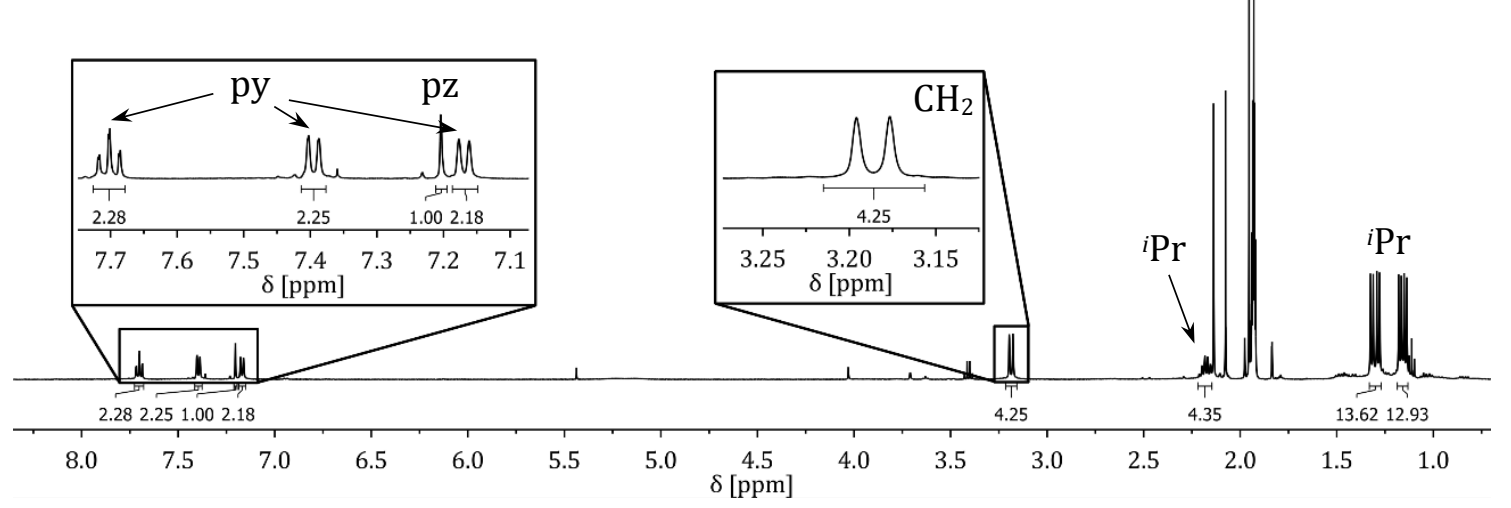

Figure 3.38: ${ }^{1} \mathrm{H}$ NMR spectrum of $\left[(\mathrm{Rh}(\mathrm{MeCN}))_{2} \mathrm{~L}^{i \mathrm{Pr}}\right]\left(\mathrm{PF}_{6}\right) 65\left(\mathrm{MeCN}-\mathrm{d}_{3}, 298 \mathrm{~K}\right)$.

In order to increase the solubility of the rhodium complexes in non-polar solvents, salt metatheses with sodium tetraphenylborate to substitute the hexafluorophosphate counterion were attempted from methanol solutions of the complexes. For $\mathbf{R h}{ }^{t \mathrm{Bu}}-\mathbf{C O}$ the desired effect was found, afterwards the complex was soluble in THF. However, neither analytically pure material nor single crystals suitable for X-ray diffraction analysis could be obtained so that the complete conversion could not be proven. When $\mathbf{R h}{ }^{t \mathrm{Bu}}-\mathbf{M e C N}$ was used in the salt metathesis reaction, the desired effect was not observed, because the precipitate from the methanol reaction mixture was still not soluble in benzene. In addition, again the isolation of clean material proved to be not possible even if the base in the complexation reaction was changed to a lithium or sodium containing base, to avoid the formation of $\mathrm{KBPh}_{4}$, which also precipitates from methanol solutions.

\subsubsection{Ligand Exchange and Flexibility}

To study the ligand exchange and bond strength behavior of $\mathbf{R h}^{t \mathrm{Bu}}-\mathbf{C O}, \mathbf{R} \mathbf{h}^{\mathrm{tBu}}-\mathbf{M e C N}$ and $\mathbf{R h}^{t{ }^{t} u_{-}} \mathbf{S M e} \mathbf{e}_{2}$, substitution reactions were performed (Scheme 3.23). The MeCN complex $\mathbf{5 8}$ can be transformed into the $\mathrm{CO}$ complex by stirring an acetone solution under an atmosphere of carbon monoxide for less than one hour. A fast color change from red to yellow and precipitation of $\mathbf{R h}{ }^{t B u}-\mathbf{C O}$ was observed. NMR spectroscopy clearly showed the complete reaction by disappearance of the $\mathbf{R h}{ }^{\mathrm{tBu}}-\mathbf{M e C N}$ resonances and the formation of those of $\mathbf{R h}^{t \mathrm{Bu}}$-CO. In the course of this project this pathway was mostly used for the synthesis of

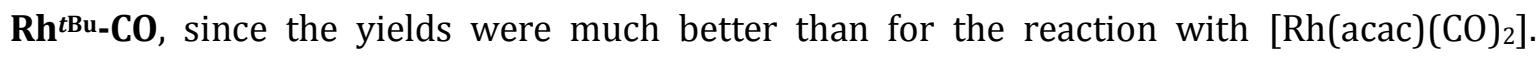
Furthermore, refluxing a THF solution of $\mathbf{R h}^{t \mathrm{Bu}}$-MeCN in the presence of an excess of $\mathrm{SMe}_{2}$ for several hours yielded quantitatively $\mathbf{R} \mathbf{h}^{t \mathrm{Bu}}-\mathbf{S} \mathbf{S} \mathbf{e}_{2}$. The reverse reaction already took place when small quantities of MeCN were present in solution, e.g., as impurity in solvents. These exchange 
reactions were not successful when $\mathbf{R h}^{\text {tBu}}$-CO was used. The complex proved to be completely inert in that context. Neither refluxing in MeCN or acetone nor irradiation with an UV lamp in the presence of several possible ligands or reagents like $\mathrm{MeCN}, \mathrm{SMe}_{2}$ or $\mathrm{MeI}$ led to a dissociation of the CO ligands. No reactions were observed if degassed solutions of $\mathbf{R h}^{t \mathrm{Bu}}-\mathbf{C O}$ or $\mathbf{R h}^{t \mathbf{B u}}-\mathbf{M e C N}$ were set under an atmosphere of ethylene gas after the course of one week at ambient and elevated temperatures. As discussed above, an ethylene and isoprene complex could be synthesized in acetone solutions. On the basis of these findings a qualitative stability order can be suggested with acetone being the weakest and CO the strongest ligand in this series of rhodium(I) complexes (Figure 3.39).

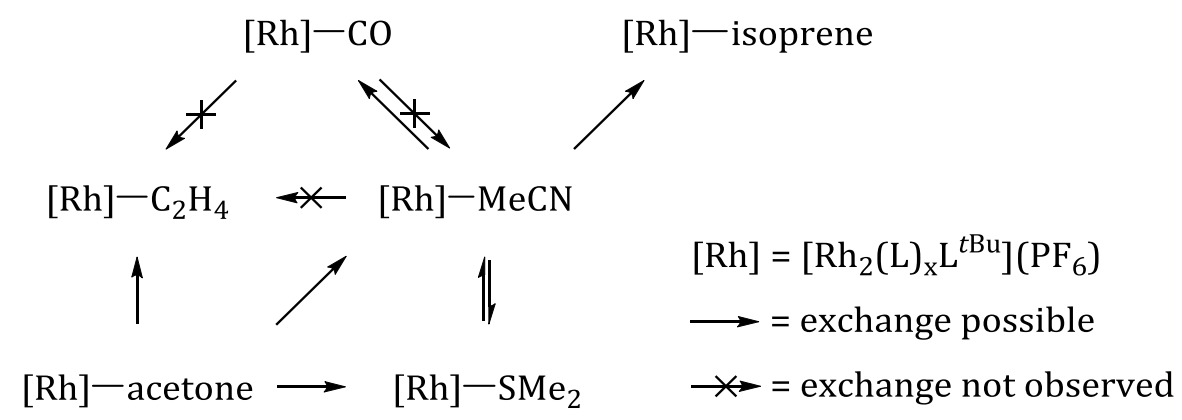

Figure 3.39: Summary of the possible and not observed ligand exchanges with rhodium(I) complexes of $\mathbf{H L}^{\mathbf{t B u}}$.

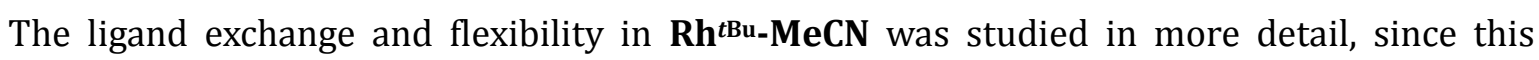
complex was mainly applied for subsequent catalysis. Firstly, a large excess of deuterated MeCN (100 equivalents) was added to a THF solution of $\mathbf{R h}^{t \mathrm{Bu}-M e C N}$ and the rate of exchange of the MeCN ligands was investigated by ${ }^{1} \mathrm{H}$ NMR spectroscopy at different temperatures $(278 \mathrm{~K}$ to $308 \mathrm{~K}$, Scheme 3.28).

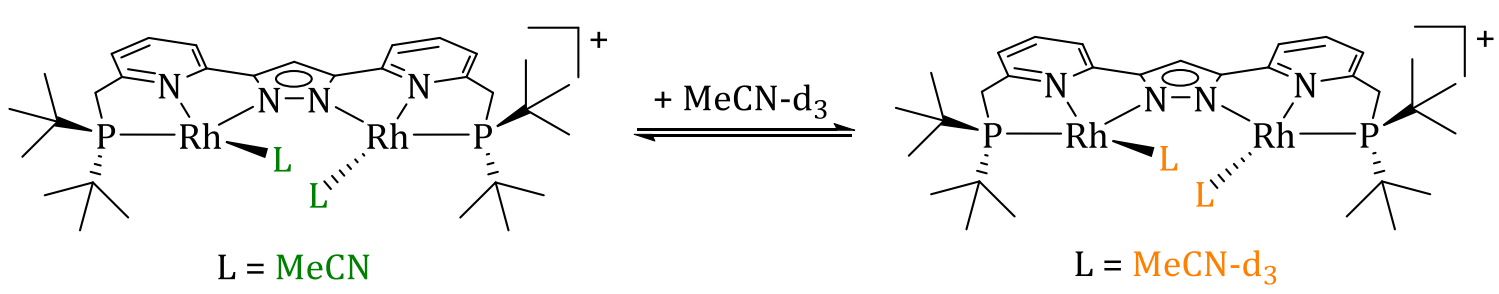

Scheme 3.28: Exchange of the MeCN (green) coligands by deuterated analogue MeCN- $\mathrm{d}_{3}$ (orange) in $\mathbf{R h} \mathbf{h}^{t \mathbf{B u}}-\mathbf{M e C N}$.

From the decrease of the resonance of bound $\mathrm{MeCN}(2.48 \mathrm{ppm})$ and increase of free MeCN $(1.94 \mathrm{ppm})$ the ratios of the deuterated and non-deuterated complexes were calculated and plotted versus time (Figure 3.40). The observed initial rate constants $\mathrm{k}_{\mathrm{obs}}(\mathrm{T})$ of the exchange at different temperatures were obtained from a linear fit of the starting phase and used for an EYRING plot $\left(1 /\right.$ T versus $\ln \left(\mathrm{k}_{\mathrm{obs}}(\mathrm{T})\right)$ to determine the thermodynamic parameters of the reaction $\Delta \mathrm{H}^{\ddagger}=29.4 \pm 6.38 \mathrm{~kJ} \mathrm{~mol}^{-1}$ and $\Delta \mathrm{S}^{\ddagger}=-201.3 \pm 22.13 \mathrm{~J} \mathrm{~mol}^{-1}$ (Figure 3.41). $\Delta \mathrm{H}^{\ddagger}$ and $\Delta \mathrm{S}^{\ddagger}$ can be

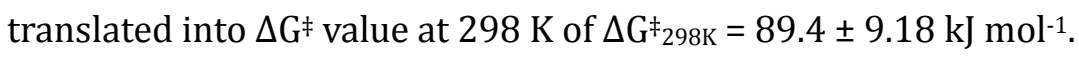




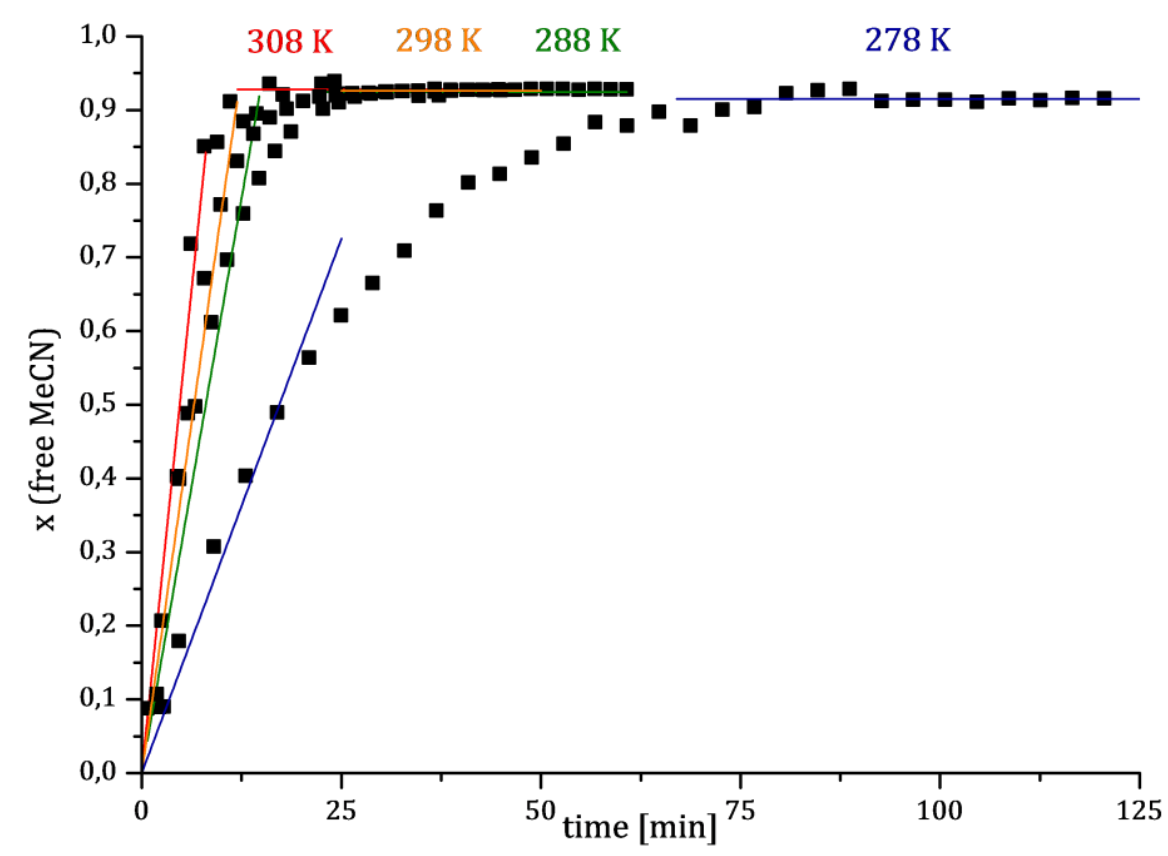

Figure 3.40: Plot of the molar ratio $\mathrm{x}$ of free MeCN $\left(=\mathrm{x}\right.$ of deuterated complex $\left.\left[\left(\mathrm{Rh}\left(\mathrm{MeCN}-\mathrm{d}_{3}\right)\right)_{2} \mathrm{~L}^{t \mathrm{Bu}}\right]\left(\mathrm{PF}_{6}\right) \mathbf{5 8}\right)$ versus time at different temperatures (blue to red).

The largely negative value for the entropy is indicative for an associative mechanism, meaning that a deuterated $\mathrm{MeCN}$ is coordinated first to form a five-fold coordinated species, which then releases MeCN.[431] Rates for the forward and the backward reactions are given in Table 3.4, derived from the rate constants and the equilibrium constants. The final ratios of the two complexes were found to be similar within the experimental error, which is in line with the expectation of a thermoneutral reaction for this kind of ligand exchange reaction.

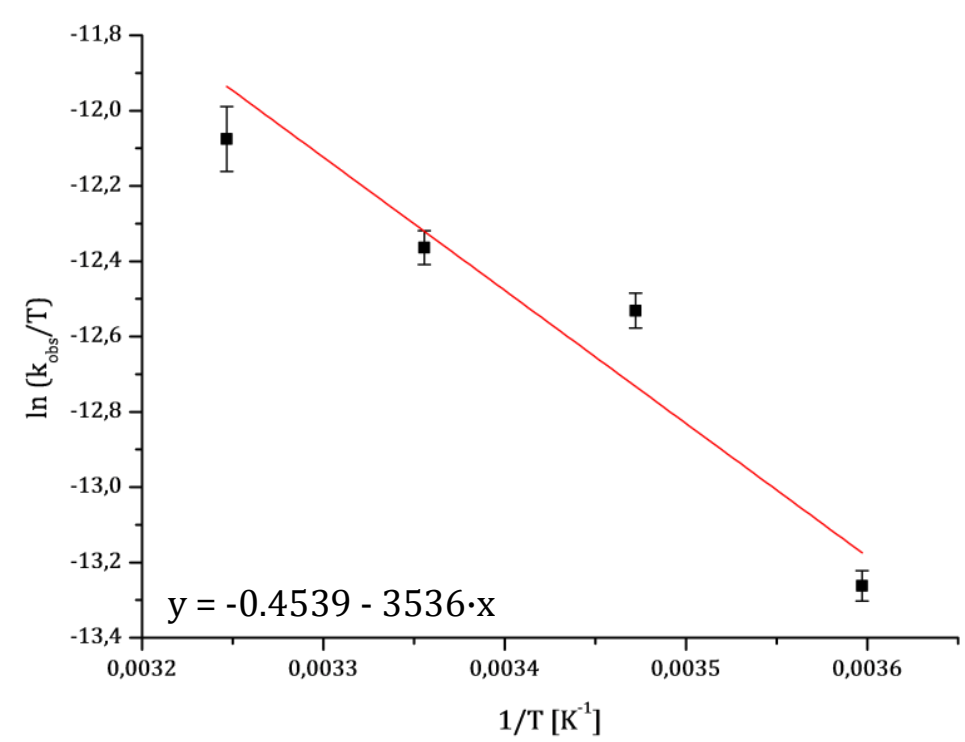

Figure 3.41: Eyring plot $\ln \left(\mathrm{k}_{\mathrm{obs}}(\mathrm{T})\right)$ versus $1 / \mathrm{T}$ of the ligand exchange reaction in $\mathbf{R h}^{t \mathbf{B u}}-\mathbf{M e C N}$ with $\mathrm{MeCN}-\mathrm{d}_{3}$. 
Table 3.4: Observed and derived rate constants $\left[10^{-2} \mathrm{~min}^{-1}\right]$ and equilibrium constant at different temperatures $[\mathrm{K}]$.

\begin{tabular}{cccccc}
\hline $\mathrm{T}$ & $\mathrm{k}_{\mathrm{obs}}$ & $\mathrm{k}_{\text {forward }}$ & $\mathrm{k}_{\text {backward }}$ & $\mathrm{X}_{\text {end, MeCN }}$ & $\mathrm{K}_{\text {eq }}$ \\
\hline 278 & 2.901 & 3.229 & -0.3276 & 0.9079 & 9.853 \\
\hline 288 & 6.240 & 11.33 & -0.5562 & 0.9244 & 12.22 \\
\hline 298 & 7.635 & 13.86 & -0.6802 & 0.9244 & 12.22 \\
\hline 308 & 10.53 & 11.70 & -1.166 & 0.9093 & 10.03 \\
\hline
\end{tabular}

In contrast to the ligand exchange, a second effect in $\mathbf{R h}^{\mathrm{tBu}}-\mathbf{M e C N}$ was investigated: The flexibility of the coligands. The MeCN ligands in the dinuclear binding pocket of $\mathbf{R h}^{t \mathrm{Bu}-\mathbf{M e C N}}$ are in steric clash and therefore pointing up and down from the ligand backbone plane (see molecular structure, Figure 3.33). In solution this arrangement is flexible and an interconversion between the two enantiomers ( $P$ and $M$ ) can occur (Figure 3.42).

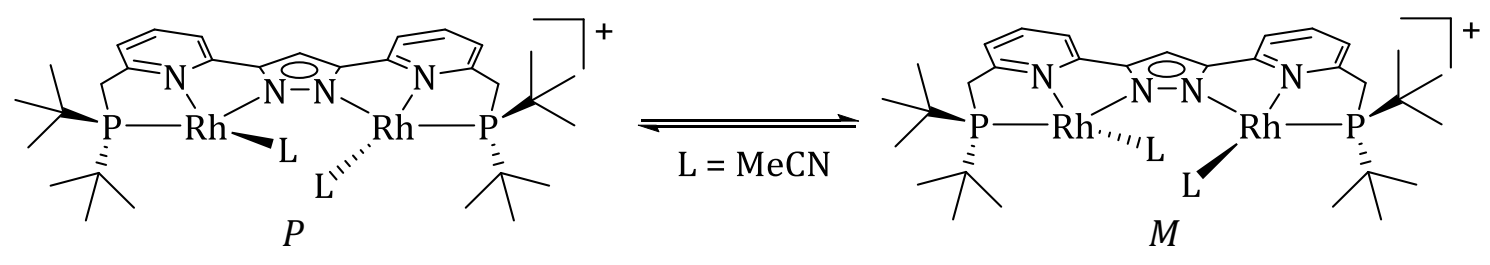

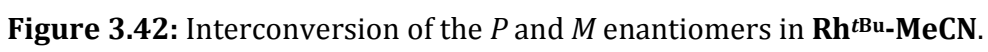

This interconversion was observed and studied by variable temperature ${ }^{1} \mathrm{H}$ NMR spectroscopy in MeCN- $\mathrm{d}_{3}$, acetone- $\mathrm{d}_{6}$ and THF- $\mathrm{d}_{8}$. The activation barriers for the interconversion were determined in considering the line width and peak separation of the tert-butyl resonances. A broadening of the signals was also observed for the $\mathrm{CH}_{2}$ groups while the resonances for the pyridine and pyrazole protons as well as the ${ }^{31} \mathrm{P}$ signals of the phosphine side arms shifted only slightly upon cooling. For the MeCN sample the coalescence point could not be observed since the freezing point of the solvent was too high (Figure 3.43). In the case of acetone and THF the coalescence points are around $\mathrm{T}_{\text {coal }} \approx 243 \mathrm{~K}$ and $\mathrm{T}_{\text {coal }} \approx 253 \mathrm{~K}$, respectively (see Appendix, Figure $6.1)$.

For the determination of the rate constants of the conversion at a given temperature the following equations were used:[432]

$$
\begin{gathered}
k(T)=\pi \cdot \Delta v_{1 / 2} \\
k(T)=\frac{\pi \cdot\left(\Delta v_{A B}\right)^{2}}{2 \cdot \Delta v_{1 / 2}}
\end{gathered}
$$




$\frac{258 \mathrm{~K}}{258 \mathrm{~K}}$

Figure 3.43: Variable temperature ${ }^{1} \mathrm{H}$ NMR measurement of $\mathbf{R h}^{\mathrm{tBu}}-\mathbf{M e C N}$ in $\mathrm{MeCN}-\mathrm{d}_{3}$.

For the slow exchange regime at low temperatures Equation 3 was used to estimate the rate of interconversion, with $\Delta v_{1 / 2}$ being the line width (full width at half maximum) neglecting the natural line broadening of the signals. When the exchange is sufficiently fast (above the coalescence point), Equation 4 becomes valid, in which $\Delta v_{A B}$ is the peak separation at temperatures below the coalescence point. From an EYRING plot that uses these calculated rate constants $\mathrm{k}_{\mathrm{T}}$ at different temperatures, the activation parameters $\Delta \mathrm{H}^{\ddagger}$ and $\Delta \mathrm{S}^{\ddagger}$ for each solvent could be derived. For a MeCN solution a single linear behavior was found in an EYRING plot (Figure 3.44, bottom), whereas for $\mathbf{R h}^{\mathrm{t}}{ }^{\mathrm{Bu}}-\mathbf{M e C N}$ in the less coordinating solvents acetone and THF two regions could be distinguished (Figure 3.44, top). A negative slope was found for temperatures from $298 \mathrm{~K}$ to $213 \mathrm{~K}$ and $223 \mathrm{~K}$ for acetone and THF, respectively. At lower temperatures a positive slope was observed which indicated two different mechanisms for the isomerization to occur at different temperatures. The $\Delta \mathrm{S}^{\ddagger}$ values for the isomerization in the high temperature regime are more negative than observed for the ligand exchange reaction but in the same order of magnitude. The free activation energies of the isomerization at $298 \mathrm{~K}$ are significantly lower than for the ligand exchange reaction $\left(\Delta \mathrm{G}^{\ddagger} 298 \mathrm{~K} \approx 90 \mathrm{~kJ} \mathrm{~mol}^{-1}\right.$ for the exchange versus $\Delta \mathrm{G}^{\ddagger}{ }_{298 \mathrm{~K}} \approx 53-63 \mathrm{~kJ} \mathrm{~mol}^{-1}$ for the interconversion at temperatures above $230 \mathrm{~K}$ ). The entropy might be decreased by a very rigid transition state. In conclusion, it was assumed that the isomerization process does not include a $\mathrm{Rh}-\mathrm{N}$ bond dissociation, as also reported for similar ruthenium complexes.[267,433]. A different mechanism seemed be involved at low 
temperatures due to opposite signs of the slopes, although the $\Delta S^{\ddagger}$ values are even more negative. This is not completely understood to date.
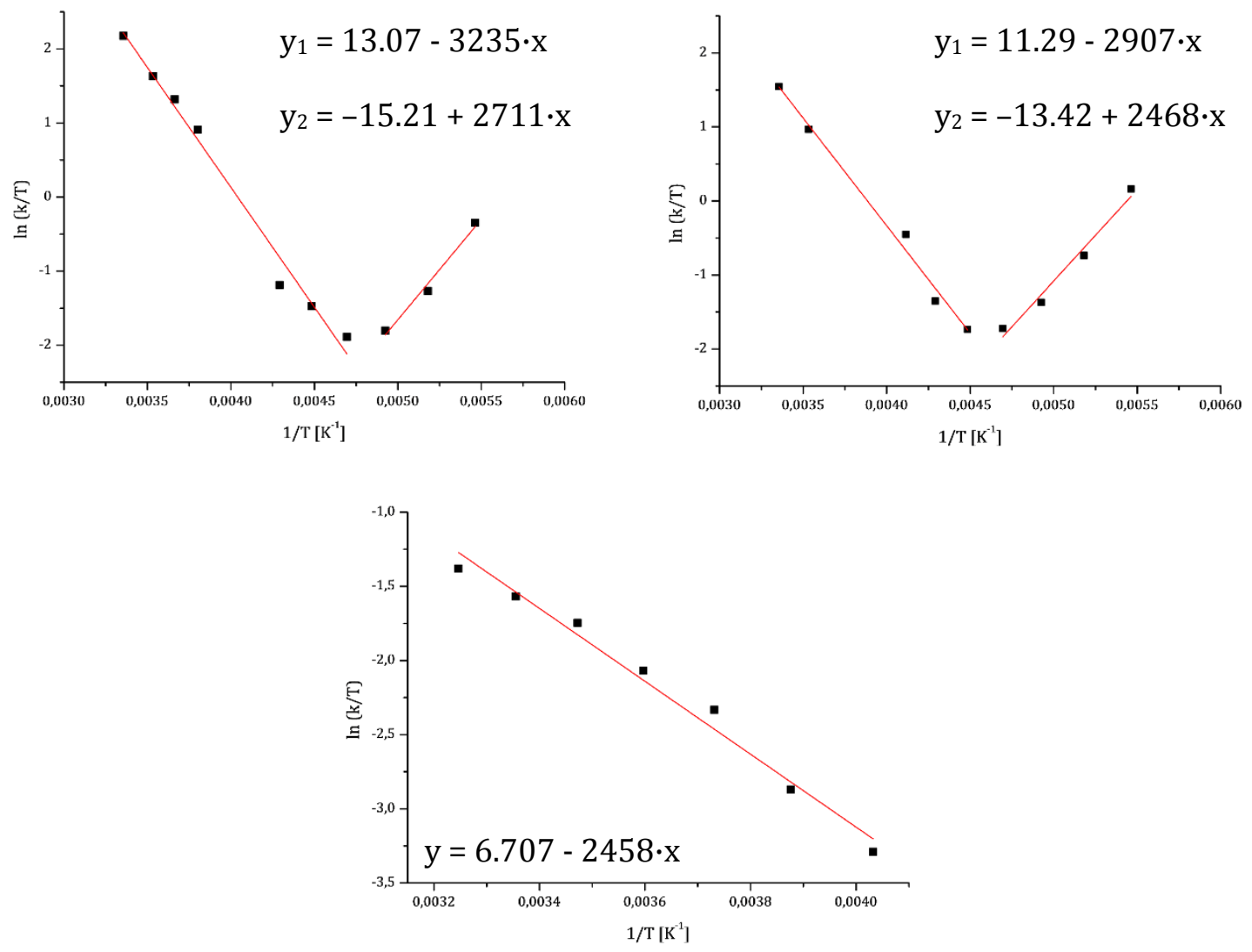

Figure 3.44: EYRING plots $\ln \left(\mathrm{k}_{\mathrm{T}}\right)$ versus $1 / \mathrm{T}$ for the interconversion of the two conformers of $\mathbf{R h}^{\text {tBu-MeCN }}$ in different solvents: Acetone- $\mathrm{d}_{6}$ (top, left), THF-d 8 (top, right) and MeCN-d 3 (bottom).

The thermodynamic parameters are summarized in Table 3.5. The $\Delta \mathrm{H}^{\ddagger}$ and $\Delta \mathrm{S}^{\ddagger}$ were translated into free activation energies $\Delta \mathrm{G}^{\ddagger}$ at $298 \mathrm{~K}$. The values for $\Delta \mathrm{H}^{\ddagger}$ in acetone and THF at higher temperatures are similar and slightly higher than in $\mathrm{MeCN}$, whereas the values for the low temperature regime were found to be negative. $\Delta S^{\ddagger}$ in MeCN is considerably more negative and $\Delta \mathrm{G}^{\ddagger_{298} \mathrm{~K}}$ is larger compared to the values for acetone and THF solutions.

Table 3.5: Thermodynamic data of the interconversion of $P$ and $M$ conformers of $\mathbf{R h}^{t \mathbf{B u}}-\mathbf{M e C N}$ in different solvents. The values for $\Delta \mathrm{H}^{\ddagger}$ and $\Delta \mathrm{G}^{\ddagger}$ are given in $\left[\mathrm{kJ} \mathrm{mol}^{-1}\right] ; \Delta \mathrm{S}^{\ddagger}$ in $\left[\mathrm{J} \mathrm{mol}^{-1} \mathrm{~K}^{-1}\right]$.

\begin{tabular}{ccccc}
\hline & & $\Delta \mathrm{H}^{\ddagger}$ & $\Delta \mathrm{S}^{\ddagger}$ & $\Delta \mathrm{G}^{\ddagger} 298 \mathrm{~K}$ \\
\hline MeCN-d $_{3}$ & & $20.43 \pm 1.20$ & $-141.8 \pm 4.37$ & $62.68 \pm 1.77$ \\
\hline \multirow{2}{*}{ acetone-d $_{6}$} & $>210 \mathrm{~K}$ & $26.90 \pm 1.40$ & $-88.86 \pm 5.59$ & $53.38 \pm 2.17$ \\
& $<210 \mathrm{~K}$ & $-22.54 \pm 2.74$ & $-324.0 \pm 14.2$ & $74.02 \pm 5.06$ \\
\hline \multirow{2}{*}{ THF-d $_{8}$} & $>220 \mathrm{~K}$ & $24.17 \pm 1.37$ & $-103.6 \pm 5.47$ & $55.05 \pm 2.13$ \\
& $<220 \mathrm{~K}$ & $-20.52 \pm 2.14$ & $-309.1 \pm 10.1$ & $71.60 \pm 3.88$ \\
\hline
\end{tabular}


When the calculations were applied for the signal broadening and splitting of the $\mathrm{CH}_{2}$ groups, very similar thermodynamic values were obtained.

In a variable temperature ${ }^{1} \mathrm{H}$ NMR measurement of the ${ }^{i} \mathrm{Pr}$ analogue $\mathbf{R h}^{\text {iPr-MeCN }}$ the interconversion of the $P$ and $M$ enantiomers was found to be fast on the NMR time scale even at low temperatures. This may reflect a larger metal-metal separation and thus less steric clash of the MeCN ligands.

\subsubsection{Synthesis of Rhodium(III) Complexes}

Low valent, electron rich late transition metals like VASKA's complex are known to easily undergo oxidative addition to the corresponding trivalent metal complexes. ${ }^{[434-436]}$ In combination with the microscopic reverse reaction, which is the reductive elimination, it plays an important role in organometallic transformations and especially in catalytic coupling reactions.[108] Many examples for oxidative additions with alkyl halides or dihydrogen of rhodium(I) pincer complexes are known in literature. ${ }^{[437-439]}$ In some cases, upon addition of dihydrogen, rather the formation of a $\mathrm{H}_{2} \sigma$-complex was observed than oxidative addition. ${ }^{[40]}$

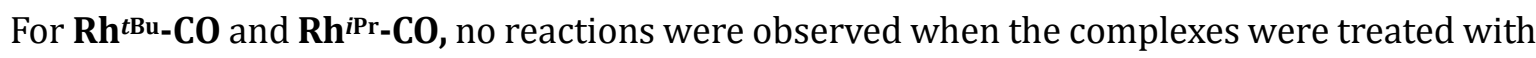
methyl iodide, phenyl iodide or dihydrogen. Even if the reaction mixtures were heated or irradiated with UV light. This observation was not surprising, since the stability of the complexes was already found before and MILSTEIN reported the reductive elimination of rhodium(III) alkyl halide species upon addition of carbon monoxide induced by the large tertbutyl groups.[439]

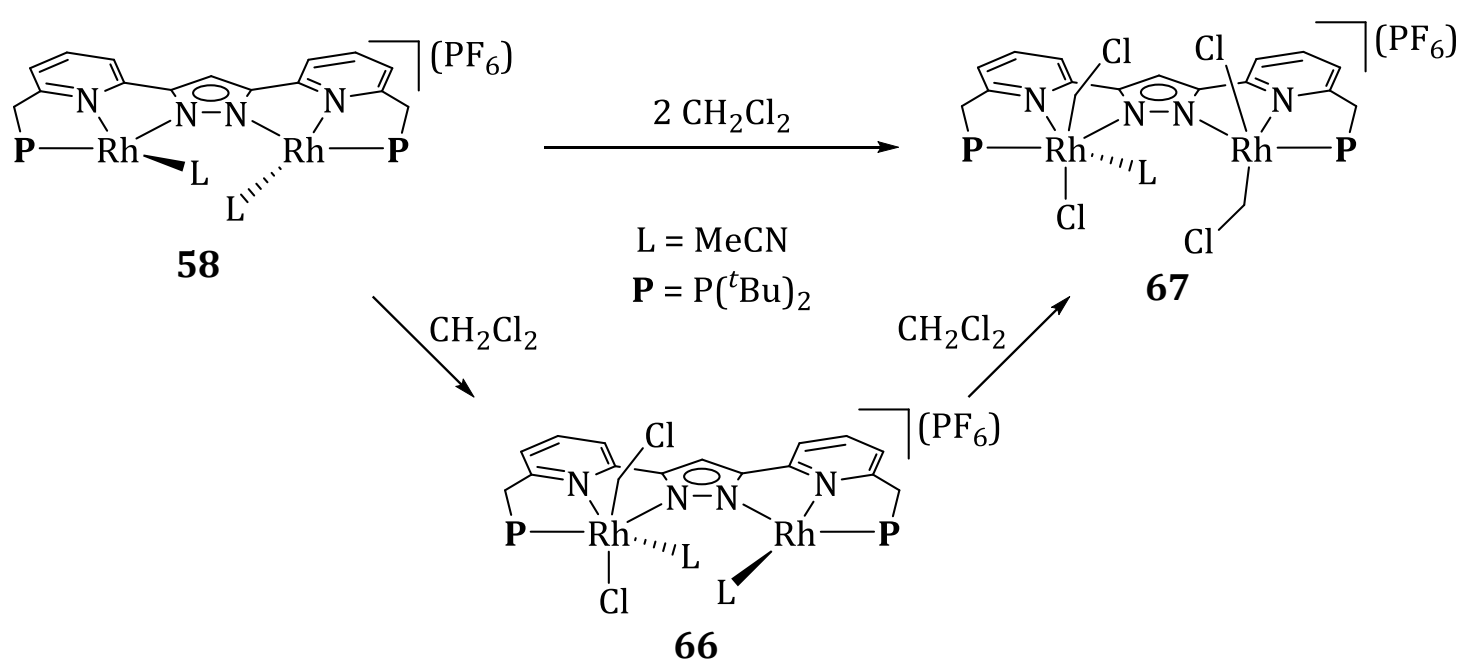

Scheme 3.29: Stepwise formation of $\left[\left(\mathrm{Rh}(\mathrm{Cl})\left(\mathrm{CH}_{2} \mathrm{Cl}\right)(\mathrm{MeCN})\right)\left(\mathrm{Rh}(\mathrm{Cl})\left(\mathrm{CH}_{2} \mathrm{Cl}\right)\right) \mathrm{L}^{t \mathrm{Bu}}\right]\left(\mathrm{PF}_{6}\right) \mathbf{6 7}$ by oxidative addition of two molecules $\mathrm{CH}_{2} \mathrm{Cl}_{2}$ to $\mathbf{R h}^{\text {tBu}}-\mathbf{M e C N}$. 
The more labile coligand $\mathrm{MeCN}$ in $\mathbf{R h}^{\mathbf{t} \mathrm{Bu}}-\mathbf{M e C N}$ was shown to enable oxidative addition reactions with methyl iodide (MeI) and dichloromethane $\left(\mathrm{CH}_{2} \mathrm{Cl}_{2}\right)$. The latter reaction was found to be slow in $\mathrm{CH}_{2} \mathrm{Cl}_{2}$ solution and proceeded within a week to completeness (Scheme 3.29). Upon standing of a $\mathrm{CH}_{2} \mathrm{Cl}_{2}$ solution of $\mathbf{R h}{ }^{t \mathrm{Bu}-} \mathbf{M e C N}$ at ambient temperature first a color change to yellow and then a precipitation of yellow needles was observed. The progress of the reaction was followed by NMR spectroscopy, which revealed a two-step process with a monooxidized intermediate $\mathbf{6 6}$. After 15 hours, resonances of a second unsymmetric species arose in a ${ }^{31} \mathrm{P}$ spectrum with one doublet having almost the same chemical shift and Rh-P coupling constant as the starting, $\mathrm{Rh}^{I} \mathrm{Rh}^{\mathrm{I}}$ complex $\mathbf{R h}^{t \mathrm{Bu}}-\mathbf{M e C N}\left(\delta=91.49 \mathrm{ppm}, J_{\mathrm{Rh}-\mathrm{P}}=178.0 \mathrm{~Hz}\right.$ ). The second doublets exhibited a significantly lower coupling constant of $J_{\mathrm{Rh}-\mathrm{P}}=128.1 \mathrm{~Hz}$ and was shifted to higher field ( $\delta=67.19 \mathrm{ppm}$ ). This is explained by the formation of a mixed valence species $\mathrm{Rh}^{\mathrm{III}} \mathrm{Rh}$, with one rhodium(I) and one rhodium(III) ion with a roughly one third smaller coupling constant as generally observed for rhodium complexes. ${ }^{[441]}$ In the further course of the reaction the doublet of the $\mathrm{Rh}^{\mathrm{I}} \mathrm{Rh}^{\mathrm{I}}$ species completely vanished while a third species appeared at $71.13 \mathrm{ppm}$ with a coupling constant of $J_{\mathrm{Rh}-\mathrm{P}}=133.0 \mathrm{~Hz}$, indicating the formation of the symmetric $\mathrm{Rh}^{\mathrm{III}} \mathrm{Rh}^{\mathrm{III}}$ complex 67 (Rh $\left.{ }^{t \mathrm{Bu}}-\mathrm{DCM}\right)$. After 5 days the reaction was finished showing only the doublet of the product in a ${ }^{31} \mathrm{P}$ NMR spectrum. The ${ }^{1} \mathrm{H}$ NMR spectrum exhibited a doublet of doublets at $4.23 \mathrm{ppm}$ for the methylene group of the $\mathrm{CH}_{2} \mathrm{Cl}$ ligand and a doublet of doublets of doublets at $3.76 \mathrm{ppm}$ for the $\mathrm{CH}_{2}$ groups of the ligand side arms.

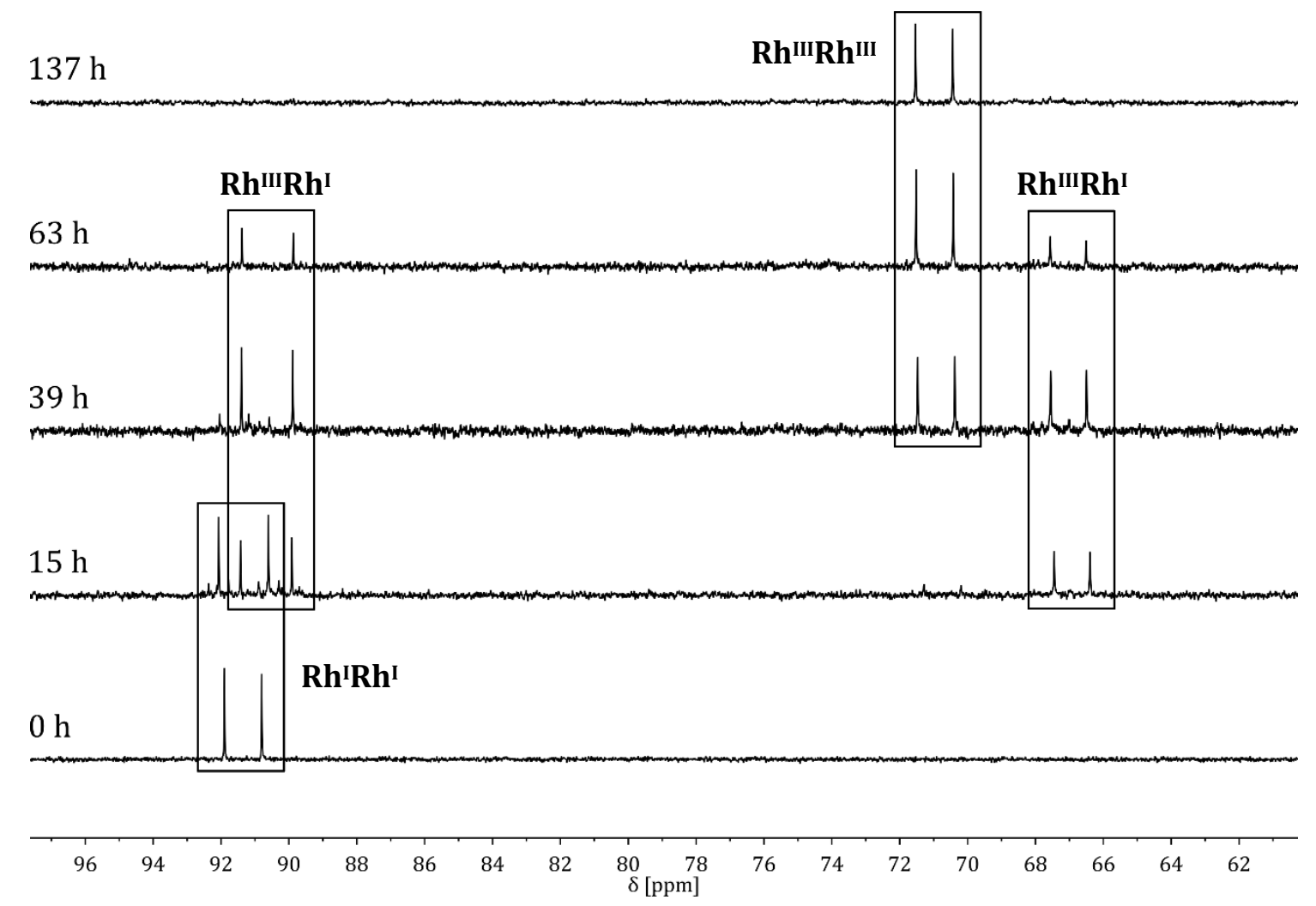

Figure 3.45: Progress of the oxidative addition of two $\mathrm{CH}_{2} \mathrm{Cl}_{2}$ to $\mathbf{R} \mathbf{h}^{t \mathrm{Bu}}-\mathbf{M e C N}$ followed by ${ }^{31} \mathrm{P}$ NMR spectroscopy showing a mono-oxidized compound as intermediate $\left(\mathrm{CD}_{2} \mathrm{Cl}_{2}, 298 \mathrm{~K}\right)$. 
$\mathbf{R h}^{\text {tBu-DCM }}$ was also characterized by mass spectrometry and X-ray diffraction analysis. An ESI+ mass spectrum of a MeCN solution of $\mathbf{R h}^{\mathrm{tBu}}-\mathbf{D C M}$ showed a dominant peak at $\mathrm{m} / \mathrm{z}=438.1$ corresponding to the doubly charged species $\left[\left(\mathrm{Rh}\left(\mathrm{CH}_{2} \mathrm{Cl}\right)\right)\left(\mathrm{Rh}(\mathrm{Cl})\left(\mathrm{CH}_{2} \mathrm{Cl}\right)\right) \mathrm{L}^{t \mathrm{Bu}}\right]^{2+}$ produced by loss of one chloride and any remaining MeCN ligands. A few crystals of $\mathbf{R} \mathbf{h}^{t \mathrm{Bu}}$-DCM were grown by layering of a yellow reaction mixture with pentane. The molecular structure showed two different rhodium centers in contrast to the solution behavior (Figure 3.46). One metal was six-coordinated having a distorted octahedral coordination geometry (Rh1), while the second metal showed a five-fold coordination in a distorted square pyramidal environment $(\mathrm{Rh} 2$, $\left.\tau_{5}=0.10\right)$.
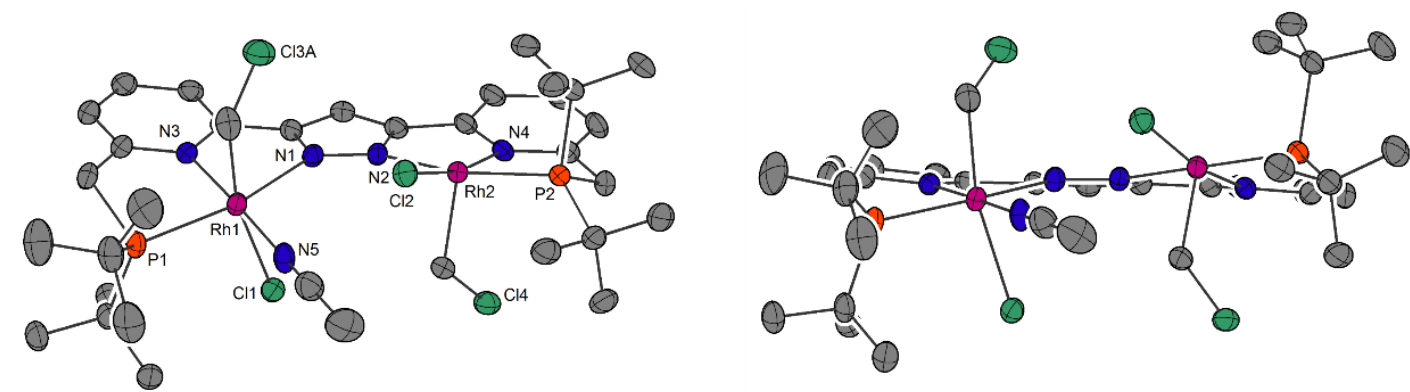

Figure 3.46: Thermal displacement ellipsoids (shown at $30 \%$ probability) of the molecular structure of $\left[\left(\mathrm{Rh}(\mathrm{Cl})\left(\mathrm{CH}_{2} \mathrm{Cl}\right)(\mathrm{MeCN})\right)\left(\mathrm{Rh}(\mathrm{Cl})\left(\mathrm{CH}_{2} \mathrm{Cl}\right)\right) \mathrm{L}^{t \mathrm{Bu}}\right]\left(\mathrm{PF}_{6}\right) 67$ in two different orientations. Counterions, hydrogen atoms and solvent molecules were omitted for clarity.

Interestingly, the chloride and chloromethylene ligands of $\mathrm{Rh} 1$ were bound in the axial positions trans to each other with a MeCN ligand in the equatorial plane completing the octahedral coordination. At the second metal site the chloride and the chloromethylene units are standing cis to each other. The metal metal distance did not change much upon oxidative addition $\left(\mathrm{d}_{\mathrm{Rh}-\mathrm{Rh}}=4.616 \AA\right)$, but the Rh-P $\left(\mathrm{d}_{\mathrm{Rh} 1-\mathrm{P}}=2.308 \AA\right.$ and $\left.\mathrm{d}_{\mathrm{Rh} 2-\mathrm{P}}=2.262 \AA\right)$ and Rh- $\mathrm{N}_{\mathrm{MeCN}}$

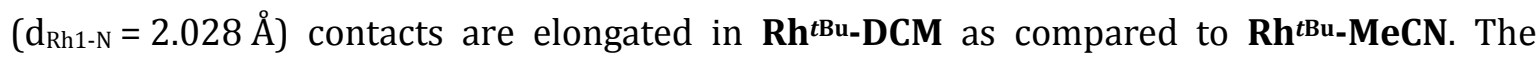
lengths of the $\mathrm{Rh}-\mathrm{Cl}$ and $\mathrm{Rh}-\mathrm{CH}_{2} \mathrm{Cl}$ bonds are similar to other rhodium(III) complexes, which

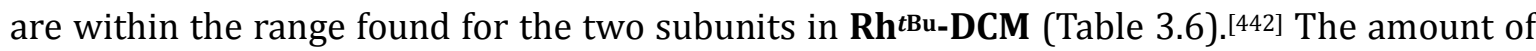
isolated crystals was too small to perform further studies on the subsequent reactivity of this complex.

The reaction with methyl iodide was fast with an instant color change from deep red to yellow occurring after the addition of MeI (Scheme 3.30). Unlike the addition of $\mathrm{CH}_{2} \mathrm{Cl}_{2}$ to $\mathbf{R h}{ }^{t \mathbf{B u}}$-MeCN no intermediary mono oxidized species could be identified by ${ }^{31} \mathrm{P}$ NMR spectroscopy, since the spectra after addition of one, two or ten equivalents of MeI showed the same three doublets (75.29 ppm, $82.86 \mathrm{ppm}$ and $85.17 \mathrm{ppm}$ ) with only minor change in the intensity ratio. All doublets had Rh-P coupling constants between $120-130 \mathrm{~Hz}$, indicating an oxidation of both rhodium(I) centers to yield assumedly 69 (Rh $\left.{ }^{\text {tBu}}-\mathbf{M e I}\right)$. This is indicative of a large experimental error for the added volume of MeI. ${ }^{1} \mathrm{H}$ NMR spectra were not as simple as expected for a mixture 102 
of potentially only two species. Numerous peaks in the region for the tert-butyl or $\mathrm{CH}_{2}$ groups were observed. Thus, resonances of the methyl groups could not be assigned. The amount of observed resonances changed upon variation of the temperature in an unexplained way. At $50{ }^{\circ} \mathrm{C}$ only the doublet at $75 \mathrm{ppm}$ remained present, but with a very low intensity. Lowering the temperature to $-50^{\circ} \mathrm{C}$ led to the appearance of new resonances.

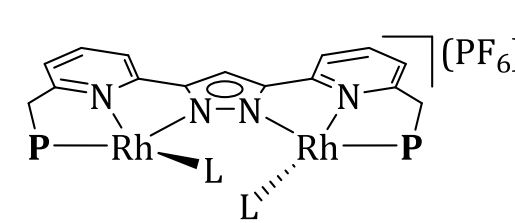

58

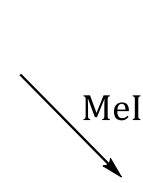

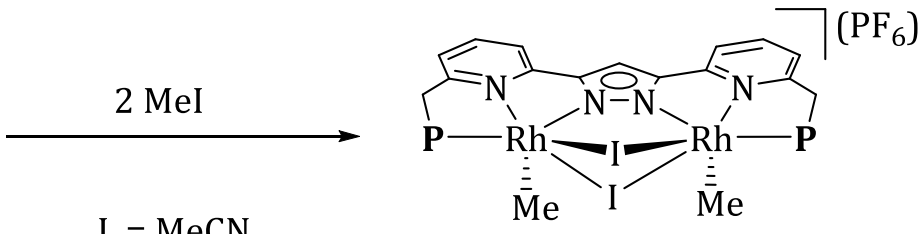

$\mathrm{L}=\mathrm{MeCN}$

$\mathbf{P}=\mathrm{P}\left({ }^{t} \mathrm{Bu}\right)_{2}$

\section{9}

MeI

$\left(\mathrm{PF}_{6}\right)$

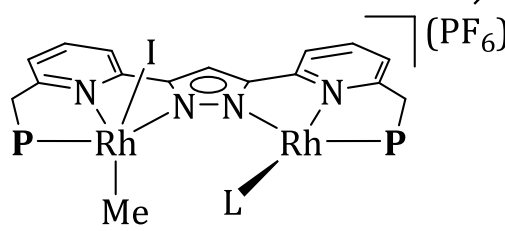

68

Scheme 3.30: Synthesis of $\left[(\mathrm{Rh}(\mathrm{Me}))_{2}(\mu-\mathrm{I})_{2} \mathrm{~L}^{t \mathrm{Bu}}\right]\left(\mathrm{PF}_{6}\right) 69$ via $\left[(\mathrm{Rh}(\mathrm{Me}) \mathrm{I})(\mathrm{Rh}(\mathrm{MeCN})) \mathrm{L}^{t \mathrm{Bu}}\right]\left(\mathrm{PF}_{6}\right) \mathbf{6 8}$ by stepwise oxidative addition of MeI to $\mathbf{R h}^{\mathrm{tBu}}-\mathbf{M e C N}$.

In ESI mass spectra a difference between the addition of one or two equivalents on MeI was observed. In both cases the dominant peak was found at $\mathrm{m} / \mathrm{z}=885.2$ corresponding to the mono oxidized compound $\left[\mathrm{Rh}_{2} \mathrm{IMeL}{ }^{t \mathrm{Bu}}\right]_{+}$(Figure 3.47). But when two equivalents were added, also a peak with an intensity of $45 \%$ at $\mathrm{m} / \mathrm{z}=1027.1$ was present, which was be assigned to the twice oxidized compound $\left[\mathrm{Rh}_{2} \mathrm{I}_{2} \mathrm{Me}_{2} \mathrm{~L}^{t \mathrm{Bu}}\right]^{+}$. Upon fragmentation in MS-MS measurements the peak of the mono oxidized compound was found again as the dominant species. However, also with a large excess of MeI, a single reaction product was not detected. These findings suggested, that the oxidative addtion of MeI is reversible under ESI conditions.
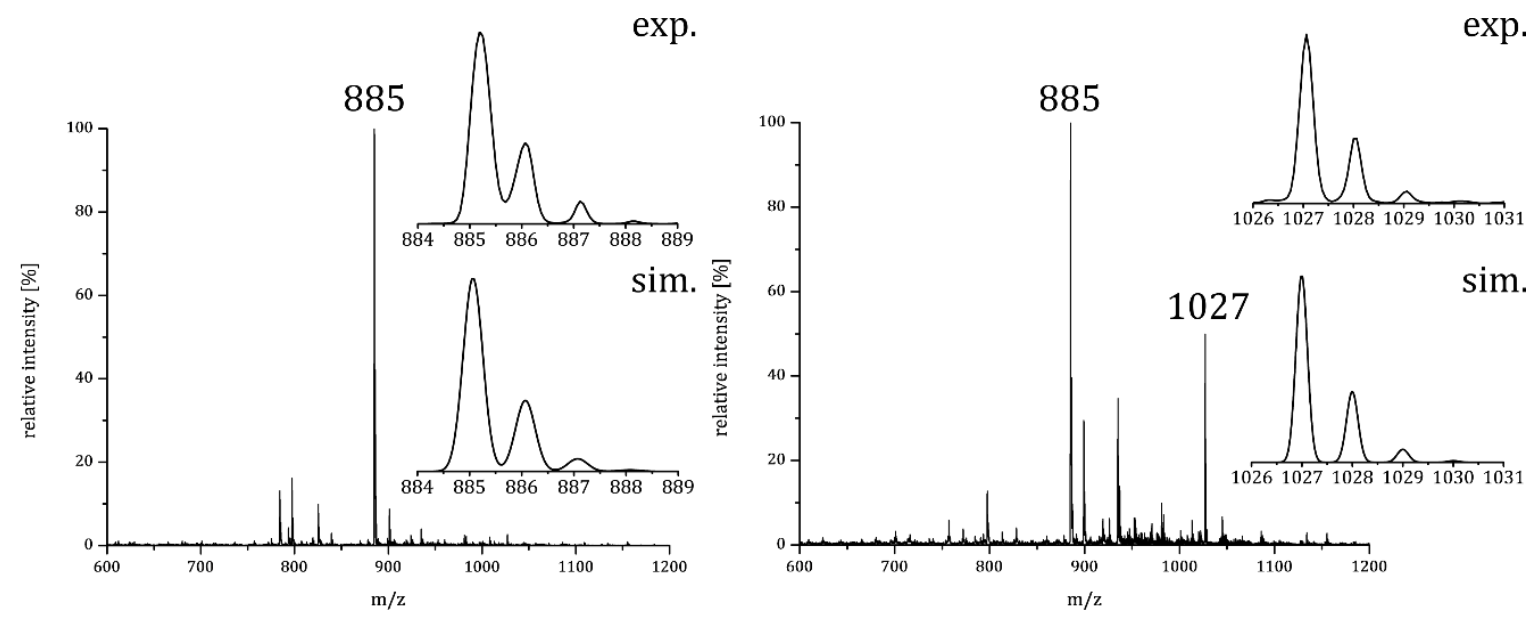

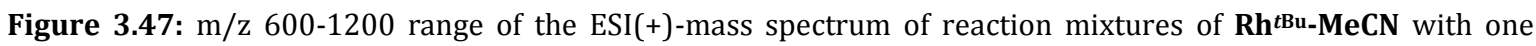
equivalent MeI (left) and two equivalents MeI (right). The insets show the experimental and simulated isotopic distribution patterns for $\left[\mathrm{Rh}_{2} \mathrm{IMeL} \mathrm{L}^{t \mathrm{Bu}}\right]^{+}$and $\left[\mathrm{Rh}_{2} \mathrm{I}_{2} \mathrm{Me}_{2} \mathrm{~L}^{t \mathrm{Bu}}\right]^{+}$. 
The solid state structure of $\mathbf{R h}{ }^{\mathrm{tBu}}$-MeI was analyzed by X-ray diffraction with single crystals obtained by standing of a MeCN solution of a reaction with a 10-fold excess of MeI. It was assumed that the cation was soluble in MeCN when hexafluorophosphate was the counterion and only precipitated as the iodide. The compound crystallized in the triclinic space group P $\overline{1}$ with four molecules in the unit cell. The molecular structure of two molecules in the unit cell are depicted in Figure 3.48. Both methyl groups are on one side of the ligand backbone plane and the iodides in bridging positions. Noticeable the two molecules (Rh ${ }^{\text {tBu-MeI A and }}$

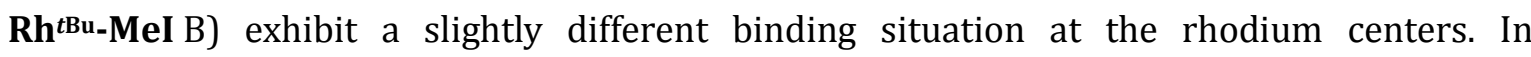
$\mathbf{R h}^{t \mathrm{Bu}-M e I} \mathrm{~A}$ both iodides bind in a bridging mode $\left(\mu^{2}-\eta^{1}-\eta^{1}\right)$ between the rhodium ions with similar distances to both metals $\left(\mathrm{d}_{\mathrm{Rh} 1-\mathrm{I} 1 \mathrm{~A}}=2.671 \AA / \mathrm{d}_{\mathrm{Rh} 2-11 \mathrm{~A}}=2.684 \AA\right.$ and $\mathrm{d}_{\mathrm{Rh} 1-\mathrm{I} 2}=3.017 \AA$ / $\mathrm{d}_{\mathrm{Rh2}-\mathrm{I} 2}=3.078 \AA$ ). In contrast to that, in the second molecule, $\mathbf{R h} \mathbf{t}^{\mathrm{tBu}}$-MeI B, one Rh-I contact is remarkably longer than the others $\left(\mathrm{d}_{\mathrm{Rh} 4-\mathrm{I} 12 \mathrm{~A}}=3.253 \AA\right.$ versus $\mathrm{d}_{\mathrm{Rh} 3-\mathrm{I} 12 \mathrm{~A}}=3.066 \AA$ ) which might be an indication for a switch in the binding modes of the iodides. This might lead to the formation of an unsymmetric species, which gives two doublets for the phosphorus atoms resulting in three resonances for the equilibrium situation (Scheme 3.31). However, to date this is an assumption. To prove the presence of an equilibrium like $\mathbf{R} \mathbf{h}^{t \mathrm{Bu}}-\mathbf{M e I} \mathrm{A} \rightleftharpoons \mathbf{R h}^{t \mathrm{Bu}}-\mathbf{M e I} \mathrm{B}$, further investigations are necessary. For example, single crystals of $\mathbf{R} \mathbf{h}^{t \mathrm{Bu}}$-MeI could be dissolved at very low temperature to follow a potential formation of an equilibrium by ${ }^{31} \mathrm{P}$ NMR spectroscopy.

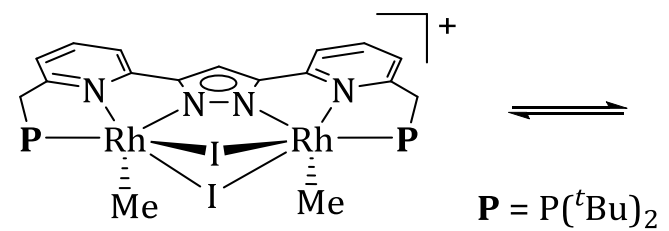

A

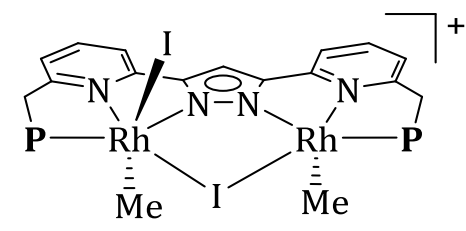

B

Scheme 3.31: Possible equilibrium in solution between $\mathbf{R h}^{t \mathrm{Bu}}-\mathbf{M e I} \mathrm{A}$ and $\mathbf{R h}^{\mathrm{tBu}}-\mathbf{M e I} \mathrm{B}$.

The other bond lengths of the two molecules are similar and summarized in Table 3.6. The rhodium-rhodium separation in $\mathbf{R h}^{t \text { Bu}}$-MeI B is little larger than in $\mathbf{R h}^{t \mathbf{B u}}$-MeI A (3.835 $\AA$ vs. $3.761 \AA$ ) and remarkably shorter as for the non-bridged $\mathbf{R h}^{\text {tBu-}}$-DCM. Another effect of the two (with the pyrazole: three) bridging units between the two rhodium centers is a more distorted octahedral coordination geometry. For Rh ${ }^{t \text { Bu}}$-MeI a stacking of the ligand backbones was observed as it was also found for $\left[(\mathrm{Zn}(\mathrm{OTf}))_{2}(\mu\right.$-OTf $\left.) \mathrm{L}^{\mathrm{tBu}}\right] \mathbf{4 9}$ (Figure 3.48, bottom right), which might explain the low solubility (only in hot DMSO) of the compound. 


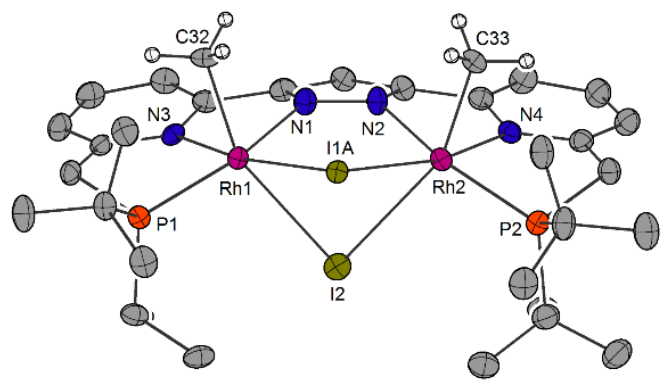

A

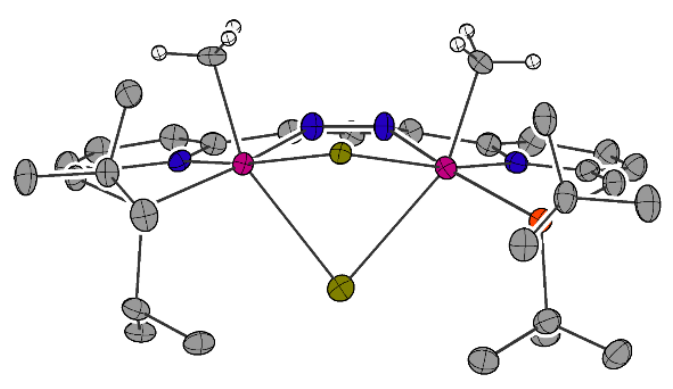

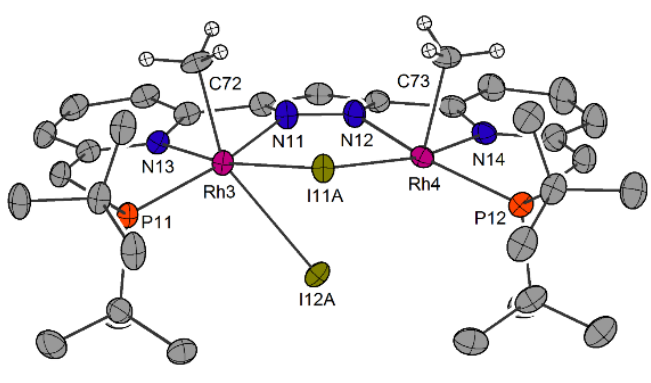

B

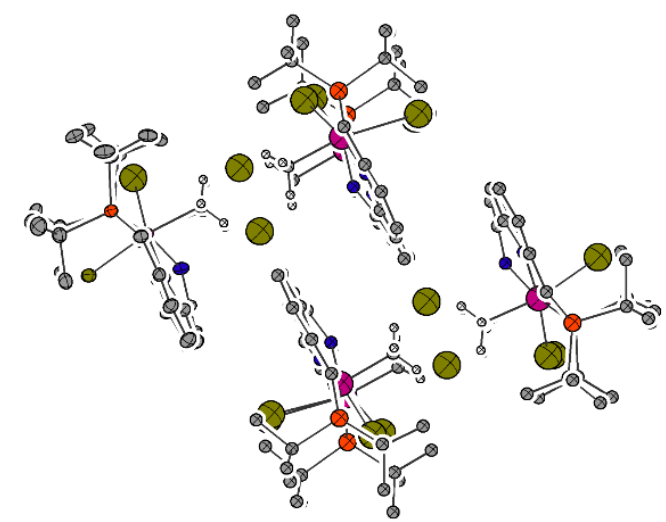

Figure 3.48: Thermal displacement ellipsoids (shown at $30 \%$ probability) of the molecular structure of $\left[\left(\mathrm{Rh}(\mathrm{Me})_{2}(\mu-\mathrm{I})_{2} \mathrm{~L}^{t \mathrm{Bu}}\right](\mathrm{I})\right.$ 69. Top: Two molecules of the unit cell with different binding situations of the iodide ligands, $\mathbf{R h}^{t \mathrm{Bu}}$-MeI A and $\mathbf{R h}^{t \mathrm{Bu}}$-MeI B. Bottom: Side view of the complex A displaying the distorted octahedral coordination geometry (left) and stacking diagram of the molecules of the unit cell (right). Counterions, hydrogen atoms except for the $\mathrm{CH}_{3}$ groups and solvent molecules were omitted for clarity.

To increase the solubility, it was tried to convert the iodide salt into the $\mathrm{PF}_{6}{ }^{-}$or $\mathrm{BPh}_{4}{ }^{-}$analogues, but crystallization of the compounds failed, so that clean material of $\mathbf{R h}^{\mathrm{t} B u}$-MeI with these anions was not be obtained.

Table 3.6: Selected bond lengths in $[\AA]$ of complexes $\left[\left(\mathrm{Rh}(\mathrm{Cl})\left(\mathrm{CH}_{2} \mathrm{Cl}\right)(\mathrm{MeCN})\right)\left(\mathrm{Rh}(\mathrm{Cl})\left(\mathrm{CH}_{2} \mathrm{Cl}\right)\right) \mathrm{L}^{t \mathrm{Bu}}\right]\left(\mathrm{PF}_{6}\right) 67$ and two molecules of the unit cell of $\left[(\mathrm{Rh}(\mathrm{Me}))_{2}(\mu-\mathrm{I})_{2} \mathrm{~L}^{t \mathrm{Bu}}\right](\mathrm{I}) 69$.

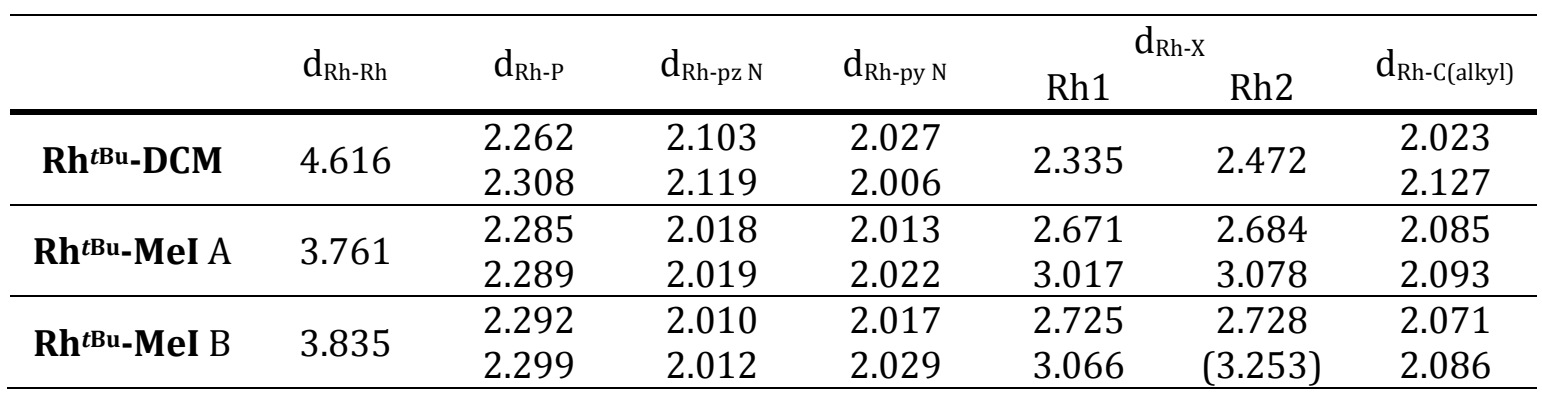

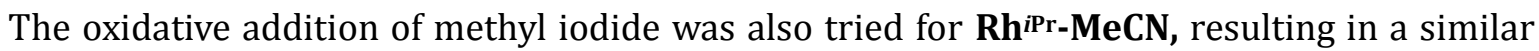
mixture of products. A series of 31P NMR spectra recorded at different temperatures showed the reversible temperature dependence of the product formation/ratio (Figure 3.49). At ambient temperature one symmetric and one asymmetric species was found with doublets at $68.28 \mathrm{ppm}\left(U_{\mathrm{Rh}-\mathrm{P}}=123.4 \mathrm{~Hz}\right)$ and $68.18 \mathrm{ppm}\left(U_{\mathrm{Rh}-\mathrm{P}}=122.7 \mathrm{~Hz}\right) / 72.29 \mathrm{ppm}\left(U_{\mathrm{Rh}-\mathrm{P}}=120.8 \mathrm{~Hz}\right)$, respectively. For all signals small coupling constants were found, which indicated the presence 
of only rhodium(III) species. The symmetric species persisted at higher temperatures while the asymmetric species was reversibly transformed into another symmetric species $\left(\delta=59.99 \mathrm{ppm}, J_{\mathrm{Rh}-\mathrm{P}}=119.7 \mathrm{~Hz}\right)$. The exact structure of these three species could not be examined, because the corresponding ${ }^{1} \mathrm{H}$ NMR spectra were characterized by the presence of many overlaying resonances and ESI mass measurements showed no dominant peak, which could be clearly assigned. Unfortunately, crystals of any of the reaction products were not obtained, so that the results of this reaction remained uncertain.

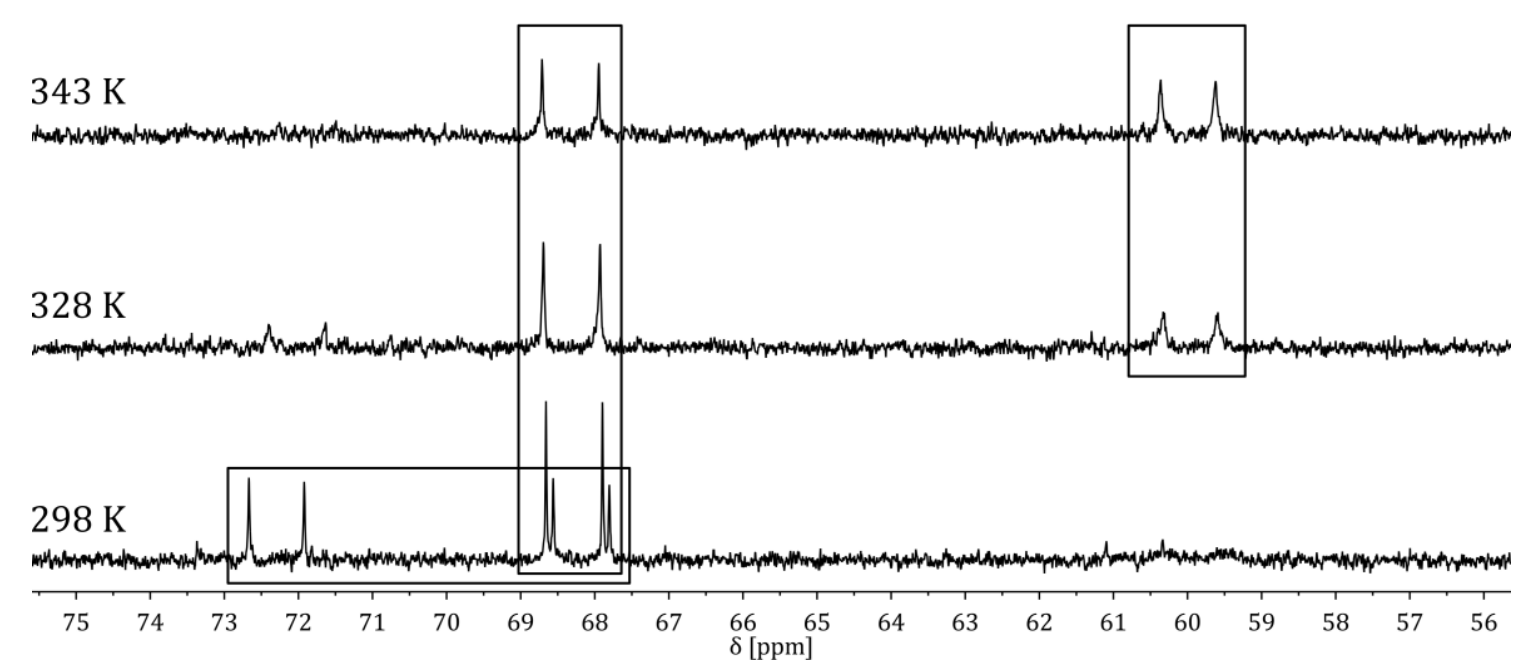

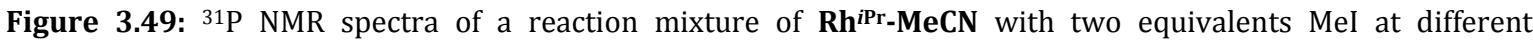
temperatures $\left(\mathrm{MeCN}-\mathrm{d}_{3}\right)$.

Most interestingly for catalytic applications would be an oxidative addition of dihydrogen. Although all synthesized rhodium(I) complexes were tested in various solvents under different dihydrogen pressures, no reaction could be observed by NMR spectroscopy even at high pressures of up to 10 bar or at low temperatures. Only in one exception two resonances low in intensity in the hydride region of a ${ }^{1} \mathrm{H}$ NMR spectrum were observed for the reaction of $\mathbf{R h}^{\text {tBu-SMe }}{ }_{2}$ in THF- $\mathrm{d}_{8}$ (Scheme 3.32, Figure 3.50). The integral of the two doublets of doublets at $-18.90 \mathrm{ppm}(J=22.0,21.0 \mathrm{~Hz})$ and $-19.02 \mathrm{ppm}(J=27.3,21.7 \mathrm{~Hz})$ corresponded to about $10 \%$ for a possible dihydride complex in relation to the $\mathrm{CH}_{2}$ group of the unreacted educt. However, in the corresponding ${ }^{31} \mathrm{P}$ NMR spectrum no new resonances were observed probably due to the low conversion rate so that the symmetry as well as the exact binding situation (cis or trans) of the newly formed dihydride species could not be determined.

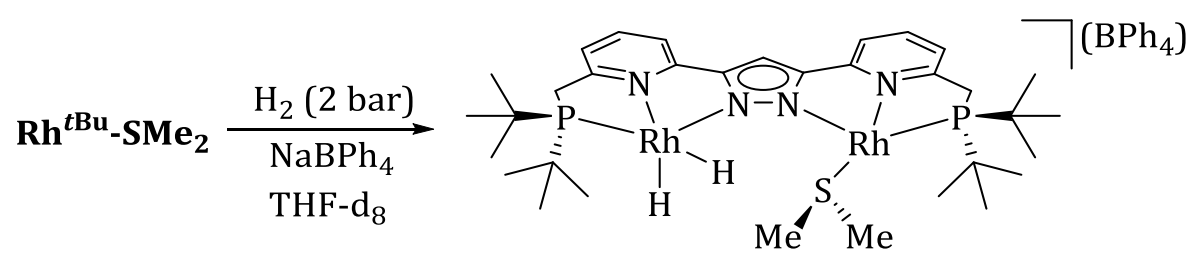

Scheme 3.32: Formation of a potential dihydride complex from $\mathbf{R h}^{t \mathrm{Bu}}-\mathbf{S M e}_{2}$ and dihydrogen. 


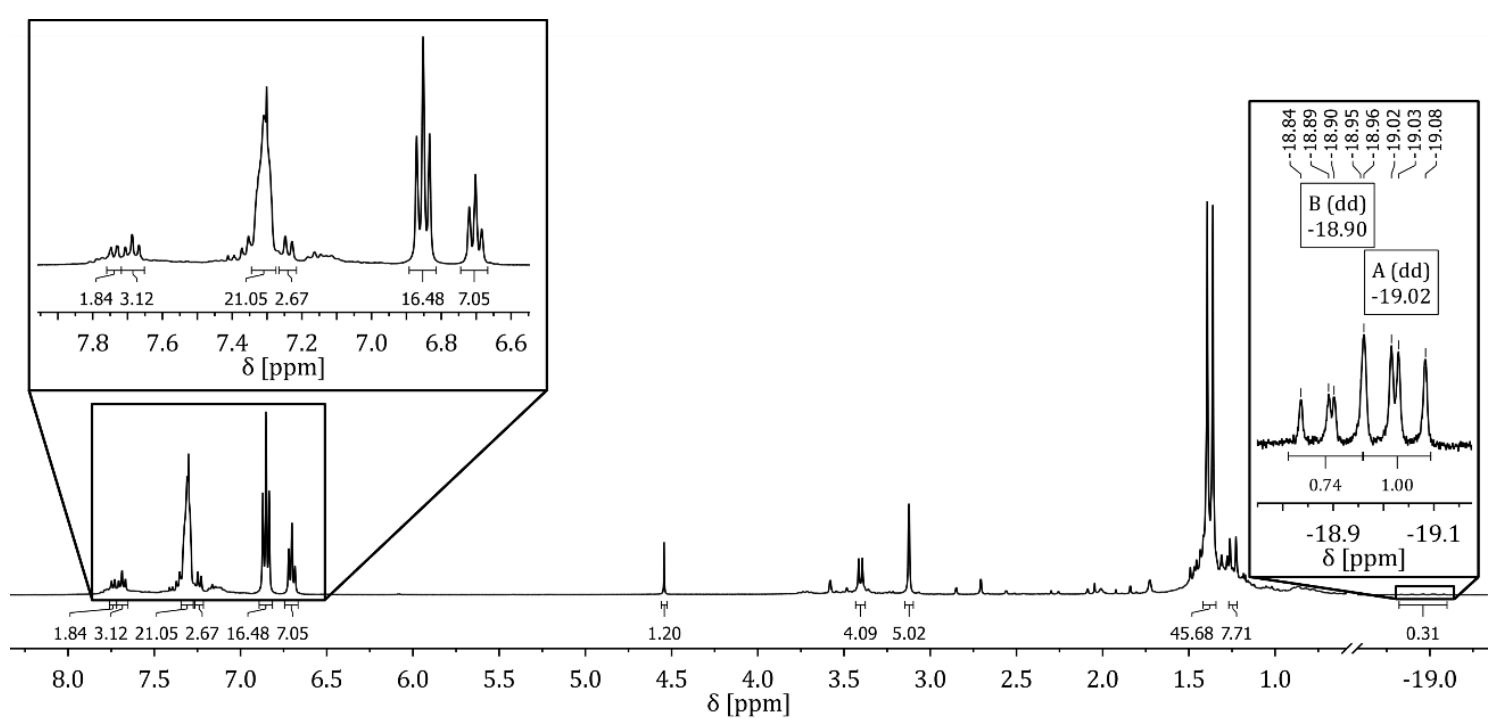

Figure 3.50: ${ }^{1} \mathrm{H}$ NMR spectrum of $\mathbf{R h}^{\mathrm{tBu}}-\mathrm{SMe}_{2}$ with $\mathrm{NaBPh}_{4}$ under an atmosphere of 2 bar of dihydrogen (THF-d8, $298 \mathrm{~K})$.

The bimetallic reactivity of related pyrazolate bridged diphosphine ligands was also discussed by the groups of BOSNICH and AKITA. ${ }^{[70,441,443]}$ BOSNICH reported the synthesis of a chlorobridged dinuclear rhodium(I) complex 70 (Figure 3.51), which was shown to undergo oxidative addition with acetyl chloride and methyl iodide.[441] However, with chloride as bridging unit only a single oxidative addition on one rhodium center was observed. When the electron density of the metal centers was increase by using a phosphide bridging unit $\left(\mathrm{PPh}_{2}^{-}\right.$, complex 71), a reversible second oxidative addition was observed. An analoguous iridium(I) complex showed a higher tendency to undergo oxidative additions (irreversible and fast reactions). It was showen, that the oxidative addition behavior could be correlated with the IR bands of the carbonyl ligands. Higher the carbonyl bands led to less facile and less complete oxidative additions to this dinuclear complexes. The IR bands of $\mathbf{R h}^{\mathbf{t B u}-\mathbf{C O}}$ were slightly lower than for 70, but higher than these of the phosphide bridged complex 71.[411] This may explain in parts, why no oxidative addition reaction was found for $\mathbf{R h}^{t \mathrm{Bu}}$-CO, although the electronic structures of the complexes are different. For $\mathbf{R h}^{\mathrm{tBu}}$-MeCN less $\pi$-backbonding of the MeCN coligands is expected, resulting in a higher electron density at the rhodium centers. This is reflected by the facile oxidative addition of both rhodium(I) ions in $\mathbf{R h} \mathbf{h}^{\mathbf{B} u-M e C N}$ with methyl iodide and methylene chloride. 


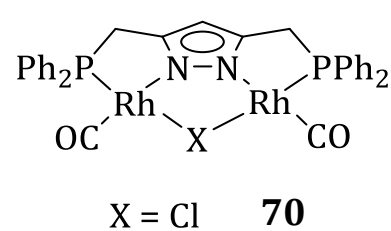

$\mathrm{PPh}_{2} \mathbf{7 1}$

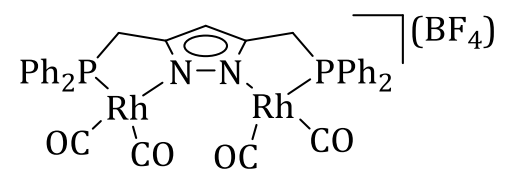

72

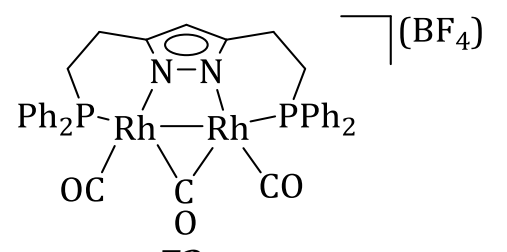

73

Figure 3.51: Related pyrazolate bridged dinuclear rhodium(I) carbonyl complexes.[70,441,443]

The group of AKITA tuned the metal-metal separation by introducing a longer side arm (complex 73; Figure 3.51). For 73 a metal separation of $d_{\mathrm{Rh}-\mathrm{Rh}}=2.71 \AA$ was observed, whereas in the monooxidized analogue of $\mathbf{7 1}$ a distance of $\mathrm{d}_{\mathrm{Rh}-\mathrm{Rh}}=3.77 \AA$ 年 was found (crystallographic data for $\mathbf{7 2}$ was not available, but the distance should be similar or longer).[441,443] This shorter

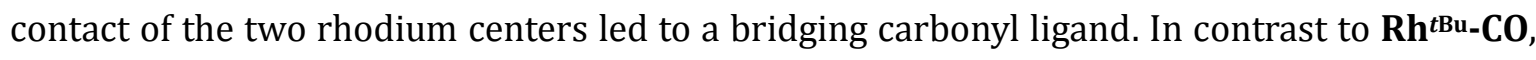
the tri- and tetracarbonyl complexes $\mathbf{7 2}$ and $\mathbf{7 3}$ showed a variable ligand substitution reactivity. Ligand dissociation of one or two carbonyl complexes formed open sites, which could react with four electron donor molecules such as $\mathrm{PPh}_{2}$. Also the reactions with acetylenes, acetylides and silanes were investigated and displayed for example an interesting coordination chemistry of $\mu_{4}$ dicarbide and $\mu_{4}$ hydride complexes.[443-445] Under similar conditions no reactions were observed for $\mathbf{R h}^{\mathrm{t} \mathbf{B u}-\mathbf{C O}}$ with silanes or lithium carbides. 


\subsection{Dearomatization/Aromatization in a Rhodium Carbonyl Complex}

A key feature of pyridine based pincer complexes is the possible deprotonation of one or both side arm $\mathrm{CH}_{2}$ or $\mathrm{NH}$ linkers, which leads to a dearomatization of the pyridine ring(s). For the abstraction of a proton, strong bases like $n$-butyl lithium ( $n \mathrm{BuLi}$ ), methyl lithium (MeLi), potassium tert-butoxide $\left(\mathrm{KO}^{\mathrm{t}} \mathrm{Bu}\right)$ or benzyl potassium $(\mathrm{BnK})$ have been used. ${ }^{2275,295,446]}$ In mononuclear PNP pincer complexes the deprotonation of the second side arm is challenging due to a high charge resulting on the ligand. This should be not so crucial in the case of the Two-in-one pincer system, since only one methylene side arm is attached per pyridine unit.

To study the deprotonation accompanied by a dearomatization of a pyridine ring in Two-in-one pincer complexes in detail, $\mathbf{R h}^{t \mathbf{B u}-\mathbf{C O}}$ was found to be a suitable compound due the high stability of the Rh-CO bonds. Attempts with $\mathbf{R} \mathbf{h}^{t \mathrm{Bu}}-\mathbf{M e C N}$ and with $\mathbf{R h}^{t \mathrm{Bu}}-\mathbf{S M} \mathbf{e}_{2}$ resulted in partial deprotonation and in the formation of a complex mixture of products. This could be due to a competing reaction of the coligands with base, e.g., the deprotonation of $\mathrm{MeCN}$, or by ligand exchange. Upon addition of one equivalent of a strong base (either $\mathrm{KO}^{t} \mathrm{Bu}$ or $\mathrm{KH}$ ) to a yellow THF suspension of $\mathbf{R h}{ }^{t \mathrm{Bu}-\mathbf{C O}}$, a color change to green was observed and a clear solution was formed (Scheme 3.33).

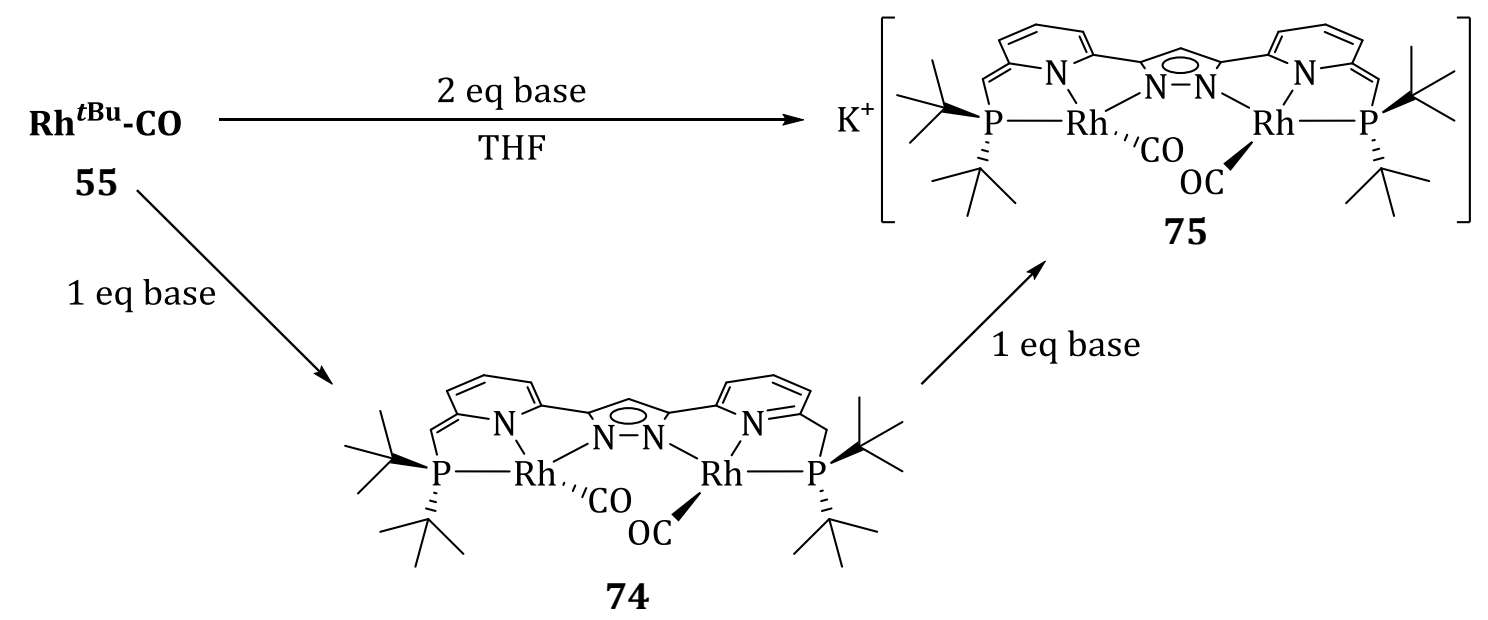

Scheme 3.33: Stepwise dearomatization of $\mathbf{R h}^{t \mathrm{Bu}}-\mathbf{C O}$ via $\left[(\mathrm{Rh}(\mathrm{CO}))_{2} \mathrm{~L}^{t \mathrm{Bu} *}\right] \mathbf{7 4}$ to yield $\left[(\mathrm{Rh}(\mathrm{CO}))_{2} \mathrm{~L}^{t \mathrm{Bu} * *}\right] \mathbf{7 5}$.

The formation of $\mathbf{R h}^{t \mathrm{Bu}}{ }^{*}$-CO (74) was established by NMR spectroscopy showing a remarkable shift of the dearomatized pyridine proton resonances of approx. $2 \mathrm{ppm}$ to higher field, whereas the aromatic pyridine signals are only slightly shifted to higher field (Figure 3.52). The resonance of the $\mathrm{CH}$ spacer is found at $3.11 \mathrm{ppm}, 0.65 \mathrm{ppm}$ highfield compared to the $\mathrm{CH}_{2}$ unit

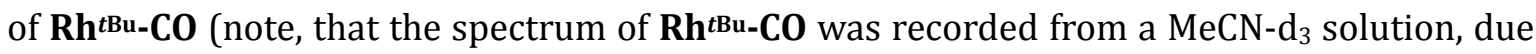
to complete insolubility of $\mathbf{R h}^{\text {tBu-CO }}$ in THF). After the second deprotonation step again a symmetric species was found with all three pyridine resonances between $5.35 \mathrm{ppm}$ and $6.35 \mathrm{ppm}$. The pyrazole 4-H signal also shifted to more high field upon dearomatization, reflecting the higher electron density on the ligand. The values for the NMR shifts of the three 
compounds are summarized in Table 3.7. When the ${ }^{1} \mathrm{H}$ NMR spectrum of $\mathbf{R h}^{\mathrm{tBu}}{ }^{* *}$-CO was recorded in $\mathrm{MeCN}-\mathrm{d}_{3}$, the $\mathrm{CH}$ group could not be observed due to a fast exchange with the deuterated solvent.

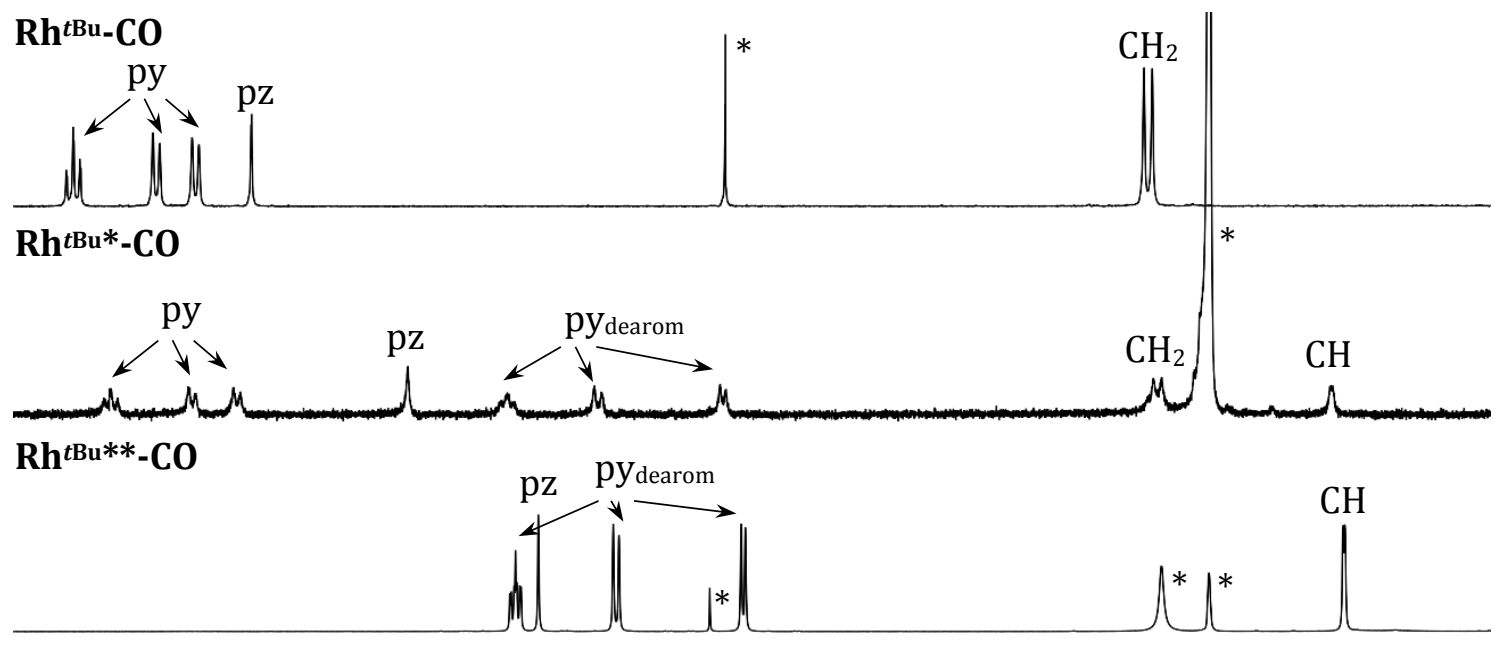

$\begin{array}{llllllllllllllllllllllllllll}8.0 & 7.8 & 7.6 & 7.4 & 7.2 & 7.0 & 6.8 & 6.6 & 6.4 & 6.2 & 6.0 & 5.8 & 5.6 & 5.4 & 5.2 & 5.0 & 4.8 & 4.6 & 4.4 & 4.2 & 4.0 & 3.8 & 3.6 & 3.4 & 3.2 & 3.0 & 2.8 & 2.6\end{array}$

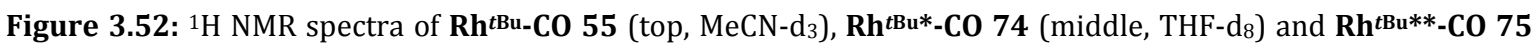
(bottom, THF- $\mathrm{d}_{8}$ ) at ambient temperatures. Residual solvent resonances are marked with an asterisk.

The dearomatization was also observed in ${ }^{31} \mathrm{P}$ NMR spectra (Figure 3.53). The resonance of the phosphine side arm shifted ca. 10 ppm to high field upon deprotonation, while the coupling constants remained essentially the same and in addition did not show a trend. The second deprotonation shifted the doublet to $87.83 \mathrm{ppm}\left(\mathrm{V}_{\mathrm{Rh}-\mathrm{P}}=152.7 \mathrm{~Hz}\right)$.

arom.

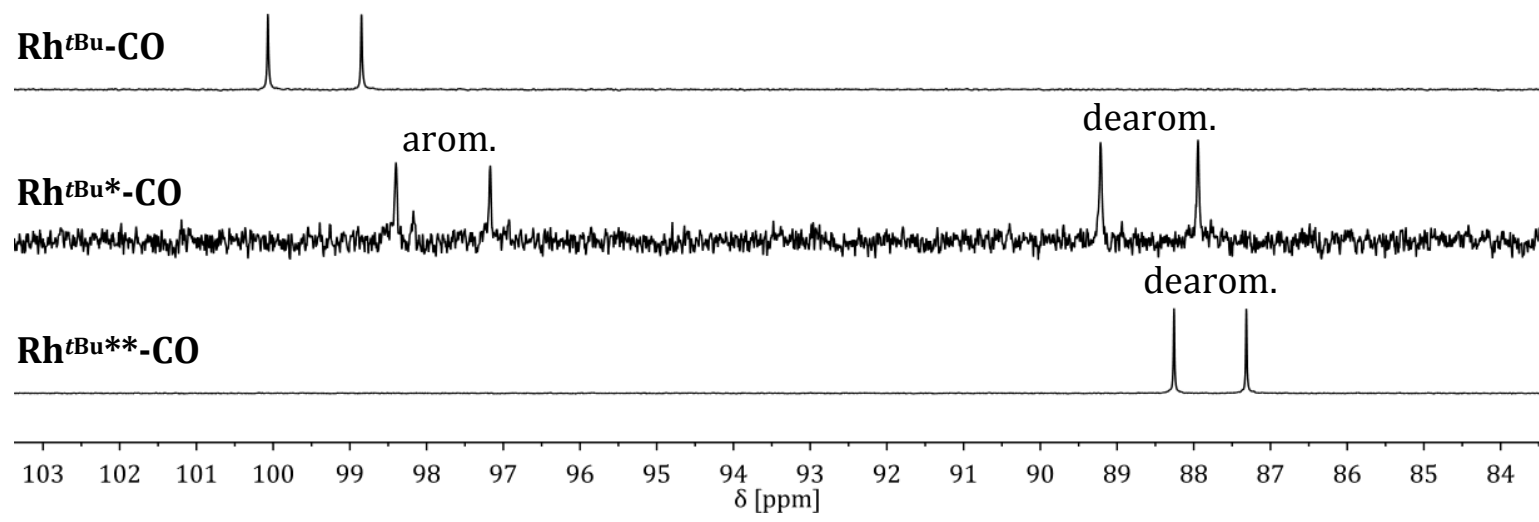

Figure 3.53: ${ }^{31} \mathrm{P}$ NMR spectra of $\mathbf{R h}^{t \mathrm{Bu}}-\mathrm{CO} 55$ (top, MeCN-d 3 ), Rh ${ }^{t \mathrm{Bu}}{ }_{-}-\mathrm{CO} \mathbf{7 4}$ (middle, THF-d 8 ) and $\mathbf{R h}^{\mathrm{tBu} * *}$-CO 75 (bottom, THF-d8) at ambient temperatures.

Unfortunately, $\mathbf{R h}^{t \mathrm{Bu}}{ }_{-}-\mathbf{C O}$ was found to be unstable in solution resulting in a grey precipitate after a short time. Therefore, it was not possible to record a ${ }^{13} \mathrm{C}$ NMR spectrum and to conduct further analytic measurements. In contrast, the twice dearomatized species $\mathbf{R h}^{t \mathbf{B u}}{ }^{* *}$-CO proved to be stable for weeks in solution under inert conditions. However, highly desired crystals of this compound were not obtained, to finally complete the characterization of the 


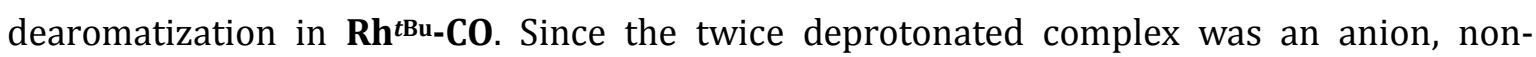
coordinating cations were added during crystallization attempts in order to increase the crystallization properties. But neither using crown ethers to coordinate the potassium cation nor the use of ylide $\mathrm{Ph}_{3} \mathrm{P}=\mathrm{CH}_{2}$ as base, which formed $\mathrm{Ph}_{3} \mathrm{PMe}^{+}$as large cation, led to better results. Obtained crystalline material was identified as starting material.

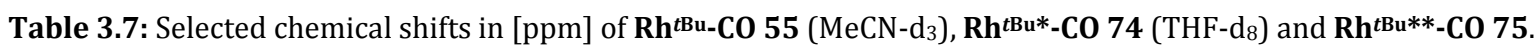

\begin{tabular}{|c|c|c|c|c|c|c|c|}
\hline & ${ }^{1} \mathrm{H} \mathrm{pz}$ & ${ }^{1} \mathrm{H}$ pyarom & ${ }^{1} \mathrm{H}$ pydearom & ${ }^{1} \mathrm{H} \mathrm{CH}_{2}$ & ${ }^{1} \mathrm{H} \mathrm{CH}$ & ${ }^{31} \mathrm{P}$ & ${ }^{13} \mathrm{C} \mathrm{CH}_{2 / 1}$ \\
\hline $\mathbf{R h}^{t B u}-\mathbf{C O}$ & 7.28 & $\begin{array}{l}7.96 \\
7.64 \\
7.49 \\
\end{array}$ & - & 3.81 & - & 99.50 & 36.7 \\
\hline $\mathbf{R h}^{t \mathrm{Bu} *-\mathrm{CO}}$ & 6.67 & $\begin{array}{l}7.82 \\
7.50 \\
7.33 \\
\end{array}$ & $\begin{array}{l}6.29 \\
5.94 \\
5.45\end{array}$ & 3.78 & 3.11 & $\begin{array}{l}97.82 \\
88.62\end{array}$ & $\begin{array}{c}\text { not } \\
\text { available }\end{array}$ \\
\hline $\mathbf{R h}^{t \mathrm{Bu} * *}-\mathrm{CO}$ & 6.17 & - & $\begin{array}{l}6.26 \\
5.87 \\
5.38 \\
\end{array}$ & - & 3.06 & 87.83 & 62.0 \\
\hline
\end{tabular}

A second analytic method for the characterization of $\mathbf{R h}^{t \mathrm{Bu}}{ }^{*}$-CO and $\mathbf{R} \mathbf{h}^{\mathrm{tBu}}{ }^{* *}$-CO was infrared spectroscopy and more precisely the vibration frequency of the carbonyl ligand. The frequency is dependent on the strength of the $\mathrm{C}$-O bond, which is correlated to the electron density at the rhodium center. The data was collected with an ATR-IR spectrometer (attenuated total reflection IR spectroscopy) in the solid state (Figure 3.54).
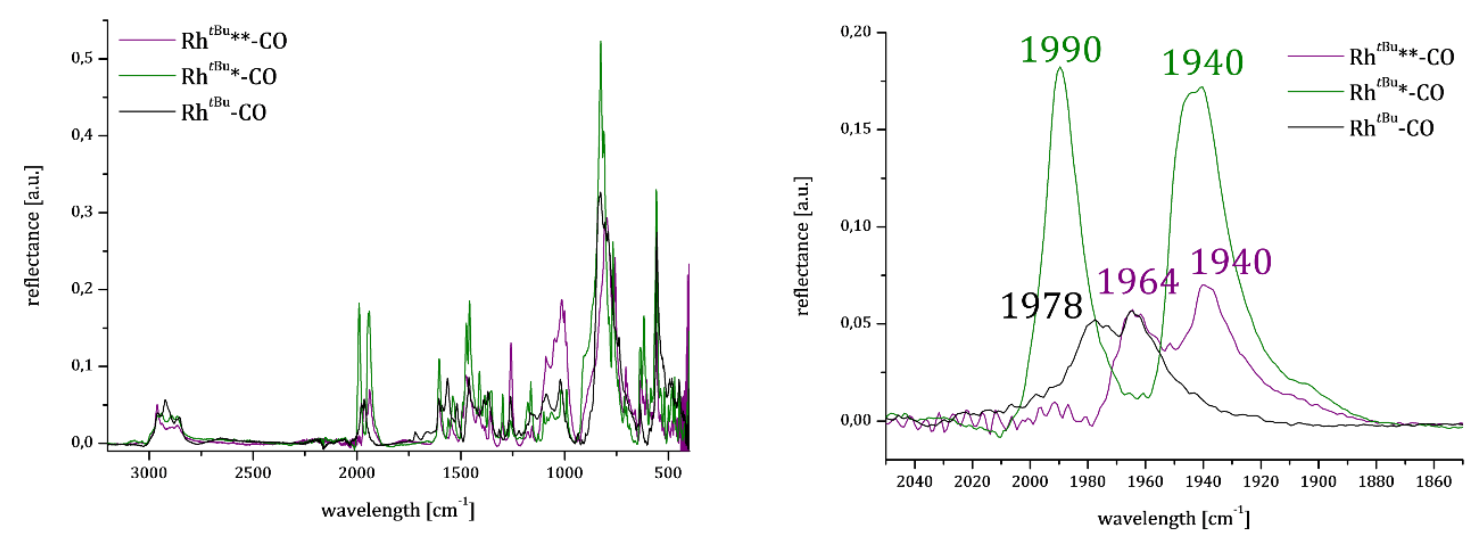

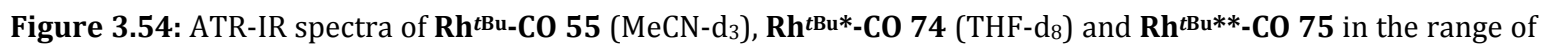
$3200-400 \mathrm{~cm}^{-1}$ (left) and in detail the region of the carbonyl vibrations (right).

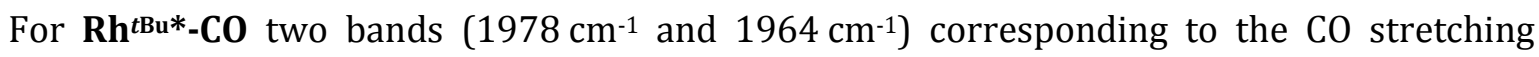
vibration were observed, due to slow interconversion of the two conformers on the IR timescale in contrast to the NMR experiments at ambient temperatures (Chapter 3.4.4.2). With addition of one equivalent of base one of the CO stretching vibrations is shifted to higher wavenumbers $\left(1990 \mathrm{~cm}^{-1}\right)$ and the other one to lower wavenumbers $\left(1940 \mathrm{~cm}^{-1}\right)$. For the twice deprotonated species, bands at $1964 \mathrm{~cm}^{-1}$ and at $1940 \mathrm{~cm}^{-1}$ were observed. The IR 
measurements reflected as well as the NMR spectra an increased $\pi$-backbonding by shifting the $\mathrm{CO}$ vibration to smaller wavenumbers.

Table 3.8: IR bands of the CO stretching vibrations in $\mathbf{R h}^{t \mathrm{Bu}}$-CO 55, $\mathbf{R h}^{t \mathrm{Bu}} *_{-}$-CO $\mathbf{7 4}$ and $\mathbf{R h}^{t \mathrm{Bu}}{ }^{* *}$-CO $\mathbf{7 5}$. The wavenumbers are given in $\left[\mathrm{cm}^{-1}\right]$.

\begin{tabular}{ll}
\hline & $v_{\mathrm{CO}}$ \\
\hline $\mathbf{R h}^{\text {tBu}}-\mathbf{C O}$ & 1978,1964 \\
\hline $\mathbf{R h}^{t \mathrm{Bu} *}$-CO & 1990,1940 \\
\hline $\mathbf{R h}^{\text {tBu**-CO }}$ & 1964,1940 \\
\hline
\end{tabular}

The dearomatized complex was used to study further reactivity. Thus protic substrates (water, methanol, para-fluoroaniline and phenylacetylene) were added but simply the reprotonation without substrate coordination to the rhodium ions was observed. The reason for this is the strength of the Rh-CO bond which inhibited a ligand exchange at the position of the coligand. Also the activation of dihydrogen under various reaction conditions failed. Thus a heterolytic splitting of $\mathrm{H}_{2}$ and with that a metal ligand cooperativity was not observed. The reaction of the

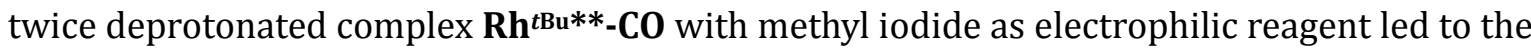
formation of the presumably tree new species as observed by ${ }^{31} \mathrm{P}$ NMR spectroscopy (Figure 3.55). The separation or assignment of the formed species was unsuccesfull to date. Thus, a certain product of this reaction could not be determined. Possibly, the methylene groups are methylated similar to reported reactions of the related mononuclear pincer complexes.[447]

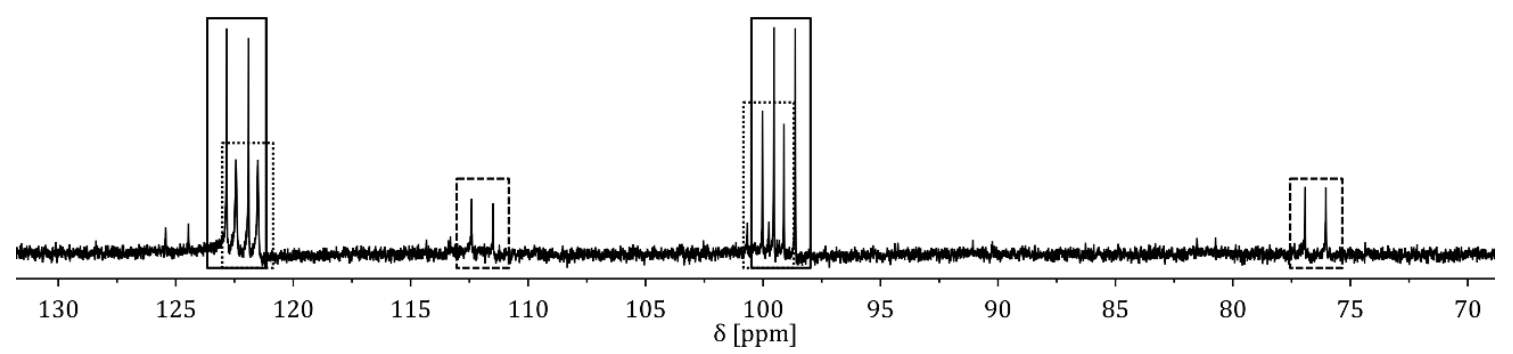

Figure 3.55: ${ }^{31} \mathrm{P}$ NMR spectrum after the reaction of $\mathbf{R h}^{t \mathbf{B u}} * *$-CO with methyl iodide (MeCN-d $\left.3,298 \mathrm{~K}\right)$. The presumably three formed species are marked with boxes (bold, dashed and dotted). 


\subsection{Catalysis}

Pincer complexes are widely used in catalysis, since they are robust, thermally stable and a variation of their electronic and steric properties is facile (see Chapter 3.1.3). With different metal complexes in hand, the catalytic activity of this ligand system was investigated in order to find interesting and maybe unusual reactivity. Inexpensive, abundant and environmentally more acceptable non-noble metals like iron, cobalt or even zinc represent attractive alternatives to common, expensive and often toxic noble metals for catalysis. ${ }^{[448-450]}$ But the development of suitable catalytic systems with these metals is still at the beginning and they often lack of activity or selectivity.[336]

\subsubsection{Zinc Catalysis}

Especially zinc catalysis is very rarely found in literature and mainly applied in polymerization reactions for example of $r a c$-lactide.[413,417,418,451,452] In recent years also other transformations were found to be mediated by zinc ions. ${ }^{[453-457]}$

In a first attempt the ring opening polymerization of rac-lactide was attempted with the used

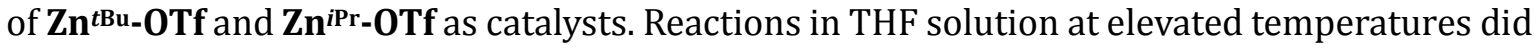
not lead to polymeric products, not even when $\mathbf{Z n}^{\text {tBu-OTf }}$ was activated with 2 equivalents of $\mathrm{MeMgBr}$. Hence, the reaction was carried out in molten monomer at $140{ }^{\circ} \mathrm{C}$ and under these conditions polylactide was produced in $52 \%$ yield after $90 \mathrm{~h}$. This is a really low activity compared to known zinc alkyl or alkoxide complexes ${ }^{[417]}$ and indeed a blind reaction using zinc triflate revealed almost the same yield and same properties of the formed polymer (PDI $=1.31$ and $\mathrm{M}_{\mathrm{w}}=9.90 \cdot 10^{3}$ ). So, zinc complexes $\mathbf{Z n}{ }^{\text {tBu}}$-OTf and $\mathbf{Z n}^{\text {iPr-OTf }}$ were not able to polymeriz raclactide in a reasonable more active way than zinc triflate.

MARDER has shown that zinc complexes are capable of mediating borylation reactions of $\mathrm{C}-\mathrm{H}$ and C-I bonds.[453] Therefore $\mathbf{Z n}^{\text {tBu-OTf }}$ was tested for this catalytic transformation in order to see, whether the two zinc ions in close proximity might increase the amount of diborylation product (Scheme 3.34).

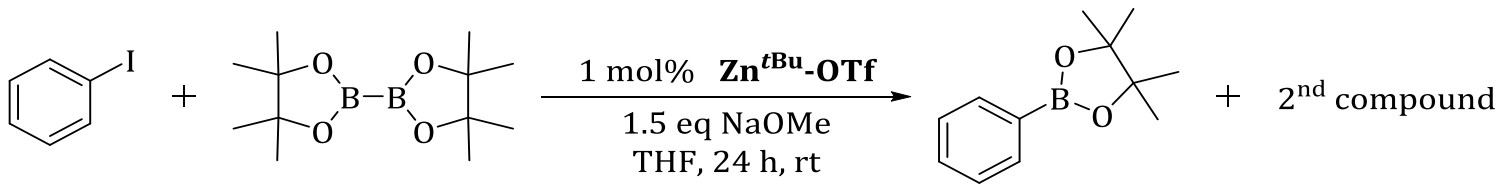

Scheme 3.34: Attempted catalytic C-I borylation by zinc complex $\mathbf{Z n}^{\text {tBu}}$-OTf.

After workup, NMR spectroscopy $\left({ }^{1} \mathrm{H},{ }^{13} \mathrm{C}\right.$ and $2 \mathrm{D}$ techniques) revealed the formation of pinacolboryl benzene and a second, unidentified compound in a 1:1 ratio. However, a second 
species could not be identified in an ESI mass spectrum. No iodo benzene was found after the reaction. Since the reaction products could not be separated with column chromatography and the identity of the second product remained unknown, zinc catalysis was not further studied.

\subsubsection{Cobalt Catalysis}

The use of cobalt for catalysis is highly desirable as discussed above (Chapter 3.1.3) and many cobalt pincer complexes are known to enable numerous catalytic transformations. ${ }^{[342,458-461]}$ For high activities, reactive cobalt species like hydride or alkyl complexes were applied or generated in situ. Since such reactive compounds could not be isolated in stoichiometric reactions before (see Chapter 3.4.2.2), it was tried to activate $\mathbf{C o}^{\mathrm{tBu}}-\mathbf{M e C N}$ with superhydride ${ }^{\circledR}$ in situ.

Co $^{t \mathrm{Bu}}-\mathrm{MeCN}$ showed no reactivity in the dehydrogenation of ammonia borane and the transfer hydrogenation of acetophenone. This was clearly indicated by the isolation of the substrates from the reaction mixtures. The hydrosilylation of 1-octene with phenylsilane produced traces of octane but no expected products of the hydrosilylation could be found by NMR spectroscopy. In hydroboration reactions of styrene and 1-octene with pinacol borane after $2.5 \mathrm{~h}$, a conversion of approx. 25\% was observed (according to the methyl resonances of the reactant and the products in ${ }^{1} \mathrm{H}$ NMR monitoring). The same conversion rate was also found for longer reactions times up to $24 \mathrm{~h}$ and for more equivalents of superhydride ${ }^{\circledR}$, indicating that the active species decomposed before. Since the active species was assumed to be formed upon addition of superhydride ${ }^{\circledR}$ and products of the stoichiometric reaction are not known, it might be that no active catalyst species were formed. Because of this issue and the found low activity of the cobalt complex $\mathbf{5 4}$ the focus was set on catalysis with rhodium complexes (see below).

\subsubsection{Rhodium Catalysis}

\subsubsection{Dimerization of Phenylacetylene}

Enynes are useful synthetic building blocks for organic synthesis. Hence, the selective synthesis of such compounds is an aim of organometallic catalysis. Many rhodium(I) complexes have been used for dimerization of terminal alkynes producing mainly the cis-, trans- or gem-isomer, depending on the catalyst. $[316,319,323]$ The dimerization products can be identified by specific ${ }^{1} \mathrm{H}$ NMR resonances and coupling constants. ${ }^{[316]} \mathrm{A}$ series of rhodium(I) complexes of the Twoin-one pincer ligand was tested for this reaction (Scheme 3.35). 
2

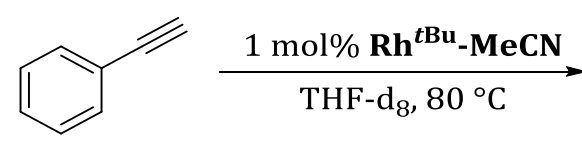

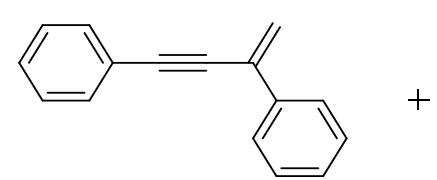

cis-isomer

trans-isomer

oligomers

Scheme 3.35: Dimerization of phenylacetylene by rhodium(I) complex $\mathbf{R h}^{t \mathrm{Bu}}$-MeCN yielding mainly gem-isomer.

It was found, that $\mathbf{R h}^{t \mathrm{Bu}}-\mathbf{M e C N}$ exhibited the best activity, while $\mathbf{R} \mathbf{h}^{\mathrm{tBu}}-\mathbf{S M e}_{2}$ was inactivated after a short period of time $(<18 \mathrm{~h})$ and no further conversion was observed. The sterically less hindered ${ }^{i} \operatorname{Pr}$ analogue $\mathbf{R h}^{\text {iPr-MeCN }}$ presumably underwent oxidative addition with phenylacetylene, observed by a fast color change to yellow upon addition of substrate and evident from a smaller coupling constant $J_{R h-P .}$ The resulting rhodium(III) species was not capable of promoting the dimerization reaction. The reaction with $\mathbf{R h}^{t \mathrm{Bu}}-\mathbf{M e C N}$ as catalyst was investigated in more detail. It was found, that the reaction was faster at $80{ }^{\circ} \mathrm{C}$ compared to $50^{\circ} \mathrm{C}$, while at ambient temperature almost no conversion was observed over days (which was important for the hydrogenation reactions, see Chapter 3.6.3.2). Due to low solubility of the complex the reaction in benzene was slower than in THF, but gave the same distribution of products. The reaction progress of a reaction in THF- $\mathrm{d}_{8}$ at $80^{\circ} \mathrm{C}$ was followed by ${ }^{1} \mathrm{H}$ NMR spectroscopy showing the increase in intensity of the resonances of the gem-and the transisomers and then after 2 days a decrease (Figure 3.57).

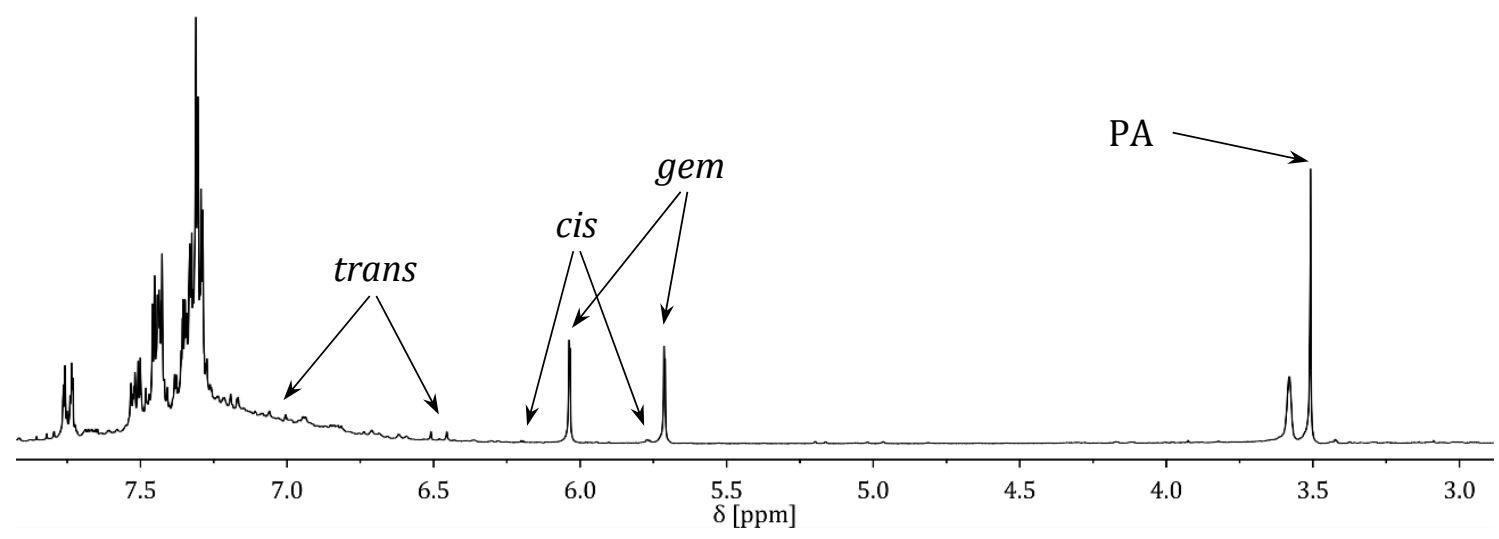

Figure 3.56: ${ }^{1} \mathrm{H}$ NMR spectrum during (after $44 \mathrm{~h}$ ) the dimerization of phenyl acetylene (PA) catalyzed by $\mathbf{R h}^{t \mathrm{Bu}}-\mathrm{MeCN}$ in THF-d8 at $80^{\circ} \mathrm{C}$.

The aromatic region broadened a lot and it was assumed that the formation of trimers and higher oligomers occurred. A reliable determination of the amount of the trans-isomer was not possible, due to overlaying aromatic signals and to a lower quality of the NMR data. Varying the reaction conditions did not lead to a more selective reaction. 


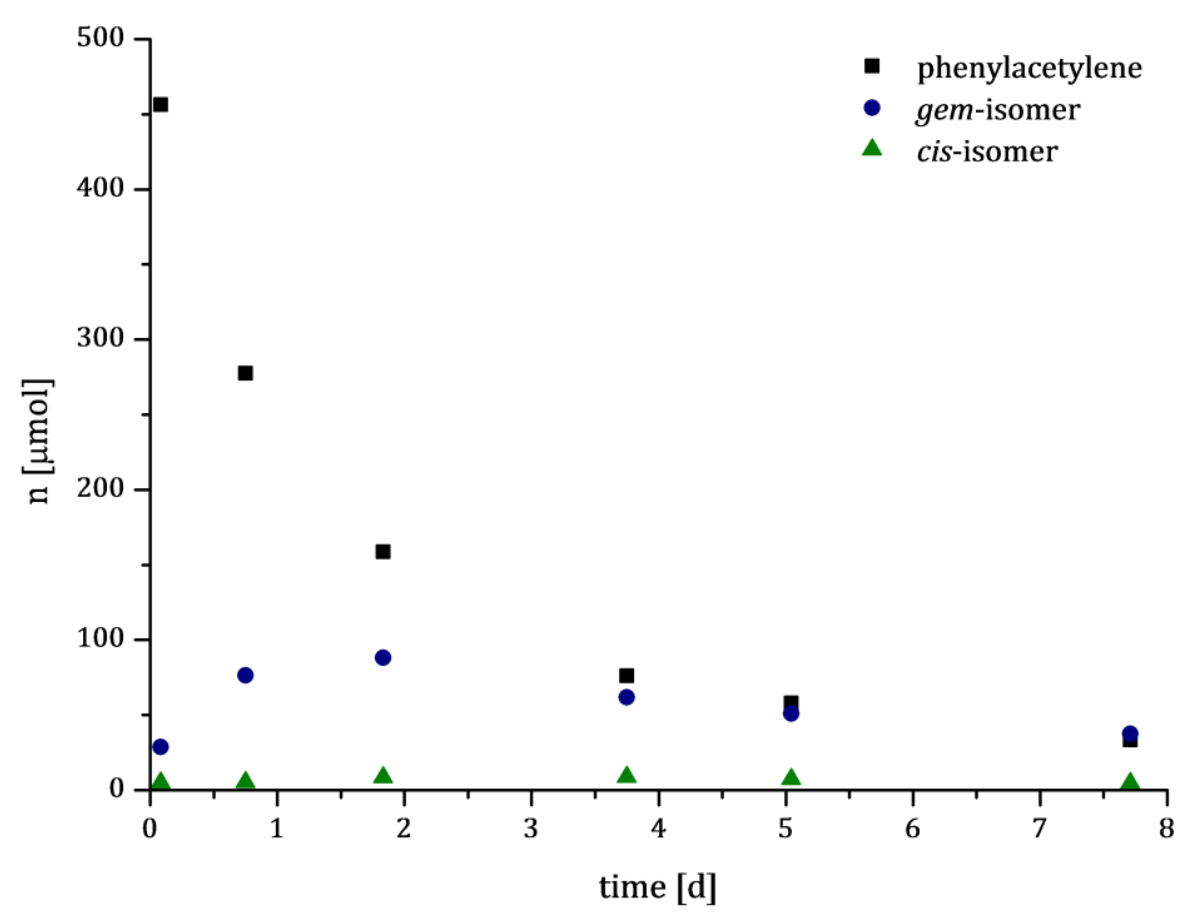

Figure 3.57: Plot of the amount $\mathrm{n}$ of the substrate (black) and products (gem: blue, trans: purple, cis: green) versus time for the catalytic dimerization of phenylacetylene with $\mathbf{R h}^{t \mathrm{Bu}}-\mathbf{M e C N} \mathbf{5 8} \mathrm{in} \mathrm{THF}-\mathrm{d} 8$ at $80^{\circ} \mathrm{C}$. Note that the amount of the trans-isomer could not be determined in a reliable way due to superimposed phenyl resonances.

The dimerization reaction was not selective and fast enough to further study this reaction. But it revealed, that the dimerization at ambient conditions slow and therefore does not led to an undesired side reaction during the hydrogenation reactions.

\subsubsection{Hydrogenation of Alkynes}

Rhodium(I) complexes as the WILKINSON catalyst are well known to promote the hydrogenation

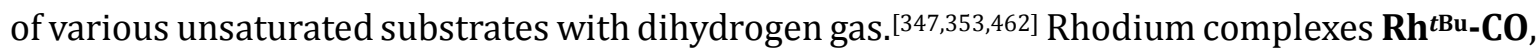
$\mathbf{R h}^{t \mathrm{Bu}}-\mathbf{M e C N}$ and $\mathbf{R h}^{t \mathrm{Bu}}-\mathrm{SMe}_{2}$ were tested in the hydrogenation of alkynes. Phenyl acetylene (PA) was chosen as a simple substrate with readily accessible substituted derivatives (Scheme 3.36).<smiles>[R]c1ccc(C#C)cc1</smiles>

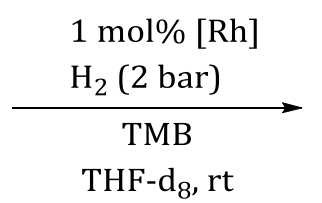<smiles>[R]c1ccc(/C=C\C)cc1</smiles><smiles>[13CH3]</smiles><smiles>[R]c1ccc(CC)cc1</smiles>

$$
\mathrm{R}=\mathrm{H}, \mathrm{Me}, \mathrm{OMe}, \mathrm{F}, \mathrm{CO}_{2} \mathrm{Me}
$$

Scheme 3.36: Catalytic hydrogenation of phenyl acetylenes to the corresponding styrenes and ethylbenzenes with different rhodium(I) complexes.

In addition, the corresponding styrene was easy to quantify using ${ }^{1} \mathrm{H}$ NMR spectroscopy, because of its well isolated peaks. The amount of formed styrenes was calculated from the 
intensity of the doublet of doublets at $5.18 \mathrm{ppm}$ (Figure 3.58) referenced by an internal standard (1,4-dioxane or 1,3,5-trimethoxybenzene) and plotted versus time. Rate constants $\mathrm{k}_{\mathrm{obs}}$ were obtained by applying the initial rate approximation method for non-optimized conditions, which are summarized in Table 3.9. The observed reaction rates were found to be very sensitive towards remaining moisture in the used hydrogen gas and reproducible results were only obtained when the dihydrogen was thoroughly dried before use (at least two hours with a cool spiral placed in liquid nitrogen). To increase the reliability of the results, every experiment was conducted three times with stock solutions of substrate, catalyst and internal standard. The rate constants $\mathrm{k}_{\mathrm{obs}}$ for the hydrogenation are weighted averages of at least two runs.

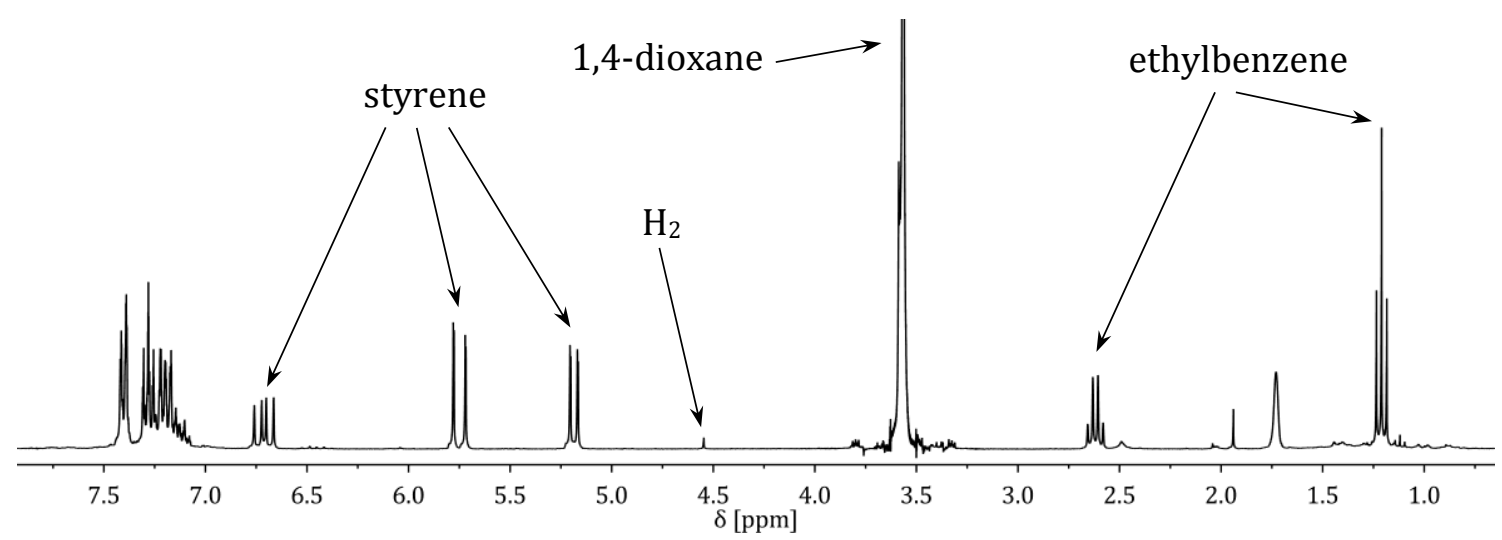

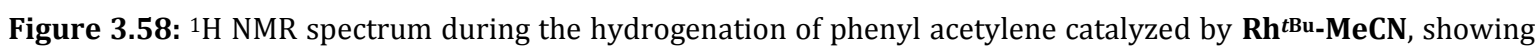
the characteristic resonances for styrene and ethylbenzene and 1,4-dioxane as internal standard (THF- $\mathrm{d}_{8}, 298 \mathrm{~K}$ ).

At first it was found that reactions in J. YounG NMR tubes resulted in different rates depending on whether the sample was shaken between the measurements or not. A long-term measurement under non-shaken conditions showed firstly a remarkable stability of the catalyst and secondly a further hydrogenation of the formed styrene to ethylbenzene (Figure 3.58). In the course of five days all phenyl acetylene was consumed and the formation of styrene was observed $\left(k_{\mathrm{obs}}(1)=22.2 \mathrm{mM} \mathrm{d}^{-1}\right)$. After that a much slower second hydrogenation reaction to ethylbenzene was observed with the appearance of ethyl resonances at $1.21 \mathrm{ppm}$ and $2.61 \mathrm{ppm}$. After 4 months further 100 equivalents of phenyl acetylene and fresh dihydrogen were added and essentially the same rate constant $\left(k_{\mathrm{obs}}(2)=22.6 \mathrm{mM} \mathrm{d}^{-1}\right)$ for the first hydrogenation was observed (Figure 3.59), highlighting an impressive stability of the catalytic system. No decomposition products of the complex were found in ${ }^{31} \mathrm{P}$ NMR spectra which could potentially lowered the rate of catalysis. For catalysis this durability is beneficial since a continuous process is conceivable, although the rate of this given catalyst was far too low for a large scale application. 


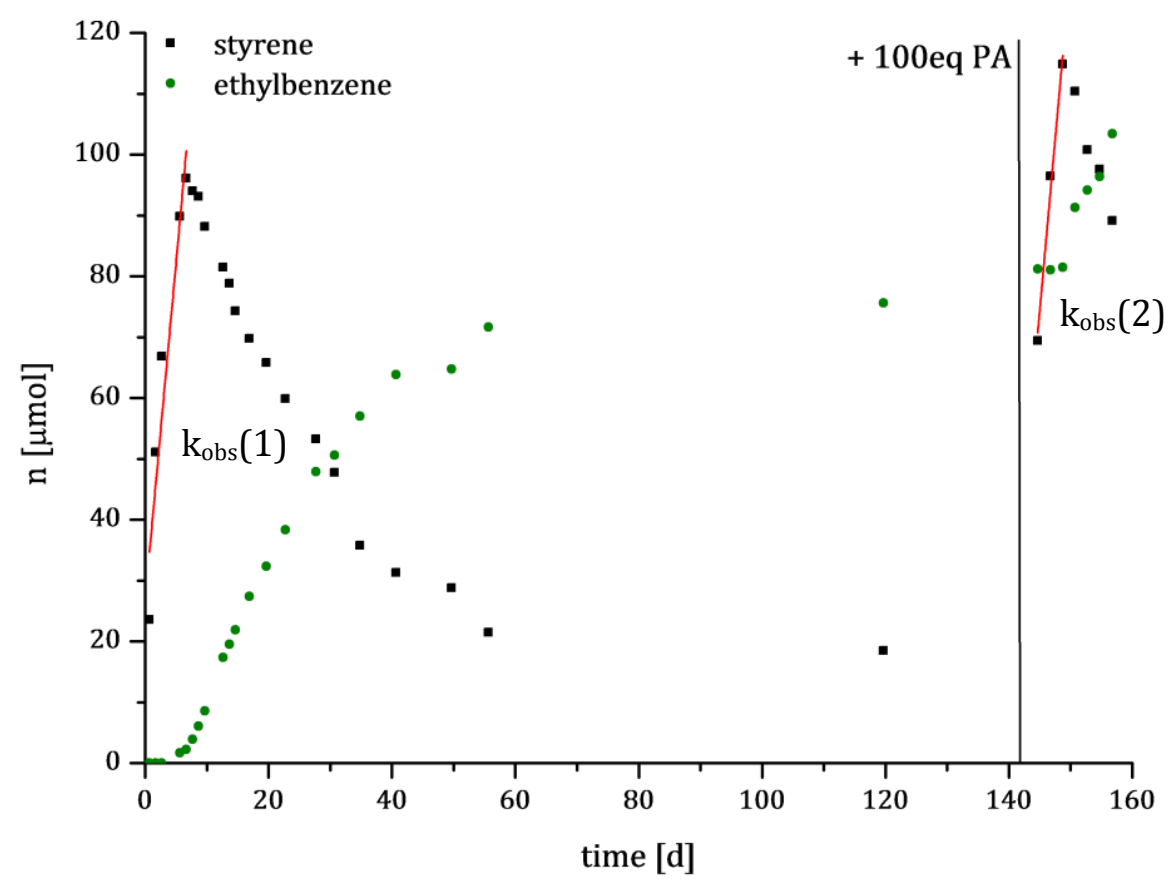

Figure 3.59: Time dependent formation of styrene (black) and ethylbenzene (green) and initial rate constant fitting for the hydrogenation of phenyl acetylene to styrene for the first and second addition of substrate.

Diphenylacetylene as an internal alkyne and 1-hexyne as an aliphatic alkyne were hydrogenated with $\mathbf{R h}^{t \mathbf{B u}}-\mathbf{M e C N}$ at ambient temperature and 2 bar dihydrogen pressure. The rate for 1-hexyne was smaller than that for phenylacetylene $\left(k_{\mathrm{obs}}=8.6 \mathrm{mmol} \mathrm{d}^{-1}\right)$ and the rate for diphenylacetylene was even lower. Here about four turnovers were observed per day $\left(k_{\text {obs }}=4.6 \mathrm{mmol} \mathrm{d}^{-1}\right)$. Nevertheless, the product was identified by NMR spectroscopy to be cis-stilbene, which indicated a cis-addition of dihydrogen to the triple bond. In a small amount also the formation of 1,2-diphenylethane was observed.

When shaking the samples between each measurement, significantly higher rates for the hydrogenation of PA were observed. This was explained by the higher concentration of dihydrogen in the reaction mixture represented by significant larger integrals of the $\mathrm{H}_{2}$ in ${ }^{1} \mathrm{H}$ NMR spectra. The shaking led to a constant $\mathrm{H}_{2}$ concentration evidenced by a constant integral of the $\mathrm{H}_{2}$ resonance at $4.54 \mathrm{ppm}$ (within the experimental error). Among the tested three precatalysts, $\mathbf{R h}^{t \mathrm{Bu}}$-MeCN exhibited the highest rate while $\mathbf{R} \mathbf{h}^{t \mathrm{Bu}}$-CO showed no reactivity at all and $\mathbf{R h}^{\mathrm{tBu}}-\mathbf{S M e}_{2}$ was less active than the acetonitrile complex (Table 3.9, entries 1 - 3). The reason for that might be that the active catalytic species is formed by decoordination of one or both coligands. The carbonyl complex is assumed to be inert towards ligand exchange under these conditions. In general, chelating ligands, as $\mathrm{SMe}_{2}$ in $\mathbf{R} \mathbf{h}^{t \mathrm{Bu}}-\mathbf{S M e}$, are more difficult to

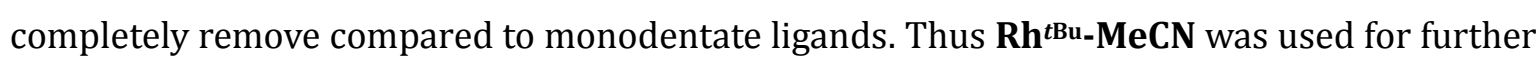
studies. 
Table 3.9: Results of the hydrogenation of different phenylacetylenes with rhodium(I) catalysts:

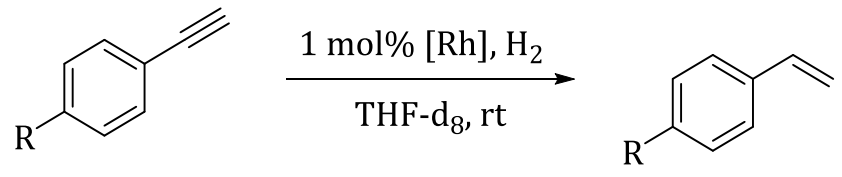

\begin{tabular}{|c|c|c|c|c|c|}
\hline entry & catalyst & $\mathrm{R}$ & eq. alkyne & $\mathrm{p}\left(\mathrm{H}_{2}\right)[\mathrm{bar}]$ & $\mathrm{k}_{\mathrm{obs}}\left[\mathrm{mM} \mathrm{h}^{-1}\right]$ \\
\hline 1 & $\mathbf{R h}^{t \mathrm{Bu}-\mathrm{CO}}$ & $\mathrm{H}$ & 100 & 2 & $-b$ \\
\hline 2 & $\mathrm{Rh}^{t \mathrm{Bu}}-\mathrm{MeCN}$ & $\mathrm{H}$ & 100 & 2 & $14.4 \pm 0.57$ \\
\hline 3 & $\mathbf{R h}^{t \mathrm{Bu}_{-} \mathrm{SMe}_{2}}$ & $\mathrm{H}$ & 100 & 2 & $9.21 \pm 0.60$ \\
\hline 5 & $\mathbf{R h}^{t \mathrm{Bu}-\mathrm{MeCN}}$ & $\mathrm{F}$ & 100 & 2 & $16.8 \pm 0.96$ \\
\hline 6 & $\mathbf{R h}^{t \mathrm{Bu}}-\mathbf{M e C N}$ & $\mathrm{Me}$ & 100 & 2 & $9.57 \pm 1.67$ \\
\hline 7 & $\mathbf{R h}^{t \mathrm{Bu}}-\mathrm{MeCN}$ & $\mathrm{OMe}$ & 100 & 2 & $13.0 \pm 0.14$ \\
\hline 8 & $\mathbf{R h}^{t \mathrm{Bu}}-\mathrm{MeCN}$ & $\mathrm{CO}_{2} \mathrm{Me}$ & 100 & 2 & $4.25 \pm 0.84$ \\
\hline 9 & $\mathrm{Rh}^{t \mathrm{Bu}}-\mathrm{MeCN}$ & $\mathrm{H}$ & 100 & $2\left(D_{2}\right)$ & $10.1 \pm 0.64$ \\
\hline 10 & $\mathrm{Rh}^{t \mathrm{Bu}}-\mathrm{MeCN}$ & $\mathrm{H}$ & 100 & 1 & $5.28 \pm 0.056$ \\
\hline 11 & $\mathbf{R h}^{t \mathrm{Bu}-\mathrm{MeCN}}$ & $\mathrm{H}$ & 100 & 1.5 & $12.1 \pm 0.56$ \\
\hline 12 & $\mathbf{R h}^{t \mathrm{Bu}-\mathrm{MeCN}}$ & $\mathrm{H}$ & 10 & 2 & $4.66 \pm 0.28$ \\
\hline 13 & $\mathbf{R h}^{t \mathrm{Bu}-\mathrm{MeCN}}$ & $\mathrm{H}$ & 30 & 2 & $8.24 \pm 0.45$ \\
\hline 14 & $\mathbf{R h}^{t \mathrm{Bu}-\mathrm{MeCN}}$ & $\mathrm{H}$ & 50 & 2 & $8.30 \pm 0.041$ \\
\hline 15 & $\mathbf{R h}^{t \mathrm{Bu}}-\mathbf{M e C N}^{\mathrm{c}}$ & $\mathrm{H}$ & 100 & 2 & $8.86 \pm 0.27$ \\
\hline 16 & $\mathbf{R h}^{t \mathrm{Bu}}-\mathbf{M e C N}^{\mathrm{d}}$ & $\mathrm{H}$ & 100 & 2 & $7.66 \pm 0.081$ \\
\hline 17 & $\mathbf{R h}^{t \mathrm{Bu}-\mathrm{MeCN}}$ & 1-hexyne & 100 & 2 & $5.49 \pm 0.69$ \\
\hline
\end{tabular}

a Amount of product was determined by ${ }^{1} \mathrm{H}$ NMR spectroscopy with 1,3,5-trimethoxybenzene as internal standard $(\delta=6.04 \mathrm{ppm})$. b No reaction was observed after one day. c $0.66 \mathrm{~mol} \%$ catalyst used. $\mathrm{d} 0.43 \mathrm{~mol} \%$ catalyst used.

The electronic structure of the substituent attached to the para-position of the alkyne moiety seemed to have a moderate effect on the reaction rate. Substrates with different electronic properties were applied in the hydrogenation reaction (for the [product] vs. time plots see Appendix 6.1). The resulting HAMmETT plot with the relative rates $\left(\mathrm{k}_{\mathrm{R}} / \mathrm{k}_{\mathrm{H}}\right)$ plotted against the HAMMETT parameters $\sigma_{\mathrm{P}}$ is shown in Figure 3.60.[463] A linear correlation of all substituents was not found, though the sterically less demanding and non-coordinating derivatives with hydrogen-, methyl- and fluoro-substituents are linear related to the $\sigma$ values with a slope of 
2.19 , indicating that a less electron rich triple bond facilitates the hydrogenation. ${ }^{[464]}$ To draw solid conclusions from this data, other substituents on the phenyl ring have to be tested in order to add more data points to the HAMmETT plot.

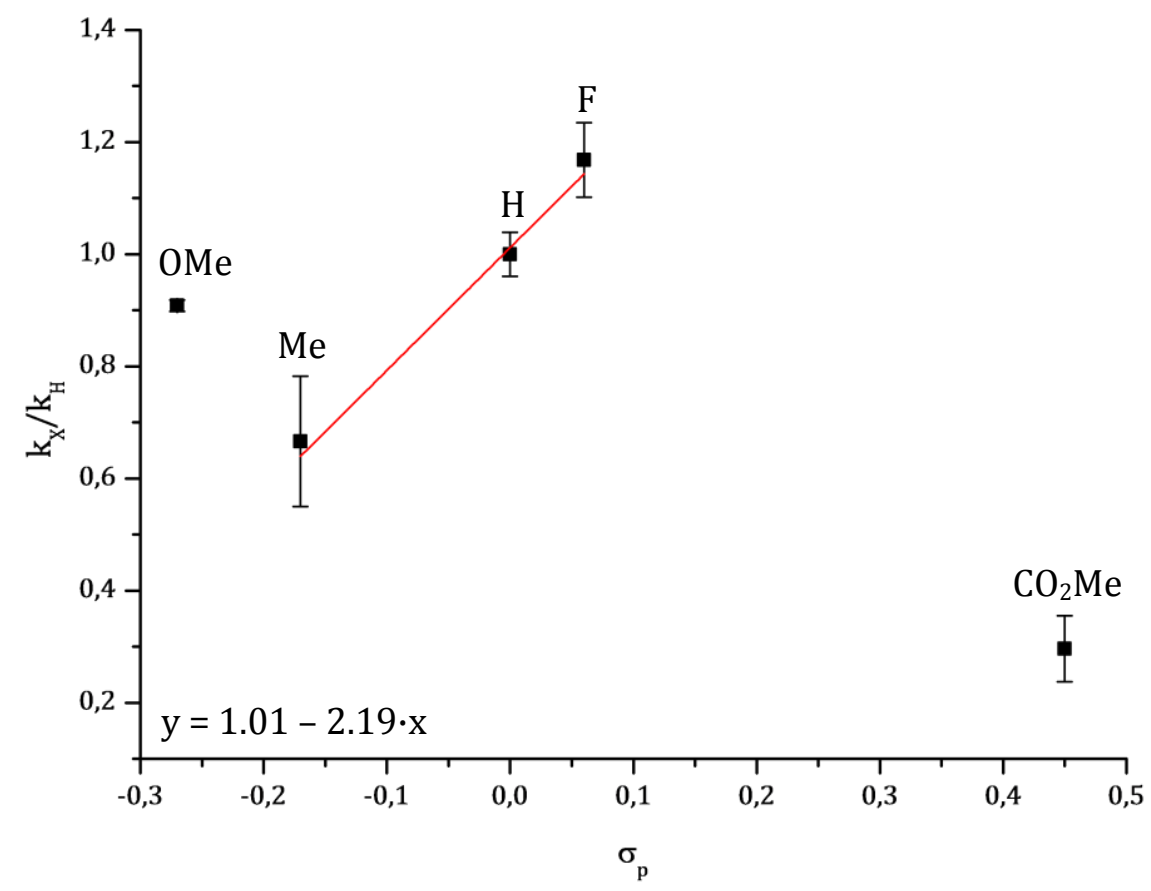

Figure 3.60: НАMмETT plot of the hydrogenation of para-substituted phenyl acetylene derivatives with $\mathbf{R h}{ }^{t \mathrm{Bu}}-\mathbf{M e C N}$ in THF-d8.

When the reaction was carried out under an atmosphere of dry deuterium gas, a slightly lower reaction rate was observed, resulting in a kinetic isotope effect (KIE) of $\mathrm{k}_{\mathrm{H}} / \mathrm{k}_{\mathrm{D}}=1.42 \pm 0.11$. The low value for the KIE showed, that the splitting of the dihydrogen bond is not directly involved in the turnover limiting step in the catalytic cycle.[465] It is possible that a pre-equilibrium involving dihydrogen or deuterium is present prior to the step with the highest barrier; for example, a reversible oxidative addition followed by the irreversible insertion of the substrate into the $\mathrm{Rh}-\mathrm{H}$ and $\mathrm{Rh}-\mathrm{D}$ bond, respectively. ${ }^{[466]}$ In addition, the hydrogenation of phenyl acetylene with deuterium indicated a cis addition of the deuterium to the alkyl triple bond. In the formed partly deuterated styrene the ${ }^{1} \mathrm{H}$ NMR resonance of the cis proton remained present, while the other two signals (trans and gem) were not observed in ${ }^{1} \mathrm{H}$ NMR spectra of the reaction mixture. For this resonance a triplet with a coupling constant of ${ }^{2} J_{\mathrm{D}-\mathrm{H}}=2.6 \mathrm{~Hz}$ was observed. 


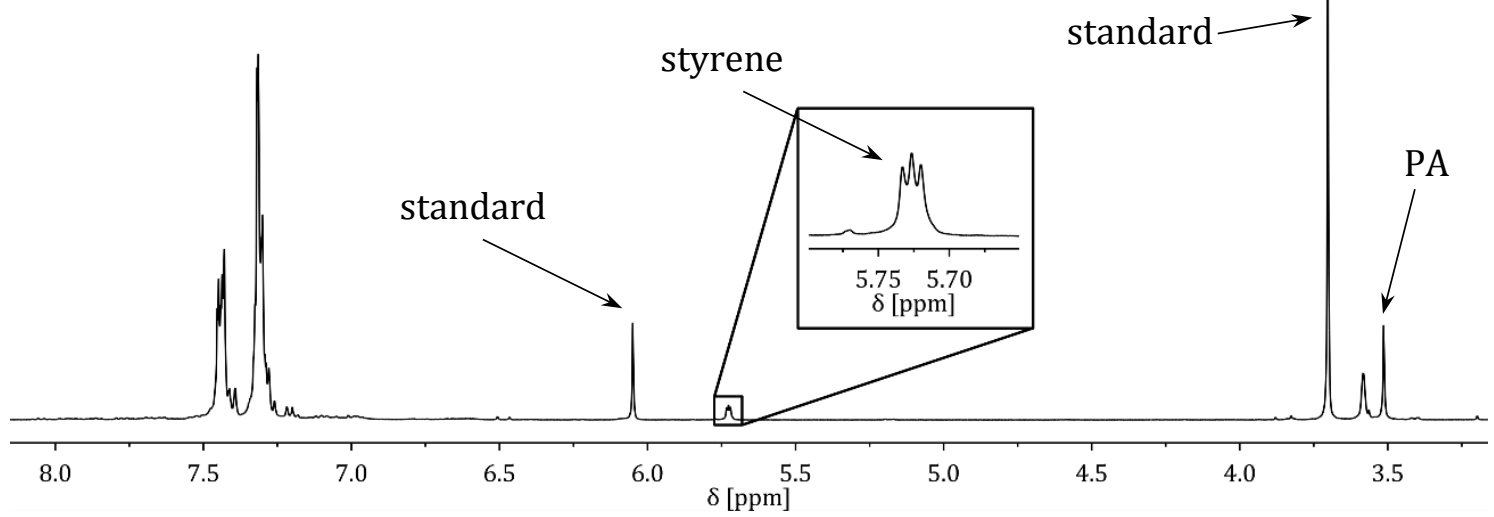

Figure 3.61: ${ }^{1} \mathrm{H}$ NMR spectrum during the hydrogenation of phenyl acetylene catalyzed by $\mathbf{R h} \mathbf{h}^{t \mathrm{Bu}}-\mathbf{M e C N}$ with $\mathrm{D}_{2}$ and 1,3,5-trimethoxybenzene as internal standard, showing the one resonance for the ethylene group of styrene (THF$\left.\mathrm{d}_{8}, 298 \mathrm{~K}\right)$.

A reaction with three equivalents of para-fluorophenylacetylene ( $p$ F-PA) under standard conditions was used to search for rhodium species appearing during the catalysis, but no species other than $\mathbf{R h}^{t \mathrm{Bu}}-\mathbf{M e C N}$ were found in ${ }^{1} \mathrm{H}$ and ${ }^{31} \mathrm{P}$ NMR spectra (Figure 3.62). The formation of the corresponding styrene derivative could nicely be seen by the increasing resonances at $5.17 \mathrm{ppm}(\mathrm{d}), 5.69 \mathrm{ppm}(\mathrm{d})$ and $6.69 \mathrm{ppm}(\mathrm{dd})$ for the alkene protons.

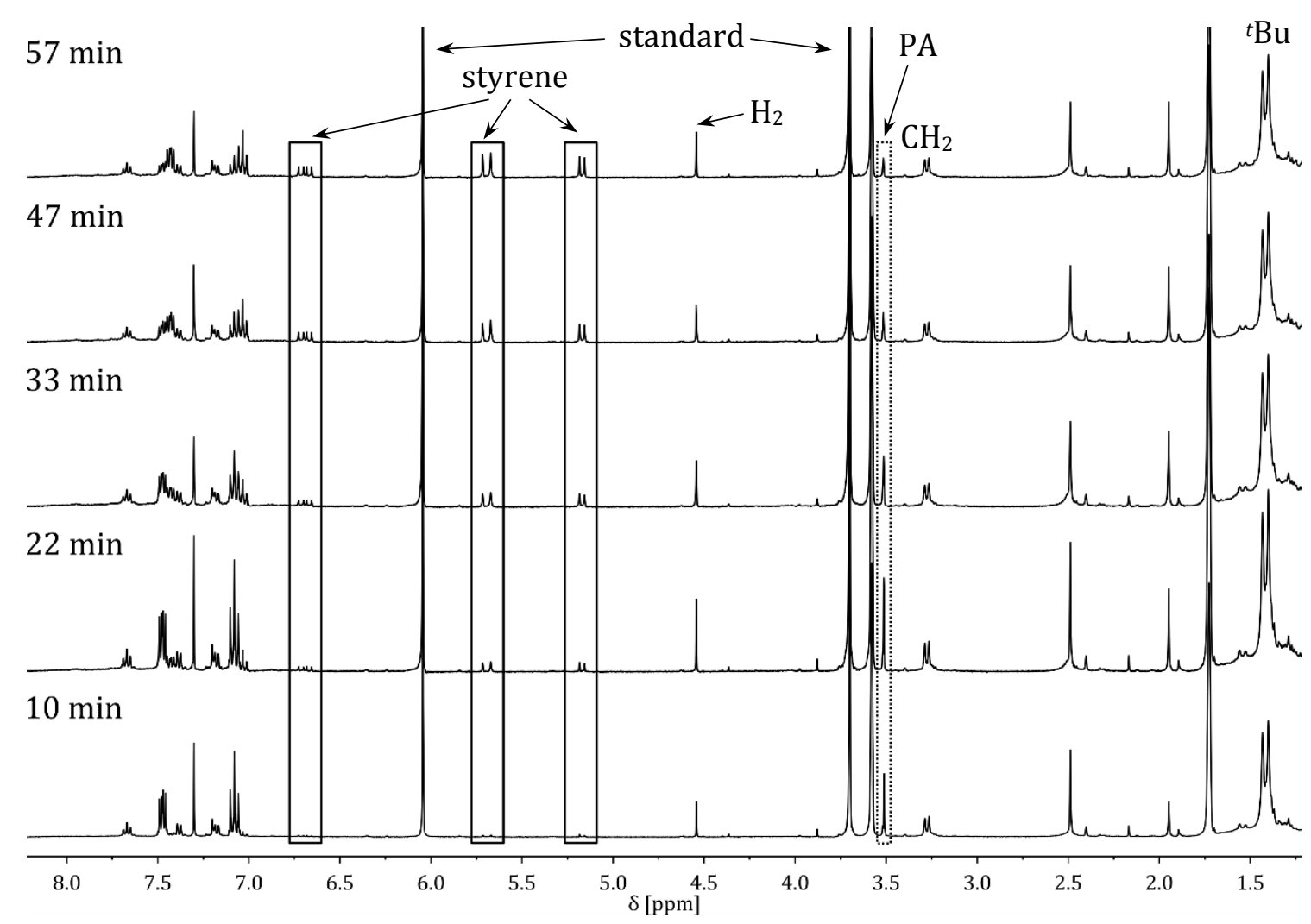

Figure 3.62: Hydrogenation of para-fluorophenylacetylene (3.0 eq.) with $\mathbf{R h}^{t \mathrm{Bu}-M e C N}$ (1.0 eq.) followed by ${ }^{1} \mathrm{H}$ NMR spectroscopy with 1,3,5-trimethoxybenzene as internal standard under $\mathrm{H}_{2}$ (2 bar) (THF- $\mathrm{d}_{8}, 298 \mathrm{~K}$ ).

In addition, ${ }^{19} \mathrm{~F}$ spectroscopy solely revealed the decrease of the substrate concentration. Thus, intermediates of the reactions could not be observed, neither a coordination of the alkyne, nor 
an oxidative addition of $\mathrm{H}_{2}$. These findings concluded that the catalytic active species were very short-lived and only present in minor amounts.

To obtain more insights into the mechanism of this hydrogenation, several experiments with varying concentrations of the substrate, the catalyst and dihydrogen were performed to study the reaction orders (Table 3.9, entries 10 -16). It was considered that the concentrations of intermediates were generally low and that intermediates reacted as soon as they were formed. To determine the reaction order of each of the reactants, the initial rates for different starting concentrations of phenyl acetylene, dihydrogen and catalyst were measured. The data was linearized and the reaction order determined from the slope of a linear fit (Figure 3.63).
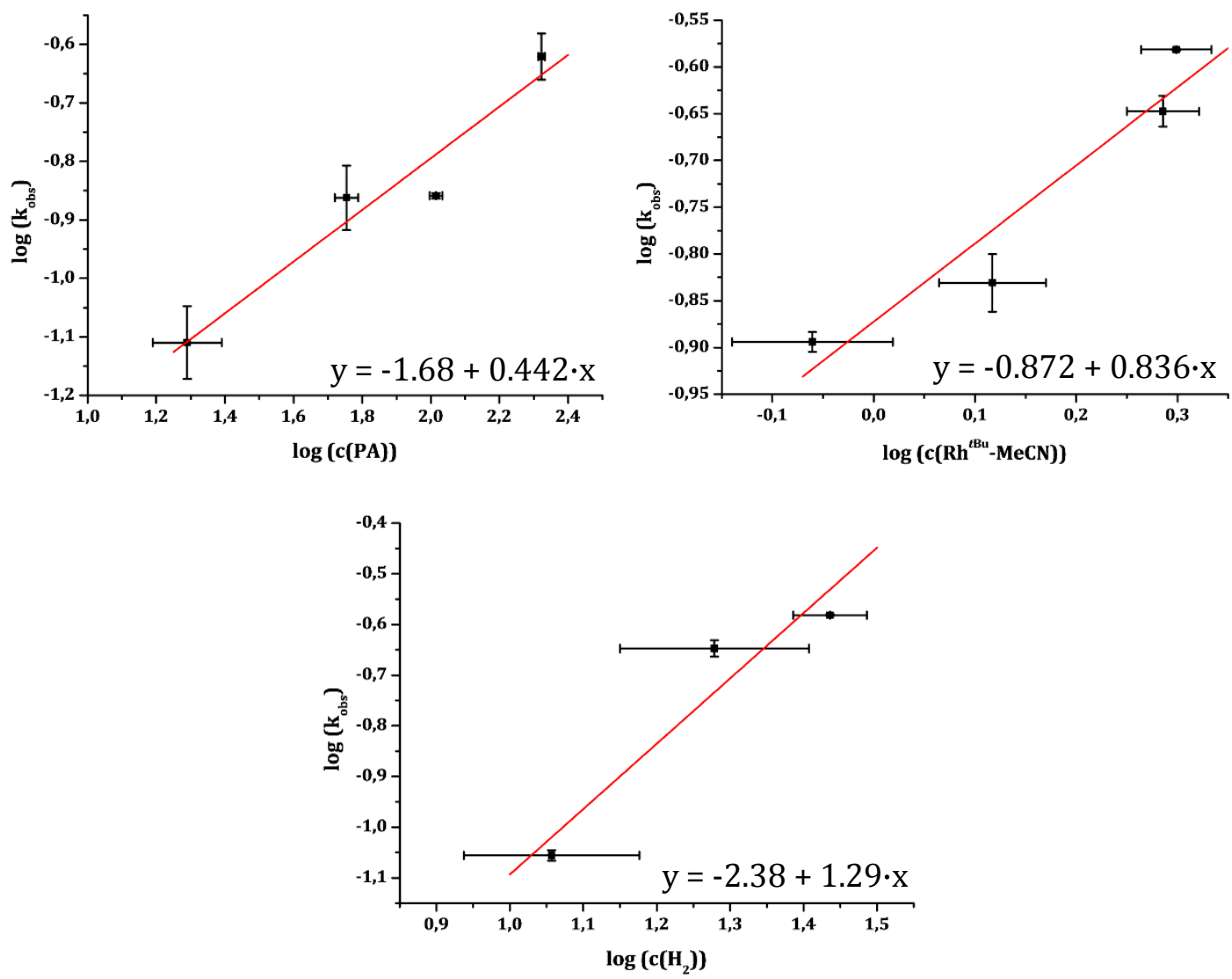

Figure 3.63: Linearized plots of the observed rate constants $\mathrm{k}_{\mathrm{obs}}$ versus varied concentrations of the reactants: Phenyl acetylene (top, left), catalyst $\mathbf{R h}^{\text {tBu}}$-Me (top, right) and dihydrogen (bottom).

Prelimenary results showed, that the reaction rate is approx. first order dependent on the concentration of dihydrogen and catalyst $\mathbf{R h}^{\text {tBu-MeCN }}$ in solution (1.29 and 0.836, respectively). The slope of the substrate dependence was found to be 0.442 , which is unusual and might indicate a more complicated mechanism. The reaction order in substrate was not in agreement with dependencies found for other rhodium hydrogenation catalyst. [354,467] The rate law of the hydrogenation of terminal phenyl acetylenes with $\mathbf{R h}^{t \mathrm{Bu}-M e C N}$ in THF- $\mathrm{d}_{8}$ might be:

$$
\frac{\partial[\text { styrene }]}{\partial t}=k_{o b s} \cdot[\text { substrate }]^{0.5} \cdot\left[\mathbf{R h}^{t \mathbf{B u}}-\mathbf{M e C N}\right] \cdot\left[\mathrm{H}_{2}\right]
$$


A catalytic rate equation could not be derived since potential intermediates were not known.

The catalytic hydrogenation of PA was also performed in the presence of additional MeCN $(\mathrm{MeCN} /$ catalyst $=10: 1)$. The observed reaction rate was significantly lower than for the reaction without additional MeCN $\left(9.76 \pm 0.37 \mathrm{mM} \mathrm{h}^{-1} v s .14 .4 \pm 0.57 \mathrm{mM} \mathrm{h}^{-1}\right)$. In pure MeCN$\mathrm{d}_{3}$ the reaction rate was found to be even smaller. The increased coligand concentration lowered the dissociation of the MeCN ligands to form a free coordination site. Thus, the concentration of the active catalyst species is lower, leading to a lower reaction rate. The same effect was found also for the WILKINSON catalyst, when additional triphenylphosphine is added. ${ }^{[349]}$ This led to the conclusion, that the first step of the catalytic cycle is the decoordination of one or both MeCN coligands in $\mathbf{R h}^{\text {tBu-MeCN. }}$

In summary the rate of the catalytic hydrogenation of alkynes with complex $\mathbf{R h}^{t \mathrm{Bu}}$-MeCN is significantly lower than known for mononuclear complexes, such as the WILKINSON catalyst.[346,353] Nonetheless it was demonstrated that hydrogenation reactions can be performed with dinuclear rhodium(I) pincer complexes. The main reason for the low catalytic activity was assumed to be the slow decoordination of the coligands to create an open binding site for the substrates.

\subsubsection{Hydrogenation of Carbon dioxide}

The development of new catalysts for the capture of carbon dioxide is of high interest and of high environmental relevance. ${ }^{[355]}$ Although the use of base metals is more desired in the sense of non-toxicity and costs, the use of noble metal catalysts is still required, since they exhibit higher activity to date. In addition, the study of mechanistic aspects of the hydrogenation of $\mathrm{CO}_{2}$ with low valent $4 \mathrm{~d}$ and $5 \mathrm{~d}$ metals have practical advantages as for example the diamagnetism of the complexes.

Excitingly, the rhodium(I) MeCN complex $\mathbf{R h}{ }^{t B u}-\mathbf{M e C N}$ was found to hydrogenate $\mathrm{CO}_{2}$ in the presence of a strong base (Scheme 3.37). As in many literature-known examples, DBU was utilized as strong base to improve the enthalpy of the hydrogenation. Triethylamine was also tried but no conversion in the presence of this weaker base was observed. The reactions were performed either in high pressure NMR tubes $\left(\mathrm{WILMAD}^{\circledR}\right)$ at ambient temperature or in a steel reactor $\left(\mathrm{BÜCHI}{ }^{\circledR}\right)$ at $50{ }^{\circ} \mathrm{C}$. For NMR experiments the sample was degassed and purged with $\mathrm{CO}_{2}$ first. The carbon dioxide was frozen out in liquid nitrogen and then $\mathrm{H}_{2}$ was added to the sample at low temperature. For performing the reaction in the steel reactor, the vessel containing the sample was purged with purified dinitrogen, the sample solution was added via syringe and purged with $\mathrm{H}_{2}$. $\mathrm{CO}_{2}$ was added afterwards. The reactions were run at $50{ }^{\circ} \mathrm{C}$ to ensure the 
tightness of the autoclave. The product formation was investigated by NMR spectroscopy with the use of an internal standard.

$$
\begin{aligned}
& \mathrm{Rh}^{t \mathrm{Bu}}-\mathrm{MeCN}
\end{aligned}
$$

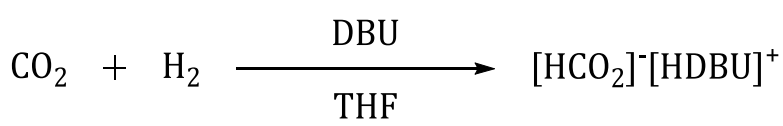

Scheme 3.37: Hydrogenation of carbon dioxide with $\mathbf{R h} \mathbf{h}^{\mathrm{B} u-M e C N}$ in the presence of a strong base.

The formation of formate as the only reaction product was proven by two dimensional NMR spectroscopy. A sharp singulet a $8.43 \mathrm{ppm}$ was observed after one day under three atmospheres of $\mathrm{H}_{2}$ and $\mathrm{CO}_{2}$ each (Figure 3.64). This resonance was coupled to a carbon resonance at $167.2 \mathrm{ppm}$. Both values are in good agreement with literature values (Figure 3.65 and Figure 3.66).[468]

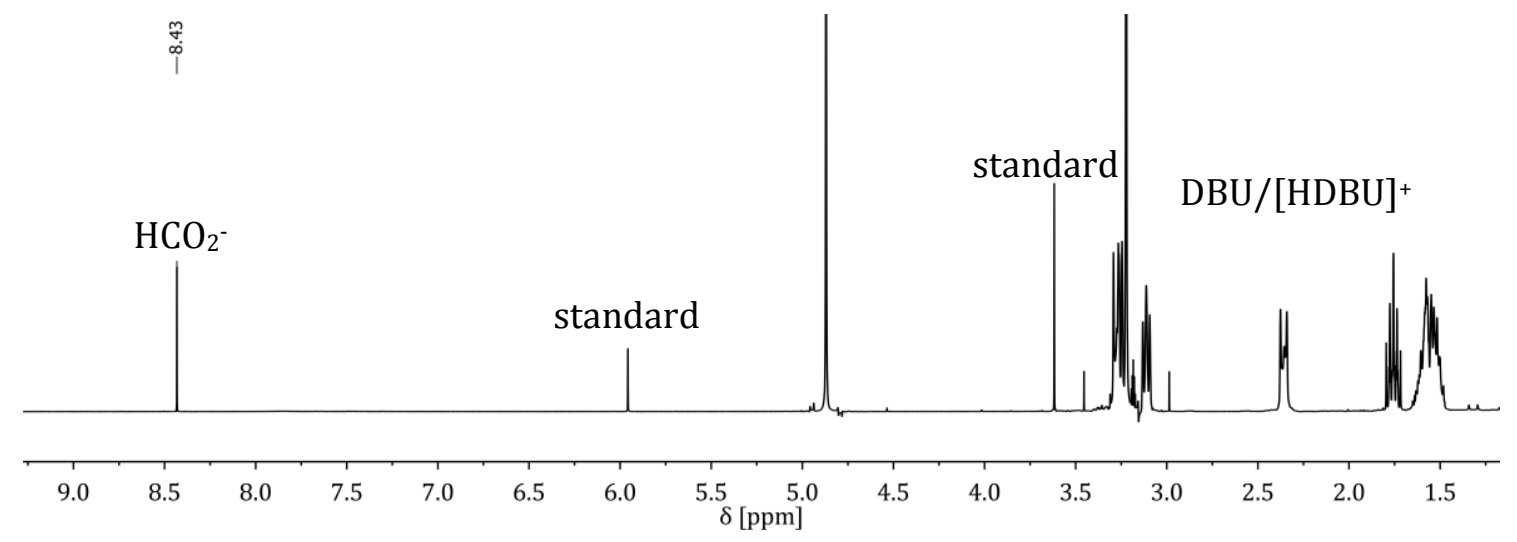

Figure 3.64: ${ }^{1} \mathrm{H}$ NMR spectrum after the workup of a reaction of $\mathrm{CO}_{2}$ and $\mathrm{H}_{2}$ with DBU catalyzed by $\mathbf{R h}^{t \mathrm{Bu}}$-MeCN in the presence of 1,3,5-trimethoxybenzene $(13.7 \mu \mathrm{mol})$ as internal standard, showing the formation of formate (MeOH-d4, $298 \mathrm{~K})$.

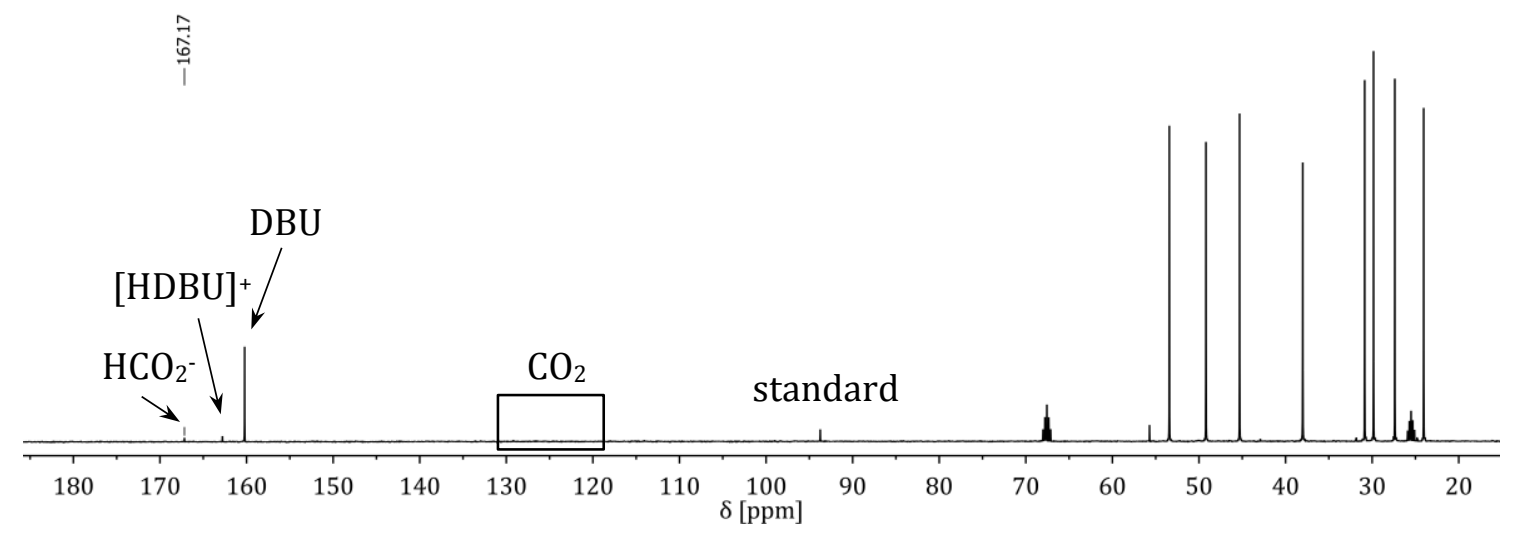

Figure 3.65: ${ }^{13} \mathrm{C}$ NMR spectrum during a reaction of $\mathrm{CO}_{2}$ and $\mathrm{H}_{2}$ with DBU catalyzed by $\mathbf{R h} \mathbf{h}^{t \mathrm{Bu}}$-MeCN in the presence of 1,3,5-trimethoxybenzene $(13.7 \mu \mathrm{mol})$ as internal standard, showing the formation of formate (THF- $\mathrm{d}_{8}, 298 \mathrm{~K}$ ). 


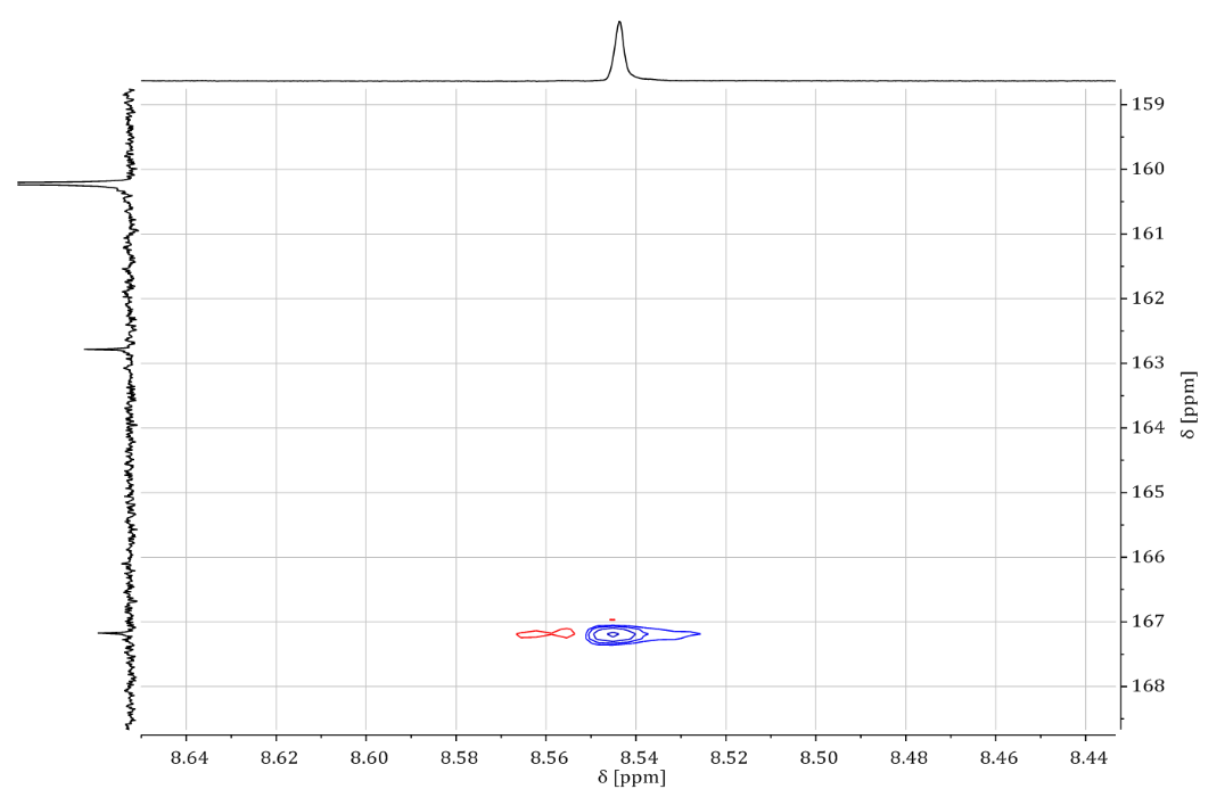

Figure 3.66: ${ }^{1} \mathrm{H}-13 \mathrm{C}$ HSQC NMR spectrum showing the correlation of resonances at $8.43 \mathrm{ppm}\left({ }^{1} \mathrm{H}\right)$ and $167.2 \mathrm{ppm}$ $\left({ }^{13} \mathrm{C}\right)$ corresponding to formate ions.

Interestingly, neither $\mathrm{H}_{2}(\delta=4.54 \mathrm{ppm})$ in a ${ }^{1} \mathrm{H}$ NMR nor $\mathrm{CO}_{2}(\delta=125.7 \mathrm{ppm})$ in a ${ }^{31} \mathrm{C} \mathrm{NMR}$ spectrum were observed in larger quantities during the NMR measurements, indicating a problem of the small volume of the high pressure NMR tube (Figure 3.65, box). LEWIS acids are known to increase the rate by enhancing the formate dissociation and this was also found in the catalysis of $\mathbf{R h}^{\text {tBu-MeCN }}$ (see Chapter 3.1.3).[283] The addition of 20 equivalents of lithium triflate (LiOTf) in relation to the precatalyst increased the conversion from 2.0 to 5.6 turnovers after $24 \mathrm{~h}$. Without $\mathbf{R h}^{\text {tBu}}$-MeCN no conversion was observed at all and again no changes of NMR resonances were observed, when only $\mathrm{H}_{2}$ or $\mathrm{CO}_{2}$ alone with DBU were mixed. Like in the case of the alkyne hydrogenation, this shows a fast reaction and very low concentrations of the reactive intermediates.

The reactions in the autoclave could be performed at higher pressure. $\mathrm{CO}_{2}$ hydrogenations to formate at high pressure (up to 100 atmospheres) and high temperatures $\left(80-120^{\circ} \mathrm{C}\right.$ ) are well-established and often used, whereas there are only few catalytic systems being active at a pressure lower than 10 atmospheres and ambient temperatures.[356,376,390] The catalysis was performed at different total pressures and a linear correlation of the conversion rate and the pressure was obtained (Figure 3.67). The observed turnover numbers and turnover frequencies are depicted in Table 3.10. The highest activity was achieved with lithium triflate as additive translating into a TON $=6.89 \pm 0.43$ after 6 hours and TOF $=1.15 \pm 0.07 \mathrm{~h}^{-1}$. 


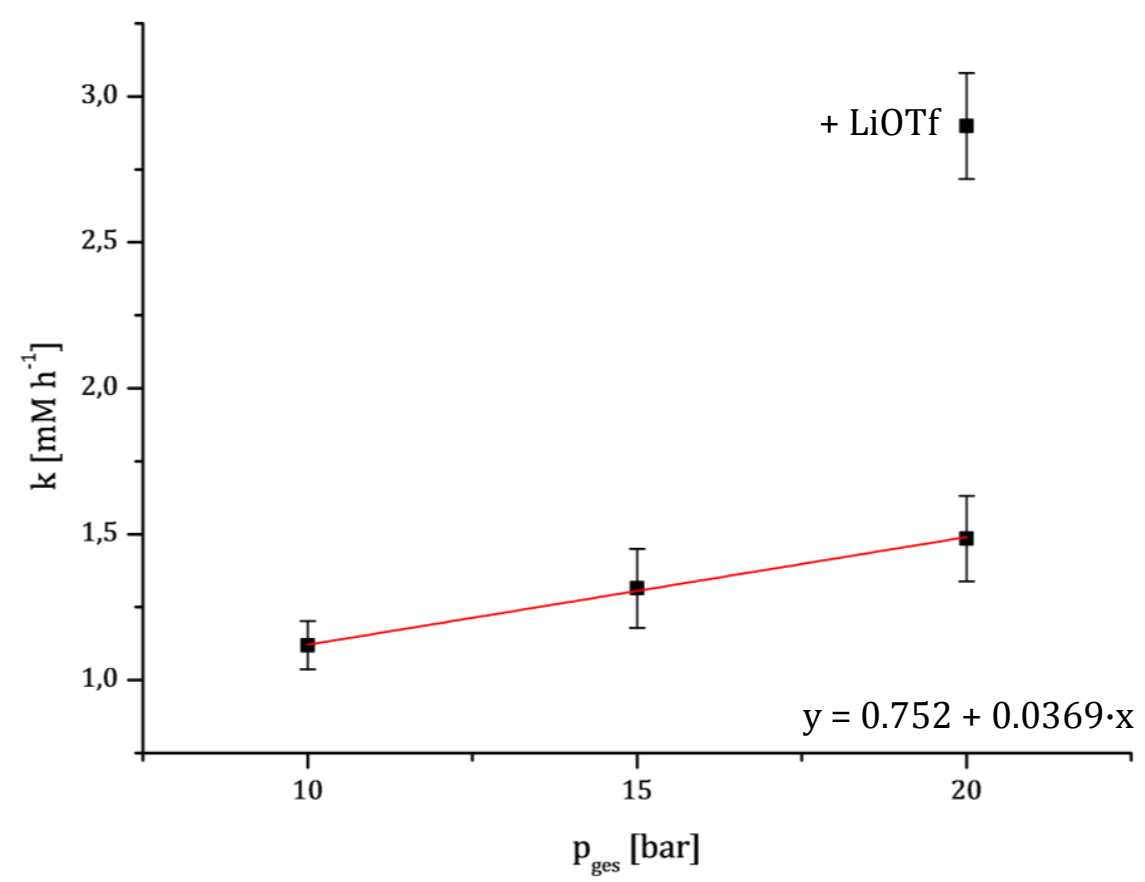

Figure 3.67: Correlation of the observed rates and total pressure pges of the reaction without additional LEWIS acid. The addition of LiOTf drastically increases the rate of conversion.

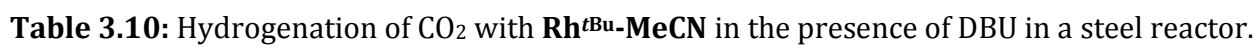

\begin{tabular}{|c|c|c|c|c|}
\hline $\begin{array}{c}\mathrm{CO}_{2} / \mathrm{H}_{2} \\
\mathrm{p}_{\text {ges }}\end{array}$ & $\begin{array}{c}+\mathrm{DBU} \\
250 \mu \mathrm{mol}\end{array}$ & $\frac{5 \mu \mathrm{mol} \mathbf{R h}^{\mathrm{tBu}}-\mathbf{M e C N}}{2 \mathrm{~mL} \text { THF }, 50^{\circ} \mathrm{C}, 6 \mathrm{~h}}$ & {$\left[\mathrm{HCO}_{2}\right]^{-}+$} & {$\left[_{\mathrm{HDBU}}\right]^{+}$} \\
\hline $\mathrm{p}_{\text {ges }}[\mathrm{bar}]$ & $\mathrm{k}\left[\mathrm{mM} \mathrm{h}^{-1}\right]$ & $\begin{array}{c}\text { final }\left[\mathrm{HCO}_{2}^{-}\right] \\
{[\mathrm{mM}]}\end{array}$ & TON & TOF $\left[\mathrm{h}^{-1}\right]$ \\
\hline 10 & $1.12 \pm 0.08$ & 6.72 & $2.62 \pm 0.19$ & $0.44 \pm 0.03$ \\
\hline 15 & $1.31 \pm 0.14$ & 7.89 & $3.15 \pm 0.32$ & $0.52 \pm 0.05$ \\
\hline 20 & $1.48 \pm 0.15$ & 8.91 & $3.48 \pm 0.34$ & $0.58 \pm 0.06$ \\
\hline $20^{a}$ & $2.90 \pm 0.18$ & 17.4 & $6.89 \pm 0.43$ & $1.15 \pm 0.07$ \\
\hline
\end{tabular}

a $66.7 \mu$ mol LiOTf added.

At a first glance, the observed values are very small in comparison to the most active, literature known catalysts,[390,393] but these catalysts were utilized under significantly harsher reaction conditions with much higher total pressures and often also at higher temperatures (Table 3.11). In general, it is difficult to compare different catalytic systems due to varying reaction conditions. 
Table 3.11: Selected examples of $\mathrm{CO}_{2}$ hydrogenation catalytic systems compared to $\mathbf{R h}^{t \mathrm{Bu}}-\mathbf{M e C N}$ (p [bar], $\mathrm{T}\left[{ }^{\circ} \mathrm{C}\right.$ ], $\mathrm{t}[\mathrm{h}]$ and final $\left.\left[\mathrm{HCO}_{2}^{-}\right][\mathrm{M}]\right)$.

\begin{tabular}{ccccccccc}
\hline $\begin{array}{c}\text { catalyst } \\
\text { precursora }\end{array}$ & solvent & $\begin{array}{c}\text { base/ } \\
\text { additive }\end{array}$ & $\begin{array}{c}\mathrm{p}\left(\mathrm{H}_{2} / \mathrm{CO}_{2}\right) \\
{[\text { bar }]}\end{array}$ & $\begin{array}{c}\mathrm{T} \\
{\left[{ }^{\circ} \mathrm{C}\right]}\end{array}$ & $\begin{array}{c}\mathrm{t} \\
{[\mathrm{h}]}\end{array}$ & TON & $\begin{array}{c}{\left[\mathrm{HCO}_{2}-\right]} \\
{[\mathrm{M}]}\end{array}$ & ref. \\
\hline $\mathbf{R h}^{\text {tBu-MeCN }}$ & $\mathrm{THF}$ & $\begin{array}{c}\text { DBU/ } \\
\text { LiOTf }\end{array}$ & $10 / 10$ & 50 & 6 & 6.9 & 0.017 & - \\
\hline $\mathrm{Ir}(\mathrm{H})_{3} \mathrm{~L}^{1}$ & $\begin{array}{c}\mathrm{H}_{2} \mathrm{O} / \\
\mathrm{THF}\end{array}$ & $\mathrm{KOH}$ & $30 / 30$ & 120 & 48 & $3.5 \cdot 106$ & 0.686 & {$[393]$} \\
\hline $\begin{array}{c}\mathrm{Ir}\left(\mathrm{Cp}^{*}\right)(\mathrm{Cl}) \\
\mathrm{L}^{2}\end{array}$ & $\mathrm{H}_{2} \mathrm{O}$ & $\mathrm{KOH}$ & $10 / 10$ & 80 & 30 & 11000 & 0.22 & {$[394]$} \\
\hline $\begin{array}{c}\mathrm{Fe}(\mathrm{H})(\mathrm{CO}) \\
\left(\mathrm{BH}_{4}\right) \mathrm{L}^{3}\end{array}$ & $\mathrm{THF}$ & $\begin{array}{c}\text { DBU/ } \\
\text { LiOTf }\end{array}$ & $35 / 35$ & 80 & 24 & 58990 & 3.54 & {$[390]$} \\
\hline $\mathrm{Rh}\left(\mathrm{L}^{4}\right)_{2} \mathrm{BF}_{4}$ & $\mathrm{THF}$ & $\begin{array}{c}\text { VERKADE's } \\
\text { base }\end{array}$ & $1 / 1$ & 21 & 1 & 406 & 2.79 & {$[469]$} \\
\hline
\end{tabular}

${ }^{a} \mathrm{~L}^{1}=2,6-\left(\mathrm{CH}_{2} \mathrm{P}\left({ }^{i} \mathrm{Pr}\right)_{2}\right)_{2} \mathrm{C}_{5} \mathrm{H}_{3} \mathrm{~N}, \mathrm{~L}^{2}=4,4^{\prime}$-dihydroxy-2,2'-bipyridine, $\mathrm{L}^{3}=\mathrm{HN}\left(\mathrm{CH}_{2} \mathrm{CH}_{2} \mathrm{P}\left({ }^{i} \mathrm{Pr}\right)_{2}\right)_{2}, \mathrm{~L}^{4}=1,5$-diaza-3,7diphospha-cyclootane derivative. ${ }^{\mathrm{b}}$ 2,8,9-triisobutyl-2,5,8,9-tetraaza-1-phospha-bicyclo[3.3.3] undecane.

To investigate the mechanism of the reaction an experiment with a low DBU loading was performed and indeed an intermediate could be detected by ${ }^{31} \mathrm{P}$ NMR spectroscopy. During the

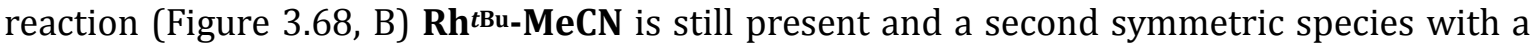
doublet at $81.66 \mathrm{ppm}$ featuring a coupling constant of a rhodium(I) compound $U_{\text {Rh-P }}=190.0 \mathrm{~Hz}$ ) was observed. After full conversion of the DBU, a single doublet at $98.84 \mathrm{ppm}$

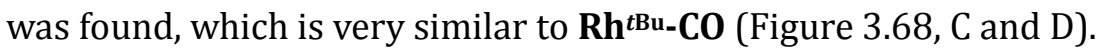

D $\quad\left\lfloor\begin{array}{l}\text { RhtBu-CO } \\ \delta=99.50 \mathrm{ppm} \\ J_{\text {Rh-P }}=148.2 \mathrm{~Hz}\end{array}\right.$

\begin{tabular}{l|l} 
C: after reaction & $\begin{array}{l}\delta=98.84 \mathrm{ppm} \\
J_{\mathrm{Rh}-\mathrm{P}}=149.0 \mathrm{~Hz}\end{array}$ \\
&
\end{tabular}

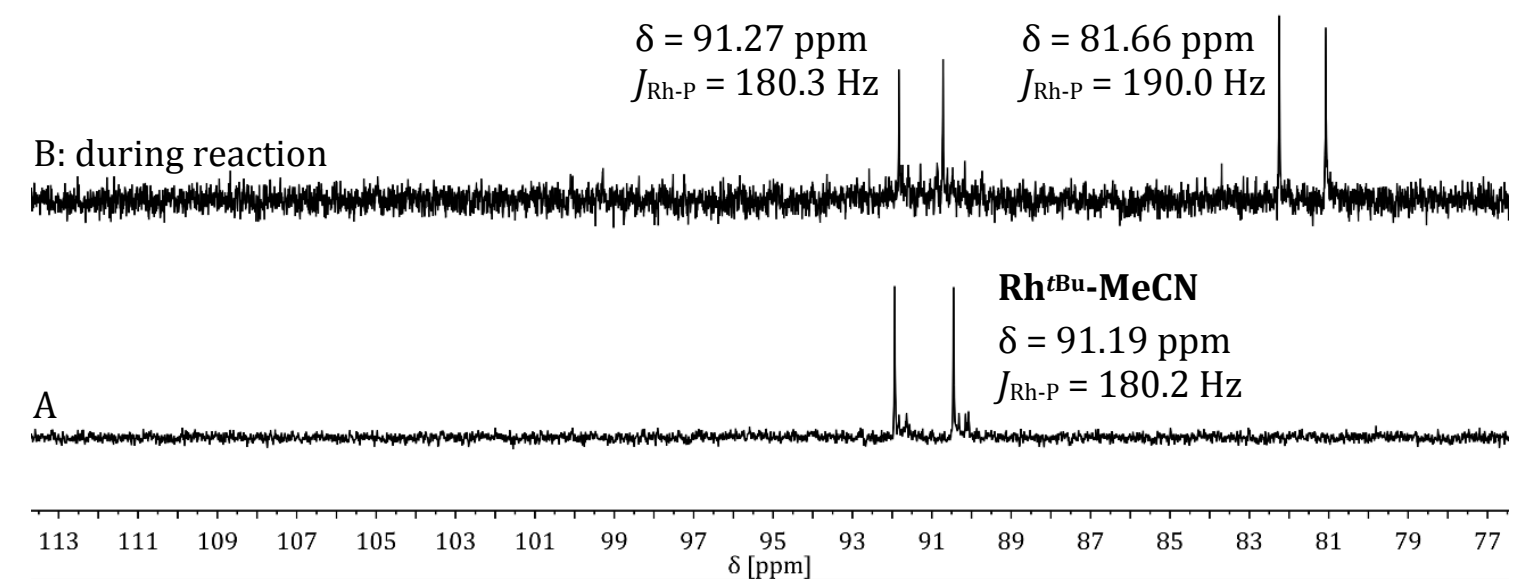

Figure 3.68: ${ }^{31} \mathrm{P}$ NMR spectra with chemical shifts and coupling constants $J_{\mathrm{Rh}-\mathrm{P}}$ of precatalyst $\mathbf{R h}^{t \mathrm{Bu}}-\mathbf{M e C N}(\mathrm{A}$, THF-d $8,500 \mathrm{MHz}$ ), the reaction mixture during catalysis (B, THF- $\mathrm{d}_{8}, 400 \mathrm{MHz}$ ), the reaction mixture after catalysis (C, THF- $\mathrm{d}_{8}, 400 \mathrm{MHz}$ ) and $\mathbf{R h}^{\mathrm{tBu}}-\mathrm{CO}\left(\mathrm{D}, \mathrm{MeCN}-\mathrm{d}_{3}, 500 \mathrm{MHz}\right.$ ) at ambient temperatures. 
The coupling constant $J_{\mathrm{Rh}-\mathrm{P}}$ of the carbonyl complexes was found before to be significantly lower than $J_{\text {Rh-P }}$ for the other rhodium(I) complexes (see Chapter 3.4.4.1). Although the data was collected in different solvents and the chemical shifts were not exactly the same as for the reference complexes, it was assumed that the final product of the catalyst was the rhodium(I) carbonyl complex. This was supported by a comparison of the reaction product's ${ }^{1} \mathrm{H}$ NMR spectrum and that of $\mathbf{R h}^{\mathrm{tBu}}$-CO (Figure 3.69). Similar chemical shifts were found for the tertbutyl, the pyrazoles and the pyridine resonances. The signal of the $\mathrm{CH}_{2}$ group is shifted to lower field after the reaction which might be a result of the use of different solvents for recording the spectra.

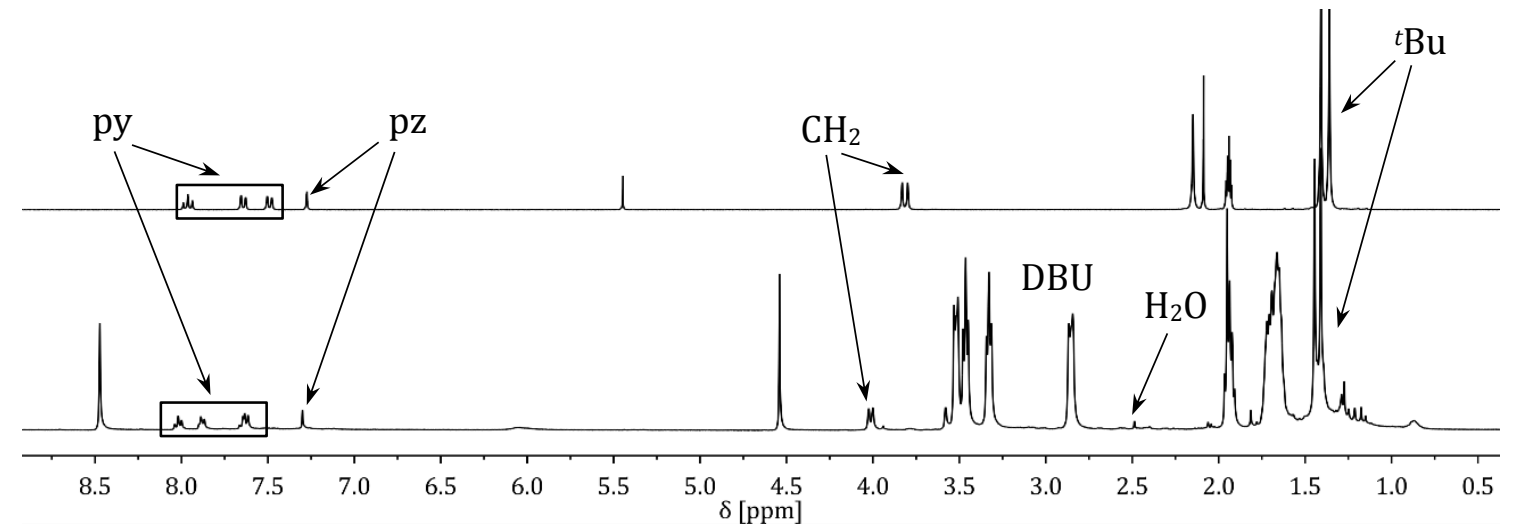

Figure 3.69: Comparison of the ${ }^{1} \mathrm{H}$ NMR spectra of $\mathbf{R h}{ }^{t \mathrm{Bu}}-\mathbf{C O}$ (top, MeCN- $\mathrm{d}_{3}$ ) and the reaction product of a $\mathrm{CO}_{2}$ hydrogenation catalysis (bottom, THF- $\mathrm{d}_{8}$ ) at ambient temperatures.

Metal assisted reduction of $\mathrm{CO}_{2}$ to $\mathrm{CO}$ can occur via several different pathways: A reductive disproportionation by the transfer of an oxygen atom to form carbonates and metal carbonyl species, [470,471] the reverse water gas shift reaction (reductive cleavage of $\mathrm{CO}_{2}$ to $\mathrm{CO}$ and $\mathrm{H}_{2} \mathrm{O}$, WGSR) ${ }^{[472,473]}$ or an oxygen transfer reaction to metal hydrides to afford intermediary metal formate complexes which can disproportionate to carbonyl complexes.[474-476] An interesting example for reductive cleavage of $\mathrm{CO}_{2}$ was reported by MILSTEIN, featuring metal ligand cooperativity.[477] An iridium hydride complex reductively cleaved carbon dioxide to form a dearomatized iridium carbonyl complex and water. This MLC mediated reaction is also conceivable for the conversion of $\mathbf{R h}^{t \mathrm{Bu}-M e C N}$ to $\mathbf{R} \mathbf{h}^{t \mathrm{Bu}}-\mathbf{C O}$ because water (ca. 0.5 eq in relation to the complex pz resonance) was found in the ${ }^{1} \mathrm{H}$ NMR spectrum after the reaction (Figure 3.69, bottom). This suggests the occurrence of two presented pathways, namely a reverse WGSR or a reductive cleavage with MLC. Further studies are needed to identify the intermediate and to investigate the possibility of such a pathways involving MLC for the formation of $\mathbf{R h}^{t \mathrm{Bu}}-\mathbf{C O}$ from catalysis of $\mathbf{R} \mathbf{h}^{t \mathrm{Bu}}-\mathbf{M e C N}$ with carbon dioxide and dihydrogen. 


\subsection{Summary}

In conclusion, the Two-in-one pincer ligand system was successfully extended to the iso-propyl analogue $\mathbf{H L}^{\text {iPr }}$ while the attempts to introduce other phosphine substituents proved the complexity of the ligand synthesis so that new strategies have to be developed for other than alkyl substituents. In addition, the yields of the established ligand precursor compounds were increased.

Different zinc(II) complexes of the Two-in-one pincer ligands $\mathbf{H L}^{t \mathbf{B u}}$ and $\mathbf{H L}^{\mathbf{i P r}}$ were synthesized and characterized. Further reactions and catalytic applications of these complexes were tested and some promising results were obtained as for example the zinc methyl complex $\mathbf{5 2}$ after reaction with $\mathrm{MeMgBr}$. Isolation of this zinc alkyl complex might lead to more active catalysts for the ring opening polymerization of $r a c$-lactide.

The reactivity of the cobalt MeCN complex $\mathbf{5 4}$ was found to be multifarious, but the isolation of any products after reactions with superhydride ${ }^{\circledR}$, strong bases, reductants or sodium borohydride failed to date. In all cases, reactions were observed by fast color changes of the reaction mixtures upon addition of reactant. Due to a very limited tool kit of suitable analytic methods for these cobalt complexes, accurate structural information of reaction products remained uncertain.

Rhodium(I) complexes of the Two-in-one pincer ligand system were synthesized with different monodentate and chelating coligands (e.g. Rh ${ }^{t \mathrm{Bu}}-\mathbf{C O}$ and $\mathbf{R h}^{t \mathrm{Bu}}-\mathbf{M e C N}$ ). Besides a full characterization including X-ray diffraction analysis, the ligand flexibility and exchange

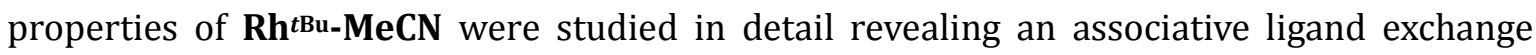
mechanism with MeCN- $\mathrm{d}_{3}$. The thermodynamics of the interconversion between the two

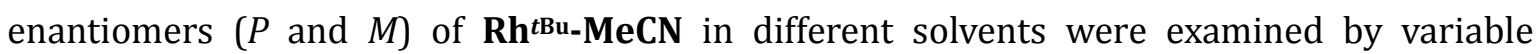
temperature ${ }^{1} \mathrm{H}$ NMR spectroscopy. Oxidative addition could be performed with methylene chloride and methyl iodide. In both cases a stepwise formation of the $\mathrm{Rh}^{\mathrm{III}} \mathrm{Rh}^{\mathrm{III}}$ species was shown by either ${ }^{31} \mathrm{P}$ NMR spectroscopy or ESI mass spectrometry.

A dearomatization/aromatization effect was found in $\mathbf{R h}{ }^{\text {tBu}}$-CO. Although the crystallization of the formed mono- and twice-deprotonated species failed, their existence was clearly indicated by NMR spectroscopy. The pyridine resonances were shifted to higher field by about $2 \mathrm{ppm}$ upon deprotonation of the methylene side arm with a strong base. $\mathbf{R h}^{\mathrm{tBu}}-\mathbf{M e C N}$, with little more labile ligands, was utilized successfully for hydrogenation reactions with dihydrogen gas. The cis hydrogenation of phenyl acetylenes at mild conditions was investigated in detail by determination of the reaction orders, KIE and initial rates $\mathrm{k}_{\mathrm{obs}}$. 
Finally and excitingly, the hydrogenation of carbon dioxide to formate proved to be possible with $\mathbf{R h}^{t \mathrm{Bu}}-\mathbf{M e C N}$ under relatively mild conditions. The reaction was followed by NMR spectroscopy and at least one intermediate was observed. However, the identity of this species could not be revealed so far. After the complete consumption of DBU, presumably $\mathbf{R h}^{\mathrm{tBu}-\mathrm{CO}}$ was found as only rhodium complex. The mechanism of the $\mathrm{CO}$ formation remained uncertain. 


\section{Conclusion}

\subsection{Summary}

Different strategies and metal complexes with different ligand scaffolds were used to investigate on the one hand their reactivity towards the activation of small molecules and on the other hand the properties of potential intermediates of these reactions.

First of all, a new tetraamine ligand $\mathbf{H}_{5} \mathbf{L}^{\text {Me }}$ was successfully synthesized. However, complexation reactions with various metal precursors failed, probably due to a complicated deprotonation behavior and steric interactions of the aryl substituents on the amine/amide side arms.

Furthermore, the synthesis of nickel(II) complexes of the macrocyclic tetracarbene ligand system was shown, while the analoguous copper and manganese complexes were not observed, presumably due to ligand decomposition. These new nickel complexes proved to be very stable towards both, oxidation and reduction. Targeted, high valent metal species could not be isolated. With $\mathrm{XeF}_{2}$ as strong oxidant a reaction was observed and a nickel-based radical could be detected by EPR spectroscopy. The low stability hampered the isolation and further characterization of the product.

In the next step, new Two-in-one pincer ligands and their metal complexes have been synthesized. Zinc(II), cobalt(II) and rhodium(I) complexes with different coligands were isolated and fully characterized. Zinc compexes could be crystallized and were used to compare

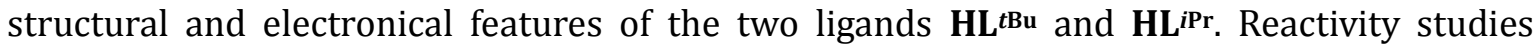
revealed a broad range of possible reactions of the cobalt(II) complex $\mathbf{C o}^{t \mathrm{Bu}}-\mathbf{M e C N}$ varying from dearomatization to reduction, but the range of spectroscopic tools suitable for analysis was too small to gain structural information about the reaction products. Crystallization attempts did not lead to single crystals suitable for X-ray diffraction analysis. Furthermore, several rhodium(I) complexes have been synthesized and characterized. For the rhodium(I) carbonyl complex $\mathbf{R h}{ }^{t \mathrm{Bu}}$-CO a dearomatization sequence was evidenced by NMR spectroscopy. Upon stepwise addition of a strong base, the pyridine and pyrazole resonances were shifted to higher field, attributed to the stepwise dearomatization of both pyridine rings of the ligand. This effect, which was first discovered for mononuclear, pyridine-based pincer complexes by MILSTEIN, is also found in the dinuclear Two-in-one pincer complex $\mathbf{R h}^{\text {tBu-CO }}$ used in this work. Complexes with $\mathrm{MeCN}$ as coligands can undergo oxidative addition with methylene chloride and methyl iodide to form dinuclear rhodium(III) alkyl halide complexes. The reaction with dihydrogen did 
not occur under the tested reaction conditions. Nevertheless, the rhodium(I) complexes were tested for hydrogenation catalysis of alkynes and carbon dioxide. $\mathbf{R h}^{\text {tBu}} \mathbf{- M e C N}$ showed the highest activity among the series of rhodium complexes of this work. Mechanistic aspects of the hydrogenation of terminal phenyl acetylenes were investigated, although intermediates could not be observed.

Exciting first results were obtained for the hydrogenation of carbon dioxide to formate salts in the presence of base. The reactions proceed under relatively mild pressure and temperature conditions compared to literature known systems. After the reaction, presumably $\mathbf{R h}{ }^{t \mathrm{Bu}}$-CO was found as single metal species, although the formation of carbon monoxide could not be explained so far.

\subsection{Prospective}

Studies regarding the mechanism of the hydrogenation catalysis with $\mathbf{R h}^{\mathrm{tBu}}-\mathbf{M e C N}$ should be continued in order to optimize the reaction conditions and gain further information, for example about the rate determining step. With this information, optimization of the ligand substitution on the phosphorus side arms should be a possibility to obtain higher activities. Additionally, the exchange of the MeCN coligands by more labile monodentate ligands should increase the decoordination of the coligands and with that increase the amount of active species in the reaction mixtures. The variation of the coordination environment around the rhodium centers (different ligands and coligands) might also lead to a higher stability of intermediates such as a potential dihydride complex after the oxidative addition of dihydrogen, allowing a characterization by X-ray diffraction analysis. Increased crystallization properties of the ligand should also facilitate the identification of reaction products of the cobalt complex reactivity studies. The prove of dearomatization of the pyridine ring by X-ray crystallography is highly desired in order to investigate the structural changes (bond lengths) upon deprotonation. The activation of substrates with dearomatized complexes with subsequent coordination of the substrate to the metal centers is an interesting effect (metal ligand cooperativity, MLC), which might be transferred to catalytic applications. The combination of MLC effect and metal metal cooperativity of pyrazolate-bridged dinuclear complexes (MMC) in a well-defined catalyst might lead to a beneficial synergism. Especially the use of heterobimetallic complexes have been shown to exhibit MMC properties.[478,479] Thus, the synthesis of heterobimetallic complexes of the Two-in-one pincer scaffold is a promising goal. For example, the use of a LEWIS acidic metal such as zinc, which activates the substrate, and a redox-active metal like cobalt(II) or rhodium(I), at which the reaction proceeds. 
Due to its relevance for transforming today's fossil fuel-base economy into an economy based on renewable energy resources, the hydrogenation of carbon dioxide has to be investigated in more detail. Reaction conditions have to be optimized and potential intermediates isolated in order to obtain a deeper understanding of the reaction. This might help to design more efficient catalysts for the use under relatively mild reaction conditions. The use of different solvents like DMSO for stabilizing formic acid should be tried. For large scale applications not only the activity of a catalyst is important, but also the stability of the complex. In this regard, positive results were obtained for $\mathbf{R h}^{\text {tBu}}$-MeCN in the hydrogenation of alkynes and this aspect should be investigated in more detail. 



\section{Experimental Section}

\subsection{General considerations}

All experiments were carried out under a dry atmosphere of purified dinitrogen in MBRAUN glove-boxes (Labmaster and Unilab) or using standard SCHLENK techniques under an atmosphere of purified argon unless otherwise noted. All solvents were used in reagent grade or better. Non-deuterated solvents were dried, distilled under an argon atmosphere and degassed with argon prior to use: $\mathrm{Et}_{2} \mathrm{O}$, THF, benzene, pentane and toluene over sodium/benzophenone ketyl; acetone over $\mathrm{B}_{2} \mathrm{O}_{3} ; \mathrm{MeOH}$, EtOH and ${ }^{i} \mathrm{PrOH}$ over Mg. Acetone- $d_{6}$ was refluxed over $\mathrm{B}_{2} \mathrm{O}_{3}$ and distilled under argon atmosphere. Other deuterated solvents were dried and stored over activated molecular sieves (3 Å). All deuterated solvents were freezepump-thaw degassed. Commercially available reagents were used as received.

\subsection{Methods}

NMR spectroscopy

NMR spectra were recorded on a Bruker Avance III HD $500\left({ }^{1} \mathrm{H} 500 \mathrm{MHz},{ }^{13} \mathrm{C} 126 \mathrm{MHz},{ }^{31} \mathrm{P}\right.$ $202 \mathrm{MHz}$ ), a Bruker Avance III HD 400, a Bruker Avance III 400 (both: ${ }^{1} \mathrm{H} 400 \mathrm{MHz},{ }^{13} \mathrm{C} 100 \mathrm{MHz}$, ${ }^{31} \mathrm{P} 162 \mathrm{MHz}$ ) and a Bruker Avance III $300\left({ }^{1} \mathrm{H} 300 \mathrm{MHz},{ }^{13} \mathrm{C} 75 \mathrm{MHz},{ }^{31} \mathrm{P} 121 \mathrm{MHz}\right.$ ) spectrometers. All spectra were measured at $298 \mathrm{~K}$ unless otherwise mentioned. ${ }^{1} \mathrm{H}$ NMR and ${ }^{13} \mathrm{C}\left\{{ }^{1} \mathrm{H}\right\}$ NMR chemical shifts are reported in parts per million downfield from tetramethylsilane were referenced to the residual hydrogen or carbon signals of the deuterated solvents according to GOLDBERG et al.[480] 31P chemical shifts are reported relative to external reference phosphoric acid $(\delta=0 \mathrm{ppm})$. Abbreviations used in the description of NMR data are as follows: b, broad; s, singlet; d, doublet; t, triplet; m, multiplet; py, pyridine; pz, pyrazole; Im imidazole; Ar aryl.

EPR spectroscopy

EPR spectra were recorded on a X band BRUKER ELEXSYS E500 spectrometer, equipped with an ER049 X microwave bridge, an ER083 CS magnet and a temperature control system ER4131 VT for liquid nitrogen as coolant. The microwave frequency was measured with a HEWLETTPACKARD frequency counter HP532B and the field control was calibrated by a BRUKER NMR field probe ER035M. 
IR spectroscopy

IR spectra were measured on an AGILENT TeChnologies Cary 630 FTIR. Intensities of the observed bands in the spectra are abbreviated as follows: s (strong), m (medium), w (weak).

\section{UV-vis spectroscopy}

UV-vis spectra of solutions were recorded on VARIAN Cary 60 and Cary 5000 (UV/vis-NIR) spectrometers using quartz cuvettes $(\mathrm{d}=1 \mathrm{~cm}$, HELLMA Analytics). For low temperature experiments a UNISOKU CoolSpek UV (USP-203-A) cryostat was used.

\section{Mass spectrometry}

ESI-MS spectra were recorded on a BRUKER HCT Ultra connected to an argon glovebox or a BRUKER maXis ESI-QTOF, EI-MS spectra on a FINNIGAN MAT 8200; MALDI experiments were conducted on a BRUKER 7 Tesla-Fourier Transform Ion Cyclotron Resonance (FTICR) mass spectrometer using a DCTB (trans-2-[3-(4-tert-butylphenyl)-2-methyl-2-propenylidene]malononitrile) matrix.

\section{Electrochemistry}

Redox potentials were determined by measurement of cyclic voltammograms using a PERKINELMER 263A potentiostat. The samples were measured using a glassy carbon working electrode, a platinum wire as counter electrode and a silver wire as pseudo-reference electrode in $0.1 \mathrm{MnBu} \mathrm{NPF}_{6} \mathrm{MeCN}$ solutions if not otherwise mentioned. Ferrocene $\left(\mathrm{Cp}_{2} \mathrm{Fe}, \mathrm{Fc}\right)$ or decamethylferrocene $\left(\mathrm{Cp}_{2}{ }_{2} \mathrm{Fe}, \mathrm{DMFc}\right)$ were used as internal reference. All potentials were referenced versus the ferrocene couple $\left(\mathrm{Fc} / \mathrm{Fc}^{+}\right)$using $\mathrm{E}_{1 / 2}(\mathrm{DMFc})=-507 \mathrm{mV}$ vs $\mathrm{Fc} / \mathrm{Fc}^{+}$.

\section{Elemental Analysis}

Elemental analyses were performed by the 'Analytical Laboratory' of the Institute of Inorganic Chemistry at Georg-August University using an ElEMENTAR Vario EL III instrument.

X-ray Diffraction Analysis

Elemental analyses X-ray data were collected on a STOE IPDS II diffractometer with an area detector (graphite monochromated Mo-K $\alpha$ radiation, $l=0.71073 \AA$ ) by use of $w$ scans at $133 \mathrm{~K}$. 136 
The structures were solved by direct methods (SHELXS-97) and refined on $\mathrm{F}^{2}$ using all reflections with SHELXL-97.[481] Non-hydrogen atoms were refined anisotropically. Most hydrogen atoms were placed in calculated positions and assigned to an isotropic displacement parameter of 1.2/1.5 $\mathrm{U}_{\mathrm{eq}}(\mathrm{C})$. Faceindexed absorption corrections were performed numerically with the program X-RED.[482] For further crystallographic details see Appendix 6.2. 


\subsection{Tetraamine ligand $\mathrm{H}_{5} \mathrm{~L}^{\mathrm{Me}}$}

3,5-Bis-(chloromethyl)-1-tetrahydropyran-2-yl-pyrazole was synthesized according to literature procedure, ${ }^{[70,71]}$ compounds $\mathbf{I V}^{[483]}$ and $\mathbf{V I I}{ }^{[72]}$ similar to reported procedures.

\subsubsection{Ligand Synthesis}

\subsubsection{N-(tert-Butyloxycarbonyl)-iminodiacetic acid (IV)}

To a solution of iminodiacetic acid III $(20.0 \mathrm{~g}, 150 \mathrm{mmol}, 1.0 \mathrm{eq})$ and sodium hydroxide $(12.0 \mathrm{~g}$, $300 \mathrm{mmol}, 2.0 \mathrm{eq})$ in a 1:1 mixture of 1,4-dioxane and water (300 mL) di-tert-butyl dicarbonate (37.9 mL, $165 \mathrm{mmol}, 1.1 \mathrm{eq}$ ) was added. The reaction mixture was stirred at ambient temperature for 6 days and then washed with $\mathrm{Et}_{2} \mathrm{O}(150 \mathrm{~mL})$. The solution was acidified with aqueous 2M hydrochloric acid (ca. $160 \mathrm{~mL}$ ) to a pH value of 1 and extracted with EtOAc (4 x $100 \mathrm{~mL}$ ). The combined organic layers were washed with saturated, aqueous $\mathrm{NaCl}$ solution (100 $\mathrm{mL}$ ), dried over $\mathrm{MgSO}_{4}$ and the solvent removed under reduced pressure. After drying the colorless oil over night in vacuo, compound IV (31.5 g, $135 \mathrm{mmol}, 90 \%$ ) was obtained as a white solid.

Formula (molecular weight) $\quad \mathrm{C}_{9} \mathrm{H}_{15} \mathrm{NO}_{6}(233.2 \mathrm{~g} / \mathrm{mol})$

${ }^{1} \mathbf{H}$ NMR $\left(300 \mathrm{MHz}\right.$, acetone- $\left.\mathrm{d}_{6}\right) \delta(\mathrm{ppm})=5.88\left(\mathrm{~s}_{\mathrm{br}}, 2 \mathrm{H}, \mathrm{CO}_{2} \mathrm{H}\right), 4.11\left(\mathrm{~s}, 2 \mathrm{H}, \mathrm{CH}_{2}\right), 4.07(\mathrm{~s}, 2 \mathrm{H}$, $\left.\mathrm{CH}_{2}\right), 1.42\left(\mathrm{~s}, 9 \mathrm{H}, \mathrm{C}\left(\mathrm{CH}_{3}\right)_{3}\right)$.

${ }^{13} \mathrm{C} \mathrm{NMR} \quad\left(75 \mathrm{MHz}, \quad\right.$ acetone- $\left.\mathrm{d}_{6}\right) \delta(\mathrm{ppm})=172.3(\mathrm{COOH}), \quad 171.9(\mathrm{COOH}), \quad 155.8(\mathrm{CO})$, $81.1\left(\mathrm{C}\left(\mathrm{CH}_{3}\right)_{3}\right), 50.8\left(\mathrm{CH}_{2}\right), 50.1\left(\mathrm{CH}_{2}\right), 28.3\left(\mathrm{C}\left(\mathrm{CH}_{3}\right)_{3}\right)$.

MS (EI) m/z (\%) = $233\left(16,[\mathrm{M}]^{+}\right), 177\left(90,\left[\mathrm{M}-\mathrm{C}\left(\mathrm{CH}_{3}\right)_{3}+\mathrm{H}\right]^{+}\right), 160\left(100,\left[\mathrm{M}-\mathrm{O}^{t} \mathrm{Bu}\right]^{+}\right)$.

\subsubsection{N-tert-Butyloxycarbonyl-bis-(N-(3,5-dimethylphenyl)-2-acetamido)-amine (V)}

A solution of IV $(11.7 \mathrm{~g}, 50.0 \mathrm{mmol}, 1.0 \mathrm{eq})$ and 3,5-dimethylaniline $(18.71 \mathrm{~mL}, 150 \mathrm{mmol}$, $3.0 \mathrm{eq})$ in THF ( $80 \mathrm{~mL})$ was cooled in an ice bath. A solution of $N, N^{\prime}$-dicyclohexylcarbodiimide ( $30.9 \mathrm{~g}, 150 \mathrm{mmol}, 3.0 \mathrm{eq}$ ) was added slowly over $1.5 \mathrm{~h}$. The reaction mixture was stirred at $0{ }^{\circ} \mathrm{C}$ for $2.5 \mathrm{~h}$ and at ambient temperature overnight. The suspension was filtered and the residue washed with THF ( $50 \mathrm{~mL})$. The filtrate was concentrated to a volume of ca. $50 \mathrm{~mL}$, layered with $\mathrm{Et}_{2} \mathrm{O}(300 \mathrm{~mL})$ and stored at $-20{ }^{\circ} \mathrm{C}$ overnight. The analytically pure product was collected by filtration. After drying in vacuo compound V (12.3 g, $28.0 \mathrm{mmol}, 56 \%)$ was obtained as a white solid. 
Formula (molecular weight) $\quad \mathrm{C}_{25} \mathrm{H}_{33} \mathrm{~N}_{3} \mathrm{O}_{4}(439.6 \mathrm{~g} / \mathrm{mol})$

${ }^{1}$ H NMR $\left(300 \mathrm{MHz}, \mathrm{CDCl}_{3}\right) \delta(\mathrm{ppm})=10.65(\mathrm{~s}, 1 \mathrm{H}, \mathrm{NH}), 9.02(\mathrm{~s}, 1 \mathrm{H}, \mathrm{NH}), 7.43(\mathrm{~s}, 2 \mathrm{H}, \mathrm{Ar} 2-\mathrm{H})$, 7.14 (s, 2H, Ar 2-H), 6.77 (s, 1H, Ar 4-H), 6.65 (s, 1H, Ar 4-H), 4.11 (s, 2H, CH $), 4.00$ (s, 2H, CH $\mathrm{CH}_{2}$, $2.32\left(\mathrm{~s}, 6 \mathrm{H}, \mathrm{Ar}-\mathrm{CH}_{3}\right), 2.22\left(\mathrm{~s}, 6 \mathrm{H}, \mathrm{Ar}-\mathrm{CH}_{3}\right), 1.42\left(\mathrm{~s}, 9 \mathrm{H}, \mathrm{C}\left(\mathrm{CH}_{3}\right)_{3}\right)$.

${ }^{13} \mathrm{C}$ NMR $\left(75 \mathrm{MHz}, \mathrm{CDCl}_{3}\right) \delta(\mathrm{ppm})=168.6(\mathrm{Ar}-\mathrm{CO}-\mathrm{N}), 168.1(\mathrm{Ar}-\mathrm{CO}-\mathrm{N}), 155.5(\mathrm{CO}), 138.6(\mathrm{Ar}$ C-2), 138.4 (Ar C-1), 138.3 (Ar C-2), 137.5 (Ar C-1), 126.0 (Ar C-4), 117.9 (Ar C-3), 117.7 (Ar C-3), $82.4\left(C\left(\mathrm{CH}_{3}\right)_{3}\right), 55.7\left(\mathrm{CH}_{2}\right), 54.5\left(\mathrm{CH}_{2}\right), 28.3\left(\mathrm{C}\left(\mathrm{CH}_{3}\right)_{3}\right), 21.6\left(\mathrm{ArCH}_{3}\right), 21.4\left(\mathrm{ArCH}_{3}\right)$.

\subsubsection{Bis-(N-(3,5-dimethylphenyl)-2-acetamido)-amine (VI)}

To a solution of $\mathbf{V}(12.3 \mathrm{~g}, 28.0 \mathrm{mmol})$ in a 1:1 mixture of MeCN and DMF (100 mL) concentrated hydroclorid acid $(50 \mathrm{~mL})$ was slowly added. The reaction mixture was stirred at ambient temperature for 3 days and neutralized with aqueous $\mathrm{NaOH}$ (ca. $25 \mathrm{~g}$ in $80 \mathrm{ml}$ ). After addition of $\mathrm{CH}_{2} \mathrm{Cl}_{2}(100 \mathrm{~mL})$ the layers were separated and the aqueous layer was extracted with $\mathrm{CH}_{2} \mathrm{Cl}_{2}$ $(2 \times 75 \mathrm{~mL})$. The combined organic layers were washed with water $(100 \mathrm{~mL})$, dried over $\mathrm{MgSO}_{4}$, filtered and the solvent removed under reduced pressure. The off-white crude product was recrystallized from EtOH. Compound VI (8.97 g, $26.3 \mathrm{mmol}, 53 \%)$ was obtained as white plates.

Formula (molecular weight) $\mathrm{C}_{20} \mathrm{H}_{25} \mathrm{~N}_{3} \mathrm{O}_{2}(339.4 \mathrm{~g} / \mathrm{mol})$

${ }^{1}$ H NMR (300 MHz, DMSO-d 6 ) $\delta(\mathrm{ppm})=9.87(\mathrm{~s}, 2 \mathrm{H}, \mathrm{NH}), 7.21(\mathrm{~s}, 4 \mathrm{H}, \mathrm{Ar} \mathrm{C}-2), 6.69(\mathrm{~s}, 2 \mathrm{H}, \mathrm{Ar}$ C-4), 3.33 (s, 4H, $\mathrm{CH}_{2}$ ), 2.20 (s, $12 \mathrm{H}, \mathrm{Ar}_{-} \mathrm{CH}_{3}$ ).

${ }^{13}$ C NMR (75 MHz, DMSO-d 6 ) $\delta(\mathrm{ppm})=170.6(C O), 138.7$ (Ar C-1), 138.2 (Ar C-2), 125.4 (Ar $\mathrm{C}-4), 117.5(\mathrm{Ar} \mathrm{C}-3), 53.1\left(\mathrm{CH}_{2}\right), 21.4\left(\mathrm{Ar}-\mathrm{CH}_{3}\right)$.

\subsubsection{3,5-Bis-(iodomethyl)-1-tetrahydropyran-2-yl-pyrazole (VII)}

A solution of 3,5-Bis-(chloromethyl)-1-tetrahydropyran-2-yl-pyrazole I (6.00 g, $24.2 \mathrm{mmol}$, $1.0 \mathrm{eq})$ in acetone $(100 \mathrm{~mL})$ was stirred over potassium iodide $(16.0 \mathrm{~g}, 96.8 \mathrm{mmol}, 4.0 \mathrm{eq})$ at ambient temperature for 3 days. The suspension was filtered and the solvent of the filtrate was removed under reduced pressure. The residue was taken up in $\mathrm{CH}_{2} \mathrm{Cl}_{2}(50 \mathrm{~mL})$ and washed with aqueous $\mathrm{Na}_{2} \mathrm{~S}_{2} \mathrm{O}_{3}$ (8 g in $40 \mathrm{~mL}$ ). The aqueous layer was extracted with $\mathrm{CH}_{2} \mathrm{Cl}_{2}(40 \mathrm{~mL})$. The combined organic layers were dried over $\mathrm{MgSO}_{4}$, filtered and the solvent removed under reduced pressure. Drying in vacuo led to the product VII (9.23 g, $21.4 \mathrm{mmol}, 88 \%)$ as yellow solid. 
Formula (molecular weight) $\quad \mathrm{C}_{10} \mathrm{H}_{14} \mathrm{I}_{2} \mathrm{~N}_{2} \mathrm{O}(432.0 \mathrm{~g} / \mathrm{mol})$

${ }^{1} \mathbf{H}$ NMR $\left(300 \mathrm{MHz}, \mathrm{CDCl}_{3}\right) \delta(\mathrm{ppm})=6.34(\mathrm{~s}, 1 \mathrm{H}, \mathrm{pz} 4-\mathrm{H}), 5.34(\mathrm{dd}, J=9.4,2.6 \mathrm{~Hz}, 1 \mathrm{H}$, thp $2-\mathrm{H})$, $4.42\left(\mathrm{~d}, J=1.0 \mathrm{~Hz}, 2 \mathrm{H}, \mathrm{pz} 3-\mathrm{CCH}_{2}\right), 4.33$ (d, $\left.J=10.5 \mathrm{~Hz}, 2 \mathrm{H}, \mathrm{pz} 5-\mathrm{CCH}_{2}\right), 4.08-3.92(\mathrm{~m}, 1 \mathrm{H}$, thp 6-H), $3.69(\mathrm{td}, J=11.1,3.0 \mathrm{~Hz}, 1 \mathrm{H}$, thp 6-H), $2.46-2.28(\mathrm{~m}, 1 \mathrm{H}, \mathrm{thp} 3-\mathrm{H}), 2.18-2.06(\mathrm{~m}, 1 \mathrm{H}, \mathrm{thp}$ 4-H), $2.02\left(\mathrm{dd}, J=13.2,3.0 \mathrm{~Hz}, 1 \mathrm{H}\right.$, thp 3-H), $1.79-1.53\left(\mathrm{~m}, 3 \mathrm{H}\right.$, thp $4-\mathrm{H}$ and thp $\left.5-\mathrm{H}_{2}\right)$.

${ }^{13}$ C NMR $\left(75 \mathrm{MHz}, \mathrm{CDCl}_{3}\right) \delta(\mathrm{ppm})=149.5$ (pz C-3), 140.9 (pz C-5), 107.3 (pz C-4), 85.1 (thp C-2), 67.8 (thp C-6), 29.3 (thp C-3), 25.0 (thp C-5), 22.5 (thp C-4), -4.1 (pz C-5 $\mathrm{CH}_{2}$ ), -9.8 (pz C-3 $\mathrm{CH}_{2}$ ).

\subsubsection{3,5-Bis-[bis-( $\mathrm{N}^{2}$-(3,5-dimethylphenyl)-2-acetamido)-methylamine]-1-tetrahydropyran- 2-yl -pyrazole $\mathrm{H}_{4} \mathrm{~L}^{\text {COMe }}$-thp (VIII)}

3,5-Bis-(iodomethyl)-1-tetrahydropyran-2-yl-pyrazole VII (1.29 g, $3.00 \mathrm{mmol}, 1.0 \mathrm{eq})$, bis-( $N$ (3,5-dimethylphenyl)-2-acetamido)-amine VI (2.04 g, $6.00 \mathrm{mmol}, 2.0 \mathrm{eq})$ and sodium carbonate (3.18 g, $30.0 \mathrm{mmol}, 10 \mathrm{eq})$ were suspended in dry $\mathrm{MeCN}(30 \mathrm{~mL})$ and stirred at $80^{\circ} \mathrm{C}$ for $24 \mathrm{~h}$. The reaction mixture was cooled to ambient temperature and the solvent was removed under reduced pressure. The residue was suspended in $\mathrm{CHCl}_{3}$ overnight and centrifuged (10 min, $2000 \mathrm{rps}$ ). The solution was carefully separated from the salt and the solvent removed under reduced pressure. After drying in vacuo, a mixture of the product VIII and sodium iodide ( $2.92 \mathrm{~g}$ ) was obtained as a yellow solid. The compound was pure due to ${ }^{1} \mathrm{H}-\mathrm{NMR}$ and attempts to completely remove the potassium salt were unsuccessful. Therefore, a full conversion was assumed and the product used for the next reaction without further purifications.

Formula (molecular weight) $\quad \mathrm{C}_{50} \mathrm{H}_{62} \mathrm{~N}_{8} \mathrm{O}_{5}(854.1 \mathrm{~g} / \mathrm{mol})$

1H NMR $\left(300 \mathrm{MHz}, \mathrm{CDCl}_{3}\right) \delta(\mathrm{ppm})=9.09(\mathrm{~s}, 2 \mathrm{H}, \mathrm{NH}), 8.91(\mathrm{~s}, 2 \mathrm{H}, \mathrm{NH}), 7.25(\mathrm{~s}, 4 \mathrm{H}, \mathrm{Ar} 2-\mathrm{H})$, 7.19 (s, 4H, Ar 2-H), 6.72 (s, 4H, Ar 4-H), 6.17 (s, 1H, pz 4-H), 5.53 (dd, J = 9.4, $1.6 \mathrm{~Hz}, 1 \mathrm{H}$, thp 2-H), 3.92 (d, $J=4.2 \mathrm{~Hz}, 2 \mathrm{H}, \mathrm{pz} 3-\mathrm{CH}_{2}$ ), 3.80 (s, 2H, pz5-CH $\mathrm{CH}_{2}$ ) 3.40 (s, 4H, CO-CH $\left.\mathrm{CH}_{2}\right), 3.23(\mathrm{~s}, 4 \mathrm{H}$, $\mathrm{CO}-\mathrm{CH}_{2}$ ), 2.26 (s, $\left.12 \mathrm{H}, \mathrm{Ar}-\mathrm{CH}_{3}\right), 2.23\left(\mathrm{~s}, 12 \mathrm{H}, \mathrm{Ar}-\mathrm{CH}_{3}\right), 1.92$ (d, J = $\left.11.9 \mathrm{~Hz}, 2 \mathrm{H}, \mathrm{thp}\right), 1.74-1.51$ (m, 4H, thp), 1.08 (qd, $J=11.9,3.2 \mathrm{~Hz}, 2 \mathrm{H}$, thp).

${ }^{13} \mathrm{C}$ NMR $\left(75 \mathrm{MHz}, \mathrm{CDCl}_{3}\right.$ ) $\delta=169.1$ (CO), 168.8 (CO), 148.0 (pz C-3), 139.6 (pz C-5), 138.8 (Ar C-2), 138.7 (Ar C-2), 137.8 (Ar C-1), 137.5 (Ar C-1), 126.4 (Ar C-3), 126.1 (Ar C-3), 117.9 (Ar C-4), 117.7 (Ar C-4), 109.3 (pz C-4), 85.0 (thp C-2), 68.1 (thp C-6), $60.0\left(\mathrm{CO}-\mathrm{CH}_{2}\right), 59.1\left(\mathrm{CO}-\mathrm{CH}_{2}\right)$, $52.1\left(\mathrm{pz} 5-\mathrm{CH}_{2}\right), 50.3\left(\mathrm{pz} 5-\mathrm{CH}_{2}\right), 34.1$ (thp C-3), 30.0 (thp C-5), 25.1 (thp C-4), $21.5\left(\mathrm{Ar}-\mathrm{CH}_{3}\right)$. 


\subsubsection{3,5-Bis-[bis-( $\mathrm{N}^{2}$-(3,5-dimethylphenyl)-2-aminoethyl)-methylamine]-1H-pyrazole $\mathrm{H}_{5} \mathrm{~L}^{\mathrm{Me}}$}

(IX)

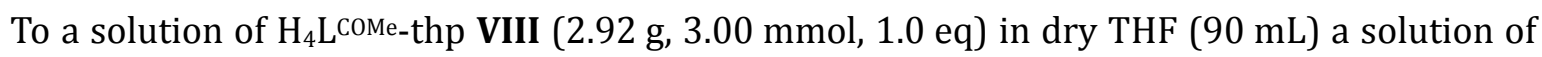
$\mathrm{BH}_{3}$. THF (30.0 mL, 1.0 M in THF, $10 \mathrm{eq}$ ) was added. The reaction mixture was stirred at $80^{\circ} \mathrm{C}$ for 3 days. The solution was cooled to ambient temperature and slowly quenched with aqueous $\mathrm{HCl}(2 \mathrm{M}, 50 \mathrm{~mL})$ and stirred for 2 days. The mixture was neutralized with aqueous $\mathrm{NaOH}(4 \mathrm{~g}$ in $40 \mathrm{~mL}$ ) to ca. $\mathrm{pH}$ 8. The aqueous layer was extracted with EtOAc ( $3 \times 75 \mathrm{~mL})$. The combined organic layers were dried over $\mathrm{MgSO}_{4}$, filtered and the solvent removed under reduced pressure. Drying in vacuo led to the desired product IX $(1.78 \mathrm{~g}, 2.48 \mathrm{mmol}, 86 \%)$ as a white, hygroscopic solid.

Formula (molecular weight) $\quad \mathrm{C}_{45} \mathrm{H}_{62} \mathrm{~N}_{8}(715.1 \mathrm{~g} / \mathrm{mol})$

${ }^{1} \mathbf{H}$ NMR $\left(300 \mathrm{MHz}, \mathrm{CDCl}_{3}\right) \delta(\mathrm{ppm})=6.37(\mathrm{~s}, 4 \mathrm{H}, \mathrm{Ar} 4-\mathrm{H}), 6.19(\mathrm{~s}, 8 \mathrm{H}, \mathrm{Ar} 2-\mathrm{H}), 6.10(\mathrm{~s}, 1 \mathrm{H}, \mathrm{pz}$

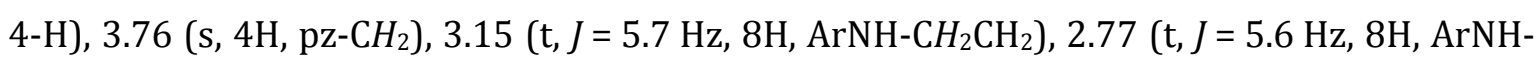
$\mathrm{CH}_{2} \mathrm{CH}_{2}$ ), $2.21\left(\mathrm{~s}, 24 \mathrm{H}, \mathrm{Ar}-\mathrm{CH}_{3}\right)$.

${ }^{13}$ C NMR $\left(75 \mathrm{MHz}, \mathrm{CDCl}_{3}\right) \delta(\mathrm{ppm})=148.4(\mathrm{Ar} \mathrm{C}-1), 139.0(\mathrm{Ar} \mathrm{C}-3), 119.8(\mathrm{Ar} \mathrm{C}-4), 111.2(\mathrm{Ar}$ C-2), 105.1 (pz C-4) $53.3\left(\mathrm{CH}_{2}\right), 50.6\left(\mathrm{pz}-\mathrm{CH}_{2}\right), 41.8\left(\mathrm{CH}_{2}\right), 25.7$ (pz C-3), 25.1 (pz C-5), 21.6 (Ar$\left.\mathrm{CH}_{3}\right)$.

MS (ESI, MeCN) m/z (\%) = $787.5\left(30,[\mathrm{M}+\mathrm{THF}+\mathrm{H}]^{+}\right), 737.5\left(10,[\mathrm{M}+\mathrm{Na}]^{+}\right), 715.3\left(100,[\mathrm{M}+\mathrm{H}]^{+}\right)$. 


\subsection{Macrocylic Tetracarbene Ligands and Complexes}

\subsubsection{Ligand Synthesis}

The following compounds were synthesized according to literature procedures: Ethan-1,2-diylbis-(trifluormethanesulfonate),[484] methylenediformamide,[485] 4,5-dimethylimidazole,[486] bisimidazol-1-yl-methane,[487] $\quad$ bis-(4,5-dimethylimidazol-1-yl)-methane,[185] $\quad 1,1^{\prime}$ methylenebis-(4,5-dimethylimidazole),[185] $\quad \mathbf{H}_{4} \mathbf{L}^{\mathbf{1}}(\mathrm{OTf})_{4} \quad \mathbf{X I I}^{[184]} \quad \mathbf{H}_{4} \mathbf{L}^{3}\left(\mathrm{PF}_{6}\right)_{4} \quad \mathbf{X X I I , [ 1 8 2 ]}$ $\left[\mathrm{Ag}_{4}\left(\mathrm{~L}^{1}\right)_{2}\right](\mathrm{OTf})_{4} \mathbf{2 5}^{[192]}$ and dimethyloxyrane ${ }^{[206]}$.

\subsubsection{3,9,14,20-tetraaza-1,6,12,17-tetraazonia-penta-cyclohexacosane-1(23),4,6(26), $10,12(25), 15,17(24), 21$-octaene tetrachloride $\mathrm{H}_{4} \mathrm{~L}^{1}(\mathrm{Cl})_{4}$ (XXIV)}

A solution of tetra- $n$-butyl-ammonium chloride (278 mg, $1.00 \mathrm{mmol}, 10 \mathrm{eq}$ ) in MeCN (1 mL) was added to a solution of $\mathbf{H}_{4} \mathbf{L}^{1}(\mathrm{OTf})_{4}(105 \mathrm{mg}, 0.10 \mathrm{mmol}, 1.0 \mathrm{eq})$ in MeCN (4 mL) and DMSO $(1.5 \mathrm{~mL})$ and stirred at ambient temperature for $18 \mathrm{~h}$. The voluminous precipitate formed was filtered off and suspended in $\mathrm{CH}_{2} \mathrm{Cl}_{2}(3 \mathrm{~mL})$. Filtration and drying in vacuo yielded $\mathbf{H}_{4} \mathbf{L}^{1}(\mathrm{Cl})_{4}$ XXIV (50 mg, $0.10 \mathrm{mmol}$, quant.) as white solid.

Formula (molecular weight) $\quad \mathrm{C}_{18} \mathrm{H}_{26} \mathrm{~N}_{8}(\mathrm{Cl})_{4}(492.2 \mathrm{~g} / \mathrm{mol})$

${ }_{1}^{1}$ NMR (300 MHz, DMSO-d 6 ) $\delta(\mathrm{ppm})=9.95\left(\mathrm{~s}, 4 \mathrm{H}, \mathrm{CH}_{2}\right), 8.17$ (s, 8H, Im 4,5-H), $6.85(\mathrm{~s}, 8 \mathrm{H}$, $\mathrm{CH}_{2} \mathrm{CH}_{2}$ ).

The imidazole $\mathrm{H}-2$ protons were not detected due to fast exchange with $\mathrm{D}_{2} \mathrm{O}$.

\subsubsection{4,5,10,11,15,16,21,22-Octamethyl-3,9,14,20-tetraaza-1,6,12,17-tetraazonia-penta- cyclohexacosane-1(23),4,6(26),10,12(25),15,17(24),21-octaene tetratriflate $\mathrm{H}_{4} \mathrm{~L}^{2}(\mathrm{OTf})_{4}$ (XXI)}

A solution of ethan-1,2-diyl-bis-(trifluormethanesulfonate) XVI (17.8 g, $54.7 \mathrm{mmol}, 1.0 \mathrm{eq})$ in MeCN (100 mL) was slowly added over a period of $6 \mathrm{~h}$ to a solution of 1,1'-methylenebis-(4,5dimethylimidazole) $\mathbf{X X}(11.2 \mathrm{~g}, 54.7 \mathrm{mmol}, 1.0 \mathrm{eq})$ in $\mathrm{MeCN}(100 \mathrm{~mL})$ at ambient temperature. The reaction mixture was heated at reflux for $2 \mathrm{~d}$. The volatiles were removed under reduced pressure and the light brown crude product recrystallized from MeCN (35 mL). The white precipitate was isolated by filtration and washed with MeCN (15 mL). The filtrate was dried under reduced pressures and the residue was recrystallized a second time from MeCN (10 mL) Drying of the combined solids in vacuo led to the ligand XXI ( $9.66 \mathrm{~g}, 9.11 \mathrm{mmol}, 33 \%$ ) as a white solid. 


\section{Formula (molecular weight) $\quad \mathrm{C}_{26} \mathrm{H}_{40} \mathrm{~N}_{8}\left(\mathrm{CF}_{3} \mathrm{SO}_{3}\right)_{4}(1060.1 \mathrm{~g} / \mathrm{mol})$}

${ }^{1}$ H NMR (300 MHz, acetonitrile- $\left.\mathrm{d}_{3}\right) \delta(\mathrm{ppm})=8.41(\mathrm{~s}, 4 \mathrm{H}, \mathrm{Im} 2-\mathrm{H}), 6.28\left(\mathrm{~s}, 4 \mathrm{H}, \mathrm{CH}_{2}\right), 4.57(\mathrm{~s}, 8 \mathrm{H}$, $\left.\mathrm{CH}_{2} \mathrm{CH}_{2}\right), 2.35\left(\mathrm{~s}, 12 \mathrm{H}, \mathrm{CH}_{3}\right), 2.28\left(\mathrm{~s}, 12 \mathrm{H}, \mathrm{CH}_{3}\right)$.

${ }^{13}$ C NMR $\left(75\right.$ MHz, acetonitrile- $\left.\mathrm{d}_{3}\right) \delta(\mathrm{ppm})=134.7$ (Im C-2), 131.0 (Im C-4/5), 129.9 (Im C4/5), $58.2\left(\mathrm{CH}_{2}\right), 47.4\left(\mathrm{CH}_{2} \mathrm{CH}_{2}\right), 9.0\left(\mathrm{CH}_{3}\right), 8.6\left(\mathrm{CH}_{3}\right)$.

MS $(\mathrm{ESI}, \mathrm{MeCN}) \mathrm{m} / \mathrm{z}(\%)=911.2\left(15,\left[\mathrm{H}_{4} \mathrm{~L}^{2}(\mathrm{OTf})_{3}\right]^{+}\right), 381.2\left(100,\left[\mathrm{H}_{4} \mathrm{~L}^{2}(\mathrm{OTf})_{2}\right]^{2+}\right)$.

\subsubsection{4,5,9,10,14,15,19,20-Octamethyl-3,8,13,18-tetraaza-1,6,11,16-tetraazoniuapenta- cylcotetracosan-1,4,6,9,11,14,16-octene-tetrakis-(hexafluorophosphate) $\mathrm{H}_{4} \mathrm{~L}^{4}\left(\mathrm{PF}_{6}\right)_{4}$ (XXIII)}

A solution of bis-(4,5-dimethyl-1H-imidazole-1-yl)-methane $\mathbf{X X}(1.38 \mathrm{~g}, 6.76 \mathrm{mmol}, 1.0 \mathrm{eq})$ and tetra- $n$-butyl-ammonium chloride $(4.00 \mathrm{~g}, 14.4 \mathrm{mmol}, 2.1 \mathrm{eq})$ in dibromomethane $(20 \mathrm{~mL}$, $287 \mathrm{mmol}, 42 \mathrm{eq}$ ) was stirred at ambient temperature for $24 \mathrm{~h}$ and then heated at reflux for $12 \mathrm{~h}$. The reaction mixture was cooled to ambient temperature, filtered and the formed yellow solid washed with MeCN (25 mL). The solid was solved in water $(4 \mathrm{~mL})$ and treated with a sat. aq. solution of potassium hexafluorophosphate. The resulting precipitate was washed with water (15 mL). Recrystallisation from methanol yielded $\mathbf{H}_{4} \mathbf{L}^{4}\left(\mathrm{PF}_{6}\right)_{4} \mathbf{X X I I I}$ (1.08 g, 1.06 mmol, $31 \%$ ) as white solid.

Formula (molecular weight) $\quad \mathrm{C}_{24} \mathrm{H}_{36} \mathrm{~N}_{8}\left(\mathrm{PF}_{6}\right)_{4}(1016.2 \mathrm{~g} / \mathrm{mol})$

${ }^{1}$ H NMR $\left(300 \mathrm{MHz}\right.$, acetonitrile-d $\left.\mathrm{d}_{3}\right) \delta(\mathrm{ppm})=9.30(\mathrm{~s}, 4 \mathrm{H}, \mathrm{Im} 2-\mathrm{H}), 6.48\left(\mathrm{~s}, 8 \mathrm{H}, \mathrm{CH}_{2}\right), 2.33$ (s, $\left.24 \mathrm{H}, \mathrm{CH}_{3}\right)$.

${ }^{13}$ C NMR $\left(75 \mathrm{MHz}\right.$, acetonitrile-d $\left.\mathrm{d}_{3}\right) \delta(\mathrm{ppm})=136.6\left(\right.$ Im C-2), $129.1(\mathrm{Im} \mathrm{C}-4, \mathrm{C}-5), 56.2\left(\mathrm{CH}_{2}\right)$, $7.6\left(\mathrm{CH}_{3}\right)$.

EA (\%) Calcd. for $\mathrm{C}_{24} \mathrm{H}_{36} \mathrm{~N}_{8} \mathrm{~F}_{24} \mathrm{P}_{4}$ : C 28.3, H 3.50, N 11.0. Found: C 28.5, H 3.82, N 11.0.

\subsubsection{Complex Synthesis}

\subsubsection{1 $\left[\mathrm{Ag}_{4}\left(\mathrm{~L}^{2}\right)_{2}\right](\mathrm{OTf})_{4}(26)$}

To a suspension of $\mathbf{H}_{4} \mathbf{L}^{2}(\mathrm{OTf})_{4}(800 \mathrm{mg}, 0.75 \mathrm{mmol}, 1.0 \mathrm{eq})$ in MeCN $(100 \mathrm{~mL})$ in the dark silver oxide (376 mg, $1.58 \mathrm{mmol}, 2.1 \mathrm{eq}$ ) was added. The suspension was heated to an oil bath temperature of $70^{\circ} \mathrm{C}$ for $24 \mathrm{~h}$ and then stirred at ambient temperature for $6 \mathrm{~h}$. The reaction mixture was filtered through Celite ${ }^{\circledR}$ and the filtrate was layered with $\mathrm{Et}_{2} \mathrm{O}(400 \mathrm{~mL})$. The 
brown crude product was filtered off. Drying in vacuo yielded $\left[\mathrm{Ag}_{4}\left(\mathrm{~L}^{2}\right)_{2}\right](\mathrm{OTf})_{4} 26(599 \mathrm{mg}$, $0.31 \mathrm{mmol}, 41 \%$ ) as light brown solid.

\section{Formula (molecular weight) $\quad \mathrm{C}_{52} \mathrm{H}_{72} \mathrm{~N}_{16} \mathrm{Ag}_{4}(\mathrm{OTf})_{4}(1949.0 \mathrm{~g} / \mathrm{mol})$}

${ }^{1}$ H NMR (300 MHz, acetonitrile- $\left.\mathrm{d}_{3}\right) \delta(\mathrm{ppm})=6.23\left(\mathrm{dt}, J=17.2,14.4,14.4 \mathrm{~Hz}, 8 \mathrm{H}, \mathrm{CH}_{2}\right), 4.51(\mathrm{~d}$, $\left.J=14.8 \mathrm{~Hz}, 4 \mathrm{H}, \mathrm{CH}_{2} \mathrm{CH}_{2}\right), 4.20\left(\mathrm{td}, J=14.3,13.5,2.5 \mathrm{~Hz}, 4 \mathrm{H}, \mathrm{CH}_{2} \mathrm{CH}_{2}\right), 4.02(\mathrm{td}, J=13.6,12.5$, $1.7 \mathrm{~Hz}, 4 \mathrm{H}, \mathrm{CH}_{2} \mathrm{CH}_{2}$ ), 3.50 (d, J = $14.3 \mathrm{~Hz}, 4 \mathrm{H}, \mathrm{CH}_{2} \mathrm{CH}_{2}$ ), 2.42 (d, $J=0.6 \mathrm{~Hz}, 12 \mathrm{H}, \mathrm{CH}_{3}$ ), 2.17 (d, $\left.J=0.6 \mathrm{~Hz}, 12 \mathrm{H}, \mathrm{CH}_{3}\right), 1.90\left(\mathrm{~d}, J=0.6 \mathrm{~Hz}, 12 \mathrm{H}, \mathrm{CH}_{3}\right), 1.51\left(\mathrm{~d}, J=0.7 \mathrm{~Hz}, 12 \mathrm{H}, \mathrm{CH}_{3}\right)$.

${ }^{13}$ C NMR $\left(75 \mathrm{MHz}\right.$, acetonitrile- $\left.\mathrm{d}_{3}\right) \delta(\mathrm{ppm})=180.4(\mathrm{Im} \mathrm{C}-2), 131.1$ (Im C-4/5), 131.0 (Im C-4/5), 129.0 (Im C-4/5), 128.9 (Im C-4/5), 128. 5 (Im C-4/5), 128.4 (Im C-4/5), 126.7 (Im C-4/5), $126.6(\mathrm{Im} \mathrm{C}-4 / 5), 61.0\left(\mathrm{CH}_{2}\right), 50.1\left(\mathrm{CH}_{2} \mathrm{CH}_{2}\right), 47.9\left(\mathrm{CH}_{2} \mathrm{CH}_{2}\right), 9.6\left(\mathrm{CH}_{3}\right), 9.0\left(5 \mathrm{CH}_{3}\right), 8.65$ $\left(5 \mathrm{CH}_{3}\right), 8.5\left(\mathrm{CH}_{3}\right)$.

MS (ESI, MeCN) m/z (\%) = $825.0\left(82,\left[\mathrm{Ag}_{2} \mathrm{~L}^{2}(\mathrm{OTf})\right]^{+}\right), 381.2\left(100,\left[\mathrm{Ag}_{2} \mathrm{~L}^{2}\left(\mathrm{H}_{2} \mathrm{O}\right)\right]^{2+}\right), 338.1(65$, $\left.\left[\mathrm{Ag}_{2} \mathrm{~L}^{2}\right]^{2+}\right), 284.6\left(24,\left[\mathrm{AgL}^{2}\right]^{2+}\right)$.

\subsubsection{2 $\left[\mathrm{Ag}_{4}\left(\mathrm{~L}^{3}\right)_{2}\right]\left(\mathrm{PF}_{6}\right)_{4}(27)$}

To a suspension of $\mathbf{H}_{4} \mathbf{L}^{3}\left(\mathrm{PF}_{6}\right)_{4}(904 \mathrm{mg}, 1.00 \mathrm{mmol}, 1.0 \mathrm{eq})$ in MeCN (70 mL) in the dark silver oxide (464 mg, $2.00 \mathrm{mmol}, 2.0 \mathrm{eq}$ ) was added. The suspension was heated to an oil bath temperature of $70^{\circ} \mathrm{C}$ for $5 \mathrm{~h}$ and then stirred at ambient temperature overnight. The reaction mixture was filtered through Celite ${ }^{\circledR}$ and the filtrate was layered with $\mathrm{Et}_{2} \mathrm{O}(100 \mathrm{~mL})$. The brown crude product was filtered off, dried in vacuo yielding $645 \mathrm{mg}$ of $\mathbf{2 7}$ and used without further purification for transmetallation attempts.

\section{Formula (molecular weight) $\quad \mathrm{C}_{32} \mathrm{H}_{32} \mathrm{~N}_{16} \mathrm{Ag}_{4}\left(\mathrm{PF}_{6}\right)_{4}(1652.0 \mathrm{~g} / \mathrm{mol})$}

1H NMR $\left(300 \mathrm{MHz}\right.$, acetonitrile- $\left.\mathrm{d}_{3}\right) \delta(\mathrm{ppm})=7.57$ (s, 8H, Im H-4/H-5), 7.42 (s, 8H, Im $\mathrm{H}-4 / \mathrm{H}-5), 6.27$ (d, $\left.J=14.0 \mathrm{~Hz}, 8 \mathrm{H}, \mathrm{CH}_{2}\right), 6.12\left(\mathrm{~d}, J=14.1 \mathrm{~Hz}, 8 \mathrm{H}, \mathrm{CH}_{2}\right)$.

\subsubsection{3 $\left[\mathrm{Ag}_{4}\left(\mathrm{~L}^{4}\right)_{2}\right]\left(\mathrm{PF}_{6}\right)_{4}(28)$}

To a suspension of $\mathbf{H}_{4} \mathbf{L}^{4}\left(\mathrm{PF}_{6}\right)_{4}(102 \mathrm{mg}, 0.10 \mathrm{mmol}, 1.0 \mathrm{eq})$ in MeCN (20 mL) in the dark silver oxide ( $46.3 \mathrm{mg}, 0.20 \mathrm{mmol}, 2.0 \mathrm{eq}$ ) was added. The suspension was heated to an oil bath temperature of $70^{\circ} \mathrm{C}$ for $5 \mathrm{~h}$ and then stirred at ambient temperature overnight. The reaction mixture was filtered through Celite ${ }^{\circledR}$ and the filtrate was layered with $\mathrm{Et}_{2} \mathrm{O}(50 \mathrm{~mL})$. The brown 
crude product was filtered off, dried in vacuo yielding $20 \mathrm{mg}$ of $\mathbf{2 8}$ and used without further purification for complexation attempts.

Formula (molecular weight) $\quad \mathrm{C}_{48} \mathrm{H}_{64} \mathrm{~N}_{16} \mathrm{Ag}_{4}\left(\mathrm{PF}_{6}\right)_{4}(1876.5 \mathrm{~g} / \mathrm{mol})$

${ }^{1} \mathbf{H}$ NMR $\left(300 \mathrm{MHz}\right.$, acetonitrile-d $\left.\mathrm{d}_{3}\right) \delta(\mathrm{ppm})=6.06\left(\mathrm{~s}, 16 \mathrm{H}, \mathrm{CH}_{2}\right), 2.26\left(2,48 \mathrm{H}, \mathrm{CH}_{2}\right)$.

\subsubsection{4 $\left[\mathrm{NiL}^{1}\right](\mathrm{OTf})_{2}(29)$}

$\left[\mathrm{Ag}_{4}\left(\mathrm{~L}^{1}\right)_{2}\right](\mathrm{OTf})_{4}(50.0 \mathrm{mg}, 29.0 \mu \mathrm{mol}, 1.0 \mathrm{eq})$ and $\mathrm{NiBr}_{2}(\mathrm{dme})(18.8 \mathrm{mg}, 60.9 \mu \mathrm{mol}, 2.1 \mathrm{eq})$ were suspended in dry MeCN $(10 \mathrm{~mL})$ for $1 \mathrm{~d}$. The reaction mixture was filtered through Celite ${ }^{\circledR}$ twice. Drying the filtrate in vacuo yielded [ $\left.\mathrm{NiL}^{1}\right](\mathrm{OTf})_{2}(13 \mathrm{mg}, 18.5 \mu \mathrm{mol}, 32 \%)$ as yellow solid. Crystals suitable for $\mathrm{X}$ ray diffraction analysis were obtained by layering a concentrated $\mathrm{MeCN}$ solution with $\mathrm{Et}_{2} \mathrm{O}$.

\section{Formula (molecular weight) $\quad \mathrm{C}_{18} \mathrm{H}_{20} \mathrm{~N}_{8} \mathrm{Ni}(\mathrm{OTf})_{2}(705.2 \mathrm{~g} / \mathrm{mol})$}

${ }^{1}$ H NMR $\left(500 \mathrm{MHz}\right.$, acetonitrile- $\left.\mathrm{d}_{3}\right) \delta(\mathrm{ppm})=7.39(\mathrm{~d}, J=2.0 \mathrm{~Hz}, 2 \mathrm{H}, \operatorname{Im} 4 / 5-\mathrm{H}), 7.18(\mathrm{~d}$, $J=2.0 \mathrm{~Hz}, 2 \mathrm{H}, \mathrm{Im} 4 / 5-\mathrm{H})$ ), $6.14\left(\mathrm{~d}, J=12.9 \mathrm{~Hz}, 2 \mathrm{H}, \mathrm{CH}_{2}\right), 6.06\left(\mathrm{~d}, J=12.9 \mathrm{~Hz}, 2 \mathrm{H}, \mathrm{CH}_{2}\right), 4.98-$ $4.89\left(\mathrm{~m}, 4 \mathrm{H}, \mathrm{CH}_{2} \mathrm{CH}_{2}\right), 4.52-4.42\left(\mathrm{~m}, 4 \mathrm{H}, \mathrm{CH}_{2} \mathrm{CH}_{2}\right)$.

${ }^{13} \mathrm{C}$ NMR $\left(75\right.$ MHz, acetonitrile- $\left.\mathrm{d}_{3}\right) \delta(\mathrm{ppm})=170.1$ (s, Im C-2), 123.7 (s, Im C-4/5), 123.1 (s, Im C-4/5), $63.2\left(\mathrm{~s}, \mathrm{CH}_{2}\right), 49.1\left(\mathrm{~s}, \mathrm{CH}_{2} \mathrm{CH}_{2}\right)$.

${ }^{19 F}$ NMR $\left(282 \mathrm{MHz}\right.$, acetonitrile- $\left.\mathrm{d}_{3}\right) \delta(\mathrm{ppm})=-79.32$ (OTf).

MS (ESI, MeCN) m/z (\%) = $555.0\left(15,\left[\mathrm{NiL}^{1}\right](\mathrm{OTf})+\right), 203.0\left[100,\left[\mathrm{NiL}^{1}\right]^{2+}\right)$.

UV/vis $(\mathrm{MeCN}): \lambda_{\max }(\mathrm{nm})\left(\epsilon\left[\mathrm{M}^{-1} \mathrm{~cm}^{-1}\right]\right)=233$ (14909), 300 (6858), 330 (8070).

EChem (MeCN, 0.1 M nBu $\mathrm{NPF}_{6}$ ): $\mathrm{E}_{1 / 2}\left[\mathrm{~V}\right.$ vs Fc/Fc $\left./ \mathrm{Fc}^{+}\right]=+1.67 \mathrm{~V}$ (irreversible, $\mathrm{Ni}^{\mathrm{II}} / \mathrm{Ni}^{\mathrm{III}}$ )

$+1.89 \mathrm{~V}$ (irreversible, $\mathrm{Ni}{ }^{\mathrm{III}} / \mathrm{Ni}^{\mathrm{IV}}$ )

$-1.48 \mathrm{~V}$ (irreversible, $\mathrm{Ni} / \mathrm{Ni}^{\mathrm{II}}$ )

EA Calcd. for $\mathrm{C}_{20} \mathrm{H}_{20} \mathrm{~F}_{6} \mathrm{~N}_{8} \mathrm{NiS}_{2} \mathrm{O}_{6}$ : C 34.06, H 2.86, N 15.89. Found: C 31.23, H 2.98, N 14.54.

\subsubsection{5 $\left[\mathrm{NiL}^{1}\right](\mathrm{Cl})_{2}(30)$}

A suspension of $\mathbf{H}_{4} \mathbf{L}^{1}(\mathrm{Cl})_{4}(145 \mathrm{mg}, 295 \mu \mathrm{mol}, 1.0 \mathrm{eq}), \mathrm{Ni}(\mathrm{OAc})_{2} \cdot 4 \mathrm{H}_{2} \mathrm{O}(73.1 \mathrm{mg}, 295 \mu \mathrm{mol}$, $1.0 \mathrm{eq})$ and sodium acetate (96.8 mg, 1,18 mmol, $4.0 \mathrm{eq})$ was refluxed in DMSO (8 mL) for $20 \mathrm{~h}$. The volatiles were removed in vacuo. The crude product was dissolved in water $(2 \mathrm{~mL})$ and 
layered with acetone. The resulting oil was taken up in $\mathrm{MeOH}(2 \mathrm{~mL})$ and layered with $\mathrm{Et}_{2} \mathrm{O}$. Isolating the yellow needles and drying the these in vacuo yielded $\left[\mathrm{NiL}^{1}\right]\left(\mathrm{Cl}_{2}(66 \mathrm{mg}\right.$, $138 \mu \mathrm{mol}, 47 \%$ ) as yellow solid. Crystals suitable for $\mathrm{X}$ ray diffraction analysis were obtained by layering a concentrated aqueous solution with acetone.

Formula (molecular weight) $\quad \mathrm{C}_{18} \mathrm{H}_{20} \mathrm{~N}_{8} \mathrm{Ni}(\mathrm{Cl})_{2}(478.0 \mathrm{~g} / \mathrm{mol})$

${ }^{1} \mathbf{H}$ NMR $\left(500 \mathrm{MHz}\right.$, acetonitrile- $\left.\mathrm{d}_{3}\right) \delta(\mathrm{ppm})=7.58(\mathrm{~d}, J=2.0 \mathrm{~Hz}, 2 \mathrm{H}, \mathrm{Im} 4 / 5-\mathrm{H}), 7.37(\mathrm{~d}$, $J=2.0 \mathrm{~Hz}, 2 \mathrm{H}, \mathrm{Im} 4 / 5-\mathrm{H}), 6.36\left(\mathrm{dd}, J=32.8,12.7 \mathrm{~Hz}, 2 \mathrm{H}, \mathrm{CH}_{2}\right), 5.17-4.99\left(\mathrm{~m}, 2 \mathrm{H}, \mathrm{CH}_{2} \mathrm{CH}_{2}\right), 4.66$ $-4.48\left(\mathrm{~m}, 2 \mathrm{H}, \mathrm{CH}_{2} \mathrm{CH}_{2}\right)$.

${ }^{13}$ C NMR $\left(75\right.$ MHz, acetonitrile- $\left.\mathrm{d}_{3}\right) \delta(\mathrm{ppm})=170.3$ (s, Im C-2), 124.0 (s, Im C-4/5), 123.1 (s, Im C-4/5), 118.2 (s, OTf-), 63.3 (s, $\left.\mathrm{CH}_{2}\right), 49.6\left(\mathrm{~s}, \mathrm{CH}_{2} \mathrm{CH}_{2}\right)$.

MS (ESI, MeCN) m/z (\%) = $441.1\left(38,\left[\mathrm{NiL}^{1}\right](\mathrm{Cl})^{+}\right), 203.0\left[100,\left[\mathrm{NiL}^{1}\right]^{2+}\right)$.

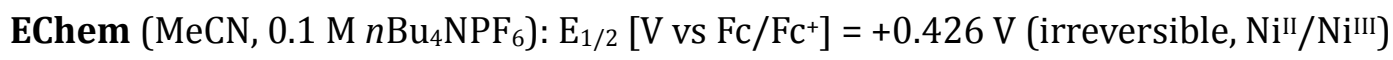
$+0.833 \mathrm{~V}$ (irreversible, $\mathrm{Ni}{ }^{I I I} / \mathrm{Ni}^{\mathrm{IV}}$ ) $-1.34 \mathrm{~V}$ (irreversible, $\mathrm{Ni}^{\mathrm{I}} / \mathrm{Ni}^{\mathrm{II}}$ ) $-1.69 \mathrm{~V}$ (irreversible, $\mathrm{Ni}^{0} / \mathrm{Ni}^{1}$ )

\subsubsection{6 $\left[\mathrm{NiL}^{2}\right](\mathrm{OTf})_{2}(31)$}

a) $\left[\mathrm{Ag}_{4}\left(\mathrm{~L}^{2}\right)_{2}\right](\mathrm{OTf})_{4}(1.53 \mathrm{~g}, 0.79 \mathrm{mmol}, 1.0 \mathrm{eq})$ and $\mathrm{NiBr}_{2}(\mathrm{dme})(487 \mathrm{mg}, 1.58 \mathrm{mmol}, 2.0 \mathrm{eq})$ were suspended in dry MeCN (30 mL) for $3 \mathrm{~d}$ and heated to $60{ }^{\circ} \mathrm{C}$ for $18 \mathrm{~h}$. The formed silver salts were separated by filtration through glas fibre filters. The volatiles were removed in vacuo, yielding the crude product (1.03 g) as yellow solid. According to ${ }^{1} \mathrm{H}$ NMR spectroscopy, there were $5 \%$ free ligand in the mixture. To obtain pure 31, a part of the crude product (300 $\mathrm{mg}$ ) was dissolved in methanol $(2 \mathrm{~mL})$ and filtered through a plug of reversed phase silica. The volatiles of the filtrate were removed in vacuo and the product extracted with $\mathrm{CH}_{2} \mathrm{Cl}_{2}(2 \times 3 \mathrm{~mL})$. Drying in vacuo yielded [NiL2](OTf) 2 (63 mg, $77.1 \mu \mathrm{mol}$, approx. $20 \%$ ) as yellow solid. Crystals suitable for $\mathrm{X}$ ray diffraction analysis were obtained by layering a concentrated EtCN solution with $\mathrm{Et}_{2} \mathrm{O}$.

b) A suspension of $\mathbf{H}_{4} \mathbf{L}^{2}$ (OTf) ${ }_{4}$ (318 mg, $0.30 \mathrm{mmol}, 1.0 \mathrm{eq}$ ), $\mathrm{NiCl}_{2}$ (39.0 mg, $0.30 \mathrm{mmol}, 1.0 \mathrm{eq}$ ) and $\mathrm{Cs}_{2} \mathrm{CO}_{3}$ (390 mg, $1.20 \mathrm{mmol}, 4.0 \mathrm{eq}$ ) in dry $\mathrm{MeCN}$ (20 mL) was stirred at ambient temperature for $20 \mathrm{~h}$. The volatiles were removed under reduced pressure and the crude product was extracted with $\mathrm{CH}_{2} \mathrm{Cl}_{2}(10 \mathrm{~mL})$ and purified by size exclusion chromatography 
(Sephadex ${ }^{\circledR}$, eluent $\mathrm{CH}_{2} \mathrm{Cl}_{2}$ ). Drying in vacuo yielded [ $\left.\mathrm{NiL}^{2}\right](\mathrm{OTf})_{2} \mathbf{3 1}(124 \mathrm{mg}, 0.15 \mathrm{mmol}, 50 \%$ ) as yellow-orange solid.

\section{Formula (molecular weight) $\quad \mathrm{C}_{26} \mathrm{H}_{36} \mathrm{~N}_{8} \mathrm{Ni}(\mathrm{OTf})_{2}(817.5 \mathrm{~g} / \mathrm{mol})$}

${ }^{1} \mathbf{H}$ NMR $\left(500 \mathrm{MHz}\right.$, acetonitrile- $\left.\mathrm{d}_{3}\right) \delta(\mathrm{ppm})=5.88\left(\mathrm{~d}, J=13.4 \mathrm{~Hz}, 2 \mathrm{H}, \mathrm{CH}_{2}\right), 5.80(\mathrm{~d}, J=13.3 \mathrm{~Hz}$, $\left.2 \mathrm{H}, \mathrm{CH}_{2}\right), 4.81-4.73\left(\mathrm{~m}, 4 \mathrm{H}, \mathrm{CH}_{2} \mathrm{CH}_{2}\right), 4.36-4.29\left(\mathrm{~m}, 4 \mathrm{H}, \mathrm{CH}_{2} \mathrm{CH}_{2}\right), 2.24-2.23\left(\mathrm{~m}, 12 \mathrm{H}, \mathrm{CH}_{3}\right)$, $2.16-2.14\left(\mathrm{~m}, 12 \mathrm{H}, \mathrm{CH}_{3}\right)$.

13C NMR (126 MHz, acetonitrile-d $\left.\mathrm{d}_{3}\right) \delta(\mathrm{ppm})=169.1$ (s, Im C-2), 127.4 (s, Im C-4/5), 126.7 (s, Im C-4/5), 120.8 (s, OTf-), $57.8\left(\mathrm{~s}, \mathrm{CH}_{2}\right), 46.0\left(\mathrm{~s}, \mathrm{CH}_{2} \mathrm{CH}_{2}\right), 8.8\left(\mathrm{~s}, \mathrm{CH}_{3}\right), 8.8\left(\mathrm{~s}, \mathrm{CH}_{3}\right)$.

${ }^{19 F}$ NMR (471 MHz, acetonitrile- $\left.\mathrm{d}_{3}\right) \delta(\mathrm{ppm})=-79.30$ (OTf)

MS $(\mathrm{ESI}, \mathrm{MeCN}) \mathrm{m} / \mathrm{z}(\%)=667.1\left(10,\left[\mathrm{NiL}^{2}\right](\mathrm{OTf})+\right), 259.1\left[100,\left[\mathrm{NiL}^{2}\right]^{2+}\right)$.

UV/vis (MeCN): $\lambda_{\max }(\mathrm{nm})\left(\epsilon\left[\mathrm{M}^{-1} \mathrm{~cm}^{-1}\right]\right)=253$ (10 163), 343 (12 044).

ATR-IR (solid sample): $v$ [ $\left.\mathrm{cm}^{-1}\right]=403(\mathrm{w}), 414(\mathrm{w}), 516(\mathrm{~s}), 533(\mathrm{w}), 541(\mathrm{w}), 557(\mathrm{w}), 572(\mathrm{~m})$, $636(\mathrm{~s}), 755(\mathrm{w}), 842(\mathrm{w}), 853(\mathrm{w}), 888(\mathrm{~m}), 977(\mathrm{w}), 1026(\mathrm{~s}), 1089$ (w), $1140(\mathrm{~s}), 1196(\mathrm{w})$, 1222 (s), 1249 (s), 1376 (m), 1392 (m), 1412 (w), 1436 (w), 1445 (w), 1468 (w), 1478 (w), $1641(w), 1660(w), 2869(w), 2925(w), 2957(w)$.

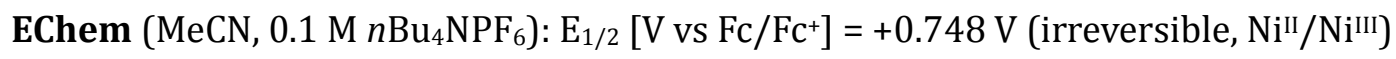
$+1.20 \mathrm{~V}$ (irreversible, $\mathrm{Ni}{ }^{\mathrm{III}} / \mathrm{Ni}^{\mathrm{IV}}$ )

$-1.25 \mathrm{~V}$ (irreversible, $\mathrm{Ni}^{\mathrm{I}} / \mathrm{Ni}^{I}$ )

$-2.41 \mathrm{~V}$ (irreversible)

$-2.51 \mathrm{~V}$ (irreversible)

EA Calcd. for $\mathrm{C}_{28} \mathrm{H}_{36} \mathrm{~F}_{6} \mathrm{~N}_{8} \mathrm{NiO}_{6} \mathrm{~S}_{2}$ : C 41.14, H 4.44, N 13.71 S 7.85. Found: C 44.28, H 5.63, N 14.73 S 6.43 .

\subsubsection{7 $\left[\mathrm{NiL}^{1}\right](\mathrm{OTf})_{3-\mathrm{x}} \mathrm{F}_{\mathrm{x}}$}

A solution of [NiL $\left.\mathrm{Ni}^{1}\right](\mathrm{OTf})_{2} 29(7.0 \mathrm{mg}, 10 \mu \mathrm{mol}, 1.0 \mathrm{eq})$ in $\mathrm{MeCN}(0.6 \mathrm{~mL})$ and $\mathrm{XeF}_{2}(1.7 \mathrm{mg}$, $10 \mu \mathrm{mol}, 1.0 \mathrm{eq})$ in $\mathrm{MeCN}(0.4 \mathrm{~mL})$ were combined at $-35^{\circ} \mathrm{C}$. An instant color change from yellow to orange was observed. A sample for an EPR measurement was directly frozen in liquid nitrogen. 


\subsection{Two-in-one Pincer Ligands and Complexes}

\subsubsection{Ligand Synthesis}

The ligand precursors $\mathbf{X X X}$ - XXXXIII as well as the final ligands were synthesized according to optimized literature procedures. ${ }^{[265,406,407]}\left[\mathrm{RhCl}\left(\mathrm{coe}_{2}\right]_{2}\right.$ was synthesized according to literature procedure.[243]

\subsubsection{6-Methyl-2-picolinic acid (XXX)}

A solution of 2,6-lutidine $(64.2 \mathrm{~g}, 600 \mathrm{mmol}, 1.0 \mathrm{eq})$ in water $(2 \mathrm{~L})$ was heated to $70^{\circ} \mathrm{C}$. $\mathrm{KMnO}_{4}$ (190 g, $1.20 \mathrm{~mol}, 2.0 \mathrm{eq}$ ) was added in portions over $48 \mathrm{~h}$. Each addition of permanganate was made after the preceding amount was consumed. The reaction mixture was stirred at $70{ }^{\circ} \mathrm{C}$ overnight, filtered hot and the residue was extracted with boiling water $(0.5 \mathrm{~L})$. The combined filtrates were concentrated to $150 \mathrm{~mL}$ and the $\mathrm{pH}$ of the solution was adjusted to 3.3 with conc. $\mathrm{HCl}$ (ca. $60 \mathrm{~mL}$ ). Toluene (300 mL) was added and the water was removed by a Dean-Stark apparatus. The toluene solution was filtered when still hot. The residue was extracted twice with boiling toluene $(250 \mathrm{~mL})$. The solvent of the combined organic layers was evaporated. After drying in vacuo, compound XXX (42.7 g, 312 mmol, 52\%) was obtained as a white solid.

Formula (molecular weight) $\quad \mathrm{C}_{7} \mathrm{H}_{7} \mathrm{NO}_{2}(137.1 \mathrm{~g} / \mathrm{mol})$

${ }^{1}$ H NMR $\left(300 \mathrm{MHz}\right.$, acetonitrile- $\left.\mathrm{d}_{3}\right) \delta(\mathrm{ppm})=7.95(\mathrm{~d}, J=7.6 \mathrm{~Hz}, 1 \mathrm{H}, \mathrm{py} 3-\mathrm{H}), 7.88(\mathrm{t}, J=7.6,1 \mathrm{H}$, py $4-\mathrm{H}), 7.51(\mathrm{~d}, J=7.6 \mathrm{~Hz}, 1 \mathrm{H}$, py $5-\mathrm{H}), 2.59\left(\mathrm{~s}, 3 \mathrm{H}, \mathrm{CH}_{3}\right)$.

${ }^{13 C}$ NMR $\left(75 \mathrm{MHz}\right.$, acetonitrile- $\left.\mathrm{d}_{3}\right) \delta(\mathrm{ppm})=165.6(\mathrm{COOH}), 159.1$ (py C-6), 146.7 (py C-2), 139.7 (py C-4), 128.7 (py C-5), 121.7 (py C-3), $23.9\left(\mathrm{CH}_{3}\right)$.

MS (EI) m/z (\%) = $137\left(8,[\mathrm{M}]^{+}\right), 93\left(100,\left[\mathrm{M}-\mathrm{CO}_{2}\right]^{+}\right)$.

\subsubsection{Methyl-6-methyl-2-picolinate (XXXI)}

To a solution of 6-methyl-2-picolinic acid XXX (40.0 g, $292 \mathrm{mmol}, 1.0 \mathrm{eq})$ in methanol (300 mL), conc. $\mathrm{H}_{2} \mathrm{SO}_{4}(50 \mathrm{~mL})$ was added dropwise over the period of $1 \mathrm{~h}$. The reaction mixture was heated at reflux for $23 \mathrm{~h}$, cooled to ambient temperature and poured onto ice ( $300 \mathrm{~mL}$ ). The solution was neutralized with $\mathrm{Na}_{2} \mathrm{CO}_{3}$. After the addition of water $(200 \mathrm{~mL})$ and $\mathrm{CH}_{2} \mathrm{Cl}_{2}$ $(200 \mathrm{~mL})$ the layers were separated and the aqueous layer extracted with $\mathrm{CH}_{2} \mathrm{Cl}_{2}(2 \times 100 \mathrm{~mL})$. The combined organic layers were dried over $\mathrm{MgSO}_{4}$, filtered and the solvent was removed 
under reduced pressure. After drying in vacuo, compound XXXI (38.3 g, $253 \mathrm{mmol}, 87 \%$ ) was obtained as a yellow oil.

Formula (molecular weight) $\quad \mathrm{C}_{8} \mathrm{H}_{9} \mathrm{NO}_{2}(151.2 \mathrm{~g} / \mathrm{mol})$

1H NMR (300 MHz, chloroform-d) $\delta(\mathrm{ppm})=7.95(\mathrm{~d}, J=7.6 \mathrm{~Hz}, 1 \mathrm{H}, \mathrm{py} 3-\mathrm{H}), 7.73(\mathrm{t}, J=7.7 \mathrm{~Hz}$, $1 \mathrm{H}$, py 4-H), 7.35 (d, J = $7.7 \mathrm{~Hz}, 1 \mathrm{H}$, py $5-\mathrm{H}), 3.99\left(\mathrm{~s}, 3 \mathrm{H}, \mathrm{OCH}_{3}\right), 2.67\left(\mathrm{~s}, 3 \mathrm{H}, \mathrm{CH}_{3}\right)$.

13C NMR (75 MHz, chloroform-d) $\delta$ (ppm) = 165.8 (COOMe), 159.1 (py C-6), 147.4 (py C-2), 137.6 (py C-4), 127.2 (py C-5), 122.7 (py C-3), $53.1\left(\mathrm{OCH}_{3}\right), 24.6\left(\mathrm{CH}_{3}\right)$.

\subsubsection{2-Acetyl-6-methylpyridine (XXXII)}

Sodium ethanolate was freshly prepared by dissolving $\mathrm{Na}(3.29 \mathrm{~g}, 143 \mathrm{mmol}, 1.6 \mathrm{eq})$ in dry ethanol $(50 \mathrm{~mL})$ and dried in vacuo overnight. Toluene $(75 \mathrm{~mL})$ and ethyl acetate $(47 \mathrm{~mL}$, $475 \mathrm{mmol}, 5.3 \mathrm{eq}$ ) were added and the mixture stirred at ambient temperature for $0.5 \mathrm{~h}$. After addition of methyl-6-methyl-2-picolinate XXXI (13.5 g, $89.5 \mathrm{mmol}, 1.0 \mathrm{eq}$ ) and further toluene $(75 \mathrm{~mL})$ the reaction mixture was heated to reflux for $6.5 \mathrm{~h}$ and stirred at ambient temperature overnight. The volatiles were removed in vacuo and the residue heated at reflux in $20 \%$ aq. $\mathrm{H}_{2} \mathrm{SO}_{4}(135 \mathrm{~mL})$ for $6 \mathrm{~h}$. The mixture was poured onto ice $(300 \mathrm{~mL})$ and neutralized with $\mathrm{Na}_{2} \mathrm{CO}_{3}$. After addition of water $(300 \mathrm{~mL})$ the aqueous layer was extracted with $\mathrm{CH}_{2} \mathrm{Cl}_{2}$ (4 x $100 \mathrm{~mL}$ ), dried over $\mathrm{MgSO}_{4}$, filtered and the solvent was removed under reduced pressure. After drying in vacuo, the compound XXXII (10.6 g, $78.7 \mathrm{mmol}, 88 \%)$ was obtained as a brown liquid.

Formula (molecular weight) $\quad \mathrm{C}_{8} \mathrm{H}_{9} \mathrm{NO}(135.2 \mathrm{~g} / \mathrm{mol})$

${ }^{1}$ H NMR $(300 \mathrm{MHz}$, chloroform-d) $\delta(\mathrm{ppm})=7.82(\mathrm{~d}, J=7.7 \mathrm{~Hz}, 1 \mathrm{H}$, py $3-\mathrm{H}), 7.69(\mathrm{t}, J=7.7 \mathrm{~Hz}$, $1 \mathrm{H}$, py $4-\mathrm{H}), 7.30(\mathrm{~d}, J=7.7 \mathrm{~Hz}, 1 \mathrm{H}$, py $5-\mathrm{H}), 2.71\left(\mathrm{~s}, 3 \mathrm{H}, \mathrm{COCH}_{3}\right), 2.61\left(\mathrm{~s}, 3 \mathrm{H}, \mathrm{CH}_{3}\right)$.

13C NMR (75 MHz, chloroform-d) $\delta(\mathrm{ppm})=200.6$ (COMe), 158.1 (py C-6), 153.2 (py C-2), 137.1 (py C-4), 126.9 (py C-5), 118.9 (py C-3), $25.9\left(\mathrm{COCH}_{3}\right), 24.5\left(\mathrm{CH}_{3}\right)$.

\subsubsection{3,5-bis-2-(6-MethylpyridyI)-pyrazole (XXXIII)}

Sodium ethanolate was freshly prepared by dissolving Na (1.29 g, $55.9 \mathrm{mmol}, 1.1 \mathrm{eq})$ in dry ethanol $(30 \mathrm{~mL})$ and dried in vacuo overnight. Toluene $(80 \mathrm{~mL})$ and methyl-6-methyl-2picolinate XXXI (7.54 g, $49.9 \mathrm{mmol}, 1.0 \mathrm{eq})$ were added and the solution was heated to $50{ }^{\circ} \mathrm{C}$. A solution of 2-acetyl-6-methylpyridine XXXII (7.02 g, $51.9 \mathrm{mmol}, 1.0 \mathrm{eq}$ ) in toluene (95 mL) was 
added dropwise over $6 \mathrm{~h}$. The dark red solution was stirred at $50^{\circ} \mathrm{C}$ for $3 \mathrm{~h}$ and then at ambient temperature overnight. The volatiles were removed in vacuo and the residue suspended in aqueous acetic acid ( $4 \mathrm{~mL}$ in $40 \mathrm{~mL}$ ). The resulting orange solid was filtered off and dried. The solid was suspended in ethanol $(80 \mathrm{~mL})$ and hydrazine monohydrate $(6.2 \mathrm{~mL}, 125 \mathrm{mmol}$, $2.5 \mathrm{eq}$ ) was added. The red solution was heated at reflux for $20 \mathrm{~h}$. The volatiles were removed in vacuo, the crude product was suspended in water $(10 \mathrm{~mL})$ and filtered. After drying in vacuo, compound XXXIII (9.50 g, $37.4 \mathrm{mmol}, 75 \%$ ) was obtained as a pale brown solid.

Formula (molecular weight) $\quad \mathrm{C}_{15} \mathrm{H}_{14} \mathrm{~N}_{4}(250.3 \mathrm{~g} / \mathrm{mol})$

${ }^{1} \mathbf{H}$ NMR $\left(300 \mathrm{MHz}, \mathrm{CDCl}_{3}\right) \delta(\mathrm{ppm})=7.69(\mathrm{~d}, J=7.5 \mathrm{~Hz}, 2 \mathrm{H}, \mathrm{py} 3-\mathrm{H}), 7.62(\mathrm{t}, J=7.6 \mathrm{~Hz}, 2 \mathrm{H}$, py 4-H), 7.41 (s, 1H, pz 4-H), 7.08 (d, J = 7.4 Hz, 2H, py 5-H), 2.60 (s, 6H, $\mathrm{CH}_{3}$ ).

${ }^{13} \mathrm{C}$ NMR $\left(75 \mathrm{MHz}, \mathrm{CDCl}_{3}\right) \delta(\mathrm{ppm})=158.3$ (py C-6), 149.5 (py C-2), 148.4 (pz C-3 and C-5), 137.3 (py C-4), 122.5 (py C-5), 117.4 (py C-3), 101.8 (pz C-4), $24.6\left(\mathrm{CH}_{3}\right)$.

\subsubsection{3-[6-(di-tert-Butylphosphino-methyl)-2-pyridyl]-5-[6-methyl-2-pyridyl]-1H-pyrazole (XXXIV)}

A solution of 3,5-bis-2-(6-methylpyridyl)-pyrazole XXXIII (4.00 g, $15.7 \mathrm{mmol}, 1.0 \mathrm{eq}$ ) in THF $(20 \mathrm{~mL})$ was degassed by three freeze-pump-thaw cycles and cooled to $-90^{\circ} \mathrm{C}$. $n$-BuLi $(2.7 \mathrm{M}$ in toluene, $14.6 \mathrm{~mL}, 39.4 \mathrm{mmol}, 2.5 \mathrm{eq}$ ) was slowly added over a period of $30 \mathrm{~min}$. The dark red solution was stirred at low temperature for $3 \mathrm{~h}$ and allowed to warm to ambient temperature overnight. Chloro-di-tert-butyl-phosphine (2.85 g, $15.7 \mathrm{mmol}, 1.0 \mathrm{eq}$ ) in THF (3 mL) was added slowly over a period of $1 \mathrm{~h}$. The reaction mixture was stirred at ambient temperature for $1 \mathrm{~d}$ and then quenched by addition of degassed water $(15 \mathrm{~mL})$. After virguous stirring for $30 \mathrm{~min}$ the aqueous layer was removed via a syringe. The organic layer was dried in vacuo overnight yielding crude XXXIV (6.02 g) which was used in the next step without further purification.

Formula (molecular weight) $\quad \mathrm{C}_{23} \mathrm{H}_{31} \mathrm{~N}_{4} \mathrm{P}(394.5 \mathrm{~g} / \mathrm{mol})$

${ }^{1} \mathbf{H}$ NMR $\left(300 \mathrm{MHz}, \mathrm{CDCl}_{3}\right) \delta(\mathrm{ppm})=11.39(\mathrm{~s}, 1 \mathrm{H}, \mathrm{pz} \mathrm{NH}), 7.62(\mathrm{t}, J=7.5 \mathrm{~Hz}, 2 \mathrm{H}, \mathrm{py} 4-\mathrm{H}), 7.34$ $(\mathrm{d}, J=8.3 \mathrm{~Hz}, 2 \mathrm{H}$, py $3-\mathrm{H}), 7.32(\mathrm{~s}, 1 \mathrm{H}, \mathrm{pz} 4-\mathrm{H}), 7.07$ (d, $J=8.0 \mathrm{~Hz}, 2 \mathrm{H}$, py $5-\mathrm{H}), 3.09\left(\mathrm{~s}, 2 \mathrm{H}, \mathrm{CH}_{2}\right)$, $2.58\left(\mathrm{~s}, 3 \mathrm{H}, \mathrm{CH}_{3}\right), 1.17\left(\mathrm{~d}, J_{P-H}=11.0 \mathrm{~Hz}, 18 \mathrm{H}, \mathrm{C}\left(\mathrm{CH}_{3}\right)_{3}\right)$.

31P NMR (121 MHz, $\left.\mathrm{CDCl}_{3}\right) \delta(\mathrm{ppm})=37.48(\mathrm{~s}), 36.50(\mathrm{~s})$. 


\subsubsection{3,5-bis-[6-(di-tert-Butylphosphino-methyl)-2-pyridyl]-1H-pyrazole $\mathrm{HL}^{\mathrm{tBu}}(\mathrm{XXXVII)}$}

The crude product XXXIV was dissolved in THF (20 mL), degassed by three freeze-pump-thaw cycles and cooled to $-90^{\circ} \mathrm{C}$. $t$-BuLi $(1.7 \mathrm{M}$ in pentane, $27.8 \mathrm{~mL}, 47.2 \mathrm{mmol}, 3.0 \mathrm{eq})$ was slowly added over a period of $1 \mathrm{~h}$. The dark red solution was stirred at low temperature for $6 \mathrm{~h}$ and allowed to warm to ambient temperature overnight. Chloro-di-tert-butyl-phosphine $(2.85 \mathrm{~g}$, $15.7 \mathrm{mmol}, 1.0 \mathrm{eq}$ ) in THF ( $3 \mathrm{~mL}$ ) was added slowly in $2 \mathrm{~h}$. The reaction mixture was stirred at ambient temperature for $1 \mathrm{~d}$ and then quenched by addition of degassed water $(15 \mathrm{~mL})$. After virguous stirring for $30 \mathrm{~min}$ the aqueous layer was removed via a syringe. The organic layer was dried in vacuo overnight. The crude product was dissolved in a minimum amount of $\mathrm{Et}_{2} \mathrm{O}$ and purified by inert column chromatography (silica, $3 \times 8 \mathrm{~cm}, \mathrm{Et}_{2} \mathrm{O}$ (ca. $200 \mathrm{~mL}$ )) and subsequent recrystallization from $\mathrm{Et}_{2} \mathrm{O}$. Ligand XXXVII (2.51 g, $\left.4.65 \mathrm{mmol}, 30 \%\right)$ was isolated by filtration as off-white solid.

Formula (molecular weight) $\quad \mathrm{C}_{31} \mathrm{H}_{48} \mathrm{~N}_{4} \mathrm{P}_{2}(538.7 \mathrm{~g} / \mathrm{mol})$

${ }_{1}^{1}$ H NMR (500 MHz, acetone- $\left.\mathrm{d}_{6}\right) \delta(\mathrm{ppm})=12.76(\mathrm{~s}, 1 \mathrm{H}, \mathrm{NH}), 7.82-7.68(\mathrm{~m}, 4 \mathrm{H}, \mathrm{py} 4-\mathrm{H}+3-\mathrm{H})$, 7.43 (s, 1H, pz 4-H), 7.38 (d, J=7.7 Hz, 2H, py 5-H), 3.13 (d, J=2.6 Hz, 4H, CH 2 ), 1.19 (d, $\left.J_{P-H}=10.8 \mathrm{~Hz}, 36 \mathrm{H}, \mathrm{C}\left(\mathrm{CH}_{3}\right)_{3}\right)$.

${ }^{13} \mathrm{C}$ NMR $\left(126 \mathrm{MHz}\right.$, acetone- $\left.\mathrm{d}_{6}\right) \delta(\mathrm{ppm})=163.0\left(\mathrm{~d}, J_{P-C}=10.5 \mathrm{~Hz}\right.$, py C-6), 137.5 (s, py C-4), 123.6 (s, py C-5), 117.1 (s, py C-3), 102.3 (s, pz C-4), $66.1\left(\mathrm{CH}_{2}\right), 32.43\left(\mathrm{~d}, J_{P-C}=24.2 \mathrm{~Hz}, \mathrm{C}\left(\mathrm{CH}_{3}\right)_{3}\right)$, $30.32\left(\mathrm{~d}, J_{P-C}=19.2 \mathrm{~Hz}, C\left(\mathrm{CH}_{3}\right)_{3}\right)$. Resonances for pz C-3/C-5 and py C-2 were not observed.

31P NMR $\left(203 \mathrm{MHz}\right.$, acetone- $\left.\mathrm{d}_{6}\right) \delta(\mathrm{ppm})=36.25(\mathrm{~s})$.

ATR-IR (solid sample): v [ $\left.\mathrm{cm}^{-1}\right]$ = $415(\mathrm{w}), 460(\mathrm{w}), 472(\mathrm{w}), 544(\mathrm{w}), 568(\mathrm{w}), 593(\mathrm{w}), 610$ (w), $637(\mathrm{~m}), 668(\mathrm{w}), 679(\mathrm{w}), 702(\mathrm{~m}), 732(\mathrm{w}), 737(\mathrm{w}), 746(\mathrm{~m}), 795(\mathrm{~s}), 811(\mathrm{~s}), 823(\mathrm{w})$, $829(\mathrm{w}), 872(\mathrm{w}), 972(\mathrm{~m}), 991(\mathrm{w}), 999(\mathrm{w}), 1014(\mathrm{w}), 1080(\mathrm{w}), 1088(\mathrm{w}), 1160(\mathrm{~m}), 1169$ (m), 1215 (w), 1256 (w), 1366 (s), 1384 (w), 1417 (m), 1438 (m), 1463 (s), 1553 (w), 1567 (m), 1591 (m), 2858 (m), 2891 (m), 2936 (m), 2971 (m), 3443 (m).

\subsubsection{3-[6-(di-iso-propylphosphino-methyl)-2-pyridyl]-5-[6-methyl-2-pyridyl]-1H-pyrazole (XXXV)}

A solution of 3,5-bis-2-(6-methylpyridyl)-pyrazole XXXIII (3.61 g, $14.2 \mathrm{mmol}, 1.0 \mathrm{eq})$ in THF $(20 \mathrm{~mL})$ was degassed by three freeze-pump-thaw cycles and cooled to $-90{ }^{\circ} \mathrm{C}$. $n$-BuLi $(1.6 \mathrm{M}$ in hexanes, $22.1 \mathrm{~mL}, 35.4 \mathrm{mmol}, 2.5 \mathrm{eq}$ ) was slowly added over a period of $30 \mathrm{~min}$. The dark red solution was stirred at low temperature for $3 \mathrm{~h}$ and allowed to warm to ambient temperature overnight. Chloro-di-iso-propyl-phosphine $(2.17 \mathrm{~g}, 14.2 \mathrm{mmol}, 1.0 \mathrm{eq})$ in THF (3 mL) was 
added slowly over a period of $1 \mathrm{~h}$. The reaction mixture was stirred at ambient temperature for $1 \mathrm{~d}$ and then quenched by addition of degassed water $(15 \mathrm{~mL})$. After virgeous stirring for 30 min the aqueous layer was removed via a syringe. The organic layer was dried in vacuo overnight yielding crude XXXIV which was used in the next step without further purification.

Formula (molecular weight) $\quad \mathrm{C}_{21} \mathrm{H}_{27} \mathrm{~N}_{4} \mathrm{P}(366.5 \mathrm{~g} / \mathrm{mol})$

${ }^{1}$ H NMR $\left(300 \mathrm{MHz}\right.$, acetone- $\left.\mathrm{d}_{6}\right) \delta(\mathrm{ppm})=11.33(\mathrm{~s}, 1 \mathrm{H}, \mathrm{NH}), 7.80(\mathrm{~s}, 1 \mathrm{H}$, py 4-H), $7.63(\mathrm{t}$, $J=7.6 \mathrm{~Hz}, 1 \mathrm{H}$, py 4-H), 7.50 (s, 2H, py 3-H, 5-H), 7.32 (s, 1H, pz 4-H), 7.22 (d, J = 7.7 Hz, 1H, py 3-H), 7.08 (d, $J=7.7 \mathrm{~Hz}, 1 \mathrm{H}$, py 5-H), $3.03\left(\mathrm{~s}, 2 \mathrm{H}, \mathrm{CH}_{2}\right), 2.59$ (s, 3H, $\left.\mathrm{CH}_{3}\right), 1.92-1.74(\mathrm{~m}, 2 \mathrm{H}$, $\left.\mathrm{CH}\left(\mathrm{CH}_{3}\right)_{2}\right), 1.18-0.98\left(\mathrm{~m}, 12 \mathrm{H}, \mathrm{CH}\left(\mathrm{CH}_{3}\right)_{2}\right)$.

${ }^{13} \mathrm{C}$ NMR $\left(75 \mathrm{MHz}\right.$, acetone- $\left.\mathrm{d}_{6}\right) \delta(\mathrm{ppm})=158.8$ (s, py C-6), 154.7 (s, py C-2), 143.3 (s, pz C-3/C-5), 137.9 (s, py C-4), 122.8 (s, py C-5), 117.5 (s, py C-3), 102.4 (s, pz C-4), 32.8 (s, $\mathrm{CH}_{2}$ ), $24.5\left(\mathrm{~s}, \mathrm{CH}_{3}\right), 24.3\left(\mathrm{~d}, J=15.4 \mathrm{~Hz}, \mathrm{CH}\left(\mathrm{CH}_{3}\right)_{2}\right), 20.1\left(\mathrm{~d}, J=15.5 \mathrm{~Hz}, \mathrm{CH}\left(\mathrm{CH}_{3}\right)_{2}\right), 19.4(\mathrm{~d}, J=10.7 \mathrm{~Hz}$, $\left.\mathrm{CH}\left(\mathrm{CH}_{3}\right)_{2}\right)$.

31P NMR (121 MHz, acetone- $\left.\mathrm{d}_{6}\right) \delta(\mathrm{ppm})=13.20(\mathrm{~s}), 12.24(\mathrm{~s})$.

\subsubsection{3,5-bis-[6-(di-iso-propylphosphino-methyl)-2-pyridyl]-1H-pyrazole $\mathrm{HL}^{\text {iPr }}$ (XXXVIII)}

The crude product XXXIV was dissolved in THF (16 mL), degassed by three freeze-pump-thaw cycles and cooled to $-90^{\circ} \mathrm{C}$. $t$-BuLi $(1.7 \mathrm{M}$ in pentane, $25.0 \mathrm{~mL}, 42.5 \mathrm{mmol}, 3.0 \mathrm{eq}$ ) was slowly added over a period of $1 \mathrm{~h}$. The dark red solution was stirred at low temperature for $6 \mathrm{~h}$ and allowed to warm to ambient temperature overnight. Chloro-di-iso-propyl-phosphine $(2.17 \mathrm{~g}$, $14.2 \mathrm{mmol}, 1.0 \mathrm{eq})$ in THF ( $3 \mathrm{~mL}$ ) was added slowly in $2 \mathrm{~h}$. The reaction mixture was stirred at ambient temperature for $1 \mathrm{~d}$ and then quenched by addition of degassed water (15 $\mathrm{mL}$ ). After virgous stirring for $30 \mathrm{~min}$ the aqueous layer was removed via a syringe. The organic layer was dried in vacuo overnight. The crude product was dissolved in a minimum amount of $\mathrm{Et}_{2} \mathrm{O}$ and purified by inert column chromatography (silica, $3.5 \times 12 \mathrm{~cm}, \mathrm{Et}_{2} \mathrm{O}$ (ca. $300 \mathrm{~mL}$ )) and subsequent recrystallization from pentane. Filtration and drying gave $130 \mathrm{mg}$ (2\%) ligand XXXVII as off-white solid.

Formula (molecular weight) $\quad \mathrm{C}_{27} \mathrm{H}_{40} \mathrm{~N}_{4} \mathrm{P}_{2}(482.6 \mathrm{~g} / \mathrm{mol})$

${ }^{1} \mathbf{H}$ NMR $\left(300 \mathrm{MHz}, \mathrm{CDCl}_{3}\right) \delta(\mathrm{ppm})=12.58(\mathrm{~s}, 1 \mathrm{H}, \mathrm{NH}), 7.64(\mathrm{~d}, J=6.2 \mathrm{~Hz}, 2 \mathrm{H}, \mathrm{py} 3-\mathrm{H}), 7.58(\mathrm{t}$, $J=7.5 \mathrm{~Hz}, 2 \mathrm{H}$, py 4-H), 7.30 (s, 1H, pz 4-H), $7.17-7.07$ (m, 2H, py 5-H), 2.91 (d, J = $1.6 \mathrm{~Hz}, 4 \mathrm{H}$, $\mathrm{CH}_{2}$ ), 1.70 (heptd, $\left.J=7.2,1.7 \mathrm{~Hz}, 4 \mathrm{H}, \mathrm{CH}\left(\mathrm{CH}_{3}\right)_{2}\right), 0.97$ (d, $\left.J=10.7,6.5 \mathrm{~Hz}, 12 \mathrm{H}, \mathrm{CH}\left(\mathrm{CH}_{3}\right)_{2}\right), 0.91$ (dd, $J=13.0,6.7 \mathrm{~Hz}, 12 \mathrm{H}, \mathrm{CH}\left(\mathrm{CH}_{3}\right)_{2}$ ). 
${ }^{13} \mathrm{C}$ NMR $\left(126 \mathrm{MHz} \mathrm{CDCl}_{3}, 243 \mathrm{~K}\right) \delta(\mathrm{ppm})=161.8$ (s, py C-6), 161.0 (s, py C-6), 153.9 (s, pz C-3 or pz C-5), 152.3 (s, py C-2), 147.8 (s, py C-2), 143.9 (s, pz C-3 or pz C-5), 138.0 (s, py C-4), 137.3 (s, py C-4), 123.5 (s, py C-5), 122.9 (s, py C-5), 117.4 (s, py C-3), 116.7 (s, py C-3), 102.1 (s, pz C-4), 32.7 (s, $\mathrm{CH}_{2}$ ), 32.4 (s, $\mathrm{CH}_{2}$ ), 24.1 (s, $\left.\mathrm{CH}\left(\mathrm{CH}_{3}\right)_{2}\right), 24.0$ (s, $\left.\mathrm{CH}\left(\mathrm{CH}_{3}\right)_{2}\right), 19.9$ (s, $\left.\mathrm{CH}\left(\mathrm{CH}_{3}\right)_{2}\right), 19.8$ (s, $\left.\mathrm{CH}\left(\mathrm{CH}_{3}\right)_{2}\right), 19.0\left(\mathrm{~s}, \mathrm{CH}\left(\mathrm{CH}_{3}\right)_{2}\right), 18.9\left(\mathrm{~s}, \mathrm{CH}\left(\mathrm{CH}_{3}\right)_{2}\right)$.

31P NMR (121 MHz, $\left.\mathrm{CDCl}_{3}\right) \delta(\mathrm{ppm})=12.20(\mathrm{~s}), 12.03(\mathrm{~s})$.

ATR-IR (solid): $v$ [cm-1] = $463(\mathrm{w}), 519(\mathrm{w}), 595(\mathrm{w}), 610(\mathrm{w}), 647(\mathrm{w}), 682(\mathrm{w}), 696(\mathrm{w}), 726$ (w), $743(\mathrm{~m}), 805(\mathrm{~s}), 872(\mathrm{w}), 879(\mathrm{w}), 983(\mathrm{~m}), 990(\mathrm{w}), 1000(\mathrm{~m}), 1083(\mathrm{w}), 1157(\mathrm{w}), 1174$ (w), 1218 (m), 1362 (m), 1417 (m), 1439 (m), 1463 (m), 1561 (m), 1574 (m), 1594 (w), 2863 (m), $2946(\mathrm{~m})$.

\subsubsection{3-[6-(diphenylphosphino-methyl)-2-pyridyl]-5-[6-methyl-2-pyridyl]-1H-pyrazole (XXXVI)}

A solution of 3,5-bis-2-(6-methylpyridyl)-pyrazole XXXIII (3.00 g, $11.8 \mathrm{mmol}, 1.0 \mathrm{eq})$ in THF $(20 \mathrm{~mL})$ was degassed by three freeze-pump-thaw cycles and cooled to $-90^{\circ} \mathrm{C}$. $n$-BuLi $(2.5 \mathrm{M}$ in hexanes, $14.2 \mathrm{~mL}, 35.4 \mathrm{mmol}, 3.0 \mathrm{eq}$ ) was slowly added over a period of $30 \mathrm{~min}$. The dark red solution was stirred at low temperature for $3 \mathrm{~h}$ and allowed to warm to ambient temperature overnight. Chloro-diphenyl-phosphine (2.60 g, $11.8 \mathrm{mmol}, 1.0 \mathrm{eq})$ in THF (4 mL) was added slowly over a period of $1 \mathrm{~h}$. The reaction mixture was stirred at ambient temperature for $1 \mathrm{~d}$ and then quenched by addition of degassed water $(15 \mathrm{~mL})$. After virgeous stirring for $30 \mathrm{~min}$ the aqueous layer was removed via a syringe. The organic layer was dried in vacuo overnight yielding crude XXXVI.

Formula (molecular weight) $\quad \mathrm{C}_{27} \mathrm{H}_{23} \mathrm{~N}_{4} \mathrm{P}(434.5 \mathrm{~g} / \mathrm{mol})$

1H NMR (300 MHz, $\left.\mathrm{CDCl}_{3}\right) \delta(\mathrm{ppm})=10.56(\mathrm{~s}, 1 \mathrm{H}, \mathrm{NH}), 7.82(\mathrm{~d}, J=7.1 \mathrm{~Hz}, 2 \mathrm{H}, \mathrm{py} \mathrm{3-H}), 7.68-$ 7.59 (m, 2H, py 4-H), $7.54-7.46$ (m, 4H, Ph), 7.39 - 7.31 (m, 6H, Ph), 7.27 (s, 1H, pz 4-H), 7.09 (d, $J=7.7 \mathrm{~Hz}, 2 \mathrm{H}$, py $5-\mathrm{H}), 3.67\left(\mathrm{~s}, 2 \mathrm{H}, \mathrm{CH}_{2}\right), 2.62\left(\mathrm{~s}, 3 \mathrm{H}, \mathrm{CH}_{3}\right)$.

31P NMR (121 MHz, $\left.\mathrm{CDCl}_{3}\right) \delta(\mathrm{ppm})=-11.22(\mathrm{~s}),-11.43(\mathrm{~s})$.

\subsubsection{Complex Synthesis}

\subsubsection{1 $\left[\mathrm{Zn}_{2}(\mu-\mathrm{OAC}) \mathrm{L}^{\mathrm{tBu}}\right]\left(\mathrm{PF}_{6}\right)_{2}(48)$}

A suspension of $\mathbf{H L}^{t \mathrm{Bu}}(25.0 \mathrm{mg}$, $46.4 \mu \mathrm{mol}, 1.0 \mathrm{eq}), \mathrm{Zn}(\mathrm{OAc})_{2} \cdot 2 \mathrm{H}_{2} \mathrm{O}(20.4 \mathrm{mg}, 92.8 \mu \mathrm{mol}, 2.0 \mathrm{eq})$ and $\mathrm{KPF}_{6}$ (16.9 mg, $\left.92.8 \mu \mathrm{mol}, 2.0 \mathrm{eq}\right)$ in dry MeCN (3 mL) was stirred until all solids were 
dissolved $(2 \mathrm{~h})$. The volatiles were removed in vacuo, the residue extracted with $\mathrm{CH}_{2} \mathrm{Cl}_{2}(2 \mathrm{~mL})$ and filtered through glas fibre filters. Drying of the light yellow filtrate in vacuo gave $40 \mathrm{mg}$ (85\%) 48 as off-white solid.

Formula (molecular weight) $\quad \mathrm{C}_{33} \mathrm{H}_{50} \mathrm{~N}_{4} \mathrm{O}_{2} \mathrm{P}_{2} \mathrm{Zn}_{2}\left(\mathrm{PF}_{6}\right)_{2}(1017.4 \mathrm{~g} / \mathrm{mol})$

${ }^{1}$ H NMR $\left(300 \mathrm{MHz}\right.$, acetone- $\left.\mathrm{d}_{6}\right) \delta(\mathrm{ppm})=7.96(\mathrm{td}, J=7.7,0.6 \mathrm{~Hz}, 2 \mathrm{H}, \mathrm{py} 4-\mathrm{H}), 7.63(\mathrm{~d}$, $J=7.7 \mathrm{~Hz}, 2 \mathrm{H}$, py 3-H), $7.42(\mathrm{dt}, J=7.8,1.0 \mathrm{~Hz}, 2 \mathrm{H}$, py $5-\mathrm{H}), 7.21(\mathrm{~s}, 1 \mathrm{H}, \mathrm{pz} 4-\mathrm{H}), 3.48(\mathrm{~d}$, $\left.J_{P-H}=8.9 \mathrm{~Hz}, 4 \mathrm{H}, \mathrm{CH}_{2}\right), 1.91(\mathrm{~s}, 3 \mathrm{H}, \mathrm{OAc}) 1.35\left(\mathrm{~d}, J=13.7 \mathrm{~Hz}, 36 \mathrm{H}, \mathrm{C}\left(\mathrm{CH}_{3}\right)_{3}\right)$.

${ }^{13}$ C NMR $\left(75\right.$ MHz, acetone- $\left.\mathrm{d}_{6}\right) \delta(\mathrm{ppm})=179.0$ (s, OAc), 156.3 (s, pz C-3/C-5), 150.2 (s, py C-6), 148.1 (s, py C-2), 142.0 (s, py C-4), 125.1 (s, py C-5), 118.41 (s, py C-3), 99.3 (s, pz C-4), 66.3 (s, $\left.\mathrm{CH}_{2}\right), 33.7\left(\mathrm{~d}, J=7.9 \mathrm{~Hz}, C\left(\mathrm{CH}_{3}\right)_{3}\right), 29.3\left(\mathrm{~d}, J=6.0 \mathrm{~Hz}, \mathrm{C}\left(\mathrm{CH}_{3}\right)_{3}\right), 26.54(\mathrm{~d}, J=20.1 \mathrm{~Hz}, \mathrm{OAc})$.

31P NMR (162 MHz, $\left.\mathrm{CD}_{3} \mathrm{CN}\right) \delta(\mathrm{ppm})=19.12(\mathrm{~s}),-144.62$ (hept, $\left.\mathrm{PF}_{6}{ }^{-}\right)$.

HR-MS (ESI+, MeCN) m/z = $364.0971\left(\left[\mathrm{Zn}_{2}(\mathrm{OAc}) \mathrm{L}^{t \mathrm{Bu}}\right]^{2+}\right.$, calcd.: 364.0969).

\subsubsection{2 $\left[(\mathrm{Zn}(\mathrm{OTf}))_{2}(\mu-\mathrm{OTf}) \mathrm{L}^{\mathrm{tBu}}\right](49)$}

A suspension of $\mathbf{H L}^{t \mathrm{Bu}}(25.0 \mathrm{mg}, 46.4 \mu \mathrm{mol}, 1.0 \mathrm{eq}), \mathrm{Zn}(\mathrm{OTf})_{2}(33.4 \mathrm{mg}, 92.8 \mu \mathrm{mol}, 2.0 \mathrm{eq})$ and $\mathrm{KO}^{t} \mathrm{Bu}(5.2 \mathrm{mg}$, $46.4 \mu \mathrm{mol}, 1.0 \mathrm{eq})$ in dry MeCN ( $3 \mathrm{~mL}$ ) was for $21 \mathrm{~h}$. The volatiles were removed in vacuo, the residue extracted with $\mathrm{CH}_{2} \mathrm{Cl}_{2}(2 \mathrm{~mL})$ and filtered through glas fibre filters. Drying of the light yellow filtrate in vacuo gave $48 \mathrm{mg}$ (93\%) $\mathbf{4 8}$ as off-white solid.

Single crystals suitable for X-ray diffraction analysis were grown by layering a MeCN solution of the crude product $(2 \mathrm{~mL})$ with $\mathrm{Et}_{2} \mathrm{O}$.

\section{Formula (molecular weight) $\quad \mathrm{C}_{31} \mathrm{H}_{47} \mathrm{~N}_{4} \mathrm{P}_{2} \mathrm{Zn}_{2}\left(\mathrm{CF}_{3} \mathrm{SO}_{3}\right)_{3}(1115.7 \mathrm{~g} / \mathrm{mol})$}

${ }^{1}$ H NMR $\left(300 \mathrm{MHz}\right.$, acetone- $\left.\mathrm{d}_{6}\right) \delta(\mathrm{ppm})=8.12(\mathrm{t}, J=7.8 \mathrm{~Hz}, 2 \mathrm{H}, \mathrm{py} 4-\mathrm{H}), 7.83(\mathrm{~d}, J=7.8 \mathrm{~Hz}, 2 \mathrm{H}$, py 3-H), 7.61 (s, 1H, pz 4-H), 7.57 (d, J = 7.6 Hz, 2H, py 5-H), 3.61 (d, J = 8.4 Hz, 4H, CH $\mathrm{CH}_{2}, 1.30$ $\left(\mathrm{d}, J=14.1 \mathrm{~Hz}, 36 \mathrm{H}, \mathrm{C}\left(\mathrm{CH}_{3}\right)_{3}\right)$.

${ }^{13}$ C NMR (126 MHz, acetone- $\left.\mathrm{d}_{6}\right) \delta(\mathrm{ppm})=155.7$ (s, pz C-3/C-5), 152.4 (s, py C-2), 147.3 (d, $J=3.0$ Hz, py C-6), 143.7 (s, py C-4), 126.2 (s, py C-5), 119.5 (s, py C-3), 102.1 (s, pz C-4), 34.0 $\left(\mathrm{d}, J=9.5 \mathrm{~Hz}, C\left(\mathrm{CH}_{3}\right)_{3}\right), 29.6\left(\mathrm{~d}, J=5.2 \mathrm{~Hz}, \mathrm{C}\left(\mathrm{CH}_{3}\right)_{3}\right), 26.4\left(\mathrm{~d}, J=18.4 \mathrm{~Hz}, \mathrm{CH}_{2}\right)$.

31P NMR (121 MHz, acetone- $\left.\mathrm{d}_{6}\right) \delta(\mathrm{ppm})=22.55(\mathrm{~s})$.

MS $(\mathrm{ESI}+, \mathrm{MeCN}) \mathrm{m} / \mathrm{z}=787.241\left(\left[\left(\mathrm{Zn}(\mathrm{MeCN})\left(\mathrm{H}_{2} \mathrm{O}\right)\right)_{2} \mathrm{~L}^{t \mathrm{Bu}}\right]^{+}\right)$. 
UV-vis $(\mathrm{MeCN}): \lambda_{\max }[\mathrm{nm}]\left(\varepsilon\left[\mathrm{M}^{-1} \mathrm{~cm}^{-1}\right]\right)=259$ (38 800), 306 (38 100).

ATR-IR (solid): v [cm-1] = $403(\mathrm{w}), 451(\mathrm{~m}), 478(\mathrm{w}), 509$ (s), $514(\mathrm{~s}), 539(\mathrm{w}), 560$ (w), 571 $(\mathrm{w}), 628(\mathrm{~s}), 636(\mathrm{~s}), 656(\mathrm{w}), 678(\mathrm{w}), 743(\mathrm{w}), 795(\mathrm{w}), 820(\mathrm{~m}), 835(\mathrm{~m}), 938(\mathrm{w}), 1007(\mathrm{~s})$, 1014 (s), 1028 (s), 1050 (w), 1077 (m), 1094 (s), 1153 (m), 1184 (m), 1210 (m), 1222 (m), 1229 (m), 1262 (s), 1274 (s), 1317 (m), 1324 (w), 1456 (m), 1475 (m), 1569 (w), 1607 (w), $2877(w), 2909(w), 2968(w)$.

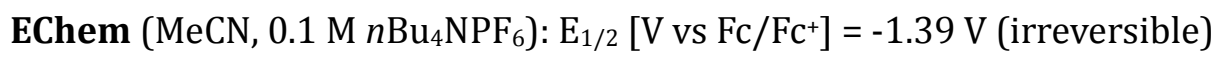
$-1.64 \mathrm{~V}$ (irreversible)

\subsubsection{3 $\left[(\mathrm{Zn}(\mathrm{OTf}))(\mathrm{Zn}(\mathrm{MeCN}))(\mu-\mathrm{OTf}) \mathrm{L}^{\mathrm{iPr}}\right](\mathrm{OTf})(50)$ and $\left[\left(\mathrm{Zn}_{2}(\mu-\mathrm{OTf}) \mathrm{L}^{\mathrm{tBu}, \mathrm{ox}}\right)_{2}(\mu-\mathrm{OH})_{2}\right](\mathrm{OTf})_{2}(51)$}

A suspension of $\mathbf{H L}^{i \mathbf{P r}}(22.2 \mathrm{mg}, 46.4 \mu \mathrm{mol}, 1.0 \mathrm{eq}), \mathrm{Zn}(\mathrm{OTf})_{2}(33.4 \mathrm{mg}, 92.8 \mu \mathrm{mol}, 2.0 \mathrm{eq})$ and $\mathrm{KO}^{t} \mathrm{Bu}(5.2 \mathrm{mg}$, $46.4 \mu \mathrm{mol}, 1.0 \mathrm{eq})$ in dry MeCN ( $5 \mathrm{~mL}$ ) was for $3 \mathrm{~h}$. The volatiles were removed in vacuo, the residue extracted with $\mathrm{CH}_{2} \mathrm{Cl}_{2}(2 \times 2 \mathrm{~mL})$ and filtered through glas fibre filters. Drying of the light yellow filtrate in vacuo gave 45 mg (92\%) 50 as off-white solid.

Single crystals suitable for X-ray diffraction analysis of $\mathbf{5 0}$ were grown by layering a MeCN solution of the crude product ( $2 \mathrm{~mL}$ ) with $\mathrm{Et}_{2} \mathrm{O}$ under inert conditions. Single crystals suitable for X-ray diffraction analysis of $\mathbf{5 1}$ were grown by layering a MeCN solution of the crude product $(2 \mathrm{~mL})$ with $\mathrm{Et}_{2} \mathrm{O}$ under aerobic conditions.

$\left[(\mathrm{Zn}(\mathrm{OTf}))(\mathrm{Zn}(\mathrm{MeCN}))(\mu-\mathrm{OTf}) \mathrm{L}^{\mathrm{iPr}}\right](\mathrm{OTf}) 50$ :

Formula (molecular weight) $\quad \mathrm{C}_{31} \mathrm{H}_{47} \mathrm{~N}_{4} \mathrm{P}_{2} \mathrm{Zn}_{2}\left(\mathrm{CF}_{3} \mathrm{SO}_{3}\right)_{3}(1059.6 \mathrm{~g} / \mathrm{mol})$

${ }^{1} \mathbf{H}$ NMR $\left(300 \mathrm{MHz}, \mathrm{CD}_{3} \mathrm{CN}\right) \delta(\mathrm{ppm})=8.11(\mathrm{t}, J=7.8 \mathrm{~Hz}, 2 \mathrm{H}$, py $4-\mathrm{H}), 7.83(\mathrm{~d}, J=7.8 \mathrm{~Hz}, 2 \mathrm{H}$, py 3-H), 7.57 (s, 1H, pz 4-H), $7.53(\mathrm{dt}, J=7.8,0.9 \mathrm{~Hz}, 2 \mathrm{H}, \mathrm{py} 5-\mathrm{H}), 3.49\left(\mathrm{~d}, J=9.1 \mathrm{~Hz}, 4 \mathrm{H}, \mathrm{CH}_{2}\right), 2.48$ - $2.34\left(\mathrm{~m}, 4 \mathrm{H}, \mathrm{CH}\left(\mathrm{CH}_{3}\right)_{2}\right), 1.20\left(\mathrm{dd}, J=7.1,2.8 \mathrm{~Hz}, 12 \mathrm{H}, \mathrm{CH}\left(\mathrm{CH}_{3}\right)_{2}\right), 1.14(\mathrm{dd}, J=7.1,0.9 \mathrm{~Hz}, 12 \mathrm{H}$, $\left.\mathrm{CH}\left(\mathrm{CH}_{3}\right)_{2}\right)$.

${ }^{13} \mathrm{C}$ NMR $\left(75 \mathrm{MHz}, \mathrm{CD}_{3} \mathrm{CN}\right) \delta(\mathrm{ppm})=155.5$ (s, py C-6), 152.1 (s, py C-2), 147.3 (s, pz C-3/C-5), 143.5 (s, py C-4), 126.2 (s, py C-5), 122.6 (s, py C-3), 119.5 (s, OTf), 101.7 (s, pz C-4), 60.8 (d, J $\left.=41.5 \mathrm{~Hz}, \mathrm{CH}_{2}\right), 26.2\left(\mathrm{~d}, J_{P-C}=20.7 \mathrm{~Hz}, \mathrm{CH}\left(\mathrm{CH}_{3}\right)_{2}\right), 22.5\left(\mathrm{~d}, J_{P-C}=15.5 \mathrm{~Hz}, \mathrm{CH}\left(\mathrm{CH}_{3}\right)_{2}\right)$.

31P NMR $\left(121 \mathrm{MHz}, \mathrm{CD}_{3} \mathrm{CN}\right) \delta(\mathrm{ppm})=6.04(\mathrm{~s})$. 
ATR-IR (solid): $v$ [cm-1] = $495(\mathrm{w}), 514(\mathrm{~m}), 541(\mathrm{w}), 572(\mathrm{w}), 588(\mathrm{w}), 633(\mathrm{~s}), 790(\mathrm{w}), 837$ (w), 1022 (s), $1104(\mathrm{w}), 1160$ (s), 1177 (m), 1206 (s), 1234 (m), $1288(\mathrm{~m}), 1312(\mathrm{~m}), 1427$ (w), $1456(\mathrm{~m}), 1470(\mathrm{w}), 1572(\mathrm{w}), 1602(\mathrm{w}), 2877(\mathrm{w}), 2968(\mathrm{w})$.

EChem (MeCN, 0.1 M nBu $\mathrm{NPF}_{6}$ ): $\mathrm{E}_{1 / 2}[\mathrm{~V}$ vs Fc/Fc'] = -1.49 V (irreversible)

$\left[\left(\mathrm{Zn}_{2}(\mu-\mathrm{OTf}) \mathrm{L}^{t \mathrm{Bu}, \mathrm{ox}}\right)_{2}(\mu-\mathrm{OH})_{2}\right](\mathrm{OTf})_{2} 51$ :

Formula (molecular weight) $\quad \mathrm{C}_{64} \mathrm{H}_{96} \mathrm{~F}_{6} \mathrm{~N}_{8} \mathrm{O}_{12} \mathrm{P}_{4} \mathrm{~S}_{2} \mathrm{Zn}_{4}\left(\mathrm{CF}_{3} \mathrm{SO}_{3}\right)_{2}(2031.3 \mathrm{~g} / \mathrm{mol})$

1H NMR $\left(400 \mathrm{MHz}, \mathrm{CD}_{3} \mathrm{CN}\right) \delta(\mathrm{ppm})=8.12(\mathrm{t}, J=7.8 \mathrm{~Hz}, 2 \mathrm{H}, \mathrm{py} 4-\mathrm{H}), 7.91(\mathrm{~d}, J=7.9 \mathrm{~Hz}, 2 \mathrm{H}, \mathrm{py}$ 3-H), $7.59-7.56$ (m, 3H, pz 4-H/py 5-H), $3.65\left(\mathrm{~d}, J_{P-H}=11.3 \mathrm{~Hz}, 4 \mathrm{H}, \mathrm{CH}_{2}\right), 2.37-2.27$ (m, 4H. $\left.\mathrm{CH}\left(\mathrm{CH}_{3}\right)_{2}\right), 1.18\left(\mathrm{dd}, J=7.2,5.2 \mathrm{~Hz}, 12 \mathrm{H}, \mathrm{CH}\left(\mathrm{CH}_{3}\right)_{2}\right), 1.13\left(\mathrm{dd}, J=7.2,5.2 \mathrm{~Hz}, 12 \mathrm{H}, \mathrm{CH}\left(\mathrm{CH}_{3}\right)_{2}\right)$.

${ }^{13}$ C NMR $\left(75 \mathrm{MHz}, \mathrm{CD}_{3} \mathrm{CN}\right) \delta(\mathrm{ppm})=154.2\left(\mathrm{~d}, J_{P-C}=8.7 \mathrm{~Hz}\right.$, py C-6), $152.1(\mathrm{~s}, \mathrm{py} \mathrm{C}-2), 149.0(\mathrm{~s}$, py py C-4) 143.3 (s, pz C-3/C-5), 127.2 (d, py C-5), 123.0 (s, py C-3), 120.4 (s, OTf), 102.2 (s, pz C-4), 29.1 (d, $J_{P-C}=50.5 \mathrm{~Hz}, \mathrm{CH}_{2}$ ), 26.3 (d, $\left.J_{P-C}=65.0 \mathrm{~Hz}, C \mathrm{H}\left(\mathrm{CH}_{3}\right)_{2}\right), 15.7$ (d, $J_{P-C}=2.3 \mathrm{~Hz}$, $\left.\mathrm{CH}\left(\mathrm{CH}_{3}\right)_{2}\right), 15.5\left(\mathrm{~d}, J_{P-C}=3.6 \mathrm{~Hz}, \mathrm{CH}\left(\mathrm{CH}_{3}\right)_{2}\right)$.

31P NMR (162 MHz, CD 3 CN) $\delta(p p m)=71.36(\mathrm{~s})$.

\subsubsection{4 $\left[\left(\mathrm{Co}(\mathrm{MeCN})_{2}\right)_{2} \mathrm{~L}^{\mathrm{tBu}}\right]\left(\mathrm{BF}_{4}\right)_{3} 54$}

A suspension of $\mathbf{H L}^{t \mathrm{Bu}}(200 \mathrm{mg}, 371 \mu \mathrm{mol}, 1.0 \mathrm{eq}), \mathrm{Co}\left(\mathrm{BF}_{4}\right)_{2} \cdot 6 \mathrm{H}_{2} \mathrm{O}(253 \mathrm{mg}, 742 \mu \mathrm{mol}, 2.0 \mathrm{eq})$ and $\mathrm{KO}{ }^{t} \mathrm{Bu}(41.6 \mathrm{mg}, 371 \mu \mathrm{mol}, 1.0 \mathrm{eq})$ in dry $\mathrm{MeCN}(10 \mathrm{~mL}$ ) was for $4 \mathrm{~h}$. The volatiles were removed in vacuo, the residue extracted with $\mathrm{CH}_{2} \mathrm{Cl}_{2}(4 \times 5 \mathrm{~mL})$ and filtered through glass fiber filters. Drying of the red-brown filtrate in vacuo gave $392 \mathrm{mg}$ (98\%) crude $\mathbf{5 4}$ as red-brown solid. Single crystals suitable for X-ray diffraction analysis were grown by layering a diluted $\mathrm{MeCN}$ solution of the crude product ( $40 \mathrm{mg}$ in $5 \mathrm{~mL}$ ) with $\mathrm{Et}_{2} \mathrm{O}$. Isolation and drying on air gave $208 \mathrm{mg}$ (52\%) as red-brown plates.

\section{Formula (molecular weight) $\quad \mathrm{C}_{39} \mathrm{H}_{59} \mathrm{~N}_{8} \mathrm{P}_{2} \mathrm{Co}_{2}\left(\mathrm{BF}_{4}\right)_{3}(1080.2 \mathrm{~g} / \mathrm{mol})$}

UV-vis (MeCN): $\lambda_{\max }[\mathrm{nm}]\left(\varepsilon\left[\mathrm{M}^{-1} \mathrm{~cm}^{-1}\right]\right)=259$ (26 500), 309 (20 900).

EChem (MeCN, 0.1 M n $n u_{4} \mathrm{NPF}_{6}$ ): $\mathrm{E}_{1 / 2}\left[\mathrm{~V}\right.$ vs $\left.\mathrm{Fc}_{\mathrm{F}} / \mathrm{Fc}^{+}\right]=-0.27 \mathrm{~V}$ (irreversible)

- $1.17 \mathrm{~V}$ (irreversible)

- $1.51 \mathrm{~V}$ (irreversible)

- $1.59 \mathrm{~V}$ (irreversible) 
ATR-IR (solid): $v$ [cm-1] = $402(\mathrm{w}), 406(\mathrm{w}), 422(\mathrm{w}), 449(\mathrm{~m}), 480(\mathrm{w}), 500(\mathrm{w}), 519(\mathrm{~m}), 587$ (w), $632(\mathrm{w}), 762(\mathrm{w}), 795(\mathrm{~m}), 810(\mathrm{w}), 842(\mathrm{~m}), 899(\mathrm{w}), 941(\mathrm{w}), 1004(\mathrm{~s}), 1024(\mathrm{~s}), 1032$ (s), 1047 (s), $1169(w), 1181(w), 1287(w), 1321(w), 1373(w), 1395(w), 1423(w), 1461$ (m), $1474(\mathrm{w}), 1563(\mathrm{~m}), 1612(\mathrm{~m}), 2283(\mathrm{w}), 2313(\mathrm{w}), 2937(\mathrm{w}), 2941(\mathrm{w})$.

EA Calcd. for $\mathrm{C}_{37} \mathrm{H}_{56} \mathrm{~B}_{3} \mathrm{Co}_{2} \mathrm{~F}_{12} \mathrm{~N}_{7} \mathrm{O}_{2} \mathrm{P}_{2}$ : C 42.77, H 5.51, N 9.44. Found: C 44.77, H 5.53, N 9.84.

\subsubsection{5 $\left[(\operatorname{Rh}(\mathrm{CO}))_{2} \mathrm{~L}^{\mathrm{tBu}}\right]\left(\mathrm{PF}_{6}\right)(55)$}

a) To a suspension of $\mathbf{H L}^{t \mathrm{Bu}}(25.0 \mathrm{mg}, 46.4 \mu \mathrm{mol}, 1.0 \mathrm{eq}), \mathrm{KO}{ }^{t} \mathrm{Bu}(5.20 \mathrm{mg}, 46.4 \mu \mathrm{mol}, 1.0 \mathrm{eq})$ and $\mathrm{KPF}_{6}(10.2 \mathrm{mg}, 55.7 \mu \mathrm{mol}, 1.2 \mathrm{eq})$ in $\mathrm{MeCN}(4 \mathrm{~mL})$ [Rh(acac)(CO) 2 ] (23.9 mg, $\left.92.8 \mu \mathrm{mol}, 2.0 \mathrm{eq}\right)$ was added as a solid. The reaction mixture was stirred at ambient temperature for $23 \mathrm{~h}$, volatiles were removed in vacuo and the residue extracted with $\mathrm{CH}_{2} \mathrm{Cl}_{2}(2 \times 3 \mathrm{~mL})$. Pure compound was obtained from $\mathrm{Et}_{2} \mathrm{O}$ diffusion into a concentrated acetonitrile solution. Drying under vacuum gave $12 \mathrm{mg}$ (28\%) 55 as yellow solid.

b) A solution of $\mathbf{5 8}(50 \mathrm{mg}, 52 \mu \mathrm{mol})$ in acetone ( $8 \mathrm{~mL}$ ) was degassed by 3 freeze-pump-thaw cycles and the solution purged with CO (ca. $1 \mathrm{~atm}$ ). The solution was stirred for $5 \mathrm{~min}$ and then the volatiles were removed under reduces pressure. Washing the residue with $\operatorname{Et}_{2} \mathrm{O}(2 \times 2 \mathrm{~mL})$ and drying in vacuo gave $42 \mathrm{mg}$ (86\%) 55 as yellow solid.

\section{Formula (molecular weight) $\quad \mathrm{C}_{33} \mathrm{H}_{47} \mathrm{~N}_{4} \mathrm{O}_{2} \mathrm{P}_{2} \mathrm{Rh}_{2}\left(\mathrm{PF}_{6}\right)(944.5 \mathrm{~g} / \mathrm{mol})$}

${ }^{1}$ H NMR (400 MHz, Acetonitrile- $\left.d_{3}\right) \delta=7.96\left(\mathrm{td}, J_{H-H}=7.9,1.1 \mathrm{~Hz}, 2 \mathrm{H}\right.$, py H-4), 7.64 (d, $J_{H-H}=7.8 \mathrm{~Hz}, 2 \mathrm{H}$, py H-3), 7.49 (d, $J_{H-H}=7.9 \mathrm{~Hz}, 2 \mathrm{H}, \mathrm{py} \mathrm{H}-5$ ), $7.27(\mathrm{~s}, 1 \mathrm{H}, \mathrm{pz}), 3.81$ (d, $J_{P-H}=9.6 \mathrm{~Hz}$, $\left.4 \mathrm{H}, \mathrm{CH}_{2}\right), 1.38\left(\mathrm{~d}, J_{P-H}=14.5 \mathrm{~Hz}, 36 \mathrm{H}, \mathrm{C}\left(\mathrm{CH}_{3}\right)_{3}\right)$.

${ }^{13} \mathrm{C}$ NMR (126 MHz, Acetonitrile- $d_{3}$ ) $\delta=194.43\left(\mathrm{dd}, J_{R h-C}=73.8 \mathrm{~Hz}, J_{P-C}=17.5 \mathrm{~Hz}, \mathrm{CO}\right), 163.91$ $\left(\mathrm{dd}, J_{R h-C}=4.8 \mathrm{~Hz}, J_{P-C}=3.2 \mathrm{~Hz}\right.$, py C-6), $155.90\left(\mathrm{dt}, J_{P-C}=3.5,1.7 \mathrm{~Hz}, \mathrm{pz} \mathrm{C}-3 / \mathrm{C}-5\right), 153.48$ (s, py C-2), 142.06 (d, $J_{P-C}=0.7 \mathrm{~Hz}$, py C-4), 122.13 (dd, $J_{R h-C}=10.4 \mathrm{~Hz}, J_{P-C}=1.0 \mathrm{~Hz}$, py C-5), 118.70 (d, $J_{P-C}=0.6 \mathrm{~Hz}$, py C-3), $103.48(\mathrm{~s}, \mathrm{pz} \mathrm{C}-4), 36.88\left(\mathrm{~d}, J_{P-C}=22.4 \mathrm{~Hz}, C\left(\mathrm{CH}_{3}\right)_{3}\right), 36.73(\mathrm{dd}$, $\left.J_{R h-C}=21.7 \mathrm{~Hz}, J_{P-C}=2.0 \mathrm{~Hz}, \mathrm{CH}_{2}\right), 29.33\left(\mathrm{dd}, J_{R h-C}=4.4 \mathrm{~Hz}, J_{P-C}=0.7 \mathrm{~Hz}, \mathrm{C}\left(\mathrm{CH}_{3}\right)_{3}\right)$.

31P NMR $\left(162 \mathrm{MHz}\right.$, Acetonitrile- $\left.d_{3}\right) \delta=99.50\left(\mathrm{~d}, J_{R h-P}=148.2 \mathrm{~Hz}\right),-144.25$ (hept, $J=706.3 \mathrm{~Hz}$, $\mathrm{PF}_{6}{ }^{-}$.

ATR-IR (solid): $v$ [ $\left.\mathrm{cm}^{-1}\right]$ = $450(\mathrm{w}), 465(\mathrm{w}), 469(\mathrm{w}), 483(\mathrm{w}), 495(\mathrm{w}), 502(\mathrm{w}), 507(\mathrm{w}), 523$ (w), $530(\mathrm{w}), 555$ (s), 739 (m), $791(\mathrm{~s}), 807$ (s), 828 (s), 1020 (m), $1088(\mathrm{w}), 1105(\mathrm{w}), 1260$ 
(m), 1371 (w), 1389 (w), 1447 (w), 1460 (m), 1564 (m), 1606 (w), 1964 (m, CO), 1978 (m, CO), $2854(\mathrm{w}), 2923(\mathrm{~m}), 2960(\mathrm{w})$.

MS (ESI+) $\mathrm{m} / \mathrm{z}=799.125\left(\left[(\mathrm{Rh}(\mathrm{CO}))_{2} \mathrm{~L}^{t \mathrm{Bu}}\right]^{+}\right)$.

EA Calcd. for $\mathrm{C}_{33} \mathrm{H}_{51} \mathrm{~N}_{4} \mathrm{O}_{2} \mathrm{P}_{2} \mathrm{Rh}_{2}$ : C 40.42, H 5.24, N 5.71. Found: C 40.44, H 5.02, N 5.40.

\subsubsection{6 $\left[(\operatorname{Rh}(\mathrm{CO}))_{2} \mathrm{~L}^{\mathrm{iPr}}\right]\left(\mathrm{PF}_{6}\right)(57)$}

To a suspension of $\mathbf{H L}^{\mathrm{iPr}}(22.2 \mathrm{mg}, 46.4 \mu \mathrm{mol}, 1.0 \mathrm{eq}), \mathrm{KO}{ }^{t} \mathrm{Bu}(5.20 \mathrm{mg}, 46.4 \mu \mathrm{mol}, 1.0 \mathrm{eq})$ and $\mathrm{KPF}_{6}(10.2 \mathrm{mg}, 55.7 \mu \mathrm{mol}, 1.2 \mathrm{eq})$ in $\mathrm{MeCN}$ (5 mL) [Rh(acac)(CO) 2 ] (23.9 mg, $\left.92.8 \mu \mathrm{mol}, 2.0 \mathrm{eq}\right)$ was added as a solid. The reaction mixture was stirred at ambient temperature for $22 \mathrm{~h}$, volatiles were removed in vacuo and the residue extracted with $\mathrm{CH}_{2} \mathrm{Cl}_{2}(2 \times 3 \mathrm{~mL})$. Pure compound was obtained from $\mathrm{Et}_{2} \mathrm{O}$ diffusion into a concentrated acetonitrile solution. Drying under vacuum gave $12 \mathrm{mg}$ (28\%) $\mathbf{5 7}$ as yellow solid.

\section{Formula (molecular weight) $\quad \mathrm{C}_{29} \mathrm{H}_{39} \mathrm{~N}_{4} \mathrm{O}_{2} \mathrm{P}_{2} \mathrm{Rh}_{2}\left(\mathrm{PF}_{6}\right)(888.4 \mathrm{~g} / \mathrm{mol})$}

1H NMR (400 MHz, Acetonitrile- $\left.d_{3}\right) \delta=7.96(\mathrm{t}, J=7.9 \mathrm{~Hz}, 2 \mathrm{H}$, py $4-\mathrm{H}), 7.64(\mathrm{~d}, J=7.9 \mathrm{~Hz}, 2 \mathrm{H}$, py 3-H), $7.48\left(\mathrm{~d}, J=7.9 \mathrm{~Hz}, 2 \mathrm{H}\right.$, py 5-H), $7.26(\mathrm{~s}, 1 \mathrm{H}, \mathrm{pz} 4-\mathrm{H}), 3.77$ (d, $\left.J=10.2 \mathrm{~Hz}, 4 \mathrm{H}_{1} \mathrm{CH}_{2}\right), 2.42-$ $2.28\left(\mathrm{~m}, 4 \mathrm{H}, \mathrm{CH}\left(\mathrm{CH}_{3}\right)_{2}\right), 1.31\left(\mathrm{dd}, J=18.0,7.1 \mathrm{~Hz}, 12 \mathrm{H}, \mathrm{CH}\left(\mathrm{CH}_{3}\right)_{2}\right), 1.19(\mathrm{dd}, J=15.7,6.9 \mathrm{~Hz}, 12 \mathrm{H}$, $\left.\mathrm{CH}\left(\mathrm{CH}_{3}\right)_{2}\right)$

${ }^{13 C}$ NMR (126 MHz, Acetonitrile- $\left.d_{3}\right) \delta=193.0$ (d, $\left.J_{\mathrm{Rh}-\mathrm{C}}=19.5 \mathrm{~Hz}, \mathrm{CO}\right), 192.4\left(\mathrm{~d}, J_{\mathrm{Rh}-\mathrm{C}}=19.3 \mathrm{~Hz}\right.$, CO), $163.0\left(\mathrm{~d}, J_{\mathrm{Rh}-\mathrm{C}}=3.4 \mathrm{~Hz}\right.$, py C-6), $163.0\left(\mathrm{~d}, J_{\mathrm{Rh}-\mathrm{C}}=3.3 \mathrm{~Hz}\right.$, py C-6), 156.1 (s, pz C-3, C-5), 153.6 (s, py C), 142.0 (s, py C-4), 122.4 (d, JRh-C $=11.2$ Hz, py C-5), 118.9 (s, py C-3), 103.7 (s, pz C-4), $36.1\left(\mathrm{~d}, J_{\mathrm{Rh}-\mathrm{C}}=25.4 \mathrm{~Hz}, \mathrm{CH}_{2}\right), 26.1$ (dd, $\left.J_{\mathrm{Rh}-\mathrm{C}}=30.1,2.0 \mathrm{~Hz}, \mathrm{CH}\left(\mathrm{CH}_{3}\right)_{2}\right), 19.2$ (d, $J_{\mathrm{Rh}-\mathrm{C}}=3.5 \mathrm{~Hz}$, $\left.\mathrm{CH}\left(\mathrm{CH}_{3}\right)_{2}\right), 18.2\left(\mathrm{~s}, \mathrm{CH}\left(\mathrm{CH}_{3}\right)_{2}\right)$.

31P NMR (162 MHz, Acetonitrile- $\left.d_{3}\right) \delta=85.29$ (d, $J=144.6 \mathrm{~Hz}$ ), -144.25 (hept, $J=706.6 \mathrm{~Hz}$, $\mathrm{PF}^{-}{ }^{-}$.

ATR-IR (solid): $v$ [cm-1] = $404(\mathrm{w}), 406(\mathrm{w}), 410(\mathrm{w}), 449(\mathrm{w}), 483(\mathrm{w}), 495(\mathrm{w}), 520(\mathrm{w}), 556$ (s), $637(\mathrm{~m}), 738(\mathrm{w}), 756(\mathrm{w}), 792(\mathrm{w}), 807(\mathrm{w}), 839(\mathrm{~s}), 1020(\mathrm{~m}), 1088(\mathrm{w}), 1103(\mathrm{w}), 1167$ (w), 1262 (w), $1371(\mathrm{w}), 1390(\mathrm{w}), 1401(\mathrm{w}), 1418(\mathrm{w}), 1458(\mathrm{~m}), 1563(\mathrm{~m}), 1605(\mathrm{~m}), 1965$ (m, CO), 1977 (m, CO), 2868 (w), 2901 (w), 2922 (w), 2963 (w).

MS (ESI+) m/z = $743.063\left(\left[(\mathrm{Rh}(\mathrm{CO}))_{2} \mathrm{~L}^{i \mathrm{Pr}}\right]^{+}\right)$. 


\subsubsection{7 $\left[(\operatorname{Rh}(\mathrm{MeCN}))_{2} \mathrm{~L}^{\mathrm{tBu}}\right] \mathrm{PF}_{6}(58)$}

a) $\left[\mathrm{RhCl}(\mathrm{coe})_{2}\right]_{2}(133 \mathrm{mg}, 186 \mu \mathrm{mol}, 1.0 \mathrm{eq})$ and $\mathrm{AgPF}_{6}(93.8 \mathrm{mg}, 371 \mu \mathrm{mol}, 2.0 \mathrm{eq})$ were suspended in MeCN ( $5 \mathrm{~mL}$ ) for $5 \mathrm{~h}$. The mixture was filtered through a glass fibre filter into a suspension of $\mathbf{H L}^{t \mathrm{Bu}}(100 \mathrm{mg}, 186 \mu \mathrm{mol}, 1.0 \mathrm{eq})$ and $\mathrm{KO}{ }^{t} \mathrm{Bu}(20.8 \mathrm{mg}, 186 \mu \mathrm{mol}, 1.0 \mathrm{eq})$ in MeCN $(5 \mathrm{~mL})$. The reaction mixture was stirred at ambient temperature for $16 \mathrm{~h}$. The volatiles were removed under reduced pressure and the residue was extracted with a benzene/MeCN 6:1 mixture (7 mL). Lyophilisation and drying in vacuo gave $170 \mathrm{mg}(94 \%) \mathbf{5 8}$ as dark red solid.

b) $\mathbf{R h}{ }^{t \mathrm{Bu}}-\mathbf{S M e}_{2}$ (42.8 mg, $45.0 \mu \mathrm{mol}$ ) was solved in MeCN (1 mL), stirred for $5 \mathrm{~min}$ and then layered with $\mathrm{Et}_{2} \mathrm{O}$. The dark red solid was separated and dried to afford $21 \mathrm{mg}$ (48\%) 58.

Formula (molecular weight) $\quad \mathrm{C}_{35} \mathrm{H}_{53} \mathrm{~N}_{6} \mathrm{P}_{2} \mathrm{Rh}_{2}\left(\mathrm{PF}_{6}\right)(970.6 \mathrm{~g} / \mathrm{mol})$

${ }_{1}^{1}$ NMR $\left(500 \mathrm{MHz}\right.$, Acetonitrile- $\left.d_{3}\right) \delta=7.72\left(\mathrm{td}, J_{H-H}=7.8,1.1 \mathrm{~Hz}, 2 \mathrm{H}\right.$, py H4), 7.39 (dd, $J_{H-H}=7.8$, $0.7 \mathrm{~Hz}, 2 \mathrm{H}$, py H3), 7.21 (s, 1H, pz H4), 7.20 (d, $J_{H-H}=7.7 \mathrm{~Hz}, 2 \mathrm{H}$, py H5), 3.28 (d, $J_{P-H}=9.1 \mathrm{~Hz}, 4 \mathrm{H}$, $\left.\mathrm{CH}_{2}\right), 1.38\left(\mathrm{~d}, J_{P-H}=13.2 \mathrm{~Hz}, 36 \mathrm{H}, \mathrm{C}\left(\mathrm{CH}_{3}\right)_{3}\right)$.

${ }^{13} \mathrm{C}$ NMR $\left(126 \mathrm{MHz}\right.$, Acetonitrile- $\left.d_{3}\right) \delta=164.3\left(\mathrm{dd}, J_{R h-C}=6.3 \mathrm{~Hz}, J_{P-C}=3.3 \mathrm{~Hz}\right.$, py C-6), 154.1 (s, pz C-3/C-5), 153.6 (s, py C-2), 126.9 (d, $J_{P-C}=11.1 \mathrm{~Hz}$, py C-4), 120.3 (dd, $J_{R h-C}=10.6 \mathrm{~Hz}, J_{P-C}=1.5$ $\mathrm{Hz}$, py C-5), 117.5 (s, py C-3), 101.6 (s, pz C-4), 36.3 (d, $J_{R h-C}=20.8 \mathrm{~Hz}, \mathrm{CH}_{2}$ ), 35.7 (d, $J_{R h-C}=16.7$ $\left.\mathrm{Hz}, \mathrm{C}\left(\mathrm{CH}_{3}\right)_{3}\right), 29.5\left(\mathrm{~d}, J_{R h-C}=5.4 \mathrm{~Hz}, \mathrm{C}\left(\mathrm{CH}_{3}\right)_{3}\right)$.

31P NMR (202 MHz, Acetonitrile- $\left.d_{3}\right) \quad \delta=91.13$ (d, $J_{R h-P}=178.2 \mathrm{~Hz}$ ), -144.25 (hept, $\left.J_{F-P}=706.4 \mathrm{~Hz}\right)$.

ATR-IR (solid): $v$ [cm-1] = $456(\mathrm{w}), 480(\mathrm{~m}), 555(\mathrm{~s}), 592(\mathrm{w}), 628(\mathrm{w}), 678(\mathrm{w}), 738(\mathrm{~m}), 750$ (w), 777 (m), 823 (s), 831 (s), $894(w), 1020$ (w), $1140(w), 1163(w), 1179(w), 1259(w)$, 1367 (w), 1387 (w), 1413 (w), 1455 (m), 1553 (w), 1603 (w), 1697 (w), 2265 (w, MeCN), 2899 (w), $2924(w), 2943(w), 2957(w)$.

MS $(\mathrm{ESI}+, \mathrm{MeCN}) \mathrm{m} / \mathrm{z}(\%)=825.307\left(100,\left[(\mathrm{Rh}(\mathrm{MeCN}))_{2} \mathrm{~L}^{t \mathrm{Bu}}\right]^{+}\right)$.

EA Calcd. for $\mathrm{C}_{35} \mathrm{H}_{53} \mathrm{~F}_{6} \mathrm{~N}_{6} \mathrm{P}_{3} \mathrm{Rh}_{2}$ : C 43.31, H 5.50, N 8.66. Found: C 42.91, H 5.30, N 8.71.

\subsubsection{8 $\left[(\operatorname{Rh}(\mathrm{MeCN}))_{2} \mathrm{~L}^{\mathrm{iPr}}\right] \mathrm{PF}_{6}(65)$}

$\left[\mathrm{RhCl}(\mathrm{coe})_{2}\right]_{2} \quad$ (66.4 mg, $\left.92.5 \mu \mathrm{mol}, 1.0 \mathrm{eq}\right)$ and $\mathrm{AgPF}_{6} \quad$ (46.8 mg, $\left.185 \mu \mathrm{mol}, 2.0 \mathrm{eq}\right)$ were suspended in MeCN ( $2 \mathrm{~mL}$ ) for $6 \mathrm{~h}$. The mixture was filtered through a glass fibre filter into a suspension of $\mathbf{H L}^{\mathrm{iPr}}(44.6 \mathrm{mg}, 92.5 \mu \mathrm{mol}, 1.0 \mathrm{eq})$ and $\mathrm{KO}^{t} \mathrm{Bu}(10.4 \mathrm{mg}, 92.5 \mu \mathrm{mol}, 1.0 \mathrm{eq})$ in 
$\operatorname{MeCN}(2 \mathrm{~mL})$. The reaction mixture was heated to $80^{\circ} \mathrm{C}$ for $17 \mathrm{~h}$. The volatiles were removed under reduced pressure and the crude product was recrystallized from acetone and pentane several times. Drying in vacuo gave $67 \mathrm{mg}(80 \%) 65$ as dark red solid.

\section{Formula (molecular weight) $\quad \mathrm{C}_{31} \mathrm{H}_{45} \mathrm{~N}_{6} \mathrm{P}_{2} \mathrm{Rh}_{2}\left(\mathrm{PF}_{6}\right)(914.5 \mathrm{~g} / \mathrm{mol})$}

${ }^{1} \mathbf{H}-N M R\left(500 \mathrm{MHz}\right.$, Acetonitrile- $\left.d_{3}\right) \delta=7.70(\mathrm{t}, J=7.8 \mathrm{~Hz}, 2 \mathrm{H}$, py $4-\mathrm{H}), 7.38(\mathrm{~d}, J=7.4 \mathrm{~Hz}, 2 \mathrm{H}$, py 3-H), 7.22 (s, 1H, pz 4-H), 7.16 (d, J = 7.7 Hz, 2H, py 5-H), 3.17 (d, J = 9.6 Hz, 4H, CH $2.11\left(\mathrm{~m}, 4 \mathrm{H}, \mathrm{CH}\left(\mathrm{CH}_{3}\right)_{2}\right), 1.26\left(\mathrm{dd}, J=16.8,7.1 \mathrm{~Hz}, 12 \mathrm{H}, \mathrm{CH}\left(\mathrm{CH}_{3}\right)_{2}\right), 1.10(\mathrm{dd}, J=14.0,6.9 \mathrm{~Hz}, 12 \mathrm{H}$, $\left.\mathrm{CH}\left(\mathrm{CH}_{3}\right)_{2}\right)$.

${ }^{13} \mathrm{C}$ NMR (126 MHz, Acetonitrile- $d_{3}$ ) $\delta=163.6$ (dd, $J=6.7,3.5 \mathrm{~Hz}, \mathrm{C}-6$ ), 154.2 (s, pz C-3/C-5), 153.7 (s, py C-2), 136.1 (s, py C-3), 120.6 (dd, $J=10.8,1.4 \mathrm{~Hz}$, py C-5), 117.8 (d, $J=0.6 \mathrm{~Hz}$, py C-4), 101.6 (s, pz C-4), 35.9 (dd, $J=23.2,1.9 \mathrm{~Hz}, \mathrm{CH}_{2}$ ), 25.6 (dd, $J=24.1,1.7 \mathrm{~Hz}, \mathrm{CH}\left(\mathrm{CH}_{3}\right)_{2}$ ), 19.53 $\left(\mathrm{d}, J=5.4 \mathrm{~Hz}, \mathrm{CH}\left(\mathrm{CH}_{3}\right)_{2}\right), 18.35\left(\mathrm{~d}, J=1.0 \mathrm{~Hz}, \mathrm{CH}\left(\mathrm{CH}_{3}\right)_{2}\right)$.

31P-NMR $\left(202 \mathrm{MHz}, \quad\right.$ Acetonitrile- $\left.d_{3}\right) \quad \delta=78.36 \quad\left(\mathrm{~d}, J_{R h-P}=176.3 \mathrm{~Hz}\right),-144.25$ (hept, $\left.J_{F-P}=706.4 \mathrm{~Hz}\right)$.

ATR-IR (solid): $v$ [ $\left.\mathrm{cm}^{-1}\right]$ = $404(\mathrm{w}), 413(\mathrm{w}), 417(\mathrm{w}), 423(\mathrm{w}), 430(\mathrm{w}), 436(\mathrm{w}), 440(\mathrm{w}), 448$ $(w), 458(w), 461(w), 464(w), 469(w), 474(w), 484(w), 487(w), 490(w), 494(w), 499(w)$ $503(\mathrm{w}), 508(\mathrm{w}), 512(\mathrm{w}), 517(\mathrm{w}), 529(\mathrm{w}), 555(\mathrm{~s}), 612(\mathrm{w}), 644(\mathrm{w}), 658(\mathrm{~m}), 738(\mathrm{w}), 751$ (w), $778(w), 787(w), 834(s), 877(w), 1024(w), 1311(w), 1362(w), 1380(w), 1405(w)$, $1408(\mathrm{w}), 1454(\mathrm{~m}), 1528(\mathrm{w}), 1552(\mathrm{w}), 1601$ (w), $2868(\mathrm{~m}), 2926(\mathrm{~m}), 2953(\mathrm{~m})$.

\subsubsection{9 $\left[\left(\operatorname{Rh}\left(\mu-\mathrm{SMe}_{2}\right)\right)_{2} \mathrm{~L}^{\mathrm{tBu}}\right] \mathrm{PF}_{6}(61)$}

a) $\left[\mathrm{RhCl}(\mathrm{coe})_{2}\right]_{2}(133 \mathrm{mg}, 186 \mu \mathrm{mol}, 1.0 \mathrm{eq})$ and $\mathrm{AgPF}_{6}$ (93.8 mg, $\left.371 \mu \mathrm{mol}, 2.0 \mathrm{eq}\right)$ were suspended in acetone $(4 \mathrm{~mL})$ for $5 \mathrm{~h}$. The mixture was filtered through a glass fibre filter into a solution of $\mathbf{H L}^{\mathrm{tBu}}(100 \mathrm{mg}, 186 \mu \mathrm{mol}, 1.0 \mathrm{eq}), \mathrm{KO}{ }^{\mathrm{Bu}}(20.8 \mathrm{mg}, 186 \mu \mathrm{mol}, 1.0 \mathrm{eq})$ and $\mathrm{SMe}_{2}$ $(55 \mu \mathrm{L}, 744 \mu \mathrm{mol}, 4.0 \mathrm{eq})$ in THF $(4 \mathrm{~mL})$. The reaction mixture was stirred at ambient temperature for $23 \mathrm{~h}$. The volatiles were removed under reduced pressure and the residue solved in acetone $(2.5 \mathrm{~mL})$ and layered with pentane to give a dark red polycrystalline solid overnight. The solid was isolated, washed with pentane and dried in vacuo to yield $143 \mathrm{mg}$ (81\%) 61.

b) A solution of $\mathbf{R h}^{t \mathrm{Bu}-M e C N}(168 \mathrm{mg}, 173 \mu \mathrm{mol}, 1.0 \mathrm{eq})$ and SMe 2 (125 mg, $\left.2.02 \mathrm{mmol}, 11.7 \mathrm{eq}\right)$ in THF ( $2 \mathrm{~mL}$ ) was heated to $60^{\circ} \mathrm{C}$ for $24 \mathrm{~h}$. The volatiles were removed in vacuo. The solid dried in vacuo to yield $143 \mathrm{mg}(87 \%) 61$. 


\section{Formula (molecular weight) $\quad \mathrm{C}_{33} \mathrm{H}_{53} \mathrm{~N}_{4} \mathrm{P}_{2} \mathrm{Rh}_{2} \mathrm{~S}\left(\mathrm{PF}_{6}\right)(950.6 \mathrm{~g} / \mathrm{mol})$}

${ }^{1}$ H-NMR $\left(500 \mathrm{MHz}\right.$, Acetone- $\left.d_{6}\right) \delta=7.92\left(\mathrm{td}, J_{H-H}=7.8,1.1 \mathrm{~Hz}, 2 \mathrm{H}, \mathrm{py} \mathrm{H}-4\right), 7.55\left(\mathrm{~d}, J_{H-H}=7.8 \mathrm{~Hz}\right.$, $2 \mathrm{H}, \mathrm{py} \mathrm{H}-3$ ), 7.50 (d, $\left.J_{H-H}=7.7 \mathrm{~Hz}, 2 \mathrm{H}, \mathrm{py} \mathrm{H}-5\right), 7.13$ (s, $\left.1 \mathrm{H}, \mathrm{pz} \mathrm{H}-4\right), 3.59$ (d, $J_{P-H}=8.6 \mathrm{~Hz}, 4 \mathrm{H}, \mathrm{CH}_{2}$ ), $3.26\left(\mathrm{t}, J_{R h-H}=1.4 \mathrm{~Hz}, 6 \mathrm{H}, \mathrm{S}\left(\mathrm{CH}_{3}\right)_{2}\right), 1.43\left(\mathrm{~d}, J_{P-H}=13.1 \mathrm{~Hz}, 38 \mathrm{H}, \mathrm{C}\left(\mathrm{CH}_{3}\right)_{3}\right)$.

${ }^{13} \mathrm{C}$ NMR $\left(126 \mathrm{MHz}\right.$, Acetone- $\left.d_{6}\right) \delta=164.3\left(\mathrm{dd}, J_{R h-C}=6.3 \mathrm{~Hz}, J_{P-C}=3.3 \mathrm{~Hz}\right.$, py C6), 154.1 (s, pz C3/C5), 153.6 (s, py C2), 136.1 (s, py C4), 126.9 (d, $J_{P-C}=11.1 \mathrm{~Hz}$, py C4), 120.3 (dd, $J_{R h-C}=10.6 \mathrm{~Hz}, J_{P-C}=1.5 \mathrm{~Hz}$, py C5), $117.5(\mathrm{~s}, \mathrm{py} \mathrm{C} 3), 101.6(\mathrm{~s}, \mathrm{pz} \mathrm{C} 4), 36.3\left(\mathrm{~d}, J_{R h-C}=20.8 \mathrm{~Hz}, \mathrm{CH}_{2}\right.$ ), $35.7\left(\mathrm{~d}, J_{R h-C}=16.7 \mathrm{~Hz}, C\left(\mathrm{CH}_{3}\right)_{3}\right), 29.5\left(\mathrm{~d}, J_{R h-C}=5.4 \mathrm{~Hz}, \mathrm{C}\left(\mathrm{CH}_{3}\right)_{3}\right)$.

31P-NMR (202 MHz, Acetone- $\left.d_{6}\right) \delta=80.76\left(\mathrm{~d}, J_{R h-P}=163.4 \mathrm{~Hz}\right),-144.26$ (hept, $\left.J_{F-P}=706.4 \mathrm{~Hz}\right)$.

ATR-IR (solid): $v$ [ $\left.\mathrm{cm}^{-1}\right]$ = $402(\mathrm{w}), 404(\mathrm{w}), 449(\mathrm{w}), 480(\mathrm{w}), 488(\mathrm{w}), 496(\mathrm{w}), 556(\mathrm{~m}), 669$ $(\mathrm{w}), 685(\mathrm{w}), 703(\mathrm{w}), 737(\mathrm{~m}), 790(\mathrm{~s}), 837$ (s), 965. (m), 1013 (s), 1062 (s), 1082 (s), 1258 (s), 2863, (w), $2907(\mathrm{w}), 2926(\mathrm{w}), 2962(\mathrm{~m})$.

EA Calcd. for $\mathrm{C}_{33} \mathrm{H}_{53} \mathrm{~F}_{6} \mathrm{~N}_{4} \mathrm{P}_{3} \mathrm{Rh}_{2} \mathrm{~S} \cdot 0.75 \mathrm{C}_{5} \mathrm{H}_{12}$ : C 43.93, H 6.22, N $5.58 \mathrm{~S}$ 3.19. Found: C 43.68, H 6.22, N 5.23 S 3.47.

\subsubsection{0 $\left[(\mathrm{Rh}(\mathrm{CO}))_{2} \mathrm{~L}^{\mathrm{tBu} *}\right](74)$}

A solution of $\mathrm{KO}^{t} \mathrm{Bu}(1.8 \mathrm{mg}, 15.9 \mu \mathrm{mol}, 1.0 \mathrm{eq})$ in THF ( $2 \mathrm{~mL}$ ) was added to solid $\mathbf{5 5}$ (15.0 mg, $15.9 \mu \mathrm{mol}, 1.0 \mathrm{eq})$. The suspension turned green within $5 \mathrm{~min}$ and was stirred at ambient temperature for $2 \mathrm{~h}$. The reaction mixture was filtered through a glass fibre filter and the volatiles were removed in vacuo to obtain $9 \mathrm{mg}$ (71\%) of $\mathbf{7 4}$ as a green solid.

\section{Formula (molecular weight) $\quad \mathrm{C}_{33} \mathrm{H}_{46} \mathrm{~N}_{4} \mathrm{P}_{2} \mathrm{Rh}_{2} \mathrm{O}_{2}(798.5 \mathrm{~g} / \mathrm{mol})$}

1H-NMR (400 MHz, THF- $\left.d_{8}\right) \delta=7.82(\mathrm{t}, J=7.6 \mathrm{~Hz}, 1 \mathrm{H}$, pyarom $4-\mathrm{H}), 7.50(\mathrm{~d}, J=7.8 \mathrm{~Hz}, 1 \mathrm{H}$, pyarom 3-H), 7.33 (d, J = 7.0 Hz, 1H, py arom 5-H), 6.67 (s, 1H, pz 4-H), 6.29 (t, J = 7.1 Hz, 1H, pydearom 4-H), $5.94(\mathrm{~d}, J=8.7 \mathrm{~Hz}, 1 \mathrm{H}$, pydearom $3-\mathrm{H}), 5.45(\mathrm{~d}, J=6.8 \mathrm{~Hz}, 1 \mathrm{H}$, pydearom $5-\mathrm{H}), 3.78(\mathrm{~d}, J=10.3 \mathrm{~Hz}, 2 \mathrm{H}$, $\mathrm{CH}_{2}$ ), $3.11(\mathrm{~d}, J=3.6 \mathrm{~Hz}, 1 \mathrm{H}, \mathrm{CH}), 1.42$ (d, $\left.J=9.9 \mathrm{~Hz}, 18 \mathrm{H}, \mathrm{C}\left(\mathrm{CH}_{3}\right)_{3}\right), 1.37$ (d, $J=9.0 \mathrm{~Hz}, 18 \mathrm{H}$, $\left.\mathrm{C}\left(\mathrm{CH}_{3}\right)_{3}\right)$.

31P-NMR (162 MHz, THF- $\left.d_{8}\right) \delta=97.82(\mathrm{~d}, J=149.2 \mathrm{~Hz}, \mathrm{P}$ arom), $88.62(\mathrm{~d}, J=154.4 \mathrm{~Hz}$, $P_{\text {dearom}}$ ), -144.26 (hept, $J_{F-P}=708.5 \mathrm{~Hz}$ ). 
ATR-IR (solid): $v$ [cm-1] = $448(\mathrm{w}), 471(\mathrm{w}), 483(\mathrm{w}), 521(\mathrm{w}), 558(\mathrm{~s}), 585(\mathrm{w}), 618(\mathrm{~m}), 635$ (m), 742 (w), 767 (m), 785 (s), 812 (s), 827 (s), 907 (w), 1162 (w), $1410(\mathrm{w}), 1458(\mathrm{~m}), 1473$ (m), 1604 (m), 1940 (s, CO), 1990 (s, CO), 2866 (w), 2892 (w), 2938 (w), $2959(w)$.

\subsubsection{1 $K\left[(\operatorname{Rh}(\mathrm{CO}))_{2} \mathrm{~L}^{\mathrm{tBu} * *}\right](75)$}

A solution of $\mathrm{KO}^{\mathrm{t}} \mathrm{Bu}(3.6 \mathrm{mg}, 31.8 \mu \mathrm{mol}, 2.0 \mathrm{eq})$ in THF ( $2 \mathrm{~mL}$ ) was added to solid $\mathbf{5 5}$ (15.0 mg, $15.9 \mu \mathrm{mol}, 1.0 \mathrm{eq})$. The suspension turned deep purple within $5 \mathrm{~min}$ and was stirred at ambient temperature for $2 \mathrm{~h}$. The reaction mixture was filtered through a glass fibre filter and the volatiles were removed in vacuo to obtain $11 \mathrm{mg}$ (83\%) 75 as a purple solid.

Formula (molecular weight) $\quad \mathrm{C}_{33} \mathrm{H}_{46} \mathrm{KN}_{4} \mathrm{P}_{2} \mathrm{Rh}_{2} \mathrm{O}_{2}(836.6 \mathrm{~g} / \mathrm{mol})$

${ }^{1}$ H-NMR $\left(400 \mathrm{MHz}, \mathrm{THF}-\mathrm{d}_{8}\right) \delta=6.26(\mathrm{ddd}, J=8.8,6.6,2.2 \mathrm{~Hz}, 2 \mathrm{H}$, pydearom $4-\mathrm{H}), 6.17(\mathrm{~s}, 1 \mathrm{H}, \mathrm{pz}$ 4-H), $5.87\left(\mathrm{~d}, J=8.8 \mathrm{~Hz}, 2 \mathrm{H}, \mathrm{y}_{\text {dearom }} 3-\mathrm{H}\right), 5.38(\mathrm{dd}, J=6.6,1.1 \mathrm{~Hz}, 2 \mathrm{H}$, pydearom $5-\mathrm{H}), 3.06(\mathrm{~d}$, $J=2.3 \mathrm{~Hz}, 2 \mathrm{H}, \mathrm{CH}), 1.36\left(\mathrm{~d}, J=13.1 \mathrm{~Hz}, 36 \mathrm{H}, \mathrm{C}\left(\mathrm{CH}_{3}\right)_{3}\right)$.

${ }^{13} \mathrm{C}$ NMR (126 MHz, THF- $\left.d_{8}\right) \delta=197.7\left(\mathrm{dd}, J_{\mathrm{Rh}-\mathrm{C}}=70.3 \mathrm{~Hz}, J_{\mathrm{P}-\mathrm{C}}=17.1 \mathrm{~Hz}, \mathrm{CO}\right), 169.84$ (dd, $J_{\mathrm{Rh}-\mathrm{C}}=18.5, J_{\mathrm{P}-\mathrm{C}}=3.7 \mathrm{~Hz}$, py C-6), 157.7 (d, $\left.J_{\mathrm{P}-\mathrm{C}}=3.0 \mathrm{~Hz}, \mathrm{pz} \mathrm{C}-3 / \mathrm{C}-5\right), 152.7$ (s, py C-2), 133.2 (s,

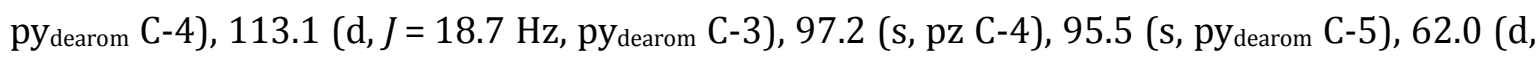
$J=56.0 \mathrm{~Hz}, \mathrm{CH}), 36.9\left(\mathrm{dd}, J=26.2,2.0 \mathrm{~Hz}, \mathrm{C}\left(\mathrm{CH}_{3}\right)_{3}\right), 30.4\left(\mathrm{~d}, J=5.2 \mathrm{~Hz}, \mathrm{C}\left(\mathrm{CH}_{3}\right)_{3}\right)$.

31P-NMR $\left(162 \mathrm{MHz}\right.$, THF- $\left.d_{8}\right) \delta=87.83(\mathrm{~d}, J=152.7 \mathrm{~Hz}),-144.25$ (hept, $\left.J=709.0 \mathrm{~Hz}, \mathrm{PF}_{6}{ }^{-}\right)$.

ATR-IR (solid): $v$ [cm-1] = $472(\mathrm{w}), 558(\mathrm{~m}), 606(\mathrm{w}), 620(\mathrm{w}), 632(\mathrm{~m}), 687(\mathrm{w}), 700(\mathrm{w}), 705$ (m), 757 (s), 766 (s), 796 (s), 804 (s), 858 (w), 991 (m), 1001 (s), 1013 (s), 1048 (m), 1068 (m), 1090 (m), 1258 (m), 1416 (w), 1458 (w), 1475 (m), 1606 (w), 1940 (m, CO),1964 (m, CO), 2865 (w), $2890(w), 2939(w), 2962(m)$.

\subsubsection{2 $\left.\left[\left(\mathrm{Rh}_{(\mathrm{CH}} \mathrm{Cl}\right) \mathrm{Cl}\right)(\mathrm{MeCN}) \mathrm{L}^{\mathrm{tBu}}\right] \mathrm{PF}_{6}(67)$}

58 (30.0 mg, $30.9 \mu \mathrm{mol})$ was solved in DCM (2 mL) and left in solution for 2 weeks. The color of the solution changed to yellow. The solution was layered with pentane and yellow crystals could be separated. Drying in vacuo gave $2 \mathrm{mg}(6 \%) 67$.

Formula (molecular weight) $\quad \mathrm{C}_{35} \mathrm{H}_{54} \mathrm{Cl}_{4} \mathrm{~N}_{5} \mathrm{P}_{2} \mathrm{Rh}_{2}\left(\mathrm{PF}_{6}\right)(1099.4 \mathrm{~g} / \mathrm{mol})$

31P NMR (121 MHz, $\left.\mathrm{CD}_{2} \mathrm{Cl}_{2}\right) \delta 71.13(\mathrm{~d}, J=133.0 \mathrm{~Hz}$ ), -144.25 (hept, $J=710.9 \mathrm{~Hz}$ ). 
${ }^{1} \mathrm{H}$ and ${ }^{13} \mathrm{C}$ NMR spectra indicated the presence of a mixture of products and single resonances could not be assigned.

ATR-IR (solid): $v$ [cm-1] = $405(\mathrm{~m}), 415(\mathrm{w}), 420(\mathrm{w}), 430(\mathrm{w}), 440(\mathrm{w}), 450(\mathrm{w}), 459(\mathrm{w}), 462$ (w), $469(w), 472(w), 484(w), 487(w), 491(w), 499(w), 512(w), 517(w), 523(w), 529(w)$, $555(\mathrm{~s}), 580(\mathrm{w}), 594(\mathrm{w}), 598(\mathrm{w}), 606(\mathrm{w}), 610(\mathrm{w}), 631(\mathrm{w}), 648(\mathrm{w}), 674(\mathrm{w}), 677(\mathrm{w}), 687$ (w), $696(\mathrm{w}), 717(\mathrm{w}), 737(\mathrm{~m}), 792(\mathrm{~m}), 829$ (s), $837(\mathrm{~s}), 871(\mathrm{w}), 1159(\mathrm{w}), 1168(\mathrm{~m}), 1371$ (w), $1387(w), 1398(w), 1458(m), 1564(w), 1605(w), 2873(w), 2905(w), 2957(w)$.

MS $(\mathrm{ESI}+) \quad \mathrm{m} / \mathrm{z}(\%)=438.0377 \quad\left(100, \quad\left[\mathrm{Rh}_{2}(\mathrm{Cl})\left(\mathrm{CH}_{2} \mathrm{Cl}\right)_{2} \mathrm{~L}^{t \mathrm{Bu}}\right]^{2+}\right), \quad 913.041 \quad(14$, $\left.\left[\mathrm{Rh}_{2}(\mathrm{Cl})_{2}\left(\mathrm{CH}_{2} \mathrm{Cl}\right)_{2} \mathrm{~L}^{t \mathrm{Bu}}\right]^{+}\right)$.

\subsubsection{3 $\left[(\mathrm{RhMe})_{2}(\mu-\mathrm{I})_{2} \mathrm{~L}^{\mathrm{tBu}}\right] \mathrm{I}(69)$}

To a solution of $58(20.0 \mathrm{mg}, 20.6 \mu \mathrm{mol}, 1.0 \mathrm{eq})$ in $\mathrm{MeCN}(0.5 \mathrm{~mL}) \mathrm{MeI}(13 \mu \mathrm{L}, 206 \mu \mathrm{mol}, 10 \mathrm{eq})$ was added. An immediately color change to yellow was observed. Yellow needles precipitated from that concentrated solution in the course of one week at ambient temperature. Isolation of the product and drying in vacuo gave $2 \mathrm{mg}(8 \%)$ of 69.

\section{Formula (molecular weight) $\quad \mathrm{C}_{33} \mathrm{H}_{53} \mathrm{I}_{2} \mathrm{~N}_{4} \mathrm{P}_{2} \mathrm{Rh}_{2}(\mathrm{I})(1154.3 \mathrm{~g} / \mathrm{mol})$}

ATR-IR (solid): $v$ [ $\left.\mathrm{cm}^{-1}\right]=404(\mathrm{~s}), 411(\mathrm{~m}), 428(\mathrm{~m}), 434(\mathrm{~m}), 450(\mathrm{~m}), 462(\mathrm{~m}), 488(\mathrm{~m}), 506$ (m), $517(\mathrm{~m}), 522(\mathrm{~m}), 528(\mathrm{~m}), 555(\mathrm{~s}), 571(\mathrm{~m}), 595(\mathrm{~m}), 609(\mathrm{~m}), 631(\mathrm{~m}), 636(\mathrm{~m}), 657(\mathrm{~m})$, $662(\mathrm{~m}), 674(\mathrm{~m}), 679(\mathrm{~m}), 708(\mathrm{~m}), 711(\mathrm{~m}), 738(\mathrm{~m}), 756(\mathrm{~m}), 829(\mathrm{~s}), 873(\mathrm{w}), 1026(\mathrm{w})$, $1084(w), 1106(w), 1110(w), 1141(w), 1148(w), 1168(m), 1262(w), 1372(w), 1396(w)$, $1406(w), 1458(m), 1560(w), 1606(w), 2872(w), 2922(w), 2955(w)$.

MS $(E S I+) \mathrm{m} / \mathrm{z}(\%)=885.308\left(100,\left[\mathrm{Rh}_{2}(\mathrm{Me})(\mathrm{I}) \mathrm{L}^{t \mathrm{Bu}}\right]^{+}\right), 1027.063\left(50,\left[\mathrm{Rh}_{2}(\mathrm{Me})_{2}(\mathrm{I})_{2} \mathrm{~L}^{t \mathrm{Bu}}\right]^{+}\right)$.

\subsubsection{Ligand Flexibility and Exchange Studies}

Activation barriers for the follow through of the acetonitrile coligands in different solvents were determined by variable-temperature ${ }^{1} \mathrm{H}$ NMR spectroscopy. While the peak separation of pairs of exchanging protons in the absence of exchange cannot be determined in the available temperature range, the rate constants for the interconversion of the two conformers in the slow exchange regime at low temperatures was estimated by using $k(T)=\pi \cdot \Delta v_{1 / 2}$, where $\mathrm{k}(\mathrm{T})$ is the rate constant at a given temperature and $\Delta v_{1 / 2}$ is the line width of the analyzed NMR signal at the same temperature. ${ }^{[488]}$ In case of fast exchange above the coalescence temperature 
$k(T)=\frac{\pi \cdot\left(\Delta v_{A B}\right)^{2}}{2 \cdot \Delta v_{1 / 2}}$ becomes valid. Here $\Delta v_{1 / 2}$ is again the line width at half height of the analyzed signal at the respective temperature, and $\Delta v_{\mathrm{AB}}$ is the peak separation below the coalescence temperature. The $\Delta v_{1 / 2}$ values were derived from line fitting using the MEstrenova program. ${ }^{[489]}$

The ligand exchange reaction of $\mathbf{R h}{ }^{t B u}-\mathbf{M e C N}$ with $M e C N-d_{3}$ to $\mathbf{R h}{ }^{t B u}-\mathbf{M e C N}-\mathbf{d}_{3}$ was performed in THF-d $\mathrm{d}_{8}$ solutions. To a solution of $\mathbf{R h}^{\text {tBu-MeCN }}$ (10 mg, $10.3 \mu \mathrm{mol}, 1.0$ eq.) in THF- $\mathrm{d}_{8}(0.5 \mathrm{~mL})$ MeCN- $\mathrm{d}_{3}\left(53.7 \mu \mathrm{L}, 1.03 \mathrm{mmol}, 100\right.$ eq.) was added at a given temperature and ${ }^{1} \mathrm{H}$ NMR spectra recorded every $1.5 \mathrm{~min}(308 \mathrm{~K}), 2 \mathrm{~min}(298 \mathrm{~K}), 2 \mathrm{~min}(288 \mathrm{~K})$ and $4 \mathrm{~min}(278 \mathrm{~K})$ until the

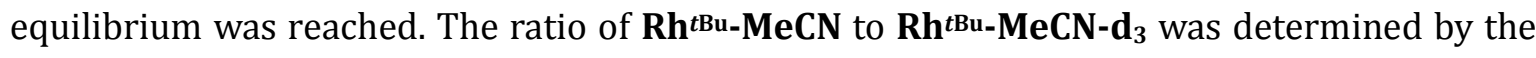
comparison of the integrals of the NMR resonances of bound MeCN in $\mathbf{R h}^{t \mathbf{B u}}-\mathbf{M e C N}$ and free

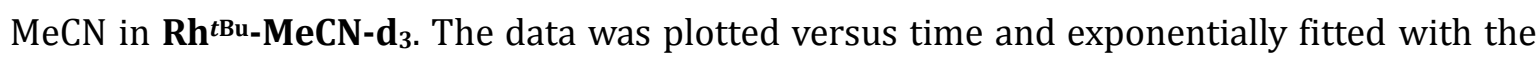
initial rate method to obtain the rate constants $\mathrm{k}_{\mathrm{obs}}(\mathrm{T})$ which were combined in an EYRING plot to determined the thermodynamic values of this exchange reaction. The equilibrium constants $\mathrm{K}_{\mathrm{eq}}$ at the end of the reactions were calculated from $\mathrm{K}_{\mathrm{eq}}=\frac{\mathrm{y}_{0}}{1-\mathrm{y}_{0}}\left(\mathrm{y}_{0}=\right.$ best-fit line at the end of the reaction) and with that, the rates for the backward $\left(\mathrm{k}_{\text {backward }}=\frac{\mathrm{k}_{\mathrm{obs}}}{1-\mathrm{K}_{e q}}\right)$ and forward $\left(\mathrm{k}_{\text {forward }}=k_{\text {obs }}-k_{\text {backward }}\right)$ reaction.

\subsubsection{Catalysis}

\subsubsection{Alkyne Dimerization}

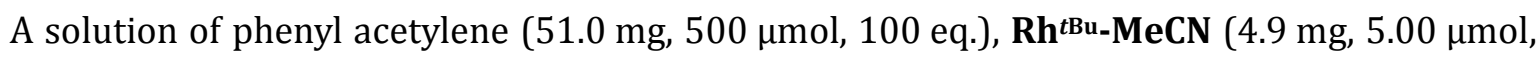
$1.0 \mathrm{eq})$ in THF- $\mathrm{d}_{8}(0.5 \mathrm{~mL})$ was heated in an $80^{\circ} \mathrm{C}$ aluminium heating block. ${ }^{1} \mathrm{H}$ NMR spectra were recorded at $298 \mathrm{~K}$ during the course of the reaction. The dimerization products were identified by typically resonances for cis- $(5.18 \mathrm{ppm}, \mathrm{d}, J=10.9 \mathrm{~Hz})$, trans- $(6.48 \mathrm{ppm}, \mathrm{d}$, $J=16.3 \mathrm{~Hz})$ and $g e m$-isomer $(5.71 \mathrm{ppm}, \mathrm{d}, J=1.0 \mathrm{~Hz})$ and remaining substrate $(3.51 \mathrm{ppm}, \mathrm{s})$.

\subsubsection{Alkyne Hydrogenation}

A solution of alkyne (10 - $100 \mathrm{eq})$, trimethoxybenzene as internal standard, catalyst (1.0 eq) and, if the complex was not soluble enough in THF (55 and 61), $\mathrm{NaBPh}_{4}\left(1.0\right.$ eq) in THF- $\mathrm{d}_{8}$ $(1.5 \mathrm{~mL})$ was portioned into three J. YounG NMR tubes, degassed by three freeze-pump-thaw cycles and purged with dried dihydrogen or deuterium gas (1.0 - 2.0 bar). The reaction was started by shaking the orange solution. The formation of styrene was monitored by ${ }^{1} \mathrm{H}$ NMR spectroscopy. Between two measurements the sample was shaken to ensure a constant $\mathrm{H}_{2}$ 
concentration in solution. The amount of formed styrene was derived from the aromatic signal of the internal standard (6.05 ppm) and plotted vs. time. Following resonances (doublet of doublets) were used for the phenyl acetylene derivatives: PA 5.19 ppm, pF-PA 5.17 ppm, pMe-PA 5.11 ppm, pOMe-PA 5.04 ppm and $\mathrm{pCO}_{2}$ Me-PA 5.34 ppm. Linear fitting of the data gave the initial rates $\mathrm{k}_{\mathrm{obs}}$.

\subsubsection{Carbon dioxide Hydrogenation}

a) NMR experiments: A solution of $\mathbf{R h}^{\mathrm{tBu}}-\mathbf{M e C N}$ (4.9 mg, $5.00 \mu \mathrm{mol}, 1.0$ eq.), DBU (38.1 mg, $250 \mu \mathrm{mol}, 50$ eq.) and if noted LiOTf (15.6 mg, $100 \mu \mathrm{mol}, 20$ eq.) in THF- $\mathrm{d}_{8}(0.2 \mathrm{~mL})$ in a high pressure NMR tube was degassed by three freeze-pump-thaw cycles and purged with $\mathrm{CO}_{2}$ ( 3 bar). The $\mathrm{CO}_{2}$ was frozen out with liquid nitrogen for 30 min and $\mathrm{H}_{2}$ (3 bar) was added at low temperatures. After warming to ambient temperatures the reaction mixture was shaken and ${ }^{1} \mathrm{H}$ NMR spectra were recorded after a certain time (e.g. $24 \mathrm{~h}$ ). The reactions were quenched by releasing the pressure and uptaking the reaction mixture in $\mathrm{MeOH}-\mathrm{d}_{4}(0.5 \mathrm{~mL})$ to ensure complete solvation of the formed formiate salt. The amount of formate formation was determined by ${ }^{1} \mathrm{H}$ NMR spectroscopy with 1,3,5-trimethoxybenzene as internal standard.

b) Autoclave experiments: Three vials ( $8 \mathrm{~mL}$ ) containing stirring bars were placed in a stainless steel autoclave (BÜCHI Picoclave with a type $3200 \mathrm{ml}$ vessel), connected to teflon tubes and evacuated over night at $80^{\circ} \mathrm{C}$. After cooling to $50^{\circ} \mathrm{C}$ the autoclave was purged with purified nitrogen gas three times. In a glove box a solution of $\mathbf{R h}^{t \mathrm{Bu}-\mathbf{M e C N}}(14.7 \mathrm{mg}, 15.0 \mu \mathrm{mol}, 1.0 \mathrm{eq}$.), DBU (114.3 mg, $750 \mu \mathrm{mol}, 50$ eq.) and if noted LiOTf (31.2 mg, $200 \mu \mathrm{mol}, 13$ eq.) in THF (6 mL) was portioned into three parts and added into the vials in the autoclave via syringes having a constant $\mathrm{N}_{2}$ flow. The teflon tubes were removed and the chamber was closed. The autoclave was pressurized with $\mathrm{H}_{2}$ and afterwards with $\mathrm{CO}_{2}$. The reaction mixture was stirred for $6 \mathrm{~h}$ during which time the pressure did not decrease more than $10 \%$ below the starting pressure. After safely releasing the pressure the reaction mixtures were dried, dissolved in $\mathrm{MeOH}-\mathrm{d}_{4}$ and $\mathrm{CH}_{2} \mathrm{Cl}_{2}(10 \mu \mathrm{L})$ was added as internal standard. 



\section{Appendix}

\subsection{Two-in-one Pincer Ligands and their Metal Complexes}
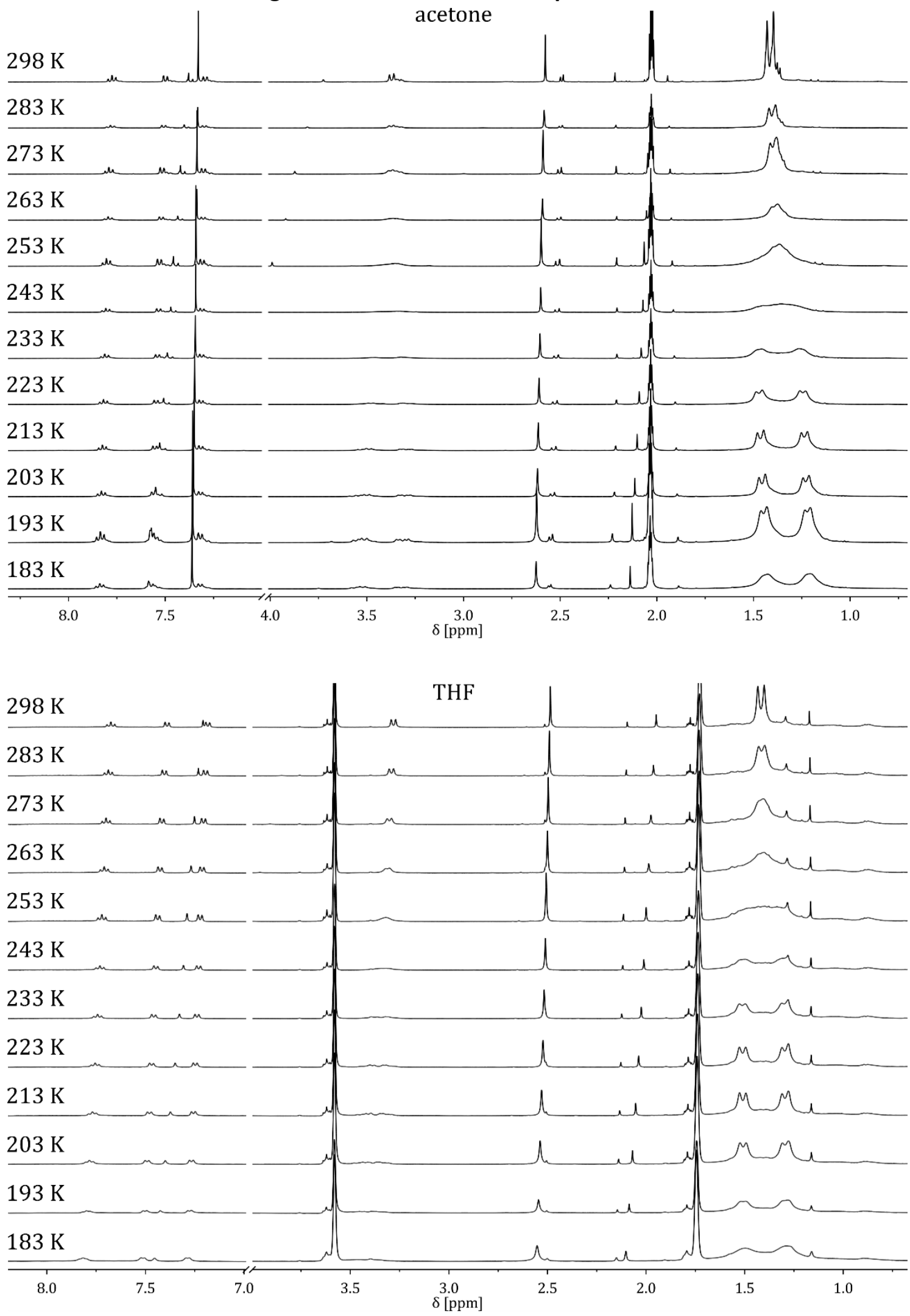

Figure 6.1: Variable temperature ${ }^{1} \mathrm{H}$ NMR of $\mathbf{R h}^{t \mathrm{Bu}}-\mathbf{M e C N}$ in acetone- $\mathrm{d}_{6}$ (top) and THF-d8 (bottom). 

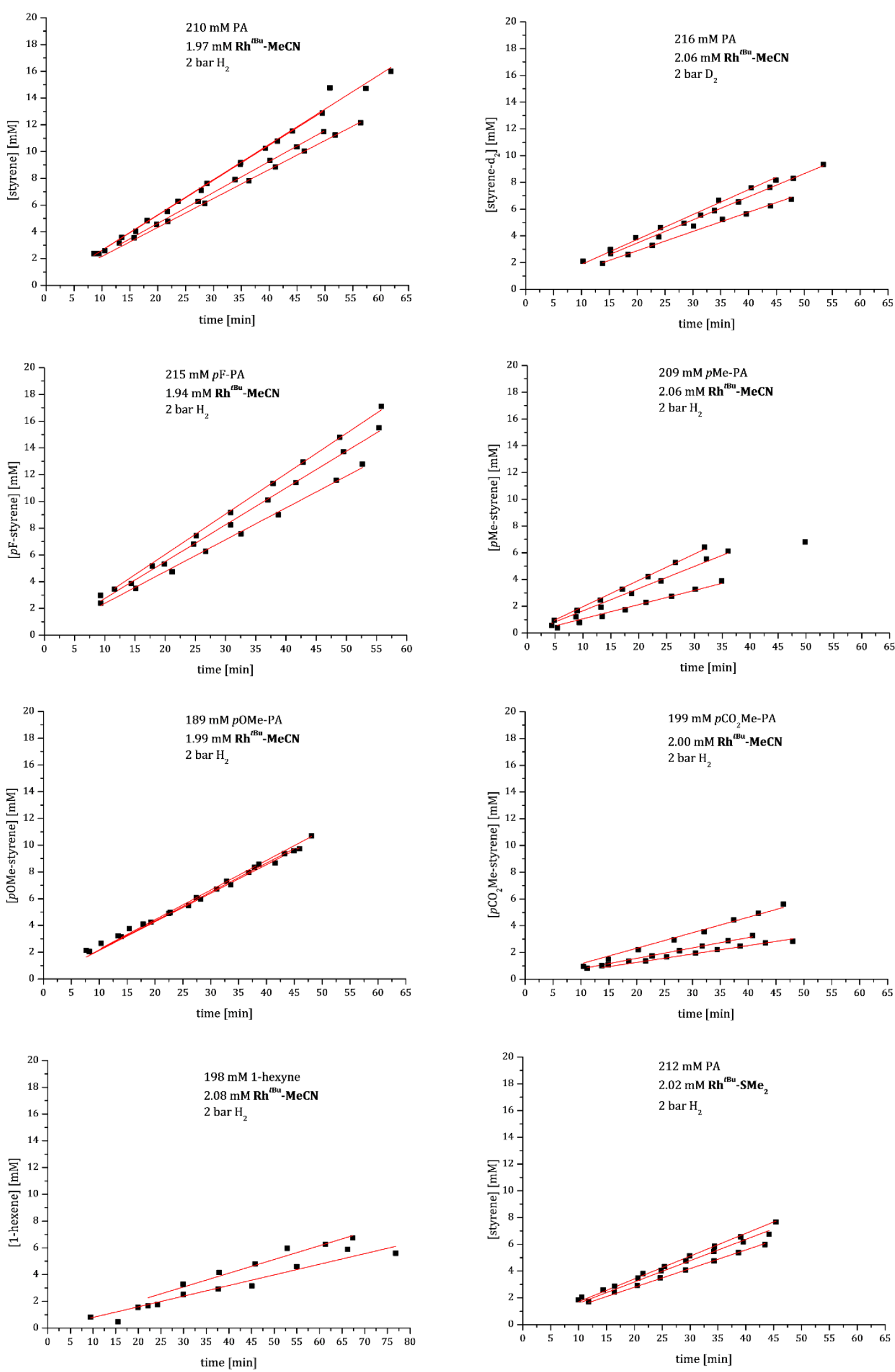

Figure 6.2: Determination of the initial rates of the hydrogenation of different phenyl acetylenes (PA, $\mathrm{PA}+\mathrm{D}_{2}$,

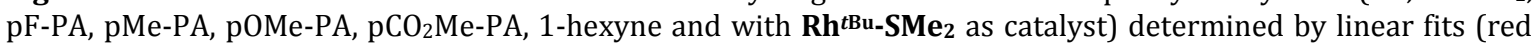
lines) of the concentration of the product versus time plots. 

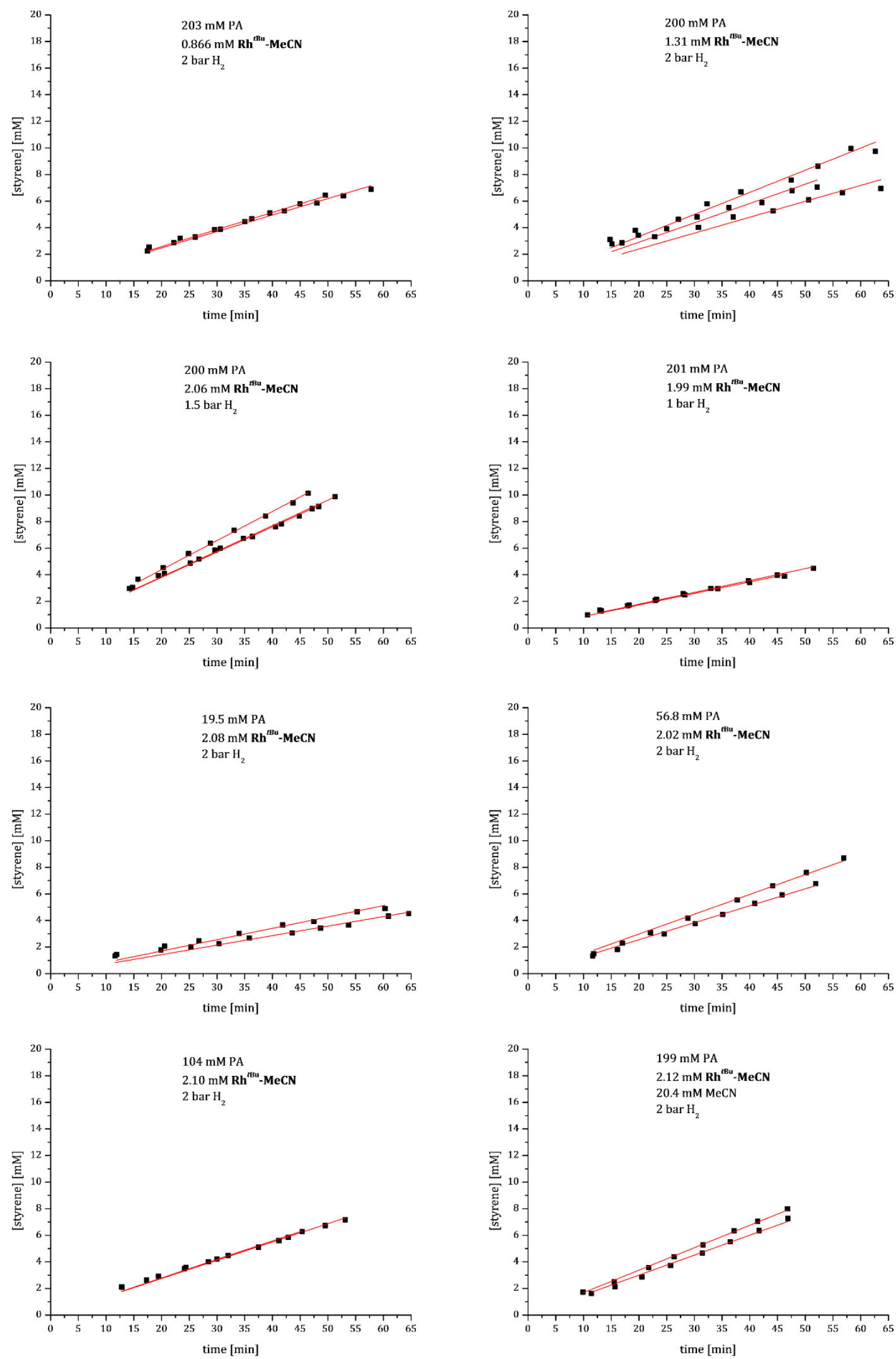

Figure 6.3: Determination of the initial rates of the hydrogenation of different catalyst, dihydrogen and substrate starting concentrations $\left(0.43 \mathrm{~mol} \% \mathbf{R h}^{t \mathrm{Bu}}\right.$-MeCN, $0.66 \mathrm{~mol} \% \mathbf{R h}^{t \mathrm{Bu}}-\mathbf{M e C N}, 1.5$ bar $\mathrm{H}_{2}, 1.0$ bar $\mathrm{H}_{2}, 10$ eq. PA, 30 eq. PA, 50 eq. PA and catalysis in the presence of additional MeCN) determined by linear fits (red lines) of the concentration of the product versus time plots. 


\subsection{Crystallographic Appendix}

Table 6.1: Crystal data and refinement details.

\begin{tabular}{|c|c|c|c|}
\hline compound & $\mathrm{H}_{4} \mathrm{~L}^{2}(\mathrm{OTf})_{4}$ XXI & {$\left[\mathrm{NiL}^{1}\right](\mathrm{OTf})_{2} \mathbf{2 9}$} & {$\left[\mathrm{NiL}^{1}\right](\mathrm{Cl})_{2} \mathbf{3 0}$} \\
\hline empirical formula & $\begin{array}{l}\mathrm{C}_{110} \mathrm{H}_{160} \mathrm{~F}_{78} \mathrm{~N}_{32} \mathrm{O}_{18} \\
\mathrm{P}_{10} \mathrm{~S}_{6}\end{array}$ & $\mathrm{C}_{20} \mathrm{H}_{20} \mathrm{~F}_{6} \mathrm{~N}_{8} \mathrm{NiO}_{6} \mathrm{~S}_{2}$ & $\mathrm{C}_{18} \mathrm{H}_{24} \mathrm{Cl}_{2} \mathrm{~N}_{8} \mathrm{NiO}_{2}$ \\
\hline formula weight & 4202.75 & 705.27 & 514.06 \\
\hline$T[\mathrm{~K}]$ & 133(2) & $133(2)$ & $133(2)$ \\
\hline crystal size $[\mathrm{mm}]$ & $0.20 \times 0.18 \times 0.17$ & $0.19 \times 0.18 \times 0.06$ & $0.45 \times 0.20 \times 0.12$ \\
\hline crystal system & triclinic & triclinic & monoclinic \\
\hline space group & $P-1$ & $P-1$ & $P 2_{1} / m$ \\
\hline$a[\AA ̊]$ & $13.9887(7)$ & $10.4109(7)$ & $7.7984(4)$ \\
\hline$b[\AA]$ & $14.5388(7)$ & $11.4028(7)$ & $12.3965(5)$ \\
\hline$c[\AA]$ & $22.1758(12)$ & $13.4097(8)$ & $11.0497(6)$ \\
\hline$\alpha\left[^{\circ}\right]$ & $100.977(4)$ & $67.363(5)$ & 90 \\
\hline$\beta\left[^{\circ}\right]$ & $90.007(4)$ & $87.553(5)$ & $105.318(4)$ \\
\hline$\gamma\left[{ }^{\circ}\right]$ & $90.020(4)$ & $65.621(5)$ & 90 \\
\hline$V\left[\AA^{3}\right]$ & $4427.6(4)$ & $1325.43(14)$ & $1030.26(9)$ \\
\hline$Z$ & 1 & 2 & 2 \\
\hline$\rho\left[\mathrm{g} / \mathrm{cm}^{3}\right]$ & 1.576 & 1.767 & 1.657 \\
\hline$F(000)$ & 2136 & 716 & 532 \\
\hline$\mu\left[\mathrm{mm}^{-1}\right]$ & 0.314 & 0.986 & 1.237 \\
\hline$T_{\min } / T_{\max }$ & $? / ?$ & $0.8491 / 0.9386$ & $0.5990 / 0.8933$ \\
\hline$\Theta$-range $\left[{ }^{\circ}\right]$ & $1.427-26.775$ & $1.66-26.88$ & $1.911-26.712$ \\
\hline$h k l$-range & $-16-17, \pm 18, \pm 28$ & $-13-11, \pm 14, \pm 16$ & $\pm 9, \pm 15, \pm 13$ \\
\hline measured refl. & 49308 & 16840 & 14614 \\
\hline unique refl. [ $\left.R_{\mathrm{int}}\right]$ & $18771[0.1615]$ & 5618 [0.0703] & $2280[0.0334]$ \\
\hline observed refl. $(I>2 \sigma(I))$ & 7793 & 3875 & 2134 \\
\hline data / restraints / param. & 18771 / 0 / 1155 & 5618 / 0 / 388 & $2280 / 0 / 163$ \\
\hline goodness-of-fit $\left(F^{2}\right)$ & 1.003 & 1.026 & 1.044 \\
\hline$R 1, w R 2(I>2 \sigma(I))$ & $0.1235,0.3044$ & $0.0638,0.1299$ & $0.0214,0.0532$ \\
\hline$R 1, w R 2$ (all data) & $0.2353,0.3703$ & $0.1033,0.1443$ & $0.0236,0.0540$ \\
\hline resid. el. dens. $\left[\mathrm{e} / \AA^{3}\right]$ & $-1.029 / 1.579$ & $-0.417 / 1.221$ & $-0.217 / 0.261$ \\
\hline
\end{tabular}


Table 6.2: Crystal data and refinement details.

\begin{tabular}{|c|c|c|c|}
\hline compound & {$\left[\mathrm{NiL}^{2}\right](\mathrm{OTf})_{2} \mathbf{3 1}$} & $\mathrm{Cu}^{\mathrm{II}}$ cube 32 & $\mathrm{Zn}^{t \mathrm{Bu}}-0 \mathrm{Tf} 49$ \\
\hline empirical formula & $\mathrm{C}_{28} \mathrm{H}_{36} \mathrm{~F}_{6} \mathrm{~N}_{8} \mathrm{NiO}_{6} \mathrm{~S}_{2}$ & $\begin{array}{l}\mathrm{C}_{53} \mathrm{H}_{77.5} \mathrm{Cu}_{4} \mathrm{~F}_{12} \mathrm{~N}_{16.5} \\
\mathrm{O}_{17.5} \mathrm{~S}_{4}\end{array}$ & $\begin{array}{l}\mathrm{C}_{34} \mathrm{H}_{47} \mathrm{~F}_{9} \mathrm{~N}_{4} \mathrm{O}_{9} \mathrm{P}_{2} \mathrm{~S}_{3} \\
\mathrm{Zn}_{2}\end{array}$ \\
\hline formula weight & 817.48 & 1836.21 & 1115.61 \\
\hline$T[\mathrm{~K}]$ & $133(2)$ & $133(2)$ & $133(2)$ \\
\hline crystal size [mm] & $0.12 \times 0.11 \times 0.09$ & $0.20 \times 0.05 \times 0.04$ & $0.29 \times 0.27 \times 0.10$ \\
\hline crystal system & monoclinic & monoclinic & triclinic \\
\hline space group & $C 2 / c$ & $C 2 / c$ & $P-1$ \\
\hline$a[\AA]$ & $21.8888(8)$ & $28.035(6)$ & $10.6909(4)$ \\
\hline$b[\AA]$ & $14.7300(4)$ & $21.620(4)$ & $12.6801(5)$ \\
\hline$c[\AA]$ & $25.7353(10)$ & $27.863(6)$ & $18.8338(7)$ \\
\hline$\alpha\left[^{\circ}\right]$ & 90 & 90 & $98.944(3)$ \\
\hline$\beta\left[{ }^{\circ}\right]$ & $106.039(3)$ & $98.43(3)$ & $94.519(3)$ \\
\hline$\gamma\left[{ }^{\circ}\right]$ & 90 & 90 & $100.670(3)$ \\
\hline$V\left[\AA^{3}\right]$ & $7974.6(5)$ & $16706(6)$ & $2463.22(17)$ \\
\hline$Z$ & 8 & 8 & 2 \\
\hline$\rho\left[\mathrm{g} / \mathrm{cm}^{3}\right]$ & 1.362 & 1.460 & 1.504 \\
\hline$F(000)$ & 3376 & 7512 & 1140 \\
\hline$\mu\left[\mathrm{mm}^{-1}\right]$ & 0.666 & 1.198 & 1.250 \\
\hline$T_{\min } / T_{\max }$ & $? / ?$ & $? / ?$ & 0.7035 / 0.8833 \\
\hline$\Theta$-range $\left[{ }^{\circ}\right]$ & $1.647-25.657$ & $1.347-25.683$ & $1.659-26.738$ \\
\hline$h k l$-range & $\pm 26,-17-16, \pm 31$ & $\pm 34, \pm 26,-33-28$ & $\pm 13,-15-16, \pm 23$ \\
\hline measured refl. & 45711 & 82246 & 34064 \\
\hline unique refl. [ $\left.R_{\text {int }}\right]$ & 7506 [0.1487] & 15759 [0.1923] & 10433 [0.0447] \\
\hline observed refl. $(I>2 \sigma(I))$ & 4980 & 8074 & 7413 \\
\hline data / restraints / param. & $7506 / 0$ / 469 & 15759 / 4 / 1007 & 10433 / 0 / 653 \\
\hline goodness-of-fit $\left(F^{2}\right)$ & 1.047 & 0.953 & 0.947 \\
\hline$R 1, w R 2(I>2 \sigma(I))$ & $0.0973,0.2166$ & $0.0816,0.1206$ & $0.0376,0.0810$ \\
\hline$R 1, w R 2$ (all data) & $0.1406,0.2403$ & $0.1665,0.1446$ & $0.0612,0.0871$ \\
\hline resid. el. dens. $\left[\mathrm{e} / \AA^{3}\right]$ & $-0.918 / 1.192$ & $-0.437 / 0.653$ & $-0.444 / 0.349$ \\
\hline
\end{tabular}


Table 6.3: Crystal data and refinement details.

\begin{tabular}{|c|c|c|c|}
\hline compound & $\mathrm{Zn}^{i P r}-0 T f 5049$ & $\mathrm{Zn}^{i \mathrm{Pr}, \mathrm{ox}-0 T f} 51$ & $\mathrm{Zn}^{\mathrm{tBu}, \mathrm{ox}-\mathrm{Br}} 53$ \\
\hline empirical formula & $\mathrm{C}_{32} \mathrm{H}_{42} \mathrm{~F}_{9} \mathrm{~N}_{5} \mathrm{O}_{9} \mathrm{P}_{2} \mathrm{~S}_{3}$ & $\mathrm{C}_{56} \mathrm{H}_{80} \mathrm{~F}_{6} \mathrm{~N}_{8} \mathrm{O}_{12} \mathrm{P}_{4} \mathrm{~S}_{2}$ & $\mathrm{C}_{35} \mathrm{H}_{53} \mathrm{Br}_{3} \mathrm{~N}_{6} \mathrm{O}_{2} \mathrm{P}_{2}$ \\
\hline & $\mathrm{Zn}_{2}$ & $\mathrm{Zn}_{4}$ & $\mathrm{Zn}_{2}$ \\
\hline formula weight & 1100.56 & 1620.76 & 1022.24 \\
\hline$T[\mathrm{~K}]$ & $133(2)$ & $133(2)$ & $133(2)$ \\
\hline crystal size [mm] & $0.29 \times 0.27 \times 0.12$ & $0.46 \times 0.34 \times 0.22$ & $0.19 \times 0.18 \times 0.06$ \\
\hline crystal system & triclinic & orthorhombic & monoclinic \\
\hline space group & $P-1$ & Cmca & $P 2_{1} / c$ \\
\hline$a[\AA]$ & $11.078(2)$ & $34.0552(6)$ & $21.4230(4)$ \\
\hline$b[\AA]$ & $14.260(3)$ & $20.5722(3)$ & $14.5404(4)$ \\
\hline$c[\AA]$ & $15.099(3)$ & $25.9600(6)$ & $28.9299(6)$ \\
\hline$\alpha\left[^{\circ}\right]$ & $92.90(3)$ & 90 & 90 \\
\hline$\beta\left[^{\circ}\right]$ & $106.47(3)$ & 90 & $109.405(2)$ \\
\hline$\gamma\left[{ }^{\circ}\right]$ & $97.89(3)$ & 90 & 90 \\
\hline$V\left[\AA^{3}\right]$ & $2255.6(9)$ & $18187.3(6)$ & $8499.7(3)$ \\
\hline$Z$ & 2 & 8 & 8 \\
\hline$\rho\left[\mathrm{g} / \mathrm{cm}^{3}\right]$ & 1.620 & 1.184 & 1.598 \\
\hline$F(000)$ & 1120 & 6672 & 4128 \\
\hline$\mu\left[\mathrm{mm}^{-1}\right]$ & 1.364 & 1.218 & 4.062 \\
\hline$T_{\min } / T_{\max }$ & $0.7062 / 0.8566$ & $? / ?$ & 0.5015 / 0.7445 \\
\hline$\Theta$-range $\left[{ }^{\circ}\right]$ & $1.413-26.929$ & $1.397-25.693$ & $1.493-25.653$ \\
\hline$h k l$-range & $\begin{array}{l}-14-13,-17-18,- \\
18-19\end{array}$ & $\pm 41,-25-22, \pm 31$ & $-26-25, \pm 17, \pm 35$ \\
\hline measured refl. & 23122 & 99323 & 104398 \\
\hline unique refl. $\left[R_{\text {int }}\right]$ & 9470 [0.0489] & 8747 [0.0667] & 16017 [0.1007] \\
\hline observed refl. $(I>2 \sigma(I))$ & 6677 & 7259 & 10561 \\
\hline data / restraints / param. & 9470 / 141 / 623 & 8747 / 3 / 426 & 16017 / 0 / 929 \\
\hline goodness-of-fit $\left(F^{2}\right)$ & 1.028 & 1.126 & 0.974 \\
\hline$R 1, w R 2(I>2 \sigma(I))$ & $0.0542,0.1231$ & $0.0814,0.2215$ & $0.0512,0.0741$ \\
\hline$R 1, w R 2$ (all data) & $0.0851,0.1349$ & $0.0953,0.2308$ & $0.0954,0.0834$ \\
\hline resid. el. dens. $\left[\mathrm{e} / \AA^{3}\right]$ & $-0.835 / 0.737$ & $-0.705 / 1.805$ & $-0.451 / 0.590$ \\
\hline
\end{tabular}


Table 6.4: Crystal data and refinement details.

\begin{tabular}{|c|c|c|c|}
\hline compound & Co ${ }^{t \mathrm{Bu}}-\mathrm{MeCN} 54$ & $\mathrm{Rh}^{t \mathrm{Bu}-\mathrm{CO}} 55$ & $\mathrm{Rh}^{t \mathrm{Bu}-M e C N} 58$ \\
\hline empirical formula & $\begin{array}{l}\mathrm{C}_{47} \mathrm{H}_{71} \mathrm{~B}_{3} \mathrm{Co}_{2} \mathrm{~F}_{12} \mathrm{~N}_{12} \\
\mathrm{P}_{2}\end{array}$ & $\mathrm{C}_{36} \mathrm{H}_{53} \mathrm{~F}_{6} \mathrm{~N}_{4} \mathrm{O}_{3} \mathrm{P}_{3} \mathrm{Rh}_{2}$ & $\mathrm{C}_{38} \mathrm{H}_{59} \mathrm{~F}_{6} \mathrm{~N}_{6} \mathrm{OP}_{3} \mathrm{Rh}_{2}$ \\
\hline formula weight & 1244.38 & 1002.55 & 1028.64 \\
\hline$T[\mathrm{~K}]$ & $133(2)$ & $133(2)$ & $133(2)$ \\
\hline crystal size [mm] & $0.49 \times 0.34 \times 0.30$ & $0.32 \times 0.29 \times 0.15$ & $0.50 \times 0.50 \times 0.39$ \\
\hline crystal system & monoclinic & orthorhombic & monoclinic \\
\hline space group & $\mathrm{P} 2 / \mathrm{n}$ & Cmca & $P 2_{1} / n$ \\
\hline$a[\AA]$ & $11.461(2)$ & $30.4622(9)$ & $15.1497(4)$ \\
\hline$b[\AA]$ & $18.378(4)$ & $17.9792(6)$ & $14.3029(3)$ \\
\hline$c[\AA]$ & $14.358(3)$ & $15.5831(6)$ & $20.9682(6)$ \\
\hline$\alpha\left[^{\circ}\right]$ & 90 & 90 & 90 \\
\hline$\beta\left[^{\circ}\right]$ & $101.10(3)$ & 90 & $98.548(2)$ \\
\hline$\gamma\left[^{\circ}\right]$ & 90 & 90 & 90 \\
\hline$V\left[\AA^{3}\right]$ & $2967.8(11)$ & $8534.6(5)$ & $4493.0(2)$ \\
\hline$Z$ & 2 & 8 & 4 \\
\hline$\rho\left[\mathrm{g} / \mathrm{cm}^{3}\right]$ & 1.392 & 1.560 & 1.521 \\
\hline$F(000)$ & 1288 & 4080 & 2104 \\
\hline$\mu\left[\mathrm{mm}^{-1}\right]$ & 0.694 & 0.951 & 0.903 \\
\hline$T_{\min } / T_{\max }$ & $0.7453 / 0.8498$ & $0.7333 / 0.8667$ & $0.6246 / 0.7899$ \\
\hline$\Theta$-range $\left[{ }^{\circ}\right]$ & $1.821-26.721$ & $1.337-26.739$ & $1.554-26.958$ \\
\hline$h k l$-range & $\begin{array}{l}-14-12, \pm 23,-18- \\
17\end{array}$ & $\pm 38, \pm 22, \pm 19$ & $\pm 19,-18-17, \pm 26$ \\
\hline measured refl. & 28593 & 54006 & 60833 \\
\hline unique refl. $\left[R_{\text {int }}\right]$ & $6287[0.0291]$ & 4614 [0.0433] & $9547[0.0461]$ \\
\hline observed refl. $(I>2 \sigma(I))$ & 5602 & 3929 & 8826 \\
\hline data / restraints / param. & $6287 / 0$ / 409 & 4614 / 68 / 301 & 9547 / 46 / 556 \\
\hline goodness-of-fit $\left(F^{2}\right)$ & 1.035 & 1.033 & 1.103 \\
\hline$R 1, w R 2(I>2 \sigma(I))$ & $0.0357,0.0927$ & $0.0302,0.0719$ & $0.0259,0.0611$ \\
\hline$R 1, w R 2$ (all data) & $0.0412,0.0956$ & $0.0408,0.0756$ & $0.0300,0.0628$ \\
\hline resid. el. dens. $\left[\mathrm{e} / \AA^{3}\right]$ & $-0.701 / 1.065$ & $-0.624 / 0.678$ & $-0.306 / 0.485$ \\
\hline
\end{tabular}


Table 6.5: Crystal data and refinement details.

\begin{tabular}{|c|c|c|c|}
\hline compound & $\mathrm{Rh}^{t \mathrm{Bu}}-\mathrm{SMe}_{2} \mathbf{6 1}$ & $\mathrm{Rh}^{\mathrm{tBu}}-\mathrm{C}_{2} \mathrm{H}_{4} 63$ & $\mathrm{Rh}^{\mathrm{tBu}-\mathrm{DCM}} 67$ \\
\hline empirical formula & $\begin{array}{l}\mathrm{C}_{39} \mathrm{H}_{65} \mathrm{~F}_{6} \mathrm{~N}_{4} \mathrm{O}_{2} \mathrm{P}_{3} \mathrm{Rh}_{2} \\
\mathrm{~S}\end{array}$ & $\mathrm{C}_{25} \mathrm{H}_{34} \mathrm{~N}_{4} \mathrm{PRh}$ & $\begin{array}{l}\mathrm{C}_{35.5} \mathrm{H}_{55} \mathrm{Cl}_{5} \mathrm{~F}_{6} \mathrm{~N}_{5} \mathrm{P}_{3} \\
\mathrm{Rh}_{2}\end{array}$ \\
\hline formula weight & 1066.74 & 524.44 & 1141.82 \\
\hline$T[\mathrm{~K}]$ & $133(2)$ & $133(2)$ & $133(2)$ \\
\hline crystal size [mm] & $0.20 \times 0.10 \times 0.08$ & $0.38 \times 0.29 \times 0.17$ & $0.38 \times 0.24 \times 0.19$ \\
\hline crystal system & monoclinic & monoclinic & monoclinic \\
\hline space group & $C 2 / c$ & $P 2_{1} / c$ & $C 2 / c$ \\
\hline$a[\AA ̊]$ & $29.8440(6)$ & $11.8259(7)$ & $38.7936(11)$ \\
\hline$b[\AA]$ & $12.0214(3)$ & $11.6485(5)$ & $9.1801(2)$ \\
\hline$c[\AA]$ & $27.3689(5)$ & $17.6108(9)$ & $32.3225(9)$ \\
\hline$\alpha\left[^{\circ}\right]$ & 90 & 90 & 90 \\
\hline$\beta\left[^{\circ}\right]$ & $110.7590(10)$ & $101.990(5)$ & $126.081(2)$ \\
\hline$\gamma\left[{ }^{\circ}\right]$ & 90 & 90 & 90 \\
\hline$V\left[\AA^{3}\right]$ & $9181.6(3)$ & $2373.0(2)$ & $9303.0(5)$ \\
\hline$Z$ & 8 & 4 & 8 \\
\hline$\rho\left[\mathrm{g} / \mathrm{cm}^{3}\right]$ & 1.543 & 1.468 & 1.630 \\
\hline$F(000)$ & 4384 & 1088 & 4616 \\
\hline$\mu\left[\mathrm{mm}^{-1}\right]$ & 0.931 & 0.807 & 1.157 \\
\hline$T_{\min } / T_{\max }$ & 0.8155 / 0.9364 & 0.7645 / 0.8957 & 0.6462 / 0.8498 \\
\hline$\Theta$-range $\left[{ }^{\circ}\right]$ & $1.459-25.627$ & $1.760-26.812$ & $1.299-25.612$ \\
\hline$h k l$-range & $-35-36, \pm 14, \pm 33$ & $\pm 14, \pm 14, \pm 22$ & $\pm 46,-11-10, \pm 39$ \\
\hline measured refl. & 51851 & 31529 & 56066 \\
\hline unique refl. [ $\left.R_{\mathrm{int}}\right]$ & 8663 [0.0473] & 5039 [0.0579] & 8756 [0.0477] \\
\hline observed refl. $(I>2 \sigma(I))$ & 7527 & 3841 & 7072 \\
\hline data / restraints / param. & 8663 / 75 / 583 & $5039 / 0 / 303$ & $8756 / 10 / 557$ \\
\hline goodness-of-fit $\left(F^{2}\right)$ & 1.018 & $0.996^{\prime}$ & 1.069 \\
\hline$R 1, w R 2(I>2 \sigma(I))$ & $0.0273,0.0610$ & $0.0340,0.0675$ & $0.0507,0.1384$ \\
\hline$R 1, w R 2$ (all data) & $0.0354,0.0634$ & $0.0550,0.0728$ & $0.0649,0.1458$ \\
\hline resid. el. dens. $\left[\mathrm{e} / \AA^{3}\right]$ & $-0.405 / 0.786$ & $-0.380 / 0.776$ & $-1.174 / 1.568$ \\
\hline
\end{tabular}


Table 6.6: Crystal data and refinement details.

\begin{tabular}{|c|c|}
\hline compound & Rh $^{t \mathrm{Bu}-M e I} 69$ \\
\hline empirical formula & $\mathrm{C}_{35} \mathrm{H}_{56} \mathrm{I}_{3} \mathrm{~N}_{5} \mathrm{P}_{2} \mathrm{Rh}_{2}$ \\
\hline formula weight & 1195.30 \\
\hline$T[\mathrm{~K}]$ & $133(2)$ \\
\hline crystal size [mm] & $0.47 \times 0.07 \times 0.05$ \\
\hline crystal system & triclinic \\
\hline space group & $P-1$ \\
\hline$a[\AA]$ & $15.6191(6)$ \\
\hline$b[\AA]$ & $15.8702(6)$ \\
\hline$c[\AA]$ & $18.7941(7)$ \\
\hline$\alpha\left[{ }^{\circ}\right]$ & $112.022(3)$ \\
\hline$\beta\left[{ }^{\circ}\right]$ & $93.953(3)$ \\
\hline$\gamma\left[{ }^{\circ}\right]$ & $97.521(3)$ \\
\hline$V\left[\AA^{3}\right]$ & $4246.1(3)$ \\
\hline$Z$ & 4 \\
\hline$\rho\left[\mathrm{g} / \mathrm{cm}^{3}\right]$ & 1.870 \\
\hline$F(000)$ & 2320 \\
\hline$\mu\left[\mathrm{mm}^{-1}\right]$ & 3.064 \\
\hline$T_{\min } / T_{\max }$ & $0.5039 / 0.7924$ \\
\hline$\Theta$-range $\left[{ }^{\circ}\right]$ & $1.404-26.805$ \\
\hline$h k l$-range & $\pm 19, \pm 20,-23-21$ \\
\hline measured refl. & 52428 \\
\hline unique refl. $\left[R_{\text {int }}\right]$ & 17964 [0.0655] \\
\hline observed refl. $(I>2 \sigma(I))$ & 13839 \\
\hline data / restraints / param. & 17964 / 26 / 936 \\
\hline goodness-of-fit $\left(F^{2}\right)$ & 1.008 \\
\hline$R 1, w R 2(I>2 \sigma(I))$ & $0.0508,0.1214$ \\
\hline$R 1, w R 2$ (all data) & $0.0714,0.1303$ \\
\hline resid. el. dens. $\left[\mathrm{e} / \AA^{3}\right]$ & $-2.368 / 2.097$ \\
\hline
\end{tabular}





\section{Biblography}

[1] J. B. Howard, D. C. Rees, Proc. Natl. Acad. Sci. 2006, 103, 17088-17093.

[2] J. Kim, D. Woo, D. C. Rees, Biochemistry 1993, 32, 7104-7115.

[3] J. B. Howard, D. C. Rees, Chem. Rev. 1996, 96, 2965-2982.

[4] O. Einsle, F. A. Tezcan, S. L. A. Andrade, B. Schmid, M. Yoshida, J. B. Howard, D. C. Rees, Science 2002, 297, 1696-700.

[5] T. Spatzal, M. Aksoyoglu, L. Zhang, S. L. A. Andrade, E. Schleicher, S. Weber, D. C. Rees, O. Einsle, Science 2011, 334, 940.

[6] K. M. Lancaster, M. Roemelt, P. Ettenhuber, Y. Hu, M. W. Ribbe, F. Neese, U. Bergmann, S. DeBeer, Science 2011, 334, 974-977.

[7] J. a Wiig, C. C. Lee, Y. Hu, M. W. Ribbe, J. Am. Chem. Soc. 2013, 135, 4982-4983.

[8] B. K. Burgess, D. J. Lowe, Chem. Rev. 1996, 96, 2983-3011.

[9] B. J. Hales, E. E. Case, J. E. Morningstar, M. F. Dzeda, L. a Mauterer, Biochemistry 1986, $25,7251-7255$.

[10] J. R. Chisnell, R. Premakumar, P. E. Bishop, J. Bacteriol. 1988, 170, 27-33.

[11] R. A. Albertys, J. Biol. Chem. 1994, 269, 7099-7102.

[12] L. C. Seefeldt, M. E. Rasche, S. a Ensign, Biochemistry 1995, 34, 5382-5389.

[13] V. Smil, Nature 1999, 400, 415-415.

[14] F. Haber, Zeitschrift für Elektrochemie und Angew. Phys. Chemie 1910, 16, 244-246.

[15] A. Mittasch, Geschichte Der Ammoniaksynthese, Verlag Chemie, Weinheim, 1951.

[16] G. Ertl, Angew. Chem. Int. Ed. 2008, 47, 3524-3535.

[17] A. Gers-Barlag, Master Thesis, Pyrazole-Based Tetraamido Ligands and Their Molybdenum Complexes - Possible Scaffolds in N2 Activation, Georg-AugustUniversität Göttingen, 2012.

[18] W. Moritz, G. Ertl, R. J. Behm, R. Imbihl, Surf. Sience 1982, 123, 129-140.

[19] A. D. Allen, C. V Senoff, Chem. Commun. 1965, 621-622.

[20] M. Hidai, Y. Mizobe, Chem. Rev. 1995, 95, 1115-1133.

[21] M. Hidai, Coord. Chem. Rev. 1999, 185-186, 99-108.

[22] M. D. Fryzuk, J. B. Love, S. J. Rettig, V. G. Young, Science. 1997, 275, 1445-1447.

[23] M. D. Fryzuk, Acc. Chem. Res. 2009, 42, 127-133.

[24] W. J. Evans, T. A. Ulibarri, J. W. Ziller, J. Am. Chem. Soc. 1988, 110, 6877-6879.

[25] K. Komori, H. Oshita, Y. Mizobe, M. Hidai, J. Am. Chem. Soc. 1989, 111, 1939-1940.

[26] C. J. Pickett, J. Talarmin, Nature 1985, 317, 652-653.

[27] A. E. Shilov, Russ. Chem. Bull. Int. Ed. 2003, 52, 2555-2562.

[28] T. A. Bazhenova, A. E. Shilov, Coord. Chem. Rev. 1995, 144, 69-145.

[29] Y. Antipin, Mikhail, L. P. Didenko, L. M. Kachapina, A. E. Shilov, A. K. Shilova, Y. T. Struchkov, J. Chem. Soc., Chem. Commun. 1989, 1467.

[30] A. E. Shilov, N. P. Luneva, S. A. Mironova, M. Y. Antipin, Y. T. Struchkov, Angew. Chem. Int. Ed. Engl. 1993, 32, 1178-1179.

[31] C. E. Laplaza, A. L. Odom, W. M. Davis, C. C. Cummins, J. D. Protasiewicz, J. Am. Chem. Soc. 1995, 117, 4999-5000.

[32] C. E. Laplaza, C. C. Cummins, Science. 1995, 268, 861-863.

[33] C. E. Laplaza, M. J. A. Johnson, J. C. Peters, A. L. Odom, E. Kim, C. C. Cummins, G. N. George, I. J. Pickering, J. Am. Chem. Soc. 1996, 118, 8623-8638.

[34] D. V Yandulov, R. R. Schrock, J. Am. Chem. Soc. 2002, 124, 6252-6253.

[35] M. W. Weatherburn, Anal. Chem. 1967, 39, 971-974.

[36] D. V Yandulov, Science. 2003, 301, 76-78.

[37] J. Chatt, J. R. Dilworth, R. L. Richards, Chem. Rev. 1978, 78, 589-625.

[38] D. V Yandulov, R. R. Schrock, Inorg. Chem. 2005, 44, 1103-1117.

[39] R. R. Schrock, Angew. Chem. Int. Ed. 2008, 47, 5512-5522.

[40] S. Schenk, B. Le Guennic, B. Kirchner, M. Reiher, Inorg. Chem. 2008, 47, 3634-3650.

[41] K. M. Wampler, R. R. Schrock, Inorg. Chem. 2007, 46, 8463-8465.

[42] J. M. Chin, R. R. Schrock, P. Müller, Inorg. Chem. 2010, 49, 7904-16. 
[43] T. Miyazaki, H. Tanaka, Y. Tanabe, M. Yuki, K. Nakajima, K. Yoshizawa, Y. Nishibayashi, Angew. Chem. Int. Ed. 2014, 53, 11488-11492.

[44] K. Arashiba, E. Kinoshita, S. Kuriyama, A. Eizawa, K. Nakajima, H. Tanaka, K. Yoshizawa, Y. Nishibayashi, J. Am. Chem. Soc. 2015, 137, 5666-5669.

[45] K. Arashiba, Y. Miyake, Y. Nishibayashi, Nat. Chem. 2011, 3, 120-125.

[46] P. Ligand, S. Kuriyama, K. Arashiba, K. Nakajima, H. Tanaka, N. Kamaru, K. Yoshizawa, Y. Nishibayashi, J. Am. Chem. Soc. 2014, 136, 9719-9731.

[47] H. Tanaka, K. Arashiba, S. Kuriyama, A. Sasada, K. Nakajima, K. Yoshizawa, Y. Nishibayashi, Nat. Commun. 2014, 5, 1-11.

[48] M. Yuki, Y. Miyake, Y. Nishibayashi, I. Wakiji, M. Hidai, Organometallics 2008, 27, 3947 3953.

[49] J. S. Anderson, J. Rittle, J. C. Peters, Nature 2013, 501, 84-7.

[50] J. S. Anderson, G. E. Cutsail, J. Rittle, B. a. Connor, W. a. Gunderson, L. Zhang, B. M. Hoffman, J. C. Peters, J. Am. Chem. Soc. 2015, 137, 7803-7809.

[51] S. E. Creutz, J. C. Peters, J. Am. Chem. Soc. 2014, 136, 1105-1115.

[52] T. J. Del Castillo, N. B. Thompson, J. C. Peters, J. Am. Chem. Soc. 2016, 138, 5341-5350.

[53] J. S. Anderson, G. E. Cutsail, J. Rittle, B. A. Connor, W. A. Gunderson, L. Zhang, B. M. Hoffman, J. C. Peters, J. Am. Chem. Soc. 2015, 137, 7803-7809.

[54] M. M. Rodriguez, E. Bill, W. W. Brennessel, P. L. Holland, Science. 2011, 334, 780-783.

[55] I. Čorić, B. Q. Mercado, E. Bill, D. J. Vinyard, P. L. Holland, Nature 2015, 526, 96-99.

[56] S. F. McWilliams, P. L. Holland, Acc. Chem. Res. 2015, 48, 2059-2065.

[57] B. Askevold, J. T. Nieto, S. Tussupbayev, M. Diefenbach, E. Herdtweck, M. C. Holthausen, S. Schneider, Nat. Chem. 2011, 3, 532-537.

[58] I. Klopsch, M. Finger, C. Würtele, B. Milde, D. B. Werz, S. Schneider, J. Am. Chem. Soc. 2014, 136, 6881-6883.

[59] I. Klopsch, M. Kinauer, M. Finger, C. Würtele, S. Schneider, Angew. Chem. Int. Ed. 2016, $55,4786-4789$.

[60] M. G. Scheibel, J. Abbenseth, M. Kinauer, F. W. Heinemann, C. Würtele, B. de Bruin, S. Schneider, Inorg. Chem. 2015, 54, 9290-9302.

[61] L. Söncksen, C. Gradert, J. Krahmer, C. Näther, F. Tuczek, Inorg. Chem. 2013, 52, 65766589.

[62] H. Broda, S. Hinrichsen, J. Krahmer, C. Näther, F. Tuczek, Dalton Trans. 2014, 43, 2007 2012.

[63] H. Broda, S. Hinrichsen, F. Tuczek, Coord. Chem. Rev. 2013, 257, 587-598.

[64] S. Hinrichsen, A. Kindjajev, S. Adomeit, J. Krahmer, C. Näther, F. Tuczek, Inorg. Chem. 2016, 55, 8712-8722.

[65] S. Buchler, PhD Thesis, Präorganisierte Zweikernkomplexe Neuer PyrazolatKompartimentliganden, Ruprecht-Karls-Univeristät Heidelberg, 2002.

[66] A. L. Gavrilova, B. Bosnich, Chem. Rev. 2004, 104, 349-384.

[67] J. Klingele, S. Dechert, F. Meyer, Coord. Chem. Rev. 2009, 253, 2698-2741.

[68] A. Prokofieva, A. I. Prikhod'ko, E. A. Enyedy, E. Farkas, W. Maringgele, S. Demeshko, S. Dechert, F. Meyer, Inorg. Chem. 2007, 46, 4298-4307.

[69] S. Buchler, F. Meyer, A. Jacobi, P. Kircher, L. Zsolnai, Zeitschrift für Naturforsch. B 1999, $54,1295-1306$.

[70] T. G. Schenck, J. M. Downes, C. R. C. Milne, P. B. Mackenzie, T. G. Boucher, J. Whelan, B. Bosnich, Inorg. Chem. 1985, 24, 2334-2337.

[71] J. C. Röder, F. Meyer, H. Pritzkow, Organometallics 2001, 20, 811-817.

[72] S. Fischer, S. Demeshko, S. Dechert, F. Meyer, Zeitschrift für Anorg. und Allg. Chemie 2012, 638, 621-627.

[73] B. Neuner, R. R. Schrock, Organometallics 1996, 15, 5-6.

[74] K. Nomura, R. R. Schrock, Inorg. Chem. 1996, 35, 3695-3701.

[75] J. S. Freundlich, R. R. Schrock, W. M. Davis, J. Am. Chem. Soc. 1996, 118, 3643-3655.

[76] R. R. Schrock, S. W. Seidel, N. C. Mösch-Zanetti, K.-Y. Shih, M. B. O’Donoghue, W. M. Davis, W. M. Reiff, J. Am. Chem. Soc. 1997, 119, 11876-11893.

[77] R. R. Schrock, C. C. Cummins, T. Wilhelm, S. Lin, S. M. Reid, M. Kol, W. M. Davis, 
Organometallics 1996, 15, 1470-1476.

[78] R. R. Schrock, S. W. Seidel, N. C. Mösch-Zanetti, D. A. Dobbs, K.-Y. Shih, W. M. Davis, Organometallics 1997, 16, 5195-5208.

[79] M. B. O’Donoghue, W. M. Davis, R. R. Schrock, Inorg. Chem. 1998, 37, 5149-5158.

[80] M. B. O’Donoghue, W. M. Davis, R. R. Schrock, W. M. Reiff, Inorg. Chem. 1999, 38, $243-$ 252.

[81] S. W. Seidel, R. R. Schrock, W. M. Davis, Organometallics 1998, 17, 1058-1068.

[82] D. M. T. Chan, M. H. Chisholm, K. Folting, J. C. Huffman, N. S. Marchant, Inorg. Chem. 1986, 25, 4170-4174.

[83] A. Wünsche von Leupoldt, C. Förster, T. J. Fiedler, N. H. Bings, K. Heinze, Eur. J. Inorg. Chem. 2013, 2013, 6079-6090.

[84] M. Sietzen, S. Batke, L. Merz, H. Wadepohl, J. Ballmann, Organometallics 2015, 34, 1118-1128.

[85] S. Batke, M. Sietzen, H. Wadepohl, J. Ballmann, Inorg. Chem. 2014, 53, 4144-4153.

[86] J. Hohenberger, K. Ray, K. Meyer, Nat. Commun. 2012, 3, 720.

[87] L. Que, Acc. Chem. Res. 2007, 40, 493-500.

[88] B. Meunier, S. P. de Visser, S. Shaik, Chem. Rev. 2004, 104, 3947-3980.

[89] I. G. Denisov, T. M. Makris, S. G. Sligar, I. Schlichting, Chem. Rev. 2005, 105, 2253-2278.

[90] S. Friedle, E. Reisner, S. J. Lippard, Chem. Soc. Rev. 2010, 39, 2768.

[91] C. E. Tinberg, S. J. Lippard, Acc. Chem. Res. 2011, 44, 280-288.

[92] R. A. Himes, K. D. Karlin, Curr. Opin. Chem. Biol. 2009, 13, 119-131.

[93] J. Shearer, Acc. Chem. Res. 2014, 47, 2332-2341.

[94] D. Evans, Coord. Chem. Rev. 2005, 249, 1582-1595.

[95] H. Dobbek, V. Svetlitchnyi, L. Gremer, R. Huber, O. Meyer, Science 2001, 293, 12811285.

[96] C. Darnault, A. Volbeda, E. J. Kim, P. Legrand, X. Vernède, P. A. Lindahl, J. C. FontecillaCamps, Nat. Struct. Biol. 2003, 10, 271-279.

[97] S. Gencic, D. A. Grahame, J. Biol. Chem. 2003, 278, 6101-6110.

[98] U. Ermler, Science. 1997, 278, 1457-1462.

[99] U. Ermler, Dalton Trans. 2005, 3451-3458.

[100] D. P. Barondeau, C. J. Kassmann, C. K. Bruns, J. A. Tainer, E. D. Getzoff, Biochemistry 2004, 43, 8038-8047.

[101] W. Lubitz, H. Ogata, O. Rudiger, E. Reijerse, Chem. Rev. 2014, 114, 4081-4148.

[102] H. Ogata, S. Hirota, A. Nakahara, H. Komori, N. Shibata, T. Kato, K. Kano, Y. Higuchi, Structure 2005, 13, 1635-1642.

[103] S. Lin, T. Agapie, Synlett 2011, 1-5.

[104] B.-T. Guan, Y. Wang, B.-J. Li, D.-G. Yu, Z.-J. Shi, J. Am. Chem. Soc. 2008, 130, 1446814470.

[105] V. P. W. Böhm, C. W. K. Gstöttmayr, T. Weskamp, W. A. Herrmann, Angew. Chem. Int. Ed. 2001, 40, 3387-3389.

[106] E. Negishi, Acc. Chem. Res. 1982, 15, 340-348.

[107] D. Milstein, J. K. Stille, J. Am. Chem. Soc. 1979, 101, 4992-4998.

[108] N. Miyaura, A. Suzuki, Chem. Rev. 1995, 95, 2457-2483.

[109] M. García-Melchor, A. A. C. Braga, A. Lledós, G. Ujaque, F. Maseras, Acc. Chem. Res. 2013, 46, 2626-2634.

[110] S. L. Zultanski, G. C. Fu, J. Am. Chem. Soc. 2013, 135, 624-627.

[111] S. Biswas, D. J. Weix, J. Am. Chem. Soc. 2013, 135, 16192-16197.

[112] N. F. Curtis, D. F. Cook, Chem. Commun. 1967, 962.

[113] P. J. Alonso, A. B. Arauzo, M. A. García-Monforte, A. Martín, B. Menjón, C. Rillo, M. Tomás, Chem. - A Eur. J. 2009, 15, 11020-11030.

[114] T.-P.-A. Cao, G. Nocton, L. Ricard, X. F. Le Goff, A. Auffrant, Angew. Chem. Int. Ed. 2014, $53,1368-1372$.

[115] C.-M. Lee, C.-H. Chen, F.-X. Liao, C.-H. Hu, G.-H. Lee, J. Am. Chem. Soc. 2010, 132, 92569258.

[116] M. I. Lipschutz, X. Yang, R. Chatterjee, T. D. Tilley, J. Am. Chem. Soc. 2013, 135, 15298- 
15301.

[117] V. M. Iluc, G. L. Hillhouse, J. Am. Chem. Soc. 2014, 136, 6479-88.

[118] B. Zheng, F. Tang, J. Luo, J. W. Schultz, N. P. Rath, L. M. Mirica, J. Am. Chem. Soc. 2014, 136, 6499-6504.

[119] F. De Rienzo, R. R. Gabdoulline, M. C. Menziani, R. C. Wade, Protein Sci. 2000, 9, 14391454.

[120] K. E. van Holde, K. I. Miller, H. Decker, J. Biol. Chem. 2001, 276, 15563-15566.

[121] E. A. Lewis, W. B. Tolman, Chem. Rev. 2004, 104, 1047-1076.

[122] R. H. Holm, P. Kennepohl, E. I. Solomon, Chem. Rev. 1996, 96, 2239-2314.

[123] S. T. Prigge, B. A. Eipper, R. E. Mains, L. M. Amzel, Science 2004, 304, 864-867.

[124] J. A. Halfen, S. Mahapatra, E. C. Wilkinson, S. Kaderli, V. G. Young, L. Que, A. D. Zuberbuhler, W. B. Tolman, Science. 1996, 271, 1397-1400.

[125] C. Citek, B.-L. Lin, T. E. Phelps, E. C. Wasinger, T. D. P. Stack, J. Am. Chem. Soc. 2014, 136, 14405-14408.

[126] L. M. Mirica, X. Ottenwaelder, T. D. P. Stack, Chem. Rev. 2004, 104, 1013-1046.

[127] J. Reim, B. Krebs, Angew. Chem. Int. Ed. Engl. 1994, 33, 1969-1971.

[128] K. E. Dalle, T. Gruene, S. Dechert, S. Demeshko, F. Meyer, J. Am. Chem. Soc. 2014, 136, 7428-7434.

[129] R. L. Lieberman, A. C. Rosenzweig, Nature 2005, 434, 177-182.

[130] R. Balasubramanian, a. C. Rosenzweig, Acc Chem Res 2007, 40, 573-580.

[131] R. Balasubramanian, S. M. Smith, S. Rawat, L. A. Yatsunyk, T. L. Stemmler, A. C. Rosenzweig, Nature 2010, 465, 115-119.

[132] M. R. Halvagar, P. V Solntsev, H. Lim, B. Hedman, K. O. Hodgson, E. I. Solomon, C. J. Cramer, W. B. Tolman, J. Am. Chem. Soc. 2014, 136, 7269-7272.

[133] Y. Shiota, K. Yoshizawa, Inorg. Chem. 2009, 48, 838-845.

[134] C. Citek, J. B. Gary, E. C. Wasinger, T. D. P. Stack, J. Am. Chem. Soc. 2015, 137, 69916994.

[135] N. Yoshikai, E. Nakamura, Chem. Rev. 2012, 112, 2339-2372.

[136] L. L. Diaddario, W. R. Robinson, D. W. Margerum, Inorg. Chem. 1983, 22, 1021-1025.

[137] P. J. M. W. L. Birker, Inorg. Chem. 1977, 16, 2478-2482.

[138] Y. Wang, T. D. P. Stack, J. Am. Chem. Soc. 1996, 118, 13097-13098.

[139] M. Akbar Ali, P. V. Bernhardt, M. A. H. Brax, J. England, A. J. Farlow, G. R. Hanson, L. L. Yeng, A. H. Mirza, K. Wieghardt, Inorg. Chem. 2013, 52, 1650-1657.

[140] J. Hanss, H.-J. Krüger, Angew. Chem. Int. Ed. Engl. 1996, 35, 2827-2830.

[141] N. Desbenoit, E. Galardon, P. Roussel, I. Artaud, A. Tomas, J. Coord. Chem. 2009, 62, 2472-2479.

[142] R. J. Phipps, M. J. Gaunt, Science. 2009, 323, 1593-1597.

[143] W. You, M. K. Brown, J. Am. Chem. Soc. 2014, 136, 14730-14733.

[144] R. A. Altman, A. Shafir, A. Choi, P. A. Lichtor, S. L. Buchwald, J. Org. Chem. 2008, 73, $284-$ 286.

[145] F. Y. Kwong, S. L. Buchwald, Org. Lett. 2003, 5, 793-796.

[146] S. R. Dubbaka, M. Kienle, H. Mayr, P. Knochel, Angew. Chem. Int. Ed. 2007, 46, 90939096.

[147] M. A. Willert-Porada, D. J. Burton, N. C. Baenziger, J. Chem. Soc. Chem. Commun. 1989, 1633-1634.

[148] R. Santo, R. Miyamoto, R. Tanaka, T. Nishioka, K. Sato, K. Toyota, M. Obata, S. Yano, I. Kinoshita, A. Ichimura, et al., Angew. Chem. Int. Ed. 2006, 45, 7611-7614.

[149] H. Furuta, H. Maeda, A. Osuka, J. Am. Chem. Soc. 2000, 122, 803-807.

[150] X. Ribas, D. a Jackson, B. Donnadieu, J. Mahía, T. Parella, R. Xifra, B. Hedman, K. 0. Hodgson, A. Llobet, T. D. P. Stack, Angew. Chem. Int. Ed. 2002, 41, 2991-2994.

[151] G. Evano, N. Blanchard, M. Toumi, Chem. Rev. 2008, 108, 3054-3131.

[152] G. Lefèvre, G. Franc, A. Tlili, C. Adamo, M. Taillefer, I. Ciofini, A. Jutand, Organometallics 2012, 31, 7694-7707.

[153] A. Casitas, A. E. King, T. Parella, M. Costas, S. S. Stahl, X. Ribas, Chem. Sci. 2010, 1, 326330. 
[154] L. M. Huffman, S. S. Stahl, J. Am. Chem. Soc. 2008, 130, 9196-9197.

[155] W. A. Herrmann, Angew. Chem. Int. Ed. 2002, 41, 1290-1309.

[156] S. P. Nolan, Acc. Chem. Res. 2011, 44, 91-100.

[157] A. Kumar, P. Ghosh, Eur. J. Inorg. Chem. 2012, 3955-3969.

[158] M. Poyatos, J. A. Mata, E. Peris, Chem. Rev. 2009, 109, 3677-3707.

[159] G. C. Fortman, S. P. Nolan, Chem. Soc. Rev. 2011, 40, 5151-5169.

[160] H.-W. Wanzlick, H.-J. Schönherr, Angew. Chem. Int. Ed. Engl. 1968, 7, 141-142.

[161] K. Öfele, J. Organomet. Chem. 1968, 12, 42-43.

[162] A. J. Arduengo, R. L. Harlow, M. Kline, J. Am. Chem. Soc. 1991, 113, 361-363.

[163] Z. Lu, S. A. Cramer, D. M. Jenkins, Chem. Sci. 2012, 3, 3081-3087.

[164] B. Liu, Q. Xia, W. Chen, Angew. Chem. Int. Ed. Engl. 2009, 48, 5513-5516.

[165] F. E. Hahn, M. C. Jahnke, Angew. Chem. Int. Ed. 2008, 47, 3122-3172.

[166] H. Jacobsen, A. Correa, A. Poater, C. Costabile, L. Cavallo, Coord. Chem. Rev. 2009, 253, 687-703.

[167] R. H. Crabtree, J. Organomet. Chem. 2005, 690, 5451-5457.

[168] C. A. Tolman, Chem. Rev. 1977, 77, 313-348.

[169] Z. L. Niemeyer, A. Milo, D. P. Hickey, M. S. Sigman, Nat. Chem. 2016, 8, 610-617.

[170] A. A. Danopoulos, N. Tsoureas, J. A. Wright, M. E. Light, Organometallics 2004, 23, 166168.

[171] L. Mercs, G. Labat, A. Neels, A. Ehlers, M. Albrecht, Organometallics 2006, 25, 56485656.

[172] J. Wu, W. Dai, J. H. Farnaby, N. Hazari, J. J. Le Roy, V. Mereacre, M. Murugesu, A. K. Powell, M. K. Takase, Dalton Trans. 2013, 42, 7404-7413.

[173] S. Meyer, C. M. Orben, S. Demeshko, S. Dechert, F. Meyer, Organometallics 2011, 30, 6692-6702.

[174] H. V. R. Dias, W. Jin, Tetrahedron Lett. 1994, 35, 1365-1366.

[175] H. Nakai, Y. Tang, P. Gantzel, K. Meyer, Chem. Commun. 2003, 24-25.

[176] U. Kernbach, M. Ramm, P. Luger, W. P. Fehlhammer, Angew. Chem. Int. Ed. Engl. 1996, $35,310-312$.

[177] R. Fränkel, C. Birg, U. Kernbach, T. Habereder, H. Nöth, W. P. Fehlhammer, Angew. Chem. Int. Ed. 2001, 40, 1907-1910.

[178] X. Hu, I. Castro-Rodrigues, K. Meyer, J. Am. Chem. Soc. 2003, 125, 12237-12245.

[179] X. Hu, I. Castro-Rodriguez, K. Meyer, Organometallics 2003, 22, 3016-3018.

[180] Z. Shi, R. P. Thummel, J. Org. Chem. 1995, 60, 5935-5945.

[181] W. W. H. Wong, M. S. Vickers, A. R. Cowley, R. L. Paul, P. D. Beer, Org. Biomol. Chem. 2005, 3, 4201-4208.

[182] Y. Chun, N. Jiten Singh, I.-C. Hwang, J. Woo Lee, S. U. Yu, K. S. Kim, Nat. Commun. 2013, 4, 1797.

[183] K. Chellappan, N. J. Singh, I.-C. Hwang, J. W. Lee, K. S. Kim, Angew. Chem. Int. Ed. 2005, 44, 2899-2903.

[184] H. M. Bass, S. A. Cramer, J. L. Price, D. M. Jenkins, Organometallics 2010, 29, 3235-3238.

[185] H. M. Bass, S. A. Cramer, A. S. McCullough, K. J. Bernstein, C. R. Murdock, D. M. Jenkins, Organometallics 2013, 32, 2160-2167.

[186] S. Meyer, I. Klawitter, S. Demeshko, E. Bill, F. Meyer, Angew. Chemie 2013, 125, 935939.

[187] W. Nam, Acc. Chem. Res. 2007, 40, 522-531.

[188] S. Meyer, PhD Thesis, Bioinspired Organometallic Analogues of Iron-Sites in Metalloproteins, Georg-August University Göttingen, 2013.

[189] C. Kupper, A. Schober, S. Demeshko, M. Bergner, F. Meyer, Inorg. Chem. 2015, 54, 3096-3098.

[190] C. Kupper, J. A. Rees, S. Dechert, S. DeBeer, F. Meyer, J. Am. Chem. Soc. 2016, 138, 78887898.

[191] S. Meyer, O. Krahe, C. Kupper, I. Klawitter, S. Demeshko, E. Bill, F. Neese, F. Meyer, Inorg. Chem. 2015, 54, 9770-9776.

[192] I. Klawitter, Phd Thesis, Makrozyklische NHC-Liganden Zur Stabilisierung 
Hochvalenter Metallionen, Georg-August Universität Göttingen, 2015.

[193] S. A. Cramer, D. M. Jenkins, J. Am. Chem. Soc. 2011, 133, 19342-19345.

[194] S. A. Cramer, R. Hernández Sánchez, D. F. Brakhage, D. M. Jenkins, Chem. Commun. 2014, 50, 13967-13970.

[195] J. R. Winkler, H. B. Gray, 2011, pp. 17-28.

[196] M. R. Anneser, S. Haslinger, A. Pöthig, M. Cokoja, J.-M. Basset, F. E. Kühn, Inorg. Chem. 2015, 150406075245005.

[197] R. McKie, J. A. Murphy, S. R. Park, M. D. Spicer, S. Zhou, Angew. Chem. Int. Ed. 2007, 46, 6525-6528.

[198] C. Brückner, C. A. Barta, R. P. Briñas, J. A. Krause Bauer, Inorg. Chem. 2003, 42, 16731680.

[199] H. Furuta, T. Ogawa, Y. Uwatoko, K. Araki, Inorg. Chem. 1999, 38, 2676-2682.

[200] S. P. Ghosh, M. C. Ghosh, E. S. Gould, Inorganica Chim. Acta 1994, 225, 83-88.

[201] N. J. Findlay, S. R. Park, F. Schoenebeck, E. Cahard, S.-Z. Zhou, L. E. a Berlouis, M. D. Spicer, T. Tuttle, J. a Murphy, J. Am. Chem. Soc. 2010, 132, 15462-4.

[202] L. Yang, D. R. Powell, R. P. Houser, Dalton Trans. 2007, 955-964.

[203] M. H. Reineke, M. D. Sampson, A. L. Rheingold, C. P. Kubiak, Inorg. Chem. 2015, 54, 3211-3217.

[204] Y. Unger, A. Zeller, M. A. Taige, T. Strassner, Dalton Trans. 2009, 4786.

[205] W. A. Herrmann, J. Schwarz, M. G. Gardiner, M. Spiegler, J. Organomet. Chem. 1999, 575, 80-86.

[206] W. Adam, Y. Y. Chan, D. Cremer, J. Gauss, D. Scheutzow, M. Schindler, J. Org. Chem. 1987, 52, 2800-2803.

[207] S. Bang, Y.-M. Lee, S. Hong, K.-B. Cho, Y. Nishida, M. S. Seo, R. Sarangi, S. Fukuzumi, W. Nam, Nat. Chem. 2014, 6, 934-940.

[208] S. Hong, F. F. Pfaff, E. Kwon, Y. Wang, M.-S. Seo, E. Bill, K. Ray, W. Nam, Angew. Chem. Int. Ed. 2014, 53, 10403-10407.

[209] E. H. Appelman, J. G. Malm, J. Am. Chem. Soc. 1964, 86, 2297-2298.

[210] Y. Gloaguen, C. Rebreyend, M. Lutz, P. Kumar, M. Huber, J. I. van der Vlugt, S. Schneider, B. de Bruin, Angew. Chem. Int. Ed. 2014, 53, 6814-6818.

[211] Z. Shi, R. P. Thummel, Tetrahedron Lett. 1995, 36, 2741-2744.

[212] F. J. Klinke, A. Das, S. Demeshko, S. Dechert, F. Meyer, Inorg. Chem. 2014, 53, $2976-$ 2982.

[213] A. Das, F. J. Klinke, S. Demeshko, S. Meyer, S. Dechert, F. Meyer, Inorg. Chem. 2012, 51, 8141-8149.

[214] P. S. Walsh, D. A. Metzger, R. Higuchi, Biotechniques 1991, 10, 506-513.

[215] C. Gunanathan, D. Milstein, Chem. Rev. 2014, 114, 12024-12087.

[216] G. Van Koten, J. Organomet. Chem. 2013, 730, 156-164.

[217] G. Van Koten, D. Milstein, Eds. , Organometallic Pincer Chemistry, Springer Verlag, Heidelberg, 2013.

[218] C. J. Moulton, B. L. Shaw, J. Chem. Soc., Dalt. Trans. 1976, 1020-1024.

[219] C. Crocker, R. J. Errington, R. Markham, C. J. Moulton, K. J. Odell, B. L. Shaw, J. Am. Chem. Soc. 1980, 102, 4373-4379.

[220] G. van Koten, Pure Appl. Chem. 1989, 61, 1681-1694.

[221] K. J. Szabó, in Top. Organomet. Chem., 2013, pp. 203-241.

[222] M. Albrecht, M. M. Lindner, Dalton Trans. 2011, 40, 8733-8744.

[223] M. E. van der Boom, D. Milstein, Chem. Rev. 2003, 103, 1759-1792.

[224] E. Peris, R. H. Crabtree, Coord. Chem. Rev. 2004, 248, 2239-2246.

[225] D. Pugh, A. A. Danopoulos, Coord. Chem. Rev. 2007, 251, 610-641.

[226] M. Albrecht, G. van Koten, Angew. Chem. Int. Ed. 2001, 40, 3750-3781.

[227] M. Asay, D. Morales-Morales, Dalton Trans. 2015, 44, 17432-17447.

[228] H. Li, B. Zheng, K.-W. Huang, Coord. Chem. Rev. 2015, 293-294, 116-138.

[229] J. Zhang, G. Leitus, Y. Ben-David, D. Milstein, J. Am. Chem. Soc. 2005, 127, 10840-10841.

[230] M. D. Fryzuk, P. A. MacNeil, S. J. Rettig, A. S. Secco, J. Trotter, Organometallics 1982, 1, 918-930. 
[231] A. Y. Verat, H. Fan, M. Pink, Y.-S. Chen, K. G. Caulton, Chem. - A Eur. J. 2008, 14, 76807686.

[232] A. A. Danopoulos, A. R. Wills, P. G. Edwards, Polyhedron 1990, 9, 2413-2418.

[233] L.-C. Liang, Coord. Chem. Rev. 2006, 250, 1152-1177.

[234] S. Schneider, J. Meiners, B. Askevold, Eur. J. Inorg. Chem. 2012, 2012, 412-429.

[235] S. C. E. Stieber, C. Milsmann, J. M. Hoyt, Z. R. Turner, K. D. Finkelstein, K. Wieghardt, S. DeBeer, P. J. Chirik, Inorg. Chem. 2012, 51, 3770-85.

[236] O. Baldovino-Pantaleón, S. Hernández-Ortega, D. Morales-Morales, Adv. Synth. Catal. 2006, 348, 236-242.

[237] D. Sieh, M. Schlimm, L. Andernach, F. Angersbach, S. Nückel, J. Schöffel, N. Šušnjar, P. Burger, Eur. J. Inorg. Chem. 2012, 2012, 444-462.

[238] T. Zell, D. Milstein, Acc. Chem. Res. 2015, 48, 1979-1994.

[239] P. Bhattacharya, J. A. Krause, H. Guan, Organometallics 2011, 30, 4720-4729.

[240] A. Hayashi, M. Okazaki, F. Ozawa, R. Tanaka, Organometallics 2007, 26, 5246-5249.

[241] T. N. Lenton, D. G. VanderVelde, J. E. Bercaw, Organometallics 2012, 31, 7492-7499.

[242] M. Basauri-Molina, S. Hernández-Ortega, D. Morales-Morales, Eur. J. Inorg. Chem. 2014, $2014,4619-4625$.

[243] C. I. Lee, N. A. Hirscher, J. Zhou, N. Bhuvanesh, O. V. Ozerov, Organometallics 2015, 34, 3099-3102.

[244] J. Takaya, N. Kirai, N. Iwasawa, J. Am. Chem. Soc. 2011, 133, 12980-12983.

[245] K. Tanoue, M. Yamashita, Organometallics 2015, 34, 4011-4017.

[246] J. I. van der Vlugt, Angew. Chem. Int. Ed. 2010, 49, 252-255.

[247] J. I. Van Der Vlugt, J. N. H. Reek, Angew. Chem. Int. Ed. 2009, 48, 8832-8846.

[248] M. Rolff, F. Tuczek, Angew. Chem. Int. Ed. 2008, 47, 2344-2347.

[249] B. Burger, S. Demeshko, E. Bill, S. Dechert, F. Meyer, Angew. Chem. Int. Ed. 2012, 51, 10045-10049.

[250] J. Wimberg, S. Meyer, S. Dechert, F. Meyer, Organometallics 2012, 31, 5025-5033.

[251] A. C. Sander, S. Maji, L. Francàs, T. Böhnisch, S. Dechert, A. Llobet, F. Meyer, ChemSusChem 2015, 8, 1697-1702.

[252] S. Neudeck, S. Maji, I. López, S. Meyer, F. Meyer, A. Llobet, J. Am. Chem. Soc. 2014, 136, 24-27.

[253] V. Lyaskovskyy, B. de Bruin, ACS Catal. 2012, 2, 270-279.

[254] J. I. van der Vlugt, Eur. J. Inorg. Chem. 2012, 2012, 363-375.

[255] A. Whitty, Nat. Chem. Biol. 2008, 4, 435-439.

[256] L. Que, W. B. Tolman, Nature 2008, 455, 333-340.

[257] D. L. J. Broere, N. P. van Leest, B. de Bruin, M. A. Siegler, J. I. van der Vlugt, Inorg. Chem. 2016, 55, 8603-8611.

[258] D. L. J. Broere, D. K. Modder, E. Blokker, M. A. Siegler, J. I. van der Vlugt, Angew. Chem. Int. Ed. 2016, 55, 2406-2410.

[259] C. M. Fafard, D. Adhikari, B. M. Foxman, D. J. Mindiola, O. V. Ozerov, J. Am. Chem. Soc. 2007, 129, 10318-10319.

[260] S. Köcher, G. P. M. van Klink, G. van Koten, H. Lang, J. Organomet. Chem. 2003, 684, 230-234.

[261] C.-W. Chan, D. M. P. Mingos, A. J. P. White, D. J. Williams, Chem. Commun. 1996, 81-83.

[262] P. Steenwinkel, H. Kooijman, W. J. J. Smeets, A. L. Spek, D. M. Grove, G. van Koten, Organometallics 1998, 17, 5411-5426.

[263] T. Tsubomura, T. Tanihata, T. Yamakawa, R. Ohmi, T. Tamane, A. Higuchi, A. Katoh, K. Sakai, Organometallics 2001, 20, 3833-3835.

[264] D. E. Herbert, O. V. Ozerov, Organometallics 2011, 30, 6641-6654.

[265] S. Samanta, S. Demesko, S. Dechert, F. Meyer, Angew. Chem. Int. Ed. 2015, 54, 583-587.

[266] D. Wang, S. V. Lindeman, A. T. Fiedler, Inorg. Chem. 2015, 54, 8744-8754.

[267] A. C. Sander, A. Schober, S. Dechert, F. Meyer, Eur. J. Inorg. Chem. 2015, 2015, 43484353.

[268] S. Romain, F. Bozoglian, X. Sala, A. Llobet, J. Am. Chem. Soc. 2009, 131, 2768-2769.

[269] S. Maji, L. Vigara, F. Cottone, F. Bozoglian, J. Benet-Buchholz, A. Llobet, Angew. Chem. 
Int. Ed. 2012, 51, 5967-5970.

[270] Y. Lee, F. T. Sloane, G. Blondin, K. a Abboud, R. García-Serres, L. J. Murray, Angew. Chem. Int. Ed. 2015, 54, 1499-1503.

[271] D. M. Ermert, I. Ghiviriga, V. J. Catalano, J. Shearer, L. J. Murray, Angew. Chem. Int. Ed. 2015, 54, 7047-7050.

[272] R. J. Eisenhart, P. A. Rudd, N. Planas, D. W. Boyce, R. K. Carlson, W. B. Tolman, E. Bill, L. Gagliardi, C. C. Lu, Inorg. Chem. 2015, 54, 7579-7592.

[273] L. J. Clouston, V. Bernales, R. K. Carlson, L. Gagliardi, C. C. Lu, Inorg. Chem. 2015, 54, 9263-9270.

[274] T. Zell, R. Langer, M. A. Iron, L. Konstantinovski, L. J. W. Shimon, Y. Diskin-Posner, G. Leitus, E. Balaraman, Y. Ben-David, D. Milstein, Inorg. Chem. 2013, 52, 9636-9649.

[275] M. Vogt, O. Rivada-Wheelaghan, M. A. Iron, G. Leitus, Y. Diskin-Posner, L. J. W. Shimon, Y. Ben-David, D. Milstein, Organometallics 2013, 32, 300-308.

[276] J. Zhang, G. Leitus, Y. Ben-David, D. Milstein, Angew. Chem. Int. Ed. 2006, 45, 11131115.

[277] L.-P. He, T. Chen, D. Gong, Z. Lai, K.-W. Huang, Organometallics 2012, 31, 5208-5211.

[278] T. Chen, L.-P. He, D. Gong, L. Yang, X. Miao, J. Eppinger, K.-W. Huang, Tetrahedron Lett. 2012, 53, 4409-4412.

[279] L.-P. He, T. Chen, D.-X. Xue, M. Eddaoudi, K.-W. Huang, J. Organomet. Chem. 2012, 700, 202-206.

[280] C. Gunanathan, B. Gnanaprakasam, M. A. Iron, L. J. W. Shimon, D. Milstein, J. Am. Chem. Soc. 2010, 132, 14763-14765.

[281] C. Gunanathan, D. Milstein, Angew. Chem. Int. Ed. 2008, 47, 8661-8664.

[282] C. Gunanathan, L. J. W. Shimon, D. Milstein, J. Am. Chem. Soc. 2009, 131, 3146-3147.

[283] E. A. Bielinski, P. O. Lagaditis, Y. Zhang, B. Q. Mercado, C. Würtele, W. H. Bernskoetter, N. Hazari, S. Schneider, J. Am. Chem. Soc. 2014, 136, 10234-10237.

[284] A. N. Marziale, A. Friedrich, I. Klopsch, M. Drees, V. R. Celinski, J. Schmedt auf der Günne, S. Schneider, J. Am. Chem. Soc. 2013, 135, 13342-13355.

[285] F. S. Schendzielorz, M. Finger, C. Volkmann, C. Würtele, S. Schneider, Angew. Chem. Int. Ed. 2016, 55, 11417-11420.

[286] M. Albrecht, M. M. Lindner, Dalton Trans. 2011, 40, 8733.

[287] Y. Chang, K. Takeuchi, M. Wakioka, F. Ozawa, Organometallics 2015, 34, 1957-1962.

[288] H. Grützmacher, Angew. Chem. Int. Ed. 2008, 47, 1814-1818.

[289] V. T. Annibale, D. Song, RSC Adv. 2013, 3, 11432-11449.

[290] C. Gunanathan, D. Milstein, Acc. Chem. Res. 2011, 44, 588-602.

[291] M. A. Iron, E. Ben-Ari, R. Cohen, D. Milstein, Dalton Trans. 2009, 9433-9439.

[292] Y. Chang, Y. Nakajima, H. Tanaka, K. Yoshizawa, F. Ozawa, 2013, 9, 1-4.

[293] E. Khaskin, M. A. Iron, L. J. W. Shimon, J. Zhang, D. Milstein, J. Am. Chem. Soc. 2010, 132, 8542-8543.

[294] S. W. Kohl, L. Weiner, L. Schwartsburd, L. Konstantinovski, L. J. W. Shimon, Y. BenDavid, M. A. Iron, D. Milstein, Science. 2009, 324, 74-77.

[295] L. Schwartsburd, M. a. Iron, L. Konstantinovski, E. Ben-Ari, D. Milstein, Organometallics 2011, 30, 2721-2729.

[296] S. M. Kloek, D. M. Heinekey, K. I. Goldberg, Angew. Chem. Int. Ed. 2007, 46, 4736-4738.

[297] S. K. Hanson, D. M. Heinekey, K. I. Goldberg, Organometallics 2008, 27, 1454-1463.

[298] M. Feller, Y. Diskin-Posner, L. J. W. Shimon, E. Ben-Ari, D. Milstein, Organometallics 2012, 31, 4083-4101.

[299] Y. Gloaguen, C. Rebreyend, M. Lutz, P. Kumar, M. Huber, J. I. van der Vlugt, S. Schneider, B. de Bruin, Angew. Chem. Int. Ed. 2014, 53, 6814-6818.

[300] E. Fogler, I. Efremenko, M. Gargir, G. Leitus, Y. Diskin-Posner, Y. Ben-David, J. M. L. Martin, D. Milstein, Inorg. Chem. 2015, 54, 2253-2263.

[301] S. P. Semproni, C. C. Hojilla Atienza, P. J. Chirik, Chem. Sci. 2014, 5, 1956-1960.

[302] C. Bianchini, A. Meli, M. Peruzzini, F. Vizza, P. Frediani, Organometallics 1990, 9, 11461155.

[303] C. Gunanathan, D. Milstein, Science. 2013, 341, 249. 
[304] S. Werkmeister, J. Neumann, K. Junge, M. Beller, Chem. - A Eur. J. 2015, 21, 1222612250.

[305] D. Milstein, Top. Catal. 2010, 53, 915-923.

[306] Y. Li, P. Sponholz, M. Nielsen, H. Junge, M. Beller, ChemSusChem 2015, 8, 804-808.

[307] P. Sponholz, D. Mellmann, C. Cordes, P. G. Alsabeh, B. Li, Y. Li, M. Nielsen, H. Junge, P. Dixneuf, M. Beller, ChemSusChem 2014, 7, 2419-2422.

[308] M. Peña-López, H. Neumann, M. Beller, ChemCatChem 2015, 7, 865-871.

[309] J. Singleton, Tetrahedron 2003, 59, 1837-1857.

[310] N. Selander, K. J. Szabó, Chem. Rev. 2011, 111, 2048-2076.

[311] F. E. Hahn, M. C. Jahnke, T. Pape, Organometallics 2007, 26, 150-154.

[312] S. Gu, W. Chen, Organometallics 2009, 28, 909-914.

[313] M. Ohff, A. Ohff, M. E. van der Boom, D. Milstein, J. Am. Chem. Soc. 1997, 119, 1168711688.

[314] O. Vechorkin, V. Proust, X. Hu, J. Am. Chem. Soc. 2009, 131, 9756-9766.

[315] S. D. Timpa, C. M. Fafard, D. E. Herbert, O. V Ozerov, Dalton Trans. 2011, 40, 54265429.

[316] C. J. Pell, O. V Ozerov, ACS Catal. 2014, 4, 3470-3480.

[317] M. Daniels, R. U. Kirss, J. Organomet. Chem. 2007, 692, 1716-1725.

[318] C. Yang, S. P. Nolan, J. Org. Chem. 2002, 67, 591-593.

[319] W. Weng, C. Guo, R. Celenligil-Cetin, B. M. Foxman, O. V Ozerov, Chem. Commun. 2006, 197-199.

[320] X. Chen, P. Xue, H. H. Y. Sung, I. D. Williams, M. Peruzzini, C. Bianchini, G. Jia, Organometallics 2005, 24, 4330-4332.

[321] C. Forsyth, W. Kerr, L. Paterson, Synlett 2013, 24, 587-590.

[322] Y. Gao, R. J. Puddephatt, Inorganica Chim. Acta 2003, 350, 101-106.

[323] K. Mochizuki, K. Sakai, T. Kochi, F. Kakiuchi, Synthesis 2013, 45, 2088-2092.

[324] O. Rivada-Wheelaghan, S. Chakraborty, L. J. W. Shimon, Y. Ben-David, D. Milstein, Angew. Chem. Int. Ed. 2016, 55, 6942-6945.

[325] T. Katagiri, H. Tsurugi, T. Satoh, M. Miura, Chem. Commun. 2008, 3405-3407.

[326] H. D. Xu, R. W. Zhang, X. Li, S. Huang, W. Tang, W. H. Hu, Org. Lett. 2013, 15, 840-843.

[327] M. Beller, J. Seayad, A. Tillack, H. Jiao, Angew. Chem. Int. Ed. 2004, 43, 3368-3398.

[328] T. E. Müller, K. C. Hultzsch, M. Yus, F. Foubelo, M. Tada, Chem. Rev. 2008, 108, 37953892.

[329] L. Zhang, Z. Zuo, X. Wan, Z. Huang, J. Am. Chem. Soc. 2014, 136, 15501-15504.

[330] C. Gunanathan, M. Hölscher, F. Pan, W. Leitner, J. Am. Chem. Soc. 2012, 134, 1434914352.

[331] C. Chen, M. B. Hecht, A. Kavara, W. W. Brennessel, B. Q. Mercado, D. J. Weix, P. L. Holland, J. Am. Chem. Soc. 2015, 137, 13244-13247.

[332] S. Chakraborty, J. A. Krause, H. Guan, Organometallics 2009, 28, 582-586.

[333] K. Kamata, A. Suzuki, Y. Nakai, H. Nakazawa, Organometallics 2012, 31, 3825-3828.

[334] D. Peng, Y. Zhang, X. Du, L. Zhang, X. Leng, M. D. Walter, Z. Huang, J. Am. Chem. Soc. 2013, 135, 19154-19166.

[335] M. D. Greenhalgh, D. J. Frank, S. P. Thomas, Adv. Synth. Catal. 2014, 356, 584-590.

[336] I. Buslov, J. Becouse, S. Mazza, M. Montandon-Clerc, X. Hu, Angew. Chem. Int. Ed. 2015, 54, 14523-14526.

[337] B. L. Tran, M. Pink, D. J. Mindiola, Organometallics 2009, 28, 2234-2243.

[338] R. Noyori, S. Hashiguchi, Acc. Chem. Res. 1997, 30, 97-102.

[339] S. E. Clapham, A. Hadzovic, R. H. Morris, Coord. Chem. Rev. 2004, 248, 2201-2237.

[340] G. Wienhöfer, F. A. Westerhaus, R. V Jagadeesh, K. Junge, H. Junge, M. Beller, Chem. Commun. 2012, 48, 4827-4829.

[341] E. Alberico, P. Sponholz, C. Cordes, M. Nielsen, H.-J. Drexler, W. Baumann, H. Junge, M. Beller, Angew. Chem. Int. Ed. 2013, 52, 14162-14166.

[342] G. Zhang, S. K. Hanson, Chem. Commun. 2013, 49, 10151.

[343] J. Zhang, G. Leitus, Y. Ben-David, D. Milstein, Angew. Chem. Int. Ed. 2006, 45, 11131115. 
[344] A. Mukherjee, D. Srimani, S. Chakraborty, Y. Ben-David, D. Milstein, J. Am. Chem. Soc. 2015, 137, 8888-8891.

[345] Y. Sun, C. Koehler, R. Tan, V. T. Annibale, D. Song, Chem. Commun. 2011, 47, 8349.

[346] J. A. Osborn, F. H. Jardine, J. F. Young, G. Wilkinson, J. Chem. Soc. A Inorganic, Phys. Theor. 1966, 1711-1732.

[347] J. F. Young, J. A. Osborn, F. H. Jardine, G. Wilkinson, Chem. Commun. 1965, 131-132.

[348] J. Luo, A. G. Oliver, J. Scott McIndoe, Dalton Trans. 2013, 42, 11312-11318.

[349] J. Halpern, Inorganica Chim. Acta 1981, 50, 11-19.

[350] C. Daniel, N. Koga, J. Han, X. Y. Fu, K. Morokuma, J. Am. Chem. Soc. 1988, 110, 37733787.

[351] S. B. Duckett, C. L. Newell, R. Eisenberg, J. Am. Chem. Soc. 1994, 116, 10548-10556.

[352] C. A. Tolman, P. Z. Meakin, D. I. Lindner, J. P. Jesson, J. Am. Chem. Soc. 1974, 96, 27622774.

[353] J. E. Perea-Buceta, I. Fernández, S. Heikkinen, K. Axenov, A. W. T. King, T. Niemi, M. Nieger, M. Leskelä, T. Repo, Angew. Chem. Int. Ed. 2015, 54, 14321-14325.

[354] J. Halpern, Science. 1982, 217, 401-407.

[355] W. Wang, Y. Himeda, J. T. Muckerman, G. F. Manbeck, E. Fujita, Chem. Rev. 2015, 115, 12936-12973.

[356] P. G. Jessop, T. Ikariya, R. Noyori, Chem. Rev. 1995, 95, 259-272.

[357] Y.-N. Li, R. Ma, L. He, Z.-F. Diao, Catal. Sci. Technol. 2014, 4, 1498-1512.

[358] G. A. Olah, G. K. S. Prakash, A. Goeppert, J. Am. Chem. Soc. 2011, 133, 12881-12898.

[359] I. Omae, Coord. Chem. Rev. 2012, 256, 1384-1405.

[360] H. Arakawa, M. Aresta, J. N. Armor, M. A. Barteau, E. J. Beckman, A. T. Bell, J. E. Bercaw, C. Creutz, E. Dinjus, D. A. Dixon, et al., Chem. Rev. 2001, 101, 953-996.

[361] A. M. Appel, J. E. Bercaw, A. B. Bocarsly, H. Dobbek, D. L. DuBois, M. Dupuis, J. G. Ferry, E. Fujita, R. Hille, P. J. A. Kenis, et al., Chem. Rev. 2013, 113, 6621-6658.

[362] K. M. K. Yu, I. Curcic, J. Gabriel, S. C. E. Tsang, ChemSusChem 2008, 1, 893-899.

[363] M. Aresta, A. Dibenedetto, A. Angelini, Chem. Rev. 2014, 114, 1709-1742.

[364] P. Markewitz, W. Kuckshinrichs, W. Leitner, J. Linssen, P. Zapp, R. Bongartz, A. Schreiber, T. E. Müller, Energy Environ. Sci. 2012, 5, 7281-7305.

[365] M. Peters, B. Köhler, W. Kuckshinrichs, W. Leitner, P. Markewitz, T. E. Müller, ChemSusChem 2011, 4, 1216-1240.

[366] E. A. Quadrelli, G. Centi, J.-L. Duplan, S. Perathoner, ChemSusChem 2011, 4, 1194-1215.

[367] C. Das Neves Gomes, O. Jacquet, C. Villiers, P. Thuéry, M. Ephritikhine, T. Cantat, Angew. Chem. Int. Ed. 2012, 51, 187-190.

[368] M. Aresta, A. Dibenedetto, Dalton Trans. 2007, 2975-2992.

[369] X. Yu, P. G. Pickup, J. Power Sources 2008, 182, 124-132.

[370] D. J. Darensbourg, Chem. Rev. 2007, 107, 2388-2410.

[371] D. B. Dell'Amico, F. Calderazzo, L. Labella, F. Marchetti, G. Pampaloni, Chem. Rev. 2003, 103, 3857-3898.

[372] M. Beley, J. Collin, R. Ruppert, J. Sauvage, J. Chem. Soc. Chem. Commun. 1984, 2, 13151316.

[373] D. C. Lacy, C. C. L. Mccrory, J. C. Peters, Inorg. Chem. 2014, 53, 4980-4989.

[374] J. D. Froehlich, C. P. Kubiak, Inorg. Chem. 2012, 51, 3932-3934.

[375] E. E. Benson, C. P. Kubiak, A. J. Sathrum, J. M. Smieja, Chem. Soc. Rev. 2009, 38, 89-99.

[376] J. Schneider, H. Jia, J. T. Muckerman, E. Fujita, Chem. Soc. Rev. 2012, 41, 2036-2051.

[377] W. Leitner, E. Dinjus, F. Gaßner, J. Organomet. Chem. 1994, 475, 257-266.

[378] S. Moret, P. J. Dyson, G. Laurenczy, Nat. Commun. 2014, 5, DOI 10.1038/ncomms5017.

[379] K. Rohmann, J. Kothe, M. W. Haenel, U. Englert, M. Hölscher, W. Leitner, Angew. Chem. Int. Ed. 2016, 55, 8966-8969.

[380] Y. Inoue, H. Izumida, Y. Sasaki, H. Hashimoto, Chem. Lett. 1976, 5, 863-864.

[381] N. N. Ezhova, N. V. Kolesnichenko, A. V. Bulygin, E. V. Slivinskii, S. Han, Russ. Chem. Bull. 2002, 51, 2165-2169.

[382] F. Gassner, W. Leitner, J. Chem. Soc. Chem. Commun. 1993, 1465-1466.

[383] F. Hutschka, A. Dedieu, M. Eichberger, R. Fornika, W. Leitner, J. Am. Chem. Soc. 1997, 
$119,4432-4443$.

[384] T. Burgemeister, F. Kastner, W. Leitner, Angew. Chem. Int. Ed. Engl. 1993, 32, 739-741.

[385] K. Tominaga, Y. Sasaki, M. Kawai, T. Watanabe, M. Saito, J. Chem. Soc. Chem. Commun. 1993, 629-631.

[386] C. Federsel, R. Jackstell, A. Boddien, G. Laurenczy, M. Beller, ChemSusChem 2010, 3, 1048-1050.

[387] C. Federsel, C. Ziebart, R. Jackstell, W. Baumann, M. Beller, Chem. - A Eur. J. 2012, 18, 72-75.

[388] C. Ziebart, C. Federsel, P. Anbarasan, R. Jackstell, W. Baumann, A. Spannenberg, M. Beller, J. Am. Chem. Soc. 2012, 134, 20701-20704.

[389] M. S. Jeletic, M. T. Mock, A. M. Appel, J. C. Linehan, J. Am. Chem. Soc. 2013, 135, 1153311536.

[390] Y. Zhang, A. D. MacIntosh, J. L. Wong, E. A. Bielinski, P. G. Williard, B. Q. Mercado, N. Hazari, W. H. Bernskoetter, Chem. Sci. 2015, 6, 4291-4299.

[391] P. G. Jessop, T. Ikariya, R. Noyori, Nature 1994, 368, 231-233.

[392] P. Munshi, A. D. Main, J. C. Linehan, C.-C. Tai, P. G. Jessop, J. Am. Chem. Soc. 2002, 124, 7963-7971.

[393] R. Tanaka, M. Yamashita, K. Nozaki, J. Am. Chem. Soc. 2009, 131, 14168-14169.

[394] Y. Himeda, S. Miyazawa, T. Hirose, ChemSusChem 2011, 4, 487-493.

[395] S. Wesselbaum, T. vom Stein, J. Klankermayer, W. Leitner, Angew. Chem. Int. Ed. 2012, $51,7499-7502$.

[396] Z. Han, L. Rong, J. Wu, L. Zhang, Z. Wang, K. Ding, Angew. Chem. Int. Ed. 2012, 51, 13041-13045.

[397] R. Tanaka, M. Yamashita, L. W. Chung, K. Morokuma, K. Nozaki, Organometallics 2011, $30,6742-6750$.

[398] J. F. Hull, Y. Himeda, W.-H. Wang, B. Hashiguchi, R. Periana, D. J. Szalda, J. T. Muckerman, E. Fujita, Nat. Chem. 2012, 4, 383-388.

[399] Y. M. Badiei, W.-H. Wang, J. F. Hull, D. J. Szalda, J. T. Muckerman, Y. Himeda, E. Fujita, Inorg. Chem. 2013, 52, 12576-12586.

[400] W.-H. Wang, J. T. Muckerman, E. Fujita, Y. Himeda, New J. Chem. 2013, 37, 1860.

[401] E. Balaraman, C. Gunanathan, J. Zhang, L. J. W. Shimon, D. Milstein, Nat. Chem. 2011, 3, 609-614.

[402] T. Zweifel, J.-V. Naubron, H. Grützmacher, Angew. Chem. Int. Ed. 2009, 48, 559-563.

[403] R. E. Rodríguez-Lugo, M. Trincado, M. Vogt, F. Tewes, G. Santiso-Quinones, H. Grützmacher, Nat. Chem. 2013, 5, 342-347.

[404] C. Gunanathan, Y. Ben-David, D. Milstein, Science. 2007, 317, 790-792.

[405] J. Zhang, E. Balaraman, G. Leitus, D. Milstein, Organometallics 2011, 30, 5716-5724.

[406] B. B. Corson, E. Depp, G. Black, J. Org. Chem. 1949, 14, 14-21.

[407] J. Pons, X. López, E. Benet, J. Casabó, F. Teixidor, F. J. Sánchez, Polyhedron 1990, 9 , 2839-2845.

[408] W. V. Dahlhoff, S. M. Nelson, J. Chem. Soc. A Inorganic, Phys. Theor. 1971, 2184-2190.

[409] E. Kossoy, M. a. Iron, B. Rybtchinski, Y. Ben-David, L. J. W. Shimon, L. Konstantinovski, J. M. L. Martin, D. Milstein, Chem. - A Eur. J. 2005, 11, 2319-2326.

[410] M. F. Semmelhack, A. Chlenov, D. M. Ho, J. Am. Chem. Soc. 2005, 127, 7759-7773.

[411] C. a Wheaton, P. G. Hayes, Chem. Commun. 2010, 46, 8404-8406.

[412] C. A. Wheaton, P. G. Hayes, Dalton Trans. 2010, 39, 3861-3869.

[413] C. A. Wheaton, P. G. Hayes, Catal. Sci. Technol. 2012, 2, 125-138.

[414] A. W. Addison, T. N. Rao, J. Reedijk, J. van Rijn, G. C. Verschoor, J. Chem. Soc., Dalt. Trans. 1984, 1349-1356.

[415] D. J. Darensbourg, M. S. Zimmer, P. Rainey, D. L. Larkins, Inorg. Chem. 1998, 37, 28522853.

[416] C. A. Wheaton, P. G. Hayes, Chem. Commun. 2010, 46, 8404.

[417] C. K. Williams, L. E. Breyfogle, S. K. Choi, W. Nam, V. G. Young, M. a. Hillmyer, W. B. Tolman, J. Am. Chem. Soc. 2003, 125, 11350-11359.

[418] M. Honrado, A. Otero, J. Fernández-Baeza, L. F. Sánchez-Barba, A. Garcés, A. Lara- 
Sánchez, J. Martínez-Ferrer, S. Sobrino, A. M. Rodríguez, Organometallics 2015, 34, 3196-3208.

[419] S. Samanta, unpublished results 2014.

[420] K. Spannhoff, N. Kuhl, G. Kehr, R. Fröhlich, G. Erker, J. Am. Chem. Soc. 2009, 131, 1783617842.

[421] N. G. Connelly, W. E. Geiger, Chem. Rev. 1996, 96, 877-910.

[422] K. Ding, T. R. Dugan, W. W. Brennessel, E. Bill, P. L. Holland, Organometallics 2009, 28, 6650-6656.

[423] C. A. Ghilardi, C. Mealli, S. Midollini, A. Orlandini, Inorg. Chem. 1985, 24, 164-168.

[424] Y. Zhang, P. G. Williard, W. H. Bernskoetter, Organometallics 2016, 35, 860-865.

[425] K. Arashiba, K. Sasaki, S. Kuriyama, Y. Miyake, H. Nakanishi, Y. Nishibayashi, Organometallics 2012, 31, 2035-2041.

[426] E. Kinoshita, K. Arashiba, S. Kuriyama, Y. Miyake, R. Shimazaki, H. Nakanishi, Y. Nishibayashi, Organometallics 2012, 31, 8437-8443.

[427] Y. Nakajima, Y. Okamoto, Y.-H. Chang, F. Ozawa, Organometallics 2013, 32, 2918-2925.

[428] M. Feller, E. Ben-Ari, T. Gupta, L. J. W. Shimon, G. Leitus, Y. Diskin-Posner, L. Weiner, D. Milstein, Inorg. Chem. 2007, 46, 10479-10490.

[429] D. Hermann, M. Gandelman, H. Rozenberg, L. J. W. Shimon, D. Milstein, Organometallics 2002, 21, 812-818.

[430] G. J. H. Nes, A. Vos, Acta Crystallogr. Sect. B Struct. Crystallogr. Cryst. Chem. 1979, 35, 2593-2601.

[431] L. Helm, A. E. Merbach, Chem. Rev. 2005, 105, 1923-1960.

[432] H. Friebolin, Ein- Und Zweidimensionale NMR-Spektroskopie, Wiley-VCH, Weinheim, 2006.

[433] N. Planas, G. J. Christian, E. Mas-Marzá, X. Sala, X. Fontrodona, F. Maseras, A. Llobet, Chem. - A Eur. J. 2010, 16, 7965-7968.

[434] J. A. Labinger, Organometallics 2015, 34, 4784-4795.

[435] L. Vaska, J. W. DiLuzio, J. Am. Chem. Soc. 1961, 83, 2784-2785.

[436] L. Vaska, J. W. DiLuzio, J. Am. Chem. Soc. 1962, 84, 679-680.

[437] D. A. Smith, D. E. Herbert, J. R. Walensky, O. V. Ozerov, Organometallics 2013, 32, 2050 2058.

[438] M. M. T. Khan, E. R. Rao, M. R. H. Siddiqui, B. T. Khan, S. Begum, S. M. Ali, J. Reddy, J. Mol. Catal. 1988, 45, 35-50.

[439] M. Feller, Y. Diskin-Posner, G. Leitus, L. J. W. Shimon, D. Milstein, J. Am. Chem. Soc. 2013, 135, 11040-11047.

[440] A. B. Chaplin, A. S. Weller, Organometallics 2011, 30, 4466-4469.

[441] T. G. Schenck, C. R. C. Milne, J. F. Sawyer, B. Bosnich, Inorg. Chem. 1985, 24, 2338-2344.

[442] Y. Jiao, W. W. Brennessel, W. D. Jones, Organometallics 2015, 34, 1552-1566.

[443] A. Gondoh, T. Koike, M. Akita, Inorganica Chim. Acta 2011, 374, 489-498.

[444] S. Tanaka, M. Akita, Angew. Chem. Int. Ed. 2001, 40, 2865-2867.

[445] T. Yamaguchi, T. Koike, M. Akita, Organometallics 2010, 29, 6493-6502.

[446] T. Simler, L. Karmazin, C. Bailly, P. Braunstein, A. A. Danopoulos, Organometallics 2016 , $35,903-912$.

[447] J. I. van der Vlugt, E. A. Pidko, D. Vogt, M. Lutz, A. L. Spek, Inorg. Chem. 2009, 48, 75137515.

[448] R. Mei, L. Ackermann, Adv. Synth. Catal. 2016, 358, 2443-2448.

[449] D. J. Tindall, H. Krause, A. Fürstner, Adv. Synth. Catal. 2016, 358, 2398-2403.

[450] W.-J. Shi, H.-W. Zhao, Y. Wang, Z.-C. Cao, L.-S. Zhang, D.-G. Yu, Z.-J. Shi, Adv. Synth. Catal. 2016, 358, 2410-2416.

[451] A. Thevenon, J. A. Garden, A. J. P. White, C. K. Williams, Inorg. Chem. 2015, 54, $11906-$ 11915.

[452] F. Drouin, P. O. Oguadinma, T. J. J. Whitehorne, R. E. Prudhomme, F. Schaper, Organometallics 2010, 29, 2139-2147.

[453] S. K. Bose, A. Deißenberger, A. Eichhorn, P. G. Steel, Z. Lin, T. B. Marder, Angew. Chem. Int. Ed. 2015, 54, 11843-11847. 
[454] S. K. Bose, K. Fucke, L. Liu, P. G. Steel, T. B. Marder, Angew. Chem. Int. Ed. 2014, 53, 1799-1803.

[455] X.-F. Wu, H. Neumann, Adv. Synth. Catal. 2012, 354, 3141-3160.

[456] T. Tsuchimoto, H. Utsugi, T. Sugiura, S. Horio, Adv. Synth. Catal. 2015, 357, 77-82.

[457] P. A. Lummis, M. R. Momeni, M. W. Lui, R. McDonald, M. J. Ferguson, M. Miskolzie, A. Brown, E. Rivard, Angew. Chem. Int. Ed. 2014, 53, 9347-9351.

[458] M. A. Nesbit, D. L. M. Suess, J. C. Peters, Organometallics 2015, 34, 4741-4752.

[459] J. V. Obligacion, P. J. Chirik, J. Am. Chem. Soc. 2013, 135, 19107-19110.

[460] S. Rösler, M. Ertl, T. Irrgang, R. Kempe, Angew. Chem. Int. Ed. 2015, 54, 15046-15050.

[461] L. Zhang, Z. Zuo, X. Leng, Z. Huang, Angew. Chem. Int. Ed. 2014, 53, 2696-2700.

[462] Y. Kita, S. Hida, K. Higashihara, H. S. Jena, K. Higashida, K. Mashima, Angew. Chem. Int. Ed. 2016, 55, 8299-8303.

[463] C. Hansch, A. Leo, R. W. Taft, Chem. Rev. 1991, 91, 165-195.

[464] J. O. Schreck, J. Chem. Educ. 1971, 48, 103.

[465] M. Gómez-Gallego, M. A. Sierra, Chem. Rev. 2011, 111, 4857-4963.

[466] E. M. Simmons, J. F. Hartwig, Angew. Chem. Int. Ed. 2012, 51, 3066-3072.

[467] M. Ahlquist, M. Gustafsson, M. Karlsson, M. Thaning, O. Axelsson, O. F. Wendt, Inorganica Chim. Acta 2007, 360, 1621-1627.

[468] A. D. Getty, C. Tai, J. C. Linehan, P. G. Jessop, M. M. Olmstead, A. L. Rheingold, Organometallics 2009, 28, 5466-5477.

[469] A. M. Lilio, M. H. Reineke, C. E. Moore, A. L. Rheingold, M. K. Takase, C. P. Kubiak, J. Am. Chem. Soc. 2015, 150617142422006.

[470] C. W. Machan, S. A. Chabolla, J. Yin, M. K. Gilson, F. A. Tezcan, C. P. Kubiak, J. Am. Chem. Soc. 2014, 136, 14598-14607.

[471] K. Kobayashi, K. Tanaka, Inorg. Chem. 2015, 54, 5085-5095.

[472] K. Garg, Y. Matsubara, M. Z. Ertem, A. Lewandowska-Andralojc, S. Sato, D. J. Szalda, J. T. Muckerman, E. Fujita, Angew. Chem. Int. Ed. 2015, 54, 14128-14132.

[473] C. Costentin, M. Robert, J.-M. Savéant, Chem. Soc. Rev. 2013, 42, 2423-2436.

[474] M. L. Scheuermann, S. P. Semproni, I. Pappas, P. J. Chirik, Inorg. Chem. 2014, 53, $9463-$ 9465.

[475] M. A. McLoughlin, N. L. Keder, W. T. A. Harrison, R. J. Flesher, H. A. Mayer, W. C. Kaska, Inorg. Chem. 1999, 38, 3223-3227.

[476] K.-W. Huang, J. H. Han, C. B. Musgrave, E. Fujita, Organometallics 2007, 26, 508-513.

[477] M. Feller, U. Gellrich, A. Anaby, Y. Diskin-Posner, D. Milstein, J. Am. Chem. Soc. 2016, $138,6445-6454$.

[478] K.-N. T. Tseng, J. W. Kampf, N. K. Szymczak, J. Am. Chem. Soc. 2016, 138, 10378-10381.

[479] I. M. Riddlestone, N. A. Rajabi, J. P. Lowe, M. F. Mahon, S. A. Macgregor, M. K. Whittlesey, J. Am. Chem. Soc. 2016, 138, 11081-11084.

[480] G. R. Fulmer, A. J. M. Miller, N. H. Sherden, H. E. Gottlieb, A. Nudelman, B. M. Stoltz, J. E. Bercaw, K. I. Goldberg, Organometallics 2010, 29, 2176-2179.

[481] G. M. Sheldrick, Acta Crystallogr. Sect. A Found. Crystallogr. 2008, 64, 112-122.

[482] STOE \& CIE GmbH, X-RED 2002.

[483] J. Cookson, M. S. Vickers, R. L. Paul, A. R. Cowley, P. D. Beer, Inorganica Chim. Acta 2008, 361, 1689-1698.

[484] E. Lindner, G. von Au, H.-J. Eberle, Chem. Ber. 1981, 114, 810-813.

[485] C. W. Sauer, R. J. Bruni, J. Am. Chem. Soc. 1955, 77, 2559-2560.

[486] C. Citek, C. T. Lyons, E. C. Wasinger, T. D. P. Stack, Nat. Chem. 2012, 4, 317-322.

[487] E. Díez-Barra, A. de la Hoz, A. Sáchez-Migallón, J. Tejeda, Heterocycles 1992, 34, 13651373.

[488] J. Chou, J. Chyn, F. L. Urbach, D. F. Gervasio, Polyhedron 2000, 19, 2215-2223.

[489] Mestrelab Research, MestReNova v. 10.0.2-15465, Santiago d Compost. Spain 2015. 



\section{Structures of Ligand precursors, Ligands and Complexes}<smiles>CC(C)(C)OC(=O)N(CC(=O)O)CC(=O)O</smiles>

IV<smiles>Cc1cc(C)cc(NC(=O)CN(CC(=O)Nc2cc(C)cc(C)c2)C(=O)OC(C)(C)C)c1</smiles>

V<smiles>[Y]c1cc(C)cc(NC(=O)CNCCCC)c1</smiles>

VI<smiles>O=C(CN(CC(=O)N[Ga])Cc1cc(CN(CC(=O)N[Al])CC(=O)N[Al])n(C2CCCCO2)n1)N[Al]</smiles>

$\mathrm{H}_{5} \mathrm{~L}^{\mathrm{Me}}$ IX<smiles></smiles><smiles></smiles>

$\mathrm{R}=\mathrm{H} \quad \mathrm{H}_{4} \mathrm{~L}^{\mathbf{1}}\left(\mathrm{OTf}_{4} \quad \mathrm{XII}\right.$

$\mathrm{R}=\mathrm{H} \quad \mathrm{H}_{4} \mathrm{~L}^{3}\left(\mathbf{P F}_{6}\right)_{4} \quad$ XXII

$\mathrm{R}=\mathrm{Me} \quad \mathrm{H}_{4} \mathrm{~L}^{2}(\mathbf{O T f})_{4} \quad \mathbf{X X I}$

$\mathrm{R}=\mathrm{Me} \quad \mathrm{H}_{4} \mathrm{~L}^{\mathbf{4}}\left(\mathbf{P F}_{6}\right)_{4} \quad$ XXIII<smiles>Cc1cccc(C(=O)O)n1</smiles>

$\mathbf{X X X}$<smiles>COC(=O)c1cccc(C)n1</smiles>

XXXI<smiles>Cc1cccc(-c2cc(-c3cccc(Cc4ccccc4)n3)n[nH]2)n1</smiles>

$\mathrm{R}={ }^{t} \mathrm{Bu} \mathbf{X X X I V}$

$\mathrm{R}={ }^{i} \operatorname{Pr} \mathbf{X X X V}$

$\mathrm{R}=\mathrm{Ph} \quad \mathbf{X X X V I}$<smiles>CC(=O)c1cccc(C)n1</smiles>

XXXII<smiles>Cc1cccc(-c2cc(-c3cccc(C)n3)[nH]n2)n1</smiles>

XXXIII<smiles>[R7]Cc1cccc(-c2cc(-c3cccc(CP)n3)n[nH]2)n1</smiles>

$\mathrm{R}={ }^{t} \mathrm{Bu} \mathbf{H L}^{t \mathrm{Bu}} \mathbf{X X X V I I}$

$\mathrm{R}={ }^{i} \operatorname{Pr} \quad$ HL $^{i \text { Pr }}$ XXXVIII 
(OTf)

29

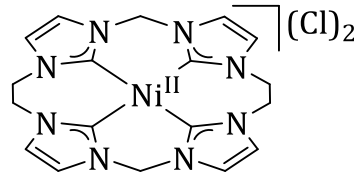

30

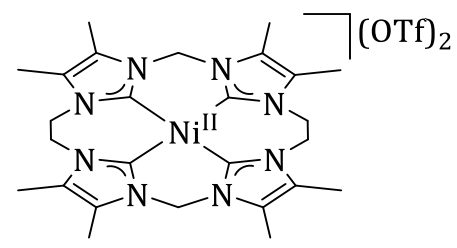

31

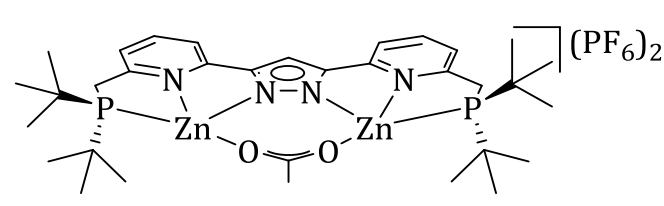

Zn ${ }^{t B u}-0 A c 48$

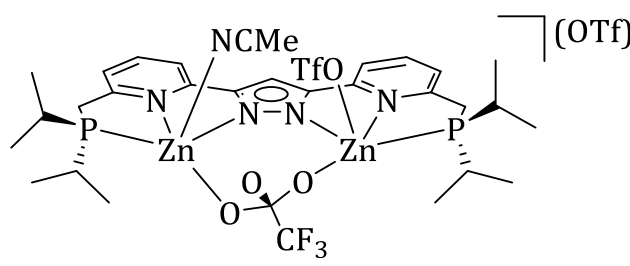

ZniPr-0Tf 50

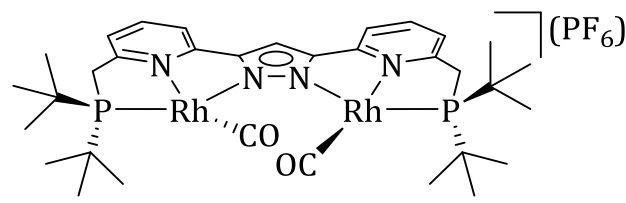

$\mathbf{R h}^{t \mathrm{Bu}}-\mathrm{CO} 55$

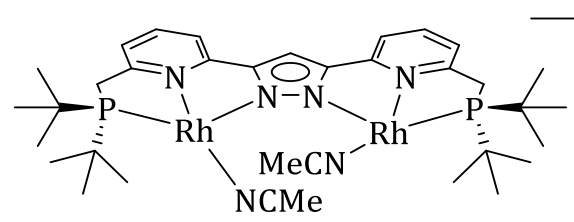

Rh $^{\text {tBu-MeCN }} 58$<smiles></smiles>

62

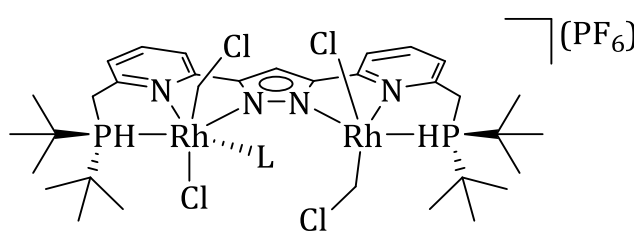

Rh $^{\text {tBu-DCM }} 67$

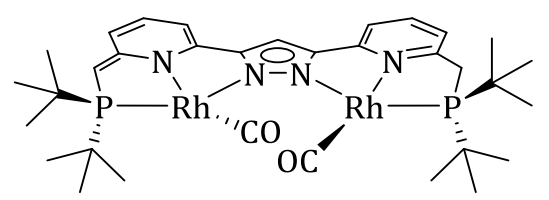

$\mathrm{Rh}^{t \mathrm{Bu}}{ }^{-\mathrm{CO}} 74$

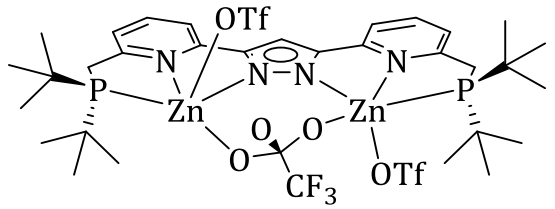

Zn $^{\text {tBu-OTf }} 49$

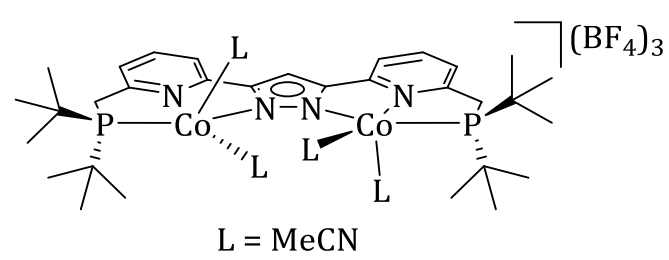

Cot ${ }^{\text {Bu }}-\mathrm{MeCN} 54$

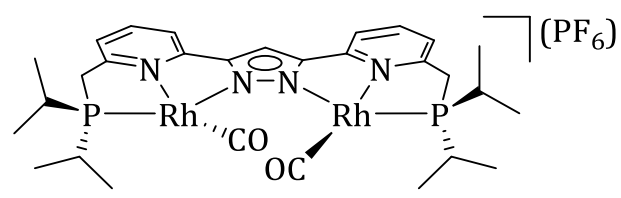

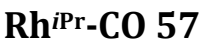

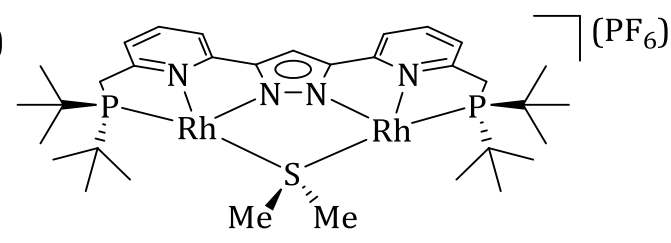

$\mathrm{Rh}^{t \mathrm{Bu}}-\mathrm{SMe}_{2} 61$

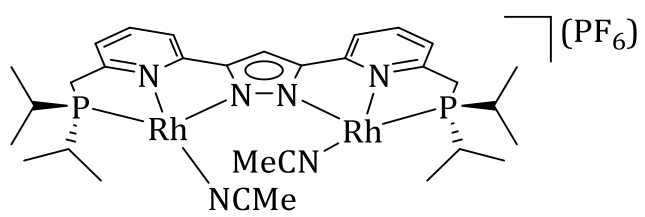

Rh $^{\text {iPr-MeCN } 65}$

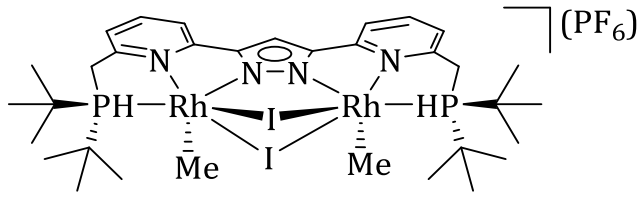

\section{Rh $^{\text {tBu-MeI }} 69$}

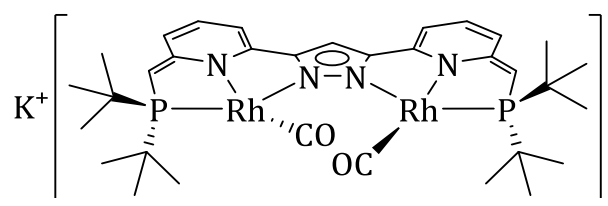

$\mathrm{Rh}^{t \mathrm{Bu} * *-\mathrm{CO}} 75$ 


\begin{tabular}{|c|c|}
\hline \multicolumn{2}{|c|}{ List of abbreviations } \\
\hline$\AA$ & Angström \\
\hline acac & acetylacetonato \\
\hline $\mathrm{ad}$ & adsorbed \\
\hline approx & approximately \\
\hline aq & aqueous \\
\hline $\operatorname{Ar}^{F}$ & $3,5-\mathrm{CF}_{3}-\mathrm{C}_{6} \mathrm{H}_{3}$ \\
\hline $\mathrm{Ar}$ & aryl \\
\hline atm & atmosphere \\
\hline Boc & tert-butyloxycarbonyl \\
\hline $\mathrm{Boc}_{2} \mathrm{O}$ & di-tert-butyldicarbonate \\
\hline bpy & 2,2'-bypridine \\
\hline cat & catalyst \\
\hline COSY & correlation spectroscopy \\
\hline $\mathrm{Cp}$ & cyclopentadienyl anion \\
\hline $\mathrm{Cp}^{*}$ & pentamethylcyclopentadienyl anion \\
\hline$d$ & distance, doublet (NMR), days (time) \\
\hline DBU & 1,8-diazabicyclo[5.4.0]undec-7-en \\
\hline DCC & dicyclohexylcarbodiimide \\
\hline DCM & dichloromethane \\
\hline DHA & dihydroanthracene \\
\hline DIBAl & di-iso-butylaluminiumhydride \\
\hline dme & dimethoxyethane \\
\hline DMFc & decamethylferrocene \\
\hline DMSO & dimethyl sulfoxide \\
\hline DOSY & diffusion ordered spectroscopy \\
\hline EA & elemental analysis \\
\hline EPR & electron paramagnetic resonance \\
\hline eq & equivalent \\
\hline ESI & electron spray ionisation \\
\hline EtCN & propionitrile \\
\hline EtOH & ethanol \\
\hline FA & formic acid \\
\hline $\mathrm{Fc}$ & ferrocene \\
\hline $\mathrm{h}$ & hour \\
\hline hept & heptet (NMR) \\
\hline HIPT & hexaisopropylterphenyl \\
\hline His & histidine \\
\hline hs & high spin \\
\hline
\end{tabular}




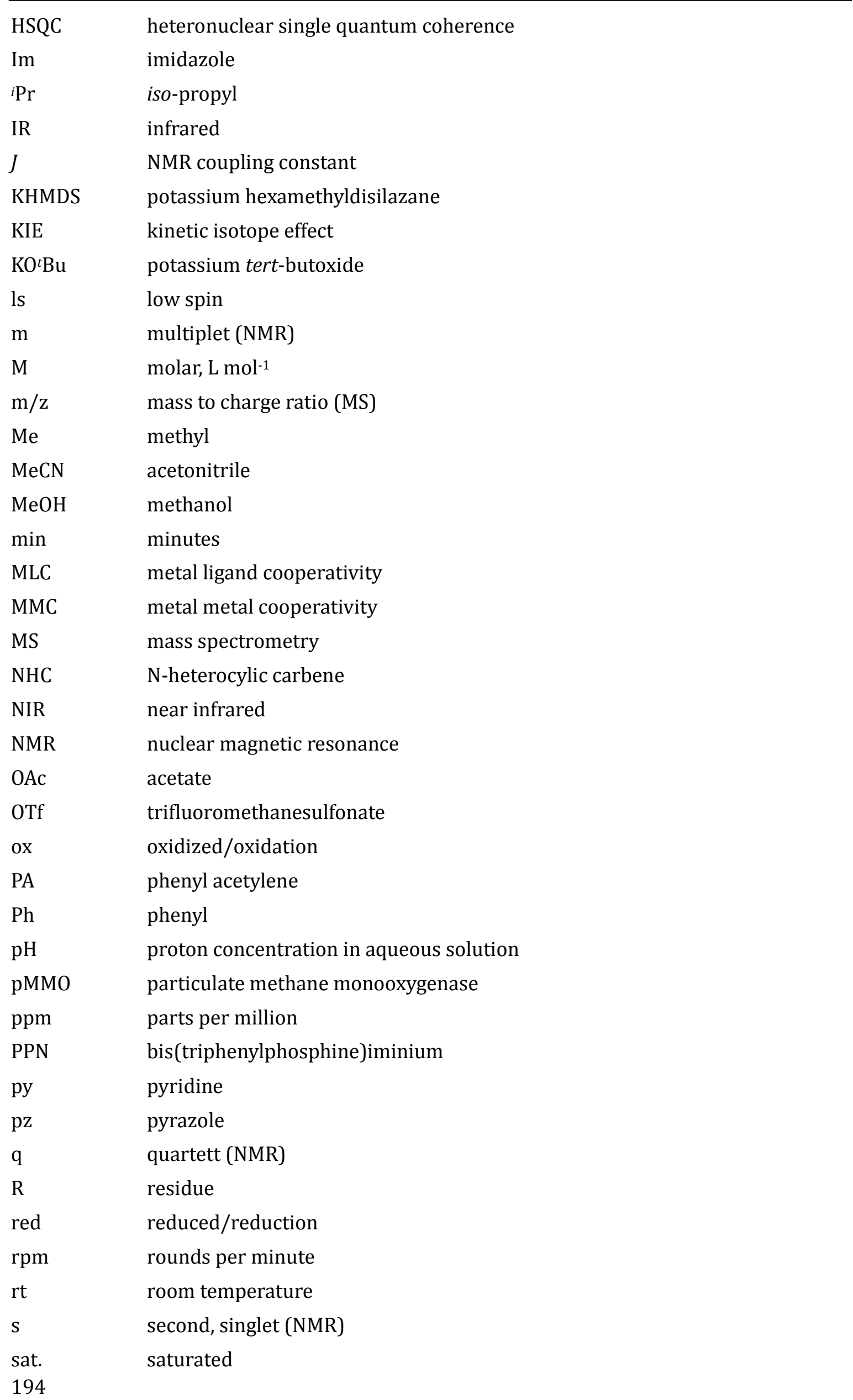




\begin{tabular}{ll}
\hline Sbr & broad singulet (NMR) \\
sPhIO & 2-(tert-butylsulfonyl)iodosobenzene \\
SQUID & superconducting quantum interference device \\
$\mathrm{t}$ & time, triplet (NMR) \\
$\mathrm{T}$ & temperature \\
${ }^{t} \mathrm{Bu}$ & tert-butyl \\
THF & tetrahydrofurane \\
thp & 2-tetrahydropyranyl \\
TMS & tetramethylsilane \\
TOF & turnover frequency \\
TON & turnover number \\
TRAN & tris-(2-amidoethyl)amine \\
Ts & tosyl \\
UV-vis & ultraviolet/visible \\
vs & versus \\
wt\% & weight percent \\
$Z$ & number of molecules per unit cell \\
$\delta$ & chemical shift \\
$\varepsilon$ & extinction coefficient \\
$v$ & wavelength \\
$\chi$ & susceptibility
\end{tabular}





\section{Acknowledgement}

First of all, I want to thank Prof. Dr. Franc Meyer for the supervision of this thesis, for his irrepressible optimism and scientific support and for providing an excellent place for research with numerous analytic methods. I thank Prof. Dr. Sven Schneider for his helpful discussions about the pincer topic and for overtaking the position of the second examiner.

I like to thank all colleagues who contributed with their expertise to this work. Dr. Sebastian Dechert, Dr. Nicole Kindermann and Anne Schober for collecting and resolving my crystallographic data as well as Dr. Claudia Stückl and Marie Bergner for EPR measurements. The NMR service department and especially Ralf Schöne for numerous measurements and technical support. The Central Analytics of the faculty of chemistry and the Analytic Laboratory of the Institute of Inorganic Chemistry for recording mass spectra and performing elemental analyses. Our technical support, Jörg Teichgräber for performing and helping with electrochemical measurements and Andreas Schwarz for providing the pyrazole building block and all other lab equipment.

Furthermore, I like to acknowledge all people from the mechanical, electrical and glass workshops for their fast and reliable work. Thanks to Petra Unger, Britta Müller and Dr. Claudia Stückl and the rest of the team for solving all administrative issues as well as to cleaners and caretakers.

For the great working atmosphere and a lot of fun in the lab I want to thank Dr. Markus Steinert and Eleonora Ferretti. Also my bachelor student Marcus Thater and research student Katharina Dabow I like to thank for their encouraged worked and discussions.

For many helpful (and also sometimes extraordinary) discussions about my topic I thank Oliver Mitevski, Dr. Markus Steinert, Stefan Resch, Alexander Brinkmeier, Anne Schober and Eleonora Ferretti.

Many thanks to Oliver Mitevski, Alexander Brinkmeier, Stefan Resch, Pierre Guorsot and Eleonora Ferretti for proof reading this thesis so attentively and quickly. In this context, I like to thank Oliver Mitevski and Anastasia Vogel for a relaxed, but nonetheless very constructive working atmosphere in our writing office down in the underground.

Many thanks to Dennis, Jojo, Julia, Jan, Olli, Anne and Rico for having a great time together in and around Göttingen. 
Finally, I want to thank my family: my parents, my sister and brother for the great time together at home and for their reliable backup and motivation. Janina for her love, her huge support, especially at the end of this work and her always open ear! And of course, Felix and Paul for making my life more vivid and worth living! 


\section{Scientific Contributions}

\section{Publication}

A. Gers-Barlag, S. Dechert, F. Meyer, "A Two-in-one Pincer Ligand and its Dinuclear Rhodium(I) complexes: Synthesis, Properties and First Catalytic Reactions”, manuscript in preparation.

\section{Posters at Conferences}

A. Gers-Barlag, S. Samanta, S. Dechert, F. Meyer, "Two-In-One Pincer Ligands and Their Transition Metal Complexes", 7. JungChemiker Forum, Göttingen, Germany, July 2015.

A. Gers-Barlag, S. Samanta, S. Dechert, F. Meyer, "Reactivity of Dinuclear Complexes of Two-InOne Pincer Ligands”, 18. Norddeutsches Doktoranden Kolloquium (18. NDDK), Goslar, Germany, September 2015.

A. Gers-Barlag, S. Dechert, F. Meyer, "Dinuclear Two-In-One Pincer Rh(I) Complexes for Catalytic Hydrogenation of Alkynes", 42 ${ }^{\text {th }}$ Internation Conference on Coordination Chemistry (ICCC 42), Brest, France, July 2016. 



\section{Curriculum Vitae}

\section{Personal Data}

\begin{tabular}{|c|c|}
\hline Name & Alexander Gers-Barlag \\
\hline Date of birth & 18th September 1987 \\
\hline Place of birth & Soest \\
\hline Nationality & German \\
\hline \multicolumn{2}{|l|}{ Academic Career } \\
\hline \multirow[t]{4}{*}{ since $11 / 2012$} & $\mathrm{PhD}$ candidate in the Department of Inorganic Chemistry, Georg-August \\
\hline & University, Göttingen \\
\hline & Advisor: Prof. Dr. Franc Meyer \\
\hline & $\begin{array}{l}\text { PhD thesis: "Two-in-one Pincer Type Ligands and Their Metal } \\
\text { Complexes for Catalysis" }\end{array}$ \\
\hline \multirow[t]{3}{*}{$04 / 2012-09 / 2012$} & $\begin{array}{l}\text { master thesis "Pyrazole-based tetraamido ligands and their } \\
\text { molybdenum complexes - possible scaffolds in } \mathrm{N}_{2} \text { activation" in the } \\
\text { Department of Inorganic Chemistry, Georg-August University, } \\
\text { Göttingen }\end{array}$ \\
\hline & Advisor: Prof. Dr. Franc Meyer \\
\hline & overall grade: 1.2 \\
\hline $10 / 2010-09 / 2012$ & graduate student \\
\hline \multirow[t]{5}{*}{$03 / 2010-07 / 2010$} & bachelor thesis "Palladiumkatalysierte Eintopfreaktionen zur Synthese \\
\hline & benzannelierter Heterocyclen" in the Department of Organic and \\
\hline & Biomolecular Chemistry, Georg-August University, Göttingen \\
\hline & Advisor: Prof. Dr. Lutz Ackermann \\
\hline & overall grade: 1.6 \\
\hline $10 / 2007-09 /$ & undergraduate student \\
\hline
\end{tabular}

\section{Pre-academic Education}

$06 / 2007$

1998-2007

1994-1998
Abitur (overall grade 1.2, major courses: chemistry and physics) Johannes-Brahms-Schule (Gymnasium), Pinneberg

Grundschule Kummerfeld 